\title{
Precision Measurements of Tau Lepton Decays
}

by

\section{Ian M. Nugent}

B.Sc., University of Victoria, 2002

M.Sc., University of Victoria, 2004

A Dissertation Submitted in Partial Fulfillment of the

Requirements for the Degree of

DOCTORATE OF PHILOSOPHY

in the Department of Physics and Astronomy

(C) Ian M. Nugent 2008

University of Victoria

All rights reserved. This Dissertation may not be reproduced in whole or in part by photocopy or other means, without the permission of the author. 


\section{Precision Measurements of Tau Lepton Decays}

by

Ian M. Nugent

B.Sc., University of Victoria, 2002

M.Sc., University of Victoria, 2004

\section{Supervisory Committee}

Dr. J. Michael Roney, Supervisor

Department of Physics and Astronomy, University of Victoria

Dr. Robert Kowalewski, Departmental Member

Department of Physics and Astronomy, University of Victoria

Dr. Dean Karlen, Departmental Member

Department of Physics and Astronomy, University of Victoria

Dr. Micaela Serra, Outside Member

Department of Computer Science, University of Victoria 


\title{
Supervisory Committee
}

\author{
Dr. J. Michael Roney, Supervisor \\ Department of Physics and Astronomy, University of Victoria \\ Dr. Robert Kowalewski, Departmental Member \\ Department of Physics and Astronomy, University of Victoria \\ Dr. Dean Karlen, Departmental Member \\ Department of Physics and Astronomy, University of Victoria \\ Dr. Micaela Serra, Outside Member \\ Department of Computer Science, University of Victoria
}

\begin{abstract}
Using data collected with the BABAR detector at the SLAC PEP-II electron-positron storage ring operating at a centre-of-mass energy near $10.58 \mathrm{GeV}$, the branching fractions $\mathcal{B}\left(\tau^{-} \rightarrow \pi^{-} \pi^{-} \pi^{+} \nu_{\tau}\right)=(8.83 \pm 0.01 \pm 0.13) \%, \mathcal{B}\left(\tau^{-} \rightarrow K^{-} \pi^{-} \pi^{+} \nu_{\tau}\right)=(0.273 \pm$ $0.002 \pm 0.009) \%, \mathcal{B}\left(\tau^{-} \rightarrow K^{-} \pi^{-} K^{+} \nu_{\tau}\right)=(0.1346 \pm 0.0010 \pm 0.0036) \%$, and $\mathcal{B}\left(\tau^{-} \rightarrow\right.$ $\left.K^{-} K^{-} K^{+} \nu_{\tau}\right)=(1.58 \pm 0.13 \pm 0.12) \times 10^{-5}$ are measured where the uncertainties are statistical and systematic, respectively. The invariant mass distribution for the $\tau^{-} \rightarrow \pi^{-} \pi^{-} \pi^{+} \nu_{\tau}, \tau^{-} \rightarrow K^{-} \pi^{-} \pi^{+} \nu_{\tau}, \tau^{-} \rightarrow K^{-} \pi^{-} K^{+} \nu_{\tau}$ and $\tau^{-} \rightarrow K^{-} K^{-} K^{+} \nu_{\tau}$ decays are unfolded to correct for detector effects. A measurement of $\mathcal{B}\left(\tau^{-} \rightarrow \phi \pi^{-} \nu_{\tau}\right)$ $=(3.42 \pm 0.55 \pm 0.25) \times 10^{-5}$, a measurement of $\mathcal{B}\left(\tau^{-} \rightarrow \phi K^{-} \nu_{\tau}\right)=(3.39 \pm 0.20 \pm 0.28) \times$ $10^{-5}$ and an upper limit on $\mathcal{B}\left(\tau^{-} \rightarrow K^{-} K^{-} K^{+} \nu_{\tau}[\right.$ ex. $\left.\phi]\right) \leq 2.5 \times 10^{-6} @ 90 \% C L$ are determined from a binned maximum likelihood fit of the $\tau^{-} \rightarrow K^{-} \pi^{-} K^{+} \nu_{\tau}$ and $\tau^{-} \rightarrow$ $K^{-} K^{-} K^{+} \nu_{\tau} \quad K^{+} K^{-}$invariant mass distributions. The branching ratio $\frac{\mathcal{B}\left(\tau^{-} \rightarrow K^{-} \nu_{\tau}\right)}{\mathcal{B}\left(\tau^{-} \rightarrow \pi^{-} \nu_{\tau}\right)}$ is measured to be $(6.531 \pm 0.056 \pm 0.093) \times 10^{-2}$ from which $\left|V_{u s}\right|$ is determined to be $0.2255 \pm 0.0023$. The branching ratio $\frac{\mathcal{B}\left(\tau^{-} \rightarrow \mu^{-} \nu_{\tau} \bar{\nu}_{\mu}\right)}{\mathcal{B}\left(\tau^{-} \rightarrow e^{-} \nu_{\tau} \bar{\nu}_{e}\right)}=(9.796 \pm 0.016 \pm 0.035) \times 10^{-1}$
\end{abstract}


is measured enabling a precision test of the Standard Model assumption of charged current lepton universality, $\frac{g_{\mu}}{g_{e}}=1.0036 \pm 0.0020$. The branching ratios $\frac{\mathcal{B}\left(\tau^{-} \rightarrow K^{-} \nu_{\tau}\right)}{\mathcal{B}\left(\tau^{-} \rightarrow e^{-} \nu_{\tau} \bar{\nu}_{e}\right)}$ $=(3.882 \pm 0.032 \pm 0.057) \times 10^{-2}$, and $\frac{\mathcal{B}\left(\tau^{-} \rightarrow \pi^{-} \nu_{\tau}\right)}{\mathcal{B}\left(\tau^{-} \rightarrow e^{-} \nu_{\tau} \bar{\nu}_{e}\right)}=(5.945 \pm 0.014 \pm 0.061) \times 10^{-1}$ are measured which provide additional tests of charged current lepton universality, $\left(\frac{g_{\tau}}{g_{\mu}}\right)_{\pi}=0.9856 \pm 0.0057$ and $\left(\frac{g_{\tau}}{g_{\mu}}\right)_{K}=0.9827 \pm 0.0086$ which can be combined to give $\left(\frac{g_{\tau}}{g_{\mu}}\right)_{\pi / K}=0.9850 \pm 0.0054$. Any deviation of these measurements from the expected Standard Model values would be an indication of new physics. 


\section{Table of Contents}

Supervisory Committee ii

Abstract

Table of Contents $\quad$ v

List of Tables $\quad$ ix

List of Figures $\quad$ XV

Acknowledgements $\quad$ xix

Dedications $\quad x x$

1 Introduction 1

2 Motivation: Strange $\tau$ Decays in the Standard Model 5

2.1 Introduction to the Standard Model of Particle Physics . . . . . . . . 5

2.2 Hadronic $\tau$ Decays and the Extraction of $\left|V_{u s}\right|$ and $m_{s} \ldots \ldots . . . \quad 14$

2.3 The Current Experimental Status of Hadronic $\tau$ Decays and the Extraction of $\left|V_{u s}\right|$ and $m_{s} \ldots \ldots \ldots \ldots$. . . . . . . . . . . . . . 42

3 The PEP-II Accelerator and the BABAR Detector 45

3.1 The PEP-II Accelerator . . . . . . . . . . . . . . . 45

3.2 The Interaction Region . . . . . . . . . . . . . . . . . . . . . 47

3.3 The BABAR Detector . . . . . . . . . . . . . . . . . . . . . . . . . . . 48 
4 Branching Fraction Measurement of $\tau^{-} \rightarrow h^{-} h^{-} h^{+} \nu_{\tau}$

4.1 Methodology of Analysis . . . . . . . . . . . . . . . . . . 65

4.2 BABAR Data Set . . . . . . . . . . . . . . . . . 66

4.3 Selection Procedure . . . . . . . . . . . . . . . . . 67

4.4 3D Dalitz Weighting . . . . . . . . . . . . . . 73

4.5 Systematic Uncertainty Studies . . . . . . . . . . . . . . . . 75

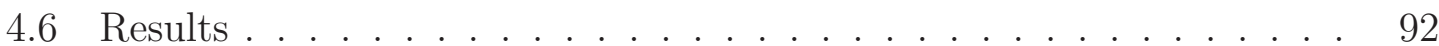

5 Fitting the $\phi$ Resonance in $\tau^{-} \rightarrow K^{-} \pi^{-} K^{+} \nu_{\tau}$ and $\tau^{-} \rightarrow K^{-} K^{-} K^{+} \nu_{\tau}$ Decays $\quad 95$

5.1 Methodology of Analysis . . . . . . . . . . . . . . . . . 95

5.2 Fitting Procedure for the $\phi$ peak for $\pi \phi$ and $K \phi \ldots . . . . . . . .96$

$5.3 \tau^{-} \rightarrow K^{-} K^{-} K^{+} \nu$ Non-resonance Upper Limit $\quad \ldots . . . . .98$

5.4 Systematic Uncertainties . . . . . . . . . . . . . . . . . . . . . . . 99

5.5 Results ......................... 101

6 Unfolding the Invariant Mass Distribution 105

6.1 Introduction to Unfolding . . . . . . . . . . . . . . . 105

6.2 Procedure ........................... 107

6.3 Systematic Uncertainty Studies . . . . . . . . . . . . . . . . 108

6.4 Results . . . . . . . . . . . . . . . . . . . 115

$7 \quad$ Measurements of $\frac{\mathcal{B}\left(\tau^{-} \rightarrow K^{-} \nu_{\tau}\right)}{\mathcal{B}\left(\tau^{-} \rightarrow \pi^{-} \nu_{\tau}\right)}, \frac{\mathcal{B}\left(\tau^{-} \rightarrow K^{-} \nu_{\tau}\right)}{\mathcal{B}\left(\tau^{-} \rightarrow e^{-} \nu_{\tau} \bar{\nu}_{e}\right)}, \frac{\mathcal{B}\left(\tau^{-} \rightarrow \pi^{-} \nu_{\tau}\right)}{\mathcal{B}\left(\tau^{-} \rightarrow e^{-} \nu_{\tau} \bar{\nu}_{e}\right)}$, and $\frac{\mathcal{B}\left(\tau^{-} \rightarrow \mu^{-} \nu_{\tau} \bar{\nu}_{\mu}\right)}{\mathcal{B}\left(\tau^{-} \rightarrow e^{-} \nu_{\tau} \bar{\nu}_{e}\right)}$

7.1 Methodology of Analysis . . . . . . . . . . . . . . . . . 119

7.2 Selection Procedure . . . . . . . . . . . . . . . 121

7.3 Systematic Uncertainty Studies . . . . . . . . . . . . . . . . . 124

7.4 Results . . . . . . . . . . . . . . . . . 134 
8.1 Results of $\tau^{-} \rightarrow h^{-} h^{-} h^{+} \nu_{\tau}$ Analysis . . . . . . . . . . . . . . . . 139

8.2 Results from the $\tau^{-} \rightarrow \phi \pi^{-} \nu_{\tau}$ and $\tau^{-} \rightarrow \phi K^{-} \nu_{\tau}$ Fit . . . . . . . . . 141

8.3 Results from the Unfolding . . . . . . . . . . . . . . . . . 143

8.4 Results from the $\frac{\mathcal{B}\left(\tau^{-} \rightarrow \mu^{-} \nu_{\tau} \bar{\nu}_{\mu}\right)}{\mathcal{B}\left(\tau^{-} \rightarrow e^{-} \nu_{\tau} \bar{\nu}_{e}\right)}$ Measurement and e- $\mu$ Universality . . . 143

8.5 Results from the $\frac{\mathcal{B}\left(\tau^{-} \rightarrow \pi^{-} \nu_{\tau}\right)}{\mathcal{B}\left(\tau^{-} \rightarrow e^{-} \nu_{\tau} \bar{\nu}_{e}\right)}$ and $\frac{\mathcal{B}\left(\tau^{-} \rightarrow K^{-} \nu_{\tau}\right)}{\mathcal{B}\left(\tau^{-} \rightarrow e^{-} \nu_{\tau} \bar{\nu}_{e}\right)}$ Measurements and $\tau-\mu$ Universality . . . . . . . . . . . . . . 146

8.6 Results from the $\frac{\mathcal{B}\left(\tau^{-} \rightarrow K^{-} \nu_{\tau}\right)}{\mathcal{B}\left(\tau^{-} \rightarrow \pi^{-} \nu_{\tau}\right)}$ Measurement and $\left|V_{u s}\right| \ldots \ldots$. . . . . . 149

9 Conclusion 152

$\begin{array}{ll}\text { A Particle Identification } & 171\end{array}$

A.1 Electron selectors . . . . . . . . . . . . . . . . . 172

A.2 Muon Selectors . . . . . . . . . . . . . . . . . . . 176

A.3 Kaon Selectors . . . . . . . . . . . . . . . . . 179

A.4 Pion Selectors . . . . . . . . . . . . . . . . . . 181

B Dalitz Weighting in the $\tau^{-} \rightarrow h^{-} h^{-} h^{+} \nu_{\tau}$ Analysis 182

C Unfolded $\tau^{-} \rightarrow \pi^{-} \pi^{-} \pi^{+} \nu_{\tau}$ Invariant Mass Spectra $\quad 188$

C.1 The Unfolded $\tau^{-} \rightarrow \pi^{-} \pi^{-} \pi^{+} \nu_{\tau} M_{123}$ Invariant Mass Spectra . . . . . 188

C.2 The Unfolded $\tau^{-} \rightarrow \pi^{-} \pi^{-} \pi^{+} \nu_{\tau} M_{13 a n d 23}$ Invariant Mass Spectra . . . 200

D Unfolded $\tau^{-} \rightarrow K^{-} \pi^{-} \pi^{+} \nu_{\tau}$ Invariant Mass Spectra 213

D.1 The Unfolded $\tau^{-} \rightarrow K^{-} \pi^{-} \pi^{+} \nu_{\tau} M_{123}$ Invariant Mass Spectra . . . . . 213

D.2 The Unfolded $\tau^{-} \rightarrow K^{-} \pi^{-} \pi^{+} \nu_{\tau} M_{13}$ Invariant Mass Spectra . . . . . 219

D.3 The Unfolded $\tau^{-} \rightarrow K^{-} \pi^{-} \pi^{+} \nu_{\tau} M_{23}$ Invariant Mass Spectra . . . . . 224

E Unfolded $\tau^{-} \rightarrow K^{-} \pi^{-} K^{+} \nu_{\tau}$ Invariant Mass Spectra

E.1 The Unfolded $\tau^{-} \rightarrow K^{-} \pi^{-} K^{+} \nu_{\tau} M_{123}$ Invariant Mass Spectra . . . . 235

E.2 The Unfolded $\tau^{-} \rightarrow K^{-} \pi^{-} K^{+} \nu_{\tau} M_{13}$ Invariant Mass Spectra . . . . . 240 
E.3 The Unfolded $\tau^{-} \rightarrow K^{-} \pi^{-} K^{+} \nu_{\tau} M_{23}$ Invariant Mass Spectra . . . . . 244

F Unfolded $\tau^{-} \rightarrow K^{-} K^{-} K^{+} \nu_{\tau}$ Invariant Mass Spectra 248

F.1 The Unfolded $\tau^{-} \rightarrow K^{-} K^{-} K^{+} \nu_{\tau} M_{123}$ Invariant Mass Spectra . . . . 248

F.2 The Unfolded $\tau^{-} \rightarrow K^{-} K^{-} K^{+} \nu_{\tau} M_{13 a n d 23}$ Invariant Mass Spectra . 252

G PID Control Samples $\quad 255$

G.1 Kaon Control Sample: $\tau^{-} \rightarrow K^{-} \pi^{-} K^{+} \nu \ldots \ldots . . . . . . . .255$

G.2 Pion Control Sample: $\tau^{-} \rightarrow \pi^{-} \pi^{-} \pi^{+} \nu$. . . . . . . . . . . . . 257

G.3 Electron Control Sample: $e^{+} e^{-} \rightarrow e^{-} e^{+} \gamma\left(\rightarrow e^{+} e^{-}\right) \quad \ldots . . . . . .259$

G.4 Muon Control Sample 1: $e^{+} e^{-} \rightarrow \mu^{-} \mu^{+} \gamma\left(\rightarrow e^{+} e^{-}\right) \quad \ldots . . . . . .260$

G.5 Muon Control Sample 2: $e^{+} e^{-} \rightarrow \mu^{-} \mu^{+} \gamma \ldots \ldots$. . . . . . . . . 261

G.6 Data MC Agreement with Control Samples . . . . . . . . . . . . . . 262

H Neutrino Pseudo-mass 265

I $\quad \frac{\mathcal{B}\left(\tau^{-} \rightarrow K^{-} \nu_{\tau}\right)}{\mathcal{B}\left(\tau^{-} \rightarrow \pi^{-} \nu_{\tau}\right)}, \frac{\mathcal{B}\left(\tau^{-} \rightarrow K^{-} \nu_{\tau}\right)}{\mathcal{B}\left(\tau^{-} \rightarrow e^{-} \nu_{\tau} \bar{\nu}_{e}\right)}, \frac{\mathcal{B}\left(\tau^{-} \rightarrow \pi^{-} \nu_{\tau}\right)}{\mathcal{B}\left(\tau^{-} \rightarrow e^{-} \nu_{\tau} \bar{\nu}_{e}\right)}$, and $\frac{\mathcal{B}\left(\tau^{-} \rightarrow \mu^{-} \nu_{\tau} \bar{\nu}_{\mu}\right)}{\mathcal{B}\left(\tau^{-} \rightarrow e^{-} \nu_{\tau} \bar{\nu}_{e}\right)}$ Backgrounds 273 


\section{List of Tables}

2.1 Fundamental Forces and Particles in the Standard Model . . . . . . . 6

3.1 Cross Sections for an $e^{+} e^{-}$Collider at 10.58GeV . . . . . . . . . . . . 47

4.1 Data and MC Integrated Luminosity. . . . . . . . . . . . . . . . . . 67

4.2 The $\tau^{-} \rightarrow h^{-} h^{-} h^{+} \nu_{\tau}$ Particle-ID Component of the Efficiency Migration Matrix . . . . . . . . . . . . . . . . . . 74

4.3 The Dominant Branching Fractions for the $\tau$ Backgrounds used for the $\tau$ Background Systematic. . . . . . . . . . . . . . . . . 90

4.4 Summary of $\tau^{-} \rightarrow h^{-} h^{-} h^{+} \nu_{\tau}$ Branching Fractions . . . . . . . . . . . 93

4.5 A Summary of the Uncertainties for the $\tau^{-} \rightarrow h^{-} h^{-} h^{+} \nu_{\tau}$ Analysis . . 94

5.1 The $K^{-} K^{+}$Invariant Mass Fit Parameters for the $K^{-} \pi^{-} K^{+}$Channel 97

5.2 The $K^{-} K^{+}$Invariant Mass Fit Parameters for the $K^{-} K^{-} K^{+}$Channel 98

5.3 The $\tau^{-} \rightarrow \phi \pi^{-} \nu_{\tau}$ and $\tau^{-} \rightarrow \phi K^{-} \nu_{\tau}$ Particle-ID Component of the Efficiency Migration Matrix . . . . . . . . . . . . . . . . . . 99

5.4 A Summary of the Uncertainties for the $\tau^{-} \rightarrow \phi \pi^{-} \nu_{\tau}$ and $\tau^{-} \rightarrow \phi K^{-} \nu_{\tau}$ Analysis . . . . . . . . . . . . . . . . . . 104

7.1 Data and MC Integrated Luminosity $\frac{\mathcal{B}\left(\tau^{-} \rightarrow K^{-} \nu_{\tau}\right)}{\mathcal{B}\left(\tau^{-} \rightarrow \pi^{-} \nu_{\tau}\right)}, \frac{\mathcal{B}\left(\tau^{-} \rightarrow K^{-} \nu_{\tau}\right)}{\mathcal{B}\left(\tau^{-} \rightarrow e^{-} \nu_{\tau} \bar{\nu}_{e}\right)}, \frac{\mathcal{B}\left(\tau^{-} \rightarrow \pi^{-} \nu_{\tau}\right)}{\mathcal{B}\left(\tau^{-} \rightarrow e^{-} \nu_{\tau} \bar{\nu}_{e}\right)}$, and $\frac{\mathcal{B}\left(\tau^{-} \rightarrow \mu^{-} \nu_{\tau} \bar{\nu}_{\mu}\right)}{\mathcal{B}\left(\tau^{-} \rightarrow e^{-} \nu_{\tau} \bar{\nu}_{e}\right)}$ Analysis. . . . . . . . . . . . . . . . . . . 121

7.2 The 1 prong Branching Ratios . . . . . . . . . . . . . . . 135 
7.3 Summary of Selected Events for Analysis . . . . . . . . . . . . . . . 138

7.4 A Summary of the Uncertainties for the $\frac{\mathcal{B}\left(\tau^{-} \rightarrow K^{-} \nu_{\tau}\right)}{\mathcal{B}\left(\tau^{-} \rightarrow \pi^{-} \nu_{\tau}\right)}, \frac{\mathcal{B}\left(\tau^{-} \rightarrow K^{-} \nu_{\tau}\right)}{\mathcal{B}\left(\tau^{-} \rightarrow e^{-} \nu_{\tau} \bar{\nu}_{e}\right)}$, $\frac{\mathcal{B}\left(\tau^{-} \rightarrow \pi^{-} \nu_{\tau}\right)}{\mathcal{B}\left(\tau^{-} \rightarrow e^{-} \nu_{\tau} \bar{\nu}_{e}\right)}$, and $\frac{\mathcal{B}\left(\tau^{-} \rightarrow \mu^{-} \nu_{\tau} \bar{\nu}_{\mu}\right)}{\mathcal{B}\left(\tau^{-} \rightarrow e^{-} \nu_{\tau} \bar{\nu}_{e}\right)}$ Analysis . . . . . . . . . . . . 138

A.1 The Definition of the Variable used for Particle Identification Part 1 . 171

A.2 The Definition of the Variable used for Particle Identification Part 2 . 172

A.3 The Electron Selector Criteria . . . . . . . . . . . . . . . . . 174

A.4 The Electron Criteria used for the Electron Veto . . . . . . . . . . . 174

A.5 The Muon Selection Criteria for the $\tau^{-} \rightarrow h^{-} h^{-} h^{+} \nu_{\tau}$ Analysis . . . . 176

A.6 The Muon Selection Criteria for the $\frac{\mathcal{B}\left(\tau^{-} \rightarrow K^{-} \nu_{\tau}\right)}{\mathcal{B}\left(\tau^{-} \rightarrow e^{-} \nu_{\tau} \bar{\nu}_{e}\right)}$ Measurement . . . . 178

A.7 The Muon Veto Included in the Standard BABAR Pion Selector . . . . 178

A.8 The Kaon Selection Criteria . . . . . . . . . . . . . . . . . . . 180

A.9 The Pion Selection Criteria . . . . . . . . . . . . . . 181

C.1 Bayesian Error Table for the $\tau^{-} \rightarrow \pi^{-} \pi^{-} \pi^{+} \nu_{\tau} M_{123}$ Invariant Mass (Part 1) . . . . . . . . . . . . . . . 190

C.2 Bayesian Error Table for the $\tau^{-} \rightarrow \pi^{-} \pi^{-} \pi^{+} \nu_{\tau} M_{123}$ Invariant Mass (Part 2) . . . . . . . . . . . . . . 191

C.3 Bayesian Total Correlation Table for the $\tau^{-} \rightarrow \pi^{-} \pi^{-} \pi^{+} \nu_{\tau} M_{123}$ Invariant Mass (Part 1) . . . . . . . . . . . . . . . . . 192

C.4 Bayesian Total Correlation Table for the $\tau^{-} \rightarrow \pi^{-} \pi^{-} \pi^{+} \nu_{\tau} M_{123}$ Invariant Mass (Part 2) . . . . . . . . . . . . . . . . 193

C.5 Bayesian Total Correlation Table for the $\tau^{-} \rightarrow \pi^{-} \pi^{-} \pi^{+} \nu_{\tau} M_{123}$ Invariant Mass $($ Part 3) . . . . . . . . . . . . . . . 194

C.6 Bayesian Total Correlation Table for the $\tau^{-} \rightarrow \pi^{-} \pi^{-} \pi^{+} \nu_{\tau} M_{123}$ Invariant Mass $($ Part 4) . . . . . . . . . . . . . . . 195 
C.7 Bayesian Total Correlation Table for the $\tau^{-} \rightarrow \pi^{-} \pi^{-} \pi^{+} \nu_{\tau} M_{123}$ Invariant Mass $($ Part 5) . . . . . . . . . . . . . . 196

C.8 Bayesian Total Correlation Table for the $\tau^{-} \rightarrow \pi^{-} \pi^{-} \pi^{+} \nu_{\tau} M_{123}$ Invariant Mass (Part 6) . . . . . . . . . . . . . . . . . 197

C.9 Bayesian Total Correlation Table for the $\tau^{-} \rightarrow \pi^{-} \pi^{-} \pi^{+} \nu_{\tau} M_{123}$ Invariant Mass (Part 7) . . . . . . . . . . . . . . . . 198

C.10 Bayesian Total Correlation Table for the $\tau^{-} \rightarrow \pi^{-} \pi^{-} \pi^{+} \nu_{\tau} M_{123}$ Invariant Mass $($ Part 8) . . . . . . . . . . . . . . 199

C.11 Bayesian Error Table for the $\tau^{-} \rightarrow \pi^{-} \pi^{-} \pi^{+} \nu_{\tau} M_{13 a n d 23}$ Invariant Mass

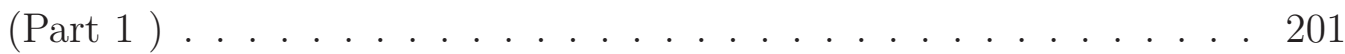

C.12 Bayesian Error Table for the $\tau^{-} \rightarrow \pi^{-} \pi^{-} \pi^{+} \nu_{\tau} M_{13 a n d 23}$ Invariant Mass

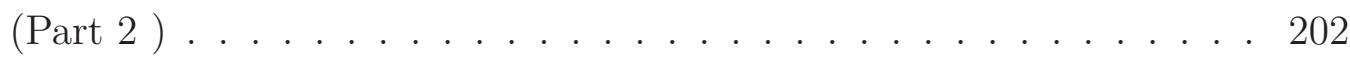

C.13 Bayesian Total Correlation Table for the $\tau^{-} \rightarrow \pi^{-} \pi^{-} \pi^{+} \nu_{\tau} M_{13 a n d 23}$ Invariant Mass $($ Part 1) . . . . . . . . . . . . . 203

C.14 Bayesian Total Correlation Table for the $\tau^{-} \rightarrow \pi^{-} \pi^{-} \pi^{+} \nu_{\tau} M_{13 a n d 23}$ Invariant Mass (Part 2) . . . . . . . . . . . . . . . . . 204

C.15 Bayesian Total Correlation Table for the $\tau^{-} \rightarrow \pi^{-} \pi^{-} \pi^{+} \nu_{\tau} M_{13 a n d 23}$ Invariant Mass (Part 3) . . . . . . . . . . . . . . 205

C.16 Bayesian Total Correlation Table for the $\tau^{-} \rightarrow \pi^{-} \pi^{-} \pi^{+} \nu_{\tau} M_{13 a n d 23}$ Invariant Mass (Part 4) . . . . . . . . . . . . . . . 206

C.17 Bayesian Total Correlation Table for the $\tau^{-} \rightarrow \pi^{-} \pi^{-} \pi^{+} \nu_{\tau} M_{13 a n d 23}$ Invariant Mass (Part 5) . . . . . . . . . . . . . 207

C.18 Bayesian Total Correlation Table for the $\tau^{-} \rightarrow \pi^{-} \pi^{-} \pi^{+} \nu_{\tau} M_{13 a n d 23}$ Invariant Mass $($ Part 6) . . . . . . . . . . . . . . . 208

C.19 Bayesian Total Correlation Table for the $\tau^{-} \rightarrow \pi^{-} \pi^{-} \pi^{+} \nu_{\tau} M_{13 a n d 23}$ Invariant Mass (Part 7) . . . . . . . . . . . . . . . . 209 
C.20 Bayesian Total Correlation Table for the $\tau^{-} \rightarrow \pi^{-} \pi^{-} \pi^{+} \nu_{\tau} M_{13 a n d 23}$ Invariant Mass (Part 8) . . . . . . . . . . . . . . . 210

C.21 Bayesian Total Correlation Table for the $\tau^{-} \rightarrow \pi^{-} \pi^{-} \pi^{+} \nu_{\tau} M_{13 a n d 23}$ Invariant Mass (Part 9) . . . . . . . . . . . . . . . . 211

C.22 Bayesian Total Correlation Table for the $\tau^{-} \rightarrow \pi^{-} \pi^{-} \pi^{+} \nu_{\tau} M_{13 a n d 23}$ Invariant Mass (Part 10) . . . . . . . . . . . . . . . . . 212

D.1 Bayesian Error Table for the $\tau^{-} \rightarrow K^{-} \pi^{-} \pi^{+} \nu_{\tau} M_{123}$ Invariant Mass

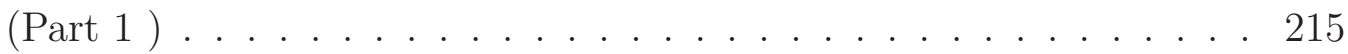

D.2 Bayesian Total Correlation Table for the $\tau^{-} \rightarrow K^{-} \pi^{-} \pi^{+} \nu_{\tau} M_{123}$ In-

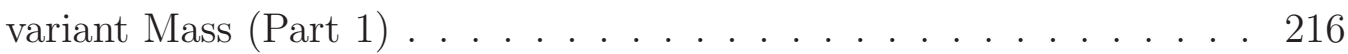

D.3 Bayesian Total Correlation Table for the $\tau^{-} \rightarrow K^{-} \pi^{-} \pi^{+} \nu_{\tau} M_{123}$ Invariant Mass (Part 2) . . . . . . . . . . . . . . 217

D.4 Bayesian Total Correlation Table for the $\tau^{-} \rightarrow K^{-} \pi^{-} \pi^{+} \nu_{\tau} M_{123}$ Invariant Mass (Part 3) . . . . . . . . . . . . . . . . 218

D.5 Bayesian Error Table for the $\tau^{-} \rightarrow K^{-} \pi^{-} \pi^{+} \nu_{\tau} M_{13}$ Invariant Mass

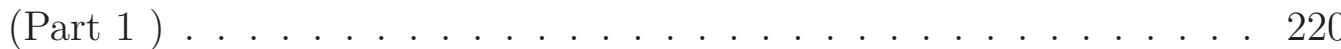

D.6 Bayesian Total Correlation Table for the $\tau^{-} \rightarrow K^{-} \pi^{-} \pi^{+} \nu_{\tau} M_{13}$ Invariant Mass (Part 1) . . . . . . . . . . . . . . . 221

D.7 Bayesian Total Correlation Table for the $\tau^{-} \rightarrow K^{-} \pi^{-} \pi^{+} \nu_{\tau} M_{13}$ Invariant Mass (Part 2) . . . . . . . . . . . . . . . . 222

D.8 Bayesian Total Correlation Table for the $\tau^{-} \rightarrow K^{-} \pi^{-} \pi^{+} \nu_{\tau} M_{13}$ Invariant Mass (Part 3) . . . . . . . . . . . . . . 223

D.9 Bayesian Error Table for the $\tau^{-} \rightarrow K^{-} \pi^{-} \pi^{+} \nu_{\tau} M_{23}$ Invariant Mass

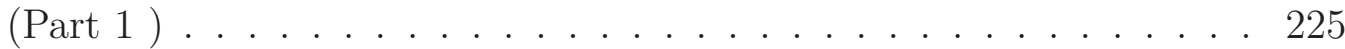


D.10 Bayesian Error Table for the $\tau^{-} \rightarrow K^{-} \pi^{-} \pi^{+} \nu_{\tau} M_{23}$ Invariant Mass

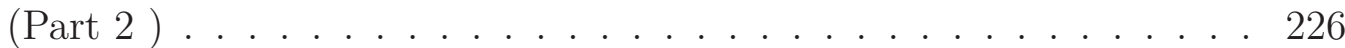

D.11 Bayesian Total Correlation Table for the $\tau^{-} \rightarrow K^{-} \pi^{-} \pi^{+} \nu_{\tau} M_{23}$ Invariant Mass (Part 1) . . . . . . . . . . . . . . . . 227

D.12 Bayesian Total Correlation Table for the $\tau^{-} \rightarrow K^{-} \pi^{-} \pi^{+} \nu_{\tau} M_{23}$ Invariant Mass (Part 2) . . . . . . . . . . . . . . 228

D.13 Bayesian Total Correlation Table for the $\tau^{-} \rightarrow K^{-} \pi^{-} \pi^{+} \nu_{\tau} M_{23}$ Invariant Mass (Part 3) . . . . . . . . . . . . . . . . . . 229

D.14 Bayesian Total Correlation Table for the $\tau^{-} \rightarrow K^{-} \pi^{-} \pi^{+} \nu_{\tau} M_{23}$ Invariant Mass (Part 4) . . . . . . . . . . . . . . 230

D.15 Bayesian Total Correlation Table for the $\tau^{-} \rightarrow K^{-} \pi^{-} \pi^{+} \nu_{\tau} M_{23}$ Invariant Mass $($ Part 5) . . . . . . . . . . . . . . . . 231

D.16 Bayesian Total Correlation Table for the $\tau^{-} \rightarrow K^{-} \pi^{-} \pi^{+} \nu_{\tau} M_{23}$ Invariant Mass $($ Part 6) . . . . . . . . . . . . . . . 232

D.17 Bayesian Total Correlation Table for the $\tau^{-} \rightarrow K^{-} \pi^{-} \pi^{+} \nu_{\tau} M_{23}$ In-

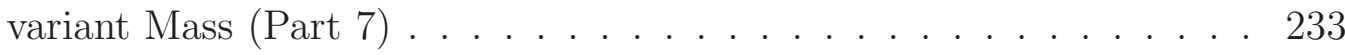

D.18 Bayesian Total Correlation Table for the $\tau^{-} \rightarrow K^{-} \pi^{-} \pi^{+} \nu_{\tau} M_{23}$ Invariant Mass $($ Part 8) . . . . . . . . . . . . . . . 234

E.1 Bayesian Error Table for the $\tau^{-} \rightarrow K^{-} \pi^{-} K^{+} \nu_{\tau} M_{123}$ Invariant Mass

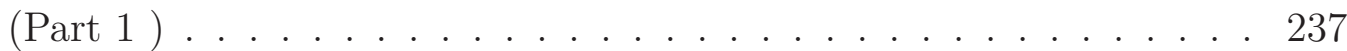

E.2 Bayesian Total Correlation Table for the $\tau^{-} \rightarrow K^{-} \pi^{-} K^{+} \nu_{\tau} M_{123}$ In-

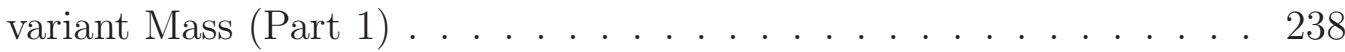

E.3 Bayesian Total Correlation Table for the $\tau^{-} \rightarrow K^{-} \pi^{-} K^{+} \nu_{\tau} M_{123}$ Invariant Mass (Part 2) . . . . . . . . . . . . . . 239 
E.4 Bayesian Error Table for the $\tau^{-} \rightarrow K^{-} \pi^{-} K^{+} \nu_{\tau} M_{13}$ Invariant Mass

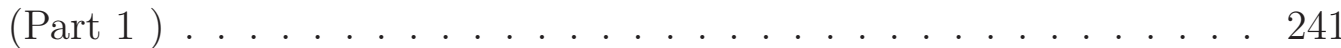

E.5 Bayesian Total Correlation Table for the $\tau^{-} \rightarrow K^{-} \pi^{-} K^{+} \nu_{\tau} M_{13}$ Invariant Mass (Part 1) . . . . . . . . . . . . . . . . 242

E.6 Bayesian Total Correlation Table for the $\tau^{-} \rightarrow K^{-} \pi^{-} K^{+} \nu_{\tau} M_{13}$ Invariant Mass $($ Part 2) . . . . . . . . . . . . . . . . . 243

E.7 Bayesian Error Table for the $\tau^{-} \rightarrow K^{-} \pi^{-} K^{+} \nu_{\tau} M_{23}$ Invariant Mass

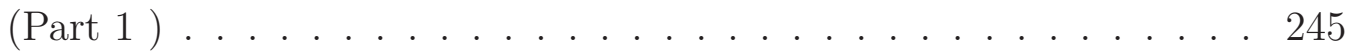

E.8 Bayesian Total Correlation Table for the $\tau^{-} \rightarrow K^{-} \pi^{-} K^{+} \nu_{\tau} M_{23}$ Invariant Mass (Part 1) . . . . . . . . . . . . . . 246

E.9 Bayesian Total Correlation Table for the $\tau^{-} \rightarrow K^{-} \pi^{-} K^{+} \nu_{\tau} M_{23}$ Invariant Mass (Part 2) . . . . . . . . . . . . . . . . . 247

F.1 Bayesian Error Table for the $\tau^{-} \rightarrow K^{-} K^{-} K^{+} \nu_{\tau} M_{123}$ Invariant Mass

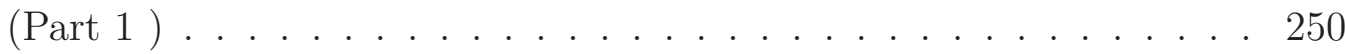

F.2 Bayesian Total Correlation Table for the $\tau^{-} \rightarrow K^{-} K^{-} K^{+} \nu_{\tau} M_{123}$

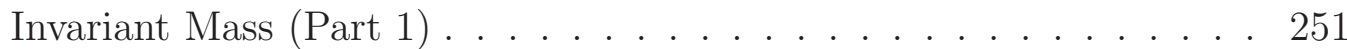

F.3 Bayesian Error Table for the $\tau^{-} \rightarrow K^{-} K^{-} K^{+} \nu_{\tau} M_{13 a n d 23}$ Invariant Mass (Part 1 ) . . . . . . . . . . . . . . 253

F.4 Bayesian Total Correlation Table for the $\tau^{-} \rightarrow K^{-} K^{-} K^{+} \nu_{\tau} M_{13 a n d 23}$

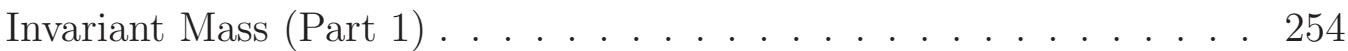

I.1 Background Summary for the $\frac{\mathcal{B}\left(\tau^{-} \rightarrow K^{-} \nu\right)}{\mathcal{B}\left(\tau^{-} \rightarrow e^{-} \nu \bar{\nu}\right)}, \frac{\mathcal{B}\left(\tau^{-} \rightarrow \pi^{-} \nu\right)}{\mathcal{B}\left(\tau^{-} \rightarrow e^{-} \nu \bar{\nu}\right)}$ and $\frac{\mathcal{B}\left(\tau^{-} \rightarrow \mu^{-} \nu \bar{\nu}\right)}{\mathcal{B}\left(\tau^{-} \rightarrow e^{-} \nu \bar{\nu}\right)}$ Analysis . . . . . . . . . . . . . . . . . . . 273 


\section{List of Figures}

2.1 Feynman Diagram for the $e^{+} e^{-} \rightarrow \tau^{+} \tau^{-}$Interaction . . . . . . . . . 9

$2.2 \tau^{-} \rightarrow e^{-} \nu_{\tau} \overline{\nu_{e}}$ Feynman Diagram . . . . . . . . . . . . . . . . . . 15

$2.3 \tau^{-} \rightarrow$ hadrons ${ }^{-} \nu_{\tau}$ Feynman Diagram . . . . . . . . . . . . . 17

2.4 The Sum Rule Integration Curve . . . . . . . . . . . . . . . . . . 25

2.5 The Lowest Order Terms of the Dimension 0 Series. . . . . . . . . . . 29

2.6 The Lowest Order Terms of the Dimension 4 Quark Condensate Series. 30

2.7 The Lowest Order Terms of Dimension 4 Gluon Condensate Series. . 30

2.8 The Lowest Order Terms of Dimension 6 Quark Operator Series. . . . 31

2.9 The Lowest Order Terms of Dimension 6 Three Gluon Operator Series. 31

2.10 World Average $m_{s} \ldots \ldots \ldots$. . . . . . . . . . . . . . . . 44

3.1 PEP-II Accelerator . . . . . . . . . . . . . . . . . . 46

3.2 BABAR Detector . . . . . . . . . . . . . . . . . . . . . . . . . . 49

3.3 SVT Cross Section in the Plane Orthogonal to the Beam Pipe . . . . 50

3.4 SVT Cross Section in the Beam Pipe Plane . . . . . . . . . . . . 50

3.5 SVT Module......................... 51

3.6 DCH Cross Section . . . . . . . . . . . . . . 52

3.7 DCH Axial and Stereo Wire Arrangement . . . . . . . . . . . . . 54

3.8 Diagram of the Isochrones in a Typical DCH Cell . . . . . . . . . . . 55

$3.9 \mathrm{DIRC} \ldots \ldots \ldots \ldots \ldots \ldots \ldots \ldots$ 
3.10 Operation of the DIRC . . . . . . . . . . . . . 57

3.11 EMC Cross Section . . . . . . . . . . . . . . . . . 59

3.12 EMC crystal . . . . . . . . . . . . . . . . . . . . 60

3.13 EMC Barrel Module . . . . . . . . . . . . . . . . . . . . . . . 61

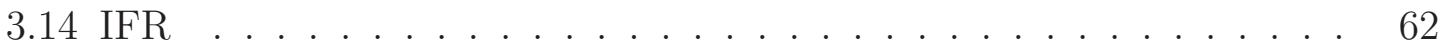

4.1 An Event Display of a Typical 1 vs 3 Prong $\tau$ Pair Event. . . . . . . 69

$4.2 \theta_{C}$ as a Function of Momentum . . . . . . . . . . . . . . . . 72

4.3 Data-MC Discrepancy in the Kaon Selector . . . . . . . . . . . 73

4.4 The Raw $\tau^{-} \rightarrow \pi^{-} \pi^{-} \pi^{+} \nu_{\tau}$ Invariant Mass Distributions . . . . . . . . 75

4.5 The Raw $\tau^{-} \rightarrow K^{-} \pi^{-} \pi^{+} \nu_{\tau}$ Invariant Mass Distributions . . . . . . . 76

4.6 The Raw $\tau^{-} \rightarrow K^{-} \pi^{-} K^{+} \nu_{\tau}$ Invariant Mass Distributions . . . . . . . 77

4.7 The Raw $\tau^{-} \rightarrow K^{-} K^{-} K^{+} \nu_{\tau}$ Invariant Mass Distributions . . . . . . 78

4.8 The 1 Prong Residual Neutral Energy Distribution for the Electron

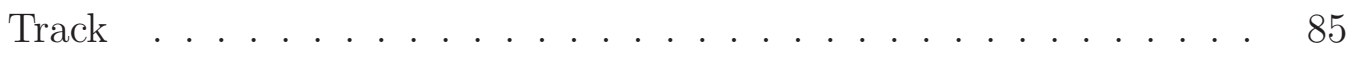

4.9 The 1 Prong Residual Neutral Energy Distribution for the Muon Track 86

4.10 The 3 Prong Residual Neutral Energy Distribution (0 GeV to $5 \mathrm{GeV}$ ) 87

4.11 The 3 Prong Residual Neutral Energy Distribution (0 GeV to $1 \mathrm{GeV}) \quad 88$

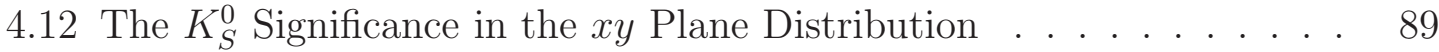

$5.1 K^{+} K^{-}$Invariant Mass in $\tau^{-} \rightarrow K^{-} \pi^{-} K^{+} \nu_{\tau}$ Decays $\ldots . . . . . . .101$

$5.2 K^{+} K^{-}$Invariant Mass in $\tau^{-} \rightarrow K^{-} K^{-} K^{+} \nu_{\tau}$ Decays . . . . . . . . 102

6.1 The Unfolded $\tau^{-} \rightarrow \pi^{-} \pi^{-} \pi^{+} \nu_{\tau}$ Invariant Mass Distributions . . . . . 115

6.2 The Unfolded $\tau^{-} \rightarrow K^{-} \pi^{-} \pi^{+} \nu_{\tau}$ Invariant Mass Distributions . . . . . 116

6.3 The Unfolded $\tau^{-} \rightarrow K^{-} \pi^{-} K^{+} \nu_{\tau}$ Invariant Mass Distributions . . . . 117

6.4 The Unfolded $\tau^{-} \rightarrow K^{-} K^{-} K^{+} \nu_{\tau}$ Invariant Mass Distributions . . . . 118 
7.1 The $\tau^{-} \rightarrow e^{-} \nu_{\tau} \bar{\nu}_{e}$ and $\tau^{-} \rightarrow \mu^{-} \nu_{\tau} \bar{\nu}_{\mu}$ c.m. Momentum . . . . . . . 136

7.2 The $\tau^{-} \rightarrow \pi^{-} \nu_{\tau}$ and $\tau^{-} \rightarrow K^{-} \nu_{\tau}$ c.m. Momentum . . . . . . . . . 137

8.1 Comparison of $\tau^{-} \rightarrow h^{-} h^{-} h^{+} \nu_{\tau}$ Branching Fractions . . . . . . . . 140

8.2 The Current Status of $\left|V_{u s}\right| \ldots \ldots \ldots$. . . . . . . . . . . 142

8.3 Comparison of $\tau^{-} \rightarrow \mu^{-} \nu_{\tau} \bar{\nu}_{\mu}, \tau^{-} \rightarrow \pi^{-} \nu_{\tau}$, and $\tau^{-} \rightarrow K^{-} \nu_{\tau}$ Branching Fractions . . . . . . . . . . . . . . . . . . 144

8.4 The Current Status of $\left(\frac{g_{\mu}}{g_{e}}\right) \ldots \ldots \ldots \ldots \ldots$. . . . . . . . 146

8.5 The Current Status of $\left(\frac{g_{\tau}}{g_{\mu}}\right) \ldots \ldots \ldots \ldots$. . . . . . . . . . 149

8.6 The Current Status of $\left|V_{s}\right|$ with $\left|V_{u s}\right|$ determined from $\frac{\mathcal{B}\left(\tau^{-} \rightarrow K^{-} \nu_{\tau}\right)}{\mathcal{B}\left(\tau^{-} \rightarrow \pi^{-} \nu_{\tau}\right)}$. . 151

A.1 The $\Delta \phi$ Cut Employed in the Electron Veto . . . . . . . . . . . . . . 175

B.1 The Raw $\tau^{-} \rightarrow \pi^{-} \pi^{-} \pi^{+} \nu_{\tau}$ Invariant Mass Distributions with Dalitz Weighted $\mathrm{MC} \ldots \ldots \ldots \ldots$. . . . . . . . . . . . 183

B.2 The Raw $\tau^{-} \rightarrow K^{-} \pi^{-} \pi^{+} \nu_{\tau}$ Invariant Mass Distributions with Dalitz Weighted $\mathrm{MC}$...................... . . 184

B.3 The Raw $\tau^{-} \rightarrow K^{-} \pi^{-} K^{+} \nu_{\tau}$ Invariant Mass Distributions with Dalitz

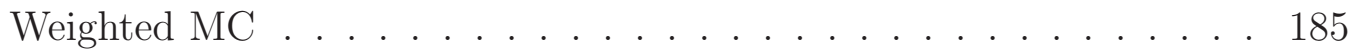

B.4 The Raw $\tau^{-} \rightarrow K^{-} K^{-} K^{+} \nu_{\tau}$ Invariant Mass Distributions with Dalitz

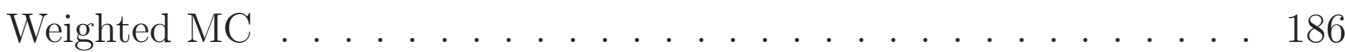

B.5 The $\tau^{-} \rightarrow h^{-} h^{-} h^{+} \nu_{\tau}$ Background Subtracted Dalitz plots . . . . . . 187

C.1 A Comparison of the $\tau^{-} \rightarrow \pi^{-} \pi^{-} \pi^{+} \nu_{\tau}$ MC Truth and Reconstructed MC for the $M_{123}$ Invariant Mass Spectra. . . . . . . . . . . . . . . . . 189

C.2 A Comparison of the $\tau^{-} \rightarrow \pi^{-} \pi^{-} \pi^{+} \nu_{\tau}$ MC Truth and Reconstructed MC for the $M_{13 a n d 23}$ Invariant Mass Spectra. . . . . . . . . . . . . . . 200 
xviii

D.1 A Comparison of the $\tau^{-} \rightarrow K^{-} \pi^{-} \pi^{+} \nu_{\tau}$ MC Truth and Reconstructed MC for the $M_{123}$ Invariant Mass Spectra. . . . . . . . . . . . . . . . . 214

D.2 A Comparison of the $\tau^{-} \rightarrow K^{-} \pi^{-} \pi^{+} \nu_{\tau}$ MC Truth and Reconstructed MC for the $M_{13}$ Invariant Mass Spectra. . . . . . . . . . . . . . . . . 219

D.3 A Comparison of the $\tau^{-} \rightarrow K^{-} \pi^{-} \pi^{+} \nu_{\tau}$ MC Truth and Reconstructed MC for the $M_{23}$ Invariant Mass Spectra. . . . . . . . . . . . . . . 224

E.1 A Comparison of the $\tau^{-} \rightarrow K^{-} \pi^{-} K^{+} \nu_{\tau}$ MC Truth and Reconstructed MC for the $M_{123}$ Invariant Mass Spectra. . . . . . . . . . . . . 236

E.2 A Comparison of the $\tau^{-} \rightarrow K^{-} \pi^{-} K^{+} \nu_{\tau}$ MC Truth and Reconstructed MC for the $M_{13}$ Invariant Mass Spectra. . . . . . . . . . . . . . . . . 240

E.3 A Comparison of the $\tau^{-} \rightarrow K^{-} \pi^{-} K^{+} \nu_{\tau}$ MC Truth and Reconstructed MC for the $M_{23}$ Invariant Mass Spectra. . . . . . . . . . . . . . . . 244

F.1 A Comparison of the $\tau^{-} \rightarrow K^{-} K^{-} K^{+} \nu_{\tau}$ MC Truth and Reconstructed MC for the $M_{123}$ Invariant Mass Spectra. . . . . . . . . . . . . . . 249

F.2 A Comparison of the $\tau^{-} \rightarrow K^{-} K^{-} K^{+} \nu_{\tau}$ MC Truth and Reconstructed MC for the $M_{13 a n d 23}$ Invariant Mass Spectra. . . . . . . . . . . . . . 252

G.1 The $\tau^{-} \rightarrow e^{-} \nu_{\tau} \bar{\nu}_{e}$ and $\tau^{-} \rightarrow \mu^{-} \nu_{\tau} \bar{\nu}_{\mu}$ Laboratory Momentum . . . . . 263

G.2 The $\tau^{-} \rightarrow \pi^{-} \nu_{\tau}$ and $\tau^{-} \rightarrow K^{-} \nu_{\tau}$ Laboratory Momentum . . . . . . 264

H.1 A 2D Projection of the $K / \pi-h h h$ Plane used for Determining the $\tau$ Direction. . . . . . . . . . . . . . . . 269

H.2 Illustration of the Neutrino Pseudo-Mass. . . . . . . . . . . . . . . . 270

H.3 The $\tau^{-} \rightarrow \pi^{-} \nu_{\tau}$ and $\tau^{-} \rightarrow K^{-} \nu_{\tau}$ Neutrino Pseudo-Mass Distributions. 271

H.4 The $\tau^{-} \rightarrow e^{-} \nu_{\tau} \bar{\nu}_{e}$ and $\tau^{-} \rightarrow \mu^{-} \nu_{\tau} \bar{\nu}_{\mu}$ Neutrino Pseudo-Mass Distribu-

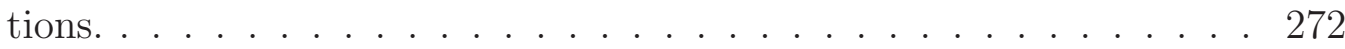




\section{Acknowledgements}

I would like to thank all the professors and students from the University of Victoria in Particle Physics, who have made this analysis possible. Special thanks goes to my supervisor, Dr. J. M. Roney, his Post-Doc, Dr. Swagato Banerjee and Dr. Kim Maltman, for his theoretical expertise and the training which he provided. I would like to thank my fellow members in the BABAR Collaboration for their contributions which have made this work possible and the extraordinary contributions of my PEPII colleagues in achieving the excellent luminosity and machine conditions for the BABAR Experiment. The success of this analysis also relied critically on the expertise and dedication of the computing organizations and facilities that support $B A B A R$, especially the University of Victoria Computing who maintained the Mercury Cluster. As a memeber of the BABAR Collaboration I wish to thank SLAC for its support and the kind hospitality extended to me. 


\section{Dedications}

This thesis is dedicated to

my parents 


\section{Chapter 1}

\section{Introduction}

This dissertation probes fundamental aspects of the charge current sector of the Standard Model using $\tau$ decays. In particular, measurements of the parameter $\left|V_{u s}\right|$ and a tests of lepton universality are performed. $\tau$ decays, which contain hadrons (h), namely pions and kaons, provide a unique opportunity to probe the relative coupling strength of the weak current to the first and second generations of quarks with unprecedented precision [1]. In particular, by measuring $\tau$ decays with an odd number of kaons (non-zero strangeness), the coupling of the $W^{ \pm}$mediator of the weak interaction to the up and strange quarks ${ }^{1},\left|V_{u s}\right|$, and the mass of the strange quark can be extracted within the framework of the Standard Model of particle physics [2-6]. This work is part of a collaborative effort within the BABAR Collaboration to measure the branching fractions and invariant mass distributions for all of the strange $\tau$ decays in order to determine $\left|V_{u s}\right|$ and the strange quark mass. A precision measurement of the ratio $\frac{\mathcal{B}\left(\tau^{-} \rightarrow \mu^{-} \nu_{\tau} \bar{\nu}_{\mu}\right)}{\mathcal{B}\left(\tau^{-} \rightarrow e^{-} \nu_{\tau} \bar{\nu}_{e}\right)}$ tests $\mu$-e charged current lepton universality, while measurements of the branching ratios $\frac{\mathcal{B}\left(\tau^{-} \rightarrow \pi^{-} \nu_{\tau}\right)}{\mathcal{B}\left(\pi^{-} \rightarrow \mu^{-}-\bar{\nu}_{\mu}\right)}$ and $\frac{\mathcal{B}\left(\tau^{-} \rightarrow K^{-} \nu_{\tau}\right)}{\mathcal{B}\left(K^{-} \rightarrow \mu^{-}-\bar{\nu}_{\mu}\right)}$ tests $\tau-\mu$ charged current lepton universality in non-strange and strange mesons respectively. In this dissertation, precision measurements of $\tau^{-} \rightarrow \pi^{-} \pi^{-} \pi^{+} \nu_{\tau}, \tau^{-} \rightarrow K^{-} \pi^{-} \pi^{+} \nu_{\tau}, \tau^{-} \rightarrow K^{-} \pi^{-} K^{+} \nu_{\tau}$,

\footnotetext{
${ }^{1}$ The relative coupling strength of the $W^{ \pm}$boson to the quark mass eigenstates is described by the Cabibbo-Kobayashi-Maskawa Matrix (CKM Matrix).
} 
$\tau^{-} \rightarrow K^{-} K^{-} K^{+} \nu_{\tau}, \tau^{-} \rightarrow \phi \pi^{-} \nu_{\tau}, \tau^{-} \rightarrow \phi K^{-} \nu_{\tau}, \tau^{-} \rightarrow K^{-} \nu_{\tau}, \tau^{-} \rightarrow \pi^{-} \nu_{\tau}$, and $\tau^{-} \rightarrow \mu^{-} \nu_{\tau} \bar{\nu}_{\mu}$ decays are made using data collected at the BABAR Detector. Deviations from the Standard Model values of $\left|V_{u s}\right|$ or lepton universality would indicate the presence of new physics. Combining recent $\tau$ branching fraction measurements from BABAR and Belle, including those described here, results in a three standard deviation difference in the value of $\left|V_{u s}\right|$ as determined using operator product expansion and finite energy sum rules. The simpler and theoretically independent approach of measuring $\left|V_{u s}\right|$ from $\frac{\mathcal{B}\left(\tau^{-} \rightarrow K^{-} \nu_{\tau}\right)}{\mathcal{B}\left(\tau^{-} \rightarrow \pi^{-} \nu_{\tau}\right)}$, presented in this dissertation, yields a result within one standard deviation of the Standard Model expectation. The lepton universality tests yield reported here are all within three standard deviations of Standard Model expectations.

Chapter 2 contains a brief introduction to the Standard Model of particle physics and an overview of the theoretical frame work required to extract the coupling parameter $\left|V_{u s}\right|$ and the strange quark mass from hadronic $\tau$ decays. In Chapter 3, a description of the experimental apparatus, the BABAR Detector and the PEP-II $e^{+} e^{-}$ collider, is given.

One of the dominant experimental uncertainties in the in the determination of $\left|V_{u s}\right|$ using $\tau$ decay arises from the uncertainty on $\mathcal{B}\left(\tau^{-} \rightarrow K^{-} \pi^{-} \pi^{+} \nu_{\tau}\right)$. To make a precision measurement of this channel, all of the $\tau^{-} \rightarrow h^{-} h^{-} h^{+} \nu_{\tau}$ events were measured in a combined analysis to account for the cross contamination. Chapter 4 presents the procedure, systematic uncertainties and results for the $\tau^{-} \rightarrow h^{-} h^{-} h^{+} \nu_{\tau}$ branching fraction measurements, where $\mathrm{h}=\mathrm{K}$ or $\pi$. The $\tau^{-} \rightarrow K^{-} \pi^{-} \pi^{+} \nu_{\tau}$ branching fraction was one of the dominant experimental uncertainties in the strange spectral density function and thus a dominant uncertainty for extracting $\left|V_{u s}\right|$. In the $\tau^{-} \rightarrow h^{-} h^{-} h^{+} \nu_{\tau}$ analysis, it was noticed that the $\tau^{-} \rightarrow K^{-} \pi^{-} K^{+} \nu_{\tau}$ and $\tau^{-} \rightarrow$ $K^{-} K^{-} K^{+} \nu_{\tau} \quad K^{+} K^{-}$invariant mass distributions had a $\phi$ resonance. Therefore, as 
described in Chapter 5, the $\phi$ peak is measured with a binned maximum likelihood fit, and the $\mathcal{B}\left(\tau^{-} \rightarrow \phi \pi^{-} \nu_{\tau}\right)$ and $\mathcal{B}\left(\tau^{-} \rightarrow \phi K^{-} \nu_{\tau}\right)$ branching fractions are extracted and an upper limit on the $\mathcal{B}\left(\tau^{-} \rightarrow K^{-} K^{-} K^{+} \nu_{\tau}[\right.$ ex. $\left.\phi]\right)$ is determined. The invariant mass distributions that correspond to the $\tau^{-} \rightarrow h^{-} h^{-} h^{+} \nu_{\tau}$ branching fractions discussed in Chapter 4 are unfolded to correct for detector effects that can bias the distribution. These are presented in Chapter 6. The invariant mass distributions are unfolded using a Bayesian Technique [7] and a complete set of systematic uncertainties are developed. When $\left|V_{u s}\right|$ was updated with the branching fraction results from the $\tau^{-} \rightarrow h^{-} h^{-} h^{+} \nu_{\tau}$ analysis, and the recent BABAR [8,9] and Belle [10] results, the extracted Cabibbo-Kobayashi-Maskawa value of $\left|V_{u s}\right|$ differed more than three standard deviations from the unitarity value required by the Standard Model. To investigate the possibility that this deviation was evidence for new physics associated with the $\tau$ lepton, a problem with the technique, or a statistical fluctuation an alternative method of determining $\left|V_{u s}\right|$ from the $\frac{\mathcal{B}\left(\tau^{-} \rightarrow K^{-} \nu_{\tau}\right)}{\mathcal{B}\left(\tau^{-} \rightarrow \pi^{-} \nu_{\tau}\right)}$ branching ratio is used. Measurements of $\frac{\mathcal{B}\left(\tau^{-} \rightarrow \mu^{-} \nu_{\tau} \bar{\nu}_{\mu}\right)}{\mathcal{B}\left(\tau^{-} \rightarrow e^{-} \nu_{\tau} \bar{\nu}_{e}\right)}, \frac{\mathcal{B}\left(\tau^{-} \rightarrow \pi^{-} \nu_{\tau}\right)}{\mathcal{B}\left(\tau^{-} \rightarrow e^{-} \nu_{\tau} \bar{\nu}_{e}\right)}$, and $\frac{\mathcal{B}\left(\tau^{-} \rightarrow K^{-} \nu_{\tau}\right)}{\mathcal{B}\left(\tau^{-} \rightarrow e^{-} \nu_{\tau} \bar{\nu}_{e}\right)}$ are also preformed as they provide competitive tests of lepton universality, an assumption, in the Standard Model, that the charged leptons all have the same coupling to the $W^{ \pm}$boson. Chapter 7 contains the procedure, systematic uncertainties and the results for the $\frac{\mathcal{B}\left(\tau^{-} \rightarrow K^{-} \nu_{\tau}\right)}{\mathcal{B}\left(\tau^{-} \rightarrow \pi^{-} \nu_{\tau}\right)}$, $\frac{\mathcal{B}\left(\tau^{-} \rightarrow K^{-} \nu_{\tau}\right)}{\mathcal{B}\left(\tau^{-} \rightarrow e^{-} \nu_{\tau} \bar{\nu}_{e}\right)}, \frac{\mathcal{B}\left(\tau^{-} \rightarrow \pi^{-} \nu_{\tau}\right)}{\mathcal{B}\left(\tau^{-} \rightarrow e^{-} \nu_{\tau} \bar{\nu}_{e}\right)}$, and $\frac{\mathcal{B}\left(\tau^{-} \rightarrow \mu^{-} \nu_{\tau} \bar{\nu}_{\mu}\right)}{\mathcal{B}\left(\tau^{-} \rightarrow e^{-} \nu_{\tau} \bar{\nu}_{e}\right)}$ branching ratio measurements. Chapter 8 discusses the results and their implications for the Standard Model, while the conclusion is in Chapter 9.

For the analysis presented in this thesis, I was the primary analyst. As the primary analyst, I developed the strategy, selection procedure, and the systematic uncertainties associated with the measurements. The software for this analysis, as with most analyses in BABAR, had two stages. First, there is the "BABAR Framework" which interfaces with the detector, reconstructs the data, generates the Monte 
Carlo simulated data, and has some initial event selection and classification. Then there is the analysis software which is developed by the primary analysts for their analysis. I was responsible for the development and validation of this software for this work. This analysis software was composed of several standalone $\mathrm{C}++$ objectoriented programs, which utilized libraries from ROOT, a data analysis program that is commonly used in particle physics, and generalized unfolding software package that was developed within the BABAR Collaboration. The systematic uncertainties, conducted for this work, are designed to account for uncertainties associated with the detector, the event selection, the Monte Carlo simulated data as well as possible errors in the software. The design and construction of the BABAR Detector had been completed before I began my participation in this experiment. However, as a member of the $B A B A R$ Collaboration, I have contributed to many other published $B A B A R$ papers by determining the tracking efficiency systematic for the collaboration and by participating in data acquisition. For the tracking efficiency, I maintained the tracking efficiency study, which I developed for my Master Thesis, and updated the results for the newly measured data. I have contributed to the acquisition of data both as the "Data Quality Manager" and as the "Shift Leader". 


\section{Chapter 2}

\section{Motivation: Strange $\tau$ Decays in the Standard Model}

The Standard Model of Particle Physics is the most accurately tested model in the history of science and it describes all of the current experimental measurements of particle physics. The Standard Model is a relativistic renormalizable quantum field theory that is based on the principles of gauge invariance which describes the elementary particles and their interactions through the electro-magnetic force, the weak force, and the strong force.

\subsection{Introduction to the Standard Model of Parti- cle Physics}

The two main classifications of particles in the Standard Model are bosons, integer spin particles, and fermions, half integer spin particles, where spin may be defined as the intrinsic quantized angular momentum of a particle and is one of the labels of the irreducible representations of the Poincaré Group ${ }^{1}$. To satisfy the principle of causality, bosons are quantized with the commutator relation ${ }^{2}$ and fermions are

\footnotetext{
${ }^{1}$ The Poincaré Group is the algebraic group that special relativity is based on.

${ }^{2}$ In quantum mechanics the commutator relation for two operators that do not commute is: $[\hat{a}, \hat{b}]=\hat{a} \hat{b}-\hat{b} \hat{a}=\imath \hbar[11]$.
} 
quantized with the anti-commutator relation ${ }^{3}$. In the Standard Model, the force carriers are described by the bosons and the particles that matter is composed of are described by fermions. Table 2.1 shows the fundamental particles of the Standard Model.

\begin{tabular}{|l|l|l|l|l|}
\hline \multicolumn{5}{|c|}{ Mediators of the Interactions (Bosons) } \\
\hline \hline Force & Particle & Charge & Mass $\left(\mathrm{GeV} / c^{2}\right)$ & Couples With \\
\hline Strong & Gluon $(\mathrm{g})$ & 0 & 0 & Quarks, g \\
\hline $\begin{array}{l}\text { Electro- } \\
\text { magnetic }\end{array}$ & Photon $(\gamma)$ & 0 & 0 & $\begin{array}{l}\text { Charged Parti- } \\
\text { cles }\end{array}$ \\
\hline Weak & $W^{ \pm}$ & \pm 1 & $(80.398 \pm 0.025)$ & $\begin{array}{l}\text { Fermions, Z } \\
\gamma\end{array}$ \\
\cline { 2 - 5 } & $Z^{0}$ & 0 & $(91.1876 \pm 0.0021)$ & Fermions, $W^{ \pm}$ \\
\hline Mass & Higgs $\left(\mathrm{H}^{0}\right)$ & 0 & $\begin{array}{l}M_{H}>114.4 \mathrm{GeV} @ 95 \% C L \\
M_{H}<211 \mathrm{GeV} @ 95 \% C L\end{array}$ & $\begin{array}{l}\text { Massive Parti- } \\
\text { cles }\end{array}$ \\
\hline
\end{tabular}

\begin{tabular}{|c|c|c|c|c|}
\hline \multicolumn{5}{|c|}{ Fundamental Fermions } \\
\hline Family & Particle & Charge & "Mass $\left(\mathrm{GeV} / c^{2}\right)$ & Couples With \\
\hline \multirow[t]{6}{*}{ Leptons } & Electron (e) & -1 & $\begin{array}{l}(5.10998910 \\
\pm 0.00000013) \times 10^{-4}\end{array}$ & $\gamma, W^{ \pm}, Z^{0}$ \\
\hline & e Neutrino $\left(\nu_{e}\right)$ & 0 & $\mathrm{~m}<2 \times 10^{-6}$ & $W^{ \pm}, Z^{0}$ \\
\hline & Muon $(\mu)$ & -1 & $(0.105658367 \pm 0.000000004)$ & $\gamma, W^{ \pm}, Z^{0}$ \\
\hline & $\mu$ Neutrino $\left(\nu_{\mu}\right)$ & 0 & $\mathrm{~m}<0.19 \times 10^{-3}, \mathrm{CL}=90 \%$ & $W^{ \pm}, Z^{0}$ \\
\hline & $\operatorname{Tau}(\tau)$ & -1 & $(1.77684 \pm 0.00017)$ & $\gamma, W^{ \pm}, Z^{0}$ \\
\hline & $\tau$ Neutrino $\left(\nu_{\tau}\right)$ & 0 & $\mathrm{~m}<18.2 \times 10^{-3}, \mathrm{CL}=95 \%$ & $W^{ \pm}, Z^{0}$ \\
\hline \multirow[t]{6}{*}{ Quarks } & $\mathrm{Up}(\mathrm{u})$ & $2 / 3$ & $(1.5$ to 3.3$) 10^{-3}$ & $\gamma, W^{ \pm}, Z^{0}, g$ \\
\hline & Down $(\mathrm{d})$ & $-1 / 3$ & $(3.5$ to 6.0$) 10^{-3}$ & $\gamma, W^{ \pm}, Z^{0}, g$ \\
\hline & Charm (c) & $2 / 3$ & $1.27_{-0.11}^{+0.07}$ & $\gamma, W^{ \pm}, Z^{0}, g$ \\
\hline & Strange (s) & $-1 / 3$ & $(0.070$ to 0.155$)$ & $\gamma, W^{ \pm}, Z^{0}, g$ \\
\hline & Top $(\mathrm{t})$ & $2 / 3$ & $171.2 \pm 2.1$ & $\gamma, W^{ \pm}, Z^{0}, g$ \\
\hline & Bottom (b) & $-1 / 3$ & $4.20_{-0.07}^{+0.17}$ & $\gamma, W^{ \pm}, Z^{0}, g$ \\
\hline
\end{tabular}

Table 2.1: The Fundamental Forces and Particles in the Standard Model [12].

In addition to these particles, the Standard Model also includes anti-particles. Anti-particles have the same mass but opposite charge and quantum numbers to

\footnotetext{
${ }^{3}$ In quantum mechanics the anti-commutator relation for two operators that do not anti-commute is: $[\hat{a}, \hat{b}]=\hat{a} \hat{b}+\hat{b} \hat{a}=\imath \hbar[11]$.
} 
that of their corresponding particle. Each of the three forces, which are relevant for subatomic particles, the electro-magnetic force, the weak force and the strong force are described in the Standard Model.

\subsubsection{Introduction to Electroweak Theory Introduction to Quantum Electrodynamics}

The electromagnetic force is described by Quantum Electrodynamics (QED), a quantized renormalizable field theory that utilizes Lorentz invariance, charge invariance, parity invariance, time reversal invariance and local gauge invariance [13, pg. 311]. The gauge symmetry that describes the electromagnetic force is the Abelian local gauge group $\mathrm{U}(1)$ where the electromagnetic current is the conserved current. In quantum electrodynamics there is one carrier of force, the photon, corresponding to the one generator in the gauge group $\mathrm{U}(1)$. Because the photon is a massless particle, it has an infinite range and travels at the maximal speed allowed by special relativity.

\section{Quantum Electrodynamics and Weak Theory}

The weak force and the electromagnetic interactions are described by the local gauge symmetries $\mathrm{SU}_{L}(2) \times \mathrm{U}(1)$. The subscript $L$ in non-Abelian gauge group $\mathrm{SU}_{L}(2)$ denotes that the force carriers can only couple to left handed fermions. The charged generators in the $\mathrm{SU}_{L}(2)$ group correspond to the $W^{+}$and $W^{-}$gauge bosons, while the photon and the $Z^{0}$ correspond to a linear combination of the neutral $\mathrm{SU}_{L}(2)$ and $\mathrm{U}(1)$ group generators. The charged $W^{ \pm}$and $Z^{0}$ force carriers have a finite mass and therefore, a short range of approximately $\frac{1}{100} \mathrm{GeV}^{-1}$. The non-commutative nature of non-Abelian groups allows the force carriers to self couple. The photon has a cou-

pling strength of $\alpha_{E M}=\frac{1}{137} \sim 10^{-2}$. In contrast, the $W^{ \pm}$and the $Z^{0}$ bosons have a weak coupling strength of $\alpha_{W} \sim 10^{-6}$. 


\section{Perturbation and Electroweak Theory}

In the Standard Model, the coupling occurring in the interaction Hamiltonian ${ }^{4}$ for the weak and electromagnetic forces is small. As a result, the interaction Hamiltonian can be used as the basis for perturbative ${ }^{5}$ expansion of the observable quantities. Observable quantities that can be calculated using this method include the cross section $^{6}$ or the decay rate ${ }^{7}$ for a particular interaction. Employing the former method, Feynman invented a method for organizing perturbative calculations called "Feynman Diagrams". Feynman Diagrams, which represent how the interaction occurs in the corresponding perturbative series, consist of a final and an initial state with a number of intermediate states, depending on the order of the corresponding term in the perturbative series. The intermediate states in a Feynman Diagram may be on shell, a "real particle", or off shell, a "virtual particle". A particle which is off shell is virtual because the particle does not satisfy $P^{2}=M^{2}$, where $M$ is the mass of the particle and $P$ is the 4-momentum of the particle. When a particle is off shell, its contribution is suppressed relative to an on shell particle. An example of a Feynman Diagram is shown in Figure 2.1, depicts the lowest order process contributing to the perturbative expansion of the amplitude for $e^{+} e^{-} \rightarrow \tau^{+} \tau^{-}$, which is the primary production mechanism for $\tau$ particles in the BABAR Detector. The amplitude for this process, when expanded in a perturbative series, yields the lowest order contribution depicted in the figure, where the Feynman Diagram occurring there is a short-hand for a product of factors, one for each of the two vertices, $e^{+} e^{-} \rightarrow \gamma$ and $\gamma \rightarrow \tau^{+} \tau^{-}$ and one for the intermediate propagating photon.

\footnotetext{
${ }^{4}$ The Hamiltonian for a given system, is the energy operator in that system.

${ }^{5}$ Perturbation theory is a method in which measurable parameters of the interactions are decomposed into a series that converges rapidly.

${ }^{6}$ The cross section, for a particular interaction, is the rate at which the interaction occurs normalized to a unit of incident flux.

${ }^{7}$ The decay rate is the mean rate of decay for a given particle.
} 


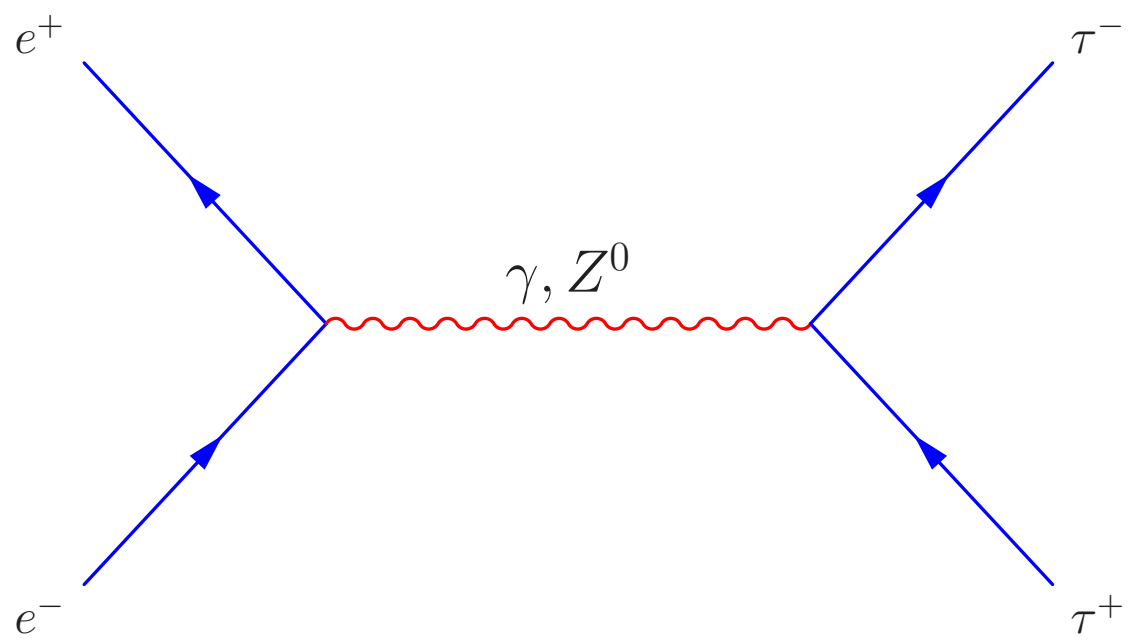

Figure 2.1: The dominant Feynman Diagram for the $e^{+} e^{-} \rightarrow \tau^{+} \tau^{-}$interaction.

The above diagram is the dominant Feynman Diagram for the $e^{+} e^{-} \rightarrow \tau^{+} \tau^{-}$ interaction. This is because the coupling constant, for the electromagnetic force, is small, thus in all electromagnetic interactions, where the terms of $n$th order are proportional to $\alpha_{E M}^{n}$, the higher order terms will be suppressed relative to the lower order terms resulting in the perturbative series converging rapidly. Similarly, the weak force has a small coupling constant causing all the perturbative series for weak interactions to also converge rapidly. However, for weak interactions that involve quarks, the reliability of the perturbative expression can be complicated by the strong interaction.

\subsubsection{Renormalization and the Standard Model}

The theories which, when unified, form the Standard Model are renormalizable theories $^{8}$. This means that there are a finite number of constants that can be redefined to remove the ultra violet divergences which result from internal particle loops in higher order Feynman Diagrams for the Greens Functions of the theory. These effec-

\footnotetext{
${ }^{8}$ In the 1970 's 't Hooft proved that all gauge theories are renormalizable [14, pg. 210].
} 
tive parameters account for the effect of the particle interactions with the vacuum. In the Standard Model, the effective parameters are the coupling constants and the masses of the particles. In addition, these effective parameters are dependent upon the energy scale of the renormalization. The energy scale at which these effective parameters are evaluated is called the renormalization scale. Thus, the higher the energy probe of a parameter, the less the vacuum effects screen the parameter changing the effective parameter value. A renormalization scheme commonly used in QCD is the Modified Minimal Subtraction (M̄S) Scheme [15].

\subsubsection{Introduction to Quantum Chromodynamics}

In the Standard Model the strong interaction is described by the non-Abelian local gauge group $\mathrm{SU}(3)$ in a renormalizable theory called Quantum Chromodynamics (QCD). The gauge group SU(3) has eight generators, and thus there are eight types of force carriers known as gluons. These gluons, although massless and electrically neutral, carry a form of charge associated with the strong force called "colour". Colour charge is the conserved charge of the $\mathrm{SU}(3)$ group. Gluons have one colour charge and one anti-colour charge, while quarks carry either one colour or one anticolour. There are three charges of colour, red (R), blue (B) and green $(\mathrm{G})$ and three complimentary colours, anti-red $(\bar{R})$, anti-blue $(\bar{B})$ and anti-green $(\bar{G})$. Because the quarks and anti-quarks can have three colour configurations and the gluons can have 8 colour configurations they correspond to multiplets, "irreducible representations of $\mathrm{SU}(3)$ ", of dimension $3,3^{*}$ and 8 respectively. When particles represented by the multiplets are bound together, they are represented by the product of their associated irreducible representation. Particular products of the previously mentioned multiplets contain the dimension 1 multiplet, or colour singlet state. This construction of colour singlets is important in QCD because only colourless objects have been 
observed in nature. This "confinement of colour" is believed to be a result of the non-Abelian and massless nature of the gluon and the scale dependence of the physical couplings, a property of renormalization. More specifically, at low momentum scales, or large distances, the coupling strength is large and the non-perturbative effects are important, while at high momentum scales, or short distance scales, QCD has a weak coupling with a asymptotic freedom. This means that the force between the two quarks grows with the distance. Eventually, there is enough energy that a quark and anti-quark pair, with the appropriate charge and colour, can be created from the quantum vacuum resulting in two colour singlets. This is called "quark confinement". These composite particles of quarks and gluons are called hadrons. At low momentum scales where the strong coupling constant is the largest, the strong force interaction can not be calculated with the perturbative technique used for the Electroweak force at all energy scales. The gluons belong to a representation of a non-Abelian group with a strong coupling constant, $\alpha_{S} \sim 1$ [16]. In QCD, the strong coupling constant depends strongly on the renormalzation scale, becoming increasingly larger as the $Q^{2}$ decreases until it diverges. The renormalization scale at which $\alpha_{S}$ diverges, is called $\Lambda_{Q C D}$. $\Lambda_{Q C D}$ has been difficult to determine experimentally and its value depends on the renormalization scheme, but it is around the typical hadronic mass, $0.1 \mathrm{GeV}<\Lambda_{Q C D}<0.5 \mathrm{GeV}$ in natural units ${ }^{9}[13-15]$.

\subsubsection{Introduction to the Lepton Sector}

The Lepton family consists of 3 generations, the electron and electron neutrino, the muon and muon neutrino, and the tau and tau neutrino. Because leptons do not interact through the strong force, they can exist as free particles. The electron is the lightest and the only stable charged lepton while the $\tau$ and $\mu$ 's are unstable particles

\footnotetext{
${ }^{9}$ Natural units are a set of measurement units in which $c=\hbar=1$.
} 
and decay weakly. The $\tau$ particle is the only lepton heavy enough to decay into hadrons.

The Standard Model description of the leptons is based on "the premise that the only physical difference among the charge leptons is that of mass" [15, pg. 145]. This premise is called Lepton Universality. Stated another way, Lepton Universality means that leptons of different generations couple with the same strength to the $W^{ \pm}$boson. [17, pg. 195-196]. This universality can be exploited experimentally, when comparing the ratio of lepton interaction rates since the mass independent terms cancel reducing the theoretical uncertainty. Moreover, the lepton flavour, or the generation, is a conserved quantity in the Standard Mode ${ }^{10}$. This conservation principle is the result of empirical evidence.

\subsubsection{Introduction to the Quark Sector}

In contrast to leptons, the quarks and anti-quarks are contained in composite particles called hadrons. The particles in hadrons consist of valence quarks, sea-quarks and gluons. There are two main types of hadrons which have been observed, mesons which consist of a valence quark anti-quark pair and baryons which consist of three valence quarks. Both the quark and the gluon fields that bind them form colour singlets. The gluon field also produces quark anti-quark pairs called "sea-quarks". These quarks are radiated and absorbed by the gluon field [13, pg. 198].

In addition to the strong force, the quarks inside the hadrons also interact via the electromagnetic and weak forces. In electromagnetic interactions with hadrons, the coupling strength of the photon to the hadron or valence quarks is unaltered by the strong interactions and sea-quarks. This is a result of the Conservation of Charge.

\footnotetext{
${ }^{10}$ The Standard Model, when extended to include massive neutrinos can allow for violation of charged lepton flavour at the $\mathcal{O}\left(10^{-54}\right)$ level.
} 
However, this is not necessarily true for the weak interactions. In the Standard Model, it is assumed that in hadronic interactions, the weak charge is conserved through interactions with the vector current and thus the coupling strength of the vector current component of the weak interaction is unaltered in hadronic interactions. This assumption is known as the Conserved Vector Current Hypothesis (CVC). The axial vector component of the weak interaction is only protected by a "partial" conservation law called the Partial Conserved Axial Current (PCAC). This means that there are small alterations to the coupling strength of the axial vector component of the weak force in interactions with hadrons. Both CVC and PCAC are based upon empirical evidence $[13-15,17]$.

For the weak force, an interaction through either an emission or an absorption of a $W^{ \pm}$boson results in a quark state which is a linear combination of the mass eigenstates mentioned in Table 2.1. In other words, the initial state and final state pairs of quarks may be written as

$$
\left[\begin{array}{l}
u \\
d^{\prime}
\end{array}\right],\left[\begin{array}{c}
c \\
s^{\prime}
\end{array}\right] \text {, and }\left[\begin{array}{c}
t \\
b^{\prime}
\end{array}\right]
$$

where $d^{\prime}, s^{\prime}$, and $b^{\prime}$, referred to as the weak eigenstates, are a linear combination of the $d, s$, and $b$ quarks. The relationship between the weak eigenstates and the mass eigenstates is described by the transforms matrix called the Cabibbo-KobayashiMaskawa Matrix (CKM Matrix)

$$
\left[\begin{array}{c}
d^{\prime} \\
s^{\prime} \\
b^{\prime}
\end{array}\right]=\left[\begin{array}{ccc}
V_{u d} & V_{u s} & V_{u b} \\
V_{c d} & V_{c s} & V_{c b} \\
V_{t d} & V_{t s} & V_{t b}
\end{array}\right]\left[\begin{array}{l}
d \\
s \\
b
\end{array}\right] .
$$

The unitarity constraints on the CKM Matrix means that there are four free pa- 
rameters including one phase, commonly described by the Wolfenstein parameterization [18]. It is the complex phase in the CKM Matrix that is responsible for the violation of the charge parity symmetry ( $\mathrm{CP}$ violation) in the Standard Model. The violation of the $\mathrm{CP}$ symmetry is the source of the asymmetry between particles and their anti-particles [19]. The charge parity symmetry may be decomposed into two simpler symmetries: charge conjugation and parity. Charge conjugation is a symmetry which connects particles to their anti-particle. Parity is a discrete symmetry about the spatial origin which "flips" the sign of the spatial coordinates, and hence the helicity ${ }^{11}$ of a particle.

\subsection{Hadronic $\tau$ Decays and the Extraction of $\left|V_{u s}\right|$ and $m_{s}$}

\subsubsection{Introduction to Hadronic $\tau$ Decays}

As discussed in Section 2.1.4, the $\tau$ lepton is the most massive lepton, and is the only lepton massive enough to decay into hadronic particles. This provides a unique opportunity for probing the coupling strength of the weak current to the first and second generation of quarks to a very high precision. At the $\tau$ mass, $(1776.84 \pm$ $0.17) \mathrm{MeV} / \mathrm{c}^{2}$, the inclusive spectral integral can be evaluated on an equivalent integral in the complex plane where the energy scale, $s$, is sufficiently large that perturbative QCD can be used. This means that experimental measurements can be compared to theoretical predictions to extract both the CKM Matrix element $\left|V_{u s}\right|$ and the strange quark mass. This section details the theoretical tools and techniques required to extract these parameters. Natural units will be assumed for the calculations in this section.

\footnotetext{
${ }^{11}$ Helicity is the projection of the spin vector of a particle onto the particle's momentum vector.
} 


\subsubsection{The Leptonic and Hadronic Decay Width of the $\tau$ Lep- ton}

The generalized differential decay width for an unpolarized $\tau$ lepton is described in terms of the matrix element and the kinematics of the decay process:

$$
\Gamma=\frac{1}{2 m_{\tau}} \sum_{f} \int \frac{d^{3} p_{\nu_{\tau}}}{(2 \pi)^{3} 2 E_{\nu_{\tau}}^{2}} \frac{|M|^{2}}{2}(2 \pi)^{4} \delta\left(p_{\tau}-p_{\nu_{\tau}}-q\right)
$$

where $m_{\tau}$ is the $\tau$ mass, $E_{\nu_{\tau}}$ is the energy of the $\tau$ neutrino, $p_{\tau}$ and $p_{\nu_{\tau}}$ are the momenta of the $\tau$ and $\tau$ neutrino and, the sum over this index, $f$, indicates a sum over all possible spin states. From Figure 2.2, it can be seen that for the leptonic decay of a $\tau$ particle into an electron, the matrix element may be written as ${ }^{12}$

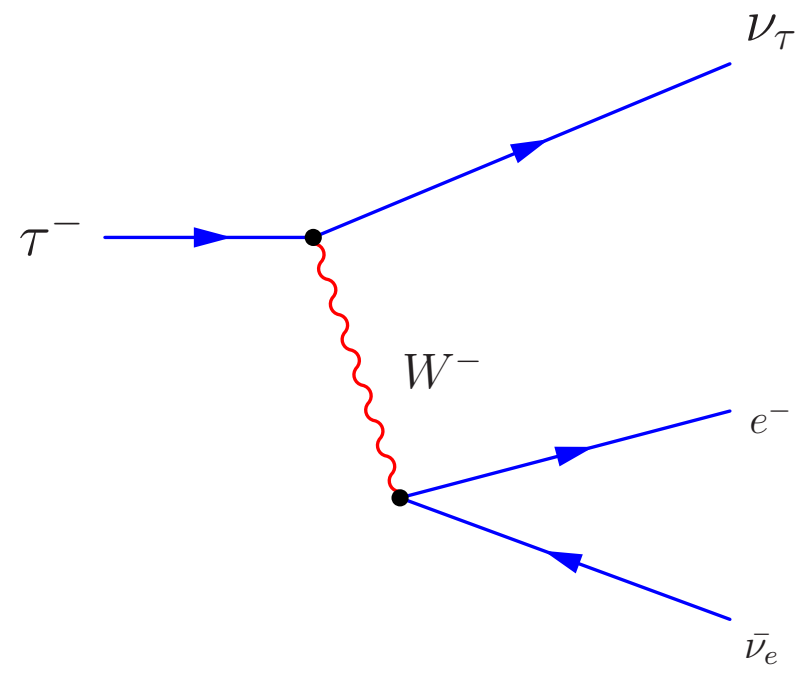

Figure 2.2: A Feynman Diagram of $\tau^{-} \rightarrow e^{-} \nu_{\tau} \overline{\nu_{e}}$.

\footnotetext{
${ }^{12}$ In the matrix element, factors of $\imath$ and -1 are commonly neglected since only the square of the matrix element is used to calculate the decay width.
} 


$$
\begin{aligned}
\mathcal{M}= & {\left[\bar{u}_{e}\left(p_{e}\right)\left(-\imath \frac{g_{W}}{2^{3 / 2}} \gamma^{\nu}\left(g_{V}-g_{A} \gamma^{5}\right)\right) v_{\overline{\nu_{e}}}\left(p_{\overline{\nu_{e}}}\right)\right] \frac{\imath g_{\mu \nu}}{M_{W}^{2}} } \\
& \times\left[\bar{u}_{\nu_{\tau}}\left(p_{\nu_{\tau}}\right)\left(-\imath \frac{g_{W}}{2^{3 / 2}} \gamma^{\mu}\left(g_{V}-g_{A} \gamma^{5}\right)\right) u_{\tau}\left(p_{\tau}\right)\right] \\
= & {\left[\bar{u}_{e}\left(p_{e}\right)\left(p_{\overline{\nu_{e}}}\right) \gamma^{\mu}\left(g_{V}-g_{A} \gamma_{5}\right) v_{\overline{\nu_{e}}}\right] \frac{g_{w}^{2}}{8 M_{W}^{2}}\left[\bar{u}_{\nu_{\tau}}\left(p_{\nu_{\tau}}\right) \gamma_{\mu}\left(g_{V}-g_{A} \gamma_{5}\right) u_{\tau}\left(p_{\tau}\right)\right] } \\
= & L_{(e)}^{\mu} \frac{g_{w}^{2}}{8 M_{W}^{2}} L_{(\tau) \mu} .
\end{aligned}
$$

$u(\bar{u})$ is the Dirac propagator for the particle (anti- particle) indicated by the relevant index, and

$$
L_{(i) \mu}=\left[\bar{u}_{\nu_{i}}\left(p_{\nu_{i}}\right) \gamma_{\mu}\left(g_{V}-g_{A} \gamma_{5}\right) u_{i}\left(p_{i}\right)\right]
$$

is the leptonic tensor where $g_{V}=g_{A}=1$ are the vector $(\mathrm{V})$ and axial vector $(\mathrm{A})$ couplings of the $W^{ \pm}$boson in the Standard Model. Noting that $p_{W}=p_{e}+p_{\nu_{e}}$, adding the electroweak radiative corrections, and utilizing Equation 2.3, the radiative inclusive decay width of the $\tau^{-} \rightarrow e^{-} \nu_{\tau} \bar{\nu}_{e}$ is

$$
\Gamma\left[\tau^{-} \rightarrow e^{-} \nu_{\tau} \bar{\nu}_{e}(\gamma)\right]=\frac{G_{F}^{2} m_{\tau}^{5}}{192 \pi^{3}} f\left(\frac{m_{e}^{2}}{m_{\tau}^{2}}\right)\left[1+\frac{3 m_{\tau}^{2}}{5 M_{W}^{2}}\right]\left[1+\frac{\alpha_{E M}\left(m_{\tau}\right)}{2 \pi}\left(\frac{25}{4}-\pi^{2}\right)\right](2.6
$$

where $G_{F}=\frac{1}{2^{5 / 2}}\left(\frac{g_{W}}{M_{W}}\right)^{2}[13,14], f(x)=1-8 x+8 x^{3}-x^{4}-12 x^{2} \log x$, under the assumption that the neutrino masses are negligible, and $m_{e}$ is the electron mass [20]. By replacing the $m_{e}$ with the muon mass, $m_{\mu}$, Equation 2.6 can also be used to describe the $\tau^{-} \rightarrow \mu^{-} \nu_{\tau} \bar{\nu}_{\mu}(\gamma)$ decay width [20].

The hadronic decay width can be derived in an analogous manner to the electron decay width by replacing the leptonic tensor for the electron and electron antineutrino and the associated Standard Model coupling constants, $g_{V}=g_{A}=1$, with the hadronic tensor 


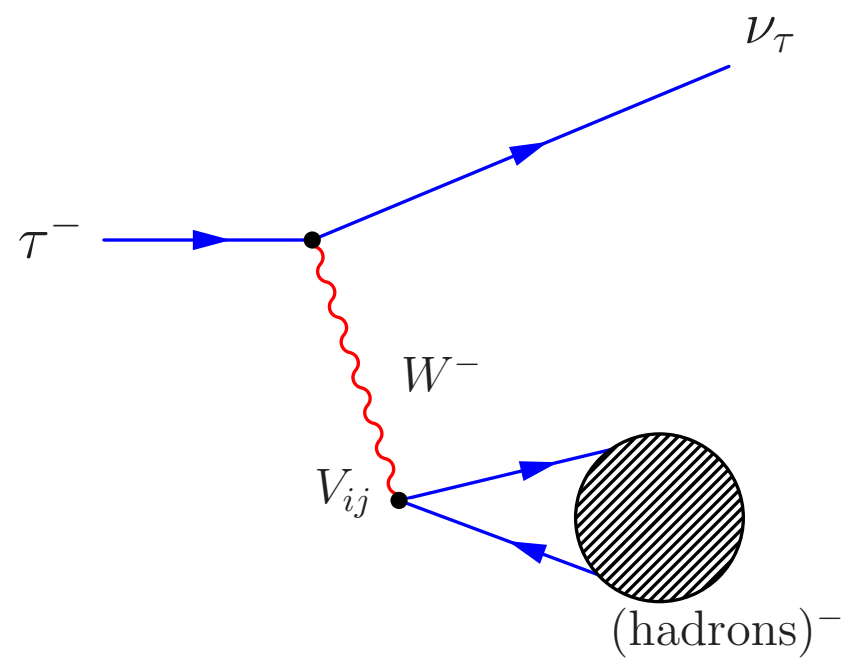

Figure 2.3: A Feynman Diagram of $\tau^{-} \rightarrow$ hadrons $^{-} \nu_{\tau}$.

$$
H^{\mu}=\left\langle h_{f}(q)\left|J^{\mu}(x)\right| 0\right\rangle=\left\langle\text { hadrons }_{f}(q)\left|V^{\mu}(x)-A^{\mu}(x)\right| 0\right\rangle
$$

and the appropriate CKM element for the coupling of the $W^{ \pm}$boson to the relevant quark pair, $i j=u d$ or $i j=u s$, which are generated from the vacuum. From this, it can be seen that the inclusive matrix element for the Feynman Diagram seen in Figure 2.3 is

$$
\mathcal{M}=\left[\left\langle\text { hadrons }_{f}(q)\left|J^{\mu}(x)\right| 0\right\rangle\right] V_{i j} \frac{1}{8 M_{W}^{2}}\left[g_{w}^{2}\right] \bar{u}_{\nu}\left(p^{\nu}\right) \gamma_{\mu}\left(1-\gamma_{5}\right) u_{\tau}\left(p_{\tau}\right) .
$$

In the hadronic matrix elements, the current $J_{\mu}$ consists of two components: the axial current $A_{\mu}$ and the vector current $V_{\mu}$. Both the axial and vector currents couple to spin 1 and spin 0 states. The expectation value of the current between the vacuum and a spin 0 state, $\left|q, \alpha_{0}\right\rangle$, necessarily has the form 


$$
\left\langle 0\left|J^{\mu}(0)\right|\left(q, \alpha_{0}\right)\right\rangle=\imath F_{\alpha_{0}}\left(m_{\alpha_{0}}^{2}\right) q^{\mu}
$$

where $F_{\alpha_{0}}\left(m_{\alpha_{0}}^{2}\right)$ is a scalar function of the only available variable, $q^{2}=m_{\alpha_{0}}^{2}$, the invariant mass squared of the hadronic state $\alpha_{0}$. Subscripts on $\alpha$ indicate the spin. The label $\alpha$ includes internal momenta for the multi-particle states, $\alpha$, and $q^{\mu}$ is the four vector of the hadronic system. Similarly, the expectation value of the current between the vacuum and a spin 1 state, $\left|q, \lambda, \alpha_{1}\right\rangle$, has to be linear in the polarization vector, $\epsilon_{\lambda}^{\mu}(q)$ of the state and therefore it necessarily has the form

$$
\left\langle 0\left|J^{\mu}(0)\right|\left(q, \lambda, \alpha_{1}\right)\right\rangle=F_{\alpha_{1}}\left(m_{\alpha_{1}}^{2}\right) \epsilon_{\lambda}^{\mu}(q) .
$$

Therefore, the hadronic matrix element squared, $H^{\mu \nu}=H^{\mu} H^{\nu \dagger}$, becomes

$$
\begin{aligned}
\sum_{f}\left\langle 0\left|J^{\mu}(0)\right| f\right\rangle\left\langle f\left|J^{\nu \dagger}(0)\right| 0\right\rangle= & \sum_{\left(q, \alpha_{0}\right)}\left|F_{\alpha_{0}}\left(m_{\alpha_{0}}\right)\right|^{2} q^{\nu} q^{\mu} \\
& +\sum_{\left(q, \alpha_{1}, \lambda\right)}\left|F_{\alpha_{1}}\left(m_{\alpha_{1}}\right)\right|^{2} \epsilon_{\lambda}^{\mu}(q) \epsilon_{\lambda}^{\nu \star}(q) \\
= & \sum_{\left(q, \alpha_{0}\right)}\left|F_{\alpha_{0}}\left(m_{\alpha_{0}}\right)\right|^{2} q^{\nu} q^{\mu} \\
& +\sum_{\left(q, \alpha_{1}\right)}\left|F_{\alpha_{1}}\left(m_{\alpha_{1}}\right)\right|^{2}\left(-g^{\mu \nu}+\frac{q^{\mu} q^{\nu}}{q^{2}}\right) .
\end{aligned}
$$

Contracting the leptonic trace, $L_{\mu \nu}=L_{\mu} L_{\nu}^{\dagger}$, with the 2 kinematic combinations $q^{\mu} q^{\nu}$ and $\left(q^{\mu} q^{\nu}-g^{\mu \nu} q^{2}\right)$ from above and using the Mandelstam variable $s=q^{2}$, yields the following expression for $\Gamma\left(\tau \rightarrow \nu_{\tau}\right.$ hadron $\left._{V / A ; i j}\right)$ : 


$$
\begin{aligned}
\Gamma\left(\tau \rightarrow \nu_{\tau} \text { hadrons }_{V / A ; i j}\right)= & \frac{G_{F}^{2} m_{\tau}^{3}\left|V_{i j}\right|^{2}}{16 \pi} \int_{0}^{m_{\tau}^{2}} d s\left(1-\frac{s}{m_{\tau}^{2}}\right)^{2}\left[\left(1+2 \frac{s}{m_{\tau}^{2}}\right)\right. \\
& \times \sum_{\left(q, \alpha_{1}\right)} \frac{1}{m_{\alpha_{1}^{2}}}\left|F_{\alpha_{1}}\left(m_{\alpha_{1}}\right)\right|^{2} \delta\left(m_{\alpha_{1}}^{2}-s\right) \\
& \left.+\sum_{\left(q, \alpha_{0}\right)}\left|F_{\alpha_{0}}\right|^{2}\left(m_{\alpha_{0}}\right) \delta\left(m_{\alpha_{0}}^{2}-s\right)\right] .
\end{aligned}
$$

The structure of the polynomial and $\left(1-\frac{s}{m_{\tau}^{2}}\right)^{2}$ appearing in $\left(1-\frac{s}{m_{\tau}^{2}}\right)^{2}\left(1+2 \frac{s}{m_{\tau}^{2}}\right)$ Equation 2.12 is a consequence of the kinematic structure of the lepton and hadronic tensors in the Standard Model. The partial decay width of $\tau$ to hadrons for the vector/axial vector currents is typically written by experimentalists using Tsai's notation $[21]$

$$
\begin{aligned}
\Gamma\left(\tau \rightarrow \nu_{\tau} \text { hadron }_{V / A ; i j}\right)= & \frac{G_{F}^{2} m_{\tau}^{3}\left|V_{i j}\right|^{2}}{32 \pi^{2}} \int_{0}^{m_{\tau}^{2}} d s\left(1-\frac{s}{m_{\tau}^{2}}\right)^{2} \\
& \times\left[\left(1+2 \frac{s}{m_{\tau}^{2}}\right) v / a_{1}^{i j}(s)+v / a_{0}^{i j}(s)\right] \\
= & \frac{G_{F}^{2} m_{\tau}^{3}\left|V_{i j}\right|^{2}}{32 \pi^{2}} \int_{0}^{m_{\tau}^{2}} d s\left(1-\frac{s}{m_{\tau}^{2}}\right)^{2} \\
& \times\left[\left(1+2 \frac{s}{m_{\tau}^{2}}\right) v / a_{0+1}^{i j}(s)-\frac{2 s}{m_{\tau}^{2}} v / a_{0}^{i j}(s)\right] 2
\end{aligned}
$$

where $v / a_{0}^{i j}(s)$ and $v / a_{1}^{i j}(s)$ are defined by

$$
\begin{array}{r}
v_{0}^{i j}(s)=\sum_{\left(\alpha_{0}\right)} 2 \pi \delta\left(m_{\alpha_{0}}^{2}-s\right)\left|F_{\alpha 0}^{V}\left(m_{\alpha_{0}}^{2}\right)\right|^{2} \\
v_{1}^{i j}(s)=\sum_{\left(\alpha_{1}\right)} 2 \pi \delta\left(m_{\alpha_{1}}^{2}-s\right) \frac{1}{m_{\alpha_{1}}^{2}}\left|F_{\alpha_{1}}^{V}\left(m_{\alpha_{1}}^{2}\right)\right|^{2} \\
a_{0}^{i j}(s)=\sum_{\left(\alpha_{0}\right)} 2 \pi \delta\left(m_{\alpha_{0}}^{2}-s\right)\left|F_{\alpha_{0}}^{A}\left(m_{\alpha_{0}}^{2}\right)\right|^{2} \\
a_{1}^{i j}(s)=\sum_{\left(\alpha_{1}\right)} 2 \pi \delta\left(m_{\alpha_{1}}^{2}-s\right) \frac{1}{m_{\alpha_{1}}^{2}}\left|F_{\alpha_{1}}^{A}\left(m_{\alpha_{1}}^{2}\right)\right|^{2}
\end{array}
$$


and $v / a_{0+1}^{i j}(s)=v / a_{0}^{i j}(s)+v / a_{1}^{i j}(s)[21]$. The significance of Tsai's notation, its physical interpretation and its relation to the current-current correlator will be discussed in the next section. For comparison of experiment and theory in later sections, it is useful to define the hadronic width as the ratio of the partial decay width of $\tau$ decaying into hadrons to the partial width of $\tau$ decaying into an electron for vector and axial vector currents and flavour content $i j$,

$$
\begin{aligned}
R_{\tau, V / A, i j}= & \frac{\Gamma\left(\tau \rightarrow \text { hadrons }_{V / A, i j}+\nu_{\tau}(\gamma)\right)}{\Gamma\left(\tau \rightarrow \mathrm{e} \overline{\mathrm{e}}_{\mathrm{\tau}} \nu_{\tau}(\gamma)\right)} \\
= & \frac{6 \pi\left|V_{i j}\right|^{2} S_{E W}}{m_{\tau}^{2}} \int_{0}^{m_{\tau}^{2}} d s\left(1-\frac{s}{m_{\tau}^{2}}\right)^{2}\left[\left(1+2 \frac{s}{m_{\tau}^{2}}\right) v / a_{0+1}^{i j}(s)\right. \\
& \left.-\frac{2 s}{m_{\tau}^{2}} v / a_{0}^{i j}(s)\right]
\end{aligned}
$$

where $S_{E W}$ is the electroweak correction factor. In addition, the differential hadronic width

$$
\begin{aligned}
\frac{d R_{\tau, V / A, i j}}{d s}= & \frac{\Gamma\left(\tau \rightarrow \text { hadrons }_{V / A, i j}+\nu_{\tau}(\gamma)\right)}{\Gamma\left(\tau \rightarrow \mathrm{e} \overline{\mathrm{e}}_{\mathrm{e}} \nu_{\tau}(\gamma)\right)} \frac{1}{N_{V / A, i j}} \frac{d N_{V / A, i j}}{d s} \\
= & \frac{6 \pi\left|V_{i j}\right|^{2} S_{E W}}{m_{\tau}^{2}}\left(1-\frac{s}{m_{\tau}^{2}}\right)^{2}\left[\left(1+2 \frac{s}{m_{\tau}^{2}}\right) v / a_{0+1}^{i j}(s)\right. \\
& \left.-\frac{2 s}{m_{\tau}^{2}} v / a_{0}^{i j}(s)\right]
\end{aligned}
$$

where $\frac{d N_{V / A, i j}}{d s}$ is the invariant mass spectra of the final state hadrons of flavour $i j$ for the relevant vector or axial vector current.

\subsubsection{The Current-Current Correlator and the Spectral Den- sity Function}

The current-current correlator or 2-point function is defined as

$$
\left\langle 0\left|T\left(J^{\mu}(x) J^{\dagger \nu}(y)\right)\right| 0\right\rangle
$$


where $\mu$ and $\nu$ are the Lorentz indices of the current. $T$ is the Time-ordering symbol. In Equation 2.21, the current $J$ is composed of construction and destruction operators. Since only terms in which the construction operator is acting on the ket state, $|0\rangle$, and the destruction operator is acting on the bra state, $\langle 0|$, are non-zero, the correlator describes the amplitude of a current $J$ creating a state at a position $y$ and propagating to a position $x$ in space-time ${ }^{13}$ [22], where it is destroyed. The time-ordered product may be written as

$$
\begin{aligned}
\left\langle 0\left|T\left(J^{\mu}(x) J^{\nu \dagger}(0)\right)\right| 0\right\rangle= & \int \frac{d^{3} p}{(2 \pi)^{3} 2 E_{p}} \sum_{f}\left[\Theta\left(x_{0}\right) e^{-\imath p \cdot x}\right. \\
& \times\left\langle 0\left|J^{\mu}(0)\right| h_{f}(p)\right\rangle\left\langle h_{f}(p)\left|J^{\nu \dagger}(0)\right| 0\right\rangle+\Theta\left(-x_{0}\right) e^{\imath p \cdot x} \\
& \left.\times\left\langle h_{f}(p)\left|J^{\nu \dagger}(0)\right| 0\right\rangle\left\langle h_{f}(p)\left|J^{\mu}(0)\right| 0\right\rangle\right]
\end{aligned}
$$

by inserting a complete set of states, $\sum_{f}\left|h_{f}(q)\right\rangle\left\langle h_{f}(q)\right|$, with $E_{p}=\left(m_{f}^{2}+|\vec{p}|^{2}\right)^{\frac{1}{2}}$. The factors $e^{\mp \imath p x}$ result from employing translation invariance on the current $J^{\mu}(x)$, $J^{\mu}(x)=e^{\imath \hat{P} \cdot x} J^{\mu}(x) e^{-\imath \hat{P} \cdot x}$. Since the state's coupling to the axial and vector current, in the sum relevant to hadronic $\tau$ decays, only run over the spin 0 and spin 1 , one can simplify the expression by inserting Equations 2.9 and 2.10. This yields

$$
\begin{aligned}
\left\langle 0\left|T\left(J^{\mu}(x) J^{\nu \dagger}(0)\right)\right| 0\right\rangle= & \sum_{\left(q, \alpha_{0}\right)} \int \frac{d^{3} p}{(2 \pi)^{3} 2 E_{p}}\left[\Theta\left(x_{0}\right) e^{-\imath p \dot{x}}+\Theta\left(-x_{0}\right) e^{\imath p \dot{x}}\right] \\
& \left|F_{\alpha_{0}}\left(m_{\alpha_{0}}\right)\right|^{2} p^{\nu} p^{\mu} \\
& +\sum_{\left(q, \alpha_{1}, \lambda\right)} \int \frac{d^{3} p}{(2 \pi)^{3} 2 E_{p}}\left[\Theta\left(x_{0}\right) e^{-\imath p \dot{x}}+\Theta\left(-x_{0}\right) e^{\imath p \dot{x}}\right] \\
& \left|F_{\alpha_{1}}\left(m_{\alpha_{1}}\right)\right|^{2}\left(-g^{\mu \nu}+\frac{p^{\mu} p^{\nu}}{p^{2}}\right),
\end{aligned}
$$

where $E_{p}$ is now defined as $E_{P}\left(m_{\alpha_{J}}\right)=\left(m_{\alpha_{J}}^{2}+|\vec{p}|^{2}\right)$. In the latter equation, the term $\int \frac{d^{3} p}{(2 \pi)^{3} 2 E_{p}}\left[\Theta\left(x_{0}\right) e^{-\imath p \dot{x}}+\Theta\left(-x_{0}\right) e^{\imath p \dot{x}}\right]$ is simply the Feynman propagator, which can

\footnotetext{
${ }^{13}$ Minkowski space-time is the four dimensional topology of Special Relativity.
} 
also be written as $\int \frac{d^{4} p}{2 \pi^{4}} \frac{\imath e^{-\imath p x}}{p^{2}-m_{\alpha}^{2}+\imath \epsilon}$. Therefore, one can insert the unity, $1=\int_{0}^{\infty} d s \delta(s-$ $\left.m_{\alpha_{J}}^{2}\right)$, into each term of the sum to obtain

$$
\begin{aligned}
\left\langle 0\left|T\left(J^{\mu}(x) J^{\nu \dagger}(0)\right)\right| 0\right\rangle= & \int_{0}^{\infty} d s \int \frac{d^{4} p}{2 \pi^{4}} \frac{\imath e^{-\imath p x}}{p^{2}-m_{\alpha}^{2}+\imath \epsilon} \times \\
& {\left[\sum_{\left(q, \alpha_{0}\right)}\left|F_{\alpha_{0}}\left(m_{\alpha_{0}}\right)\right|^{2} p^{\nu} p^{\mu}\right.} \\
& \left.+\sum_{\left(q, \alpha_{1}\right)}\left|F_{\alpha_{1}}\left(m_{\alpha_{1}}\right)\right|^{2}\left(-g^{\mu \nu}+\frac{p^{\mu} p^{\nu}}{p^{2}}\right)\right] .
\end{aligned}
$$

Performing a Fourier Transform to get the momentum space current-current 2-point function yields

$$
\begin{aligned}
\Pi^{\mu \nu} \equiv & \imath \int d^{4} x e^{\imath q x}\left\langle 0\left|T\left(J^{\mu}(x) J^{\nu \dagger}(0)\right)\right| 0\right\rangle \\
= & q^{\mu} q^{\nu}\left(\int_{0}^{\infty} d s \frac{\rho_{i j ; V / A}^{\prime(0)}(s)}{s-q^{2}-\imath \epsilon}\right) \\
& +\left(-g^{\mu \nu}+\frac{q^{\mu} q^{\nu}}{q^{2}}\right)\left(\int_{0}^{\infty} d s \frac{\rho_{i j ; V / A}^{(1)}(s)}{s-q^{2}-\imath \epsilon}\right) \\
= & q^{\mu} q^{\nu}\left(\int_{0}^{\infty} d s \frac{\rho_{i j ; V / A}^{(0)}(s)}{s-q^{2}-\imath \epsilon}\right) \\
& +\left(-g^{\mu \nu} q^{2}+q^{\mu} q^{\nu}\right)\left(\int_{0}^{\infty} d s \frac{\rho_{i j ; V / A}^{(1)}(s)}{s-q^{2}-\imath \epsilon}\right)
\end{aligned}
$$

where $\rho^{J}(s)$ are the Kallen-Lehmann Spectral Density Functions, which represent the coupling strength of all the states of spin $J$ to the given currents as a function of invariant mass squared of the system $s$. The relevant spectral density functions appearing in Equation 2.26 in hadronic $\tau$ decays are 


$$
\begin{array}{r}
\rho_{i j ; V}^{(0)}(s)=\frac{v_{0}^{i j}(s)}{2 \pi}=\sum_{\left(\alpha_{0}\right)} \delta\left(m_{\alpha_{0}}^{2}-s\right)\left|F_{\alpha 0}^{V}\left(m_{\alpha_{0}}^{2}\right)\right|^{2} \\
\rho_{i j ; V}^{(1)}(s)=\frac{v_{1}^{i j}(s)}{2 \pi}=\sum_{\left(\alpha_{1}\right)} \delta\left(m_{\alpha_{1}}^{2}-s\right) \frac{1}{m_{\alpha_{1}}^{2}}\left|F_{\alpha_{1}}^{V}\left(m_{\alpha_{1}}^{2}\right)\right|^{2} \\
\rho_{i j ; A}^{(0)}(s)=\frac{a_{0}^{i j}(s)}{2 \pi}=\sum_{\left(\alpha_{0}\right)} \delta\left(m_{\alpha_{0}}^{2}-s\right)\left|F_{\alpha_{0}}^{A}\left(m_{\alpha_{0}}^{2}\right)\right|^{2} \\
\rho_{i j ; A}^{(1)}(s)=\frac{a_{1}^{i j}(s)}{2 \pi}=\sum_{\left(\alpha_{1}\right)} \delta\left(m_{\alpha_{1}}^{2}-s\right) \frac{1}{m_{\alpha_{1}}^{2}}\left|F_{\alpha_{1}}^{A}\left(m_{\alpha_{1}}^{2}\right)\right|^{2} .
\end{array}
$$

At this point, it is useful to define the $\mathrm{J}=1$ and 0 scalar correlator functions:

$$
\begin{aligned}
\Pi^{\mu \nu}\left(q^{2}\right) & =q^{\mu} q^{\nu} \Pi^{\prime(0)}\left(q^{2}\right)+\left(\frac{q^{\mu} q^{\nu}}{q^{2}}-g^{\mu \nu}\right) \Pi^{\prime(1)}\left(q^{2}\right) \\
& =q^{\mu} q^{\nu} \Pi^{(0)}\left(q^{2}\right)+\left(q^{\mu} q^{\nu}-g^{\mu \nu} q^{2}\right) \Pi^{(1)}\left(q^{2}\right)
\end{aligned}
$$

where $\Pi^{(1)}\left(q^{2}\right)$ and $\Pi^{\prime(1)}\left(q^{2}\right)$ are two different conventions that are related by $1 / q^{2}$. The scalar correlators are directly related to the spectral density function

$$
\Pi^{\prime(0 / 1)}\left(q^{2}\right) \sim \int_{t h}^{\infty} d s \frac{\rho^{(0 / 1)}(s)}{s-q^{2}-\imath \epsilon}
$$

where $\sim$ indicates that one or more subtractions may be required for the integral containing the spectral density function to converge. The existence of these spectral representations, in Equation 2.34, follow from the completeness of states and Poincare invariance. From the definition of the spectral density function in Equations 2.28 and 2.30, it can be seen that the isolated single particle state in the spectral density function corresponds to a pole in the positive real $q^{2}$ axis in the scalar correlator, while the multi-particle states correspond to a branch cut on the positive real $q^{2}$ axis in the scalar correlator. To see this latter point note that, from Cauchy's Theorem, we have the identity that: 


$$
\begin{array}{r}
\frac{1}{s-q^{2} \pm \imath \epsilon}=\mathcal{P} \frac{1}{s-q^{2}} \pm \imath \pi \delta\left(s-q^{2}\right) \\
\Rightarrow \operatorname{Im}\left(\Pi^{\prime}\left(q^{2} \pm \imath \epsilon\right)\right)= \pm \pi \rho\left(q^{2}\right)
\end{array}
$$

for the scalar correlator functions. Equivalently,

$$
\rho^{(0 / 1)}\left(q^{2}\right)=\frac{1}{\pi} \Im m\left(\Pi^{\prime(0 / 1)}\left(q^{2}+\imath \epsilon\right)\right) .
$$

This also means that the Tsai's spectral density function notation corresponds to the discontinuity across the real axis in the imaginary part of the correlator.

\subsubsection{Vector and Axial Vector Contributions to the Spectral Density Function}

The states contributing to the spectral density function for the vector current are

$$
\begin{aligned}
& v_{0 / 1}^{u d}: 2 \pi, 4 \pi, 6 \pi, K \bar{K}, K \bar{K} \pi, \ldots \\
& v_{0 / 1}^{u s}: K \pi, K 2 \pi, K 3 \pi, K 4 \pi, K 5 \pi, K K \bar{K}, K K \bar{K} \pi, \ldots
\end{aligned}
$$

For axial vector current, the states contributing to the spectral density function are

$$
\begin{aligned}
& a_{0 / 1}^{u d}: \pi, 3 \pi, 5 \pi, K \bar{K} \pi, \ldots \\
& a_{0 / 1}^{u s}: K, K 2 \pi, K 3 \pi, K 4 \pi, K 5 \pi, K K \bar{K} \pi, \ldots
\end{aligned}
$$

The assignment of decay modes consisting entirely of an even or an odd number of pions to the vector or axial vector current induced transitions is a result of Gparity $^{14}$ and its conservation in QCD. The G-parity of the QCD vacuum and vector current is even, $(+1)$, while the G-parity of the pions and the axial currents are odd, $(-1)$.

\footnotetext{
${ }^{14} \mathrm{G}$-parity is the combination of the charge conjugation operator and a rotation of $\pi$ about the $I_{2}$ axis in isospin space $\left(G=C e^{-\imath \pi I_{2}}\right)$ and is conserved in strong interactions. The isospin space utilized in G-parity is only relevant to mesons formed from $u$ and $d$ quarks. Therefore, mesons that contain a $s, c, b$, or $t$ quark are not eigenstates of G-parity.
} 


\subsubsection{QCD Sum Rules in Hadronic $\tau$ Decays}

When the correlator is defined, so that the only singularities are from the poles and discontinuities caused from physical states along the positive $q^{2}$ axis, then from Cauchy's Theorem the integral about the curve C illustrated in Figure 2.4 is equal to

$$
\oint_{C} d s w(s) \Pi(s)=\oint_{|s|=s_{0}} d s w(s) \Pi(s)+2 \pi \imath \int_{s_{t h}}^{s_{0}} d s \rho(s) w(s)=0
$$

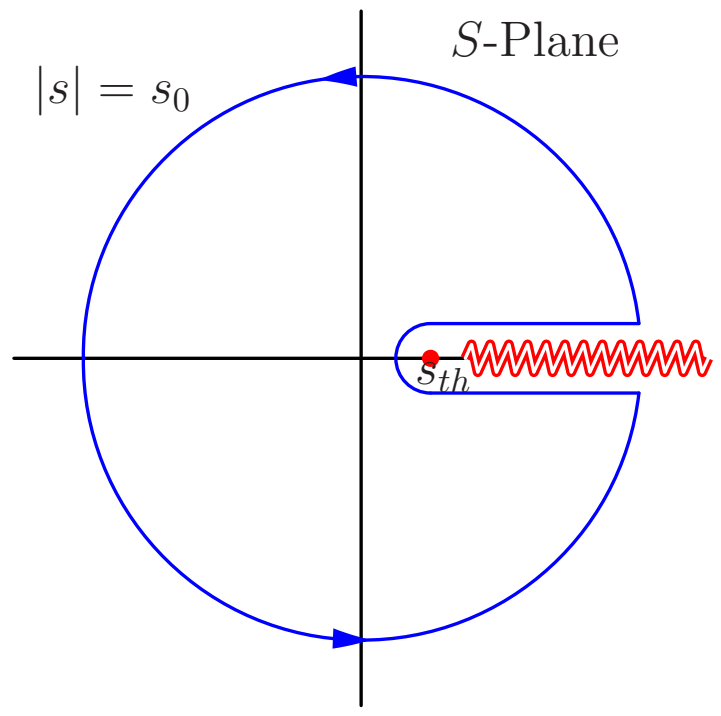

Figure 2.4: The Sum Rule Integration Curve for the current-current correlator in hadronic $\tau$ decays. The curve represents the integration path, while the single dot and zigzag line represents the pole and the discontinuity starting at $s=s_{t h}$.

where $w(s)$ is any analytic function, since there are no poles or branch cuts inside the curve C. From this, one obtains the Finite Energy Sum Rules (FESR):

$$
-\frac{1}{2 \pi \imath} \oint_{|s|=s_{0}} d s w(s) \Pi(s)=\int_{s_{t h}}^{s_{0}} d s \rho(s) w(s) .
$$


QCD Sum Rules are Sum Rules in which one has also included the constraints of asymptotic freedom and kinematic restrictions, $q^{2}>>\Lambda_{Q C D}^{2}$, allowing the Operator Product Expansion (OPE) representation of the correlator to be employed on the LHS of the equation with coefficients calculated using perturbative QCD.

Since, in QCD, the scalar correlators $\Pi_{V / A ; i j}^{(0,1)}$ defined in Equation 2.35 are known to have kinematic singularities, it is necessary to work with combinations which do not have such singularities if one wishes to employ the basic FESR relation from Equation 2.43. It is thus common to see FESRs involving hadronic $\tau$ decay data written using the combinations $\Pi_{V / A ; i j}^{(0+1)} \equiv \Pi_{V / A ; i j}^{(0)}+\Pi_{V / A ; i j}^{(1)}$ and $s \Pi_{V / A ; i j}^{(0)}$, which are known explicitly not to have kinematic singularities in $\mathrm{QCD}{ }^{15}$ for both the axial and vector cases. The combination $\Pi_{V / A ; i j}^{(0+1)} \equiv \Pi_{V / A ; i j}^{(0)}+\Pi_{V / A ; i j}^{(1)}$ is known as the longitudinal plus transverse component of the correlator while $s \Pi_{V / A ; i j}^{(0)}$ is referred to as the longitudinal component of the correlator. For $q^{2}>>\Lambda_{Q C D}^{2}$, these components of the correlator can be computed by perturbative QCD with a theoretical technique called the Operator Product Expansion (OPE) [23, 24].

\subsubsection{The Operator Product Expansion}

The OPE is an operator relation that is employed for short distance expansions to write a product of operators at small separations, $x$, in terms of a standard linearly independent basis of local operators whose coefficients carry the $x$ dependence of the original operators [22]. Explicitly,

\footnotetext{
${ }^{15}$ Kinematic singularities are singularities in the scalar parts of the correlator not associated with the existence of a real physical state. Such singularities can result from the use of a particular algebraic representation for the correlator structure. For example, in QCD, the scalar correlator $\Pi_{V}^{\prime(0)}\left(q^{2}\right)$ only has singularities associated with physical states, but the unprimed convention for the correlator $\Pi_{V}^{(1)}\left(q^{2}\right)=\Pi_{V}^{\prime(1)}\left(q^{2}\right) / q^{2}$ has a singularity at $q^{2}=0$ where there is no zero mass vector state.
} 


$$
\mathcal{O}_{1}(x, \mu) \mathcal{O}_{2}(0, \mu)=\sum_{n=0}^{\infty} \mathcal{C}_{12}^{n}(x, \mu) \mathcal{O}_{n}(\mu)
$$

where $\mu$ is the normalization scale, the energy scale at which the renormalizable parameters are evaluated. $\mathcal{C}_{12}^{n}(x, \mu)$ is the Wilson Coefficient for the $n$th operator. For small $x$, the Wilson Coefficient describes the high energy effects and therefore can be calculated perturbatively in an expansion of the strong coupling constant, $\alpha_{s}(\mu)$ [22]. The matrix element of the corresponding $n$th operator, $\mathcal{O}_{n}(\mu)$, describes the low energy effects. The standard linearly independent basis is comprised of the set of all Lorentz invariant and gauge invariant operators having the quantum numbers of the original operator product. Fourier transforming to momentum space, the OPE may be written as

$$
\imath \int d^{4} x e^{\imath q x} \mathcal{O}_{1}(x, \mu) \mathcal{O}_{2}(0, \mu)=\sum_{n=0}^{\infty} \mathcal{C}_{12}^{n}(s, \mu) \mathcal{O}_{n}(\mu)
$$

for large $|s|$ since only the Wilson Coefficient depends on the position $x$. By applying dimensional analysis, and using the notation $s=q^{2}=-Q^{2}$, it can be seen that the Wilson Coefficient may be redefined in terms of a dimensionless parameter $\overline{\mathcal{C}}_{n}\left(-Q^{2}, \mu\right)$ :

$$
\mathcal{C}_{12}^{n}\left(-Q^{2}, \mu\right) \mathcal{O}_{n}(\mu)=\frac{\overline{\mathcal{C}}_{12}^{n}\left(-Q^{2}, \mu\right) \mathcal{O}_{n}(\mu)}{Q^{d_{n}-d_{1}-d_{2}}}
$$

where $d_{1}$ is the dimension of the operator $\mathcal{O}_{1}(x, \mu), d_{2}$ is the dimension of the operator $\mathcal{O}_{2}(0, \mu), d_{n}$ is the dimension of the operator $\mathcal{O}(\mu)_{n}[22]$ and the Mandelstam variable $s=q^{2}=-Q^{2}$.

The scalar parts of vector and axial vector correlators, $\Pi_{V / A}^{(j)}\left(q^{2}\right)$, are dimensionless and therefore their OPE has the form 


$$
\Pi^{(J)}\left(-Q^{2}\right)=\sum_{D=0,2, \ldots} \frac{1}{Q^{D}}\left[\sum_{n=1}^{n_{\max }^{D}} \overline{\mathcal{C}}_{12}^{n,(J)}\left(-Q^{2}, \mu\right)\left\langle 0\left|\mathcal{O}(\mu)_{n}\right| 0\right\rangle\right]_{d i m \mathcal{O}_{n}=D}
$$

where $n_{\max }^{D}$ is the number of operators of dimension $D=d_{n}-d_{1}-d_{2}$. This form of the correlator depends on the scale $s=-Q^{2}$ at which it is evaluated. If $s \gg \Lambda_{Q C D}$ then the perturbative series within the OPE converge sufficiently that the matrix element of the operator product can be evaluated reliably given $\alpha_{s}$ at some reference scale [25]. However, if non-perturbative corrections are included it is also necessary to have the values of the condensates, which will be defined later, as input.

In the perturbative evaluation of the coefficients of the OPE, it is assumed that all the gluons carry a large momentum, so that the gluon lines in the Feynman Diagrams are perturbative. However, this neglects the effects of soft collective phenomena (for example, topologically non-trivial gluon configurations called instantons) where large momenta are transferred by multiple gluons with small momenta. Such additional contributions are exponentially suppressed for spacelike $Q^{2}$ but can become oscillatory with only power suppression in the timelike direction. FESRs retain part of the exponential suppression over most of the contour, but still must work at sufficiently large scales that the integrated versions of these collective contributions are small. Model and empirical studies indicate that, if the weights employed have at least a double zero at $s=s_{0}$ then it is sufficient to work with $s_{0}>2 \mathrm{GeV}^{2}[16]$.

The FESR, defined in Equation 2.43, relates the $\Pi(s)$ on the left-hand side to the spectral density function on the right-hand side. From Equation 2.25, it can be seen that the correlator, $\Pi^{\mu \nu}$, involves products of the currents and thus has an OPE representation for $q^{2} \gg \Lambda_{Q C D}$. Hence, the scalar correlators from the FESR also have an OPE representation for $q^{2} \gg \Lambda_{Q C D}$. These latter OPE representations involve vacuum expectation values of operators constructed from gluon field tensors 
and quark bilinear functions $[23,24]$.

The dimension 0 term in the OPE representation of the scalar correlators, $\Pi_{V / A, i j}$, consists of the Naive Parton Model prediction and higher order terms in $\alpha_{s}$. The Naive Parton Model predicts that the correlator for hadronic $\tau$ decays is

$$
\begin{aligned}
& \Pi_{A, i j}^{(1)}(s)=\Pi_{V, i j}^{(1)}(s)=\frac{N_{c}}{12 \pi^{2}} \log (-s)+\mathrm{constant} \\
& \Pi_{A, i j}^{(0)}(s)=\Pi_{V, i j}^{(0)}(s)=0
\end{aligned}
$$

where $N_{c}$ is the number of colour degree of freedom [25]. Figure 2.5 illustrates some of the Feynman Diagrams for the perturbative massless quark QCD correction series.

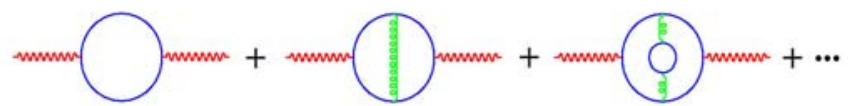

Figure 2.5: The lowest order terms of the dimension 0 series. The solid blue lines represent the quark lines while the curved red line represents the relevant currents and the coiled green lines represent the gluon lines.

There are no Lorentz invariant and gauge invariant operators of dimension 2 [25]. However, the mass corrections proportional to $m_{q}^{2}, m_{q}^{4}, \cdots$ to the dimension 0 operator, in the conventional terminology used in the literature, are treated as being of dimension $2,4, \ldots$. The explicit forms for the $\mathrm{d}=2$ and 4 mass corrections can be found in $[26,27]$.

At dimension 4, in addition to the $\mathcal{O}\left(m_{\alpha}^{4}\right)$ perturbative contributions there are contributions proportional to the quark condensate, $\left\langle m_{j} \bar{\psi}_{i} \psi_{i}\right\rangle$, and the gluon condensate, $\left\langle\frac{\alpha_{s}(\mu)}{\pi} G G\right\rangle$. Feynman Diagrams relevant to obtaining the perturbative expansion of the Wilson Coefficients of the quark condensate and gluon condensate series 
in $\alpha_{s}(\mu)$ can be seen can be seen in Figures 2.6 and 2.7 [26].

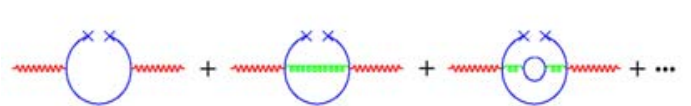

Figure 2.6: The lowest order terms of dimension $4 \propto\left\langle m_{j} \bar{\psi}_{i} \psi_{i}\right\rangle$. The solid blue lines represent the quark lines while the curved red line represents the relevant currents and the coiled green lines represent the gluon lines. The crosses in the Feynman Diagrams represent the creation or annihilation of a particle into the vacuum.

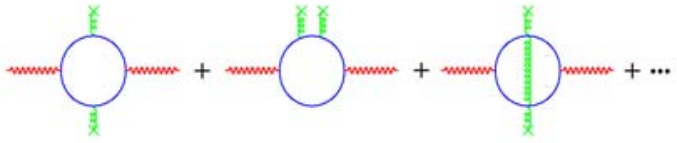

Figure 2.7: The lowest order terms of the dimension $4 \propto\left\langle\frac{\alpha_{s}(\mu)}{\pi} G G\right\rangle$. The solid blue lines represent the quark lines while the curved red line represents the relevant currents and the coiled green lines represent the gluon lines. The crosses in the Feynman Diagrams represent the creation or annihilation of a particle into the vacuum.

At dimension 6 there are 17 quark and gluon operator combinations. The dominant contributions are from the three gluon operators, $\left\langle{ }^{a b c} \mathrm{G}_{\mu}^{a \nu} \mathrm{G}_{\nu}^{b \lambda} \mathcal{G}_{\lambda}^{c \mu}\right\rangle$, and 4 quark operators, $\left\langle\bar{\psi}_{i} \Gamma \psi_{j} \bar{\psi}_{k} \Gamma \psi_{l}\right\rangle$ where $\Gamma$ is the product of the $S U(3)$ colour matrix with a Dirac matrix. The dominant Feynman Diagrams for the 4 quark operators and the three gluon operators can be seen in Figures 2.8 and 2.9. The other 15 operators are formed from combinations of the gluon and quark operators. However, the vacuum expectation values of these operators are believed to be very small relative to the former dimension 6 operators, because of suppression due to the light quark masses. This enables one to safely neglect these terms in hadronic $\tau$ decays [25].

The higher dimension terms for hadronic $\tau$ decay are most commonly neglected since the values of the relevant condensates are typically not known. However, in some cases, it is possible to determine the value of the appropriate linear combination of condensates of a given dimension by fitting to data. It is also possible to suppress such unknown or poorly known condensate contributions through judicious choices of the weights $w(s)$ appearing in the FESRs employed [28]. 


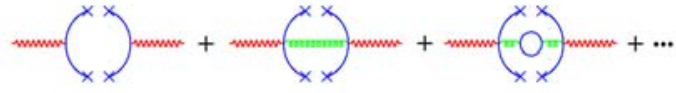

Figure 2.8: The lowest order terms of dimension $6 \mathcal{O}\left(q^{-4}\right)$ Quark Operator Series. The solid blue lines represent the quark lines while the curved red line represents the relevant currents and the coiled green lines represent the gluon lines. The crosses in the Feynman Diagrams represent the creation or annihilation of a particle into the vacuum.

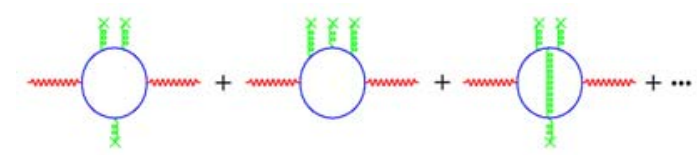

Figure 2.9: The lowest order terms of dimension $6 \mathcal{O}\left(q^{-4}\right)$ three gluon operator series. The solid blue lines represent the quark lines while the curved red line represents the relevant currents and the coiled green lines represent the gluon lines. The crosses in the Feynman Diagrams represent the creation or annihilation of a particle into the vacuum.

\subsubsection{Evaluation of the Correlator and Weights in the FESR}

Taking $\Pi(s)$ in Equation 2.43 to be one of the combinations having no kinematic singularities, namely $\Pi(s)=\Pi_{V / A, i j}^{(0+1)}(s)$, and employing the OPE for this combination as well as a polynomial weight $w(s)=\sum_{m} P_{m}\left(\frac{s}{s_{0}}\right)^{m}$, the left-hand side of the FESR relation becomes

$$
\begin{gathered}
-\frac{1}{2 \pi \imath} \oint_{|s|=s_{0}} d s w(s) \Pi_{V / A, i j}^{(0+1) O P E}(s) \\
=-\frac{1}{2 \pi \imath} \oint_{|s|=s_{0}} d s\left[\sum_{m} P_{m}\left(\frac{s}{s_{0}}\right)^{m}\right] \Pi_{V / A, i j}^{(0+1) O P E}\left(-Q^{2}\right)
\end{gathered}
$$

where the OPE of the correlator has been defined in Equation 2.47. If the leading order contributions to the higher dimension OPE contributions are written in the form $C_{D} / Q^{D}$, with the constant $C_{D}$ representing the effective combination of all condensates of dimension $D$, the weighted integral of this expression becomes

$$
\begin{aligned}
& -\frac{1}{2 \pi \imath} \oint_{|s|=s_{0}} d s w(s) \Pi_{V / A, i j, D}^{(0+1) O P E}(s) \\
= & -\frac{1}{2 \pi \imath} \oint_{|s|=s_{0}} d s\left[\sum_{m} P_{m}\left(\frac{s}{s_{0}}\right)^{m}\right] \frac{C_{D}}{Q^{D}}
\end{aligned}
$$


where $C_{D}$ is the effective condensate. Using the notation $y=\frac{s}{s_{0}}=-x=\frac{-Q^{2}}{s_{0}}$, this reduced to

$$
\begin{aligned}
-\frac{1}{2 \pi \imath} \oint_{|s|=s_{0}} d s w(s)\left[\Pi^{O P E}(s)\right]_{D}= & \frac{s_{0}}{2 \pi \imath} \oint_{|x|=1} d x \sum_{m} P_{m}(-1)^{m} x^{m} \\
& \times \frac{C_{D}}{s_{0}^{k+1} x^{k+1}} \\
= & \frac{1}{2 \pi \imath} \frac{\sum_{m} P_{m}(-1)^{m} C_{D}}{s_{0}^{k}} \\
& \times \oint_{|x|=1} d x \frac{x^{m}}{x^{k+1}}
\end{aligned}
$$

for dimension D. Solving for the contour integral, one obtains

$$
\begin{aligned}
-\frac{1}{2 \pi \imath} \oint_{|s|=s_{0}} d s w(s) \Pi^{O P E}(s) & =\frac{1}{2 \pi \imath} \frac{\sum_{m} P_{m}(-1)^{m} \sum_{D} C_{D}}{s_{0}^{k}}\left[2 \pi \imath \delta_{m k}\right] \\
& =\sum_{k} \frac{P_{k}(-1)^{k} C_{D}}{s_{0}^{k}}
\end{aligned}
$$

From this, it can be seen that if one uses a polynomial weight in the FESR, the term $x^{k}$ will pick out the $\mathrm{D}=2 \mathrm{k}+2$ terms from the OPE. Uncertainties associated with the neglect of $D=8$ and higher OPE contributions can thus be controlled by working with weights having a degree less than 3. Alternately, since the integrated contribution of different dimensions are seen to scale differently with $s_{0}$, one may work with weights of higher degree, neglecting $D>6$ contributions, but test empirically the reliability of this assumption by studying the FESR in question as a function of $s_{0}$.

\subsubsection{Longitudinal Subtraction for Hadronic $\tau$ Decays}

Currently, for all scales kinematically accessible in hadronic $\tau$ decays, the series of known contributions to the $D=2$ term in the OPE of the longitudinal part of the axial and vector current-current correlator is extremely non-convergent. This 
poor convergence of the longitudinal component can be illustrated by looking at the spectral moments

$$
\begin{aligned}
R_{V / A, i j}^{k l}= & 12 \pi^{2}\left|V_{i j}\right|^{2} S_{E W} \int_{0}^{m_{\tau}} \frac{d s}{m_{\tau}^{2}}\left(1-\frac{s}{m_{\tau}^{2}}\right)^{k+2}\left(\frac{s}{m_{\tau}^{2}}\right)^{l} \\
& \times\left[\left(1+2 \frac{s}{m_{\tau}^{2}}\right) \rho_{V / A, i j}^{1+0}-2 \frac{s}{m_{\tau}^{2}} \rho_{V / A, i j}^{0}\right] \\
= & \int_{0}^{m_{\tau}} \frac{d s}{m_{\tau}^{2}}\left(1-\frac{s}{m_{\tau}^{2}}\right)^{k}\left(\frac{s}{m_{\tau}^{2}}\right)^{l} \frac{d R_{\tau, V / A, i j}}{d s}
\end{aligned}
$$

which have historically been used in the QCD Sum Rules for hadronic $\tau$ decays $[5,26,29]$. In the longitudinal component of the spectral moment,

$$
R_{V / A, i j}^{0, k l}=12 \pi^{2}\left|V_{i j}\right|^{2} S_{E W} \int_{0}^{m_{\tau}} \frac{d s}{m_{\tau}^{2}}\left(1-\frac{s}{m_{\tau}^{2}}\right)^{k+2}\left(\frac{s}{m_{\tau}^{2}}\right)^{l}\left[-2 \frac{s}{m_{\tau}^{2}} \rho_{V / A, i j}^{0}\right]
$$

both the kinematic weights and the spectral density function are positive definite where $s$ is defined for the range $\left[0, m_{\tau}^{2}\right]$. Since $0 \leq \frac{s}{m_{\tau}^{2}} \leq 1$ and $0 \leq\left(1-\frac{s}{m_{\tau}^{2}}\right) \leq 1$ over the entire range of integration, $\left|L^{k+1, l}\right|<\left|L^{k, l}\right|$ and $\left|L^{k+1, l}\right|<\left|L^{k, l}\right|$ for $k=$ $0,1,2, \ldots$ and $l=0,1,2, \ldots$ However, when any of the truncation schemes, used in the literature, are employed in the computation of the poorly converged integrated $D=2$ longitudinal series of the OPE corresponding to the spectral moments, this relation is not satisfied. This is a direct "violation of spectral positivity". Since it is not possible to use the OPE reliably to represent $\Pi_{V / A ; i j}^{(0)}(s)$, the contribution of the longitudinal spectral function to the experimentally measured spectral function must be removed, in order to utilize the FESR based on the well behaved $\Pi_{V / A ; i j}^{(0+1)}$ and to extract parameters from the OPE using data. However, there are no current experimental measurements, other than those for the kaon and pion poles, to allow one to perform an experimental longitudinal subtraction ${ }^{16}[4]$. Fortunately, there are

\footnotetext{
${ }^{16} \mathrm{An}$ experimental measurement setting an upper limit on the non-K pole contribution to the strange spectral density function would be useful.
} 
rigorous theoretical constraints from QCD which can help us deal with this problem. In $\mathrm{QCD}$, the divergences of the axial and vector currents are related to the scalar and pseudo-scalar densities by means of the Ward identities

$$
\begin{array}{r}
\partial_{\mu} V_{i j}^{\mu}=\left(m_{i}-m_{j}\right) \bar{q}_{i} q_{j}=\left(m_{i}-m_{j}\right) J_{i j}^{S} \equiv S_{i j} \\
\partial_{\mu} A_{i j}^{\mu}=\left(m_{i}+m_{j}\right) \bar{q}_{i} \imath \gamma_{5} q_{j}=\left(m_{i}+m_{j}\right) J_{i j}^{P} \equiv P_{i j}
\end{array}
$$

where $J_{i j}^{S}$ and $J_{i j}^{P}$ are the flavour $i j$ scalar and pseudo-scalar densities. Thus, in the $\mathrm{SU}(3)$ chiral limit the axial and vector currents are all conserved, $\partial_{\mu} J^{\mu}=0$ where $J^{\mu}$ is either the axial or vector current. With the scalar "decay constants", $F_{\alpha_{0}}\left(q^{2}\right)$, introduced earlier, then

$$
\left\langle 0\left|J^{\mu}(x)\right| \alpha_{0}, q\right\rangle=\imath F_{\alpha_{0}}\left(m_{\alpha_{0}}^{2}\right) q^{\mu} e^{-\imath q \cdot x} .
$$

Since $J^{\mu}$ is a conserved current in the $\mathrm{SU}(3)$ chiral limit, one obtains

$$
\left\langle 0\left|\partial_{\mu} J^{\mu}(x)\right| \alpha_{0}, 0\right\rangle=F_{\alpha_{0}}\left(m_{\alpha_{0}}^{2}\right) q^{2}=F_{\alpha_{0}}\left(m_{\alpha_{0}}^{2}\right) m_{\alpha_{0}}^{2}=0 .
$$

This means that either $F_{\alpha_{0}}\left(m_{\alpha_{0}}^{2}\right)=0$ or $m_{\alpha_{0}}^{2}=0$. In the SU(3) chiral limit, the only massless states are the 8 Goldstone bosons, $\pi^{ \pm}, \pi^{0}, \eta, K^{ \pm}, K^{0}$ and $\bar{K}^{0}$. Hadronic $\tau$ decays only involve the pion, $\pi^{ \pm}$, or kaon, $K^{ \pm}$, state since the other members of the octet do not couple to the $W^{ \pm}$boson. Because the vector current does not couple to the pseudo-scalar states, $F_{\alpha_{0}}=0$ for all spin 0 states in the $\mathrm{SU}(3)$ chiral limit, and hence $\rho_{V, i j}^{(0)}(s)=0$ for all $s$ in the $\mathrm{SU}(3)$ chiral limit. For the axial vector current, in the chiral SU(3) limit, $m_{u}=m_{d}=m_{s}=0$, the pion and the kaon are Goldstone bosons with $m_{\pi}^{2}=m_{K}^{2}=0$ and the corresponding $F_{\alpha_{0}}\left(m_{\alpha_{0}}^{2}\right)$, referred to as $F_{\pi}$ and $F_{K}$ respectively, are non-zero. The decay strength of the pion pole, $F_{\pi}$, and the decay strength of the kaon pole, $F_{K}$, are well known from $\pi_{l 2}$ and $K_{l 2}$ measurements. Excluding these two states, $F_{\alpha_{0}}=0$ for all other pseudo-scalar states since all such 
states have $m_{\alpha_{0}}>0$ in the $\mathrm{SU}(3)$ chiral limit. Away from the $\mathrm{SU}(3)$ chiral limit, the divergence of the axial and vector current of flavour $i j$ in Equations 2.57 and 2.58 become of order $O\left(m_{i} \mp m_{j}\right)$. Therefore, for all states which had $m_{\alpha_{0}}>0$ in the $\mathrm{SU}(3)$ chiral limit, have $F_{\alpha_{0}}=O\left(m_{i} \mp m_{j}\right)$ away from the $\mathrm{SU}(3)$ chiral limit, except the $\pi$ and $\mathrm{K}$ terms. Thus, the contributions to the spectral density function, $\rho_{V / A ; i j}^{0}$, which are proportional to $\left|F_{\alpha_{0}}\right|^{2}$, are of order $O\left(\left(m_{i} \mp m_{j}\right)^{2}\right)$ and hence all double chirally suppressed ${ }^{17}$ compared to the pion and kaon pole contributions.

Hence, away from the chiral limit, the longitudinal subtraction of $-2 y_{\tau}(1-$ $\left.y_{\tau}\right)^{2} \rho_{V / A ; i j}^{(0)}$, contains the unsuppressed pseudo-scalar Goldstone boson contributions and in addition the double chirally suppressed components from the scalar and other pseudo-scalar states. From Equations 2.57, 2.58 and 2.60 the spectral density functions of $\left(m_{i} \mp m_{j}\right)^{2} \Pi_{i j}^{S / P}\left(q^{2}\right)$ and $\rho_{i j}^{S / P}\left(q^{2}\right)$, are equal to $\rho_{i j}^{V / A}\left(q^{2}\right)$. This implies that the FESR for scalar and pseudo-scalar spectral density functions can be used to constrain the FESR of the longitudinal vector and axial vector spectral functions. More precisely, since in $\mathrm{QCD} q^{\mu} q^{\nu} \Pi_{V / A ; i j}^{\mu \nu}=q^{4} \Pi_{V / a ; i j}^{0}\left(q^{2}\right)$ then it can be shown that

$$
\begin{aligned}
& q^{4} \Pi_{V, i j}^{(0)}\left(q^{2}\right)=q_{\mu} q_{\nu} \Pi_{\mu \nu, i j}^{V,(0)}\left(q^{2}\right)=\left(m_{i}-m_{j}\right)^{2}\left[\Pi_{i j}^{S}\left(q^{2}\right)+\left(\left\langle\bar{\psi}_{i} \psi_{i}\right\rangle-\left\langle\bar{\psi}_{j} \psi_{j}\right\rangle\right)\right] \\
& q^{4} \Pi_{A, i j}^{(0)}\left(q^{2}\right)=q_{\mu} q_{\nu} \Pi_{\mu \nu, i j}^{A,(0)}\left(q^{2}\right)=\left(m_{i}+m_{j}\right)^{2}\left[\Pi_{i j}^{P}\left(q^{2}\right)+\left(\left\langle\bar{\psi}_{i} \psi_{i}\right\rangle+\left\langle\bar{\psi}_{j} \psi_{j}\right\rangle\right)\right]
\end{aligned}
$$

where the scalar correlators,

$$
\Pi_{i j}^{S}\left(q^{2}\right)=\imath \int d x e^{\imath q x}\left\langle 0\left|T\left[J_{i j}^{S}(x)\left(J_{i j}^{S}\right)^{\dagger}(0)\right]\right| 0\right\rangle,
$$

and pseudo-scalar correlators,

$$
\Pi_{i j}^{P}\left(q^{2}\right)=\imath \int d x e^{\imath q x}\left\langle 0\left|T\left[J_{i j}^{P}(x)\left(J_{i j}^{P}\right)^{\dagger}(0)\right]\right| 0\right\rangle,
$$

\footnotetext{
${ }^{17}$ Chiral suppression is the suppression of a physical value by some factor dependent on the light quark mass(es) which vanishes in the relevant chiral limit.
} 
are defined with respect to the scalar current $J_{i j}^{S}(x)$ and the pseudo-scalar current $J_{i j}^{2}(x)$. These equations show that $\rho^{(0)}\left(q^{2}\right)=\left(1 / q^{4}\right) \rho_{S, P}\left(q^{2}\right)$, which means that if the scalar and pseudo-scalar spectral contributions excluding the kaon and pion pole contributions can be obtained, the longitudinal spectral function in $\tau$ decay follow automatically. The $\Pi_{i j}^{S, P}$, can be computed theoretically using the OPE. Because the FESR based on $\left(m_{i}-m_{j}\right)^{2} \Pi^{S}$ and $\left(m_{i}+m_{j}\right)^{2} \Pi^{S}$ are known to have a good convergence, the Sum Rules based on the scalar and pseudo-scalar correlators can be utilized to constrain the decay constants of the state other than the pion and kaon poles. From the magnitude of the quark masses, it can be seen that the $J=0$ contribution to the spectral density function is highly suppressed and can therefore be neglected. In addition to the constraint mentioned here, there are other QCD constraints that can be used in the case of the strange scalar channel [30-33].

\subsubsection{Flavour Breaking Finite Energy Sum Rules}

As the Finite Energy Sum Rule is true for all the appropriately defined spin components, current components and flavour components of the spectral density function and correlator, one can take the difference between two pairs of Finite Energy Sum Rules containing different quark flavours, namely $s$ and $d$ quarks. This procedure, which may be seen in Equation 2.65, is known as the Flavour Breaking Finite Energy Sum Rule (FBFESR) $[2,27,28,34]$.

$$
\delta R_{\tau, O P E}^{w_{0+1}, w_{0}}\left(s_{0}\right)=\frac{R_{\tau, u d}^{w_{0+1}, w_{0}}\left(s_{0}\right)}{\left|V_{u d}\right|^{2}}-\frac{R_{\tau, u s}^{w_{0+1}, w_{0}}\left(s_{0}\right)}{\left|V_{u s}\right|^{2}}
$$

where 


$$
\begin{aligned}
R_{\tau, i j}^{w_{0+1}, w_{0}} & =12 \pi^{2}\left|V_{i j}\right|^{2} S_{E W} \int_{0}^{s_{0}} d s\left[w_{0+1}(s) \rho_{0+1}^{i j}(s)-w_{0}(s) \rho_{0}^{i j}(s)\right] \\
& =\int_{0}^{m_{\tau}^{2}} d s\left[w_{0+1}(s) \frac{d R_{\tau, i j}^{0+1}(s)}{d s}-w_{0}(s) \frac{d R_{\tau, i j}^{0}(s)}{d s}\right]
\end{aligned}
$$

is composed of the experimentally measured quantities from Equation 2.19 and 2.20 and have a corresponding OPE representation. The weighted non-strange and strange current integrals $R_{\tau, u d}^{w_{0+1}, w_{0}}$ and $R_{\tau, u s}^{w_{0+1}, w_{0}}$ involve explicit factors of $\left|V_{u d}\right|^{2}$ and $\left|V_{u s}\right|^{2}$ which are divided out in order to get a difference of correlators involving different mass quarks which are normalized in a common way and hence have to produce zero difference in the SU(3) flavour limit.

$$
\begin{aligned}
\delta R_{\tau, O P E}^{w}\left(s_{0}\right)= & \imath 6 \pi S_{E W} \oint_{|s|=s_{0}} d s\left[w_{0+1}(s)\left(\Pi_{0+1}^{u d}(s)-\Pi_{0+1}^{u s}(s)\right)\right. \\
& \left.-w_{0}(s)\left(\Pi_{0}^{u d}(s)-\Pi_{0}^{u s}(s)\right)\right]
\end{aligned}
$$

is calculated using the OPE. From Equations 2.65 to 2.69 it can be trivially seen that the FBFESR also hold for the longitudinal plus transverse component, which can be computed theoretically.

\section{Flavour Breaking Finite Energy Sum Rules and the OPE}

When the FBFESR is employed for the longitudinal plus transverse component, all of the mass independent terms cancel $[25,26]$ leaving the dimension 2 mass correction as the lowest order term. Neglecting the $u$ and $d$ quark masses which are small (see Table $2.1)$, this term is proportional to the square of the strange quark mass, $m_{s}^{2}[3,28,29]$. More specifically, the dimension 2 term in the flavour breaking difference $\delta R_{O P E}^{w}\left(s_{0}\right)$ is usually written as

$$
\left.\delta R_{O P E}^{w}\left(s_{0}\right)\right|_{D=2}=24 S_{e w} \frac{m_{s}^{2}\left(m_{\tau}^{2}\right)}{m_{\tau}^{2}}\left(1-\epsilon_{d}^{2}\right) \Delta_{w, D=2}^{(0+1)}\left(\frac{\alpha_{s}(\tau)}{\pi}\right)
$$


where $\epsilon_{d}$ is the ratio of the down quark mass to the strange quark mass, $\frac{m_{d}}{m_{s}}$, and $\Delta_{w, D=2}^{(0+1)}\left(\frac{\alpha_{s}(\tau)}{\pi}\right)$ is the dimension 2 perturbative QCD correction factor which one determines from the OPE [35].

The dimension 4 quark condensates are dominated by the Renormalization Group invariant strange quark condensate, which is calculated using the Gell-Mann-OakesRenner (GMOR) relation and Chiral Perturbation Theory $(\mathrm{CHPT})^{18}$ quark mass ratios $[25,36]$. GMOR is a relation between the mass of the quark current, $m_{u}+m_{d}$, the pion mass, $m_{\pi}$, and the pion decay constant, $f_{\pi}$, which defines the light quark condensate, $\left\langle\left(m_{u}+m_{d}\right) \bar{u} u\right\rangle[37,38]$ :

$$
\left\langle\left(m_{u}+m_{d}\right) \bar{u} u\right\rangle=-f_{\pi}^{2} m_{\pi}^{2} .
$$

With the GMOR relation, the strange quark condensate $\left\langle m_{s} \bar{s} s\right\rangle$ is readily obtained in terms of $f_{\pi}, m_{\pi}$, the CHPT quark mass ratio $\frac{m_{s}}{m_{u}+m_{d}}$ and the Renormalization Group Invariation Condensate Ratio $\frac{\langle\bar{s} s\rangle}{\langle\bar{u} u\rangle},[25,28,36]$,

$$
\left\langle m_{s} \bar{s} s\right\rangle=\left[\frac{m_{s}}{m_{u}+m_{d}}\right]\left[\frac{\langle\bar{s} s\rangle}{\langle\bar{u} u\rangle}\right]\left\langle\left(m_{u}+m_{d}\right) \bar{u} u\right\rangle .
$$

The remaining term in the dimension 4 series flavour breaking difference, is the dimension $0 m_{s}$ correction term which is proportional to $m_{s}^{4}$ and turns out to be numerically tiny compared to the strange quark condensate contribution [28].

The contribution of the dimension 6 contribution is estimated with the Vacuum Saturation Approximation (VSA) [28]. The VSA is a method of approximating the expectation value of a four quark operator based on the assumption that when inserting all possible intermediate states into the expectation value the vacuum state dominates. When this approximation is made, only vacuum matrix elements of products of currents/densities, which are known empirically, remain [15].

\footnotetext{
${ }^{18} \mathrm{CHPT}$ is an effective field theory that utilizes the broken chiral symmetry of QCD.
} 


\subsubsection{Determination of $m_{s}$ and $V_{u s}$ from FBFESR Extracting $\left|V_{u s}\right|$ from FBFESR}

From the longitudinal plus transverse FBFESR,

$$
\delta R_{\tau, O P E}^{(0+1), w}=\frac{R_{\tau, u d, e x p}^{(0+1), w}}{\left|V_{u d}^{2}\right|}-\frac{R_{\tau, u s, e x p}^{(0+1), w}}{\left|V_{u s}^{2}\right|}
$$

the CKM Matrix element $\left|V_{u s}\right|$ can be isolated and written in terms of the theoretical components, the weighted experimentally measured ud and us flavoured hadronic widths, $R_{\tau, u d, e x p}^{(0+1), w}$ and $R_{\tau, u s, e x p}^{(0+1), w}$, and current world average of $\left|V_{u d}\right|$,

$$
\left|V_{u s}\right|=\sqrt{\frac{R_{\tau, u s, e x p}^{(0+1), w}}{\frac{R_{\tau, u d, e x p}^{(0+1),}}{\left|V_{u d}\right|^{2}}-\delta R_{\tau, O P E}^{(0+1), w}}} .
$$

In the denominator of Equation 2.73 the experimental component, $\frac{R_{\tau, u d, e x p}^{(0+1), w}}{\left|V_{u d}\right|^{2}}$, dominates over the term determined with the OPE integral, $\delta R_{\tau, O P E}^{(0+1), w}$. As a result, it is possible to make highly precise measurements of $V_{u s}$ without knowing the OPE integral to the same level of precision $[2,27,28,34]$.

\section{Extracting $m_{s}$ from FBFESR}

In the OPE representation of the flavour breaking difference, $\delta R_{O P E}^{w}\left(s_{0}\right)$, from the FBFESR, for hadronic $\tau$ decays the dominant term that depends on the strange quark mass is the dimension 2 mass correction terms. Solving for $m_{s}$ yields

$$
m_{s}=\sqrt{\frac{M_{\tau}^{2}}{24 S_{E W}\left(1-\epsilon_{d}^{2}\right) \Delta_{D=2}^{(0+1), w}\left(\frac{\alpha_{s}\left(M_{\tau}\right)}{\pi}\right)}\left(\delta R_{\tau, \exp }^{(0+1), w}-\delta R_{\tau, D>2}^{(0+1), w, O P E}\right)}
$$

$[2,27,28,34]$. If the dimension 4 mass correction term is included in the analytical solution for $m_{s}^{2}$ instead of being treated as a constant with $m_{s}$ fixed to the PDG value [39], Equation 2.65 can be written as a quadratic equation of $m_{s}^{2}$, 


$$
a m_{s}^{4}+b m_{s}^{2}+c=0
$$

where

$$
\begin{aligned}
a & =\left.\frac{\delta R_{D=4, m_{s}}^{w, L+T}\left(m_{s}\right)}{m_{s}^{4}}\right|_{m_{s} \text { from PDG }} \\
b & =\frac{24 S_{e w}\left(1-\epsilon_{d}^{2}\right) \Delta_{w, D=2}^{(0+1)}\left(\frac{\alpha_{s}\left(M_{\tau}\right)}{\pi}\right)}{M_{\tau}^{2}} \\
c & =\delta R_{C H P T}^{w, L+T}+\delta R_{G M O R}^{w, L+T}+\delta R_{V S A}^{w, L+T}-\delta R_{e x p}^{w, L+T} .
\end{aligned}
$$

\section{Truncation and Convergence Issues of the FBFESR for the Extraction of $\left|V_{u s}\right|$ and $m_{s}$}

In addition to the higher dimension terms, which are neglected from the OPE term in the FBFESR, the dimension 2 term of the transverse plus longitudinal FBFESR is known to have a slow convergence $[27,28]$ for the spectral weights used in the literature, namely the weight for the spectral moments which are defined in Equation 2.54:

$$
w^{k l}=\left(1-\frac{s}{m_{\tau}^{2}}\right)^{k+2}\left(\frac{s}{m_{\tau}^{2}}\right)^{l}
$$

for the longitudinal plus transverse SDF. If these terms are non-negligible at $s_{0}=m_{\tau}^{2}$, their contribution will increase with a decrease in $s_{0}$ causing the OPE representation in the FBFESR to become more unreliable. Therefore, if the truncation in the OPE is correctly described by the theoretical uncertainties and the experimental measurements are reliable, there should be a region of stability with respect to $s_{0}$, within the relevant uncertainties, for the quantities $\left(\left|V_{u s}\right|\right.$ and $\left.m_{s}\right)$ extracted at values

of $s_{0}$ around $s_{0}=m_{\tau}^{2}$ [28]. The effect, due to the slow convergence of the truncated integrated $\mathrm{D}=2$ series, can be mitigated by the choice of the weights [34]. These 
weights are defined as:

$$
\begin{aligned}
w_{10}(y)= & {[1-y]^{4}[1+y]^{2}\left[1+y^{2}\right]\left[1+y+y^{2}\right] } \\
= & 1-y-y^{2}+2 y^{5}-y^{8}-y^{9}+y^{10} \\
\hat{w}_{10}(y)= & \hat{w}\left(r=1.2, \cos \left(\theta_{1}\right)=0.5, \cos \left(\theta_{2}\right)=0.1, y\right) \\
= & {[1-y]^{4}\left[1+\frac{y}{r}\right]^{2}\left[1+2 \cos \left(\theta_{1}\right) \frac{y}{r}+\frac{y^{2}}{r^{2}}\right]\left[1+2 \cos \left(\theta_{2}\right) \frac{y}{r}+\frac{y^{2}}{r^{2}}\right] } \\
w_{20}(y)= & (1-y)^{6}\left(1+4.2451 y+9.4682 y^{2}+14.4155 y^{3}+16.4589 y^{4}\right. \\
& +14.6598 y^{5}+10.2818 y^{6}+5.5567 y^{7}+2.1157 y^{8}+0.3520 y^{9} \\
& -0.2065 y^{10}-0.2154 y^{11}-0.1040 y^{12}-0.03040 y^{13} \\
& \left.-0.0045 y^{14}\right)
\end{aligned}
$$

where $y=\frac{s}{s_{0}}$ [34]. The reason, for the improved convergence in these weights, is because they have been constructed to emphasize the regions of the complex plane where the $\mathrm{D}=2 \mathrm{~J}=0+1$ correlator series are, where the convergence is known to be better [16].

\subsubsection{Determining $\left|V_{u s}\right|$ from $\tau^{-} \rightarrow \pi^{-} \nu_{\tau}$ and $\tau^{-} \rightarrow K^{-} \nu_{\tau}$}

In addition to the FBFESR approach, $\left|V_{u s}\right|$ can be determined from the ratio of the $\tau^{-} \rightarrow K^{-} \nu_{\tau}$ and $\tau^{-} \rightarrow \pi^{-} \nu_{\tau}$ decay widths. From Equation 2.11, it can be seen that the ratio of the decay width $\Gamma\left(\tau^{-} \rightarrow K^{-} \nu_{\tau}\right)$ relative to the decay width $\Gamma\left(\tau^{-} \rightarrow \pi^{-} \nu_{\tau}\right)$ decay may be written as:

$$
\frac{\Gamma\left(\tau^{-} \rightarrow K^{-} \nu_{\tau}\right)}{\Gamma\left(\tau^{-} \rightarrow \pi^{-} \nu_{\tau}\right)}=\frac{\mathcal{B}\left(\tau^{-} \rightarrow K^{-} \nu_{\tau}\right)}{\mathcal{B}\left(\tau^{-} \rightarrow \pi^{-} \nu_{\tau}\right)}=\frac{f_{k}^{2}\left|V_{u s}\right|^{2}}{f_{\pi}^{2}\left|V_{u d}\right|^{2}}\left(\frac{1-m_{K}^{2} / m_{\tau}^{2}}{1-m_{\pi}^{2} / m_{\tau}^{2}}\right)^{2}
$$

where $m_{\pi}$ and $m_{K}$ are the pion and kaon masses, and $\frac{f_{k}}{f_{\pi}}$ may be determined theortically [40]. 


\subsection{The Current Experimental Status of Hadronic $\tau$ Decays and the Extraction of $\left|V_{u s}\right|$ and $m_{s}$}

\subsubsection{Experimental Status of Hadronic $\tau$ Decays}

The spectral density function of the hadronic $\tau$ decays have been measured by the ALEPH Collaboration [41-44], the CLEO Collaboration [45-50], and the OPAL Collaboration $[51,52]$. The ALEPH and OPAL experiments were located on the LEP $e^{+} e^{-}$Ring at CERN and provided an extremely clean sample of $\tau^{+} \tau^{-}$pairs with a high selection efficiency, while CLEO, which had higher statistics, could measure higher multiplicity modes and investigate the structure of the hadronic $\tau$ decays [53]. Before this work, the most precise determinations of $\left|V_{u s}\right|$ from hadronic $\tau$ were $0.2223 \pm 0.0032 \exp \pm 0.0038$ th [28], $0.2209 \pm 0.0031$ [54] and $0.2225 \pm 0.0034[55,56]$. However, the latter measurement may have an underestimation of the theoretical uncertainties due to the issue discussed in Section 2.2.10. The extracted values of $m_{s}$ are sensitive to the absolute normalization of strange spectral density function because of the large cancellation between the strange and non-strange spectral integrals and the convergence of the dimension 2 series. However, most analyses neglect uncertainties related to the truncation and convergence of the dimension 2 OPE series and therefore underestimate the total uncertainty on the strange quark mass [53]. With the new data measured at the B-factories $B A B A R[8,9]$ and Belle [10], it is expected that $\left|V_{u s}\right|$ can be measured to an accuracy greater than $\sim 0.0010$, a value which is competitive with the world average $[35,54,57]$.

\subsubsection{Status of $\left|V_{u s}\right|$ and $m_{s}$}

The current world average of $\left|V_{u s}\right|$ determined by averaging the experimentally measured values from $K_{l 2}$ decays [58-61], $K_{l 3}$ decays [62-72], hyperon decays [73,74] and 
hadronic $\tau$ decays $[2,27,28,34,41,51,53,54]$ with the unitarity constraint,

$$
\left|V_{u s}\right|=\sqrt{1-V_{u d}^{2}-V_{u b}^{2}},
$$

is $0.226 \pm 0.001$ [39]. The extraction of $\left|V_{u s}\right|$ from $K_{l 2}$ decays uses the ratio of decay rates $\frac{\Gamma(K \rightarrow \mu \nu \mu}{\Gamma(\gamma))} \Gamma \frac{\left|V_{u s}\right|^{2} f_{K}^{2}}{\left|V_{u d}\right|^{2} f_{\pi}^{2}}$, where the ratio of the form factors $f_{K}$ and $f_{\pi}$ are determined with Lattice $\mathrm{QCD}{ }^{19}$. The $K_{l 3}$ analyses use the experimentally measured and theoretical formulation of the decay rate for $K \rightarrow \pi e \nu_{e}$ and/or $K \rightarrow \pi \mu \nu_{\mu}$ to compute $\left|V_{u s}\right|$. In the past there was a $2-2.5 \sigma$ discrepancy between the unitarity value of $\left|V_{u s}\right|$ and the value extracted from the $K_{l 3}$ decays, namely $K \rightarrow \pi e \nu$ and $K \rightarrow \pi \mu \nu$ decays. Recent measurements at BNL E865 [62], KTeV [63,64], KLOE [65-68], NA48 [69-71], and ISTRA + [72] using improved radiative corrections, are consistent with unitarity [39]. In hyperon decays, $\left|V_{u s}\right|$ is extracted from the semileptonic decays $\Lambda \rightarrow p e^{-} \overline{\nu_{e}}, \Sigma^{-} \rightarrow n e^{-} \overline{\nu_{e}}, \Xi^{-} \rightarrow \Lambda e^{-} \overline{\nu_{e}}, \Xi^{-} \rightarrow \Sigma^{0} e^{-} \overline{\nu_{e}}$, and $\Xi^{0} \rightarrow \Sigma^{+} e^{-} \overline{\nu_{e}}$ by means of a fit to the differential decay rate.

The most precise method of determining the mass is a theoretical calculation using Lattice QCD [75-84]. The strange quark mass has also been experimentally measured using pseudo-scalar Sum Rules $[32,33,85]$ and scalar [31,32] Sum Rules as well as Sum Rules based on $e^{+} e^{-} \rightarrow$ hadrons and hadronic $\tau$ decay data $[3,29,86,87]$. The current world average, $m_{s}=95 \pm 25 \mathrm{MeV} / \mathrm{c}^{2}$ [39], and the extracted values which the world average is based on can be seen in Figure 2.10.

\footnotetext{
${ }^{19}$ Lattice QCD is a theoretical simulation of the strong force computed numerically on a grid or lattice that uses a minimal amount of experimentally measured quantities, namely the meson and baryon masses, as input.
} 


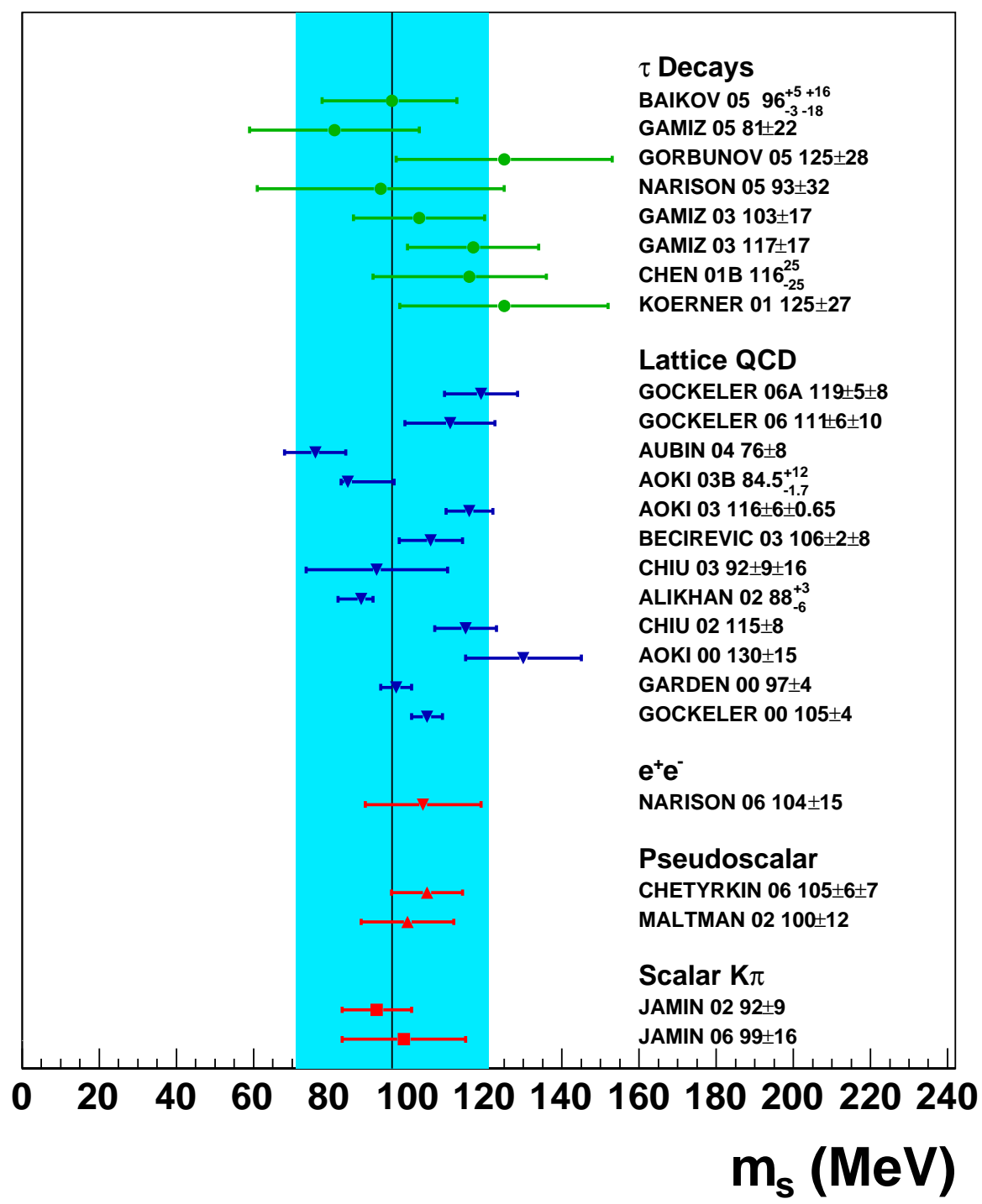

Figure 2.10: The extracted values of $m_{s}$ that are used to determine the world average. The solid band represents the current world average [39]. 


\section{Chapter 3}

\section{The PEP-II Accelerator and the BABAR Detector}

\subsection{The PEP-II Accelerator}

The PEP-II Ring is an upgrade of the original PEP Ring to enable CP Violation to be studied in the $\mathrm{B}^{0}$ and $\overline{\mathrm{B}}^{0}$ mesons. The PEP-II Accelerator, seen in Figure 3.1, is an asymmetrical electron and positron collider. It consists of two rings, a high energy storage ring $(9.0 \mathrm{GeV})$ for the electrons and a low energy ring $(3.1 \mathrm{GeV})$ for the positrons. The former ring is an upgrade of the existing PEP ring to accommodate the high currents while the latter ring is a new addition enabling asymmetric beam energies. Since many of the interactions, which are expected to have CP Violation in the $\mathrm{B}^{0}$ and $\overline{\mathrm{B}}^{0}$ mesons, have a small branching ratio, approximately $10^{-5}$ or smaller, the PEP-II upgrade required an "unprecedented" nominal luminosity ${ }^{1}$ of $10^{-34} \mathrm{~cm}^{-2} \mathrm{~s}^{-1}$. The PEP-II ring utilizes the linac to inject the electron and positron into the PEP-II ring at colliding energies. In the centre-of-mass (c.m.) frame, the collision energy corresponds to the mass of the $\Upsilon(4 \mathrm{~s})$ particle, a resonant state that is composed of a $b$ and anti- $b$ quark with a mass of $10.58 \mathrm{GeV} / \mathrm{c}^{2}$. The $\Upsilon(4 \mathrm{~s})$ particle is slightly above the threshold energy of $\mathrm{B}^{0}$ and $\overline{\mathrm{B}}^{0}$ production, resulting in a decay

\footnotetext{
${ }^{1}$ Luminosity is defined as the number of incident particles per unit area per unit time.
} 
rate greater than $96 \%$ into $\mathrm{B}^{0}, \overline{\mathrm{B}}^{0}, \mathrm{~B}^{-}$and $\mathrm{B}^{+}$particles. If the c.m. energy of the collisions were higher, there would be both an increase in the background and a reduced cross section for $\mathrm{B}^{0}$ and $\overline{\mathrm{B}}^{0}$ production. The boost ${ }^{2}$ between the c.m. frame and the laboratory frame enables time dependent CP Violation to be studied. This is because a time delay between $\mathrm{B}^{0}$ and $\overline{\mathrm{B}}^{0}$ decays, which are nearly at rest in the c.m. frame, translates into a measurable displacement along the beam axis.

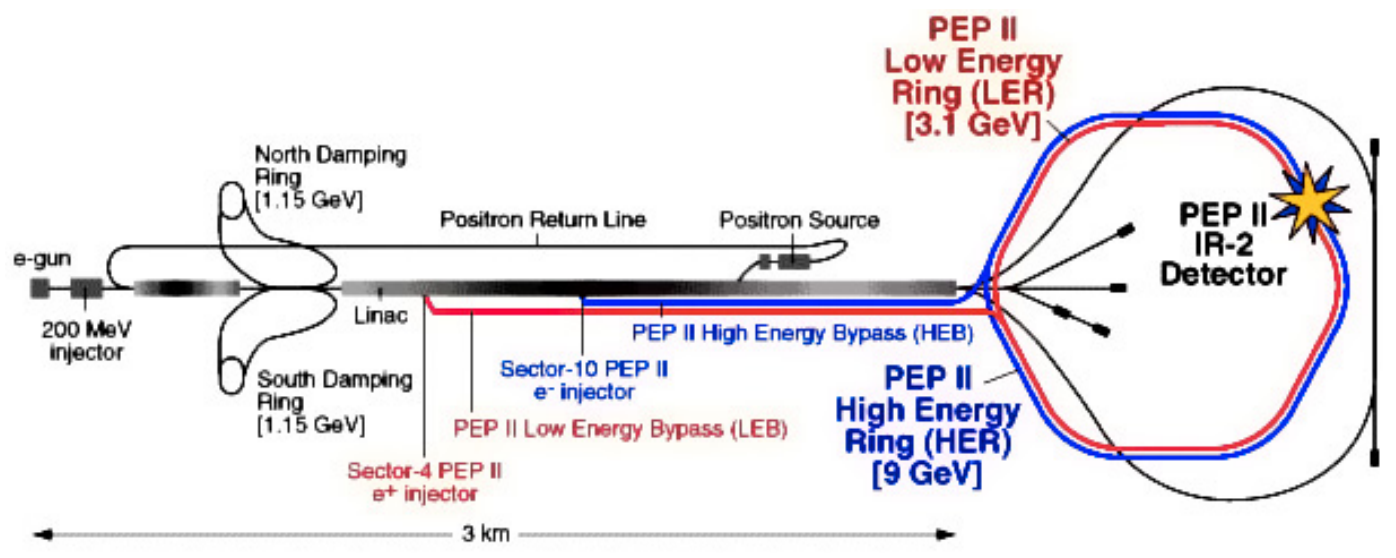

Figure 3.1: The diagram of the PEP-II Accelerator. [88, BABAR Detector Image Gallery].

The high luminosity of the PEP-II accelerator coupled with the relatively large cross section for $\tau$ pairs at the c.m. energy $10.58 \mathrm{GeV}$ results in the BABAR facilities doubling as a $\tau$ factory. Below is a table of the relative cross sections at a c.m. energy $10.58 \mathrm{GeV}[89]$.

\footnotetext{
${ }^{2}$ In special relativity, the transformation between two reference frames, which differ only by a relative velocity, is referred to as a boost.
} 


\begin{tabular}{|c|c|}
\hline Interaction & $\overline{\sigma(n b)}$ \\
\hline \multicolumn{2}{|l|}{ Leptonic Interactions } \\
\hline$e^{+} e^{-} \rightarrow e^{+} e-(17<\theta<160)$ & 21.2 \\
\hline$e^{+} e^{-} \rightarrow e^{+} e-(20<\theta<120)$ & 14.4 \\
\hline$e^{+} e^{-} \rightarrow \mu^{+} \mu-$ & 1.147 \\
\hline$e^{+} e^{-} \rightarrow \tau^{+} \tau-$ & 0.919 \\
\hline \multicolumn{2}{|c|}{ Semi-Hadronic Interactions } \\
\hline$e^{+} e^{-} \rightarrow u \bar{u}$ & 1.39 \\
\hline$e^{+} e^{-} \rightarrow d \bar{d}$ & 0.35 \\
\hline$e^{+} e^{-} \rightarrow s \bar{s}$ & 0.35 \\
\hline$e^{+} e^{-} \rightarrow u \bar{u} / d \bar{d} / s \bar{s}$ & 2.09 \\
\hline$e^{+} e^{-} \rightarrow c \bar{c}$ & 1.30 \\
\hline$e^{+} e^{-} \rightarrow b \bar{b}$ & 1.05 \\
\hline
\end{tabular}

Table 3.1: The cross sections for an $e^{+} e^{-}$collider at $10.58 \mathrm{GeV}[89]$

\subsection{The Interaction Region}

The PEP-II ring has one detector, the BABAR Detector, located at the second interaction region on the ring. The electron and positron beams are manipulated through bending, by dipole magnets, and focused, by quadrapole magnets so that they collide approximately along the central axis of the $B A B A R$ Detector at the "interaction point" or IP. $\mathrm{CsI}(\mathrm{Tl})$ crystals, a scintillating material, which are positioned beside the beam pipe are utilized to monitor the focusing of the beams. This interaction region is incapsulated inside a "low mass beryllium cylinder". The low $z$ of beryllium minimizes the interactions of the subatomic particles with the detector support tube to a radiation length ${ }^{3}$ of $0.005 \mathrm{X}_{0}$.

Since the PEP-II ring is a high current machine, the interaction region produces several significant sources of background. The quadrapole and dipole magnets, which are located close to the interaction region to maximize the focusing of the beam, pro-

\footnotetext{
${ }^{3}$ Radiation length of a material, $\mathrm{X}_{0}$, is defined as the distance in which energy of a particle is reduced by $e^{-1}$ through electromagnetic interactions with that material.
} 
duce sychrotron radiation ${ }^{4}$ as the trajectory of the beams is altered. Production of an $e^{+} e^{-}$pair, referred to as Bhabha scattering, cause electrons and positions to enter the detector. Beam particles are "lost" through bremsstrahlung 5 and Coulomb scattering $^{6}$ with residual gas molecules in the beam. The beam particles, not at the correct momentum for stable storage in the ring, also interact with the magnets and the beam pipe to produce additional upstream background. The accumulation of dose, which causes a high occupancy and radiation damage inside the active components of the detector, is monitored with pin diodes and diamond detectors located around the detector. To reduce the accumulation of unnecessary dose to the detector, potential high radiation regions are suppressed by the addition of extra material [89].

\subsection{The BABAR Detector}

The BABAR Detector is composed of five main components. An illustration of the BABAR Detector and its components can be seen in Figure 3.2. They are the Silicon Vertex Tracker (Vertex Detector), the Drift Chamber (Tracking Chamber), the DIRC (Detector of Internally Reflected Cherenkov light), Electromagnetic Calorimeter (Electron/Photon Detector) and the Instrumented Flux Return (Muon/Hadron Detector). Signals measured by the BABAR detector are filtered through a trigger to select physic events which are of interest. The NbTi 1.5 Tesla superconducting solenoid magnet produces a magnetic field parallel to the detector axis in the tracking region which bends the trajectory of the charged subatomic particle through the Lorentz Force. The uncertainty in the BABAR Detector for determining the transverse momentum $\mathrm{p}_{t}$, the momentum orthogonal to the initial electron beam direction, with

\footnotetext{
${ }^{4}$ Sychrotron radiation is the radiation that is emitted by a relativistic charged particle in a circular path due to the acceleration.

${ }^{5}$ Bremsstrahlung scattering is the scattering of an electron through the emission of a photon.

${ }^{6}$ Coulomb scattering is the scattering of an electron by a photon.
} 
this method is

$$
\frac{\sigma_{\mathrm{P}_{t}}}{\mathrm{P}_{t}}=(0.13 \pm 0.01) \% \cdot \mathrm{P}_{t} \oplus(0.45 \pm 0.03) \%
$$

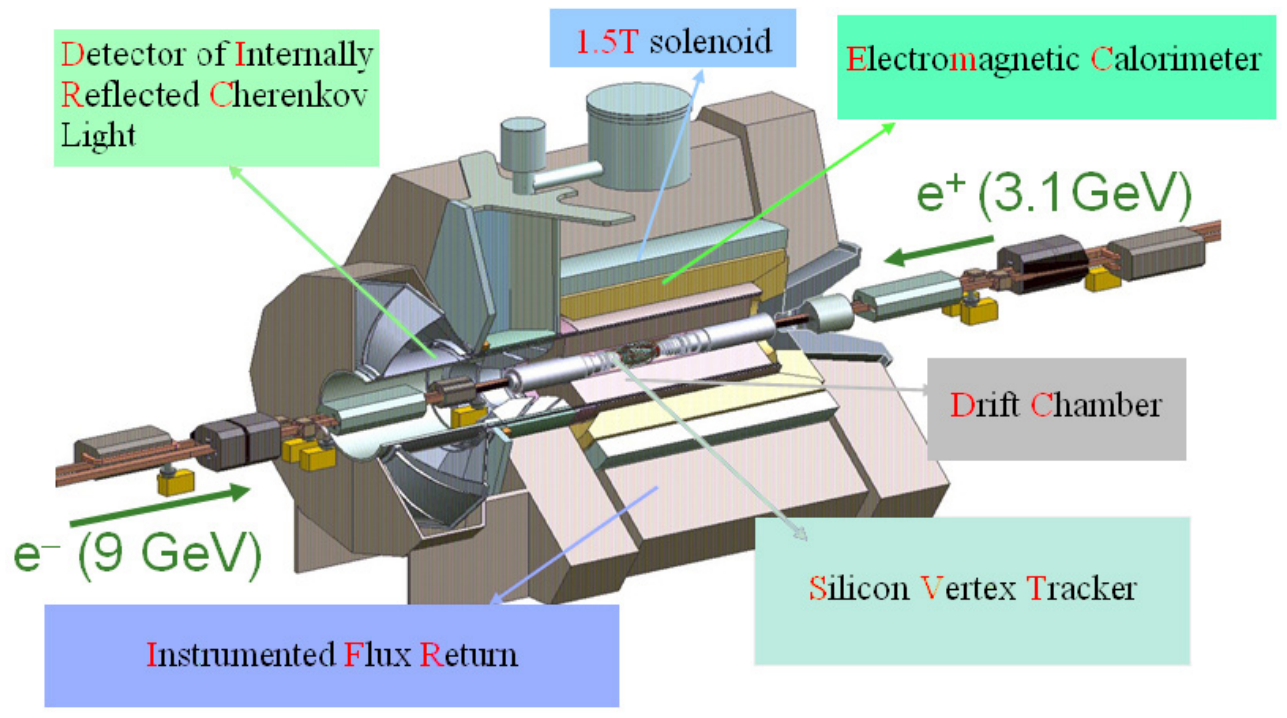

Figure 3.2: The BABAR Detector [88, BABAR Detector Image Gallery].

The $z$ axis is defined as the central axis of the detector which is within $100 \mathrm{mrad}$ of the direction of the high energy electron beam. The $y$ axis is in the vertical direction toward the zenith, while the $x$ axis is in the horizontal direction pointing away from the centre of the PEP-II ring. The interaction point is the origin of the BABAR coordinate system [89]. From these cartesian coordinates, the spherical angles $\theta$ and $\phi$ are derived. $\theta$ is the polar angle defined relative to the $z$ axis, while $\phi$ is the angle relative to the $x$ axis in the $x y$ plane.

\subsubsection{The Silicon Vertex Tracker}

The Silicon Vertex Tracker (SVT) is one of the two tracking sub-detectors in the BABAR Detector. As seen in Figure 3.2, it is the innermost detector positioned around the beam pipe. The purpose of the SVT is to "reconstruct the decay vertices of two 
primary B mesons in order to determine the time between the two decays" [89, pg. 81]. This allows for time-dependent $\mathrm{CP}$ asymmetries to be studied. Moreover, the SVT is capable of reconstructing low momentum tracks that do not enter the Drift Chamber.

The SVT is constructed from 52 "double-sided" silicon modules. These modules are positioned in a 5 layer configuration as seen in Figures 3.3 and 3.4. The detector consists of readout strips to give information in $z$ and $\phi$. The inner two layers are primarily designed to determine the $z$ location of the vertex, while the outer two layers are designed to merge the reconstructed tracks with the Drift Chamber. The third or middle layer enables the reconstruction of trajectories using only SVT tracks, such as low momentum tracks that do not reach the Drift Chamber. The intrinsic resolution of the silicon detectors in the three inner most layers is $10 \mu \mathrm{m}$ in the $\phi$ direction and $12 \mu \mathrm{m}$ in the $z$ direction. In the two outer layers, the intrinsic resolution in $\phi$ is $10-12 \mu \mathrm{m}$ and in the $z$ direction $25 \mu \mathrm{m}$.

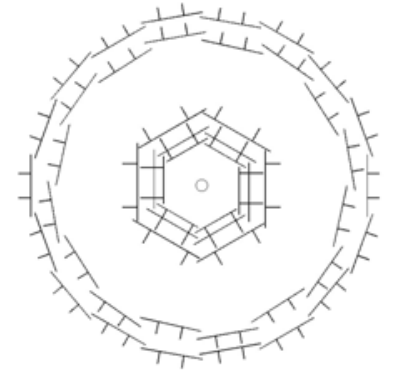

Figure 3.3: A cross section view of the SVT in the plane orthogonal to the beam pipe $[90$, Figure 4-3].

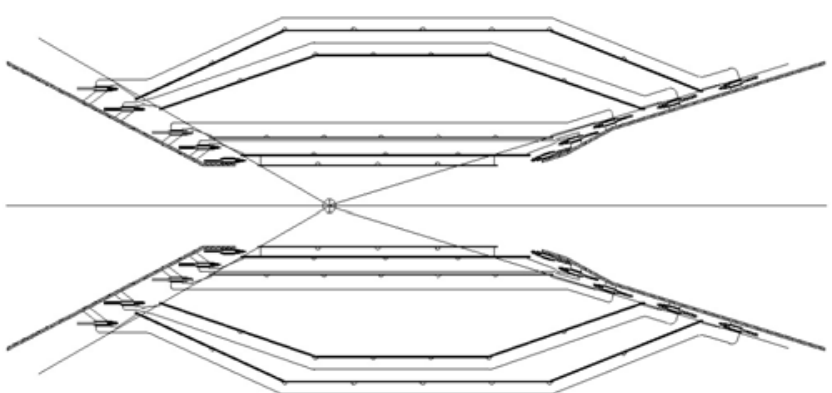

Figure 3.4: A cross section view of the SVT in the beam pipe plane [90, Figure 4-2].

The above geometry has a solid angle coverage designed to maximize the geometric acceptance. However, this is constrained by the location of the bending magnets. As a result, the solid angle coverage is constrained to be between the polar angles 
$20.1^{\circ}$ and $150.2^{\circ}$. This corresponds to $29.5^{\circ}$ and $161.8^{\circ}$ in the c.m. frame. The exterior two layers of the SVT, which are kinked in $z$ to minimize the incident angles, are arranged in an overlapping geometry, while the interior layers are in a pin-wheel arrangement. This ensures that an incident particle crosses through a module at each layer it transverses.

The modules consist of a composite fiber frame upon which the silicon detector and kevlar support ribs are mounted. The frame is constructed of carbon fiber to minimize the radiation length. The silicon detectors are connected with wire bonds into two "half modules". These half modules are composed of two to four silicon detectors and separate the modules into electrically isolated forward and backward sections. The outward pointing side of the silicon detectors have strips parallel to the $z$ direction for measurements in the $\phi$ direction. On the inward side of the silicon detectors, the strips are orthogonal to the $z$ direction for measurements in the $z$ direction. The data is read out through high density interconnect electronic hybrid devices mounted at each end of the modules [89,90].

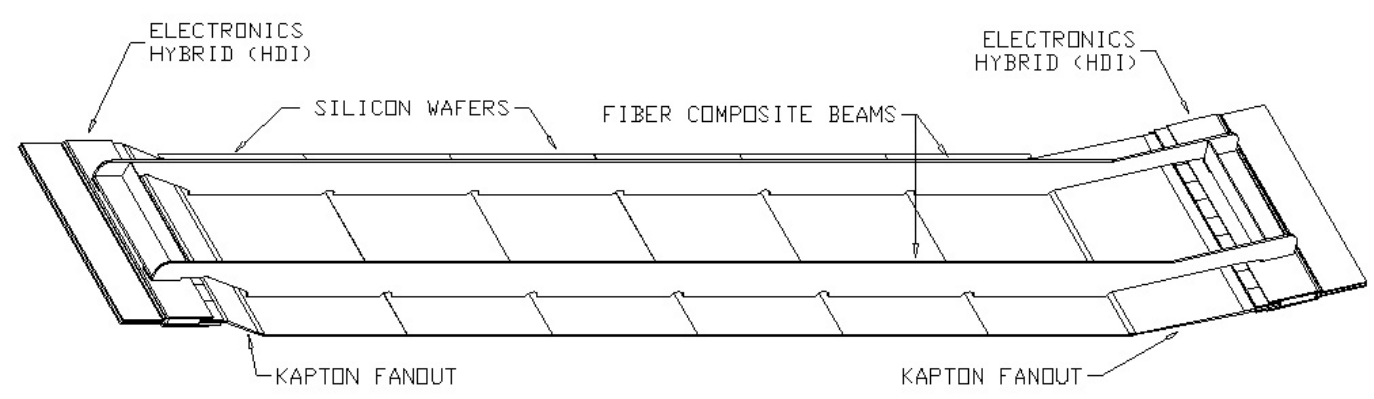

Figure 3.5: A diagram of a Module in the SVT [90, Figure 4-15]. 


\subsubsection{The Drift Chamber}

Exterior to the SVT is the second tracking detector, the Drift Chamber (DCH). The DCH is the primary tracking detector in the BABAR Detector. The chamber is composed of two end plates made of carbon fiber and an inner and outer support tube. The forward and backward end plates are $1.2-2.4 \mathrm{~cm}$ and $2.4 \mathrm{~cm}$ respectively. The forward end plate has a reduced thickness, near the outer radius, to minimize the radiation length a particle must travel before entering the calorimeter system. The outer support tube, which is composed of two carbon fiber layers around a Nomex core, is the structural support that carries the load of the internal wire. The inner support tube is made of beryllium, again, to minimize the radiation length. The chamber, which is filled with $80 \%$ helium and $20 \%$ ISO-butane, contains an array of sense and field wires. A diagram of the DCH can be seen in Figure 3.6.

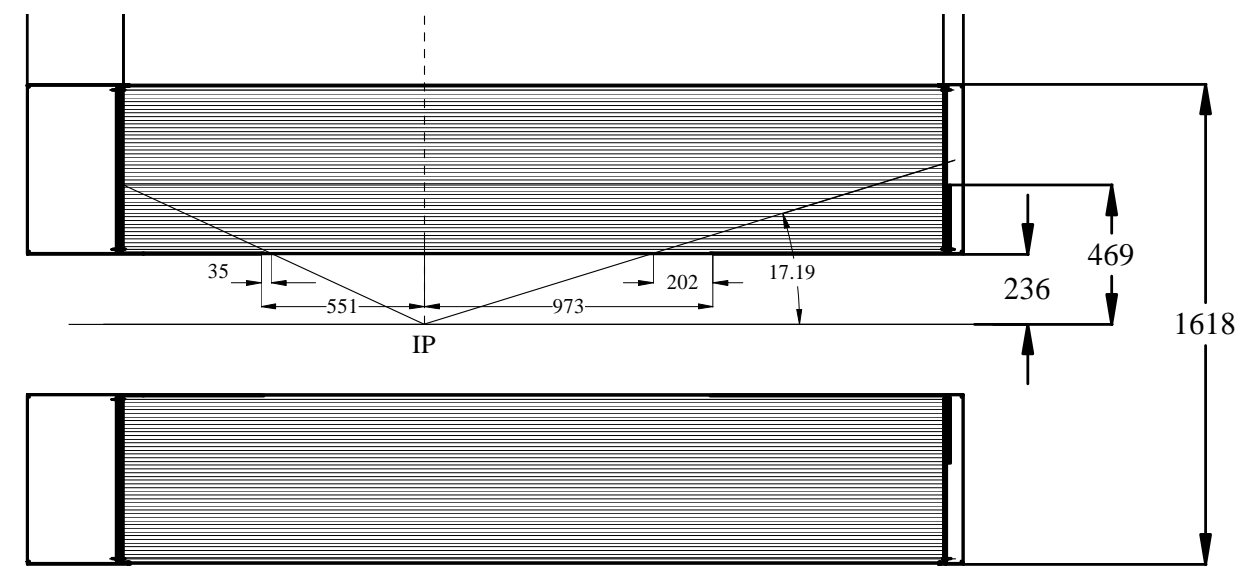

Figure 3.6: A cross sectional view of the DCH [89, Figure 3-7].

The charged sense wires, which detect the signal, are $20 \mu \mathrm{m}$ gold plated tungstenrhenium wires. Although the sense wires were maintained at a potential of $1900 \mathrm{~V}$ and $1960 \mathrm{~V}$ at the beginning of the experiment, the majority of the data was recorded with a potential of $1930 \mathrm{~V}$ on the sense wires. The wires employed to produce the 
electric field pattern, the field wires, are $120 \mu \mathrm{m}$ and $80 \mu \mathrm{m}$ gold plated aluminum. The field wires surrounding the sense wires are grounded while the other field wires have a potential of $340 \mathrm{~V}$. The array of sense and field wires consists of 40 stereo (U and V) and axial (A) layers, with the charged sense wires positioned between the super-layers. In the axial layers the field wires and sense wires are parallel to the $z$ axis, yielding a position measurement in the axial or $x y$ plane. In contrast, the stereo wires have an angle relative to the $z$ axis. In addition to this, the stereo angle varies with the radial distance of the wires from the beam axis to maintain the shape of the cells. The absolute magnitude of the stereo angle is between of $42.0 \mathrm{mrad}$ to $66.5 \mathrm{mrad}$. These layers are arranged in sets of four, where each layer has a unique stereo angle and twist that increases in magnitude with the radial distance from the interaction region. Figure 3.7 illustrates the stereo axial pattern employed in the Drift Chamber. The U stereo layers correspond to the wire arrangements with both a positive stereo angle and a positive twist angle, while the $\mathrm{V}$ stereo layers correspond to a negative stereo angle and a negative twist angle. This difference in stereo angle between the U, V and A layers set up a non-orthogonal basis for the solid angle that allows the particles path to be reconstructed in terms of $\theta$ in addition to $\phi$ and the radius $r$.

The field wires within the layers form hexagonal cells around the sense wires, and, thus the potential difference between the field and sense wires produces an electric field. When a subatomic particle travels through a cell, it ionizes the gas. The free electrons, attracted by the positive charge, drift with a mean velocity of $v_{\text {drift }}$ towards the sense wire. The length of the path that the electrons travel, $\mathrm{L}_{\text {path }}$, is dependent upon the position, where the ionization took place, and the electric and magnetic field in that cell. Due to the strong electric field, the electrons in the gas undergo amplification when within a distance of several wire radii from the sense 


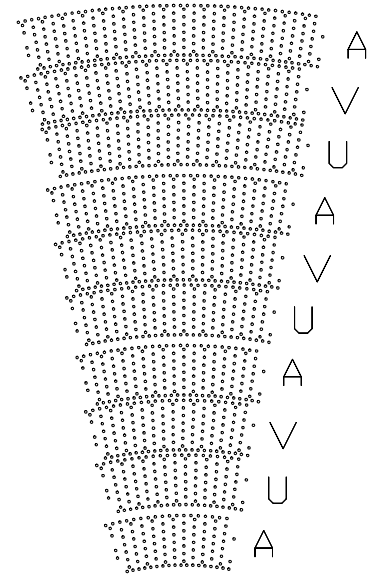

Figure 3.7: A diagram of the axial and stereo arrangement [89, Figure 3-8].

wire. Positive ions are produced in an avalanche close to the sense wire, and their movement away from the wire creates a measurable signal on the sense wire. This signal is proportional to the ionization created by the subatomic particle travelling through the gas. Hence, the $\frac{d E}{d x} 7$ information is obtained from summing the total charge deposited on the wire and correcting for the incident angle of the charged particle. In order to obtain a precise measurement of the trajectory, the drift time is measured and converted to a drift distance from prior knowledge of the drift velocity. To use the "time-to-distance relationship" the time that the particle transversed the cell, $t_{0}$, is required. The $t_{0}$ time is obtained from a knowledge of the collision times of the beams and the distance that the particle travelled from the interaction point to the location of inonization in the cell. The time-to-distance relations can be depicted graphically in terms of "isochrones", or surfaces of equal drift time. The maximum time for the electrons to drift to the sense wire is approximately 600ns. Figure 3.8 is a diagram of a typical cell with lines of equal isochrone superimposed $[89,90]$. The intrinsic resolution of a DCH cell is $\sim 100 \mu \mathrm{m}$.

\footnotetext{
$7 \frac{d E}{d x}$ is the average energy loss per unit length.
} 


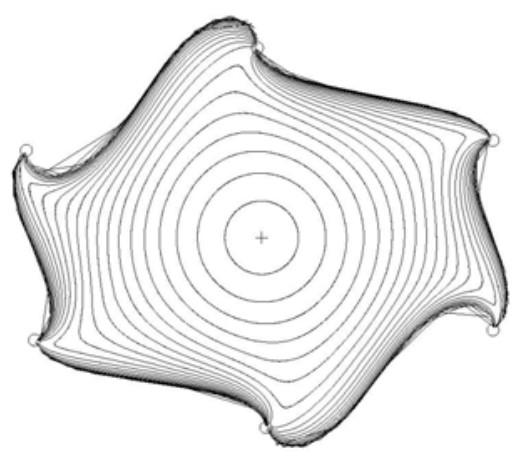

Figure 3.8: A diagram of the isochrones in a typical DCH cell. The isochrones are spaced by 50 ns $[89$, Figure $3-9]$.

The Drift Chamber was constructed at TRIUMF in Vancouver, Canada as the Canadian contribution to the experiment.

\subsubsection{The DIRC}

The DIRC, the third detector from the centre in Figure 3.2, is a "new kind" of ring Cherenkov detector and is intended for particle identification. The DIRC was designed to distinguish between kaons and pions which have a momentum of $1.7 \mathrm{GeV}$ to $4.2 \mathrm{GeV}$, and to tag the flavour of a B meson decay through a $b \rightarrow c \rightarrow s$ cascade [91, Section 8.1]. The DIRC has a geometric acceptance of $25.5^{\circ}$ to $147^{\circ}$ in the laboratory frame. A schematic of the DIRC can be seen in Figure 3.9 [90, Chapter 6].

The DIRC has two major components that measure the Cherenkov Cone: the Standoff Box and the set of twelve Bar Boxes. The Standoff Box, which is composed of stainless steel, is a toroidal structure with 12 cone shaped sectors. Each sector has 896 Photo Multiplier Tubes at a radial distance of $\approx 1.17 \mathrm{~m}$ from the end of the Fused Silica Bars. The Standoff Box is filled with $6000 \mathrm{~L}$ of purified water, a substance with an index of refraction similar to that of fused silica, to minimize the total internal reflection at the Fused Silica/water surface. Each of the Bar Boxes, 


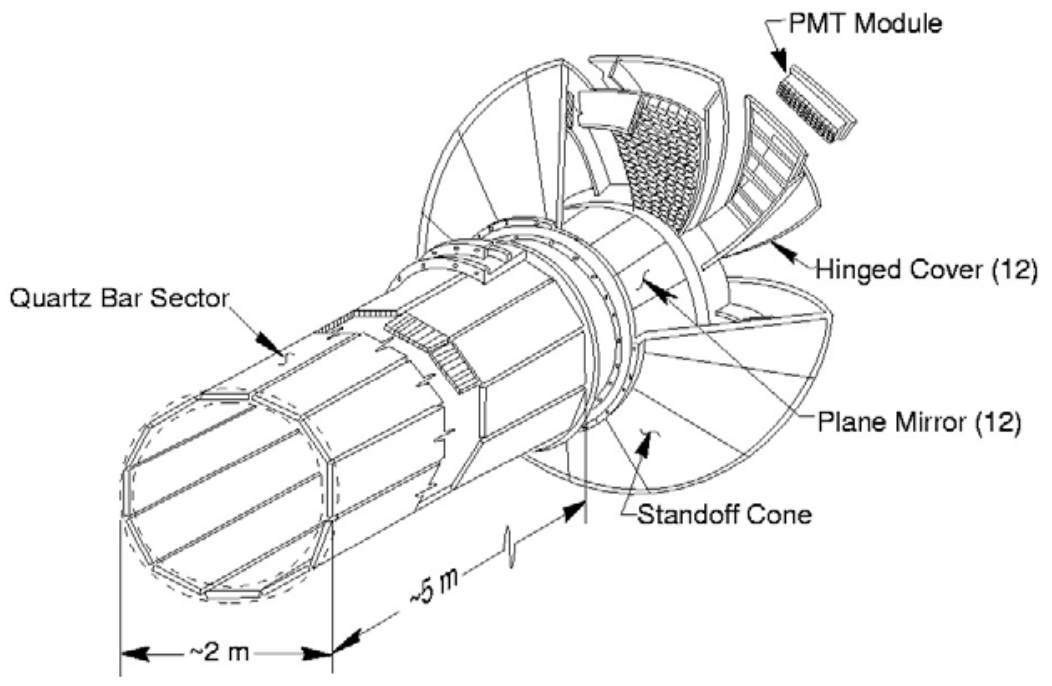

Figure 3.9: A diagram of the components in the DIRC [90, Figure 6-10].

composed of thin aluminium-hexel panels, are connected to an individual sector of the Standoff Box with a 10mm thick Fused Silica window. Inside the Bar Box, the Fused Silica window is glued to a Fused Silica wedge, which, in turn, is glued to twelve optically isolated Fused Silica Bars with mirrors attached on the opposing end. It is in these 144 optically isolated Fused Silica Bars that the Cherenkov light ${ }^{8}$ is produced. The 144 Fused Silica Bars are composed of four 1.225m bars of spectrosil, a fused silica [91, Chapter 8].

When a charged particle travelling above $0.679 c$, the speed of light in fused silica, enters one of the 144 Fused Silica Bars, it emits an electromagnetic radiation wave front, called a Cherenkov Cone. The angle of this Cherenkov Cone is

$$
\theta_{C}=\arccos \left(\frac{1}{\beta \sqrt{\epsilon(\omega)}}\right),
$$

where the $\beta$ is the ratio of the particles velocity to the speed of light in the vacuum,

\footnotetext{
${ }^{8}$ Cherenkov light is the resulting light produced by a particle travelling faster than the speed of light in a medium.
} 
and $\epsilon(\omega)$ is the frequency dependent dielectric constant of the medium through which the particle is travelling [92, eq.13.50]. Part or all of the cone will be transmitted through total internal reflection or specular reflection at the bar's end down the bar and into the Standoff Box. The geometry of the bar and wedge are such that the angle of the light is preserved. The light then travels to the detector surface where the incident number of photons is counted by the Photo Multiplier Tubes. An illustration of this can be seen in Figure 3.10.

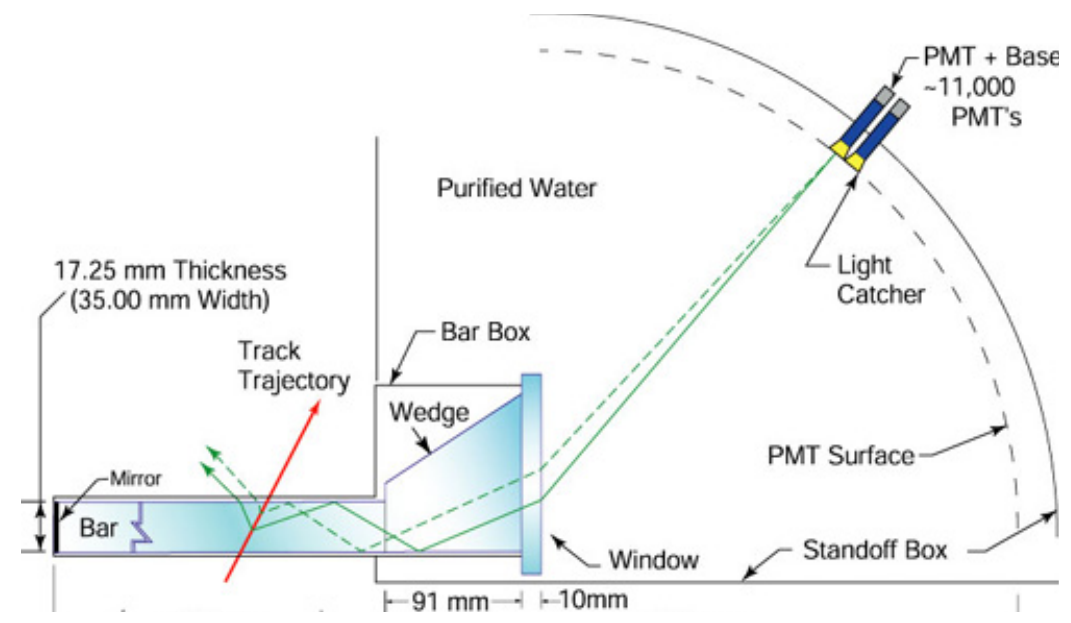

Figure 3.10: An illustration of the operation of the DIRC [88, DIRC Detector Image Collection].

\subsubsection{The Electromagnetic Calorimeter}

The Electromagnetic Calorimeter (EMC) is the fourth sub-detector in the BABAR Detector. The EMC is designed to measure the energy and momentum of photons and electrons. It is therefore useful for the measurement of photons that may have come from $\pi^{0} \rightarrow \gamma \gamma$ and to assist in distinguishing electrons from muons as well as electrons from charged pions. To achieve this, the EMC has both a high angular and energy resolution. The angular resolution of the EMC is 


$$
\sigma_{\theta}=\frac{3 m r}{\sqrt{E}} \oplus 2 m r
$$

where $\mathrm{E}$ is the energy in units of $\mathrm{GeV}$. The angular resolution in Equation 3.3 is a result of the "transverse crystal size and (the) average distance to the interaction point" [90, pg. 248]. The energy resolution, which is defined for a photon with an angle of $\theta=\pi / 2$, is

$$
\frac{\sigma_{E}}{E}=\frac{1 \%}{E^{\frac{1}{4}}} \oplus 1.2 \%
$$

Again, the energy $\mathrm{E}$ is in units of $\mathrm{GeV}$. The systematic error is a result of internal calibration errors, inhomogeneous light collection and leakage in the front and rear components.

The EMC is composed of a barrel section and conical endcap. The barrel and endcap have a combined geometric acceptance region from $15.8^{\circ}$ to $140.8^{\circ}$ in the laboratory frame. This corresponds to a geometric acceptance of $26.5^{\circ}$ to $156.3^{\circ}$ in the c.m. frame. The barrel section contains 5880 trapezoidal CsI(Tl) crystals in 49 rows of 120 crystals. Each row has a specific size and shape so that the face of the crystal, in the row, points toward the interaction point. This is to minimize the leakage due to both the gaps between the crystals and the material through which the particle must transverse to enter the crystals. The CsI(Tl) crystals also vary in radiation length from $16.0 \mathrm{X}_{0}$ in the backward direction to $17.5 \mathrm{X}_{0}$ in the forward direction by $0.5 \mathrm{X}_{0}$ increments. In contrast, the endcap is composed of $900 \mathrm{CsI}(\mathrm{Tl})$ crystals with a radiation length of $17.5 \mathrm{X}_{0}$. These crystals are also trapezoidal in shape. These crystals are arranged into nine rows so that the crystal dimensions are approximately uniform. The three outermost rows have 120 crystals, while the middle three rows and inner three rows have 100 and 80 crystals respectively. Figure 
3.11 is a schematic of the crystal arrangement in the EMC.

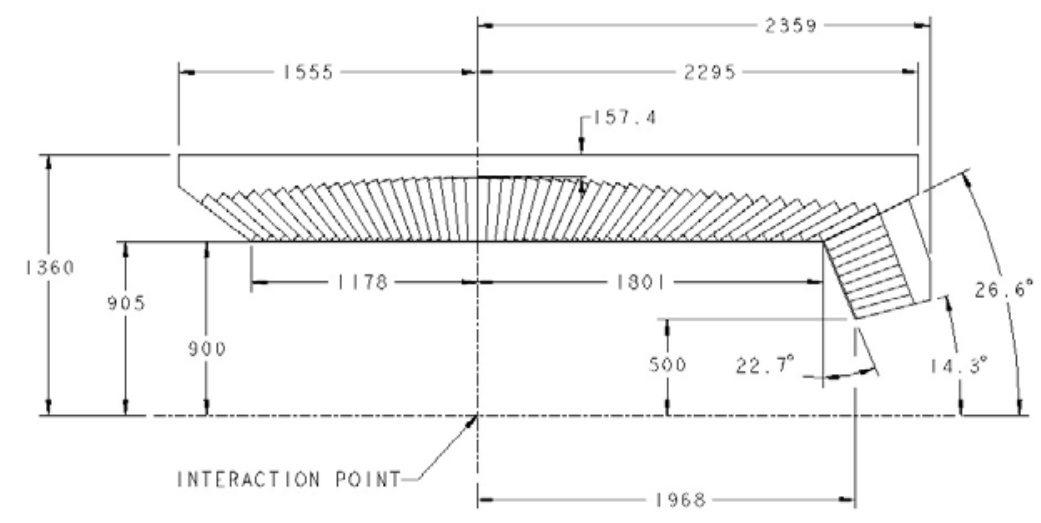

Figure 3.11: A schematic of the EMC [90, Figure 7-4].

CsI(Tl) crystals are scintillators, that fluoresce when valence electrons, which are excited into the band gap by subatomic particles, decay into the valence band. Each crystal is connected to a wavelength shifter that shifts the fluoresce radiation scintillated from the $\mathrm{CsI}(\mathrm{Tl})$ crystal, a peak wavelength of $565 \mathrm{~nm}$ to $960 \mathrm{~nm}$. This is within the absorption wavelength range of the photo diodes, which have a quantum efficiency of $75 \%$. The two photo diodes are not affected by the 1.5 Tesla magnetic field. The crystals are covered with Aluminum Mylar on the front, and Teflon AF, Diffused Reflector and Aluminum Mylar on the sides to ensure all the light is directed onto the photo diodes. A schematic of a $\mathrm{CsI}(\mathrm{Tl})$ crystal and photo diodes can be seen in Figure 3.12. The CsI (Tl) crystals are then mounted inside modules that house the signal cables and the cooling system, as shown in Figure 3.13. These modules are attached to a carbon fiber support cylinder with an aluminum frame $[89,90]$. 


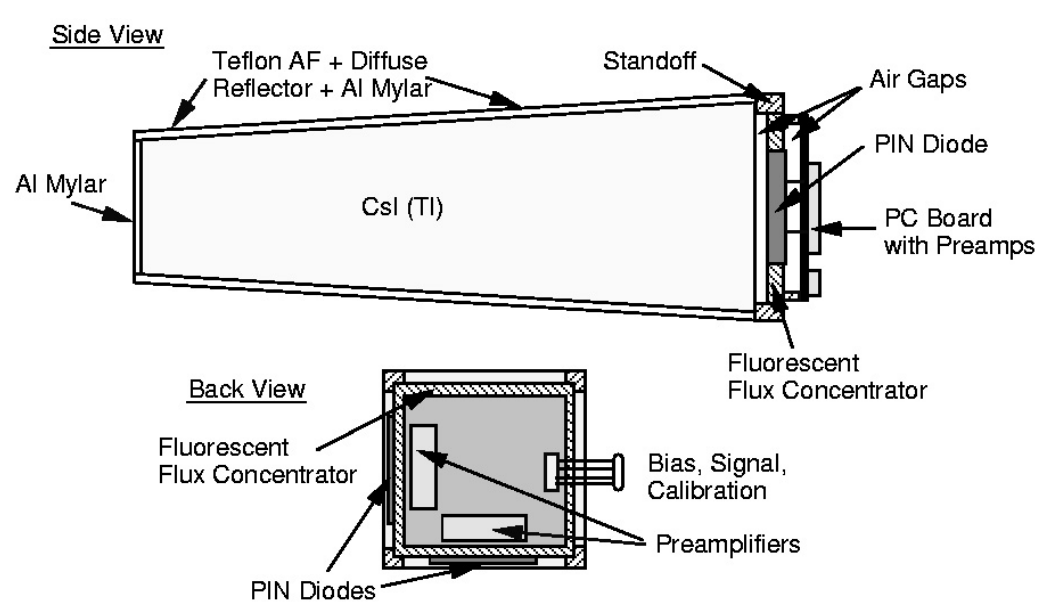

Figure 3.12: A schematic of a $\operatorname{CsI}(\mathrm{Tl})$ crystal and photo diodes [90, Figure 7-19].

\subsubsection{The Instrumented Flux Return}

The Instrumented Flux Return (IFR) facilitates a dual purpose as the magnetic flux return for the 1.5 Tesla super conduction magnet, and as a neutral hadron and muon detector. The IFR is composed of three components, the barrel and two endcaps. The barrel consists of six sextants each $3.75 \mathrm{~m}$ in length and between $1.88 \mathrm{~m}$ and $3.23 \mathrm{~m}$ width while the endcaps are hexagonal plates. The IFR barrel is constructed with 18 layers of iron plates where the first nine interior plates are $2 \mathrm{~cm}$ in thickness, the next four plates are $3 \mathrm{~cm}$ thick, followed by two $5 \mathrm{~cm}$ thick iron plates and the two exterior plates are $10 \mathrm{~cm}$ thick. The endcaps have a similar arrangement of iron plates except there are three $5 \mathrm{~cm}$ thick plates and only one $10 \mathrm{~cm}$ thick exterior plate. The variation in the plate sizes with the radius is to allow low momentum muons to be detected, while insuring that hadrons are completely absorbed. An illustration of the IFR iron structure can be seen in Figure 3.14.

The IFR has 21 layers of Resistive Plate Counters (RPC) located in the 17 gaps between the plates, on the outside of the detector, between the solenoid magnet 


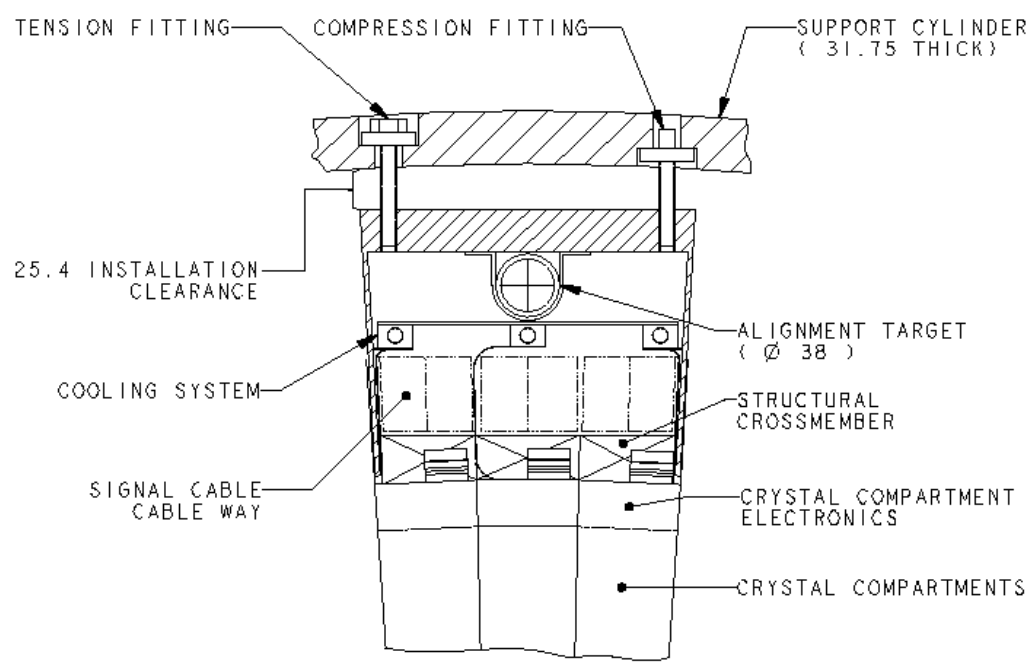

Figure 3.13: A schematic of the barrel module in the EMC [90, Figure 7-5].

and IFR iron plates and in a double layer around the outside of the EMC. The $\mathrm{RPC}$ are segmented along the $z$ and $\phi$ directions in the barrel section, and along the $x$ and $y$ direction in the end plates. Therefore, with the layer in which the hit occurred identified, the position can be reconstructed. Moreover, the hits are matched with the SVT and DCH tracks during reconstruction. RPC are a type of capacitor, a device in which two conducting plates, with different potentials, are separated by a dielectric material. In the RPC, the dielectric material is composed of a two layers of Bakelite along the conductors with a gas mixture, $134 \mathrm{~A}\left(\mathrm{C}_{2} \mathrm{H}_{2} \mathrm{~F}_{6}\right)$, Freon $\left(\mathrm{C}_{x} \mathrm{Cl}_{y} \mathrm{~F}_{z}\right)$ [93], Argon and a small amount of ISO-butane in the central layer. When a particle travels through the gas filled layer, the gas becomes ionized, breaking down the dielectric resulting in a signal [90]. Unfortunately, it was found that the RPC degraded rapidly. Therefore, the muon detection system was upgraded with Limited Streamer Tubes (LST) during the detector shutdown periods from 2004-2006. The RPC's, in the inner 18 layers of the detector, were replaced with 12 layers of LST detectors and 6 layers of brass to compensate for the loss of absorbing material by 


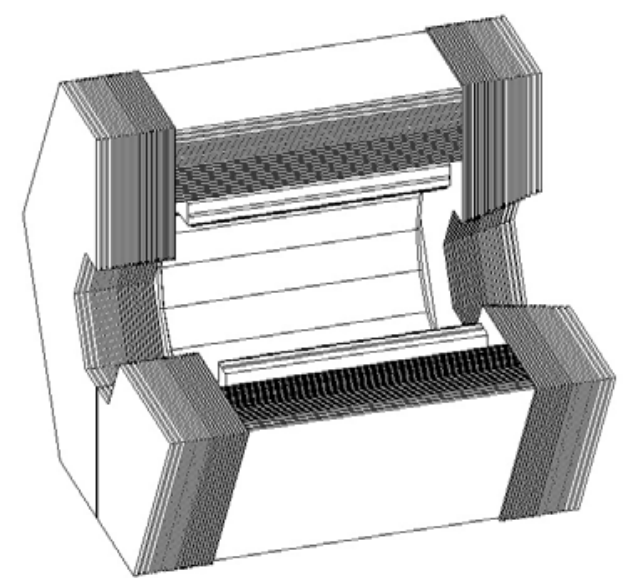

Figure 3.14: A schematic of the IFR [90, Figure 8-9].

removing the RPC detectors. The brass was positioned in every other layer starting in the 5th innermost layer. The LST detector consists of 7 to 8 LST cells which are $380 \mathrm{~mm}$ long, $15 \mathrm{~mm}$ high and $17 \mathrm{~mm}$ wide. The cells are composed of PVC plastic coated with a graphite paint that is maintined at a grounded potential, a central high voltage gold plated anode which is held in place by 6 wire holders, and are filled with a (89:8:3) gas mixture of CO2, ISO-butane, and Argon. When a particle travels through a LST cell, it ionizes the gas causing a current to flow between the anode and the grounded exterior. The resulting decrease in the anode voltage while the current flows is the signal [94].

\subsubsection{Trigger Selection}

The selection of events of interest in the BaBar Detector has been decomposed into two stages: the hardware based level 1 Trigger (L1) and the software based Level 3 Trigger (L3). An additional trigger, Level 2 which was not implemented, was also included in the data-flow design in case the luminosity produced a higher background in the detector than expected. These triggers are designed to select events of interest 
while reducing the background and random noise [95].

\section{Level 1 Trigger}

The Level 1 Trigger accepts information from the DCH, EMC and IFR. A signal consisting of one bit from each of the 7104 cells in the DCH is processed by the Drift Chamber Trigger (DCT) to identify tracks. The Electromagnetic Trigger (EMT) receives input from the 280 towers $^{9}$ in the EMC, and identifies the energy deposits in the EMC. The IFR is decomposed into ten sections from which eight of the $\phi$ readout layers provide input for the IFR Trigger (IFT). These sections are the six sextants and the two half doors. The primary functions of the IFT are to veto cosmic events and to identify muons from the interaction $e^{+} e^{-} \rightarrow \mu^{+} \mu^{-}$which can be used for measuring detector parameters such as the luminosity. The output of the DCT, and the EMT and the IFT are utilized to determine if the signal contains a physics event by a Global Level Trigger. The frequency at which the events are accepted by the Level 1 Trigger is approximately $1 \mathrm{kHz}$, a result of the luminosity and the background rate of the detector [95].

\section{Level 3 Trigger}

The Level 3 Trigger, which runs on a farm of Linux machines, has a primary purpose of reducing the frequency of selected events from its maximum input rate, $2 \mathrm{KHz}$, to less than $120 \mathrm{~Hz}$ with a high efficiency in physics events of interest. The L3 Trigger also supports the online fast monitoring, and selects calibration samples. The L3 Trigger receives input from the L1 Trigger and two subdetectors, the DCH and the EMC. After an initial reconstruction of the charged tracks in the DCH and the

\footnotetext{
${ }^{9}$ The Barrel section of the EMC contains 240 towers each consisting of 24 crystals in a 8 by 3 array defined along the $\theta$ and $\phi$ axis respectively. While the End Cap contains 40 towers with 19-22 crystals arranged in wedges. The threshold for a signal in the CsI(Tl) crystals is $15 \mathrm{MeV}$. This threshold energy in the $\mathrm{CsI}(\mathrm{Tl})$ crystals was changed from $20 \mathrm{MeV}$ to $15 \mathrm{MeV}$ in 2000.
} 
neutral cluster in the EMC, the physics content of the event is evaluated. The "Physics Filters" are designed to select $\tau^{+} \tau^{-}$, two photon, $\mathrm{B} \overline{\mathrm{B}}$ and $c \overline{\mathrm{c}}$ events with a high efficiency. In addition to the "Physics Filters", the "Bhabha Filters" isolate the unique topology of electron-positron scattering and rejects them from the selected events. This selection reduces frequency to a rate in which the entire event from all of the subdetectors and the two triggers can be written to tape for storage and later, a complete reconstruction of the events [95]. 


\section{Chapter 4}

\section{Branching Fraction Measurement of $\tau^{-} \rightarrow h^{-} h^{-} h^{+} \nu_{\tau}$}

\subsection{Methodology of Analysis}

The strategy for the $\tau^{-} \rightarrow h^{-} h^{-} h^{+} \nu_{\tau}$ analysis is to select a pure sample of $\tau^{-} \rightarrow$ $h^{-} h^{-} h^{+} \nu_{\tau}$ decays from $e^{+} e^{-} \rightarrow \tau^{+} \tau^{-}$events by requiring $\tau^{+}$partner to decay leptonically: $\tau^{+} \rightarrow e^{+} \nu_{\tau} \bar{\nu}_{e}$ or $\tau^{+} \rightarrow \mu^{+} \nu_{\tau} \bar{\nu}_{\mu}$. Charge conjugation ${ }^{1}$ is implied throughout this thesis. The selected hadron tracks, $h^{ \pm}$tracks, are then identified uniquely as a kaon or pion and the decays are categorized as $\tau^{-} \rightarrow \pi^{-} \pi^{-} \pi^{+} \nu_{\tau}, \tau^{-} \rightarrow K^{-} \pi^{-} \pi^{+} \nu_{\tau}$, $\tau^{-} \rightarrow K^{-} \pi^{-} K^{+} \nu_{\tau}$, and $\tau^{-} \rightarrow K^{-} K^{-} K^{+} \nu_{\tau}$. The number of signal events are then determined by means of an efficiency migration matrix, $\epsilon_{i j}$, obtained from Monte Carlo (MC) Simulations, that takes into account cross-feed between the four channels where $i$ is the selected decay index and $j$ is the true decay index. Data control samples of kaons and pions from $D^{*+} \rightarrow \pi^{+} D^{0}$ and $D^{0} \rightarrow K^{-} \pi^{+}$decays are used to modify the efficiency migration matrix to correct for small data/MC differences in the modelling of the particle identification. The number of signal events for decay mode $j$ is:

\footnotetext{
${ }^{1}$ Charge conjugation operator is the operator which changes a particle into its antiparticle. When charge conjugation is assumed for a decay, it means that the decay is assumed to be invariant under the charge conjugation operator.
} 


$$
\mathbf{N}_{j}^{S i g}=\sum_{i}\left(\epsilon^{-1}\right)_{j i}\left(\mathbf{N}_{i}^{D a t a}-\mathbf{N}_{i}^{B k g}\right)
$$

where $\mathbf{N}_{i}^{\text {Data }}$ is the number of data events selected as decay mode $i$, and $\mathbf{N}_{i}^{B k g}$ is the number of MC background events selected as decay mode $i$ excluding the $\tau^{-} \rightarrow$ $h^{-} h^{-} h^{+} \nu$ cross-feed channels. Accounting for the integrated luminosity, $\mathcal{L}$, and the $\tau$ pair cross section, $\sigma_{\tau^{+} \tau^{-}}$, one obtains the branching fraction

$$
\mathbf{B r}_{j}=\frac{\mathbf{N}_{j}^{S i g}}{2 \mathcal{L} \sigma_{\tau^{+} \tau^{-}}}
$$

for decay mode $j$, where the number of $\tau$ pairs produced in the collision is $\mathcal{L} \sigma_{\tau^{+} \tau^{-}}$.

\subsection{BABAR Data Set}

The analysis employs a BABAR data sample from runs 1-5 which corresponds to an integrated luminosity of $\mathcal{L}=342 \mathrm{fb}^{-1}$ and includes both on-resonance and offresonance data ${ }^{2}$. The most significant MC samples are those describing the $e^{+} e^{-} \rightarrow$ $\tau^{+} \tau^{-}$events. The $e^{+} e^{-} \rightarrow \tau^{+} \tau^{-}$events were simulated with the " $e^{+} e^{-} \rightarrow \tau^{+} \tau^{-}$" KK2F Monte Carlo Generator $[96,97]$ which employs the TAUOLA Software Package [98]. The $\tau^{-} \rightarrow K^{-} \pi^{-} \pi^{+} \nu$ and $\tau^{-} \rightarrow K^{-} K^{-} K^{+} \nu$ generators were incorporated into the TAUOLA simulation by means of the $\mathrm{K}(1650)$ resonance decaying into $\phi \mathrm{K}$ and by an improved form factor for $\tau \rightarrow K^{-} \pi^{-} \pi^{+} \nu$, respectively. The improved form factors for the $\tau \rightarrow K^{-} \pi^{-} \pi^{+} \nu \mathrm{MC}$ use the modelling parameters [99]: $\mathrm{M}(1270)=$ $1.254 \mathrm{GeV} / c^{2}, \mathrm{M}(1400)=1.463 \mathrm{GeV} / c^{2}, \Gamma(1270)=0.26 \mathrm{GeV}, \Gamma(1400)=0.30 \mathrm{GeV}, \mathrm{F} 1$ $=\mathrm{BW}[\mathrm{K} 1(1270)] \mathrm{X} \mathrm{BW}[\rho(770)], \mathrm{F} 2=(\eta \mathrm{BW}[\mathrm{K} 1(1270)]+\mathrm{BW}[\mathrm{K} 1(1400)]) \mathrm{X} \mathrm{BW}\left[\mathrm{K}^{*}\right]$

\footnotetext{
${ }^{2}$ For the BABAR experiment, the PEP-II storage rings operate with a c.m. energy around the $\Upsilon(4 S)$ resonance, $10.58 \mathrm{GeV}$. Data that is collected on the $\Upsilon(4 S)$ is referred to as on-resonance and data that is collected slightly below the $\Upsilon(4 S)$ resonance is referred to as off-resonance.
} 
and $\eta=0.33[100]^{3}$. The non- $\tau$ backgrounds were simulated for: $e^{+} e^{-} \rightarrow \mu^{+} \mu^{-}(\gamma)[96]$, $e^{+} e^{-} \rightarrow \Upsilon(4 S) \rightarrow B^{0} \bar{B}^{0}, e^{+} e^{-} \rightarrow \Upsilon(4 S) \rightarrow B^{+} B^{-}, e^{+} e^{-} \rightarrow c \bar{c}, e^{+} e^{-} \rightarrow u \bar{u}, e^{+} e^{-} \rightarrow$ $d \bar{d}$, and $e^{+} e^{-} \rightarrow s \bar{s}[101,102]$. The MC Simulation included a complete simulation of the BABAR Detector response by means of the GEANT4 Software Package [103]. The simulated events are then reconstructed in the same manner as data. The run-by-run integrated luminosities for the MC and data are displayed in Table 4.1.

\begin{tabular}{|l|r|r|r|r|r|r|}
\hline & Run 1 & Run 2 & Run 3 & Run 4 & Run 5 & Total \\
\hline Data & 23.0 & 68.1 & 34.8 & 110.4 & 106.0 & 342.4 \\
\hline$\tau^{+} \tau^{-}$ & 39.8 & 151.1 & 74.4 & 100.3 & 99.8 & 465.3 \\
$\mu^{+} \mu^{-}$ & 41.7 & 136.7 & 75.9 & 101.0 & 105.6 & 461.0 \\
$u d s$ & 41.6 & 120.61 & 74.0 & 101.5 & 101.9 & 439.6 \\
$c \bar{c}$ & 63.3 & 216.7 & 103.429 & 120.8 & 98.8 & 602.9 \\
$B^{+} B^{-}$ & 126.5 & 452.7 & 216.724 & 306.0 & 306.9 & 1409.0 \\
$B^{0} \bar{B}^{0}$ & 125.9 & 461.4 & 226.2 & 303.8 & 302.5 & 1419.7 \\
\hline$\tau^{-} \rightarrow K^{-} \pi^{-} \pi^{+} \nu$ & $210 \mathrm{~K}$ & $628 \mathrm{~K}$ & $330 \mathrm{~K}$ & $1012 \mathrm{~K}$ & $730 \mathrm{~K}$ & $2910 \mathrm{~K}$ \\
$\tau^{-} \rightarrow K^{-} K^{-} K^{+} \nu$ & $166 \mathrm{~K}$ & $500 \mathrm{~K}$ & $264 \mathrm{~K}$ & $806 \mathrm{~K}$ & $586 \mathrm{~K}$ & $2322 \mathrm{~K}$ \\
$\tau^{-} \rightarrow K^{-} \pi^{-} \pi^{+} \pi^{0} \nu$ & $20 \mathrm{~K}$ & $20 \mathrm{~K}$ & $20 \mathrm{~K}$ & $20 \mathrm{~K}$ & $20 \mathrm{~K}$ & $100 \mathrm{~K}$ \\
$\tau^{-} \rightarrow K^{-} \pi^{-} K^{+} \pi^{0} \nu$ & $20 \mathrm{~K}$ & $20 \mathrm{~K}$ & $20 \mathrm{~K}$ & $20 \mathrm{~K}$ & $20 \mathrm{~K}$ & $100 \mathrm{~K}$ \\
$\tau^{-} \rightarrow K^{-} K^{-} K^{+} \pi^{0} \nu$ & $20 \mathrm{~K}$ & $20 \mathrm{~K}$ & $20 \mathrm{~K}$ & $20 \mathrm{~K}$ & $20 \mathrm{~K}$ & $100 \mathrm{~K}$ \\
\hline
\end{tabular}

Table 4.1: The Data (on-resonance+off-resonance) and equivalent MC integrated luminosity for Runs 1-5 in $\mathrm{fb}^{-1}$. For the $\tau^{-} \rightarrow K^{-} \pi^{-} \pi^{+} \nu_{\tau}$ and $\tau^{-} \rightarrow K^{-} K^{-} K^{+} \nu_{\tau}$ MC the total number of events produced is quoted, while the $\tau^{-} \rightarrow \pi^{-} \pi^{-} \pi^{+} \nu_{\tau}$ and $\tau^{-} \rightarrow K^{-} \pi^{-} K^{+} \nu_{\tau}$ are included in the generic $\tau^{+} \tau^{-}$MC.

\subsection{Selection Procedure}

\subsubsection{Characteristic Event Shape}

The event selection of $\tau^{-} \rightarrow h^{-} h^{-} h^{+} \nu_{\tau}$ begins by selecting events which have an event shape consistent with the kinematic constraint from $e^{+} e^{-} \rightarrow \tau^{+} \tau^{-}$events in which one $\tau$ decays leptonically and the other decays into $\tau^{-} \rightarrow h^{-} h^{-} h^{+} \nu_{\tau}$. Therefore,

\footnotetext{
${ }^{3} \mathrm{BW}$ stands for Breit-Wigner function.
} 
events are required to have four well reconstructed tracks, which do not originate from conversions in the material of the detectors, and have a net charge of zero. In $e^{+} e^{-} \rightarrow \tau^{+} \tau^{-}$at the BABAR collison energy, $\sim \sqrt{s}=10.58 \mathrm{GeV}$, the individual $\tau$ decays are well separated in the c.m. frame. This makes it convenient to select events by splitting the event into hemispheres in the c.m. frame, with the plane orthogonal to the thrust axis ${ }^{4}$ with three of the tracks in one hemisphere and the other track isolated in the opposite hemisphere. These events are referred to as 1 verses 3 prong events. An event display of a typical 1 verses 3 prong $\tau$ decay can be seen in Figure 4.1.

To remove contamination from $q \bar{q}$ and two photon backgrounds, the event is required to have kinematic distributions that are consistent with coming from $\tau^{+} \tau^{-}$ events. Because the $\tau^{+} \tau^{-}$pairs have a high momentum in the c.m. frame, the events have a jetty shape, and therefore a thrust higher than 0.85 is required to remove $q \bar{q}$ and two photon backgrounds which have a more spherically symmetrical shape and lower thrust. In addition, the transverse momentum in the c.m. frame of the event, the momentum orthogonal to the beam axis, is required to be greater than $0.9 \%$ of the c.m. energy to remove events that do not have missing particles, namely neutrinos.

To allow for a clean particle identification of the selected tracks in the event, the track must be within the acceptance of the relevant sub-detectors. Therefore, the tracks in the event are required to be within the geometric acceptance of both the DIRC and the EMC, $(-0.76<\cos (\theta)<0.80)$. Furthermore, the track must have a minimum transverse momentum of $250 \mathrm{MeV} / c$, enabling them to reach the DIRC.

\footnotetext{
${ }^{4}$ The thrust axis is defined by the unit vector that maximizes the projected momentum from all the charged and neutral reconstructed particles in the event. The thrust axis gives the axis of energy flow in the event. The thrust of an event is a shape parameter determined from the normalized projection of all the momenta from the relevant reconstructed particles onto the thrust axis $[104,105]$.
} 

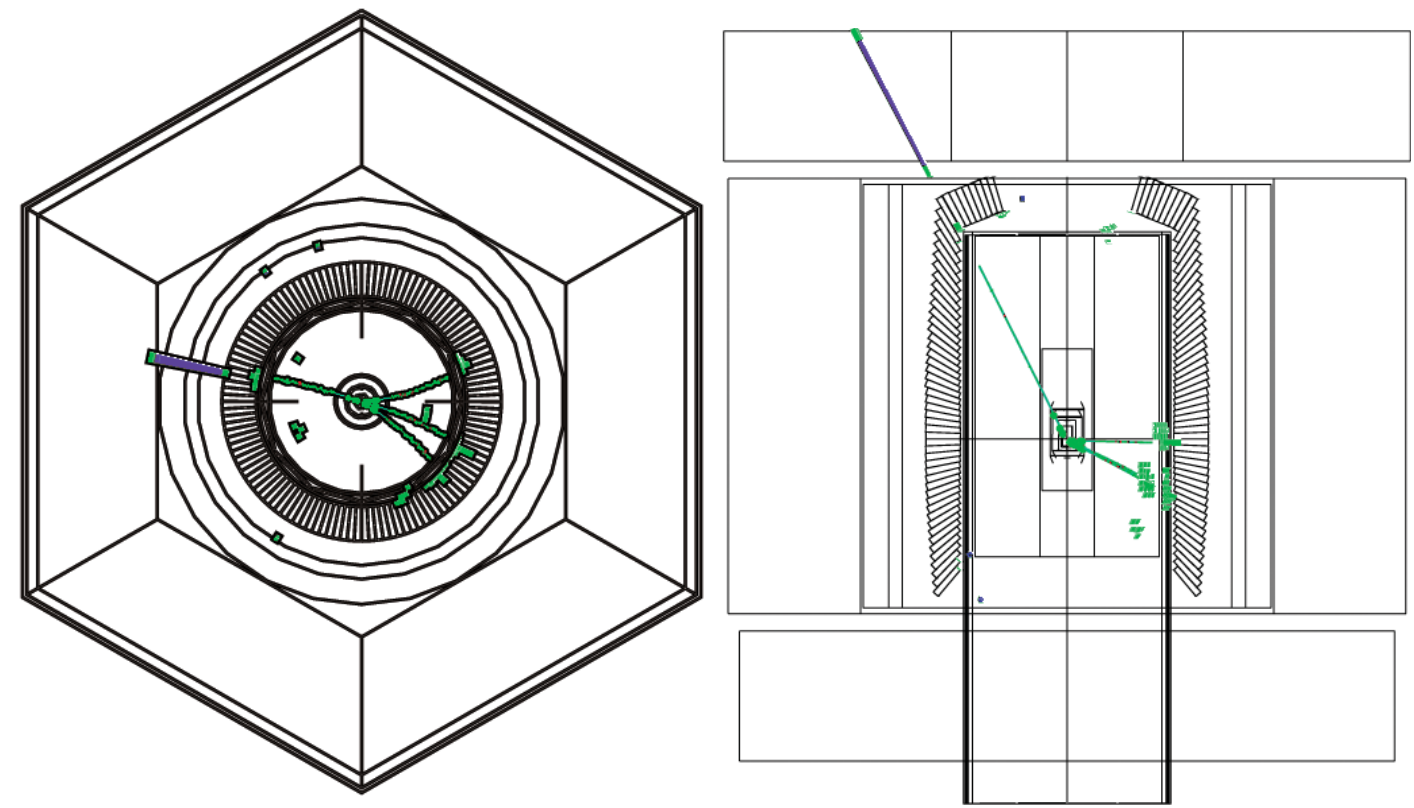

Figure 4.1: An event display of a typical 1 vs 3 prong $\tau$ pair event $\left(\tau^{+} \rightarrow \mu^{+} \nu \bar{\nu}\right.$ and $\tau^{-} \rightarrow \pi^{-} \pi^{-} \pi^{+} \nu$ ). The image on the left-hand side is a front view of the BABAR Detector, while the image on the right-hand side is a lateral view of the event. The two inner most detectors are the Silicon Vertex Tracker (SVT) and the drift Chamber (DCH). In these detectors, you can see the measured ionization from four particles that transversed the detector. The energy per unit of distance $(d E / d x)$ left by a particle can be used to identify the tracks. Exterior to the DCH are the 12 quartz bars from the DIRC. As the high energy particles transverse the quartz bars, the Cherenkov light is collected and reflected into the standoff box. The Cherenkov angle is measured in the standoff box component of the DIRC and used for particle identification. The outside of the DIRC is the Electromagnetic Calorimeter (EMC). The green blocks near the tracks in the EMC indicate that the particles have only deposited a small amount of energy. The solid purple hit on the isolated track in the outermost detector, the IFR and the small signal in the EMC clearly indicates that the isolated particle was a muon. With the additional information from the SVT, the DCH and the DIRC, the three tracks that do not produce a signal in the IFR and have a small signal in the electromagnetic calorimeter are identified as pion tracks.

\subsubsection{Tag Selection}

The isolated track or "tag track" is required to be identified as either an electron or a muon. A detailed description of these selectors can be found in Appendix A. To 
remove contamination from Bhabha events, $e^{+} e^{-} \rightarrow e^{+} e^{-}(\gamma)$, and $e^{+} e^{-} \rightarrow \mu^{+} \mu^{-}(\gamma)$, in which an undetected photon converts into an $e^{+} e^{-}$pair, the tag track is required to have a maximum momentum of less than $80 \%$ of $\sqrt{s} / 2 c$.

\subsubsection{Prong Selection}

To further reduce the Bhabha contamination, the 3 prong tracks must fail the standard BABAR electron selector. The remaining backgrounds are primarily from $\tau$ decays with $\pi^{0}$ and $K_{S}^{0}$. The $\tau$ events containing $K_{S}^{0}$ are considered to be backgrounds because the reconstruction efficiency of tracks originating from a $K_{S}^{0}$ decays differs from that of tracks originating from the interaction point in the detector. Identified pairs of oppositely charged tracks which have a mass consistent with being that of the $K_{S}^{0}$ are removed, if the vertex of the track pair is more than six standard deviations from the interaction point.

\subsubsection{Neutral Rejection}

To reduce contamination from $\pi^{0}$ events and non- $\tau$ backgrounds, a neutral veto is required. The neutral veto removes any events with well reconstructed $\pi^{0}$ candidates or events with large unassociated net neutral energies. The neutral association employed in this analysis is based on the proximity of the neutrals to the track or tracks in the same c.m. hemisphere as the neutral and the energy of the neutral. Reconstructed neutrals are classified as being associated with a track if they have: an $E_{c a l}<0.200 \mathrm{GeV}$ or an $E_{c a l}<1 G e V-0.016 \mathrm{GeV} / \mathrm{cm} * d$ for an electron; $E_{\text {cal }}<0.200 \mathrm{GeV}$ for a muon; or $E_{\text {cal }}<0.200 \mathrm{GeV}$ and $d<50 \mathrm{~cm}$ for a hadron. $d$ is defined as the distance in centimetres between the cluster associated with the track and the nearest neutral at the inner surface of the calorimeter. The remaining unassociated neutrals with an energy greater than $0.050 \mathrm{GeV}$ are then consid- 
ered. In the electron tag hemisphere, the event is vetoed if a residual neutral has an energy $E_{\text {res }}^{T a g} \geq 1.0 \mathrm{GeV}$. Similarly, the muon hemisphere is vetoed if a residual neutral has an energy $E_{r e s}^{T a g} \geq 0.5 \mathrm{GeV}$. For the 3 prong hemisphere, the event is vetoed if the total residual energy is $E_{\text {res }}^{3 p r o n g} \geq 0.200 \mathrm{GeV}$. After applying the neutral cuts, the $e^{+} e^{-} \rightarrow q \bar{q}$ background in the $\tau^{-} \rightarrow h^{-} h^{-} h^{+} \nu_{\tau}$ sample is reduced to $\sim 0.1 \%$. Contribution from $\tau$ decays with an extra $\pi^{0}$ to each of the four decay modes $\tau^{-} \rightarrow \pi^{-} \pi^{-} \pi^{+} \nu_{\tau}, \tau^{-} \rightarrow K^{-} \pi^{-} \pi^{+} \nu_{\tau}, \tau^{-} \rightarrow K^{-} \pi^{-} K^{+} \nu_{\tau}$, and $\tau^{-} \rightarrow K^{-} K^{-} K^{+} \nu_{\tau}$ are estimated to be $(3.6 \pm 0.3) \%,(2.3 \pm 0.4) \%,(0.4 \pm 0.1) \%$, and less than $5.0 \%$ respectively.

\subsubsection{Selection of the $\pi^{-} \pi^{-} \pi^{+}, K^{-} \pi^{-} \pi^{+}, K^{-} \pi^{-} K^{+}$and $K^{-} K^{-} K^{+}$ Channels.}

Once the events have been selected, the signal tracks are then identified as either a kaon or a pion to classify the event into the four decay modes: $\tau^{-} \rightarrow \pi^{-} \pi^{-} \pi^{+} \nu_{\tau}$; $\tau^{-} \rightarrow K^{-} \pi^{-} \pi^{+} \nu_{\tau} ; \tau^{-} \rightarrow K^{-} \pi^{-} K^{+} \nu_{\tau} ;$ and $\tau^{-} \rightarrow K^{-} K^{-} K^{+} \nu_{\tau}$. The particle identification of kaons and pions by the BABAR Detector utilizes information from the DIRC, namely the Cherenkov angle $\left(\theta_{C}\right)$, the SVT, and the Drift Chamber, specifically the differential ionization energy loss per unit length $\left(\frac{d E}{d x}\right)$, to distinguish the two particle types. This information is employed in a likelihood approach to identify kaon and pion tracks with a standard BABAR particle identification selector defined in Appendix A. After selecting the pions and kaons with the standard BABAR kaon selector, there is a discrepancy between data and $\mathrm{MC}$ around $P_{l a b}=0.7 \mathrm{GeV} / c$ for events that are in the $\theta_{C}$ vs $\mathrm{P}$ regions $\mathrm{A}$ and $\mathrm{B}$ as defined in Figure 4.2. This discrepancy can be seen in the pion control sample selected as a kaon illustrated in Figure 4.3 (left). These regions correspond to when the information from the Cherenkov angle is not usable and where the separation between pion and kaon $\frac{d E}{d x}$ curves is minimal. Therefore, a 
track that satisfies the kaon criteria is required to be in region $\mathrm{C}$ of the $\theta_{C}$ vs $P_{l a b}$ or have $P_{l a b}>0.8 \mathrm{GeV} / c$ to be identified as a kaon, while a track that fails the standard kaon selector is classified as a pion. The improved agreement in the pion control sample selected as a kaon after the extra selection criteria are applied can be seen in Figure 4.3 (right). The characteristic kaon identification efficiency is $\sim 80 \%$ and pion misidentification rate is $\sim 1 \%$.

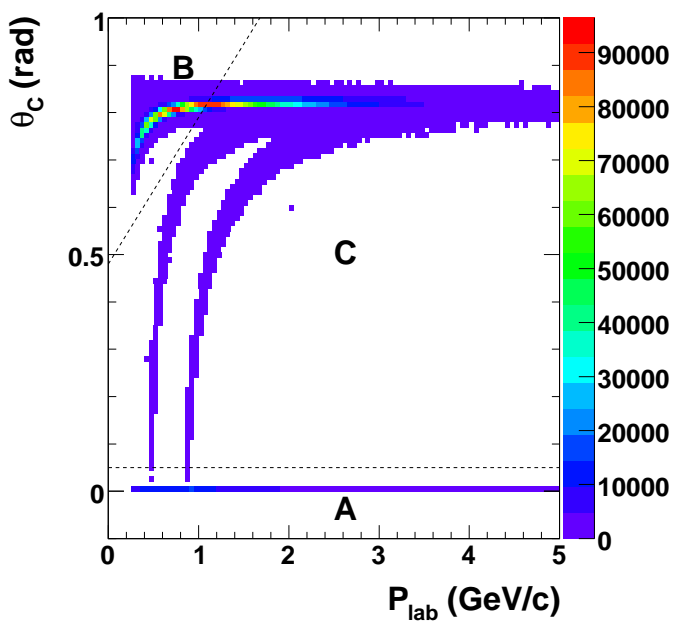

Figure 4.2: The particle identification regions for $\theta_{C}$ as a function of momentum. The lower rectangular region is referred to as region $\mathrm{A}$, the upper triangular region is referred to as region $\mathrm{B}$ and the remaining region is referred to as region $\mathrm{C}$.

An event is selected as $\tau^{-} \rightarrow \pi^{-} \pi^{-} \pi^{+} \nu_{\tau}$ if all three hadron tracks are classified as pions. If there are two oppositely charged pions and one kaon, the event is selected as $\tau^{-} \rightarrow K^{-} \pi^{-} \pi^{+} \nu_{\tau}$; if there are two oppositely charged kaons and one pion, the event is selected as $\tau^{-} \rightarrow K^{-} \pi^{-} K^{+} \nu_{\tau}$. Lastly, if all the hadrons are classified as kaons, the event is selected as $\tau^{-} \rightarrow K^{-} K^{-} K^{+} \nu_{\tau}$. The component of the efficiency matrix, $M_{i j}$, associated with the pion-kaon particle separation and the classification of the events can be seen in Table 4.2 . 

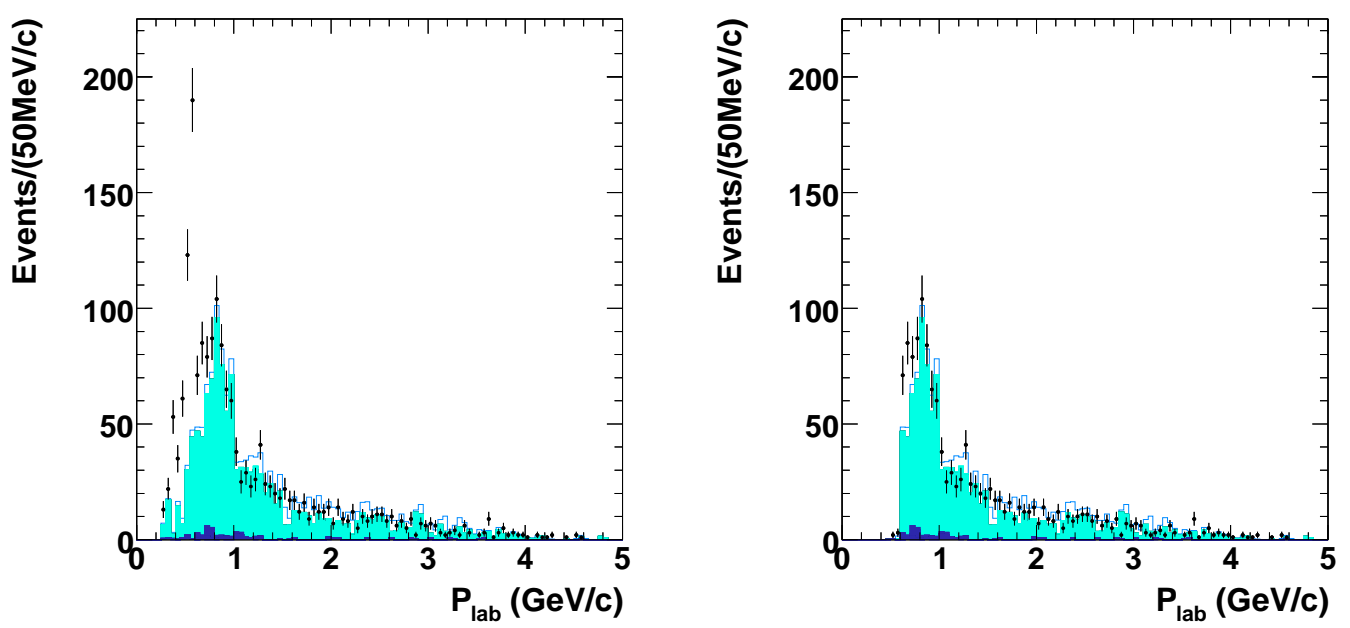

Figure 4.3: The Data-MC discrepancy for the standard BABAR kaon selector before (left) and after (right) the selection criteria that a track selected as a kaon is required to be in region $\mathrm{C}$ of the $\theta_{C}$ vs $P_{l a b}$ or have $P_{l a b}>0.8 \mathrm{GeV} / c$ has been applied the pion control sample. This control sample selects $\tau^{-} \rightarrow h^{-} h^{-} h^{+} \nu_{\tau}$ cross-feed events using the $\tau^{-} \rightarrow K^{-} \pi^{-} K^{+} \nu_{\tau}$ selection criteria in this analysis with the additional constraint that the invariant mass of the selected events is between $1.65 \mathrm{GeV} / c^{2}-1.82 \mathrm{GeV} / c^{2}$ and the corresponding particle identification for the kaon. The data is represented by the points with the error bars representing the statistical uncertainty. The open histogram represents the $\tau^{-} \rightarrow K^{-} \pi^{-} \pi^{+} \nu_{\tau} \mathrm{MC}$, while the lightly shaded histogram represents the $\tau^{-} \rightarrow \pi^{-} \pi^{-} \pi^{+} \nu_{\tau}$ cross-feed. The remaining $\tau$ backgrounds and the non- $\tau$ backgrounds are represented by the dark histogram.

The 3 prong hemisphere is required to have a total invariant mass, determined with the selected particle masses, consistent with coming from a $\tau$ decay, $M_{3 p r o n g}<$ $1.82 \mathrm{GeV} / c^{2}$. The mass cut is conservatively placed above the $\tau$ mass to account for resolution effects.

\subsection{D Dalitz Weighting}

In the invariant mass distributions, seen in Figures 4.4 to 4.7, there are small discrepancies between the data and $\mathrm{MC}$ due to the modelling of resonance structure in 
Chapter 4. Branching Fraction Measurement of $\tau^{-} \rightarrow h^{-} h^{-} h^{+} \nu_{\tau}$

\begin{tabular}{|l|llll|}
\hline Candidates & \multicolumn{4}{|c|}{ Decay modes } \\
& $\pi \pi \pi$ & $K \pi \pi$ & $K \pi K$ & $K K K$ \\
\hline$\pi \pi \pi$ & 97.40 & 22.49 & 4.73 & 1.02 \\
$K \pi \pi$ & 1.42 & 74.87 & 16.43 & 6.38 \\
$K \pi K$ & 0.01 & 0.49 & 59.63 & 25.54 \\
$K K K$ & - & - & 0.26 & 50.87 \\
\hline
\end{tabular}

Table 4.2: The component of the efficiency migration matrix associated with pionkaon particle identification represented as percentages for the $\tau^{-} \rightarrow h^{-} h^{-} h^{+} \nu_{\tau}$ analysis. Note: this efficiency matrix includes efficiency losses resulting from the cross-feed of the wrong charge combinations and small factors associated with data control sample corrections to the MC cross-feed efficiencies. As a result, the columns in this table do not add up to $100 \%$.

the decays. This means that the kinematic distributions of the signal tracks in the MC, namely $P_{l a b}$ and $\theta$ which detector acceptance and efficiency depend on, will have slight deviations from the data. As a result, the efficiency of the event selection may be biased by the poor modelling of the resonance structure in the $\tau^{-} \rightarrow h^{-} h^{-} h^{+} \nu_{\tau}$ decays. To correct for this, the reconstructed MC events are weighted so that their three dimensional Daltiz distribution ${ }^{5}$ match the measured three dimensional Daltiz distribution in the data for the $\tau^{-} \rightarrow \pi^{-} \pi^{-} \pi^{+} \nu_{\tau}, \tau^{-} \rightarrow K^{-} \pi^{-} \pi^{+} \nu_{\tau}$, and $\tau^{-} \rightarrow K^{-} \pi^{-} K^{+} \nu_{\tau}$ decays, thereby constraining all the kinematic variables in the decay. This weighting was done in an iterative process to account for shifts in the cross-feed. Due to limitations from statistics, the reconstructed $\mathrm{MC}$ events for the $\tau^{-} \rightarrow K^{-} K^{-} K^{+} \nu_{\tau}$ decay are weighted to have the same invariant mass distribution as the measured data. The invariant mass distributions after the Dalitz weighting has been applied to the MC can be found in Appendix B.

\footnotetext{
${ }^{5}$ For a three body decay, the kinematic structure of the event is described by two variables in the Dalitz plot. Typically, the two variables used in Dalitz plots are $M_{13}$ and $M_{23}$. However, due to the missing neutrino in $\tau$ decays, the invariant mass of the hadronic system, $M_{123}$, must be included in the Daltiz distribution if the kinematics of the decay are to be described completely.
} 

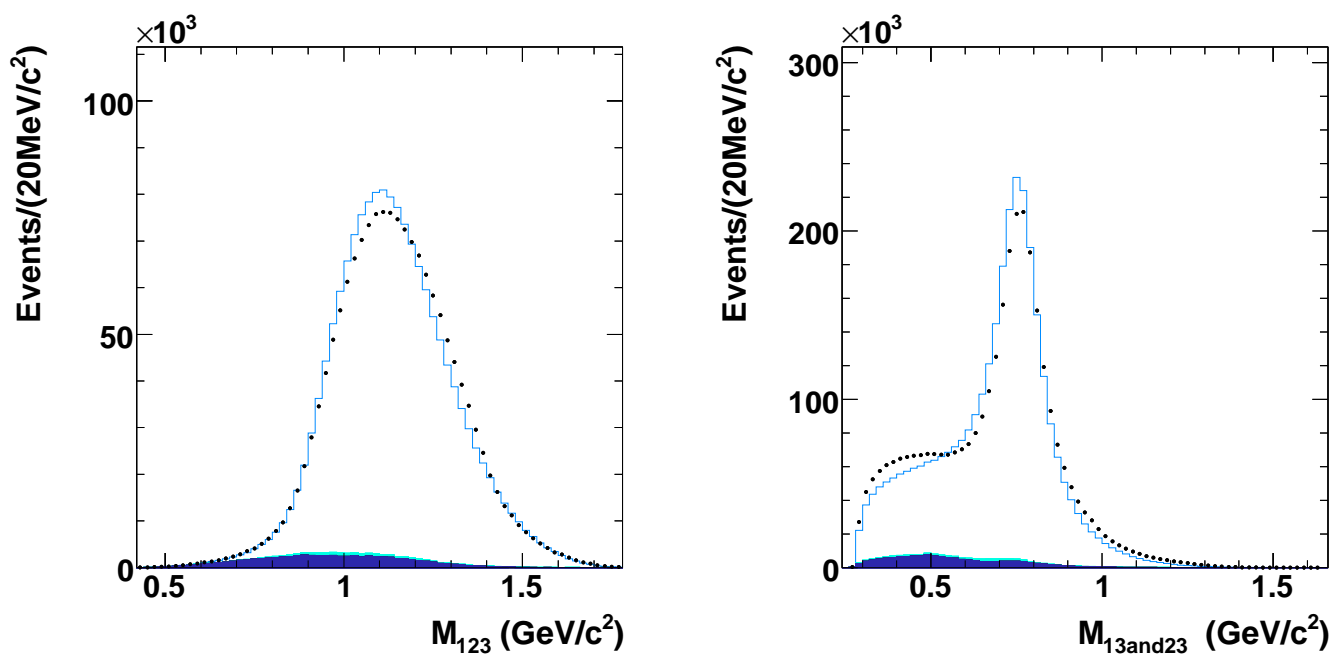

Figure 4.4: The Raw $\tau^{-} \rightarrow \pi^{-} \pi^{-} \pi^{+} \nu_{\tau}$ invariant mass distributions for $M_{123}$ (left), $M_{13}$ and $M_{23}$ (right). The data is represented by the points with the error bars representing the statistical uncertainty. The open histogram represents the signal $\mathrm{MC}$ for the respective channels, while the lightly shaded histogram represents the cross-feed from the other $\tau^{-} \rightarrow h^{-} h^{-} h^{+} \nu_{\tau}$ channels. The remaining $\tau$ backgrounds and the non- $\tau$ backgrounds are represented by the dark histogram.

\subsection{Systematic Uncertainty Studies}

The systematic uncertainties in this analysis originate from event reconstruction and selection, the MC simulation modelling of the background, the detector response, and the signal efficiency.

\subsubsection{Normalization Systematic Uncertainty}

- The systematic uncertainty associated with the normalization has two components: the luminosity uncertainty and the cross-section uncertainty. The uncertainty in the cross-section is $\sigma_{e^{+} e^{-} \rightarrow \tau^{+} \tau^{-}}=(0.919 \pm 0.003)$ [106]. The luminosity uncertainty is determined from Bhabha events, $\mu$ pair events and two photon events $[107,108]$. After removing the $\mu$-pair theoretical uncertainty, which can- 

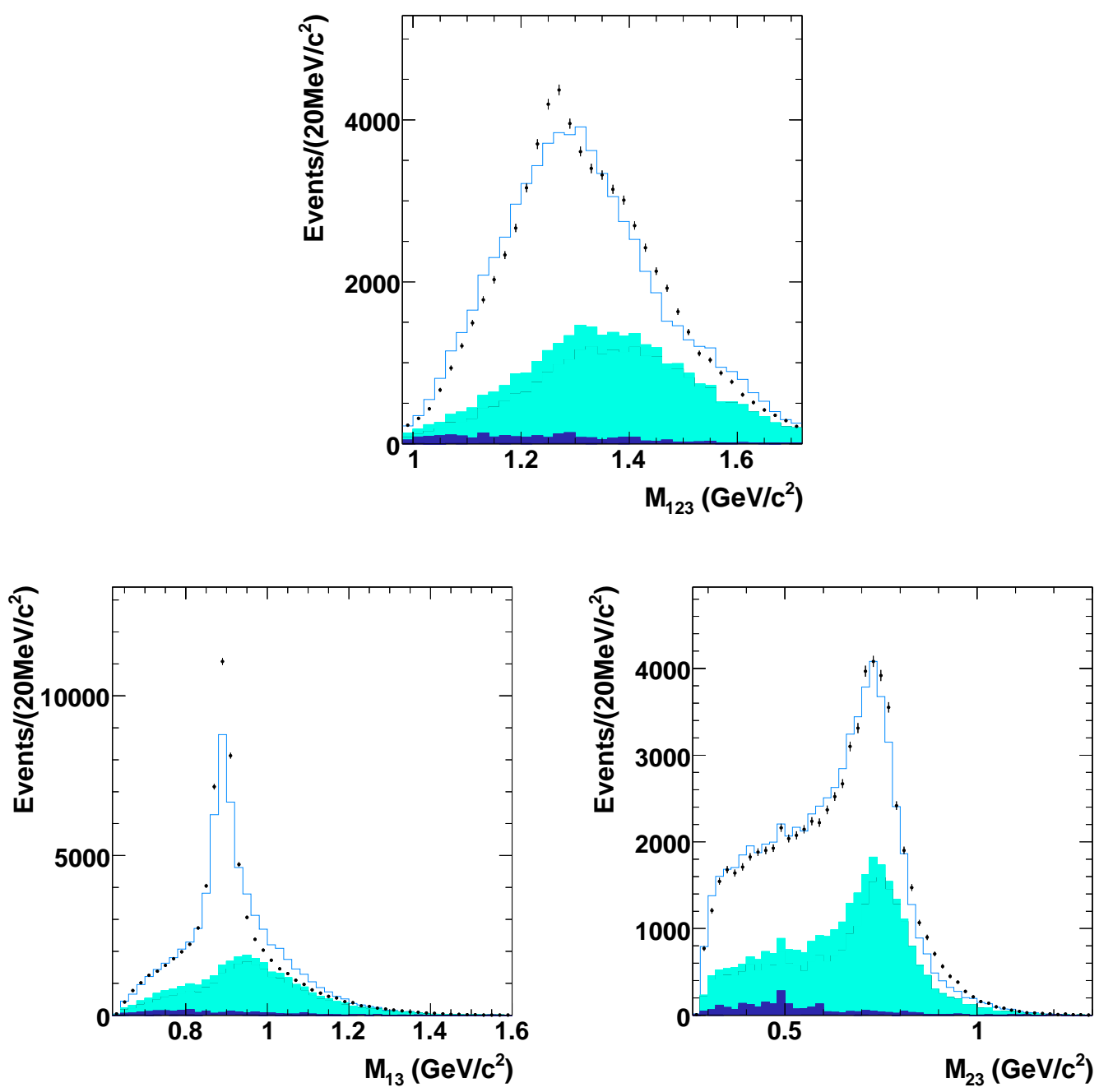

Figure 4.5: The Raw $\tau^{-} \rightarrow K^{-} \pi^{-} \pi^{+} \nu_{\tau}$ invariant mass distributions for $M_{123}$ (top), $M_{13}$ (bottom-left), and $M_{23}$ (bottom-right). The data is represented by the points with the error bars representing the statistical uncertainty. The open histogram represents the signal MC for the respective channels, while the lightly shaded histogram represents the cross-feed from the other $\tau^{-} \rightarrow$ $h^{-} h^{-} h^{+} \nu_{\tau}$ channels. The remaining $\tau$ backgrounds and the non- $\tau$ backgrounds are represented by the dark histogram.

cels in $\sigma \mathcal{L}$, the total luminosity uncertainty is $0.94 \%^{6}[107,108]$. Therefore, the

\footnotetext{
${ }^{6}$ The total luminosity uncertainty of $0.94 \%$ is the run by run luminosity uncertainty. This systematic uncertainty results in a $\sim 20 \%$ probability for the probability of the run variations between runs 2 to 5 .
} 

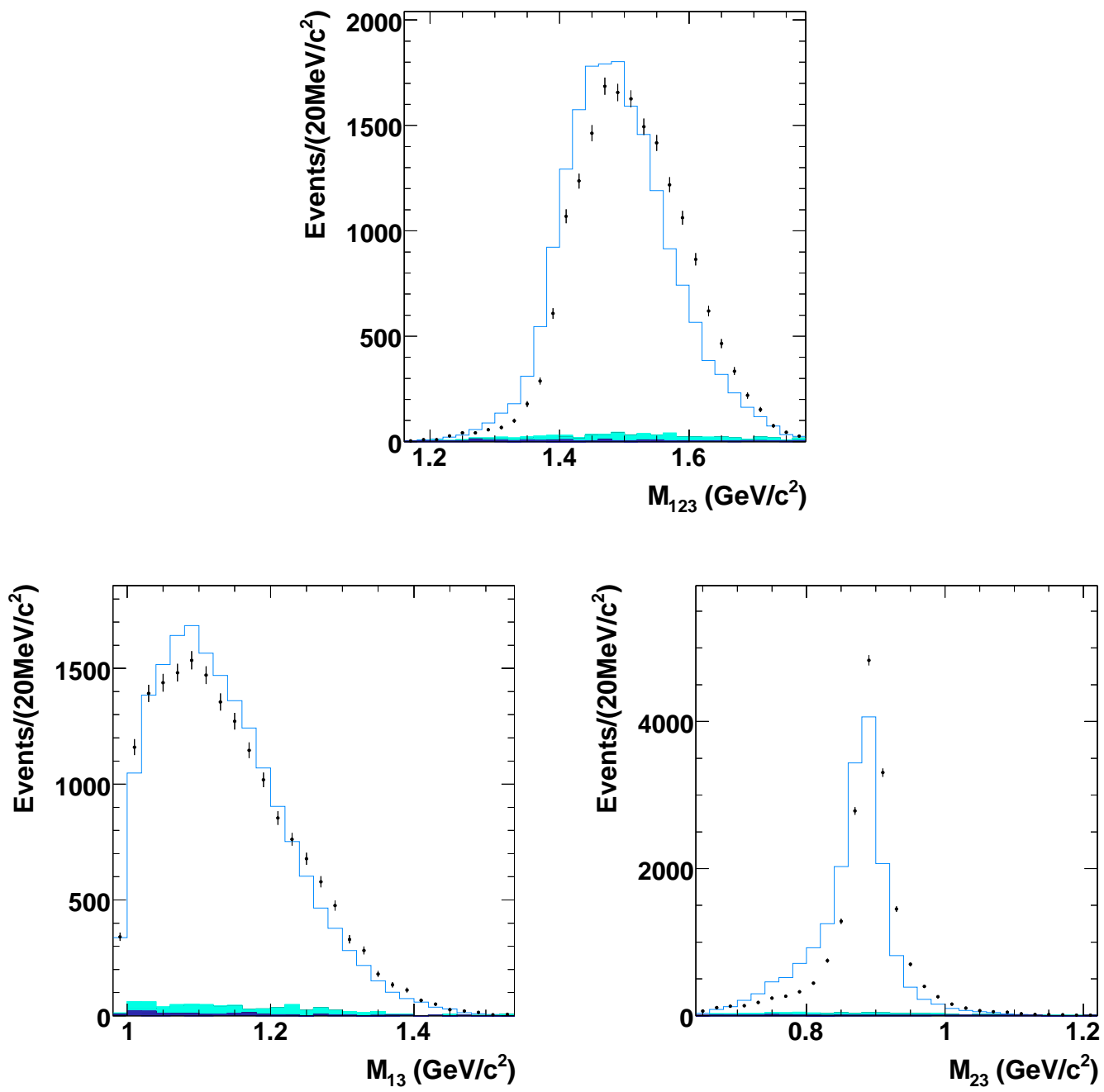

Figure 4.6: The Raw $\tau^{-} \rightarrow K^{-} \pi^{-} K^{+} \nu_{\tau}$ invariant mass distributions for $M_{123}$ (top), $M_{13}$ (bottom-left), and $M_{23}$ (bottom-right). The data is represented by the points with the error bars representing the statistical uncertainty. The open histogram represents the signal MC for the respective channels, while the lightly shaded histogram represents the cross-feed from the other $\tau^{-} \rightarrow$ $h^{-} h^{-} h^{+} \nu_{\tau}$ channels. The remaining $\tau$ backgrounds and the non- $\tau$ backgrounds are represented by the dark histogram.

systematic uncertainty due to normalization is $1.0 \%$. 

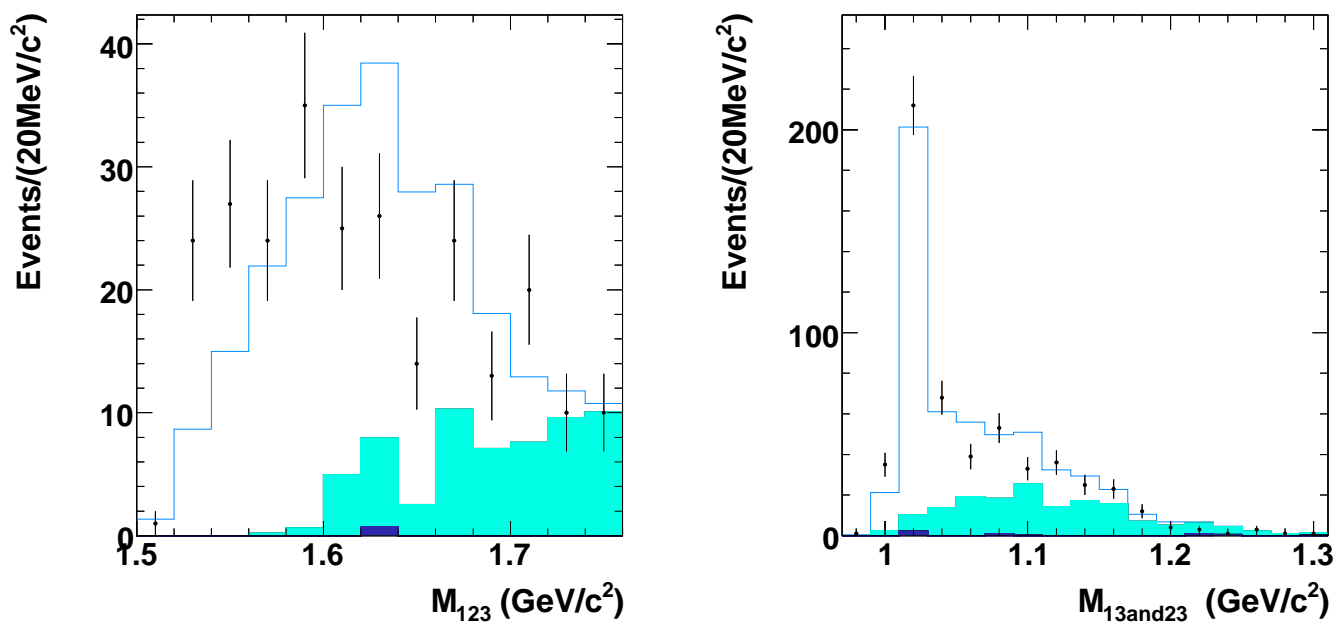

Figure 4.7: The Raw $\tau^{-} \rightarrow K^{-} K^{-} K^{+} \nu_{\tau}$ invariant mass distributions for $M_{123}$ (left), $M_{13}$ and $M_{23}$ (right). The data is represented by the points with the error bars representing the statistical uncertainty. The open histogram represents the signal MC for the respective channels, while the lightly shaded histogram represents the cross-feed from the other $\tau^{-} \rightarrow h^{-} h^{-} h^{+} \nu_{\tau}$ channels. The remaining $\tau$ backgrounds and the non- $\tau$ backgrounds are represented by the dark histogram.

\subsection{2 $M_{i j}$ and Particle-ID}

- The components of the efficiency migration matrix, which are determined from the $\tau$ pair MC, have a statistical uncertainty associated with the MC number of events generated. The propagation of these uncertainties to the measured branching fractions are determined from a Toy $\mathrm{MC}^{7}$ program with 10,000 trials.

- The migration matrix $M_{i j}$ is modified, using data control samples of kaons and pions from $D^{*+} \rightarrow \pi^{+} D^{0}, D^{0} \rightarrow \pi^{+} K^{-}$decays, to account for the small differences between $\mathrm{MC}$ and data. There are two sources of uncertainties arising from the particle identification corrections.

\footnotetext{
${ }^{7} \mathrm{~A}$ program which uses the Monte Carlo Technique to compute the variation in a measurement from an initial set of measured uncertainties is commonly referred to as a Toy MC program.
} 
1. There is a systematic uncertainty related to statistical uncertainties on the entries from the data and $\mathrm{MC}$ control samples used to produce the particle identification correction. The statistical uncertainties for the data and MC control samples, on the measured branching fractions, is determined from a Toy MC program with 100 trials.

2. There is a systematic uncertainty associated with the difference between the event environment of the control sample events used to create the standard BABAR particle-ID table corrections and the environment of the events selected in the $\tau^{-} \rightarrow h^{-} h^{-} h^{+} \nu_{\tau}$ analysis. Such differences could arise from track and photon multiplicity differences. To account for the possible differences in the efficiency from the environment, the particle identification efficiency for each element, in the efficiency matrix, is determined from the selected $\tau^{-} \rightarrow h^{-} h^{-} h^{+} \nu_{\tau} \mathrm{MC}$ events, using the truth information and compared to the average particle identification efficiency determined from the $D^{*+} \rightarrow \pi^{+} D^{0}, D^{0} \rightarrow \pi^{+} K^{-}$pion and kaon MC control samples. As an estimate, the percent difference, of the efficiency determined from the $\tau^{-} \rightarrow h^{-} h^{-} h^{+} \nu_{\tau} \mathrm{MC}$ events relative to the efficiency from the particle-ID $\mathrm{MC}$ control samples, was taken as the systematic uncertainty.

- In Section 4.3.5, it was shown that there is a discrepancy in the standard kaon selector. This results in a data-MC discrepancy in the amount of pions feeding down into the channels that have more kaons. To quantify the uncertainty associated with the discrepancy in the rate of pions being misidentified as kaons with the modified kaon selector, the $\tau^{-} \rightarrow \pi^{-} \pi^{-} \pi^{+} \nu_{\tau}$ events selected as $\tau^{-} \rightarrow K^{-} \pi^{-} \pi^{+} \nu_{\tau}$ events, that had an invariant mass above the $\tau^{-} \rightarrow K^{-} \pi^{-} \pi^{+} \nu_{\tau}$ signal, were used as a control sample. The reduction in the 
data-MC disagreement, from the extra selection criteria, is quantified by taking the ratio of excess data events before and after the extra selection criteria has been applied to the control sample in Figure 4.3. The systematic uncertainty associated with a data-MC discrepancy is then the difference between the branching fraction measured with the modified kaon selector and the standard kaon selector multiplied by the ratio which describes the reduction in the data-MC disagreement.

- A similar particle identification discrepancy of kaons being mis-identified as pions was observed in the $\tau^{-} \rightarrow \pi^{-} \pi^{-} K^{+} \nu_{\tau}$ and $\tau^{-} \rightarrow K^{-} K^{-} \pi^{+} \nu_{\tau}$ decay modes which are highly suppressed ${ }^{8}$. Since, there is no evidence for these decay modes in the data, they are utilized as control samples to determine a systematic uncertainty associated with a kaon being identified as a pion. This data-MC discrepancy in the selected $\tau^{-} \rightarrow \pi^{-} \pi^{-} K^{+} \nu_{\tau}$ and $\tau^{-} \rightarrow K^{-} K^{-} \pi^{+} \nu_{\tau}$ events originates from the same the problematic region as the data-MC discrepancy of pions being selected as kaons. From these control samples, a very conservative upper limit of $40 \%$ is assigned as the systematic uncertainty on the selected events with kaon mis-identification as pion rate for the $\tau^{-} \rightarrow \pi^{-} \pi^{-} \pi^{+} \nu_{\tau}$, and $\tau^{-} \rightarrow K^{-} \pi^{-} K^{+} \nu_{\tau}$ channels. For the $\tau^{-} \rightarrow K^{-} \pi^{-} \pi^{+} \nu_{\tau}$ decay mode the systematic uncertainty is the difference in the $\tau^{-} \rightarrow K^{-} \pi^{-} \pi^{+} \nu_{\tau}$ branching fraction with and without the pion being required to satisfy the extra selection criteria that the track be in region $\mathrm{C}$ of the $\theta_{C}$ vs $P_{l a b}$ or have $P_{l a b}>0.8 \mathrm{GeV} / c$.

\footnotetext{
${ }^{8}$ The Feynman Diagram for these decay modes requires an extra weak interaction relative to the other $\tau^{-} \rightarrow h^{-} h^{-} h^{+} \nu_{\tau}$ decay modes.
} 


\subsubsection{Signal Modelling}

- In Section 4.4, the reconstructed MC is weighted to correct the modelling of resonance structure in the $\tau^{-} \rightarrow h^{-} h^{-} h^{+} \nu_{\tau}$ decays. The systematic uncertainty assigned for the modelling of the resonance structure is taken at the full shift in the branching fractions determined with and without the 3D Dalitz weighting.

\subsubsection{EMC and DCH Response}

- The systematic uncertainty associated with the scale and resolution modelling of the reconstructed tracks in the tracking detectors is estimated by varying the scale and resolution of the momentum in the MC using a control sample of $e^{+} e^{-} \rightarrow \mu^{+} \mu^{-} \gamma$ events. The measured momentum from the tracking detectors can be written in terms of the momentum MC Truth, $P_{M C}^{T r u t h}$, the measured momentum, $P_{M C}^{r e c o}$, a momentum resolution, $R_{P}$, is the Data-MC momentum resolutions ratio, and $S_{P}$ is the Data-MC momentum scale ratio for the momentum.

$$
P_{M C}^{r e c c^{\prime}}\left(P_{M C}^{T r u t h}, P_{M C}^{r e c o}, S_{P}\right)=\left(P_{M C}^{T r u t h}+R_{P}\left(P_{M C}^{T r u t h}-P_{M C}^{r e c o}\right)\right) S_{P} .
$$

For positive tracks $S_{P}=0.99994 \pm 0.00033$ and $R_{P}=0.9488 \pm 0.0031$ and

for negative tracks $S_{P}=0.99928 \pm 0.00035$ and $R_{P}=0.9425 \pm 0.0032$ [109]. The uncertainty associated with the momentum scale is taken as the change in the branching fractions when the momentum scale in the MC is modified using Equation 4.3. Similarly, the uncertainty on the momentum resolution is taken as the change in the branching fractions when the momentum resolution in the MC is modified using Equation 4.3.

- The determination of the energy scale and resolution systematic is determined 
with an approach analogous to that used for the momentum energy scale and resolution. The EMC energy, when parameterized in terms of the data MC energy resolution, $R_{E}=0.95 \pm 0.14$, and the energy scale offset, $S_{E}=-0.0020 \pm$ $0.0031 \mathrm{GeV}$, is

$$
E_{M C}^{r e c o^{\prime}}\left(E_{M C}^{\text {Truth }}, E_{M C}^{r e c o}, R_{E}\right)=\left(E_{M C}^{\text {Truth }}+R_{E}\left(E_{M C}^{\text {Truth }}-E_{M C}^{r e c o}\right)\right)+S_{E}
$$

where $E_{M C}^{r e c o}$ is the reconstructed $\mathrm{MC}$ energy, and $E_{M C}^{T r u t h}$ is the energy $\mathrm{MC}$ Truth [109]. The uncertainty on the energy resolution is taken as the change in the branching fractions when the energy resolution in the $\mathrm{MC}$ is modified using Equation 4.4 and the uncertainty associated with the energy scale is taken as the change in the branching fractions when the energy scale in the $\mathrm{MC}$ is modified using Equation 4.4.

- The modelling of $\theta$ in the $\mathrm{MC}$ is accurate to $0.000897 \pm 0.000005 \mathrm{rad}$ [109]. Therefore, the $\theta$ is shifted by $\pm 0.000897 \mathrm{rad}$ in the $\mathrm{MC}$ and the maximum change in the branching ratios is taken as the systematic uncertainty.

- The c.m. energy is known to an accuracy of $\pm 2 M e V$ [110]. The systematic resulting from the c.m. energy scale and energy spread was determined as the maximum shift resulting from varying the c.m. energy in the $\mathrm{MC}$ by $\pm 2 \mathrm{MeV}$.

- The systematic uncertainty related to the modelling of the tracking efficiency was determined with a 1 versus 3 prong $\tau$ pair control sample [111-113]. Because the data and MC tracking efficiencies were consistent within the uncertainties of the control sample no correction was applied. The uncertainty on the ratio of data and $\mathrm{MC}$ efficiencies determined from the 1 versus 3 prong $\tau$ pair control sample was taken as the systematic uncertainty on each track. 
- There is a $3 \%$ systematic uncertainty associated with the efficiency of the $\pi^{0}$ rejection [114]. Therefore, a systematic uncertainty of $3 \%$ is applied to all of the remaining backgrounds that contain $\pi^{0}$ 's.

- There is a systematic uncertainty associated with the modelling of the signal distributions for which cuts were applied. For each of the cuts applied in the $\tau^{-} \rightarrow h^{-} h^{-} h^{+} \nu_{\tau}$ analysis associated with tracking or the EMC, the mean of the selected distribution of the cut parameter for the data and $\mathrm{MC}$ were compared to verify the modelling in the MC. If the means on the data and MC have a statistical deviation of more than 2 standard deviations, a systematic uncertainty was assigned to account for possible modelling bias of the MC signal associated with the cut variable. The systematic uncertainty is evaluated as the change in the branching fractions, caused by altering the cut value applied to the $\mathrm{MC}$, to account for the deviation between the data and MC means for the cut variable. The cut variable that had this modelling bias uncertainty applied are:

1. The cut applied to the residual neutral energy of the 1 prong lepton tracks. The difference between the data and MC means used to shift the signal MC distribution for the 1 prong residual neutral energy cut is $0.00039 \mathrm{GeV}$ for the electron track and $0.00021 \mathrm{GeV}$ for the muon track. Figures 4.8 and 4.9 show the data $\mathrm{MC}$ agreement in the 1 prong residual neutral energy distribution. The systematic uncertainty is calculated for the electron and muon cases separately and then combined using the standard propagation of errors.

2. The cut applied to the residual neutral energy of the 3 prong signal tracks. The difference between the data and MC means used to shift the MC distribution for the 3 prong residual neutral energy cut is $0.00019 \mathrm{GeV}$. 
The data MC agreement in the 3 prong residual neutral energy distribution is shown in Figures 4.10 and 4.11 .

3. The cut applied to remove contamination from $\tau$ decays containing $K_{S}^{0}$. The difference between the data and MC means used to shift the MC distribution for the $K_{S}^{0}$ significance cut is 0.0311 . The distribution of the $K_{S}^{0}$ significance in the $x y$ plane can be seen in Figure 4.12.

\subsubsection{Trigger}

- The systematic uncertainty associated with the trigger is estimated from running the selection criteria with and without the trigger for $\tau^{-} \rightarrow K^{-} \pi^{-} \pi^{+} \nu_{\tau}$ and $\tau^{-} \rightarrow K^{-} K^{-} K^{+} \nu_{\tau} \mathrm{MC}$ events. Using the conservative estimate that the trigger is correctly modelled to the $10 \%$ level [109], the systematic uncertainty applied to the branching fractions is defined as $10 \%$ of the largest relative shift in the number of selected MC events. This turned out to be a negligible systematic uncertainty and is therefore applied to the $\tau^{-} \rightarrow \pi^{-} \pi^{-} \pi^{+} \nu_{\tau}$ and $\tau^{-} \rightarrow K^{-} \pi^{-} K^{+} \nu_{\tau}$ channels as well.

\subsubsection{Backgrounds}

- The systematic uncertainty from the $\tau$ backgrounds was determined for all the major background modes by shifting branching fraction in the MC by one standard deviation about their values [39]. The branching fraction for the dominant backgrounds can be seen in Table 4.3. For the $\tau^{-} \rightarrow K^{-} K^{-} K^{+} \pi^{0} \nu_{\tau}$ background, there is only an upper limit on the branching ratio. Therefore, the uncertainty, associated with this background mode, is defined as the change in the measured branching fraction caused by subtracting the $\tau^{-} \rightarrow K^{-} K^{-} K^{+} \pi^{0} \nu_{\tau}$ MC background, where the branching fraction is normalized to the $\tau^{-} \rightarrow$ 

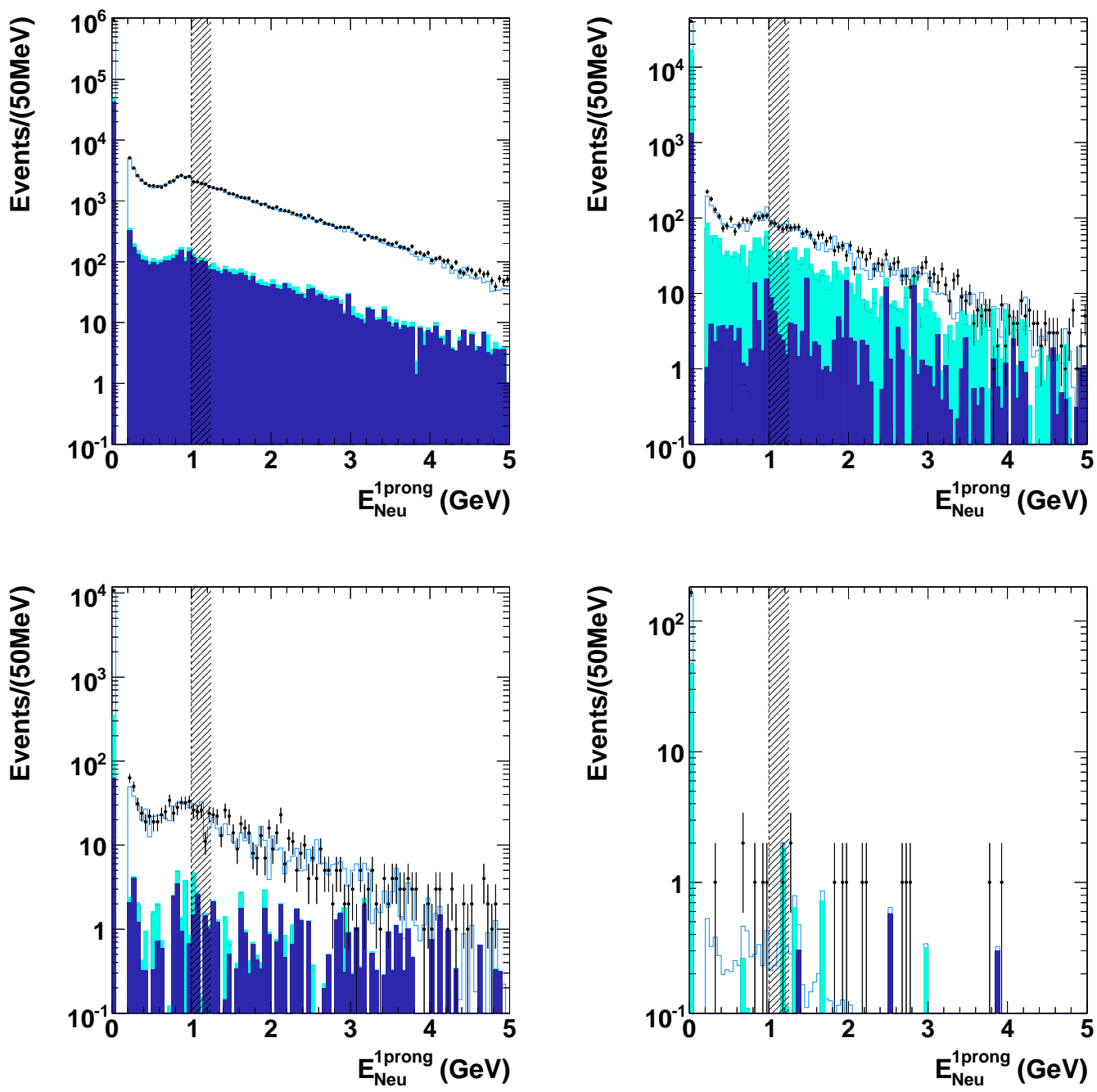

Figure 4.8: The 1 prong residual neutral energy distribution for the electron track vs the $\tau^{-} \rightarrow \pi^{-} \pi^{-} \pi^{+} \nu_{\tau}$ (top-left), $\tau^{-} \rightarrow K^{-} \pi^{-} \pi^{+} \nu_{\tau}$ (top-right), $\tau^{-} \rightarrow$ $K^{-} \pi^{-} K^{+} \nu_{\tau}$ (bottom-left), and $\tau^{-} \rightarrow K^{-} K^{-} K^{+} \nu_{\tau}$ (bottom-right) channels. The data is represented by the points with the error bars representing the statistical uncertainty. The open histogram represents the signal MC for the respective channels, while the lightly shaded histogram represents the cross-feed from the other $\tau^{-} \rightarrow h^{-} h^{-} h^{+} \nu_{\tau}$ channels. The remaining $\tau$ backgrounds and the non- $\tau$ backgrounds are represented by the dark histogram. The dotted line represents the cut value while the hatching indicated which side of the cut is rejected. The sharp discontinuity in these distributions is a result of the neutral association definitions. 

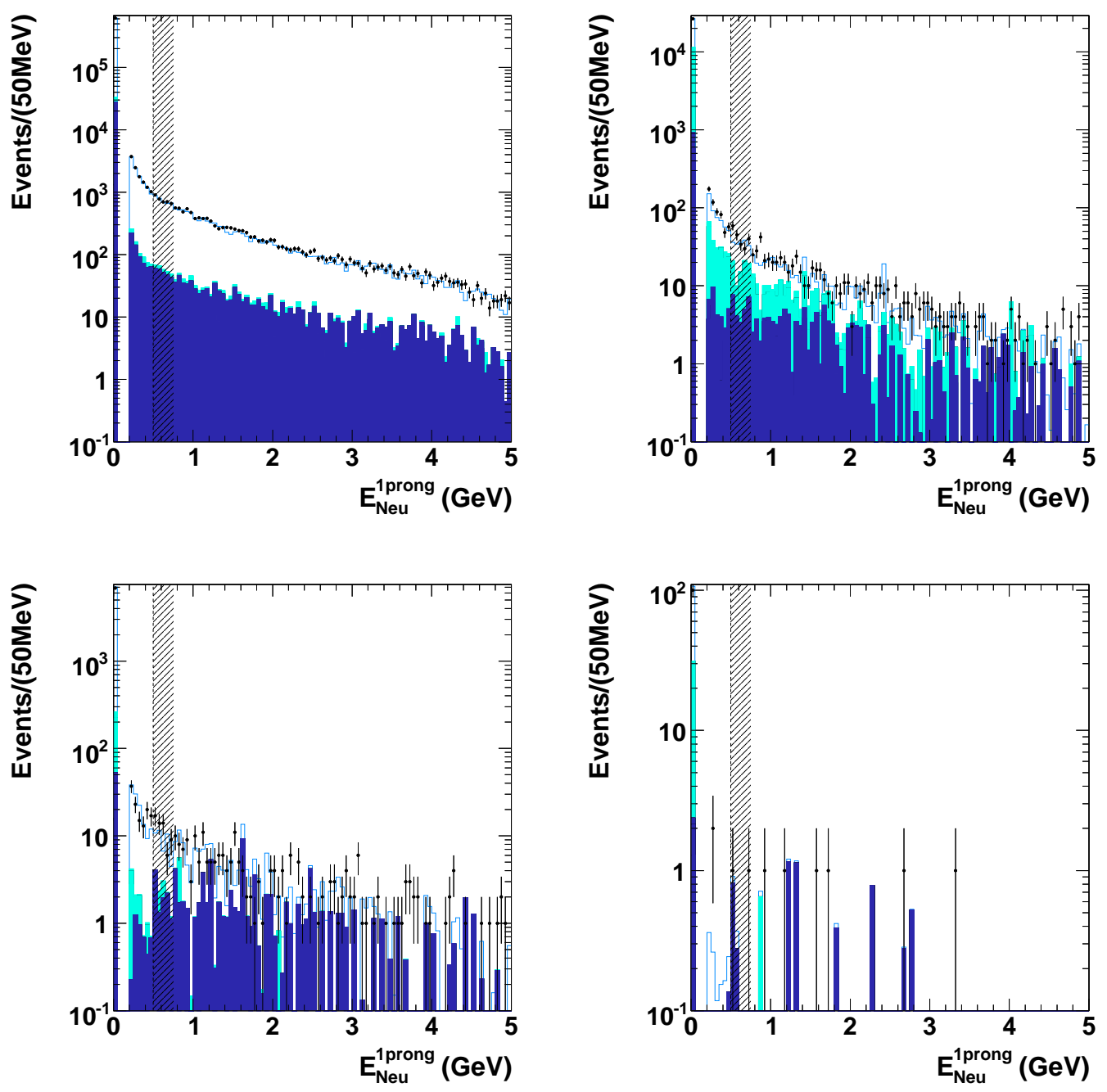

Figure 4.9: The 1 prong residual neutral energy distribution for the muon track vs the $\tau^{-} \rightarrow \pi^{-} \pi^{-} \pi^{+} \nu_{\tau}$ (top-left), $\tau^{-} \rightarrow K^{-} \pi^{-} \pi^{+} \nu_{\tau}$ (top-right), $\tau^{-} \rightarrow$ $K^{-} \pi^{-} K^{+} \nu_{\tau}$ (bottom-left), and $\tau^{-} \rightarrow K^{-} K^{-} K^{+} \nu_{\tau}$ (bottom-right) channels. The data is represented by the points with the error bars representing the statistical uncertainty. The open histogram represents the signal MC for the respective channels, while the lightly shaded histogram represents the cross-feed from the other $\tau^{-} \rightarrow h^{-} h^{-} h^{+} \nu_{\tau}$ channels. The remaining $\tau$ backgrounds and the non- $\tau$ backgrounds are represented by the dark histogram. The dotted line represents the cut value while the hatching indicated which side of the cut is rejected. The sharp discontinuity in these distributions is a result of the neutral association definitions. 

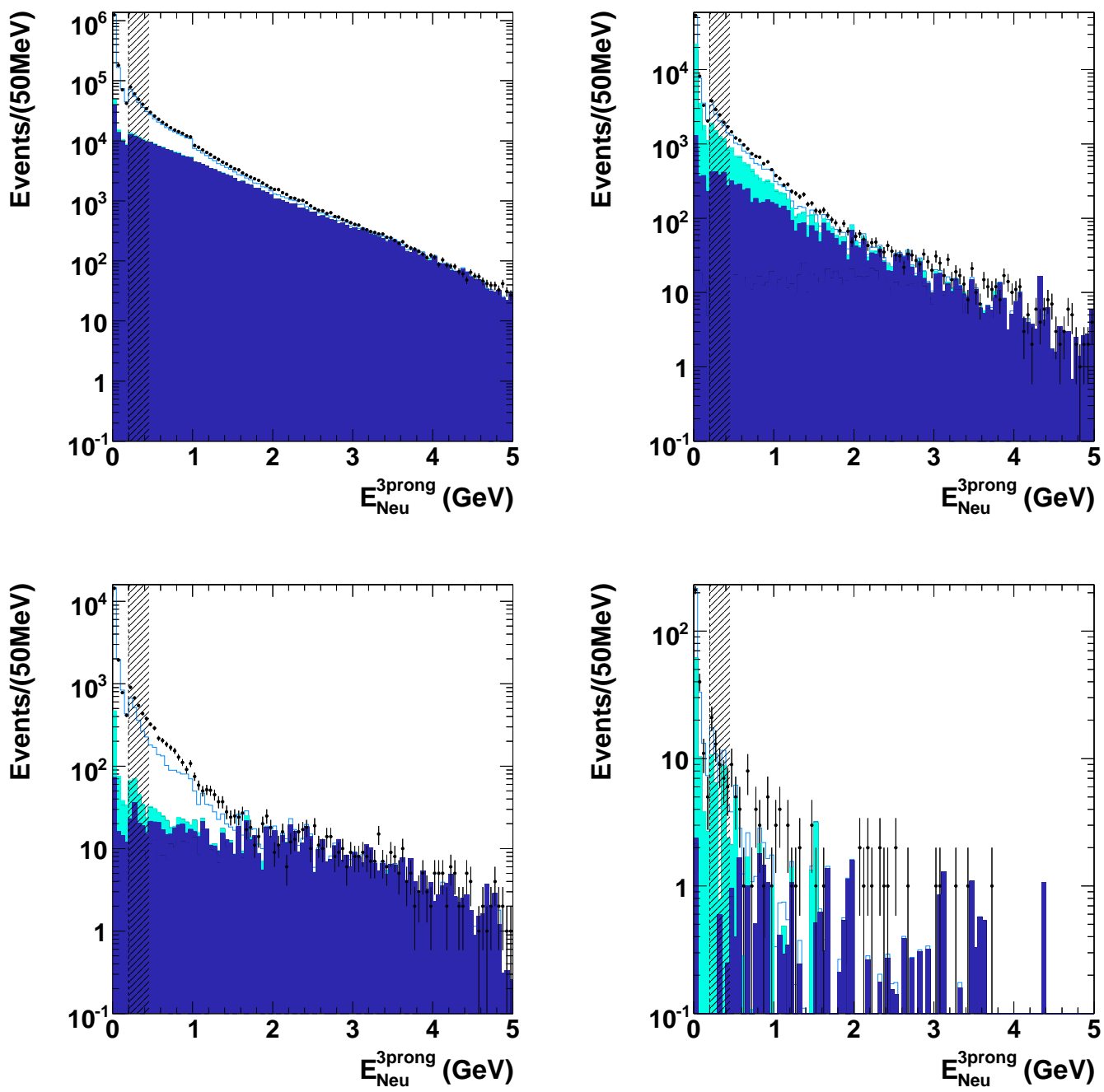

Figure 4.10: The 3 prong residual neutral energy distribution from $0 \mathrm{GeV}$ to $5 \mathrm{GeV}$ for $\tau^{-} \rightarrow \pi^{-} \pi^{-} \pi^{+} \nu_{\tau}$ (top-left), $\tau^{-} \rightarrow K^{-} \pi^{-} \pi^{+} \nu_{\tau}$ (top-right), $\tau^{-} \rightarrow$ $K^{-} \pi^{-} K^{+} \nu_{\tau}$ (bottom-left), and $\tau^{-} \rightarrow K^{-} K^{-} K^{+} \nu_{\tau}$ (bottom-right). The data is represented by the points with the error bars representing the statistical uncertainty. The open histogram represents the signal MC for the respective channels, while the lightly shaded histogram represents the cross-feed from the other $\tau^{-} \rightarrow h^{-} h^{-} h^{+} \nu_{\tau}$ channels. The remaining $\tau$ backgrounds and the non- $\tau$ backgrounds are represented by the dark histogram. The dotted line represents the cut value while the hatching indicated which side of the cut is rejected. The sharp discontinuity in these distributions is a result of the neutral association definitions. 

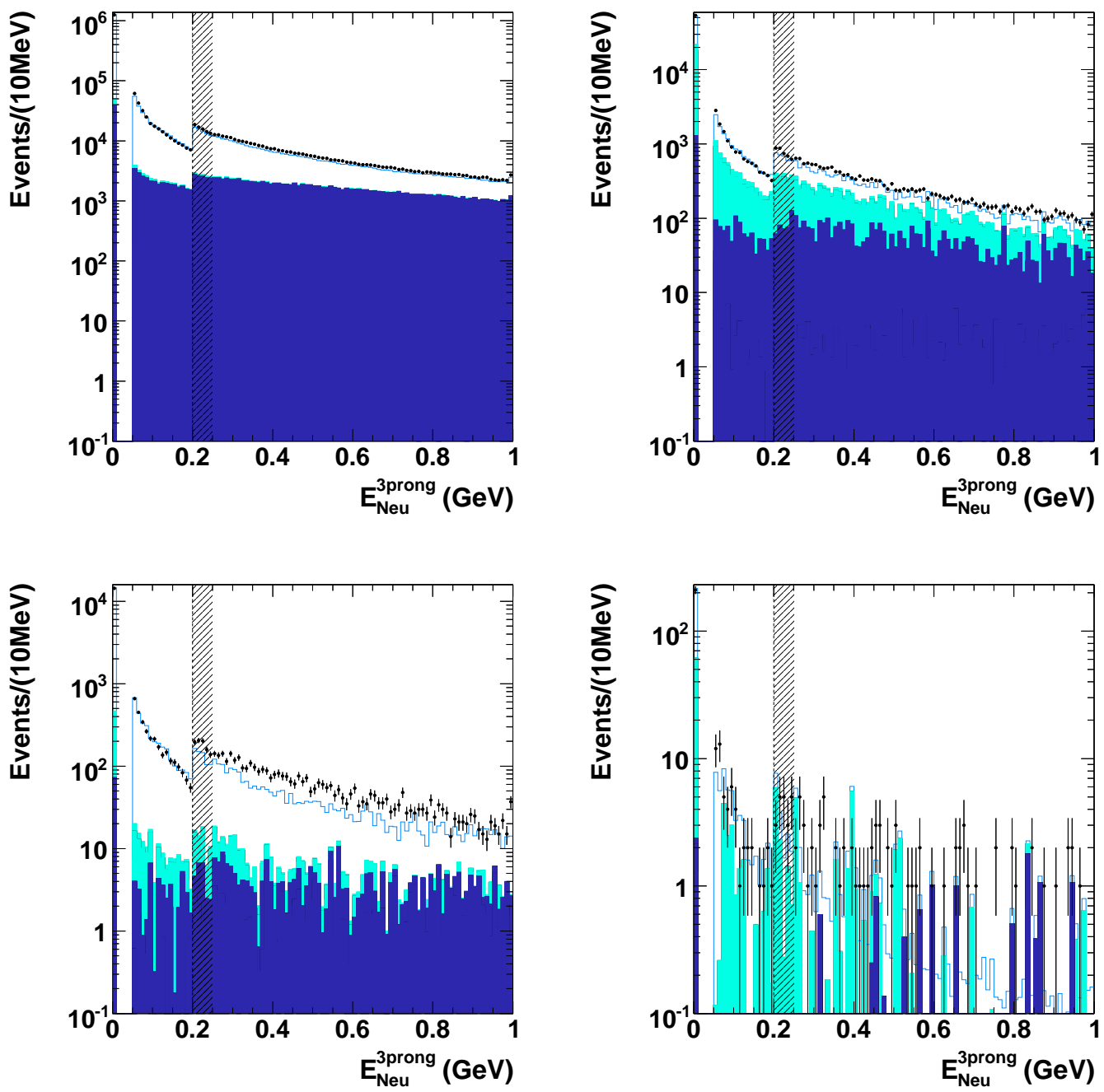

Figure 4.11: The 3 prong residual neutral energy distribution from $0 \mathrm{GeV}$ to $1 \mathrm{GeV}$ for $\tau^{-} \rightarrow \pi^{-} \pi^{-} \pi^{+} \nu_{\tau}$ (top-left), $\tau^{-} \rightarrow K^{-} \pi^{-} \pi^{+} \nu_{\tau}$ (top-right), $\tau^{-} \rightarrow$ $K^{-} \pi^{-} K^{+} \nu_{\tau}$ (bottom-left), and $\tau^{-} \rightarrow K^{-} K^{-} K^{+} \nu_{\tau}$ (bottom-right). The data is represented by the points with the error bars representing the statistical uncertainty. The open histogram represents the signal MC for the respective channels, while the lightly shaded histogram represents the cross-feed from the other $\tau^{-} \rightarrow h^{-} h^{-} h^{+} \nu_{\tau}$ channels. The remaining $\tau$ backgrounds and the non- $\tau$ backgrounds are represented by the dark histogram. The dotted line represents the cut value while the hatching indicated which side of the cut is rejected. The sharp discontinuity in these distributions is a result of the neutral association definitions. 

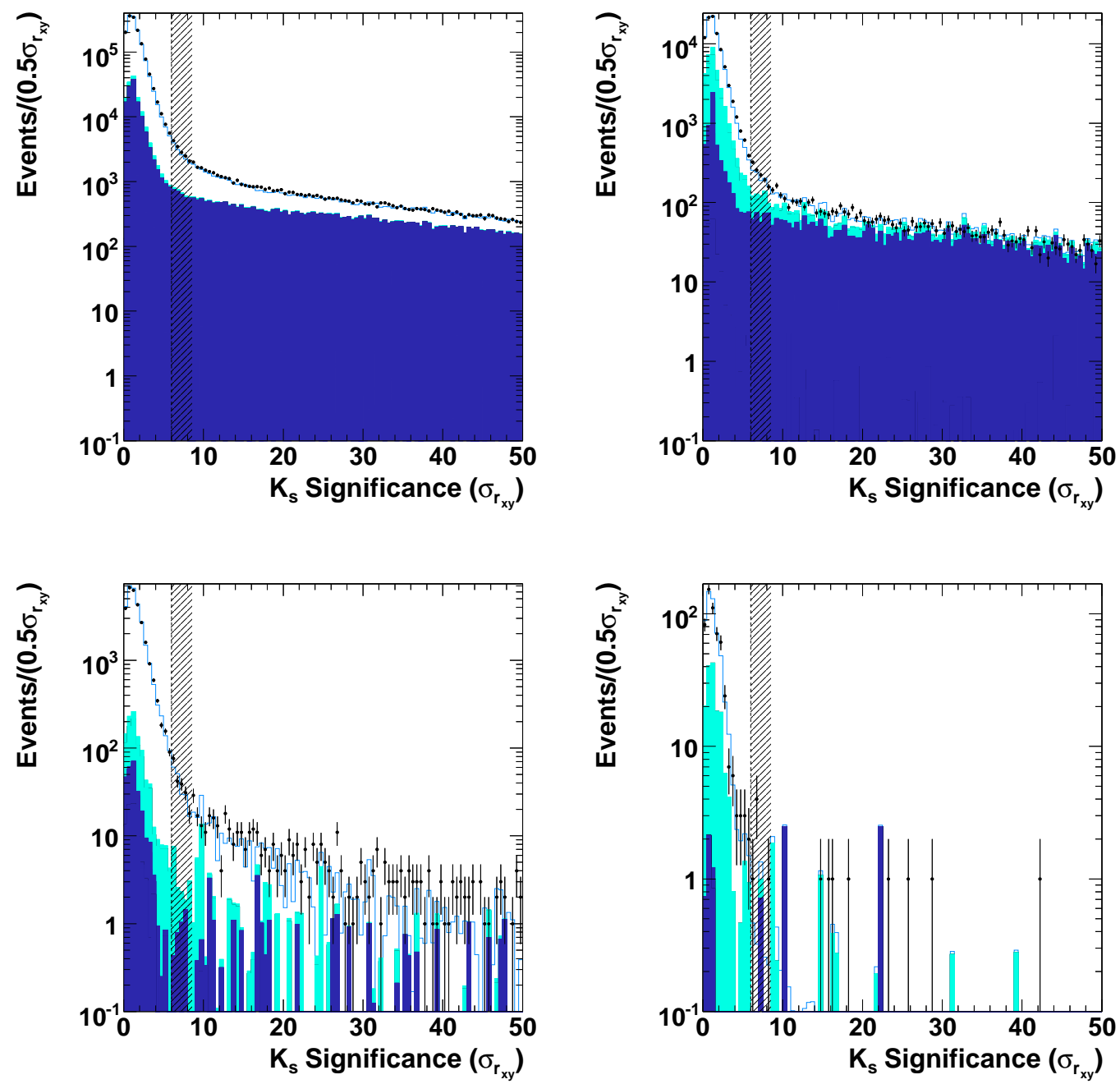

Figure 4.12: The $K_{S}^{0}$ significance in the $x y$ plane distribution for $\tau^{-} \rightarrow$ $\pi^{-} \pi^{-} \pi^{+} \nu_{\tau}$ (top-left), $\tau^{-} \rightarrow K^{-} \pi^{-} \pi^{+} \nu_{\tau}$ (top-right), $\tau^{-} \rightarrow K^{-} \pi^{-} K^{+} \nu_{\tau}$ (bottomleft), and $\tau^{-} \rightarrow K^{-} K^{-} K^{+} \nu_{\tau}$ (bottom-right). The data is represented by the points with the error bars representing the statistical uncertainty. The open histogram represents the signal MC for the respective channels, while the lightly shaded histogram represents the cross-feed from the other $\tau^{-} \rightarrow h^{-} h^{-} h^{+} \nu_{\tau}$ channels. The remaining $\tau$ backgrounds and the non- $\tau$ backgrounds are represented by the dark histogram. The dotted line represents the cut value while the hatching indicated which side of the cut is rejected.

$K^{-} K^{-} K^{+} \pi^{0} \nu_{\tau}$ branching fraction estimated, from the $\tau^{-} \rightarrow K^{-} K^{-} K^{+} \pi^{0} \nu_{\tau}$ upper-limit [115], compared to when there is no $\tau^{-} \rightarrow K^{-} K^{-} K^{+} \pi^{0} \nu_{\tau}$ back- 
ground subtraction.

\begin{tabular}{|l|l|}
\hline Decay & Br. \\
\hline \hline$\tau^{-} \rightarrow \pi^{-} \pi^{-} \pi^{+} \pi^{0} \nu_{\tau}$ & $4.46 \pm 0.06$ \\
$\tau^{-} \rightarrow K^{-} \pi^{-} \pi^{+} \pi^{0} \nu_{\tau}$ & $(7.9 \pm 1.2)^{-4}$ \\
$\tau^{-} \rightarrow K^{-} \pi^{-} K^{+} \pi^{0} \nu_{\tau}$ & $(6.4 \pm 2.1)^{-5}$ \\
$\tau^{-} \rightarrow K^{-} K^{-} K^{+} \pi^{0} \nu_{\tau}$ & $(2.9 \pm 2.9) \times 10^{-6}\left(4.8 \times 10^{-6} @ 90 \%\right.$ CL [115]) \\
$\tau^{-} \rightarrow K_{S}^{0} \pi^{-} \nu_{\tau} \rightarrow \pi^{-} \pi^{-} \pi^{+} \nu_{\tau}$ & $(8.9 \pm 0.4) \times 10^{-3}$ \\
$\tau^{-} \rightarrow K^{-} \bar{K}^{0} \gamma \nu_{\tau}$ & $(1.54 \pm 0.16) \times 10^{-3}$ \\
$\tau^{-} \rightarrow \rho^{-} \nu_{\tau}$ & $(25.50 \pm 0.10) \times 10^{-2}$ \\
\hline
\end{tabular}

Table 4.3: The dominant branching fractions for the $\tau$ backgrounds used for determining the $\tau$ background systematic [39].

- The dominant systematic uncertainty associated with $\mu$ pairs is the cross-section of the $\mu$ pairs. Therefore, the systematic uncertainty associated with the $\mu$ pairs is determined from the shift in the branching fraction caused by shifting the $\mu$ pairs by one standard deviation of the cross-section. This results in a negligible uncertainty.

- The systematic uncertainty associated with the $q \bar{q}$ background utilizes the side band region above the 3 prong invariant mass cut, $M>1.82 \mathrm{GeV} / \mathrm{c}^{2}$, to determine the deviation between data and the $q \bar{q}$ background. The $q \bar{q} \mathrm{MC}$ is then scaled to match the data in this region and the resulting shift in the branching fraction is taken as the systematic uncertainty. The scale factors used in this analysis were: 3.91 for the $q \bar{q}$ background in the $\tau^{-} \rightarrow \pi^{-} \pi^{-} \pi^{+} \nu_{\tau}$ channel; 1.78 for the $q \bar{q}$ background in the $\tau^{-} \rightarrow K^{-} \pi^{-} \pi^{+} \nu_{\tau}$ channel; 1.79 for the $q \bar{q}$ background in the $\tau^{-} \rightarrow K^{-} \pi^{-} K^{+} \nu_{\tau}$ channel; and -0.32 for the $q \bar{q}$ background in the $\tau^{-} \rightarrow K^{-} K^{-} K^{+} \nu_{\tau}$ channel.

- In the $\tau^{-} \rightarrow h^{-} h^{-} h^{+} \nu_{\tau}$ analysis, there is no Bhabha MC available, therefore a systematic uncertainty is required to account for possible biases caused by 
Bhabha contamination in this work. In the default analysis, none of the three hadron candidates on the 3 prong side are allowed to pass the electron veto. To estimate the Bhabha contamination, the number of electron vetoes is loosened by requiring only 0,1 or 2 electron vetoes and then measuring the percent deviation between the data and $\mathrm{MC}$ in the region $0.6 c^{-1}<P_{c m} / \sqrt{s}<0.8 c^{-1}$, where, due to kinematics, the Bhabha contamination is the highest. It is assumed that the percent deviation is the result of Bhabha contamination. From these percent deviations for 0,1 , and 2 electron vetoes, a least square fit is used to estimate the percent Bhabha contamination when all three electron vetoes are applied. The resulting estimate of the percent Bhabha contamination is used to set a conservative systematic for the Bhabha contamination.

- There is a systematic uncertainty associated with the modelling of the background distributions for which cuts were applied. For each of the cuts applied in the $\tau^{-} \rightarrow h^{-} h^{-} h^{+} \nu_{\tau}$ analysis associated with tracking or the EMC, the validity modelling of the background $\mathrm{MC}$ was characterized by comparing the mean of the data and $\mathrm{MC}$ distributions in the region being rejected by the cut. If the means on the data and MC differ by more than 2 standard deviations, a systematic uncertainty was assigned to account for possible modelling bias of the MC backgrounds associated with the cut variable. The systematic uncertainty is evaluated as the change in the branching fractions caused by altering the cut value applied to the MC, to account for the deviation between the data and MC means for the cut variable. The cut variables which have a background modelling uncertainty assigned are:

1. The cut applied to the residual neutral energy of the 1 prong lepton tracks. The difference between the data and MC means used to shift the back- 
ground MC distributions for the 1 prong residual neutral energy cut on the electron track is $0.025 \mathrm{GeV}$ for the $\tau^{-} \rightarrow \pi^{-} \pi^{-} \pi^{+} \nu_{\tau}$ channel. The MC for the other channels were not altered because they were not of statistical significance.

2. The cut applied to the residual neutral energy of the 3 prong signal tracks. The difference between the data and MC means used to shift the background MC distributions for the 3 prong residual neutral energy cut are: $0.0108 \mathrm{GeV}$ for the $\tau^{-} \rightarrow \pi^{-} \pi^{-} \pi^{+} \nu_{\tau}$ channel; $0.016 \mathrm{GeV}$ for the $\tau^{-} \rightarrow K^{-} \pi^{-} \pi^{+} \nu_{\tau}$ channel; and $-0.073 \mathrm{GeV}$ for the $\tau^{-} \rightarrow K^{-} \pi^{-} K^{+} \nu_{\tau}$ channel. The background $\tau^{-} \rightarrow K^{-} K^{-} K^{+} \nu_{\tau}$ cut was not altered for the MC because the data MC deviation is less than 1 standard deviation.

3. The cut applied to remove contamination from $\tau$ decays containing $K_{S}^{0}$. The difference between the data and MC means used to shift the background MC distributions for the $K_{S}^{0}$ significance cut is -0.17 ; for the $\tau^{-} \rightarrow \pi^{-} \pi^{-} \pi^{+} \nu_{\tau}$ channel; -0.67 for the $\tau^{-} \rightarrow K^{-} \pi^{-} \pi^{+} \nu_{\tau}$ channel; and 2.10 for the $\tau^{-} \rightarrow K^{-} \pi^{-} K^{+} \nu_{\tau}$ channel. The background $\tau^{-} \rightarrow K^{-} K^{-} K^{+} \nu_{\tau}$ cut was not altered for the MC because the data MC deviation is less than 1 standard deviation.

- The MC Backgrounds have a statistical uncertainty associated with the number of MC events generated. The propagation of these uncertainties to the measured branching fractions are determined from a Toy MC program with 10,000 trials.

\subsection{Results}

The branching fraction results for the $\tau^{-} \rightarrow h^{-} h^{-} h^{+} \nu_{\tau}$ analysis are presented in Table 4.4 with the Particle Data Group (PDG) [39] for comparison. These measurements 
represent a significant improvement in our understanding of these branching fractions and are consistent with the previous PDG world averages.

\begin{tabular}{|l|c|c|}
\hline & $\tau^{-} \rightarrow \pi^{-} \pi^{-} \pi^{+} \nu_{\tau}$ & $\tau^{-} \rightarrow K^{-} \pi^{-} \pi^{+} \nu_{\tau}$ \\
\hline$\epsilon$ & 0.028 & 0.031 \\
$N^{\text {Data }}$ & $(1.5953 \pm 0.0013) \times 10^{6}$ & $(6.956 \pm 0.0026) \times 10^{4}$ \\
$N^{\text {Bkg }}$ & $(0.0642 \pm 0.0002) \times 10^{6}$ & $(0.226 \pm 0.0064) \times 10^{4}$ \\
$N^{\text {Sig }}$ & $(55.59 \pm 0.05) \times 10^{6}$ & $(171.5 \pm 1.2) \times 10^{4}$ \\
$\mathcal{B}$ (this work) & $(8.83 \pm 0.01 \pm 0.13) \%$ & $(0.273 \pm 0.002 \pm 0.009) \%$ \\
\hline $\mathcal{B}$ (PDG fit) & $(9.02 \pm 0.08) \%$ & $(0.333 \pm 0.035) \%[51,116,117]$ \\
\hline \multicolumn{2}{|c|}{} \\
\hline$\epsilon$ & $\tau^{-} \rightarrow K^{-} \pi^{-} K^{+} \nu_{\tau}$ & $\tau^{-} \rightarrow K^{-} K^{-} K^{+} \nu_{\tau}$ \\
\hline$N^{\text {Data }}$ & 0.035 & 0.039 \\
$N^{\text {Bkg }}$ & $(1.819 \pm 0.013) \times 10^{4}$ & $275 \pm 17$ \\
$N^{\text {Sig }}$ & $(0.0145 \pm 0.0008) \times 10^{4}$ & $2.5 \pm 1.5$ \\
$\mathcal{B}$ (this work) & $(0.1346 \pm 0.0010 \pm 0.0036) \%$ & $(1.58 \pm 0.13 \pm 0.84) \times 10^{4}$ \\
\hline $\mathcal{B}$ (PDG fit) & $(0.153 \pm 0.010) \%$ & $\leq 3.7 \times 10^{-5} @ 90 \% C L$ \\
\hline
\end{tabular}

Table 4.4: These tables summarize the $\tau^{-} \rightarrow h^{-} h^{-} h^{+} \nu_{\tau}$ branching fraction results. The first row contains the characteristic efficiencies before the application of particle identification. The number of selected events, background events and signal events determined using Equation 4.1 are displayed in rows 2 through 4 . In the 5th line, the branching fractions from this work are presented where the first uncertainty is statistical and the second uncertainty is the systematic uncertainty. For comparison, the bottom row contains the corresponding world average branching fractions [39].

A decomposition of the uncertainties related to the measured $\tau^{-} \rightarrow h^{-} h^{-} h^{+} \nu_{\tau}$ branching ratios can be found in Table 4.5. The decay modes $\tau^{-} \rightarrow \pi^{-} \pi^{-} \pi^{+} \nu_{\tau}$, $\tau^{-} \rightarrow K^{-} \pi^{-} \pi^{+} \nu_{\tau}$, and $\tau^{-} \rightarrow K^{-} \pi^{-} K^{+} \nu_{\tau}$ are systematically limited. For $\tau^{-} \rightarrow$ $\pi^{-} \pi^{-} \pi^{+} \nu_{\tau}$, the dominant uncertainties are the luminosity uncertainty and the EMC and DCH response uncertainty. The main contribution to the EMC and DCH response uncertainty is from the modelling of neutrals and their association to the hadron tracks in the EMC for the 3 prong hemisphere. For the $\tau^{-} \rightarrow K^{-} \pi^{-} \pi^{+} \nu_{\tau}$ and $\tau^{-} \rightarrow K^{-} \pi^{-} K^{+} \nu_{\tau}$ decay modes, the dominant uncertainty is related to the mod- 


\begin{tabular}{|c|c|c|c|c|}
\hline & $\pi \pi \pi$ & $K \pi \pi$ & $K \pi K$ & $K K K$ \\
\hline \multicolumn{5}{|c|}{ Statistical Uncertainties } \\
\hline data statistics & 0.08 & 0.66 & 0.74 & 8.24 \\
\hline \multicolumn{5}{|c|}{ Systematic Uncertainties } \\
\hline $\mathcal{L} \sigma_{e^{+} e^{-} \rightarrow \tau^{+} \tau^{-}}$ & 1.0 & 1.0 & 1.0 & 1.0 \\
\hline$M_{i j}$ and particle ID & 0.4 & 3.0 & 1.9 & 4.9 \\
\hline signal modelling & 0.2 & 0.2 & 1.3 & 2.0 \\
\hline EMC and DCH response & 0.8 & 0.9 & 0.8 & 1.2 \\
\hline trigger & 0.1 & 0.1 & 0.1 & 0.1 \\
\hline backgrounds & 0.4 & 0.7 & 0.4 & 5.5 \\
\hline Total & 1.4 & 3.4 & 2.7 & 7.8 \\
\hline \multicolumn{5}{|c|}{ Error Correlation Matrix } \\
\hline$\pi \pi \pi$ & & 0.544 & 0.390 & 0.031 \\
\hline$K \pi \pi$ & & & 0.177 & 0.093 \\
\hline$K \pi K$ & & & & 0.087 \\
\hline
\end{tabular}

Table 4.5: A summary of the uncertainties for the $\tau^{-} \rightarrow h^{-} h^{-} h^{+} \nu_{\tau}$ analysis expressed in percentages. The individual systematic uncertainties are added in quadrature to obtain the total systematic uncertainty. The bottom of the table contains the error correlation matrix which includes both statistical and systematic components.

elling of the kaon and pion separation. These decay modes also have a significant contribution to the total systematic uncertainty from the modelling of neutrals and their association to the hadron tracks in the EMC for the 3 prong hemisphere. In contrast to the latter modes, the $\tau^{-} \rightarrow K^{-} K^{-} K^{+} \nu_{\tau}$ decay mode is statistically limited. However, a significant contribution to the uncertainty comes from the uncertainty in the $\tau^{-} \rightarrow K^{-} K^{-} K^{+} \pi^{0} \nu_{\tau}$ background, described in Section 4.5.6, and the MC statistical uncertainty of the migration matrix elements. 


\section{Chapter 5}

\section{Fitting the $\phi$ Resonance in $\tau^{-} \rightarrow K^{-} \pi^{-} K^{+} \nu_{\tau}$ and $\tau^{-} \rightarrow K^{-} K^{-} K^{+} \nu_{\tau}$ Decays}

\subsection{Methodology of Analysis}

In the $\tau^{-} \rightarrow h^{-} h^{-} h^{+} \nu_{\tau}$ analysis, the $\tau$ decays $\tau^{-} \rightarrow K^{-} \pi^{-} K^{+} \nu_{\tau}$ and $\tau^{-} \rightarrow K^{-} K^{-} K^{+} \nu_{\tau}$ were observed to have a $\phi$ resonance contribution in their $K^{-} K^{+}$invariant mass spectra seen in Figures 4.6 and 4.7. The $\mathcal{B}\left(\tau^{-} \rightarrow \phi \pi^{-} \nu_{\tau}\right)$ is useful in understanding the mixing of the $\omega-\phi$ and Okubo-Zweig-Iizuka (OZI) suppression [118], while a complete investigation of the non-resonance and resonance component of the $\tau^{-} \rightarrow K^{-} K^{-} K^{+} \nu_{\tau}$ has never been studied before. A binned maximum likelihood fit is used to measure the $\phi$ resonance in the background subtracted $\tau^{-} \rightarrow$ $K^{-} \pi^{-} K^{+} \nu_{\tau}$ and $\tau^{-} \rightarrow K^{-} K^{-} K^{+} \nu_{\tau} K^{-} K^{+}$invariant mass spectra and thereby determine $\mathcal{B}\left(\tau^{-} \rightarrow \phi \pi^{-} \nu_{\tau}\right), \mathcal{B}\left(\tau^{-} \rightarrow \phi K^{-} \nu_{\tau}\right)$ and $\mathcal{B}\left(\tau^{-} \rightarrow K^{-} K^{-} K^{+} \nu_{\tau}[\right.$ ex. $\left.\phi]\right)$. The number of $\tau^{-} \rightarrow \phi \pi^{-} \nu_{\tau}$ and $\tau^{-} \rightarrow \phi K^{-} \nu_{\tau}$ signal events are then determined using an efficiency migration matrix, $\epsilon_{i j}$, obtained from MC as in the $\tau^{-} \rightarrow h^{-} h^{-} h^{+} \nu_{\tau}$ analysis, where again, the modelling of the particle identification in the efficiency matrix has been corrected using data control samples of kaons and pions from $D^{*+} \rightarrow K^{+} D^{0}$ and $D^{0} \rightarrow K^{-} \pi^{+}$decays. The number of signal events for decay mode $j$ is then 
Chapter 5. Fitting the $\phi$ Resonance in $\tau^{-} \rightarrow K^{-} \pi^{-} K^{+} \nu_{\tau}$ and $\tau^{-} \rightarrow K^{-} K^{-} K^{+} \nu_{\tau}$ Decays

$$
\mathbf{N}_{j}^{S i g}=\sum_{i}\left(\epsilon^{-1}\right)_{j i}\left(\mathbf{N}_{i}^{F i t}\right)
$$

where $\mathbf{N}_{i}^{F i t}$ is the number of $\phi$ resonance events extracted from the binned maximum likelihood fit. The branching fractions are obtained by accounting for the luminosity, $\mathcal{L}$, and the $\tau$ pair cross-section, $\sigma_{\tau^{+} \tau^{-}}$as described in Equation 4.2. The non-resonance $\tau^{-} \rightarrow K^{-} K^{-} K^{+} \nu_{\tau}$ contribution is determined from the $\tau^{-} \rightarrow$ $K^{-} K^{-} K^{+} \nu_{\tau}$ binned maximum likelihood fit. After correcting for the non-resonance $\tau^{-} \rightarrow K^{-} K^{-} K^{+} \nu_{\tau}$ efficiency obtained from MC simulation, an upper-limit on the branching fraction $\mathcal{B}\left(\tau^{-} \rightarrow K^{-} K^{-} K^{+} \nu_{\tau}[\right.$ ex. $\left.\phi]\right)$ is made.

\subsection{Fitting Procedure for the $\phi$ peak for $\pi \phi$ and $K \phi$}

The statistics for both of the $K^{-} K^{+}$invariant mass spectra were enhanced by loosening the kaon selection algorithm compared to that used for the $\tau^{-} \rightarrow h^{-} h^{-} h^{+} \nu_{\tau}$ analysis as this provides a significantly higher signal-to-background ratio. The component of the migration matrix associated with particle identification for the loosened kaon selector can be found in Table 5.3. The $\tau$ and non- $\tau$ backgrounds were removed from the $K^{-} K^{+}$invariant mass distribution for $\tau^{-} \rightarrow K^{-} \pi^{-} K^{+} \nu_{\tau}$ and $\tau^{-} \rightarrow K^{-} K^{-} K^{+} \nu_{\tau}$ channels. In the $\tau^{-} \rightarrow K^{-} \pi^{-} K^{+} \nu_{\tau}$ channel, the $\phi$ peak in the $K^{-} K^{+}$invariant mass distribution was fitted with Breit-Wigner convoluted with a Gaussian to describe that $\phi$ peak, while the non- $\pi \phi$ background was modelled with a polynomial of 3rd order. A summary of the parameters used in this fit can be found in Table 5.1. For the $\tau^{-} \rightarrow K^{-} K^{-} K^{+} \nu_{\tau}$ channel, the $K^{-} K^{+}$invariant mass distribution with both combinations of $K^{-} K^{+}$included, was fitted with a Breit-Wigner convoluted with a Gaussian to describe that $\phi$ peak, while the combinatoric background was modelled 
Chapter 5. Fitting the $\phi$ Resonance in $\tau^{-} \rightarrow K^{-} \pi^{-} K^{+} \nu_{\tau}$ and $\tau^{-} \rightarrow K^{-} K^{-} K^{+} \nu_{\tau}$ Decays

\begin{tabular}{|l|r|l|}
\hline Parameter & Value & Explanation \\
\hline & $K^{-} \pi^{-} K^{+}$Signal Function (Breit-Wigner) \\
\hline$m_{\phi}$ & $(1019.78 \pm 0.33) \mathrm{MeV} / c^{2}$ & $\begin{array}{l}\text { The } \phi \text { mass was floated in the fit and } \\
\text { is consistent with the world average } \\
\text { value, }(1019.460 \pm 0.019) \mathrm{MeV} / \mathrm{c}^{2}[39] .\end{array}$ \\
\hline$\Gamma_{\phi}$ & $(4.2600 \pm 0.50) \mathrm{MeV}$ & The $\phi$ width $[39]$ is fixed in this fit. \\
\hline \multicolumn{3}{|c|}{$K^{-} \pi^{-} K^{+}$Detector Resolution Function (Gaussian) } \\
\hline$\sigma$ & $K^{-} \pi^{-} K^{+}$Signal Normalization \\
\hline$n_{\text {sig }}$ & $(4.50 \pm 0.55) \times 10^{-2}$ & $\begin{array}{l}\text { The percent of signal events in the dis- } \\
\text { tribution measured in the fit. }\end{array}$ \\
\hline \multicolumn{3}{|c|}{$K^{-} \pi^{-} K^{+}$Background Function (3rd Order Polynomial) } \\
\hline$p_{0}$ & $(5.150 \pm 0.040) \times 10^{5}$ & This value is determined from the fit. \\
\hline$p_{1}$ & $(-9.737 \pm 0.067) \times 10^{5}$ & This value is determined from the fit. \\
\hline$p_{2}$ & $(4.565 \pm 0.036) \times 10^{5}$ & This value is determined from the fit. \\
\hline
\end{tabular}

Table 5.1: The fit parameters for the $\phi$ resonance in the $K^{-} K^{+}$invariant mass distribution for the $K^{-} \pi^{-} K^{+}$channel.

with the function:

$$
f(m)= \begin{cases}{\left[m-2 m_{K}\right] e^{\left[a *\left(m^{2}-\left(2 m_{K}\right)^{2}\right]\left[m^{2}-\left(2 m_{K}\right)^{2}\right]^{b}\right.},} & \text { if } m>2 m_{K} \\ 0, & \text { if } m \leq 2 m_{K}\end{cases}
$$

where $m_{K}$ is the kaon mass [119]. Table 5.2 contains a summary of the parameters used in this fit. For both the $\tau^{-} \rightarrow K^{-} \pi^{-} K^{+} \nu_{\tau}$ and the $\tau^{-} \rightarrow K^{-} K^{-} K^{+} \nu_{\tau}$ fits, the $\sigma$ for the Gaussian, which describes the detector resolution, was fixed to a value determined from fitting the $\mathrm{MC}$ signal, $1.28 \mathrm{MeV} / c^{2}$, and the mass of the $\phi$ peak was fitted with a Gaussian constraint using the $\phi$ mass from [39]. 
Chapter 5. Fitting the $\phi$ Resonance in $\tau^{-} \rightarrow K^{-} \pi^{-} K^{+} \nu_{\tau}$ and $\tau^{-} \rightarrow K^{-} K^{-} K^{+} \nu_{\tau}$ Decays

\begin{tabular}{|c|c|c|}
\hline Parameter & Value & Explanation \\
\hline \multicolumn{3}{|c|}{$K^{-} K^{-} K^{+}$Signal Function (Breit-Wigner) } \\
\hline$m_{\phi}$ & $(1019.64 \pm 0.23) \mathrm{MeV} / c^{2}$ & $\begin{array}{l}\text { The } \phi \text { mass was floated in the fit and } \\
\text { is consistent with the world average } \\
\text { value, }(1019.460 \pm 0.019) \mathrm{MeV} / \mathrm{c}^{2}[39] \text {. }\end{array}$ \\
\hline$\Gamma_{\phi}$ & $(4.2600 \pm 0.50) \mathrm{MeV}$ & The $\phi$ width [39] is fixed in this fit. \\
\hline \multicolumn{3}{|c|}{$K^{-} K^{-} K^{+}$Detector Resolution Function (Gaussian) } \\
\hline$\sigma$ & $(1.28) \mathrm{MeV} / c^{2}$ & $\begin{array}{l}\text { The detector resolution value is fixed } \\
\text { for the fit. }\end{array}$ \\
\hline \multicolumn{3}{|c|}{$K^{-} K^{-} K^{+}$Signal Normalization } \\
\hline$n_{\text {sig }}$ & $(4.94 \pm 0.29) \times 10^{-1}$ & $\begin{array}{l}\text { The percent of signal events in the dis- } \\
\text { tribution. This value is determined } \\
\text { from the fit. }\end{array}$ \\
\hline \multicolumn{3}{|c|}{$K^{-} \pi^{-} K^{+}$Background Function (Equation 5.2) } \\
\hline$a$ & $-0.77 \pm 0.15$ & $\begin{array}{l}\text { Phase space parameter } 1 . \text { This value is } \\
\text { determined from the fit. }\end{array}$ \\
\hline$b$ & $-3.4 \pm 1.5$ & $\begin{array}{l}\text { Phase space parameter } 2 \text {. This value is } \\
\text { determined from the fit. }\end{array}$ \\
\hline$m_{K}$ & $(493.677 \pm 0.016) \mathrm{MeV} / \mathrm{c}^{2}$ & $\begin{array}{l}\text { The kaon mass is fixed to the world av- } \\
\text { erage value [39] for this fit. }\end{array}$ \\
\hline
\end{tabular}

Table 5.2: The fit parameters for the $\phi$ resonance in the $K^{-} K^{+}$invariant mass distribution for the $K^{-} K^{-} K^{+}$channel.

\section{$5.3 \quad \tau^{-} \rightarrow K^{-} K^{-} K^{+} \nu$ Non-resonance Upper Limit}

The upper limit of the $\tau^{-} \rightarrow K^{-} K^{-} K^{+} \nu_{\tau}$ non-resonance branching fraction is determined from the $\tau^{-} \rightarrow \phi K^{-} \nu_{\tau}$ fit ${ }^{1}$. The number of $\tau^{-} \rightarrow K^{-} K^{-} K^{+} \nu_{\tau}$ non-resonance events is the number of events $\tau^{-} \rightarrow K^{-} K^{-} K^{+} \nu_{\tau}$ events in the $\tau^{-} \rightarrow \phi K^{-} \nu_{\tau}$ fit minus the number of measured $\tau^{-} \rightarrow \phi K^{-} \nu_{\tau}$ events from the fit. After correcting for the $\tau^{-} \rightarrow K^{-} K^{-} K^{+} \nu_{\tau}$ non-resonance selection efficiency, (2.5456 \pm 0.13$) \%$, the luminosity, and the $\tau$ pair cross-section, the upper limit was calculated with both the

\footnotetext{
${ }^{1}$ An upper limit on the $\tau^{-} \rightarrow K^{-} K^{-} K^{+} \nu_{\tau}$ non-resonance branching fraction was also determined by selecting events from the non- $\phi$ regions of the Dalitz plane, but the upper limit extracted with this method was not competitive with the upper limit extracted from the $\tau^{-} \rightarrow \phi K^{-} \nu_{\tau}$ fit.
} 
Chapter 5. Fitting the $\phi$ Resonance in $\tau^{-} \rightarrow K^{-} \pi^{-} K^{+} \nu_{\tau}$ and $\tau^{-} \rightarrow K^{-} K^{-} K^{+} \nu_{\tau}$ Decays

\begin{tabular}{|l|ll|}
\hline Candidates & \multicolumn{2}{|c|}{ Decay modes } \\
& $K \pi K$ & $K K K$ \\
\hline$\phi \pi$ & 72.54 & 19.20 \\
$\phi K$ & 0.83 & 66.06 \\
\hline
\end{tabular}

Table 5.3: The component of the efficiency migration matrix associated with pionkaon particle identification represented as percentages for the $\tau^{-} \rightarrow \phi \pi^{-} \nu_{\tau}$ and $\tau^{-} \rightarrow$ $\phi K^{-} \nu_{\tau}$ measurements. Note: this efficiency matrix includes efficiency losses resulting from the events being misidentified as $\tau^{-} \rightarrow \pi^{-} \pi^{-} \pi^{+} \nu_{\tau}, \tau^{-} \rightarrow K^{-} \pi^{-} \pi^{+} \nu_{\tau}$ events, wrong charge combinations, and small factors associated with data control sample corrections to the $\mathrm{MC}$ cross-feed efficiencies. As a result, the columns in this table do not add up to $100 \%$.

Gaussian assumption ${ }^{2}$ and the method defined in [120].

\subsection{Systematic Uncertainties}

The systematic uncertainties for the $\tau^{-} \rightarrow \phi \pi^{-} \nu_{\tau}, \tau^{-} \rightarrow \phi K^{-} \nu_{\tau}$ and non-resonance $\tau^{-} \rightarrow K^{-} K^{-} K^{+} \nu_{\tau}$ decays include the systematic uncertainties from the $\tau^{-} \rightarrow$ $h^{-} h^{-} h^{+} \nu_{\tau}$ analysis as well as the uncertainties originating from the $\phi \rightarrow K^{+} K^{-}$ branching fraction, the binned maximum likelihood fit and the backgrounds associated with these channels.

\subsection{1 $\mathcal{B}\left(\phi \rightarrow K^{+} K^{-}\right)$Uncertainty}

- The systematic uncertainty for the $\mathcal{B}\left(\phi \rightarrow K^{+} K^{-}\right)$is given in [39].

\subsubsection{Fit Parameterization}

- The systematic uncertainty associated with the fit parameterization was estimated by varying the model used to describe the background in the binned

\footnotetext{
${ }^{2}$ If one assumes that the uncertainty can be approximated by a Gaussian, the upper limit can be calculated $x+\sqrt{2} e r f^{-1}(0.90) \sigma$ for a $90 \%$ C.L. where $x$ is the measured value and $\sigma$ is the uncertainty on the measured value.
} 
Chapter 5. Fitting the $\phi$ Resonance in $\tau^{-} \rightarrow K^{-} \pi^{-} K^{+} \nu_{\tau}$ and $\tau^{-} \rightarrow K^{-} K^{-} K^{+} \nu_{\tau}$ Decays

maximum likelihood fit and comparing the stability of the result. In these studies, polynomials of 4 th and 5 th order were used to model the backgrounds in the $\tau^{-} \rightarrow \phi \pi^{-} \nu_{\tau}$ fit and a 4 th order polynomial was used to model the combinatoric background in the $\tau^{-} \rightarrow \phi K^{-} \nu_{\tau}$ fit.

\subsubsection{Fit Bias}

- The fit bias was determined using MC studies. In the $\tau^{-} \rightarrow \phi \pi^{-} \nu_{\tau}$ fit, the bias was determined as the difference between the number of fitted $\phi$ events and the number of $\phi$ events in the MC. The $\tau^{-} \rightarrow \phi \pi^{-} \nu_{\tau}$ fit bias was determined to be negligible. For the $\tau^{-} \rightarrow \phi K^{-} \nu_{\tau}$ fit, the bias was determined as the shift in $n_{\text {sig }}$ measured from the fit relative to the known amount in the MC. The $\tau^{-} \rightarrow \phi K^{-} \nu_{\tau}$ fit bias was determined to be $1.3 \%$.

\subsubsection{Additional Backgrounds}

- The systematic uncertainty on the peaking $q \bar{q}$ background was estimated by doubling the peaking $q \bar{q}$ background in the background subtraction. This scaling factor was estimated from the 3 prong invariant mass $M>1.82 \mathrm{GeV} / \mathrm{c}^{2}$ region as documented in Section 4.5.6.

- The contamination from $\tau^{-} \rightarrow \phi \pi^{-} \pi^{0} \nu$ is estimated with the $\tau^{-} \rightarrow K^{-} \pi^{-} K^{+} \pi^{0} \nu$ contamination in the $\tau^{-} \rightarrow K^{-} \pi^{-} K^{+} \nu$ channel. The $\tau^{-} \rightarrow \phi \pi^{-} \pi^{0} \nu$, like the $\tau^{-} \rightarrow \phi \pi^{-} \nu$ channel, is OZI suppressed. Therefore, the suppression of $\tau^{-} \rightarrow \phi \pi^{-} \pi^{0} \nu$ is estimated as being equivalent to the suppression of the $\tau^{-} \rightarrow \phi \pi^{-} \nu$. To account for any difference in the OZI suppression and the resonance structure, this estimate of the $\tau^{-} \rightarrow \phi \pi^{-} \pi^{0} \nu$ is increased by a factor of 10 . 
Chapter 5. Fitting the $\phi$ Resonance in $\tau^{-} \rightarrow K^{-} \pi^{-} K^{+} \nu_{\tau}$ and $\tau^{-} \rightarrow K^{-} K^{-} K^{+} \nu_{\tau}$ Decays

\subsection{Results}

The $\phi$ peak in the $\tau^{-} \rightarrow K^{-} \pi^{-} K^{+} \nu_{\tau}$ can clearly be seen in $K^{+} K^{-}$invariant mass distribution Figure 5.1 with the corresponding binned maximum likelihood fit located in the inset. After background subtraction of the non- $\tau^{-} \rightarrow K^{-} \pi^{-} K^{+} \nu_{\tau}$ events, the

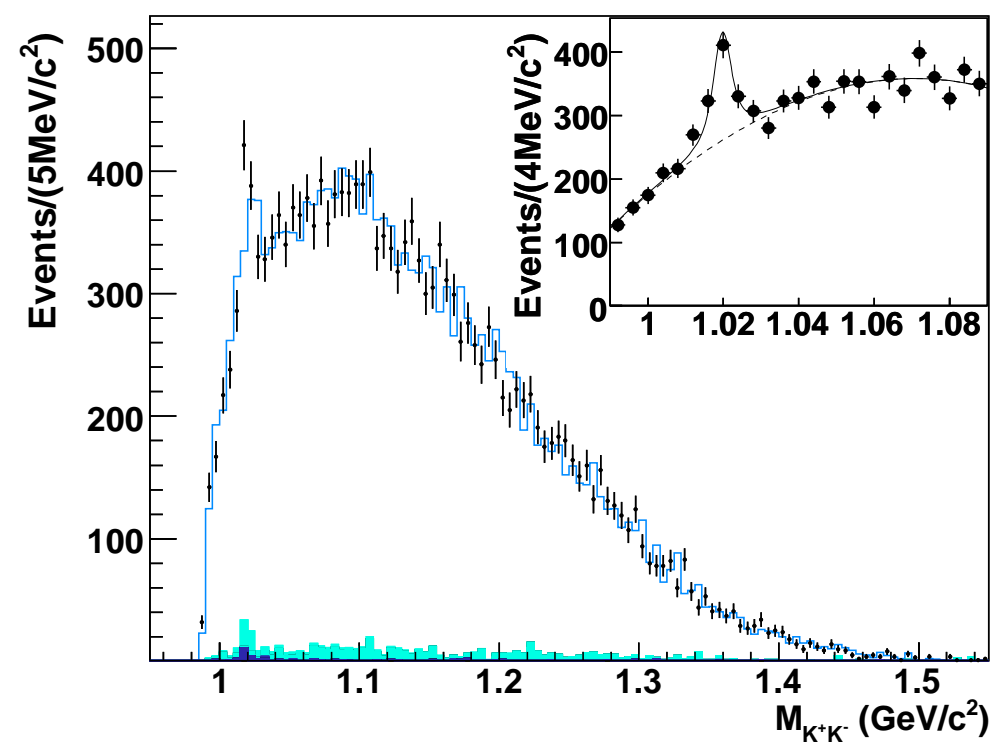

Figure 5.1: The $K^{+} K^{-}$invariant mass in $\tau^{-} \rightarrow K^{-} \pi^{-} K^{+} \nu_{\tau}$ decays. The data is represented by the points with the error bars representing the statistical uncertainty. The open histogram represents the $\tau^{-} \rightarrow K^{-} \pi^{-} K^{+} \nu_{\tau} \mathrm{MC}$, while the lightly shaded histogram represents the $\tau^{-} \rightarrow h^{-} h^{-} h^{+} \nu_{\tau}$ cross-feed. The non- $\tau$ backgrounds are represented by the dark histogram. The inset shows the fit results for $\tau^{-} \rightarrow \phi \pi^{-} \nu_{\tau}$ overlaid upon the background subtracted data. The solid line represents the complete fit function, while the dotted line represents the fit function for the non-resonant component. The points represent the data and the error bars represent the statistical uncertainty and the MC statistical uncertainty from the background subtraction.

$K^{+} K^{-}$mass distribution, below $1.09 \mathrm{GeV} / c^{2}$, from the $\tau^{-} \rightarrow \phi \pi^{-} \nu_{\tau}$ decay is well described by a Breit-Wigner convoluted with a Gaussian resolution function for the signal and a 3rd order polynomial for the background. The $\chi^{2}$ per degree of freedom 
Chapter 5. Fitting the $\phi$ Resonance in $\tau^{-} \rightarrow K^{-} \pi^{-} K^{+} \nu_{\tau}$ and $\tau^{-} \rightarrow K^{-} K^{-} K^{+} \nu_{\tau}$ Decays

for the binned maximum likelihood fit is $26.28 / 21$, a probability value of $19.6 \%$. The signal yields from the fit is $344 \pm 42 \tau^{-} \rightarrow \phi \pi^{-} \nu_{\tau}$ candidates.

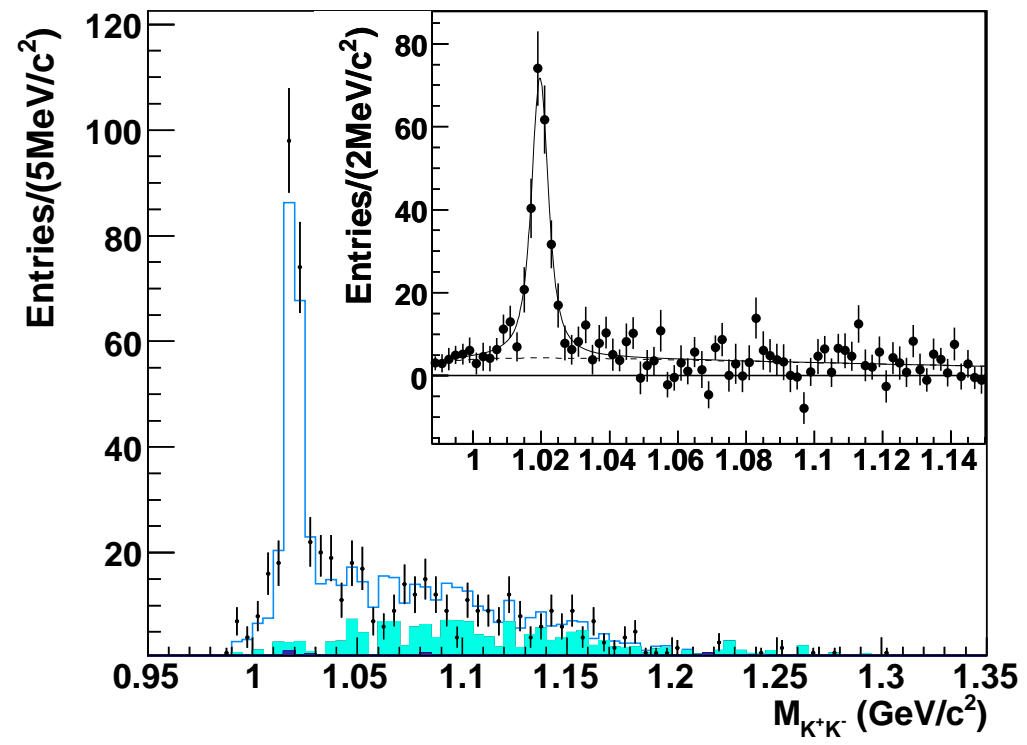

Figure 5.2: The $K^{+} K^{-}$invariant mass in $\tau^{-} \rightarrow K^{-} K^{-} K^{+} \nu_{\tau}$ decays with both $K^{+} K^{-}$ combination per event. The data is represented by the points with the error bars representing the statistical uncertainty. The open histogram represents the $\tau^{-} \rightarrow$ $K^{-} K^{-} K^{+} \nu_{\tau} \mathrm{MC}$, while the lightly shaded histogram represents the $\tau^{-} \rightarrow h^{-} h^{-} h^{+} \nu_{\tau}$ cross-feed. The non- $\tau$ backgrounds are represented by the dark histogram. The inset shows the fit results for $\tau^{-} \rightarrow \phi K^{-} \nu_{\tau}$ overlaid upon the background subtracted data. The solid line represents the complete fit function, while the dotted line represents the fit function for the combinatoric background. The points represent the data and the error bars represent the statistical uncertainty and the MC statistical uncertainty from the background subtraction.

Similarly, Figure 5.2 illustrates the $\phi$ peak in the $\tau^{-} \rightarrow K^{-} \pi^{-} K^{+} \nu_{\tau}$ decay and the corresponding binned maximum likelihood fit in the inset. Below $1.15 \mathrm{GeV} / c^{2}$ in the $K^{+} K^{-}$invariant mass distribution for the $\tau^{-} \rightarrow K^{-} K^{-} K^{+} \nu_{\tau}$ decay, the $\phi$ resonance is well described by a Breit-Wigner convoluted with a Gaussian resolution function and the combinatoric background is well described by Equation 5.2. The $\chi^{2}$ 
Chapter 5. Fitting the $\phi$ Resonance in $\tau^{-} \rightarrow K^{-} \pi^{-} K^{+} \nu_{\tau}$ and $\tau^{-} \rightarrow K^{-} K^{-} K^{+} \nu_{\tau}$ Decays

per degree of freedom is $140 / 176$ resulting in a fit probability of $97.9 \%$. The number of $\tau^{-} \rightarrow \phi K^{-} \nu_{\tau}$ signal events determined from this fit is $274 \pm 16$.

The resulting branching fractions for the $\tau^{-} \rightarrow \phi \pi^{-} \nu_{\tau}$ and $\tau^{-} \rightarrow \phi K^{-} \nu_{\tau}$ decays are $(3.42 \pm 0.55 \pm 0.25) \times 10^{-5}$ and $(3.39 \pm 0.20 \pm 0.28) \times 10^{-5}$ respectively, with a correlation of -0.07 . In addition to these branching fractions, the $\tau^{-} \rightarrow \phi K^{-} \nu_{\tau}$ fit is used to set the first upper limit on $\mathcal{B}\left(\tau^{-} \rightarrow K^{-} K^{-} K^{+} \nu_{\tau}[\right.$ ex. $\left.\phi]\right) \leq 2.5 \times 10^{-6} @ 90 \% C L$ using the prescription defined in Section 5.3. A summary of the systematic uncertainties for these values can be found in Table 5.4. The $\mathcal{B}\left(\tau^{-} \rightarrow \phi \pi^{-} \nu_{\tau}\right)$ is statistically limited while the $\mathcal{B}\left(\tau^{-} \rightarrow \phi K^{-} \nu_{\tau}\right)$ is limited by both the statistical uncertainty and the systematic uncertainty. In both modes, the dominant systematic uncertainty is due to backgrounds. For the $\tau^{-} \rightarrow \phi K^{-} \nu_{\tau}$ decay mode this is a result of the lack of information available for the $\tau^{-} \rightarrow K^{-} K^{-} K^{+} \pi^{0} \nu_{\tau}$ decay, while in the $\tau^{-} \rightarrow \phi \pi^{-} \nu_{\tau}$ decay mode the background is $q \bar{q}$ events and potential $\tau^{-} \rightarrow K^{-} \pi^{-} K^{+} n \pi^{0} \nu_{\tau}$ events which peak at the $\phi$. 
Chapter 5. Fitting the $\phi$ Resonance in $\tau^{-} \rightarrow K^{-} \pi^{-} K^{+} \nu_{\tau}$ and $\tau^{-} \rightarrow K^{-} K^{-} K^{+} \nu_{\tau}$ Decays

\begin{tabular}{|l|c|c|}
\hline & $K \pi K$ & $K K K$ \\
\hline \multicolumn{2}{|c|}{ Statistical Uncertainties } \\
\hline fit statistics & 16.1 & 5.9 \\
\hline \multicolumn{2}{|c|}{ Systematic Uncertainties } \\
\hline $\mathcal{B}\left(\phi \rightarrow K^{+} K^{-}\right)$ & 1.2 & 1.2 \\
fit parameterization & 1.0 & 2.0 \\
fit bias & na & 1.3 \\
$\mathcal{L} \sigma_{e^{+} e^{-} \rightarrow \tau^{+} \tau^{-}}$ & 1.0 & 1.0 \\
$M_{i j}$ and particle ID & 1.9 & 4.9 \\
signal modelling & 1.3 & 2.0 \\
EMC and DCH response & 0.8 & 1.2 \\
trigger & 0.1 & 0.1 \\
backgrounds & 6.7 & 5.5 \\
\hline Total & 7.3 & 8.3 \\
\hline
\end{tabular}

Table 5.4: A summary of the uncertainties for the $\tau^{-} \rightarrow \phi \pi^{-} \nu_{\tau}$ and $\tau^{-} \rightarrow \phi K^{-} \nu_{\tau}$ analysis expressed in percentages. The individual systematic uncertainties are added in quadrature to obtain the total systematic uncertainty. 


\section{Chapter 6}

\section{Unfolding the Invariant Mass Distribution}

\subsection{Introduction to Unfolding}

When measuring a physical observable, the imperfections of the apparatus generally cause distortions in the measured observable. These distortions are typically caused by the finite resolution of the detector, the non-linear response of the scale and/or the resolution of the detector over the distribution of the observable, and the probability of acceptance which may vary over the distribution of the observable. In order to compare an experimental measurement of an observable to the theoretical prediction or to another experimental measurement, the distortions, in the measured observable, must be corrected to obtain the "true" distribution of the observable. This process is referred to as "unfolding" or "deconvolution". The unfolding of an observable requires the sources of the distortions, caused by the detector, during the process of measurement to be understood, well modelled, and should be numerically stable independent of the physics model used to describe the observable. Unfolding methods that are commonly employed in particle physics include: Bayesian Unfolding [7], Singular Value Decomposition [121], parameterization of the detectors response with spline interpolations [122], and simple migration where each bin is assumed to be 
uncorrelated. The unfolding technique used in this analysis is the Bayesian Unfolding.

\subsubsection{Bayesian Unfolding}

Bayesian Unfolding is an iterative unfolding method that is based on Bayes Theorem. Bayes' Theorem states that conditional probability of the cause, $C_{i}$, given the event, $E$, referred to as the posterior probability is

$$
P\left(C_{i} \mid E\right)=\frac{P\left(E \mid C_{i}\right) P\left(C_{i}\right)}{\sum_{l=1}^{n_{c}} P\left(E \mid C_{l}\right) P\left(C_{l}\right)}
$$

where $P\left(C_{i}\right)$ is the prior probability for the cause $C_{i}$ and $P\left(E \mid C_{i}\right)$ is the conditional probability of the event $E$ given the cause $C_{i}$. The denominator is a normalization condition of the probability $P(E)$. For the measurement of an observable, the cause corresponds to the "true" distribution before the distorting effect of the measurement, and the event corresponds to the distribution of the measured observable. The conditional probability $P\left(E \mid C_{i}\right)$ takes into account the effects of measuring the observable with the detector and is commonly determined using a full MC simulation of the detector. The iterative process of Bayesian Unfolding begins with the assumption of the prior probability $P\left(C_{i}\right)$ which represents the best knowledge of the initial or "true" distribution. Then, using the Bayes' Theorem and the conditional probability $P\left(E \mid C_{i}\right)$ the posterior probability can be determined. From the posterior probability the number of events for each cause is calculated, $\hat{n}^{\prime}\left(C_{i}\right)=n(E) P\left(C_{i} \mid E\right)$, and therefore a prior probability $P^{\prime}\left(C_{i}\right)=\hat{n}^{\prime} / N_{\text {total }}$, where $N_{\text {total }}=\sum_{i=1}^{n_{c}} \hat{n}\left(C_{i}\right)$ is the total number of events. This new prior probability will be between the original prior and the true probability for each cause. By replacing the original prior with $P^{\prime}\left(C_{i}\right)$ and repeating the process, the unfolded distribution can be brought closer to the true distribution in an iterative process. The number of iterations is chosen to minimize the statistical uncertainty and the bias, where the bias is the deviation 
between the unfolded distribution and the "true" distribution which results from the dependence of the unfolding method on the physics model used to simulate the detector's response [7].

\subsection{Procedure}

The unfolding of the invariant mass distributions, $M_{123}, M_{13}$ and $M_{23}$, begin with the data and $\mathrm{MC}$ data sets and selection criteria from the branching fraction measurement defined in Section 4.1. The 3 dimensional Dalitz re-weighting of the $\mathrm{MC}$ is not applied to allow for the evaluation of the bias caused by Bayesian Unfolding technique. The details on the determination of the bias due to the unfolding can be found in Section 6.3.3. Before unfolding, the backgrounds, including the cross-feed from the other signal channels, is subtracted from the data. The Bayesian Unfolding procedure is applied to the given invariant mass data distribution for a range of iterations, $n=1 \rightarrow 30$. The optimal numbers of iterations for the unfolding is the number of iterations with the minimum total mean square error, $\sigma_{M E S}=\sqrt{\sum_{i} \sigma_{\text {stat }, i}^{2}+\sigma_{\text {bias }, i}^{2}}$, where $\sigma_{\text {stat }, i}$ is the statistical error in the $i$ th bin of the invariant mass distribution and $\sigma_{\text {bias }, i}$ is the unfolding bias in the $i$ th bin of the invariant mass distribution. Using the invariant mass distribution unfolded with the optimized number of iterations, the bin by bin efficiency correction, which is determined from MC truth, is applied and the distribution is normalized to unity. The statistical uncertainty was determined using a 1,000 trial MC distributions which simulated the mean and statistical uncertainty on the measured data distribution. The systematic uncertainties are then determined for the normalized invariant mass distribution. 


\subsection{Systematic Uncertainty Studies}

The systematic uncertainties relevant for the unfolding are those which can alter the shape of the invariant mass distributions. These uncertainties originate from event reconstruction and selection, the $\mathrm{MC}$ simulation modelling of the backgrounds, the signal efficiency and the detector resolution.

\subsubsection{Normalization Systematic Uncertainty}

- There is a systematic uncertainty associated with the luminosity $[107,108]$ and the cross-section [106] for the subtracted backgrounds. This systematic uncertainty is only determined for the $\tau$ backgrounds because the uncertainty associated with the non- $\tau$ backgrounds is determined with control samples, and is described in Section 6.3.6. The backgrounds are increased by the normalization uncertainty, $1.0 \%$, and then subtracted from the measured invariant mass distributions and unfolded. The resulting shift in each bin relative to the default unfolded distribution is taken as the systematic uncertainty.

\subsection{2 $M_{i j}$ and Particle-ID}

- The MC statistical uncertainty for the signal and backgrounds are estimated separately with Toy-MC simulations for the backgrounds and signal. Each of the Toy-MC studies had 1,000 trials where the Toy-MC simulated the mean, the statistical uncertainty, and the correlation on the respective distributions.

- The particle-ID Statistical Systematic was estimated with a Toy-MC study with 100 trials. In each trial, the Toy-MC particle-ID tables were generated from the standard BABAR particle-ID tables, with the particle-ID efficiency being modelled by a Gaussian distribution. The MC events were then selected and 
weighted with the Toy-MC particle-ID tables. The invariant mass distribution, after being background subtracted, was then unfolded. The RMS of each bin and the associated correlations were then calculated from 100 unfolded distributions which used the Toy-MC for unfolding.

- The systematic uncertainty related to pions being mis-identified as kaons has a similar methodology to that of the branching fraction measurement in Section 4.5.2. The event selection is run without the extra selection critera requirements so that the kaon tracks are not in the problematic region for particle identification described in Sections 4.3.5 and 4.5.2. After the invariant mass distributions are unfolded, the difference in the bin values, relative to the default unfolded invariant mass distributions, are scaled by the ratio of data-MC disagreement from the pion cross-feed sample with and without the extra particle-ID requirements, described in Section 4.5.2, to obtain the systematic uncertainty.

- The systematic uncertainty associated with the mis-identification of a kaon as a pion is based on the same strategy that the $\tau^{-} \rightarrow h^{-} h^{-} h^{+} \nu_{\tau}$ analysis used in Section 4.5.2, the use of the $\tau^{-} \rightarrow \pi^{-} \pi^{-} K^{+} \nu_{\tau}$ and $\tau^{-} \rightarrow K^{-} K^{-} \pi^{+} \nu_{\tau}$ decay modes to estimate the systematic uncertainty. For the $\tau^{-} \rightarrow \pi^{-} \pi^{-} \pi^{+} \nu_{\tau}$, and $\tau^{-} \rightarrow K^{-} \pi^{-} K^{+} \nu_{\tau}$ channels, the MC cross-feeds for decay modes, with an additional kaon to the mode being studied, are scaled by $140 \%$ and used in the background subtraction. Again, the invariant mass distribution is unfolded and the difference between the default unfolded distributions and the invariant mass distributions are taken as the systematic uncertainty. For the $\tau^{-} \rightarrow$ $K^{-} \pi^{-} K^{+} \nu_{\tau}$ channels, the cross-feed decay modes are scaled to account for the full shift in the branching ratio measurement. The selection of the MC is run with the extra requirements that the pion tracks are not in the problematic 
region for particle identification descibed in Sections 4.3.5 and 4.5.2. Again, the systematic uncertainty is taken as the deviation between the resulting unfolded distribution and the default unfolded distribution.

\subsubsection{Unfolding Bias}

- The bias in the unfolding is estimated by unfolding the signal MC spectra, that has had the 3D Dalitz weighting described in Section 4.4 applied, as if it were the data and comparing the results to the $\mathrm{MC}$ truth of the weighted invariant mass spectra. The deviation in each bin is the bais due to the unfolding and is taken as the systematic uncertainty.

\subsubsection{EMC and DCH Response}

- The systematic uncertainty associated with the momentum scale in the tracking detectors was determined using the data-MC scale ratio determined from a control sample [109] in a similar procedure to Section 4.5.4. The momentum scale in the $\mathrm{MC}$ is adjusted by the data-MC scale ratio from the control sample and the selection criteria was applied to the MC. The invariant mass distributions were then unfolded using the modified MC. The systematic uncertainty in each bin is taken as the shift relative to the unfolded distribution determined from the unmodified MC. The same method was applied for the momentum resolution of the tracking detectors where the $\mathrm{MC}$ track resolution was scaled by the data-MC momentum resolution ratio from [109].

- The systematic uncertainty for the scale and resolution in the EMC was determined using an analogous procedure to the systematic for the scale and resolution in the tracking detectors. The energy scale in the MC was modified by the data-MC scale ratio from [109] and then the selection was applied to the 
MC. After unfolding, the shift in each bin of the unfolded spectra, relative to the default unfolded spectra, is taken as the systematic uncertainty. For the energy resolution, a similar procedure is applied where the MC energy resolution is modified with the data-MC energy resolution ratio [109].

- The systematic resulting from the $\theta$ scale was determined by shifting the polar angle by one standard deviation, $\pm 0.000897 \mathrm{rad}$, about the mean value in the MC. Two modified MC sets are obtained, one for the positive shift and one for the negative shift of the polar angle. After applying the event selection and unfolding, the systematic uncertainty for each bin is taken as half of the deviation between the two modified MC sets.

- There is a systematic uncertainty associated with the uncertainty on the beam collision energy [110]. The same procedure used for determining the $\theta$ distribution was applied to obtain the systematic uncertainty for the beam energy.

- The tracking efficiency and correction factor are dependent on the $\cos (\theta)$ and momentum distribution of the tracks, variables that the invariant mass is also dependent on. Therefore, the tracking uncertainty associated with variation in $\cos (\theta)$ and momentum, $0.16 \%$ [111-113], is applied as a flat correction factor for each of the 3 hadron tracks and the lepton tag.

- There is a systematic uncertainty associated with the modelling of the signal distributions to which the cuts were applied. For each of the cuts applied in the $\tau^{-} \rightarrow h^{-} h^{-} h^{+} \nu_{\tau}$, analysis associated with tracking or the EMC, the mean of the selected distribution of the cut parameter for the data and $\mathrm{MC}$ were compared to verify the modelling in the MC. If the means on the data and MC have a statistical deviation of more than 2 standard deviations, a systematic 
uncertainty was assigned to account for the possible modelling biasing of the $\mathrm{MC}$ signal associated with the cut variable. The systematic uncertainty is evaluated by altering the cut value applied to the $\mathrm{MC}$ to account for the deviation between the data and $\mathrm{MC}$ means for the cut variable. After the event selection and the unfolding have been applied, the deviation in each bin of the unfolded spectra, relative to the default unfolded spectra, is taken as the systematic uncertainty. The cut variables that had this modelling bias uncertainty applied are:

1. The cut applied to the residual neutral energy of the 1 prong lepton tracks.

2. The cut applied to the residual neutral energy of the 3 prong signal tracks.

3. The cut applied to remove contamination from $\tau$ decays containing $K_{S}^{0}$.

\subsubsection{Trigger}

- The affects of the trigger on the unfolding was determined by comparing the unfolded distribution on the MC selected with and without the trigger applied to the $\tau^{-} \rightarrow K^{-} \pi^{-} \pi^{+} \nu_{\tau}$ and $\tau^{-} \rightarrow K^{-} K^{-} K^{+} \nu_{\tau}$ MC. Using the conservative estimate that the trigger is correctly modelled to the $10 \%$ level, [109] the systematic uncertainty applied to each bin, in the unfolded distribution, is defined as $10 \%$ of the largest relative shift in the number of selected MC events from the $\tau^{-} \rightarrow K^{-} \pi^{-} K^{+} \nu_{\tau}$ and $\tau^{-} \rightarrow K^{-} K^{-} K^{+} \nu_{\tau}$ channels. The mean value of the systematic uncertainty in the $\tau^{-} \rightarrow K^{-} \pi^{-} K^{+} \nu_{\tau}$ unfolded distribution is taken as the uncertainty for each bin in the $\tau^{-} \rightarrow \pi^{-} \pi^{-} \pi^{+} \nu_{\tau}$ and $\tau^{-} \rightarrow K^{-} \pi^{-} K^{+} \nu_{\tau}$ unfolded distribution. 


\subsubsection{Backgrounds}

- The systematic uncertainty from the $\tau$ backgrounds was determined for all the major background modes by shifting branching fractions in the MC by one standard deviation about their measured values. For the $\tau^{-} \rightarrow h^{-} h^{-} h^{+} \nu_{\tau}$ channels, the values measured in this paper are used, while for the other branching fractions, the world average values [39] are used. The remaining background modes are shifted together by their combined uncertainty

$$
\sigma_{i}^{\tau, B k g}=\sum_{j} w_{i j} \frac{\sigma_{j}}{\mathcal{B}_{j}}
$$

where $w_{i j}$ is the amount of contamination from mode $j$ in channel $i$, and $\frac{\sigma_{j}}{\mathcal{B}_{j}}$ is the percent uncertainty on the $j$ th background mode. The background subtraction is applied to the data and the unfolding procedure is applied. The deviation caused by the modified background in the unfolded spectra is taken as the systematic uncertainty.

- The correction factor for the $q \bar{q}$ background was determined from the 3 prong invariant mass region, $M_{3 \text { prong }}>1.82 \mathrm{GeV} / c^{2}$ from the same procedure as in Section 4.5.6. The deviation between the unfolded invariant mass distribution with the correction factor applied to the subtracted non- $\tau$ backgrounds and the unfolded invariant mass from the default procedure is defined as the systematic uncertainty.

- There is a $3 \%$ systematic uncertainty associated with the modelling of the $\pi^{0}$ rejection efficiency [114]. To assess the systematic uncertainty associated with this, all the backgrounds that contain $\pi^{0}$ 's are increased by $3 \%$ before being subtracted from the data. After unfolding, the systematic uncertainty is deter- 
mined from the resulting shift relative to the default unfolded distributions.

- The systematic uncertainty from Bhabha contamination was estimated by allowing an increase on the Bhabha contamination into the invariant mass distributions to account for the increased data statistics and the biasing of the shape in the invariant mass distributions. More specifically, the 3 electron vetoes in the 3 prong hemisphere were removed, and the resulting data distribution was normalized to the default number of events in the data plus a correction factor to account for the extra Bhabha background events. The correction factor is $3 \times$ the Bhabha systematic uncertainty defined in Section 4.5.6. This normalization corrects for the efficiency difference caused by removing the electron vetoes. The data is then background subtracted and unfolded using the default MC. The systematic uncertainty is defined as $1 / 3$ the difference between the modified unfolded invariant mass distributions and the default unfolded distributions.

- There is a systematic uncertainty associated with the modelling of the background distributions for which cuts were applied. For each of the cuts applied in the $\tau^{-} \rightarrow h^{-} h^{-} h^{+} \nu_{\tau}$ analysis associated with tracking or the EMC, the means of the $\tau^{-} \rightarrow h^{-} h^{-} h^{+} \nu_{\tau}$ distribution of the cut parameter for the data and MC were compared to verify the modelling in the MC. If the means on the data and MC have a statistical deviation of more than 2 standard deviations, a systematic uncertainty was assigned to account for possible modelling biasing of the MC signal associated with the cut variable. The systematic uncertainty is evaluated by altering the cut value applied to the MC in all of the $\tau^{-} \rightarrow h^{-} h^{-} h^{+} \nu_{\tau}$ channels to account for the deviation between the data and MC means for the cut variable. After the events selection and the unfolding have been applied, 
the deviation in each bin of the unfolded spectra relative to the default unfolded spectra is taken as the systematic uncertainty. The cut variables that had this modelling bias uncertainty applied are:

1. The cut applied to the residual neutral energy of the 1 prong lepton tracks.

2. The cut applied to the residual neutral energy of the 3 prong signal tracks.

3. The cut applied to remove contamination from $\tau$ decays containing $K_{S}^{0}$.

\subsection{Results}

The unfolded invariant mass distributions can be seen in Figures 6.1 to 6.4.
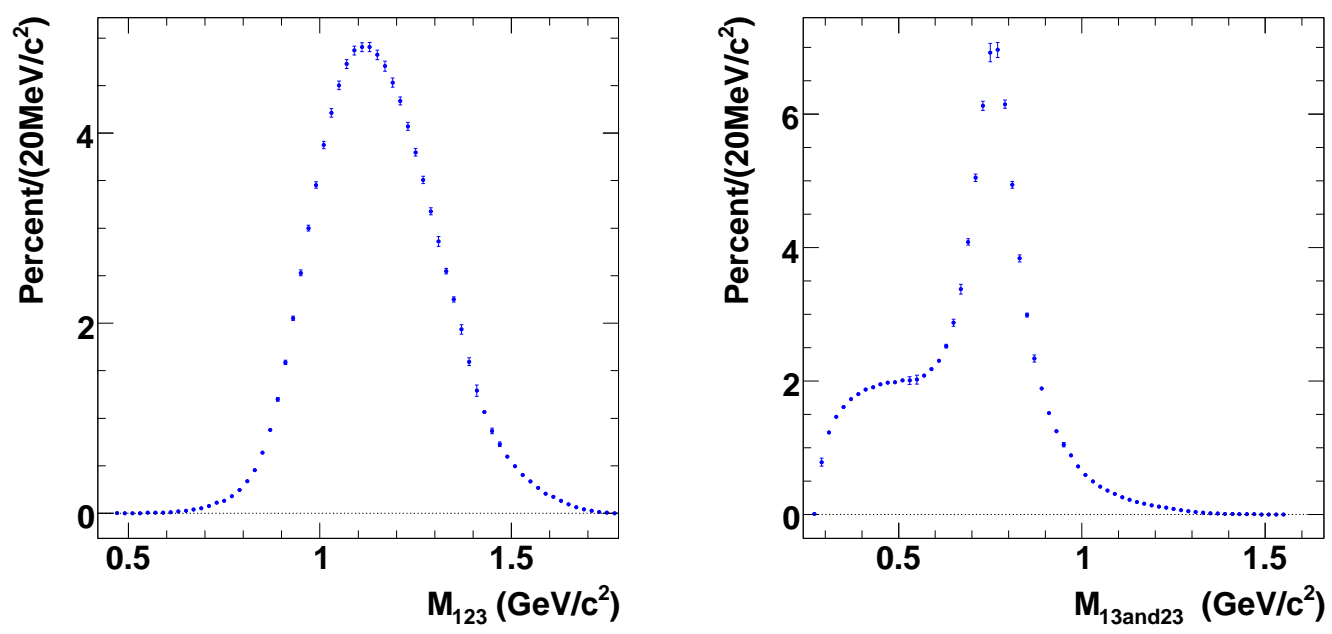

Figure 6.1: The unfolded $\tau^{-} \rightarrow \pi^{-} \pi^{-} \pi^{+} \nu_{\tau}$ invariant mass distributions for $M_{123}$ (left), $M_{13}$ and $M_{23}$ (right). The data is represented by the points with the inner error bars representing the statistical uncertainty and the outer error bars representing the total uncertainty. The integral of the unfolded distributions have been normalized to $100 \%$.

The numerical values and the corresponding correlation tables can be found in Appendices $\mathrm{C}$ to $\mathrm{F}$. The largest uncertainties in the unfolding of the $\tau^{-} \rightarrow \pi^{-} \pi^{-} \pi^{+} \nu_{\tau}$ 

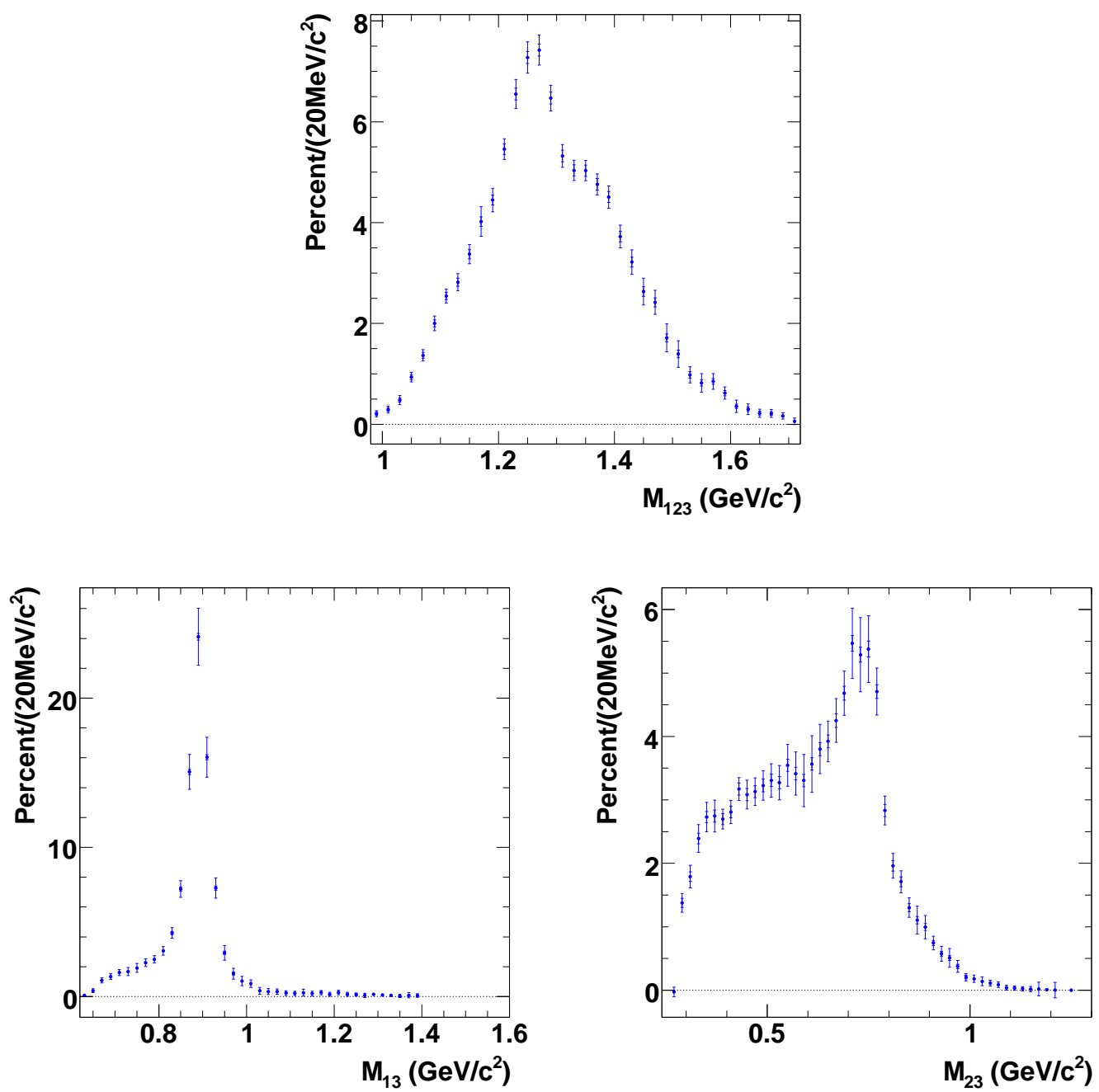

Figure 6.2: The unfolded $\tau^{-} \rightarrow K^{-} \pi^{-} \pi^{+} \nu_{\tau}$ invariant mass distributions for $M_{123}$ (top), $M_{13}$ (bottom-left) and $M_{23}$ (bottom-right). The data is represented by the points with the inner error bars representing the statistical uncertainty and the outer error bars representing the total uncertainty. The integral of the unfolded distributions have been normalized to $100 \%$.

invariant mass spectras originate from, the bias uncertainty, the momentum scale uncertainty, the uncertainty associated with kaons being mis-identified as pions, and the limited MC statistics for the $\tau^{-} \rightarrow \pi^{-} \pi^{-} \pi^{+} \nu_{\tau}$ decay. For the $\tau^{-} \rightarrow K^{-} \pi^{-} \pi^{+} \nu_{\tau}$ the dominant uncertainties originate from the limited background and signal MC statis- 

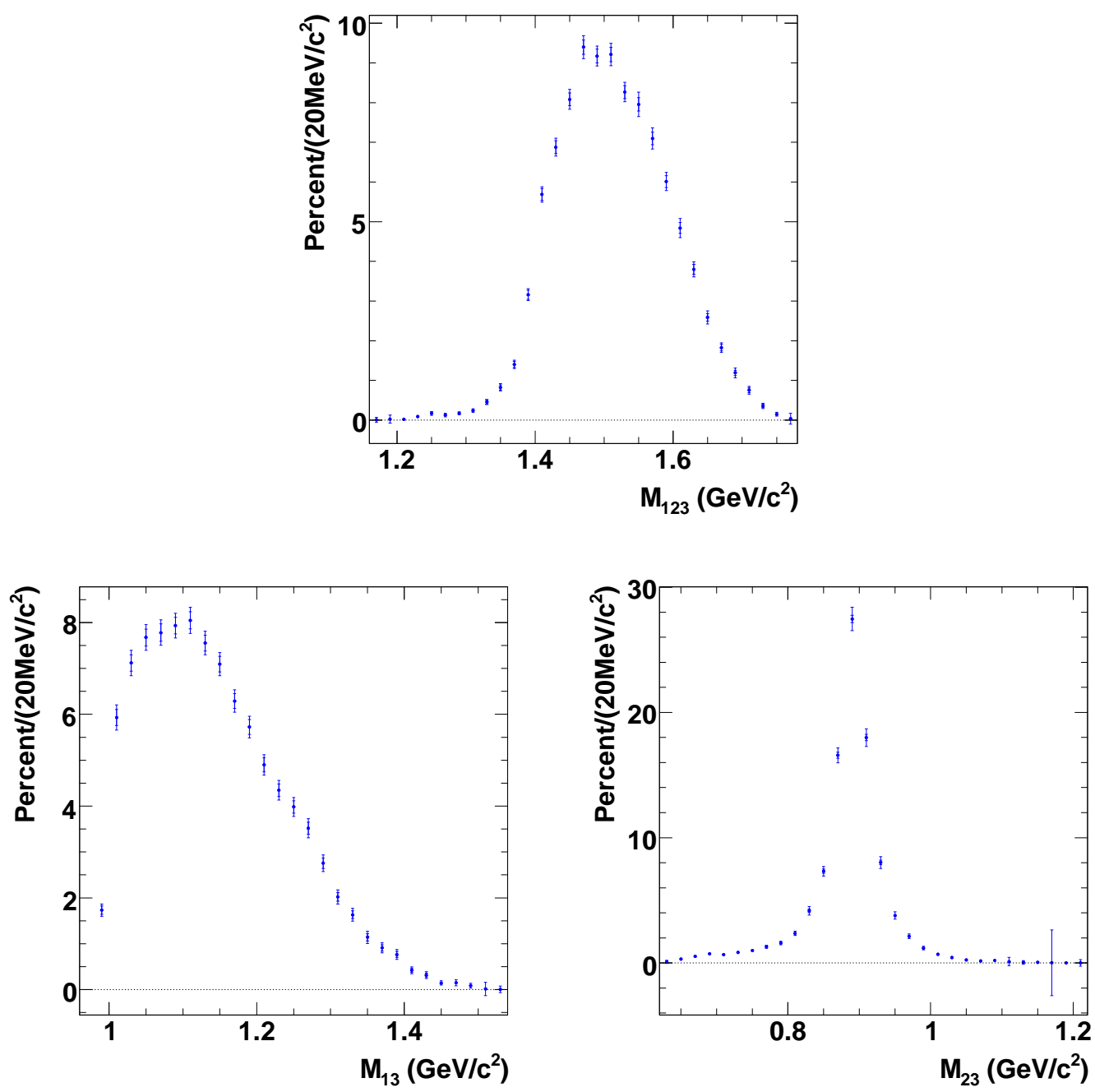

Figure 6.3: The unfolded $\tau^{-} \rightarrow K^{-} \pi^{-} K^{+} \nu_{\tau}$ invariant mass distributions for $M_{123}$ (top), $M_{13}$ (bottom-left) and $M_{23}$ (bottom-right). The data is represented by the points with the inner error bars representing the statistical uncertainty and the outer error bars representing the total uncertainty. The integral of the unfolded distributions have been normalized to $100 \%$.

tics, the uncertainty associated with kaons being mis-identified as pions, the particleID Table statistical uncertainty, and the bias uncertainty. In the $\tau^{-} \rightarrow K^{-} \pi^{-} K^{+} \nu_{\tau}$ unfolded spectra, the largest uncertainties come from the statistical uncertainty and the MC signal statistical uncertainty. The $\tau^{-} \rightarrow K^{-} K^{-} K^{+} \nu_{\tau}$ unfolded spectra are 

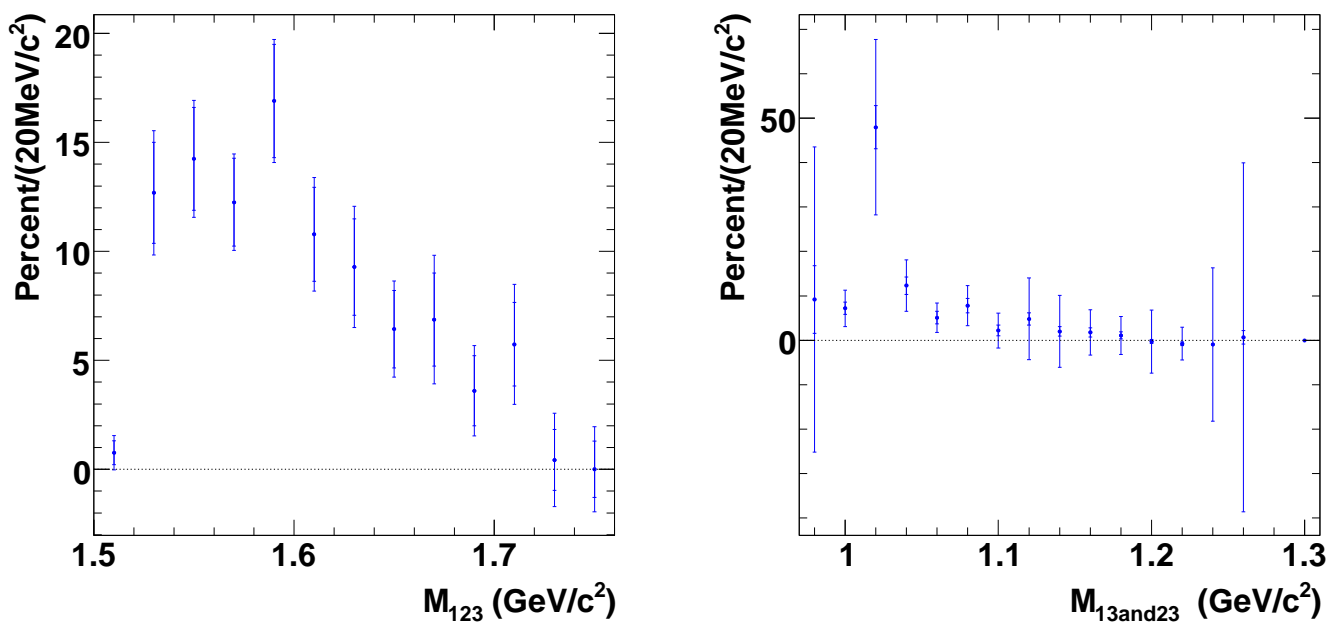

Figure 6.4: The unfolded $\tau^{-} \rightarrow K^{-} K^{-} K^{+} \nu_{\tau}$ invariant mass distributions for $M_{123}$ (left), $M_{13}$ and $M_{23}$ (right). The data is represented by the points with the inner error bars representing the statistical uncertainty and the outer error bars representing the total uncertainty. The integral of the unfolded distributions have been normalized to $100 \%$.

statistically limited. 


\section{Chapter 7}

Measurements of $\frac{\mathcal{B}\left(\tau^{-} \rightarrow K^{-} \nu_{\tau}\right)}{\mathcal{B}\left(\tau^{-} \rightarrow \pi^{-} \nu_{\tau}\right)}$,

$$
\begin{aligned}
& \frac{\mathcal{B}\left(\tau^{-} \rightarrow K^{-} \nu_{\tau}\right)}{\mathcal{B}\left(\tau^{-} \rightarrow e^{-} \nu_{\tau} \bar{\nu}_{e}\right)}, \frac{\mathcal{B}\left(\tau^{-} \rightarrow \pi^{-} \nu_{\tau}\right)}{\mathcal{B}\left(\tau^{-} \rightarrow e^{-} \nu_{\tau} \bar{\nu}_{e}\right)}, \text { and } \\
& \frac{\mathcal{B}\left(\tau^{-} \rightarrow \mu^{-} \nu_{\tau} \bar{\nu}_{\mu}\right)}{\mathcal{B}\left(\tau^{-} \rightarrow e^{-} \nu_{\tau} \bar{\nu}_{e}\right)}
\end{aligned}
$$

\subsection{Methodology of Analysis}

The approach for this analysis is to select a pure sample of $1 \operatorname{prong} \tau^{-}$decays with no neutrals using a 3 prong $\tau^{+} \rightarrow \pi^{+} \pi^{+} \pi^{-} \nu_{\tau}$ decay of the $\tau^{+}$. After selection, the corrected number of decays produced in the experiment are calculated for each channel separately using the efficiencies determined from MC simulations. The efficiency, $\mathcal{E}_{i}$, of each channel is modified using data control samples of kaons and pions from $D^{*+} \rightarrow \pi^{+} D^{0}, D^{0} \rightarrow \pi^{+} K^{-}$decays, electrons from $e^{+} e^{-} \rightarrow e^{+} e^{-} \gamma$ events, and muons from $e^{+} e^{-} \rightarrow \mu^{+} \mu^{-} \gamma$ to account for small differences between MC and data. The

number of decay mode $i$ signal events measured in the sample, $\mathbf{N}_{i}^{S i g}$, is then:

$$
\mathbf{N}_{i}^{\text {Sig }}=\left(\mathcal{E}^{-1}\right)_{i}\left(\mathbf{N}_{i}^{\text {Data }}-\mathbf{N}_{i}^{B k g}\right)
$$


Chapter 7. Measurements of $\frac{\mathcal{B}\left(\tau^{-} \rightarrow K^{-} \nu_{\tau}\right)}{\mathcal{B}\left(\tau^{-} \rightarrow \pi^{-} \nu_{\tau}\right)}, \frac{\mathcal{B}\left(\tau^{-} \rightarrow K^{-} \nu_{\tau}\right)}{\mathcal{B}\left(\tau^{-} \rightarrow e^{-} \nu_{\tau} \bar{\nu}_{e}\right)}, \frac{\mathcal{B}\left(\tau^{-} \rightarrow \pi^{-} \nu_{\tau}\right)}{\mathcal{B}\left(\tau^{-} \rightarrow e^{-} \nu_{\tau} \bar{\nu}_{e}\right)}$, and $\frac{\mathcal{B}\left(\tau^{-} \rightarrow \mu^{-} \nu_{\tau} \bar{\nu}_{\mu}\right)}{\left.\operatorname{B(\tau }-e^{-} \nu^{-} \bar{\nu}\right)}$

where $\mathbf{N}_{i}^{\text {Data }}$ is the number of data events selected in decay channel $i$ and $\mathbf{N}_{i}^{B k g}$ is the estimated number of background events in decay channel $i$. From this, both the branching fraction, $\mathcal{B}_{i}=\frac{\mathbf{N}_{i}^{S i g}}{2 \mathcal{L} \sigma_{e^{+} e^{-} \rightarrow \tau^{+} \tau^{-}}}$, and the branching ratio relative to $\tau^{-} \rightarrow$ $e^{-} \nu_{\tau} \bar{\nu}_{e}, \mathcal{B}_{\text {ratio }, i}=\frac{\mathbf{N}_{i}^{\text {Sig }}}{\mathbf{N}_{\tau^{-} \rightarrow e^{-} \nu_{\tau} \bar{\nu}_{e}}^{\text {Si }}}$, can be computed for each decay mode $i$. The measurements in this analysis were blinded by normalizing the data in the histograms to the MC and scaling the number of selected events in each channel by a separate random number from $0.95-1.05$ in a reproducible manner until the selection procedure and systematic uncertainties were completed and approved by an internal BABAR review committee.

\subsubsection{BABAR Data Set}

The analysis employs $B A B A R$ data recorded in runs 1-6 and includes data that was recorded with the collision energy on the $\Upsilon(4 S)$ resonance, $10.58 \mathrm{GeV}$, and below the $\Upsilon(4 S)$ resonance at $10.54 \mathrm{GeV}$. This data sample corresponds to an integrated luminosity for $\mathcal{L}=467 \mathrm{fb}^{-1}$ where $423 \mathrm{fb}^{-1}$ is recorded on the $\Upsilon(4 S)$ resonance and $44 \mathrm{fb}^{-1}$ is recorded below the $\Upsilon(4 S)$. The generic MC samples employed in this analysis were produced in a similar manner to the $\tau^{-} \rightarrow h^{-} h^{-} h^{+} \nu_{\tau}$ analysis. The most significant generic MC was the $e^{+} e^{-} \rightarrow \tau^{+} \tau^{-}$events simulated with the KK2F Monte Carlo Generator [96,97] which employs the TAUOLA Software Package [98]. The non- $\tau$ backgrounds were simulated for: $e^{+} e^{-} \rightarrow \mu^{+} \mu^{-}(\gamma)[96], e^{+} e^{-} \rightarrow \Upsilon(4 S) \rightarrow$ $B^{0} \bar{B}^{0}, e^{+} e^{-} \rightarrow \Upsilon(4 S) \rightarrow B^{+} B^{-} e+e-\rightarrow c \bar{c}, e+e-\rightarrow u \bar{u}$, and $e+e-\rightarrow d \bar{d}$, $e+e-\rightarrow s \bar{s}[101,102]$. The signal MC, $\tau^{\mp} \rightarrow \pi^{\mp} \nu_{\tau}-\tau^{ \pm} \rightarrow \pi^{ \pm} \pi^{ \pm} \pi^{\mp} \nu_{\tau}, \tau^{\mp} \rightarrow K^{\mp} \nu_{\tau}$ - $\tau^{ \pm} \rightarrow \pi^{ \pm} \pi^{ \pm} \pi^{\mp} \nu_{\tau}$, and $\tau^{\mp} \rightarrow(1 \text { prong })^{\mp} \nu_{\tau}-\tau^{ \pm} \rightarrow \pi^{ \pm} \pi^{ \pm} \pi^{\mp} \nu_{\tau}$ events were simulated with the " $e^{+} e^{-} \rightarrow \tau^{+} \tau^{-}$" KK2F Monte Carlo Generator [96,97]. The GEANT4 Software Package [103] was used to simulate the BABAR Detector response for the MC. The integrated luminosities for the MC and data are displayed in Table 7.1. 
Chapter 7. Measurements of $\frac{\mathcal{B}\left(\tau^{-} \rightarrow K^{-} \nu_{\tau}\right)}{\mathcal{B}\left(\tau^{-} \rightarrow \pi^{-} \nu_{\tau}\right)}, \frac{\mathcal{B}\left(\tau^{-} \rightarrow K^{-} \nu_{\tau}\right)}{\mathcal{B}\left(\tau^{-} \rightarrow e^{-} \nu_{\tau} \bar{\nu}_{e}\right)}, \frac{\mathcal{B}\left(\tau^{-} \rightarrow \pi^{-} \nu_{\tau}\right)}{\mathcal{B}\left(\tau^{-} \rightarrow e^{-} \nu_{\tau} \bar{\nu}_{e}\right)}$, and

\begin{tabular}{|l|r|r|r|r|r|r|r|}
\hline & Run 1 & Run 2 & Run 3 & Run 4 & Run 5 & Run 6 & Total \\
\hline Data & 23.0 & 68.0 & 34.7 & 110.4 & 147.7 & 83.4 & 467.3 \\
\hline$\tau^{+} \tau^{-}$ & 22.1 & 60.4 & 30.5 & 98.0 & 143.9 & 75.1 & 430.0 \\
$\mu^{+} \mu^{-}$ & 22.6 & 62.1 & 32.1 & 101.0 & 148.7 & 77.7 & 444.2 \\
$u d s$ & 22.5 & 62.3 & 31.9 & 101.6 & 151.4 & 76.2 & 445.8 \\
$c \bar{c}$ & 45.3 & 129.9 & 64.6 & 194.5 & 282.1 & 153.4 & 869.8 \\
$B^{+} B^{-}$ & 67.2 & 187.5 & 90.5 & 305.4 & 444.0 & 232.9 & 1327.6 \\
$B^{0} \bar{B}^{0}$ & 67.6 & 187.9 & 91.9 & 300.5 & 441.0 & 237.4 & 1326.4 \\
\hline$(1 \text { prong })^{-}$ & $1687 \mathrm{~K}$ & $4740 \mathrm{~K}$ & $2644 \mathrm{~K}$ & $8095 \mathrm{~K}$ & $10629 \mathrm{~K}$ & $5541 \mathrm{~K}$ & $33336 \mathrm{~K}$ \\
$(1 \text { prong })^{-}$ & $1687 \mathrm{~K}$ & $5030 \mathrm{~K}$ & $2644 \mathrm{~K}$ & $8025 \mathrm{~K}$ & $10649 \mathrm{~K}$ & $5437 \mathrm{~K}$ & $33472 \mathrm{~K}$ \\
$\tau^{-} \rightarrow \pi^{-} \nu_{\tau}$ & $2112 \mathrm{~K}$ & $6288 \mathrm{~K}$ & $3306 \mathrm{~K}$ & $10120 \mathrm{~K}$ & $13321 \mathrm{~K}$ & $4490 \mathrm{~K}$ & $39637 \mathrm{~K}$ \\
$\tau^{-} \rightarrow \pi^{-} \nu_{\tau}$ & $2112 \mathrm{~K}$ & $6288 \mathrm{~K}$ & $3306 \mathrm{~K}$ & $10118 \mathrm{~K}$ & $13088 \mathrm{~K}$ & $4918 \mathrm{~K}$ & $39830 \mathrm{~K}$ \\
$\tau^{-} \rightarrow K^{-} \nu_{\tau}$ & $528 \mathrm{~K}$ & $1570 \mathrm{~K}$ & $827 \mathrm{~K}$ & $2531 \mathrm{~K}$ & $3325 \mathrm{~K}$ & $1222 \mathrm{~K}$ & $10003 \mathrm{~K}$ \\
$\tau^{-} \rightarrow K^{-} \nu_{\tau}$ & $528 \mathrm{~K}$ & $1570 \mathrm{~K}$ & $827 \mathrm{~K}$ & $2531 \mathrm{~K}$ & $3329 \mathrm{~K}$ & $1234 \mathrm{~K}$ & $10019 \mathrm{~K}$ \\
\hline
\end{tabular}

Table 7.1: The Data (on-resonance and off-resonance) and equivalent MC integrated luminosity for Runs 1-6 in $f b^{-1}$. For the generated signal MC the total number of events produced is quoted.

\subsection{Selection Procedure}

Events that have a total net charge of zero and four well reconstructed tracks, which are not consistent with originating from the conversion of a photon in the material of the detector, are selected. The four tracks are required 1) to be within the angular acceptance of the DIRC and EMC and 2) that they reach the DIRC, $p_{t}>250 \mathrm{MeV} / \mathrm{c}$, to insure good particle identification from the EMC, DIRC and DCH. The plane orthogonal to the thrust axis $[104,105]$ is used to divide the event into two hemispheres in the c.m., where each hemisphere is associated with one of the $\tau$ decays. The "signal" hemisphere is required to have a single track and the other tracks are in the "3 prong" hemisphere. To ensure a clean separation between the particles, the signal track momentum is required to be between $1 \mathrm{GeV} / c$ to $4 \mathrm{GeV} / c$.

To remove two photon and Bhabha background, the event must have a missing energy in the c.m. between $10 \%$ and $70 \%$ of $\sqrt{s}$. The angle of the missing momentum 
Chapter 7. Measurements of $\frac{\mathcal{B}\left(\tau^{-} \rightarrow K^{-} \nu_{\tau}\right)}{\mathcal{B}\left(\tau^{-} \rightarrow \pi^{-} \nu_{\tau}\right)}, \frac{\mathcal{B}\left(\tau^{-} \rightarrow K^{-} \nu_{\tau}\right)}{\mathcal{B}\left(\tau^{-} \rightarrow e^{-} \nu_{\tau} \bar{\nu}_{e}\right)}, \frac{\mathcal{B}\left(\tau^{-} \rightarrow \pi^{-} \nu_{\tau}\right)}{\mathcal{B}\left(\tau^{-} \rightarrow e^{-} \nu_{\tau} \bar{\nu}_{e}\right)}$, and $\frac{\mathcal{B}\left(\tau^{-} \rightarrow \mu^{-} \nu_{\tau} \bar{\nu}_{\mu}\right)}{\mathcal{B}\left(\tau^{-} \rightarrow e^{-} \nu_{\tau} \bar{\nu}_{e}\right)}$

in the c.m., $\theta_{m i s s}^{c m}$, is constrained to satisfy the relation $\left|\cos \left(\theta_{m i s s}^{c m}\right)\right|<0.7$. To further reduce the two photon contamination, the thrust $[104,105]$ of the event is required to be above 0.9 , and the net missing transverse momentum in the c.m. is greater than $0.009 \sqrt{s} / c$. The 3 prong tracks have an electron veto applied to them to further reduce the Bhabha contamination. This results in less than $0.025 \%$ contamination from two photon events and less than $0.031 \%$ contamination from Bhabha events.

To increase the purity of the $\tau^{+} \tau^{-}$pair sample, the tracks in the 3 prong hemisphere are required to be consistent with being pions. Events which contain track pairs consistent with coming from a $K_{S}^{0}$ are vetoed. Large unassociated net neutral energy $>0.200 \mathrm{MeV}$ in the 3 prong hemisphere is also removed to reduce the non- $\tau$ backgrounds.

Once a sample of 1 prong $\tau$ events has been selected, the events are then classified as $\tau^{-} \rightarrow K^{-} \nu_{\tau}, \tau^{-} \rightarrow \pi^{-} \nu_{\tau}, \tau^{-} \rightarrow e^{-} \nu_{\tau} \bar{\nu}_{e}$, or $\tau^{-} \rightarrow \mu^{-} \nu_{\tau} \bar{\nu}_{\mu}$ based upon particle identification information from the five sub-detectors at $B A B A R$. The 1 prong track in the $\tau^{-} \rightarrow K^{-} \nu_{\tau}$ channel is required to be consistent with being a kaon, to have a ratio of deposited electromagnetic energy to the particle momentum in the lab frame $(\mathrm{E} / \mathrm{p})$ less than $0.85 \mathrm{c}$, and for momentums above $3 \mathrm{GeV} / c$, in the laboratory frame, they must be inconsistent with being a muon. For the $\tau^{-} \rightarrow \pi^{-} \nu_{\tau}$ channel, the 1 prong track must be consistent with being pion and not a muon and have an $\mathrm{E} / \mathrm{p}<0.85 \mathrm{c}$. In the $\tau^{-} \rightarrow e^{-} \nu_{\tau} \bar{\nu}_{e}$ channels, the signal track must be consistent with being an electron, and in the $\tau^{-} \rightarrow \mu^{-} \nu_{\tau} \bar{\nu}_{\mu}$ channel, the signal track is required to be consistent with being a muon and not an electron. The particle identification and mis-identification of kaons, pions and muons were verified and/or corrected with a $K^{-}$control track from a $\tau^{-} \rightarrow K^{-} \pi^{-} K^{+} \nu_{\tau}$ control sample, a $\pi^{+}$control track from a $\tau^{-} \rightarrow \pi^{-} \pi^{-} \pi^{+} \nu_{\tau}$ control sample, and the low momentum muon control track from the $e^{+} e^{-} \rightarrow \mu^{+} \mu^{-} \gamma$ and $e^{+} e^{-} \rightarrow \mu^{+} \mu^{-} \gamma \rightarrow \mu^{+} \mu^{-} e^{+} e^{-}$samples. Similarly, the 
Chapter 7. Measurements of $\frac{\mathcal{B}\left(\tau^{-} \rightarrow K^{-} \nu_{\tau}\right)}{\mathcal{B}\left(\tau^{-} \rightarrow \pi^{-} \nu_{\tau}\right)}, \frac{\mathcal{B}\left(\tau^{-} \rightarrow K^{-} \nu_{\tau}\right)}{\mathcal{B}\left(\tau^{-} \rightarrow e^{-} \nu_{\tau} \bar{\nu}_{e}\right)}, \frac{\mathcal{B}\left(\tau^{-} \rightarrow \pi^{-} \nu_{\tau}\right)}{\mathcal{B}\left(\tau^{-} \rightarrow e^{-} \nu_{\tau} \bar{\nu}_{e}\right)}$, and $\frac{\mathcal{B}\left(\tau^{-} \rightarrow \mu^{-} \nu_{\tau} \bar{\nu}_{\mu}\right)}{\mathcal{B}\left(\tau^{-} \rightarrow e^{-} \nu_{\tau} \bar{\nu}_{e}\right)}$

electron particle identification is validated using an $e^{+} e^{-} \rightarrow e^{+} e^{-} \gamma$ control sample for data compared to the selected $\tau^{-} \rightarrow e^{-} \nu_{\tau} \bar{\nu}_{e}$ signal MC. A detailed description of the control samples with plots of the momentum spectra after the corrections, in Figure G.1 and G.2, have been applied can be found in Appendix G. Channel specific selection criteria are then applied. For $\tau^{-} \rightarrow e^{-} \nu_{\tau} \bar{\nu}_{e}$, and $\tau^{-} \rightarrow \mu^{-} \nu_{\tau} \bar{\nu}_{\mu}$ events, the remaining Bhabha and $e^{+} e^{-} \rightarrow \mu^{+} \mu^{-}$background is vetoed by requiring the signal track to have a momenta less than $80 \%$ of $\sqrt{s} / 2 \mathrm{c}$. Events with an unassociated neutral energy $>1.0 \mathrm{GeV}(>0.5 \mathrm{GeV})$ in the 1 prong hemisphere are removed from the $\tau^{-} \rightarrow e^{-} \nu_{\tau} \bar{\nu}_{e}\left(\tau^{-} \rightarrow \mu^{-} \nu_{\tau} \bar{\nu}_{\mu}\right)$ channel to reduce the non- $\tau$ backgrounds. In the $\tau^{-} \rightarrow K^{-} \nu_{\tau}$ and $\tau^{-} \rightarrow \pi^{-} \nu_{\tau}$ channels $\tau^{-} \rightarrow \rho^{-} \nu_{\tau}, \tau^{-} \rightarrow K^{-} \pi^{0} \nu_{\tau}$ and non- $\tau$ backgrounds are reduced by rejecting the net unassociated energy $>0.200 \mathrm{MeV}$ in the 1 prong hemisphere.

The primary background for the $\tau^{-} \rightarrow K^{-} \nu_{\tau}$ channel are $\tau^{-} \rightarrow K^{-} K_{L}^{0} \nu_{\tau}, \tau^{-} \rightarrow$ $K^{-} \pi^{0} \nu_{\tau}, \tau^{-} \rightarrow \rho^{-} \nu_{\tau}, \tau^{-} \rightarrow \pi^{-} \nu_{\tau}$ and non- $\tau$ backgrounds and for the $\tau^{-} \rightarrow \pi^{-} \nu_{\tau}$ channel are $\tau^{-} \rightarrow \rho^{-} \nu_{\tau}, \tau^{-} \rightarrow \mu^{-} \nu_{\tau} \bar{\nu}_{\mu}$ and non- $\tau$ backgrounds. To suppress these events, an algorithm that uses an approximation of the $\tau$ directions to determine the "pseudo-mass" of the neutrino is employed. The c.m. direction of the $\tau$ that produced the signal track is approximated from the 3 prong system by assuming the $\tau^{+} \tau^{-}$pairs are back-to-back. The angle between the $\tau$ that decayed to the 3 prong system and direction of the hadronic system in the c.m. is

$$
\alpha=\arccos \left(\frac{\sqrt{s} E_{\pi \pi \pi}^{c m}-m_{\tau}^{2} c^{4}-m_{\pi \pi \pi}^{2} c^{4}}{2 P_{\tau}^{c m} P_{\pi \pi \pi}^{c m} c^{2}}\right)
$$

where $E_{\pi \pi \pi}^{c m}, m_{\pi \pi \pi}$, and $P_{\pi \pi \pi}$ are the energy, mass, and momentum of the 3 prong hadronic system in c.m., $P_{\tau}^{c m}$ is the momentum of the $\tau$ particle in the c.m. and $m_{\tau}$ is the mass of the $\tau$ [123]. The projection angle of the $\tau$ out of the plane of the 
Chapter 7. Measurements of $\frac{\mathcal{B}\left(\tau^{-} \rightarrow K^{-} \nu_{\tau}\right)}{\mathcal{B}\left(\tau^{-} \rightarrow \pi^{-} \nu_{\tau}\right)}, \frac{\mathcal{B}\left(\tau^{-} \rightarrow K^{-} \nu_{\tau}\right)}{\mathcal{B}\left(\tau^{-} \rightarrow e^{-} \nu_{\tau} \bar{\nu}_{e}\right)}, \frac{\mathcal{B}\left(\tau^{-} \rightarrow \pi^{-} \nu_{\tau}\right)}{\mathcal{B}\left(\tau^{-} \rightarrow e^{-} \nu_{\tau} \bar{\nu}_{e}\right)}$, and $\frac{\mathcal{B}\left(\tau^{-} \rightarrow \mu^{-} \nu_{\tau} \bar{\nu}_{\mu}\right)}{\mathcal{B}\left(\tau^{-} \rightarrow e^{-} \nu_{\tau} \bar{\nu}_{e}\right)}$

signal track and 3 prong hadronic system, $\delta$, is fixed to an optimized value. From this approximate $\tau$ direction, and the c.m. direction of the signal track, the invariant mass of the neutrino, the "pseudo-mass", is evaluated. The angle $\delta$ and the cut on the "pseudo-mass" are optimized to minimize the statistical and background systematic uncertainties. This approach is taken instead of a cone matching algorithm [123] to reduce the sensitivity of the calculation to the modelling of the angular resolution of the detector and initial-final-state-radiation. A detailed description of this method can be found in Appendix $\mathrm{H}$.

\subsection{Systematic Uncertainty Studies}

The systematic uncertainties in the $\tau^{-} \rightarrow K^{-} \nu_{\tau}$ analysis originate from event reconstruction and selection, the MC simulation modelling of the background, the detector response, and the signal efficiency.

\subsubsection{Normalization Systematic Uncertainty}

- The normalization of the MC events for the background subtraction does not cancel in the ratio of branching fraction, therefore a systematic uncertainty is required. Taking the luminosity uncertainty on this data set, $0.64 \%$, in quadrature with the uncertainty in the cross-section, $\sigma_{e^{+} e^{-} \rightarrow \tau^{+} \tau^{-}}=(0.919 \pm$ 0.003) [106], we have a normalization uncertainty of $0.69 \%$ on the background normalization. Because the non- $\tau$ MC is scaled, using a control sample, and the control sample is used to determine the corresponding systematic uncertainty which includes the normalization, this systematic is only applied to the $\tau$ pair MC background events.

- The branching fraction of $\tau^{-} \rightarrow \pi^{-} \pi^{-} \pi^{+} \nu_{\tau}$ and the other 3 prong branching 
Chapter 7. Measurements of $\frac{\mathcal{B}\left(\tau^{-} \rightarrow K^{-} \nu_{\tau}\right)}{\mathcal{B}\left(\tau^{-} \rightarrow \pi^{-} \nu_{\tau}\right)}, \frac{\mathcal{B}\left(\tau^{-} \rightarrow K^{-} \nu_{\tau}\right)}{\mathcal{B}\left(\tau^{-} \rightarrow e^{-} \nu_{\tau} \bar{\nu}_{e}\right)}, \frac{\mathcal{B}\left(\tau^{-} \rightarrow \pi^{-} \nu_{\tau}\right)}{\mathcal{B}\left(\tau^{-} \rightarrow e^{-} \nu_{\tau} \bar{\nu}_{e}\right)}$, and

fractions which contribute to the normalization uncertainty also contributes to the normalization of the backgrounds. The $\tau^{-} \rightarrow \pi^{-} \pi^{-} \pi^{+} \nu_{\tau}$ is the most significant decay mode in the 3 prong hemisphere, resulting in the branching fraction uncertainty from $\tau^{-} \rightarrow \pi^{-} \pi^{-} \pi^{+} \nu_{\tau}$ dominating the normalization uncertainty. This analysis uses the $\tau^{-} \rightarrow \pi^{-} \pi^{-} \pi^{+} \nu_{\tau}$ branching fraction determined in [8].

\subsubsection{Efficiency Statistical and Particle-ID Systematic Un- certainty}

- The signal efficiencies have a statistical uncertainty associated with the number of MC events generated.

- The particle-ID Statistical Systematic was determined with a Toy-MC study using a similar method defined in Section 4.5.2. In the Toy-MC Study, 100 Toy particle-ID tables were produced using a Gaussian distribution, where the 100 Toy-MC particle-ID tables were generated from the standard BABAR particleID tables, and where the particle-ID efficiency was taken as the mean of the Gaussian and the uncertainty on the particle-ID efficiency was the RMS of the Gaussian. The analysis was then run for each of the Toy particle-ID tables and the resulting RMS of the measured quantities was taken as the systematic uncertainty.

- To validate the correction factors applied to correct the data MC particle-ID discrepancy from $D^{*+} \rightarrow \pi^{+} D^{0}, D^{0} \rightarrow \pi^{+} K^{-}, e^{+} e^{-} \rightarrow \mu^{+} \mu^{-} \gamma, e^{+} e^{-} \rightarrow e^{+} e^{-} \gamma$ particle-ID control samples and the $E / p$ cut, additional particle-ID control samples were developed for this analysis which have event characteristics that are more similar to the events selected in this analysis. The $\tau^{-} \rightarrow \pi^{-} \pi^{-} \pi^{+} \nu_{\tau}$ events are used for the pion control sample, $\tau^{-} \rightarrow K^{-} \pi^{-} K^{+} \nu_{\tau}$ events are used 
Chapter 7. Measurements of $\frac{\mathcal{B}\left(\tau^{-} \rightarrow K^{-} \nu_{\tau}\right)}{\mathcal{B}\left(\tau^{-} \rightarrow \pi^{-} \nu_{\tau}\right)}, \frac{\mathcal{B}\left(\tau^{-} \rightarrow K^{-} \nu_{\tau}\right)}{\mathcal{B}\left(\tau^{-} \rightarrow e^{-} \nu_{\tau} \bar{\nu}_{e}\right)}, \frac{\mathcal{B}\left(\tau^{-} \rightarrow \pi^{-} \nu_{\tau}\right)}{\mathcal{B}\left(\tau^{-} \rightarrow e^{-} \nu_{\tau} \bar{\nu}_{e}\right)}$, and $\frac{\mathcal{B}\left(\tau^{-} \rightarrow \mu^{-} \nu_{\tau} \bar{\nu}_{\mu}\right)}{\mathcal{B}\left(\tau^{-} \rightarrow e^{-} \nu_{\tau} \bar{\nu}_{e}\right)}$

for the kaon control sample, $e^{+} e^{-} \rightarrow e^{+} e^{-} \gamma$ events, where the photon converts, are used as a control sample for the electrons, and $e^{+} e^{-} \rightarrow \mu^{+} \mu^{-} \gamma$ events, where the photon is detected in the EMC and where the photon converts, are used as control samples for the muon. For the $\tau^{-} \rightarrow \pi^{-} \pi^{-} \pi^{+} \nu_{\tau}$ control sample, the odd sign track is used as the control track, while for the $\tau^{-} \rightarrow K^{-} \pi^{-} K^{+} \nu_{\tau}$ control sample the like sign track is used for the kaon control track. The $e^{+} e^{-} \rightarrow e^{+} e^{-} \gamma, e^{+} e^{-} \rightarrow \mu^{+} \mu^{-} \gamma$ control sample's track which is closest to the photon or the conversion in the c.m. frame, is used for the control track. The systematic uncertainties determined from the control samples for the kaon particle identification including the $\mathrm{E} / \mathrm{p}$ cut are:

1. In the kaon control track selected as a kaon, the data/MC ratio from the control sample is consistent with 1.0. Because this particle-ID study included the $\mathrm{E} / \mathrm{p}$ cut, the systematic uncertainty is taken as the statistical uncertainty on the control sample. This yields a $0.66 \%$ uncertainty.

2. In the muon control track selected as a kaon, the data/MC ratio from the control sample is inconsistent with 1.0 after being weighted to the muon background. Therefore the muon background is corrected and the systematic uncertainty is taken as the statistical uncertainty on the control sample. This gives a $0.33 \%$ uncertainty.

3. In the pion control track selected as a kaon, the data/MC ratio from the control sample is inconsistent with 1.0 after being weighted to the pion background. Therefore the pion background is corrected and the systematic uncertainty is taken as the statistical uncertainty on the control sample. This produces a $0.35 \%$ uncertainty.

The systematic uncertainties determined from the control samples for the pion 
Chapter 7. Measurements of $\frac{\mathcal{B}\left(\tau^{-} \rightarrow K^{-} \nu_{\tau}\right)}{\mathcal{B}\left(\tau^{-} \rightarrow \pi^{-} \nu_{\tau}\right)}, \frac{\mathcal{B}\left(\tau^{-} \rightarrow K^{-} \nu_{\tau}\right)}{\mathcal{B}\left(\tau^{-} \rightarrow e^{-} \nu_{\tau} \bar{\nu}_{e}\right)}, \frac{\mathcal{B}\left(\tau^{-} \rightarrow \pi^{-} \nu_{\tau}\right)}{\mathcal{B}\left(\tau^{-} \rightarrow e^{-} \nu_{\tau} \bar{\nu}_{e}\right)}$, and $\frac{\mathcal{B}\left(\tau^{-} \rightarrow \mu^{-} \nu_{\tau} \bar{\nu}_{\mu}\right)}{\mathcal{B}\left(\tau^{-} \rightarrow e^{-} \nu_{\tau} \bar{\nu}_{e}\right)}$

particle identification including the $\mathrm{E} / \mathrm{p}$ cut are:

1. In the pion control track selected as a pion, the data/MC ratio from the control sample is inconsistent with 1.0. Therefore the pion efficiency is corrected and the systematic uncertainty is taken as the statistical uncertainty on the control sample. This means there is a $0.061 \%$ uncertainty.

2. In the muon control track selected as a pion, the data/MC ratio from the control sample is inconsistent with 1.0 after being weighted to the muon background. Therefore the muon background is corrected and the systematic uncertainty is taken as the statistical uncertainty on the control sample. This gives a $0.36 \%$ uncertainty.

The systematic uncertainties determined from the control samples for the muon particle identification are:

1. In the muon control tracks selected as a muon, the data/MC ratios from the control sample are consistent with 1.0. Therefore, no systematic uncertainty is applied.

2. In the pion control track selected as a muon, the data/MC ratio from the control sample is inconsistent with 1.0 after being weighted to the pion background. Therefore the pion background is corrected and the systematic uncertainty is taken as the statistical uncertainty on the control sample. This yields a $0.06 \%$ uncertainty.

The systematic uncertainties determined from the control samples for the electron particle identification are:

1. In the electron control track selected as an electron, the data/MC ratio from the control sample deviates from 1 by two standard deviation. 
Chapter 7. Measurements of $\frac{\mathcal{B}\left(\tau^{-} \rightarrow K^{-} \nu_{\tau}\right)}{\mathcal{B}\left(\tau^{-} \rightarrow \pi^{-} \nu_{\tau}\right)}, \frac{\mathcal{B}\left(\tau^{-} \rightarrow K^{-} \nu_{\tau}\right)}{\mathcal{B}\left(\tau^{-} \rightarrow e^{-} \nu_{\tau} \bar{\nu}_{e}\right)}, \frac{\mathcal{B}\left(\tau^{-} \rightarrow \pi^{-} \nu_{\tau}\right)}{\mathcal{B}\left(\tau^{-} \rightarrow e^{-} \nu_{\tau} \bar{\nu}_{e}\right)}$, and

Because the control sample does not cover the entire $\cos (\theta)$ range, the full data/MC deviations from 1.0 is taken as the systematic uncertainty, $0.21 \%$.

The total systematic uncertainty associated with the particle identification in each channel is the quadratic sum of the signal particle identification and the particle mis-identification uncertainties. From this, the particle identification uncertainty on the branching ratios is determined using normal error propagation. To validate the correction factors determined from the control samples developed in this analysis, the analysis was run with an alternative set of particle identification selectors. The alternative selectors have a substantially different signal efficiency and mis-identification rates from the default selectors for this analysis. For the kaon and pion selectors, the mis-identification rates were approximately $\sim 1.5-2$ times larger than the default selectors. Before applying the particle identification correction factors determined from the control samples developed in this work, the $\frac{\mathcal{B}\left(\tau^{-} \rightarrow \pi^{-} \nu_{\tau}\right)}{\mathcal{B}\left(\tau^{-} \rightarrow e^{-} \nu_{\tau} \bar{\nu}_{e}\right)}$ deviated by $\sim 5 \%$ between the two selectors, the $\frac{\mathcal{B}\left(\tau^{-} \rightarrow K^{-} \nu_{\tau}\right)}{\mathcal{B}\left(\tau^{-} \rightarrow e^{-} \nu_{\tau} \bar{\nu}_{e}\right)}$ deviated by $\sim 3 \%$ between the two selectors and the $\frac{\mathcal{B}\left(\tau^{-} \rightarrow \mu^{-} \nu_{\tau} \bar{\nu}_{\mu}\right)}{\mathcal{B}\left(\tau^{-} \rightarrow e^{-} \nu_{\tau} \bar{\nu}_{e}\right)}$ deviated by $\sim 1.5 \%$. After applying the correction factors, all the branching ratios were consistent within the assigned systematic uncertainty for particle identification added in quadrature with the correlated statistical uncertainty.

\subsection{3 $\tau^{-} \rightarrow h^{-} h^{-} h^{+} \nu_{\tau}$ Modelling}

- The determination of the pseudo-neutrino mass uses the 3 prong four vector, and therefore is sensitive to the kinematic resonance structure of the $\tau^{-} \rightarrow$ $\pi^{-} \pi^{-} \pi^{+} \nu_{\tau}$ decay. A systematic uncertainty associated with the modelling of 
Chapter 7. Measurements of $\frac{\mathcal{B}\left(\tau^{-} \rightarrow K^{-} \nu_{\tau}\right)}{\mathcal{B}\left(\tau^{-} \rightarrow \pi^{-} \nu_{\tau}\right)}, \frac{\mathcal{B}\left(\tau^{-} \rightarrow K^{-} \nu_{\tau}\right)}{\mathcal{B}\left(\tau^{-} \rightarrow e^{-} \nu_{\tau} \bar{\nu}_{e}\right)}, \frac{\mathcal{B}\left(\tau^{-} \rightarrow \pi^{-} \nu_{\tau}\right)}{\mathcal{B}\left(\tau^{-} \rightarrow e^{-} \nu_{\tau} \bar{\nu}_{e}\right)}$, and $\frac{\mathcal{B}\left(\tau^{-} \rightarrow \mu^{-} \nu_{\tau} \bar{\nu}_{\mu}\right)}{\mathcal{B}\left(\tau^{-} \rightarrow e^{-} \nu_{\tau} \bar{\nu}_{e}\right)}$

the $\tau^{-} \rightarrow \pi^{-} \pi^{-} \pi^{+} \nu_{\tau}$ decay is determined from the full shift in the branching fractions determined with and without the 3D Dalitz weighting described in Section 4.4

\subsubsection{EMC and DCH Responses}

- The systematic uncertainties associated with the DCH and EMC response are:

1. the momentum scale and resolution modelling;

2. the energy scale and resolution modelling;

3. the modelling of $\theta$;

4. the modelling of the beam energy scale and spread.

To determine these systematic uncertainties, this analysis uses the same procedure as the $\tau^{-} \rightarrow h^{-} h^{-} h^{+} \nu_{\tau}$ analysis which is described in Section 4.5.4.

- The systematic uncertainty related to the modelling of the tracking efficiency in the MC does not cancel for the subtracted background events in the branching ratio. Therefore, the tracking efficiency uncertainty [111-113] is applied to $\tau$ pair MC background events and propagated into the branching ratio. This systematic uncertainty is not applied to the non- $\tau$ background events because the non- $\tau$ MC is scaled using a control sample and a systematic uncertainty is applied.

- There is a systematic uncertainty associated with the modelling of the signal distributions for which cuts were applied. In the $\tau^{-} \rightarrow h^{-} h^{-} h^{+} \nu_{\tau}$ analysis it was found that there are possible modelling biases associated with tracking or the EMC. A systematic uncertainty was assigned to account for possible modelling biasing of the MC signal associated with the cut variable. The systematic 
Chapter 7. Measurements of $\frac{\mathcal{B}\left(\tau^{-} \rightarrow K^{-} \nu_{\tau}\right)}{\mathcal{B}\left(\tau^{-} \rightarrow \pi^{-} \nu_{\tau}\right)}, \frac{\mathcal{B}\left(\tau^{-} \rightarrow K^{-} \nu_{\tau}\right)}{\mathcal{B}\left(\tau^{-} \rightarrow e^{-} \nu_{\tau} \bar{\nu}_{e}\right)}, \frac{\mathcal{B}\left(\tau^{-} \rightarrow \pi^{-} \nu_{\tau}\right)}{\mathcal{B}\left(\tau^{-} \rightarrow e^{-} \nu_{\tau} \bar{\nu}_{e}\right)}$, and $\frac{\mathcal{B}\left(\tau^{-} \rightarrow \mu^{-} \nu_{\tau} \bar{\nu}_{\mu}\right)}{\mathcal{B}\left(\tau^{-} \rightarrow e^{-} \nu_{\tau} \bar{\nu}_{e}\right)}$

uncertainty is evaluated as the change in the branching fractions, caused by altering the cut value applied to the MC, to account for the deviation between the data and MC means for the cut variable. The cut variables that had this modelling bias uncertainty applied are:

1. The cut applied to the residual neutral energy of the 1 prong lepton tracks. The difference between the data and MC means used to shift the signal MC distribution for the 1 prong residual neutral energy cut are: $-0.00013 \mathrm{GeV}$ for the $\tau^{-} \rightarrow e^{-} \nu_{\tau} \bar{\nu}_{e}$ decay channel; $0.00010 \mathrm{GeV}$ for the $\tau^{-} \rightarrow \mu^{-} \nu_{\tau} \bar{\nu}_{\mu}$ decay channel; $0.0017 \mathrm{GeV}$ for the $\tau^{-} \rightarrow \pi^{-} \nu_{\tau}$ decay channel; and $0.0016 \mathrm{GeV}$ for the $\tau^{-} \rightarrow K^{-} \nu_{\tau}$ decay channel.

2. The cut applied to the residual neutral energy of the 3 prong tracks associated with the signal. The difference between the data and MC means used to shift the signal MC distribution was $0.00019 \mathrm{GeV}$ for the most significant decay mode, $\tau^{-} \rightarrow \pi^{-} \pi^{-} \pi^{+} \nu_{\tau}$.

3. The cut applied to remove contamination from $\tau$ decays containing $K_{S}^{0}$. The difference between the data and MC means used to shift the signal MC distribution was -0.0052 for the most significant decay mode, $\tau^{-} \rightarrow$ $\pi^{-} \pi^{-} \pi^{+} \nu_{\tau}$

\section{$E / p$ Background Uncertainty}

- In addition to the $E / p$ cut efficiency systematic which is included in the particleID systematic uncertainty, there is a systematic uncertainty associated with the modelling of the electron background in the $E / p$ cut. The region $0.95 c<$ $E / p<1.05 c$ is used as a side band region to obtain a data/MC scaling factor for the electron background. The resulting shift in the branching ratios caused 
Chapter 7. Measurements of $\frac{\mathcal{B}\left(\tau^{-} \rightarrow K^{-} \nu_{\tau}\right)}{\mathcal{B}\left(\tau^{-} \rightarrow \pi^{-} \nu_{\tau}\right)}, \frac{\mathcal{B}\left(\tau^{-} \rightarrow K^{-} \nu_{\tau}\right)}{\mathcal{B}\left(\tau^{-} \rightarrow e^{-} \nu_{\tau} \bar{\nu}_{e}\right)}, \frac{\mathcal{B}\left(\tau^{-} \rightarrow \pi^{-} \nu_{\tau}\right)}{\mathcal{B}\left(\tau^{-} \rightarrow e^{-} \nu_{\tau} \bar{\nu}_{e}\right)}$, and

by scaling the $\tau^{-} \rightarrow e^{-} \nu_{\tau} \bar{\nu}_{e} \mathrm{MC}$ background in the $\tau^{-} \rightarrow K^{-} \nu_{\tau}$ and $\tau^{-} \rightarrow$ $\pi^{-} \nu_{\tau}$ channel by the ratio of data/MC in the side band region is taken as the systematic uncertainty.

\subsubsection{Backgrounds}

- The default analysis requires that none of the three hadron candidates on the 3 prong side pass an electron veto. To study the Bhabha contamination we loosen the electron vetoes by only requiring $z=0,1$ or 2 of the vetoes on the hadrons. The branching ratios with $z$ electron vetoes $\mathcal{B}_{i, h h h=z}$ are fit with a linear least-square fit, and the resulting linear equation is used to estimate the Bhabha contamination when all three electron vetoes are applied.

- The two-photon background is not modelled by the MC. Therefore, it is identified as an excess of data in the side band regions: $-\log \frac{2 \sqrt{\left(p_{x}^{t o t, c m}\right)^{2}+\left(p_{y}^{t o t, c m}\right)^{2}}}{E_{c m}^{P E P}}$; thrust; $E_{M i s s}^{C M} / E_{P E P}^{C M}$; and $\cos \left(\theta_{m i s s}^{C M}\right)$. To quantify the contamination from twophoton events, the $-\log \frac{2 \sqrt{\left(p_{x}^{t o t, c m}\right)^{2}+\left(p_{y}^{t o t, c m}\right)^{2}}}{E_{c m}^{P E P}}<4.0$ cut is changed to $-\log \frac{2 \sqrt{\left(p_{x}^{t o t, c m}\right)^{2}+\left(p_{y}^{t o t, c m}\right)^{2}}}{E_{c m}^{P E P}}>3.0$ to select two-photon events. Then the number of two-photon events are determined as the excess number of selected events relative to the MC. Since, the $\tau^{-} \rightarrow K^{-} \nu_{\tau}$ channel had the largest number of excess events, 3, relative to the number of signal events, we set an upper limit of 5.01 events at $95 \%$ confidence. Therefore, the systematic associated with two-photon background is less than $0.025 \%$ before applying the $-\log \frac{2 \sqrt{\left(p_{x}^{t o t, c m}\right)^{2}+\left(p_{y}^{t o t, c m}\right)^{2}}}{E_{c m}^{P E P}}$ cut.

- The branching ratios of $\tau$ decays are set to the PDG 2007 values [12]. The percent uncertainty of these branching ratios is taken as the uncertainty on the $\tau$ backgrounds. 
Chapter 7. Measurements of $\frac{\mathcal{B}\left(\tau^{-} \rightarrow K^{-} \nu_{\tau}\right)}{\mathcal{B}\left(\tau^{-} \rightarrow \pi^{-} \nu_{\tau}\right)}, \frac{\mathcal{B}\left(\tau^{-} \rightarrow K^{-} \nu_{\tau}\right)}{\mathcal{B}\left(\tau^{-} \rightarrow e^{-} \nu_{\tau} \bar{\nu}_{e}\right)}, \frac{\mathcal{B}\left(\tau^{-} \rightarrow \pi^{-} \nu_{\tau}\right)}{\mathcal{B}\left(\tau^{-} \rightarrow e^{-} \nu_{\tau} \bar{\nu}_{e}\right)}$, and

$$
\sigma_{i}^{\tau, B k g}=\sqrt{\sum_{j}\left(w_{i j} \frac{\sigma_{j}}{\mathcal{B}_{j}}\right)^{2}}
$$

where $w_{i j}$ is the amount of contamination from mode $j$ in channel $i$, and $\mathcal{B}_{j}$ branching fraction of $\tau$ background events of type $j$ and $\sigma_{j}$ is the uncertainty on the branching fraction of $\tau$ background events of type $j$.

- The $q \bar{q}$ background is corrected by employing a control sample where $m_{h h h}>$ $1.82 \mathrm{GeV} / c^{2}$. The control sample has the same cuts applied as the signal except the mass cut on the 3 prong side. The non- $\tau$ MC is scaled by ratio of data to $\mathrm{MC}$ after the $\tau$ pair and $\mu$ pair $\mathrm{MC}$ have been subtracted in the control sample. The statistical uncertainty on the correction factor is taken as the uncertainty on the non- $\tau$ background correction factor. To determine the possible Bhabha contamination in the $m_{h h h}>1.82 \mathrm{GeV}$ control sample, the control sample was selected before the electron vetoes on the 3 prong side were applied. The resulting difference for $\tau^{-} \rightarrow K^{-} \nu_{\tau}$ control sample is $0.32 \%$, for the $\tau^{-} \rightarrow \pi^{-} \nu_{\tau}$ control sample was $1.2 \%$, for the $\tau^{-} \rightarrow e^{-} \nu_{\tau} \bar{\nu}_{e}$ control sample was $137 \%$ and for the $\tau^{-} \rightarrow \mu^{-} \nu_{\tau} \bar{\nu}_{\mu}$ control sample was $8.8 \%$. The resulting shift in the branching ratio caused by assuming that the scaling factor is off by $1 / 3$ of the latter numbers results in a negligible uncertainty, less than $0.1 \%$ for the worst case. For the $\tau^{-} \rightarrow K^{-} \nu_{\tau}$ channel, the correction factor was also determined using the sideband regions $\cos \left(\theta^{\text {miss }}\right)>0.9$ and $0.6<$ Thrust $<0.8$. These values were consistent with the $m_{h h h}>1.82 \mathrm{GeV} / c^{2}$ control sample, however, due to the known two-photon contamination in these regions, these results are only used as a cross check.

- There is statistical uncertainty associated with the number of background MC 
Chapter 7. Measurements of $\frac{\mathcal{B}\left(\tau^{-} \rightarrow K^{-} \nu_{\tau}\right)}{\mathcal{B}\left(\tau^{-} \rightarrow \pi^{-} \nu_{\tau}\right)}, \frac{\mathcal{B}\left(\tau^{-} \rightarrow K^{-} \nu_{\tau}\right)}{\mathcal{B}\left(\tau^{-} \rightarrow e^{-} \nu_{\tau} \bar{\nu}_{e}\right)}, \frac{\mathcal{B}\left(\tau^{-} \rightarrow \pi^{-} \nu_{\tau}\right)}{\mathcal{B}\left(\tau^{-} \rightarrow e^{-} \nu_{\tau} \bar{\nu}_{e}\right)}$, and $\frac{\mathcal{B}\left(\tau^{-} \rightarrow \mu^{-} \nu_{\tau} \bar{\nu}_{\mu}\right)}{\mathcal{B}\left(\tau^{-} \rightarrow e^{-} \nu_{\tau} \bar{\nu}_{e}\right)}$

of events generated.

- There is a systematic uncertainty associated with the modelling of the background distributions for which cuts were applied. In the $\tau^{-} \rightarrow h^{-} h^{-} h^{+} \nu_{\tau}$ analysis it was found that there are possible modelling biases associated with tracking or the EMC. A systematic uncertainty was assigned to account for possible modelling biasing of the MC signal associated with the cut variable. The systematic uncertainty is evaluated as the change in the branching fractions, caused by altering the cut value applied to the MC, to account for the deviation between the data and MC means for the cut variable. The cut variables which have a background modelling uncertainty assigned are:

1. The cut applied to the residual neutral energy of the 1 prong signal tracks. The difference between the data and MC means used to shift the background $\mathrm{MC}$ distributions for the residual neutral energy of the 1 prong cut are: $0.0039 \mathrm{GeV}$ for the $\tau^{-} \rightarrow e^{-} \nu_{\tau} \bar{\nu}_{e}$ decay channel; $-0.010 \mathrm{GeV}$ for the $\tau^{-} \rightarrow \mu^{-} \nu_{\tau} \bar{\nu}_{\mu}$ decay channel; $0.032 \mathrm{GeV}$ for the $\tau^{-} \rightarrow \pi^{-} \nu_{\tau}$ decay channel; and $0.011 \mathrm{GeV}$ for the $\tau^{-} \rightarrow K^{-} \nu_{\tau}$ decay channel.

2. The cut applied to the residual neutral energy of the 3 prong lepton tracks. The difference between the data and MC means used to shift the background $\mathrm{MC}$ distributions for the residual neutral energy of the 3 prong cut are: $-0.021 \mathrm{GeV}$.

3. The cut applied to remove contamination from $\tau$ decays containing $K_{S}^{0}$. The difference between the data and MC means used to shift the background MC distributions for the $K_{S}^{0}$ significance cut is 0.17 . 
Chapter 7. Measurements of $\frac{\mathcal{B}\left(\tau^{-} \rightarrow K^{-} \nu_{\tau}\right)}{\mathcal{B}\left(\tau^{-} \rightarrow \pi^{-} \nu_{\tau}\right)}, \frac{\mathcal{B}\left(\tau^{-} \rightarrow K^{-} \nu_{\tau}\right)}{\mathcal{B}\left(\tau^{-} \rightarrow e^{-} \nu_{\tau} \bar{\nu}_{e}\right)}, \frac{\mathcal{B}\left(\tau^{-} \rightarrow \pi^{-} \nu_{\tau}\right)}{\mathcal{B}\left(\tau^{-} \rightarrow e^{-} \nu_{\tau} \bar{\nu}_{e}\right)}$, and $\frac{\mathcal{B}\left(\tau^{-} \rightarrow \mu^{-} \nu_{\tau} \bar{\nu}_{\mu}\right)}{\mathcal{B}\left(\tau^{-}-e^{-} \nu_{\tau} \bar{\nu}\right)}$

\subsubsection{Trigger}

- In the $\tau^{-} \rightarrow h^{-} h^{-} h^{+} \nu_{\tau}$ analysis, the trigger efficiency was determined with a MC study where the trigger selection was removed, and the difference in the number of selected events was used to determine the trigger systematic under the assumption that the trigger is correctly modelled to $10 \%$. In this study, the trigger systematic for electron and muon tag tracks were similar. Because the selection characteristics of pions and kaons are between electrons and muons, a conservative upper limit of $0.1 \%$ is applied to the branching ratios with the assumption of a $50 \%$ correlation between the trigger systematic for the different 1 prong particles.

\subsection{Results}

The branching ratios measured in this analysis are shown in Table 7.2 with the world averages [12] for comparison. The $\frac{\mathcal{B}\left(\tau^{-} \rightarrow K^{-} \nu_{\tau}\right)}{\mathcal{B}\left(\tau^{-} \rightarrow e^{-} \nu_{\tau} \bar{\nu}_{e}\right)}$ and $\frac{\mathcal{B}\left(\tau^{-} \rightarrow K^{-} \nu_{\tau}\right)}{\mathcal{B}\left(\tau^{-} \rightarrow \pi^{-} \nu_{\tau}\right)}$ branching ratios are a significant improvement over the current world average. The $\frac{\mathcal{B}\left(\tau^{-} \rightarrow \mu^{-} \nu_{\tau} \bar{\nu}_{\mu}\right)}{\mathcal{B}\left(\tau^{-} \rightarrow e^{-} \nu_{\tau} \bar{\nu}_{e}\right)}$ is consistent and more precise than the current world average, while $\frac{\mathcal{B}\left(\tau^{-} \rightarrow \pi^{-} \nu_{\tau}\right)}{\mathcal{B}\left(\tau^{-} \rightarrow e^{-} \nu_{\tau} \bar{\nu}_{e}\right)}$ branching ratio is competitive and consistent with the world average. The c.m. momentum for the $\tau^{-} \rightarrow e^{-} \nu_{\tau} \bar{\nu}_{e}$ and $\tau^{-} \rightarrow \mu^{-} \nu_{\tau} \bar{\nu}_{\mu}$ decays are shown in Figure 7.1 while the $\tau^{-} \rightarrow \pi^{-} \nu_{\tau}$ and $\tau^{-} \rightarrow K^{-} \nu_{\tau}$ c.m. momentum are in Figure 7.2. A summary of the selected events in this analysis can be found in Table 7.3, while a more detailed discussion of the MC backgrounds can be found in Appendix I. The background modes which dominate the $\tau^{-} \rightarrow K^{-} \nu_{\tau}, \tau^{-} \rightarrow \pi^{-} \nu_{\tau}$ and $\tau^{-} \rightarrow \mu^{-} \nu_{\tau} \bar{\nu}_{\mu}$ channels are $\tau$-pair events. These events are selected as a result of particle mis-identification and/or neutral particles that were not measured.

A summary of the systematic uncertainties are shown in Table 7.4. The dominate 
Chapter 7. Measurements of $\frac{\mathcal{B}\left(\tau^{-} \rightarrow K^{-} \nu_{\tau}\right)}{\mathcal{B}\left(\tau^{-} \rightarrow \pi^{-} \nu_{\tau}\right)}, \frac{\mathcal{B}\left(\tau^{-} \rightarrow K^{-} \nu_{\tau}\right)}{\mathcal{B}\left(\tau^{-} \rightarrow e^{-} \nu_{\tau} \bar{\nu}_{e}\right)}, \frac{\mathcal{B}\left(\tau^{-} \rightarrow \pi^{-} \nu_{\tau}\right)}{\mathcal{B}\left(\tau^{-} \rightarrow e^{-} \nu_{\tau} \bar{\nu}_{e}\right)}$, and $\mathcal{B}\left(\tau^{-} \rightarrow \mu^{-} \nu_{\tau} \bar{\nu}_{\mu}\right)$

\begin{tabular}{|l|c|c|}
\hline & $\frac{\mathcal{B}\left(\tau^{-} \rightarrow K^{-} \nu_{\tau}\right)}{\mathcal{B}\left(\tau^{-} \rightarrow e^{-} \nu_{\tau} \bar{\nu}_{e}\right)}$ & $\frac{\mathcal{B}\left(\tau^{-} \rightarrow \pi^{-} \nu_{\tau}\right)}{\mathcal{B}\left(\tau^{-} \rightarrow e^{-} \nu_{\tau} \bar{\nu}_{e}\right)}$ \\
\hline $\mathcal{B}$ (this work) & $(3.882 \pm 0.032 \pm 0.057) \times 10^{-2}$ & $(5.945 \pm 0.014 \pm 0.061) \times 10^{-1}$ \\
$\mathcal{B}$ (PDG fit) & $(3.84 \pm 0.13) \times 10^{-2}$ & $(6.076 \pm 0.061) \times 10^{-1}$ \\
\hline
\end{tabular}

\begin{tabular}{|l|c|c|}
\hline & $\frac{\mathcal{B}\left(\tau^{-} \rightarrow \mu^{-} \nu_{\tau} \bar{\nu}_{\mu}\right)}{\mathcal{B}\left(\tau^{-} \rightarrow e^{-} \nu_{\tau} \bar{\nu}_{e}\right)}$ & $\frac{\mathcal{B}\left(\tau^{-} \rightarrow K^{-} \nu_{\tau}\right)}{\mathcal{B}\left(\tau^{-} \rightarrow \pi^{-} \nu_{\tau}\right)}$ \\
\hline $\mathcal{B}$ (this work) & $(9.796 \pm 0.016 \pm 0.035) \times 10^{-1}$ & $(6.531 \pm 0.056 \pm 0.093) \times 10^{-2}$ \\
$\mathcal{B}$ (PDG fit) & $(9.725 \pm 0.039) \times 10^{-1}$ & $(6.33 \pm 0.21) \times 10^{-2}$ \\
\hline
\end{tabular}

Table 7.2: The 1 prong branching ratios measured in this analysis and the corresponding world averages for the branching ratios [12].

systematic uncertainties originate from particle identification and backgrounds. In the $\tau^{-} \rightarrow K^{-} \nu_{\tau}$ channel, the most significant background uncertainties originated from the uncertainty on the $\tau^{-} \rightarrow K^{-} K_{L}^{0} \nu_{\tau}$ branching fraction, and the statistics of the sideband region used to scale the non- $\tau$ backgrounds. For the $\tau^{-} \rightarrow \pi^{-} \nu_{\tau}$ channel, the most significant uncertainty related to the background comes from the $\tau^{-} \rightarrow$ $e^{-} \nu_{\tau} \bar{\nu}_{e}$ background correction factor determined from the $E / p$ sideband region. For the $\tau^{-} \rightarrow e^{-} \nu_{\tau} \bar{\nu}_{e}$ channel, the uncertainty associated with the Bhabha contamination is the largest background uncertainty, while in the $\tau^{-} \rightarrow \mu^{-} \nu_{\tau} \bar{\nu}_{\mu}$ channel the limited statistics in the non- $\tau$ is the largest background uncertainty. In the $\frac{\mathcal{B}\left(\tau^{-} \rightarrow \pi^{-} \nu_{\tau}\right)}{\mathcal{B}\left(\tau^{-} \rightarrow e^{-} \nu_{\tau} \bar{\nu}_{e}\right)}$ and the $\frac{\mathcal{B}\left(\tau^{-} \rightarrow K^{-} \nu_{\tau}\right)}{\mathcal{B}\left(\tau^{-} \rightarrow e^{-} \nu_{\tau} \bar{\nu}_{e}\right)}$ branching ratios, the modelling of the neutrals is the most significant contribution to the EMC and DCH response uncertainty. 
Chapter 7. Measurements of $\frac{\mathcal{B}\left(\tau^{-} \rightarrow K^{-} \nu_{\tau}\right)}{\mathcal{B}\left(\tau^{-} \rightarrow \pi^{-} \nu_{\tau}\right)}, \frac{\mathcal{B}\left(\tau^{-} \rightarrow K^{-} \nu_{\tau}\right)}{\mathcal{B}\left(\tau^{-} \rightarrow e^{-} \nu_{\tau} \bar{\nu}_{e}\right)}, \frac{\mathcal{B}\left(\tau^{-} \rightarrow \pi^{-} \nu_{\tau}\right)}{\mathcal{B}\left(\tau^{-} \rightarrow e^{-} \nu_{\tau} \bar{\nu}_{e}\right)}$, and $\frac{\mathcal{B}\left(\tau^{-} \rightarrow \mu^{-} \nu_{\tau} \bar{\nu}_{\mu}\right)}{\mathcal{B}\left(\tau^{-} \rightarrow e^{-} \nu_{\tau} \bar{\nu}_{e}\right)}$
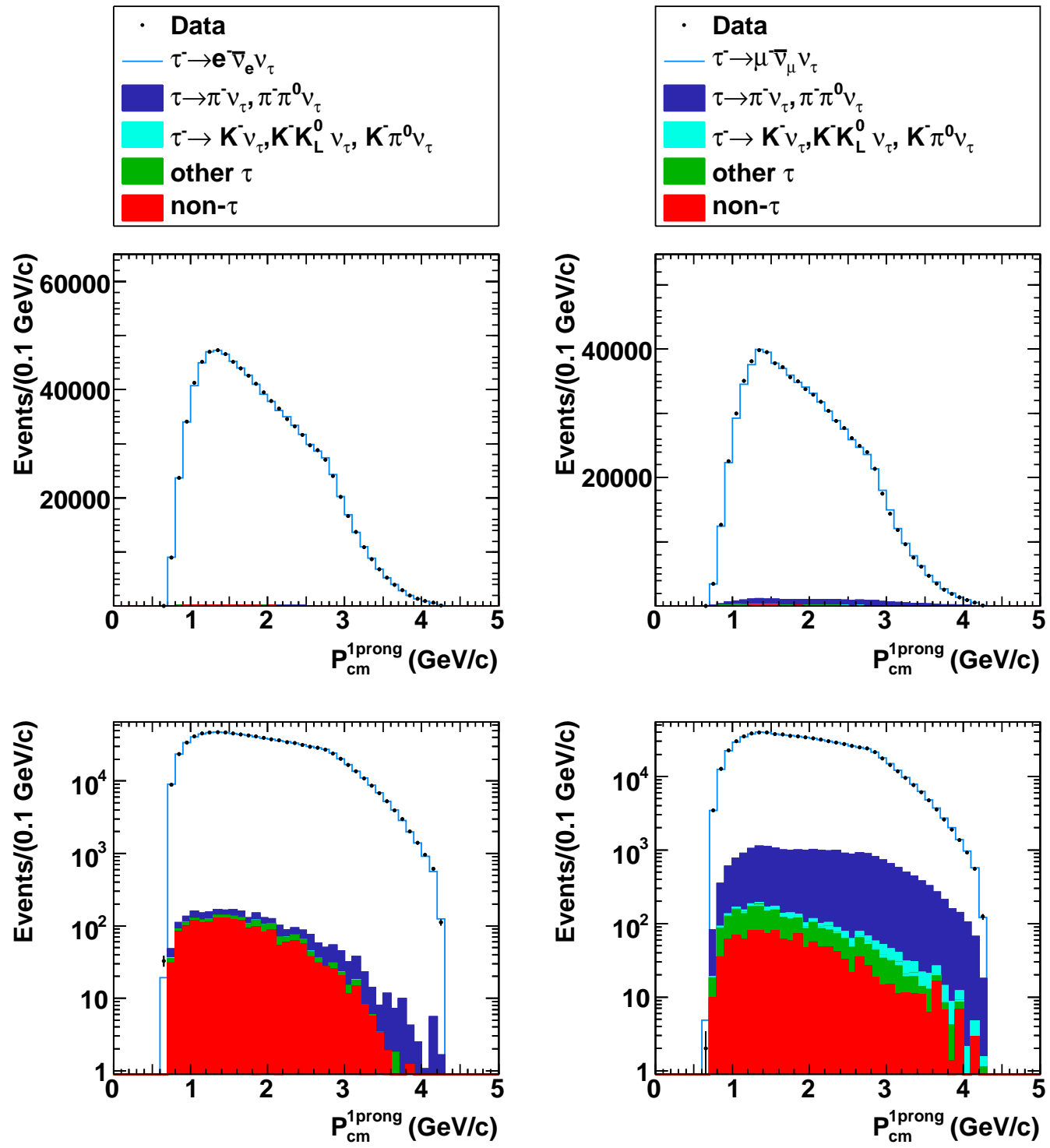

Figure 7.1: The $\tau^{-} \rightarrow e^{-} \nu_{\tau} \bar{\nu}_{e}$ (left) and $\tau^{-} \rightarrow \mu^{-} \nu_{\tau} \bar{\nu}_{\mu}$ (right) c.m. momentum shown both linear (top) and logarithmic scale(bottom). The legends for the $\tau^{-} \rightarrow e^{-} \nu_{\tau} \bar{\nu}_{e}$ and $\tau^{-} \rightarrow \mu^{-} \nu_{\tau} \bar{\nu}_{\mu}$ channels are shown above the respective plots. 
Chapter 7. Measurements of $\frac{\mathcal{B}\left(\tau^{-} \rightarrow K^{-} \nu_{\tau}\right)}{\mathcal{B}\left(\tau^{-} \rightarrow \pi^{-} \nu_{\tau}\right)}, \frac{\mathcal{B}\left(\tau^{-} \rightarrow K^{-} \nu_{\tau}\right)}{\mathcal{B}\left(\tau^{-} \rightarrow e^{-} \nu_{\tau} \bar{\nu}_{e}\right)}, \frac{\mathcal{B}\left(\tau^{-} \rightarrow \pi^{-} \nu_{\tau}\right)}{\mathcal{B}\left(\tau^{-} \rightarrow e^{-} \nu_{\tau} \bar{\nu}_{e}\right)}$, and
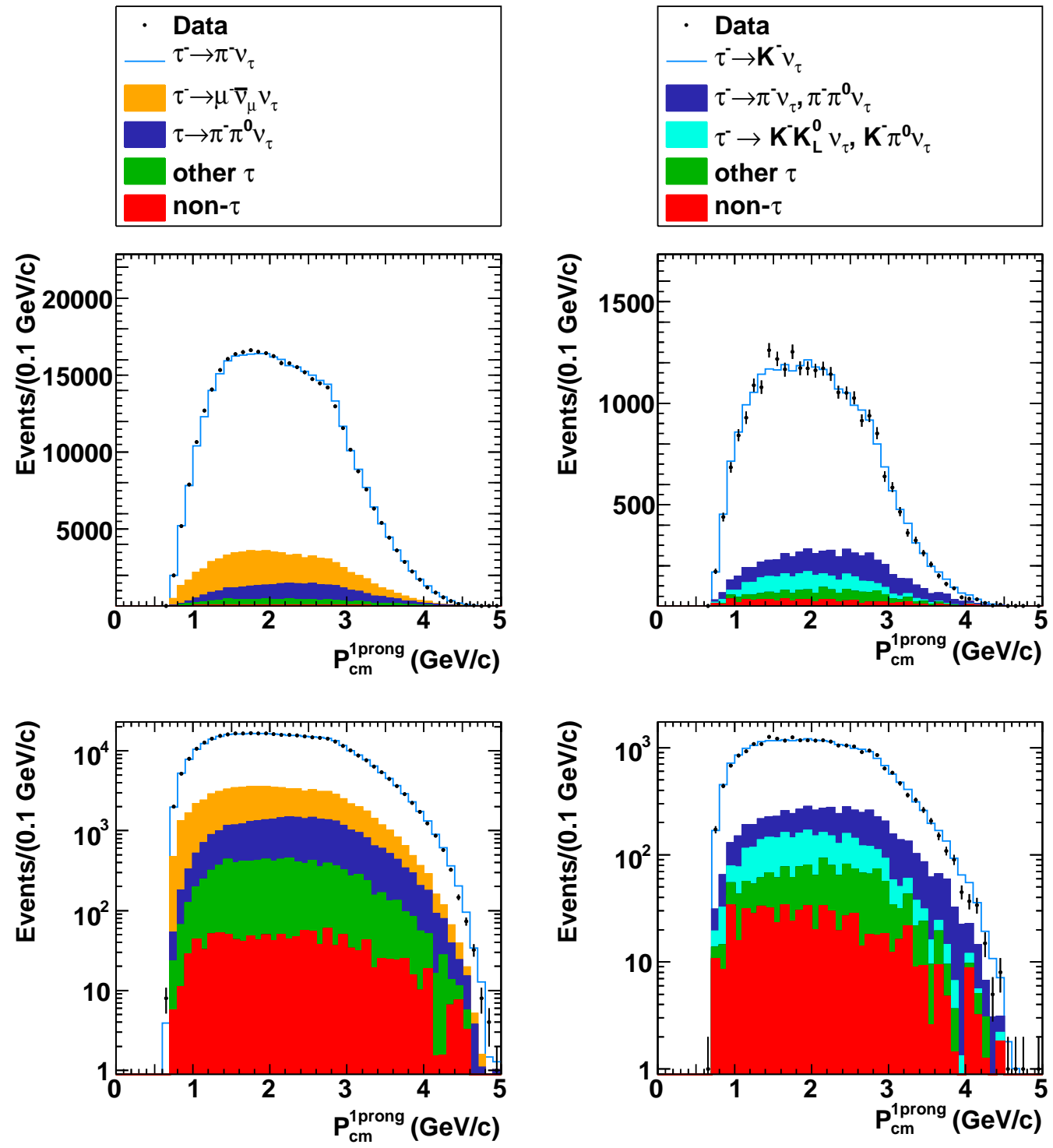

Figure 7.2: The $\tau^{-} \rightarrow \pi^{-} \nu_{\tau}$ (left) and $\tau^{-} \rightarrow K^{-} \nu_{\tau}$ (right) c.m. momentum shown both linear (top) and logarithmic scale(bottom). The legends for the $\tau^{-} \rightarrow \pi^{-} \nu_{\tau}$ and $\tau^{-} \rightarrow K^{-} \nu_{\tau}$ channels are shown above the respective plots. 
Chapter 7. Measurements of $\frac{\mathcal{B}\left(\tau^{-} \rightarrow K^{-} \nu_{\tau}\right)}{\mathcal{B}\left(\tau^{-} \rightarrow \pi^{-} \nu_{\tau}\right)}, \frac{\mathcal{B}\left(\tau^{-} \rightarrow K^{-} \nu_{\tau}\right)}{\mathcal{B}\left(\tau^{-} \rightarrow e^{-} \nu_{\tau} \bar{\nu}_{e}\right)}, \frac{\mathcal{B}\left(\tau^{-} \rightarrow \pi^{-} \nu_{\tau}\right)}{\mathcal{B}\left(\tau^{-} \rightarrow e^{-} \nu_{\tau} \bar{\nu}_{e}\right)}$, and

\begin{tabular}{|l|c|c|}
\hline Channel & $\tau^{-} \rightarrow K^{-} \nu_{\tau}$ & $\tau^{-} \rightarrow \pi^{-} \nu_{\tau}$ \\
\hline$\epsilon$ & 0.003297 & 0.003240 \\
Purity & 0.7662 & 0.7871 \\
$N^{\text {Data }}$ & $(2.512 \pm 0.016) \times 10^{4}$ & $(3.691 \pm 0.006) \times 10^{5}$ \\
$N^{\text {Bkg }}$ & $5963 \pm 56$ & $80700 \pm 304$ \\
$N^{\text {Sig }}$ & $(1.916 \pm 0.016) \times 10^{4}$ & $(2.884 \pm 0.006) \times 10^{5}$ \\
\hline
\end{tabular}

\begin{tabular}{|l|c|c|}
\hline Channel & $\tau^{-} \rightarrow e^{-} \nu_{\tau} \bar{\nu}_{e}$ & $\tau^{-} \rightarrow \mu^{-} \nu_{\tau} \bar{\nu}_{\mu}$ \\
\hline$\epsilon$ & 0.005892 & 0.004925 \\
Purity & 0.9969 & 0.9732 \\
$N^{\text {Data }}$ & $(8.844 \pm 0.009) \times 10^{5}$ & $(7.311 \pm 0.009) \times 10^{5}$ \\
$N^{\text {Bkg }}$ & $2772 \pm 43$ & $20138 \pm 84$ \\
$N^{\text {Sig }}$ & $(8.817 \pm 0.009) \times 10^{5}$ & $(7.110 \pm 0.009) \times 10^{5}$ \\
\hline
\end{tabular}

Table 7.3: Summary of selected events for the $\frac{\mathcal{B}\left(\tau^{-} \rightarrow K^{-} \nu_{\tau}\right)}{\mathcal{B}\left(\tau^{-} \rightarrow e^{-} \nu_{\tau} \bar{\nu}_{e}\right)}, \frac{\mathcal{B}\left(\tau^{-} \rightarrow \pi^{-} \nu_{\tau}\right)}{\mathcal{B}\left(\tau^{-} \rightarrow e^{-} \nu_{\tau} \bar{\nu}_{e}\right)}$, $\frac{\mathcal{B}\left(\tau^{-} \rightarrow \mu^{-} \nu_{\tau} \bar{\nu}_{\mu}\right)}{\mathcal{B}\left(\tau^{-} \rightarrow e^{-} \nu_{\tau} \bar{\nu}_{e}\right)}$, and $\frac{\mathcal{B}\left(\tau^{-} \rightarrow K^{-} \nu_{\tau}\right)}{\mathcal{B}\left(\tau^{-} \rightarrow \pi^{-} \nu_{\tau}\right)}$ analysis.

\begin{tabular}{|l|c|c|c|c|}
\hline & $\frac{\mathcal{B}\left(\tau^{-} \rightarrow K^{-} \nu_{\tau}\right)}{\mathcal{B}\left(\tau^{-} \rightarrow e^{-} \nu_{\tau}\right)}$ & $\frac{\mathcal{B}\left(\tau^{-} \rightarrow \pi^{-} \nu_{\tau}\right)}{\mathcal{B}\left(\tau^{-} \rightarrow e^{-} \nu_{\tau}\right)}$ & $\frac{\mathcal{B}\left(\tau^{-} \rightarrow \mu^{-} \nu_{\tau} \bar{\nu}_{\mu}\right)}{\mathcal{B}\left(\tau^{-} \rightarrow e^{-} \nu_{\tau} \bar{\nu}_{e}\right)}$ & $\frac{\mathcal{B}\left(\tau^{-} \rightarrow K^{-} \nu_{\tau}\right)}{\mathcal{B}\left(\tau^{-} \rightarrow \pi^{-} \nu_{\tau}\right)}$ \\
\hline \multicolumn{5}{|c|}{ Statistical Uncertainties } \\
\hline data statistics & 0.84 & 0.24 & 0.16 & 0.85 \\
\hline \multicolumn{5}{|c|}{ Systematic Uncertainties } \\
\hline normalization & 0.45 & 0.44 & 0.05 & 0.06 \\
$\epsilon$ and particle ID & 0.94 & 0.51 & 0.32 & 0.99 \\
$\tau^{-} \rightarrow \pi^{-} \pi^{-} \pi^{+} \nu_{\tau}$ modelling & 0.27 & 0.07 & 0.01 & 0.29 \\
EMC and DCH response & 0.54 & 0.64 & 0.08 & 0.25 \\
trigger & 0.10 & 0.10 & 0.10 & 0.10 \\
backgrounds & 0.85 & 0.44 & 0.08 & 0.95 \\
radiation modelling & 0.04 & 0.10 & 0.04 & 0.10 \\
\hline Total & 1.5 & 1.0 & 0.36 & 1.4 \\
\hline
\end{tabular}

Table 7.4: A summary of the uncertainties for the $\frac{\mathcal{B}\left(\tau^{-} \rightarrow K^{-} \nu_{\tau}\right)}{\mathcal{B}\left(\tau^{-} \rightarrow \pi^{-} \nu_{\tau}\right)}, \frac{\mathcal{B}\left(\tau^{-} \rightarrow K^{-} \nu_{\tau}\right)}{\mathcal{B}\left(\tau^{-} \rightarrow e^{-} \nu_{\tau} \bar{\nu}_{e}\right)}$, $\frac{\mathcal{B}\left(\tau^{-} \rightarrow \pi^{-} \nu_{\tau}\right)}{\mathcal{B}\left(\tau^{-} \rightarrow e^{-} \nu_{\tau} \bar{\nu}_{e}\right)}$, and $\frac{\mathcal{B}\left(\tau^{-} \rightarrow \mu^{-} \nu_{\tau} \bar{\nu}_{\mu}\right)}{\mathcal{B}\left(\tau^{-} \rightarrow e^{-} \nu_{\tau} \bar{\nu}_{e}\right)}$ analysis expressed in percentages. The individual systematic uncertainties are added in quadrature to obtain the total systematic uncertainty. 


\section{Chapter 8}

\section{Discussion}

In this section, the implications of the experimental results presented in this work on the Standard Model of Particle Physics are discussed.

\subsection{Results of $\tau^{-} \rightarrow h^{-} h^{-} h^{+} \nu_{\tau}$ Analysis}

The $\tau^{-} \rightarrow h^{-} h^{-} h^{+} \nu_{\tau}$ branching fractions, compared to the previous measurements which are included in the world average [39] of these decay modes, can be seen in Figure 8.1. The $\mathcal{B}\left(\tau^{-} \rightarrow \pi^{-} \pi^{-} \pi^{+} \nu_{\tau}\right)$ measurement from this work is also consistent with a precision measurement of $\mathcal{B}\left(\tau^{-} \rightarrow \pi^{-} \pi^{-} \pi^{+} \nu_{\tau}(\right.$ ex. $\left.\omega)\right)$ [41] when the $\omega$ contribution is taken into account. The $\mathcal{B}\left(\tau^{-} \rightarrow K^{-} \pi^{-} \pi^{+} \nu_{\tau}\right)$ measurement disagrees with $[51,116]$ by more than 2 standard deviations, but is in agreement with [117]. The $\mathcal{B}\left(\tau^{-} \rightarrow K^{-} K^{-} K^{+} \nu_{\tau}\right)$ measurement is the first measurement of the $\tau^{-} \rightarrow K^{-} K^{-} K^{+} \nu_{\tau}$ decay in which no resonance structure is assumed with a significance of $8.2 \sigma$. The $\mathcal{B}\left(\tau^{-} \rightarrow h^{-} h^{-} h^{+} \nu_{\tau}\right)=(9.24 \pm 0.14) \%$, determined by combining

the four $\tau^{-} \rightarrow h^{-} h^{-} h^{+} \nu_{\tau}$ branching fractions, is consistent with the world average $(9.44 \pm 0.14) \%[39]$

With these new branching fractions, and the recent $\mathcal{B}\left(\tau^{-} \rightarrow \pi^{-} K_{S}^{0} \nu_{\tau}\right)$ from BABAR [8,9] and Belle [10], the strange spectral density function can be updated 

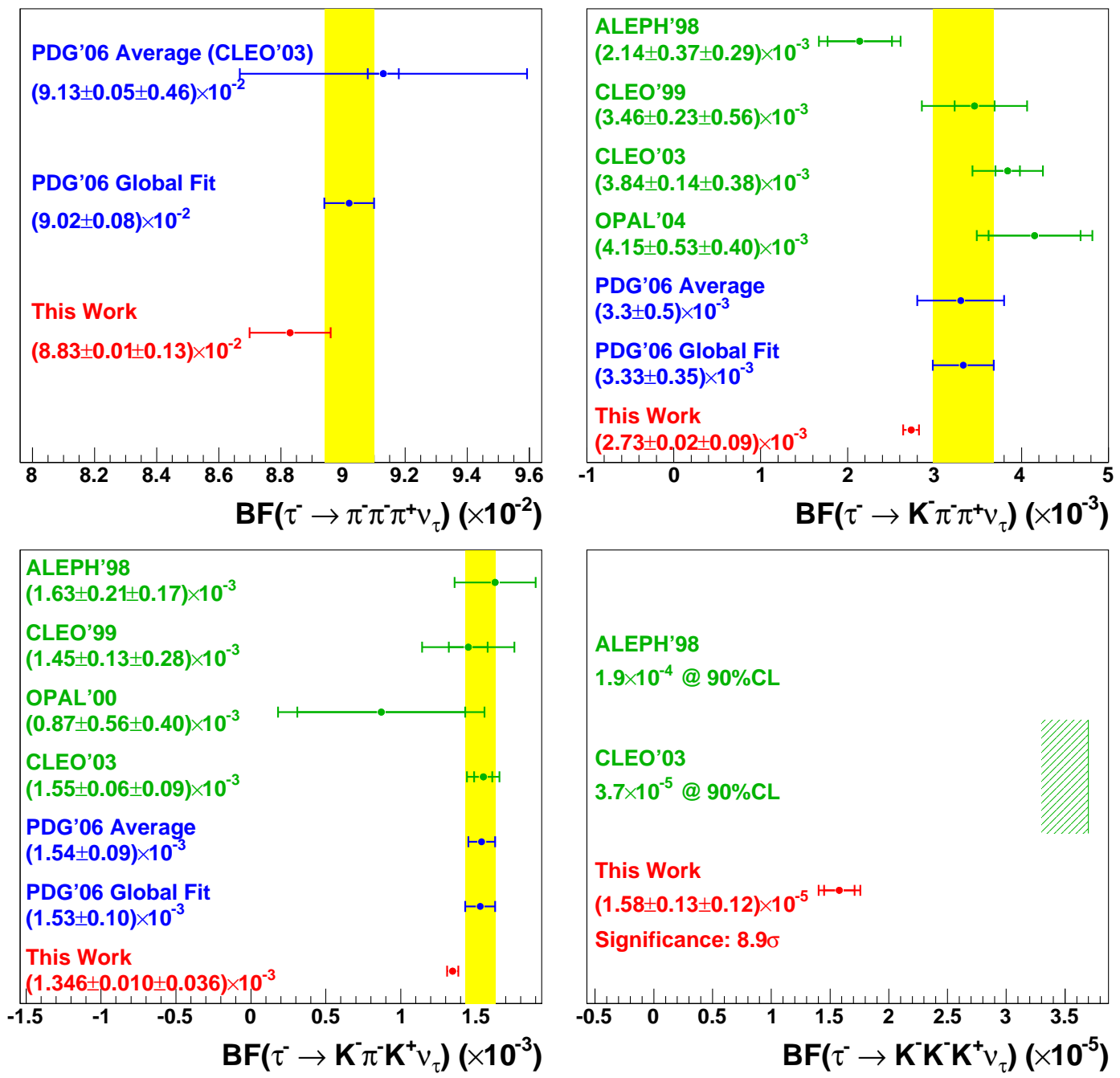

Figure 8.1: A comparison of $\tau^{-} \rightarrow \pi^{-} \pi^{-} \pi^{+} \nu_{\tau}$ (top-left), $\tau^{-} \rightarrow K^{-} \pi^{-} \pi^{+} \nu_{\tau}$ (top-right), $\tau^{-} \rightarrow K^{-} \pi^{-} K^{+} \nu_{\tau}$ (bottom-left), and $\tau^{-} \rightarrow K^{-} K^{-} K^{+} \nu_{\tau}$ (bottomright) branching fractions measured in this thesis to the the world average and global fit from the PDG [39]. The points represent the measured values, while the inner error bars represent the statistical uncertainty and the outer error bars represent the total uncertainty.

yielding a measurement of $\left|V_{u s}\right|$. Figure 8.2 includes the updated values of $\left|V_{u s}\right|$ from [124]. Although the $R^{(0,0)}$ spectral moments are known to have theoretical issues with convergence, the other weights do not exhibit this problem to the same extent. The deviation of $\left|V_{u s}\right|$ extracted with $\tau$ decays using FESR and the $R^{(0,0)}$ moment from 
the unitarity value raises the possibility of new physics involving the 3rd generation of charged leptons. This makes a complete measurement of the strange spectral density function from hadronic $\tau$ decays, independent from previous measurements very important. Furthermore, it strongly motivated the use of $\tau^{-} \rightarrow K^{-} \nu_{\tau}$ as a powerful precision probe of $\left|V_{u s}\right|$ involving the $\tau$ lepton, but with both theoretical and experimental errors that are independent of $\left|V_{u s}\right|$ obtained from the FESR and the $R^{(0,0)}$ moment.

\subsection{Results from the $\tau^{-} \rightarrow \phi \pi^{-} \nu_{\tau}$ and $\tau^{-} \rightarrow \phi K^{-} \nu_{\tau}$ Fit}

The $\mathcal{B}\left(\tau^{-} \rightarrow \phi \pi^{-} \nu_{\tau}\right)=(3.42 \pm 0.55 \pm 0.25) \times 10^{-5}$ measurement is the first measurement of this Okubo-Zweig-Iizuka (OZI) suppressed [118] decay mode and is consistent with the upper limit of $\mathcal{B}\left(\tau^{-} \rightarrow \phi \pi^{-} \nu_{\tau}\right)<(1.2-2.0) \times 10^{-4}$ from CLEO [128]. This measurement deviates from the value predicted by a vector dominance model, $\mathcal{B}\left(\tau^{-} \rightarrow \phi \pi^{-} \nu_{\tau}\right)<(1.20 \pm 0.28) \times 10^{-5}$ at $90 \%$ C.L. [118], by 3.3 standard deviations. The $\mathcal{B}\left(\tau^{-} \rightarrow \phi \pi^{-} \nu_{\tau}\right)$, when compared to the $\mathcal{B}\left(\tau^{-} \rightarrow \omega \pi^{-} \nu_{\tau}\right)$, can provide important information about $\phi-\omega$ mixing [118].

The measurement of $\mathcal{B}\left(\tau^{-} \rightarrow \phi K^{-} \nu_{\tau}\right)=(3.39 \pm 0.20 \pm 0.28) \times 10^{-5}$ is consistent with a recent Belle result of $\mathcal{B}\left(\tau^{-} \rightarrow \phi K^{-} \nu_{\tau}\right)=(4.05 \pm 0.25 \pm 0.26) \times 10^{-5}$ [129], and is similar to the $\sim 2.1 \times 10^{-5}$ expected from a naive prediction [128]. Because there was no evidence of $\tau^{-} \rightarrow K^{-} K^{-} K^{+} \nu_{\tau}$ excluding $\phi$ in the fit, an upper limit of $\mathcal{B}\left(\tau^{-} \rightarrow K^{-} K^{-} K^{+} \nu_{\tau}(\right.$ ex. $\left.\phi)\right)<2.5 \times 10^{-6}$ at $90 \%$ C.L. was set. The $\mathcal{B}\left(\tau^{-} \rightarrow\right.$ $\left.\phi \pi^{-} \nu_{\tau}\right)$ and $\mathcal{B}\left(\tau^{-} \rightarrow \phi K^{-} \nu_{\tau}\right)$ have been reproduced theoretically using the $\mathrm{SU}(3)$ flavour symmetry, the ideal $\omega-\phi$ mixing. The "post-dicted" values are $\mathcal{B}\left(\tau^{-} \rightarrow\right.$

$\left.\phi \pi^{-} \nu_{\tau}\right)=(3.64 \pm 0.93) \times 10^{-5}$, and $\mathcal{B}\left(\tau^{-} \rightarrow \phi K^{-} \nu_{\tau}\right)=(4.0 \pm 1.2) \times 10^{-5}[130]$. 


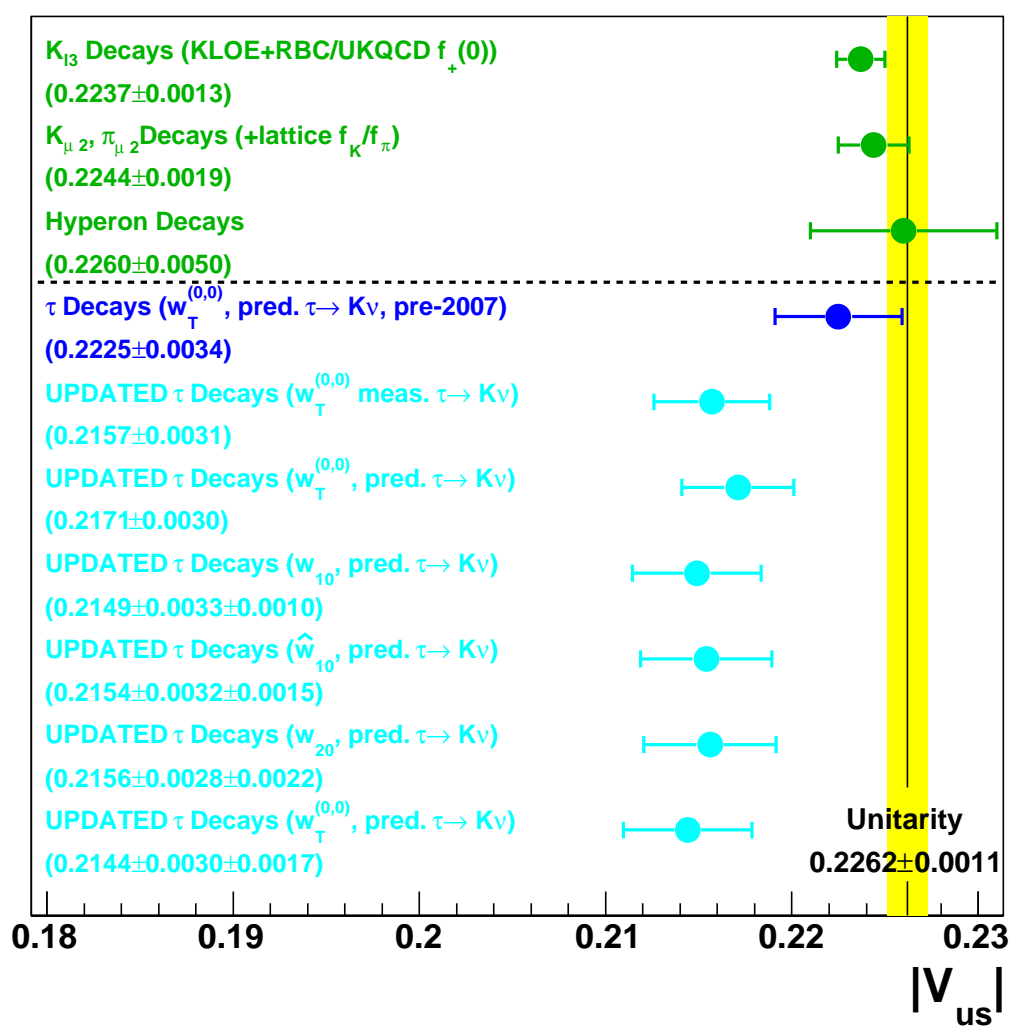

Figure 8.2: The current status of the $\left|V_{u s}\right|$ compared to the unitarity value [39]. The recent measurements from kaon decays, $K_{l 3}$ [125] and $K_{l 2}$ [40], and the hyperon decay data [126] are located above the dashed line, while the values of $\left|V_{u s}\right|$ from hadronic $\tau$ decays are below the dashed line. The upper most value of $\left|V_{u s}\right|$ from $\tau$ decays is extracted with the $R^{0,0}$ moment [55] and does not include the recent hadronic measurements from this work or BABAR [9] and Belle [10]. The next two values of $\left|V_{u s}\right|$ were extracted in [127] using the PDG [39] value of $\tau^{-} \rightarrow K^{-} \nu_{\tau}$ and the predicted value. The remaining values of $\left|V_{u s}\right|$ were measured by [124] and includes a branching ratio re-scaling of the $u s+u d$ ALEPH99 spectral density functions [41].

The measured ratio for this analysis of $\frac{\mathcal{B}\left(\tau^{-} \rightarrow \phi K^{-} \nu_{\tau}\right)}{\mathcal{B}\left(\tau^{-} \rightarrow \phi \pi^{-} \nu_{\tau}\right)}$ is $0.99 \pm 0.21$ which is consistent with a vector meson dominance model value $\frac{\mathcal{B}\left(\tau^{-} \rightarrow \phi K^{-} \nu_{\tau}\right)}{\mathcal{B}\left(\tau^{-} \rightarrow \phi \pi^{-} \nu_{\tau}\right)} \sim 1.08[131$, and references within]. Assuming CVC $[13-15,17]$, the $\mathcal{B}\left(\tau^{-} \rightarrow \phi \pi^{-} \nu_{\tau}\right)$ branching fraction can be predicted from $\mathcal{B}\left(e^{+} e^{-} \rightarrow \phi \pi^{0}\right)[132]$. This $e^{+} e^{-}$measurement predicts $\mathcal{B}_{C V C}\left(\tau^{-} \rightarrow\right.$ $\left.\phi \pi^{-} \nu_{\tau}\right)=(3.8 \pm 0.9 \pm 0.2) \times 10^{-5}[132,133]$, which is consistent with the measurement presented in this work. 


\subsection{Results from the Unfolding}

The $\tau^{-} \rightarrow \pi^{-} \pi^{-} \pi^{+} \nu_{\tau} M_{123}$ invariant mass is dominated by the $\mathrm{a}_{1}(1260)$, while the $M_{13 a n d 23}$ invariant mass spectra show a strong $\rho(770)$ resonance. The resonance structure in the $\tau^{-} \rightarrow K^{-} \pi^{-} \pi^{+} \nu_{\tau} M_{13}$ invariant mass appears to be dominated by the $K^{*}(892)$ resonance while the $M_{13}$ invariant mass is dominated by the $\rho(770)$ resonance. The $\tau^{-} \rightarrow K^{-} \pi^{-} \pi^{+} \nu_{\tau} M_{123}$ invariant mass spectra contains both a strong peak from the $K_{1}(1270)$ resonance and a peak from the $K_{1}(1400)$ resonance. From the unfolded $\tau^{-} \rightarrow K^{-} \pi^{-} K^{+} \nu_{\tau} M_{23}$ invariant mass distribution, it can be seen that $\tau^{-} \rightarrow$ $K^{-} K^{*}(892) \nu_{\tau}$ is the primary decay mode. The assumption of a Conserved Vector Current can be tested by comparing the vector component of the $\tau^{-} \rightarrow K^{-} K^{*}(892) \nu_{\tau}$ branching ratio measured from the $\tau^{-} \rightarrow K^{-} \pi^{-} K^{+} \nu_{\tau}$ channel to the corresponding $e^{+} e^{-}$decay mode, $e^{+} e^{-} \rightarrow K^{-} K^{*}(892)$. In order to make this comparison, the axialvector and vector components of the $\tau^{-} \rightarrow K^{-} \pi^{-} K^{+} \nu_{\tau}$ branching fraction must be separated. This is beyond the scope of this work.

\subsection{Results from the $\frac{\mathcal{B}\left(\tau^{-} \rightarrow \mu^{-} \nu_{\tau} \bar{\nu}_{\mu}\right)}{\mathcal{B}\left(\tau^{-} \rightarrow e^{-} \nu_{\tau} \bar{\nu}_{e}\right)}$ Measurement and e- $\mu$ Universality}

The $\frac{\mathcal{B}\left(\tau^{-} \rightarrow \mu^{-} \nu_{\tau} \bar{\nu}_{\mu}\right)}{\mathcal{B}\left(\tau^{-} \rightarrow e^{-} \nu_{\tau} \bar{\nu}_{e}\right)}$ branching ratio was measured to be $(9.796 \pm 0.016 \pm 0.035) \times$ $10^{-1}$. This measurement has a slightly smaller uncertainty than the world average of $(9.725 \pm 0.039) \times 10^{-1}[12]$ which combines measurements of $\tau^{-} \rightarrow e^{-} \nu_{\tau} \bar{\nu}_{e}$ and measurements $\tau^{-} \rightarrow \mu^{-} \nu_{\tau} \bar{\nu}_{\mu}$ from five different experiments. The branching fraction $\mathcal{B}\left(\tau^{-} \rightarrow \mu^{-} \nu_{\tau} \bar{\nu}_{\mu}\right)$ can be determined from the branching ratio using the world average value of $\mathcal{B}\left(\tau^{-} \rightarrow e^{-} \nu_{\tau} \bar{\nu}_{e}\right)=(17.82 \pm 0.05) \times 10^{-2}$ [12]. This results in a branching fraction of $\mathcal{B}\left(\tau^{-} \rightarrow \mu^{-} \nu_{\tau} \bar{\nu}_{\mu}\right)=(17.46 \pm 0.03 \pm 0.08) \times 10^{-2}$. A comparison of the $\mathcal{B}\left(\tau^{-} \rightarrow \mu^{-} \nu_{\tau} \bar{\nu}_{\mu}\right)$ branching fraction to the previous measurements which are included 
in the world average [12] can be seen in Figure 8.3. The $\mathcal{B}\left(\tau^{-} \rightarrow \mu^{-} \nu_{\tau} \bar{\nu}_{\mu}\right)$ branching fraction is both consistent with and competitive with the previous measurements which are used in the world averages.
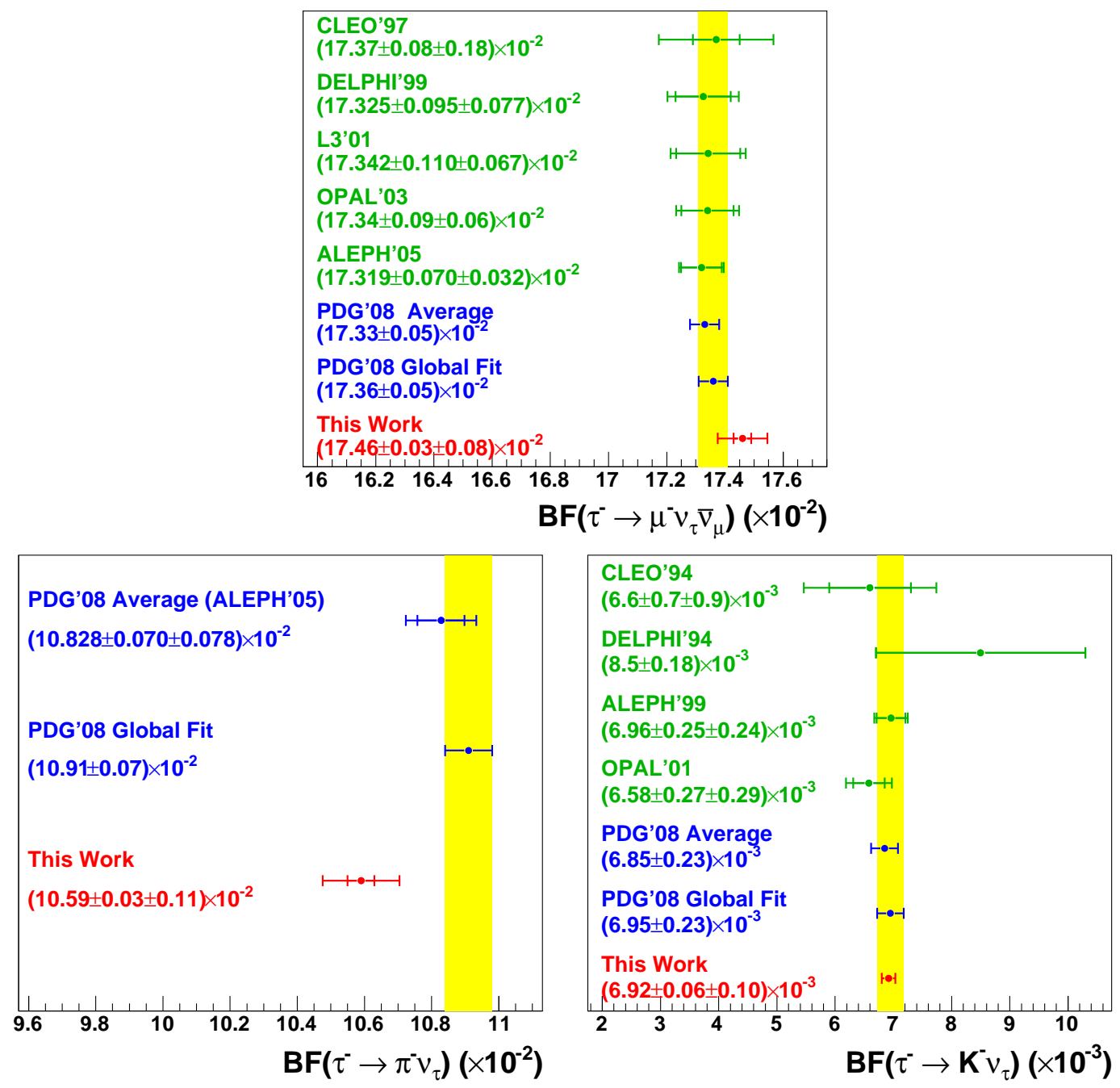

Figure 8.3: A comparison of $\tau^{-} \rightarrow \mu^{-} \nu_{\tau} \bar{\nu}_{\mu}$ (top), $\tau^{-} \rightarrow \pi^{-} \nu_{\tau}$ (bottom-left), and $\tau^{-} \rightarrow K^{-} \nu_{\tau}$ (bottom-right) branching fractions measured in this thesis to the previous measurements and the world average from the PDG [12] where the world average of $\tau^{-} \rightarrow \pi^{-} \nu_{\tau}$ comes from the ALEPH measurement [41]. The points represent the measured values, while the inner error bars represent the statistical uncertainty and the outer error bars represent the total uncertainty.

The dominant uncertainties in the $\frac{\mathcal{B}\left(\tau^{-} \rightarrow \mu^{-} \nu_{\tau} \bar{\nu}_{\mu}\right)}{\mathcal{B}\left(\tau^{-} \rightarrow e^{-} \nu_{\tau} \bar{\nu}_{e}\right)}$ branching ratio measurement 
originate from particle identification uncertainty, the data statistics and the limited signal MC statistics. Since the particle identification uncertainty is limited by the statistics of the control samples, the uncertainty for this analysis is almost entirely statistical. This means that proposed new experiments, which are expected to have substantially larger data sets, namely Super B [134] and Super KEK B [135], can expect to reduce the systematic associated with the statistical limitation of the control samples uncertainty on this measurement by approximately a factor of 10 .

The $\frac{\mathcal{B}\left(\tau^{-} \rightarrow \mu^{-} \nu_{\tau} \bar{\nu}_{\mu}\right)}{\mathcal{B}\left(\tau^{-} \rightarrow e^{-} \nu_{\tau} \bar{\nu}_{e}\right)}$ branching ratio may be used to make a precision test of $\mu$-e lepton universality as shown in Equation 8.1.

$$
\left(\frac{g_{\mu}}{g_{e}}\right)_{\tau}=\sqrt{\frac{\mathcal{B}\left(\tau^{-} \rightarrow \mu^{-} \nu_{\tau} \bar{\nu}_{\mu}\right) f\left(m_{e}^{2} / m_{\tau}^{2}\right)}{\mathcal{B}\left(\tau^{-} \rightarrow e^{-} \nu_{\tau} \bar{\nu}_{e}\right) f\left(m_{e}^{2} / m_{\tau}^{2}\right)}}
$$

where $f(x)=1-8 x+8 x^{3}-x^{4}-12 x^{2} \log x$, under the assumption that the neutrino masses are negligible [20]. Applying Equation 8.1 to the branching ratio measured in this analysis yields

$$
\left(\frac{g_{\mu}}{g_{e}}\right)_{\tau}=1.0036 \pm 0.0020
$$

a value which is consistent with the Standard Model's assumption of lepton universality. Combining this with the world average from $\tau$ decay, $\left(\frac{g_{\mu}}{g_{e}}\right)_{\tau}=1.0000 \pm 0.0020$ [12], one obtains $\left(\frac{g_{\mu}}{g_{e}}\right)_{\tau}=1.0018 \pm 0.0014$. This is consistent with: $\left(\frac{g_{\mu}}{g_{e}}\right)_{\pi}=$ $1.0023 \pm 0.0016$ [136-138] determined from $\pi^{-} \rightarrow \mu^{-} \bar{\nu}_{\mu}$ and $\pi^{-} \rightarrow e^{-} \bar{\nu}_{e}$ decays; $\left(\frac{g_{\mu}}{g_{e}}\right)_{K_{l 2}}=1.004 \pm 0.007[39,139-141]$ determined from $K^{-} \rightarrow \mu^{-} \bar{\nu}_{\mu}$ and $K^{-} \rightarrow e^{-} \bar{\nu}_{e}$ decays; $\left(\frac{g_{\mu}}{g_{e}}\right)_{K_{l 3}}=1.0021 \pm 0.0025[142,143]$ determined from $K \rightarrow \pi \mu \bar{\nu}_{\mu}$ and $K \rightarrow \pi e \bar{\nu}_{e}$ decays; and $\left(\frac{g_{\mu}}{g_{e}}\right)_{W}=0.997 \pm 0.010$ [144] determined from $W^{-} \rightarrow \mu^{-} \bar{\nu}_{\mu}$ and $W^{-} \rightarrow e^{-} \bar{\nu}_{e}$ decays. Assuming that these values of $\left(\frac{g_{\mu}}{g_{e}}\right)$ can be combined with the new average from $\tau$ decays and that they are uncorrelated, the new world aver- 
age is $\left(\frac{g_{\mu}}{g_{e}}\right)=1.0020 \pm 0.0010$. This is 2.0 standard deviations away from the lepton universality assumption. Figure 8.4 shows the status of $\left(\frac{g_{\mu}}{g_{e}}\right)$ with the updated value of $\left(\frac{g_{\mu}}{g_{e}}\right)_{\tau}$ and the new world average.

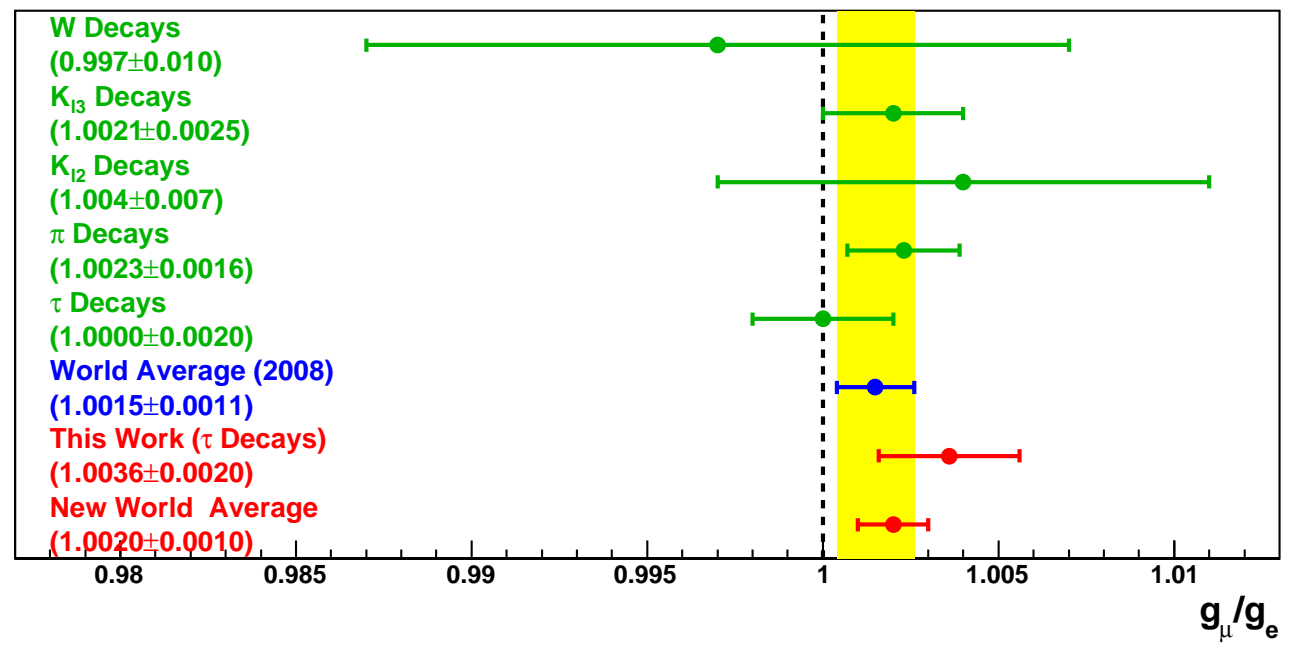

Figure 8.4: The current status of the $\left(\frac{g_{\mu}}{g_{e}}\right)$ determined from $W^{ \pm}$decays [144], $K_{l 3}$ decays $[142,143], K_{l 2}$ decays $[39,139-141], \pi^{-} \rightarrow \mu^{-} \bar{\nu}_{\mu}$ and $\pi^{-} \rightarrow e^{-} \bar{\nu}_{e}$ decays [136-138], and $\tau$ decays [12], with the updated value of $\left(\frac{g_{\mu}}{g_{e}}\right)_{\tau}$ and the new world average.

\subsection{Results from the $\frac{\mathcal{B}\left(\tau^{-} \rightarrow \pi^{-} \nu_{\tau}\right)}{\mathcal{B}\left(\tau^{-} \rightarrow e^{-} \nu_{\tau} \bar{\nu}_{e}\right)}$ and $\frac{\mathcal{B}\left(\tau^{-} \rightarrow K^{-} \nu_{\tau}\right)}{\mathcal{B}\left(\tau^{-} \rightarrow e^{-} \nu_{\tau} \bar{\nu}_{e}\right)}$ Measurements and $\tau-\mu$ Universality}

The $\frac{\mathcal{B}\left(\tau^{-} \rightarrow \pi^{-} \nu_{\tau}\right)}{\mathcal{B}\left(\tau^{-} \rightarrow e^{-} \nu_{\tau} \bar{\nu}_{e}\right)}$ branching ratio is measured to be $(5.945 \pm 0.014 \pm 0.061) \times 10^{-1}$, and is consistent with the world average $(6.076 \pm 0.061) \times 10^{-1}[12]$, which is based on the measurement by [41] and the electron world average. The branching fraction $\mathcal{B}\left(\tau^{-} \rightarrow\right.$ $\left.\pi^{-} \nu_{\tau}\right)=(10.59 \pm 0.03 \pm 0.11) \times 10^{-2}$ is determined from the $\frac{\mathcal{B}\left(\tau^{-} \rightarrow \pi^{-} \nu_{\tau}\right)}{\mathcal{B}\left(\tau^{-} \rightarrow e^{-} \nu_{\tau} \bar{\nu}_{e}\right)}$ branching ratio using the electron branching fraction world average value [12]. Figure 8.3 shows 
a comparison of the $\mathcal{B}\left(\tau^{-} \rightarrow \pi^{-} \nu_{\tau}\right)$ branching fraction to the world average value [41]. The $\mathcal{B}\left(\tau^{-} \rightarrow \pi^{-} \nu_{\tau}\right)$ branching ratio is consistent within 1.5 standard deviations and competitive with the only previous measurements used in the world average [41]. The dominant systematic uncertainties for the $\frac{\mathcal{B}\left(\tau^{-} \rightarrow \pi^{-} \nu_{\tau}\right)}{\mathcal{B}\left(\tau^{-} \rightarrow e^{-} \nu_{\tau} \bar{\nu}_{e}\right)}$ branching ratio are the neutral energy modelling, the normalization, and the particle identification.

For $\frac{\mathcal{B}\left(\tau^{-} \rightarrow K^{-} \nu_{\tau}\right)}{\mathcal{B}\left(\tau^{-} \rightarrow e^{-} \nu_{\tau} \bar{\nu}_{e}\right)}$, the measured branching ratio, $\frac{\mathcal{B}\left(\tau^{-} \rightarrow K^{-} \nu_{\tau}\right)}{\mathcal{B}\left(\tau^{-} \rightarrow e^{-} \nu_{\tau} \bar{\nu}_{e}\right)}=(3.882 \pm 0.032 \pm$ $0.057) \times 10^{-2}$, is a significant improvement compared to the world average $(3.84 \pm$ $0.13) \times 10^{-2}[12]$. Using the electron branching fraction world average value [12] the branching fraction $\mathcal{B}\left(\tau^{-} \rightarrow K^{-} \nu_{\tau}\right)$ was determined to be $(6.92 \pm 0.06 \pm 0.10) \times$ $10^{-3}$. From Figure 8.3 it can be seen that this $\mathcal{B}\left(\tau^{-} \rightarrow K^{-} \nu_{\tau}\right)$ branching fraction is consistent with and two times more precise than the current world average. The two largest systematic uncertainties for the $\frac{\mathcal{B}\left(\tau^{-} \rightarrow K^{-} \nu_{\tau}\right)}{\mathcal{B}\left(\tau^{-} \rightarrow e^{-} \nu_{\tau} \bar{\nu}_{e}\right)}$ are the statistical uncertainty and particle identification uncertainty. However, there are also significant uncertainties related to the backgrounds, the neutral energy modelling and the normalization. As a result, the $\frac{\mathcal{B}\left(\tau^{-} \rightarrow \pi^{-} \nu_{\tau}\right)}{\mathcal{B}\left(\tau^{-} \rightarrow e^{-} \nu_{\tau} \bar{\nu}_{e}\right)}$ and $\frac{\mathcal{B}\left(\tau^{-} \rightarrow K^{-} \nu_{\tau}\right)}{\mathcal{B}\left(\tau^{-} \rightarrow e^{-} \nu_{\tau} \bar{\nu}_{e}\right)}$ systematic uncertainties are highly correlated.

The $\mathcal{B}\left(\tau^{-} \rightarrow \pi^{-} \nu_{\tau}\right)$ and $\mathcal{B}\left(\tau^{-} \rightarrow K^{-} \nu_{\tau}\right)$ branching fractions can be used to test the $\tau-\mu$ lepton universality both in strange and non-strange decays. For the non-strange decay modes,

$$
\left(\frac{g_{\tau}}{g_{\mu}}\right)_{\pi}=\sqrt{\frac{\mathcal{B}\left(\tau^{-} \rightarrow \pi^{-} \nu_{\tau}\right) 2 m_{\pi} m_{\mu}^{2} \tau_{\pi}}{\mathcal{B}\left(\pi^{-} \rightarrow \mu^{-} \bar{\nu}_{\mu}\right) \delta_{\tau / \pi} m_{\tau}^{3} \tau_{\tau}}\left(\frac{1-m_{\mu}^{2} / m_{\pi}^{2}}{1-m_{\pi}^{2} / m_{\tau}^{2}}\right)^{2}}
$$

where $m_{\pi}$ is the pion mass, $m_{\mu}$ is the muon mass, $m_{\tau}$ is the $\tau$ mass, $\tau_{\pi}$ is the pion life time, $\tau_{\tau}$ is the $\tau$ life time, and $\delta_{\tau / \pi}=(1.0016 \pm 0.0014)$ [145] is the electroweak radiative correction. Similarly, for the strange decay modes, 


$$
\left(\frac{g_{\tau}}{g_{\mu}}\right)_{K}=\sqrt{\frac{\mathcal{B}\left(\tau^{-} \rightarrow K^{-} \nu_{\tau}\right) 2 m_{K} m_{\mu}^{2} \tau_{K}}{\mathcal{B}\left(K^{-} \rightarrow \mu^{-} \bar{\nu}_{\mu}\right) \delta_{\tau / K} m_{\tau}^{3} \tau_{\tau}}\left(\frac{1-m_{\mu}^{2} / m_{K}^{2}}{1-m_{K}^{2} / m_{\tau}^{2}}\right)^{2}}
$$

where $m_{K}$ is the kaon mass, and $\delta_{\tau / K}=(1.0090 \pm 0.0022)$ [145] is the radiative correction. Using the world average values for the masses and life times [12],

$$
\left(\frac{g_{\tau}}{g_{\mu}}\right)_{\pi}=0.9856 \pm 0.0057
$$

for the non-strange decay modes and

$$
\left(\frac{g_{\tau}}{g_{\mu}}\right)_{K}=0.9827 \pm 0.0086
$$

for the strange decay modes. These are consistent with each other and can be averaged. After taking into account the correlations between $\frac{\mathcal{B}\left(\tau^{-} \rightarrow \pi^{-} \nu_{\tau}\right)}{\mathcal{B}\left(\tau^{-} \rightarrow e^{-} \nu_{\tau} \bar{\nu}_{e}\right)}$ and $\frac{\mathcal{B}\left(\tau^{-} \rightarrow K^{-} \nu_{\tau}\right)}{\mathcal{B}\left(\tau^{-} \rightarrow e^{-} \nu_{\tau} \bar{\nu}_{e}\right)}$ one obtains an averaged

$$
\left(\frac{g_{\tau}}{g_{\mu}}\right)_{\pi / K}=0.9850 \pm 0.0054
$$

The previous world average values of $\left(\frac{g_{\tau}}{g_{\mu}}\right)$ for non-strange and strange $\tau$ decays are $0.996 \pm 0.005[12]$ and $0.976 \pm 0.017$ [12] respectively. Taking into account the correlations from the masses and the lifetimes, these values are combined with the measurements from this paper to obtain $\left(\frac{g_{\tau}}{g_{\mu}}\right)_{\pi}=0.9914 \pm 0.0046$ for non-strange $\tau$ decays and $\left(\frac{g_{\tau}}{g_{\mu}}\right)_{K}=0.9820 \pm 0.0079$ for strange $\tau$ decays. The new world average values $\left(\frac{g_{\tau}}{g_{\mu}}\right)$ determined from non-strange and strange $\tau$ decays, are combined to give a new world average $\left(\frac{g_{\tau}}{g_{\mu}}\right)_{\pi / K}=0.9904 \pm 0.0045$ for $\tau$ decay, where the correlations from the lifetime and masses have been accounted for. $\left(\frac{g_{\tau}}{g_{\mu}}\right)$ is also measured from $\tau^{-} \rightarrow e^{-} \nu_{\tau} \bar{\nu}_{e}$ decay using the $\tau, \mu$ lifetimes and masses, $\left(\frac{g_{\tau}}{g_{\mu}}\right)_{\tau^{-} \rightarrow e^{-} \nu_{\tau} \bar{\nu}_{e}}=1.0006 \pm$ $0.0022[12]$, and from $W^{-} \rightarrow \tau^{-} \bar{\nu}_{\tau}$ and $W^{-} \rightarrow \mu^{-} \bar{\nu}_{\mu}$ decays, $\left(\frac{g_{\tau}}{g_{\mu}}\right)_{W}=1.039 \pm 0.013$ [144]. These values deviate within 2.3 and 3.5 standard deviations respectively from 
the new world average determined from $\tau^{-} \rightarrow \pi^{-} \nu_{\tau}$ and $\tau^{-} \rightarrow K^{-} \nu_{\tau}$ decays. The current status of $\left(\frac{g_{\tau}}{g_{\mu}}\right)$ can be seen in Figure 8.5.

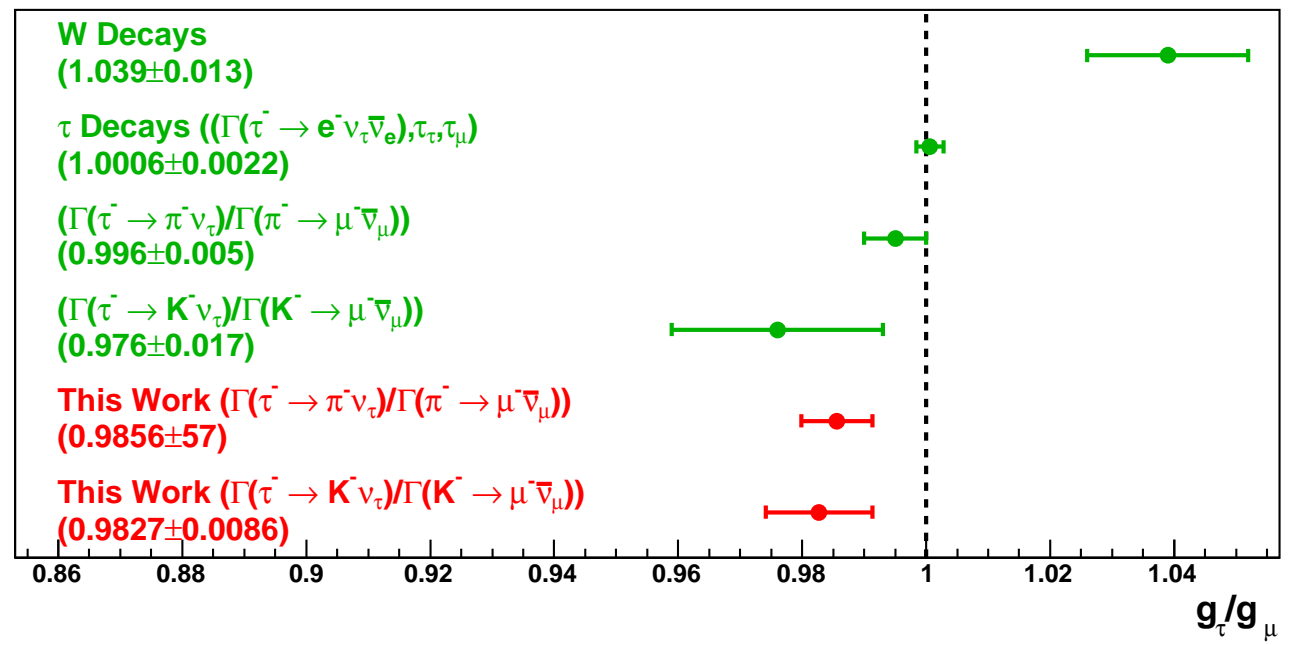

Figure 8.5: The current status of the $\left(\frac{g_{\tau}}{g_{\mu}}\right)$ determined from $W^{ \pm}$decays [144], $\tau^{-} \rightarrow$ $e^{-} \nu_{\tau} \bar{\nu}_{e}$ decays [12], and the non-strange and strange decays from [12] and this work.

\subsection{Results from the $\frac{\mathcal{B}\left(\tau^{-} \rightarrow K^{-} \nu_{\tau}\right)}{\mathcal{B}\left(\tau^{-} \rightarrow \pi^{-} \nu_{\tau}\right)}$ Measurement and $\left|V_{u s}\right|$}

The branching ratio $\frac{\mathcal{B}\left(\tau^{-} \rightarrow K^{-} \nu_{\tau}\right)}{\mathcal{B}\left(\tau^{-} \rightarrow \pi^{-} \nu_{\tau}\right)}$ was measured to be $(6.531 \pm 0.056 \pm 0.093) \times 10^{-2}$, which is a significant improvement compared to the world average, $(6.33 \pm 0.21) \times 10^{-2}$ [12]. The limiting systematic uncertainties in this branching ratio came from particle identification uncertainty and the background uncertainty. Because the particle identification uncertainty is limited by the control samples, and the backgrounds are dominated by $\tau^{-} \rightarrow K^{-} K_{L}^{0} \nu_{\tau}$ and the limited statistics in the non- $\tau$ sideband region, future experiments, such as Super KEK B [135] and Super B [134], with significantly 
higher statistics will be able to improve this measurement to the $0.5 \%$ level.

From Equation 2.81, $\frac{\mathcal{B}\left(\tau^{-} \rightarrow K^{-} \nu_{\tau}\right)}{\mathcal{B}\left(\tau^{-} \rightarrow \pi^{-} \nu_{\tau}\right)}$ branching ratio can be written in terms of the decay strengths, the CKM matrix elements $V_{u s}$ and $V_{u d}$ and a mass correction factor. Using the QCD Lattice value $\frac{f_{k}}{f_{\pi}}=1.189 \pm 0.007$ [40] we obtain

$$
\left|V_{u s}\right|=0.2255 \pm 0.0023
$$

a value which is consistent with the unitarity value of $\left|V_{u s}\right|=0.2262 \pm 0.0011$. A comparision of $\left|V_{u s}\right|$ extracted using $\frac{\mathcal{B}\left(\tau^{-} \rightarrow K^{-} \nu_{\tau}\right)}{\mathcal{B}\left(\tau^{-} \rightarrow \pi^{-} \nu_{\tau}\right)}$ to the previous measurements of $\left|V_{u s}\right|$ can be seen in Figure 8.6. 


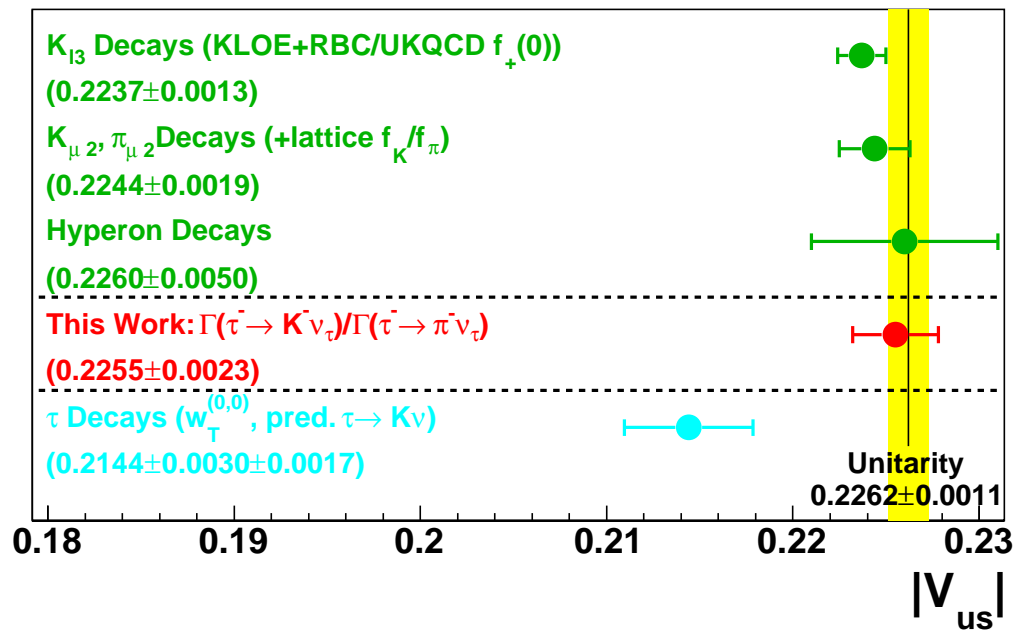

Figure 8.6: The current status of the $\left|V_{u s}\right|$ with $\left|V_{u s}\right|$ determined from $\frac{\mathcal{B}\left(\tau^{-} \rightarrow K^{-} \nu_{\tau}\right)}{\mathcal{B}\left(\tau^{-} \rightarrow \pi^{-} \nu_{\tau}\right)}$. The recent measurements from kaon decays, $K_{l 3}$ [125] and $K_{l 2}$ [40], and the hyperon decay data [126] are located above the dashed lines, while the value of $\left|V_{u s}\right|$ from $\frac{\mathcal{B}\left(\tau^{-} \rightarrow K^{-} \nu_{\tau}\right)}{\mathcal{B}\left(\tau^{-} \rightarrow \pi^{-} \nu_{\tau}\right)}$ is located between the dashed lines. Below the dashed lines is $W_{T}^{0,0}$ measured in [124] using measurements from this work, BABAR [9] and Belle [10], which has been included to represent the current status of $\left|V_{u s}\right|$ extracted using hadronic $\tau$ decays. 


\section{Chapter 9}

\section{Conclusion}

A number of branching fractions and ratios of the 3rd generation of charged leptons were measured and interpreted in the context of the Standard Model of particle physics. The $\mathcal{B}\left(\tau^{-} \rightarrow \pi^{-} \pi^{-} \pi^{+} \nu_{\tau}\right)$ is measured to be $(8.83 \pm 0.01 \pm 0.13) \%$, a value more precise than the previous measurement that directly identified the pions [116]. The $\tau^{-} \rightarrow K^{-} \pi^{-} \pi^{+} \nu_{\tau}$ and $\tau^{-} \rightarrow K^{-} \pi^{-} K^{+} \nu_{\tau}$ branching fractions were measured to be $(0.273 \pm 0.002 \pm 0.009) \%$ and $(0.1346 \pm 0.0010 \pm 0.0036) \%$ respectively, both of which are significantly more precise than the world average. This first measurement of $\tau^{-} \rightarrow K^{-} K^{-} K^{+} \nu_{\tau}$ branching fraction, in which no resonance structure is assumed, yields a value of $(1.58 \pm 0.13 \pm 0.12) \times 10^{-5}$.

The $\tau^{-} \rightarrow \phi \pi^{-} \nu_{\tau}$ and $\tau^{-} \rightarrow \phi K^{-} \nu_{\tau}$ decay modes were observed in the $M_{K^{+} K^{-}}$ invariant mass spectrum. The $\mathcal{B}\left(\tau^{-} \rightarrow \phi \pi^{-} \nu_{\tau}\right)$ and $\mathcal{B}\left(\tau^{-} \rightarrow \phi K^{-} \nu_{\tau}\right)$ were extracted from the $M_{K^{+} K^{-}}$invariant mass spectrum by means of a binned maximum likelihood fit and then using the corresponding efficiency matrix to account for the cross-feed. The first measurement of $\mathcal{B}\left(\tau^{-} \rightarrow \phi \pi^{-} \nu_{\tau}\right)$ was determined to be $(3.42 \pm 0.55 \pm 0.25) \times$ $10^{-5}$, while $\mathcal{B}\left(\tau^{-} \rightarrow \phi K^{-} \nu_{\tau}\right)$ was measured to be $(3.39 \pm 0.20 \pm 0.28) \times 10^{-5}$, a value

which is both competitive and consistent with the concurrent result by Belle [129]. From the binned maximum likelihood of the $M_{K^{+} K^{-}}$invariant mass spectrum, no 
evidence was found for $\tau^{-} \rightarrow K^{-} K^{-} K^{+} \nu_{\tau}$ excluding $\phi$ so the first upper limit on $\mathcal{B}\left(\tau^{-} \rightarrow K^{-} K^{-} K^{+} \nu_{\tau}(\right.$ ex. $\left.\phi)\right)$ was determined to be $\mathcal{B}\left(\tau^{-} \rightarrow K^{-} K^{-} K^{+} \nu_{\tau}(\right.$ ex. $\left.\phi)\right)<$ $2.5 \times 10^{-6}$ at $90 \%$ C.L.

Updating the current spectral density functions with these measured $\tau^{-} \rightarrow$ $h^{-} h^{-} h^{+} \nu_{\tau}$ branching fractions and the recent results from Belle [10] the value of $\left|V_{u s}\right|$ extracted using FESR is approximately 3 standard deviations below unitarity. This indicated a possible deviation of $\left|V_{u s}\right|$ from the unitarity value in $\tau$ decays. To investigate the possibility for new physics, related to $\left|V_{u s}\right|$ in $\tau$ decays, an alternative method for measuring $\left|V_{u s}\right|$ the branching ratio $\frac{\mathcal{B}\left(\tau^{-} \rightarrow K^{-} \nu_{\tau}\right)}{\mathcal{B}\left(\tau^{-} \rightarrow \pi^{-} \nu_{\tau}\right)}$ was used. This method has a theoretical uncertainty which is completely independent from the FESR approach and does not have problems related to the convergence of OPE series. The $\frac{\mathcal{B}\left(\tau^{-} \rightarrow K^{-} \nu_{\tau}\right)}{\mathcal{B}\left(\tau^{-} \rightarrow \pi^{-} \nu_{\tau}\right)}$ was measured to be $(6.531 \pm 0.056 \pm 0.093) \times 10^{-2}$. Using the QCD Lattice value $\frac{f_{k}}{f_{\pi}}=1.189 \pm 0.007$ and $\left|V_{u d}\right|=[40],\left|V_{u s}\right|$ is determined to be $\left|V_{u s}\right|=0.2255 \pm 0.0023$, a value which is consistent with the expected value determined from unitarity $\left|V_{u s}\right|=0.2262 \pm 0.0011$.

In the 1 prong branching ratio analysis, $\frac{\mathcal{B}\left(\tau^{-} \rightarrow \mu^{-} \nu_{\tau} \bar{\nu}_{\mu}\right)}{\mathcal{B}\left(\tau^{-} \rightarrow e^{-} \nu_{\tau} \bar{\nu}_{e}\right)}$ was determined to be $(9.796 \pm 0.016 \pm 0.035) \times 10^{-1}$. Using this branching ratio, a lepton universality test between the muon and the electron was performed. $\frac{g_{\mu}}{g_{e}}$ was measured to be $1.0036 \pm 0.0020$, a value which is consistent with the lepton universality assumption in the Standard Model. Using the world average [39] electron branching fraction, the $\mathcal{B}\left(\tau^{-} \rightarrow \mu^{-} \nu_{\tau} \bar{\nu}_{\mu}\right)$ was determined to be $(17.42 \pm 0.03 \pm 0.08) \times 10^{-2}$, a value that is both competitive with and consistent with previous measurements. For the $\frac{\mathcal{B}\left(\tau^{-} \rightarrow \pi^{-} \nu_{\tau}\right)}{\mathcal{B}\left(\tau^{-} \rightarrow e^{-} \nu_{\tau} \bar{\nu}_{e}\right)}$, the branching ratio was determined to be $(5.945 \pm 0.014 \pm 0.061) \times 10^{-1}$. Using the world average for the electron branching fraction [39], $\mathcal{B}\left(\tau^{-} \rightarrow \pi^{-} \nu_{\tau}\right)$ is determined to be $(10.59 \pm 0.03 \pm 0.11) \times 10^{-2}$ a value which is competitive with and consistent with the previous measurements $[41,146]$. Comparing this branching fraction to 
the $\pi^{-} \rightarrow \mu \nu_{\mu}$ branching fraction after correcting for the masses and the lifetimes, one obtains $\left(\frac{g_{\tau}}{g_{\mu}}\right)_{\pi}=0.9856 \pm 0.0057$. This value is within 2.5 standard deviations of the prediction for lepton universality in the Standard Model. The $\frac{\mathcal{B}\left(\tau^{-} \rightarrow K^{-} \nu_{\tau}\right)}{\mathcal{B}\left(\tau^{-} \rightarrow e^{-} \nu_{\tau} \bar{\nu}_{e}\right)}$ branching ratio was measured to be $(3.882 \pm 0.032 \pm 0.057) \times 10^{-2}$, which results in a $\mathcal{B}\left(\tau^{-} \rightarrow K^{-} \nu_{\tau}\right)$ fraction of $(6.92 \pm 0.06 \pm 0.10) \times 10^{-3}$. This branching fraction is both consistent with and a significant improvement relative to the world average [39]. From this, $\left(\frac{g_{\tau}}{g_{\mu}}\right)_{K}=0.9827 \pm 0.0086$ was determined to be $0.9856 \pm 0.0057$. After taking into account the correlations between $\mathcal{B}\left(\tau^{-} \rightarrow K^{-} \nu_{\tau}\right)$ and $\mathcal{B}\left(\tau^{-} \rightarrow \pi^{-} \nu_{\tau}\right)$ the combined $\frac{g_{\tau}}{g_{\mu}}$ ratio was determined to be $\left(\frac{g_{\tau}}{g_{\mu}}\right)_{\pi / K}=0.9850 \pm 0.0054$. This value is within 2.8 standard deviations from the universality assumption. The $\frac{\mathcal{B}\left(\tau^{-} \rightarrow K^{-} \nu_{\tau}\right)}{\mathcal{B}\left(\tau^{-} \rightarrow \pi^{-} \nu_{\tau}\right)}$, $\frac{\mathcal{B}\left(\tau^{-} \rightarrow K^{-} \nu_{\tau}\right)}{\mathcal{B}\left(\tau^{-} \rightarrow e^{-} \nu_{\tau} \bar{\nu}_{e}\right)}$, and $\frac{\mathcal{B}\left(\tau^{-} \rightarrow \mu^{-} \nu_{\tau} \bar{\nu}_{\mu}\right)}{\mathcal{B}\left(\tau^{-} \rightarrow e^{-} \nu_{\tau} \bar{\nu}_{e}\right)}$ branching fractions were limited by statistics of the data set and the control samples. Therefore, one should be able to significantly improve the precision of these measurements at future experiments such as SuperB [134] and Super KEK B [135] .

The $\tau^{-} \rightarrow h^{-} h^{-} h^{+} \nu_{\tau}$ invariant mass spectra is determined in this paper using a Bayesian Unfolding algorithm [7]. The $\tau^{-} \rightarrow \pi^{-} \pi^{-} \pi^{+} \nu_{\tau}$ invariant mass spectra is competitive with the previously measured invariant mass distributions $[41,52,53]$, while the $\tau^{-} \rightarrow K^{-} \pi^{-} \pi^{+} \nu_{\tau}$ and $\tau^{-} \rightarrow K^{-} \pi^{-} K^{+} \nu_{\tau}$ are significant improvements relative to the current ALEPH and OPAL measurements $[42,51,53]$. The $\tau^{-} \rightarrow$ $K^{-} K^{-} K^{+} \nu_{\tau}$ invariant mass distribution is the first unfolded distribution. When the invariant mass spectra being measured by other members of the BABAR Collaboration are available, these invariant mass spectra will result in a significant improvement in the strange and non-strange spectral density function and are required to fully examine the existing $3 \sigma$ deviation from unitarity of the $\left|V_{u s}\right|$ as determined using the FESR formalism. 


\section{Bibliography}

[1] N. Cabibbo. Unitary Symmetry and Leptonic Decays. Phys. Rev. Lett., 10:531532, 1963.

[2] Elvira Gamiz, Matthias Jamin, Antonio Pich, Joaquim Prades, and Felix Schwab. $V_{u s}$ and $m_{s}$ from hadronic $\tau$ decays. Phys. Rev. Lett., 94:011803, 2005.

[3] S. Chen et al. Strange quark mass from the invariant mass distribution of Cabibbo-suppressed tau decays. Eur. Phys. J., C22:31-38, 2001.

[4] Kim Maltman and Joachim Kambor. On the longitudinal contributions to hadronic $\tau$ decay. Phys. Rev., D64:093014, 2001.

[5] Kim Maltman. Problems With Extracting $m_{s}$ from Flavor Breaking in Hadronic $\tau$ Decays. Phys. Rev., D58:093015, 1998.

[6] Stephan Narison. Strange-quark mass from combined $e^{+} e^{-}$and tau-decay data:test of the isospin symmetry and implications on $\epsilon^{\prime} / \epsilon$ and $m_{u, d}$. Phys. Lett., B466:345-354, 1999.

[7] G. D'Agostini. A Multidimensional unfolding method based on Bayes' theorem. Nucl. Instrum. Meth., A362:487-498, 1995. 
[8] B. Aubert et al. Exclusive branching fraction measurements of semileptonic tau decays into three charged hadrons, $\tau^{-} \rightarrow \phi \pi^{-} \nu_{\tau}$ and $\tau^{-} \rightarrow \phi K^{-} \nu_{\tau}$. Phys. Rev. Lett., 100:011801, 2008.

[9] B. Aubert et al. Measurement of the $\tau^{-} \rightarrow K^{-} \pi^{0} \nu_{\tau}$ Branching Fraction. Phys. Rev., D76:051104, 2007.

[10] D. Epifanov et al. Study of $\tau^{-} \rightarrow K_{S} \pi^{-} \nu_{\tau}$ decay at Belle. Phys. Lett., B654:65$73,2007$.

[11] Richard L. Liboff. Introductory quantum mechanics 3rd Edition. USA: Addison-Wesley Publishing Company, Inc. (1998) 874p.

[12] C. Amsler et al. Review of particle physics. Phys. Lett., B667:1, 2008.

[13] F. Halzen and Alan D. Martin. Quarks and leptons: an introductory course in modern particle physics. New York, USA: Wiley ( 1984) 396p.

[14] David J. Griffiths. Introduction to elementary particles. New York, USA: Wiley (1987) 392p.

[15] J. F. Donoghue, E. Golowich, and Barry R. Holstein. Dynamics of the standard model. Camb. Monogr. Part. Phys. Nucl. Phys. Cosmol., 2:1-540, 1992.

[16] Kim Maltman. Private Communications.

[17] D. h. Perkins. Introduction to high-energy physics 4th Edition. Cambridge University Press, England, 2001.

[18] Lincoln Wolfenstein. Parametrization of the Kobayashi-Maskawa Matrix. Phys. Rev. Lett., 51:1945, 1983. 
[19] Yosef Nir. CP violation: A new era. 2001, hep-ph/0109090.

[20] W. J. Marciano and A. Sirlin. Electroweak Radiative Corrections to tau Decay. Phys. Rev. Lett., 61:1815-1818, 1988.

[21] Yung-Su Tsai. Decay Correlations of Heavy Leptons in $e^{+} e^{-} \rightarrow$ Lepton ${ }^{+}$Lepton $^{-}$. Phys. Rev., D4:2821, 1971.

[22] Michael Edward Peskin and Daniel V. Schroeder. An introduction to quantum field theory. Reading, USA: Addison-Wesley (1995) 842p.

[23] E. Braaten. QCD Predictions for the Decay of the $\tau$ Lepton. Phys. Rev. Lett., 60:1606-1609, 1988.

[24] Eric Braaten. The Perturbative QCD Corrections to the Ratio R for tau Decay. Phys. Rev., D39:1458, 1989 .

[25] E. Braaten, Stephan Narison, and A. Pich. QCD analysis of the tau hadronic width. Nucl. Phys., B373:581-612, 1992.

[26] K. G. Chetyrkin and A. Kwiatkowski. Mass corrections to the tau decay rate. Z. Phys., C59:525-532, 1993.

[27] P. A. Baikov, K. G. Chetyrkin, and J. H. Kuhn. Strange quark mass from tau lepton decays with $O\left(\right.$ alpha $\left._{s}^{3}\right)$ accuracy. Phys. Rev. Lett., 95:012003, 2005.

[28] Kim Maltman and Carl E. Wolfe. $V_{u s}$ from hadronic $\tau$ decays. Phys. Lett., B639:283-289, 2006.

[29] Antonio Pich and Joaquim Prades. Strange quark mass determination from Cabibbo-suppressed tau decays. JHEP, 10:004, 1999. 
[30] Matthias Jamin, Jose Antonio Oller, and Antonio Pich. Strangeness-changing scalar form factors. Nucl. Phys., B622:279-308, 2002.

[31] Matthias Jamin, Jose Antonio Oller, and Antonio Pich. Light quark masses from scalar sum rules. Eur. Phys. J., C24:237-243, 2002.

[32] M. Jamin, J. A. Oller, and A. Pich. Scalar K $\pi$ form factor and light quark masses. Phys. Rev., D74:074009, 2006.

[33] Kim Maltman and Joachim Kambor. Decay constants, light quark masses and quark mass bounds from light quark pseudoscalar sum rules. Phys. Rev., D65:074013, 2002.

[34] Joachim Kambor and Kim Maltman. The strange quark mass from flavor breaking in hadronic $\tau$ decays. Phys. Rev., D62:093023, 2000.

[35] E. Gamiz, M. Jamin, A. Pich, J. Prades, and F. Schwab. Determination of $m_{s}$ and $\left|V_{u s}\right|$ from hadronic $\tau$ decays. JHEP, 01:060, 2003.

[36] A. Pich. QCD Predictions for the Tau Hadronic Width: Determination of $\alpha_{s}\left(M_{\tau}^{2}\right)$. Nucl. Phys. Proc. Suppl., 39BC:326, 1995.

[37] Murray Gell-Mann, R. J. Oakes, and B. Renner. Behavior of current divergences under SU(3) x SU(3). Phys. Rev., 175:2195-2199, 1968.

[38] K. Langfeld and C. Kettner. The quark condensate in the GMOR relation. Mod. Phys. Lett., A11:1331-1338, 1996.

[39] W. M. Yao et al. Review of particle physics. J. Phys., G33:1-1232, 2006.

[40] E. Follana, C. T. H. Davies, G. P. Lepage, and J. Shigemitsu. High Precision 
determination of the pi, K, D and $\mathrm{D}_{s}$ decay constants from lattice QCD. Phys. Rev. Lett., 100:062002, 2008.

[41] S. Schael et al. Branching ratios and spectral functions of $\tau$ decays: Final ALEPH measurements and physics implications. Phys. Rept., 421:191-284, 2005 .

[42] R. Barate et al. Study of $\tau$ decays involving kaons, spectral functions and determination of the strange quark mass. Eur. Phys. J., C11:599-618, 1999.

[43] R. Barate et al. Measurement of the spectral functions of axial-vector hadronic tau decays and determination of $\alpha_{S}\left(M_{\tau}^{2}\right)$. Eur. Phys. J., C4:409-431, 1998.

[44] R. Barate et al. Measurement of the spectral functions of vector current hadronic tau decays. Z. Phys., C76:15-33, 1997.

[45] T. E. Coan et al. Wess-Zumino Current and the Structure of the Decay $\tau^{-}$toK $K^{-} K^{+} \pi^{-} \nu_{\tau}$. Phys. Rev. Lett., 92:232001, 2004.

[46] S. Anderson et al. Hadronic structure in the decay $\tau^{-} \rightarrow \pi^{-} \pi^{0} \nu_{\tau}$. Phys. Rev., D61:112002, 2000.

[47] K. W. Edwards et al. Resonant structure of $\tau \rightarrow 3 \pi p i^{0} \nu_{\tau}$ and $\tau \rightarrow \omega \pi \nu_{\tau}$ decays. Phys. Rev., D61:072003, 2000.

[48] T. E. Browder et al. Structure functions in the decay $\tau^{\mp} \rightarrow \tau^{\mp} \pi^{0} \pi^{0} \nu_{\tau}$. Phys. Rev., D61:052004, 2000.

[49] D. M. Asner et al. Hadronic structure in the decay $\tau^{-} \rightarrow \nu_{\tau} \pi^{-} \pi^{0} \pi^{0}$ and the sign of the $\tau$ neutrino helicity. Phys. Rev., D61:012002, 2000. 
[50] T. Coan et al. Measurement of $\alpha_{s}$ from $\tau$ decays. Phys. Lett., B356:580-588, 1995

[51] G. Abbiendi et al. Measurement of the strange spectral function in hadronic $\tau$ decays. Eur. Phys. J., C35:437-455, 2004.

[52] K. Ackerstaff et al. Measurement of the strong coupling constant $\alpha_{s}$ and the vector and axial-vector spectral functions in hadronic tau decays. Eur. Phys. J., C7:571-593, 1999.

[53] Michel Davier, Andreas Hocker, and Zhiqing Zhang. The physics of hadronic tau decays. Rev. Mod. Phys., 78:1043-1109, 2006.

[54] K. Maltman and C. E. Wolfe. Joint extraction of $m_{s}$ and $V_{u s}$ from hadronic $\tau$ decays. Phys. Lett., B650:27-32, 2007.

[55] M. Jamin. Status of Vus. presented at XLIInd Rencontres de Moriond: Electroweak Interactions and Unified Theories 2007.

[56] Elvira Gamiz, Matthias Jamin, Antonio Pich, Joaquim Prades, and Felix Schwab. $\left|V_{u s}\right|$ and $m_{s}$ from hadronic tau decays. Nucl. Phys. Proc. Suppl., 169:85-89, 2007.

[57] K. Maltman and C. E. Wolfe. $V_{u s}$ (and $m_{s}$ ) From Hadronic $\tau$ Decays. hep$\mathrm{ph} / 0703314$.

[58] F. Ambrosino et al. Measurement of the absolute branching ratio for the $K^{+} \rightarrow$ $\mu^{+} \nu(\gamma)$ decay with the KLOE detector. Phys. Lett., B632:76-80, 2006.

[59] C. Aubin et al. Light pseudoscalar decay constants, quark masses, and low energy constants from three-flavor lattice QCD. Phys. Rev., D70:114501, 2004. 
[60] C. Bernard et al. Update on $\pi$ and K physics. PoS, LAT2005:025, 2006.

[61] William J. Marciano. Precise determination of $\left|V_{u s}\right|$ from lattice calculations of pseudoscalar decay constants. Phys. Rev. Lett., 93:231803, 2004.

[62] A. Sher et al. New, high statistics measurement of the $K^{+} \rightarrow \pi^{0} e^{+} \nu\left(K_{e 3}^{+}\right)$ branching ratio. Phys. Rev. Lett., 91:261802, 2003.

[63] T. Alexopoulos et al. A determination of the CKM parameter $\left|V_{u s}\right|$. Phys. Rev. Lett., 93:181802, 2004.

[64] T. Alexopoulos et al. Measurements of $\mathrm{K}(\mathrm{L})$ branching fractions and the CP violation parameter $\left|\eta^{ \pm}\right|$. Phys. Rev., D70:092006, 2004.

[65] F. Ambrosino et al. Measurements of the absolute branching ratios for the dominant $K_{L}$ decays, the $K_{L}$ lifetime, and $V_{u s}$ with the KLOE detector. Phys. Lett., B632:43-50, 2006.

[66] F. Ambrosino et al. Measurement of the branching ratio of the $K_{L} \rightarrow \pi^{+} \pi^{-}$ decay with the KLOE detector. Phys. Lett., B638:140-145, 2006.

[67] F. Ambrosino et al. Measurement of the branching fraction and charge asymmetry for the decay $K_{S} \rightarrow \pi e \nu$ with the KLOE detector. Phys. Lett., B636:173182,2006

[68] Barbara Sciascia. KLOE extraction of $V_{u s}$ from kaon decays and lifetimes. PoS, HEP2005:287, 2006.

[69] A. Lai et al. Measurement of the branching ratio of the decay $K_{L} \rightarrow \pi^{ \pm} e^{\mp} \nu$ and extraction of the CKM parameter $\left|V_{u s}\right|$. Phys. Lett., B602:41-51, 2004. 
[70] A. Lai et al. Measurement of the Ratio $\Gamma\left(K_{L} \rightarrow \pi^{+} \pi^{-}\right) / \Gamma\left(K_{L} \rightarrow \pi e \nu\right)$ and Extraction of the CP Violation Parameter $\left|\eta^{ \pm}\right|$. Phys. Lett., B645:26-35, 2007.

[71] J. R. Batley et al. Measurements of Charged Kaon Semileptonic Decay Branching Fractions $K^{ \pm} \rightarrow \pi^{0} \mu^{ \pm} \nu$ and $K^{ \pm} \rightarrow \pi^{0} e^{ \pm} \nu$ and Their Ratio. Eur. Phys. J., C50:329-340, 2007.

[72] V. I. Romanovsky et al. Measurement of $K_{e 3}^{-}$branching ratio. 2007.

[73] Nicola Cabibbo, Earl C. Swallow, and Roland Winston. Semileptonic hyperon decays and CKM unitarity. Phys. Rev. Lett., 92:251803, 2004.

[74] V. Mateu and A. Pich. $V_{u s}$ determination from hyperon semileptonic decays. JHEP, 10:041, 2005.

[75] M. Gockeler et al. Determination of light and strange quark masses from full lattice QCD. Phys. Lett., B639:307-311, 2006.

[76] M. Gockeler et al. Estimating the unquenched strange quark mass from the lattice axial Ward identity. Phys. Rev., D73:054508, 2006.

[77] C. Aubin et al. First determination of the strange and light quark masses from full lattice QCD. Phys. Rev., D70:031504, 2004.

[78] S. Aoki et al. Light hadron spectroscopy with two flavors of O(a)- improved dynamical quarks. Phys. Rev., D68:054502, 2003.

[79] S. Aoki et al. Light hadron spectrum and quark masses from quenched lattice QCD. Phys. Rev., D67:034503, 2003.

[80] Damir Becirevic, Vittorio Lubicz, and Cecilia Tarantino. Continuum determi- 
nation of light quark masses from quenched lattice QCD. Phys. Lett., B558:69$78,2003$.

[81] Ting-Wai Chiu and Tung-Han Hsieh. Light quark masses, chiral condensate and quark-gluon condensate in quenched lattice QCD with exact chiral symmetry. Nucl. Phys., B673:217-237, 2003.

[82] S. Aoki et al. Quenched light hadron spectrum. Phys. Rev. Lett., 84:238-241, 2000 .

[83] Joyce Garden, Jochen Heitger, Rainer Sommer, and Hartmut Wittig. Precision computation of the strange quark's mass in quenched QCD. Nucl. Phys., B571:237-256, 2000.

[84] M. Gockeler et al. A lattice determination of light quark masses. Phys. Rev., D62:054504, 2000.

[85] Stephan Narison. Strange quark mass from $e^{+} e^{-}$revisited and present status of light quark masses. Phys. Rev., D74:034013, 2006.

[86] J. G. Korner, F. Krajewski, and A. A. Pivovarov. Determination of the strange quark mass from Cabibbo suppressed tau decays with resummed perturbation theory in an effective scheme. Eur. Phys. J., C20:259-269, 2001.

[87] K. G. Chetyrkin, Johann H. Kuhn, and A. A. Pivovarov. Determining the strange quark mass in Cabibbo suppressed tau lepton decays. Nucl. Phys., B533:473-493, 1998.

[88] BaBar WebSite: http://www.slac.stanford.edu/BFROOT. Internal Website (BaBar Documentation) Last Accessed: 2008. 
[89] ed. Harrison, P. F. and ed. Quinn, Helen R. The BaBar physics book: Physics at an asymmetric B factory. Papers from Workshop on Physics at an Asymmetric B Factory (BaBar Collaboration Meeting), Rome, Italy, 11-14 Nov 1996, Princeton, NJ, 17-20 Mar 1997, Orsay, France, 16-19 Jun 1997 and Pasadena, CA, 22-24 Sep 1997.

[90] D. Boutigny et al. BaBar technical design report. SLAC-R-0457.

[91] B. Aubert et al. The BaBar detector. Nucl. Instrum. Meth., A479:1-116, 2002.

[92] J. D. Jackson. Classical Electrodynamics' 3rd Ed. John Wiley \& Sons, Inc. United States of America, 2001.

[93] http://www.bctf.bc.ca/bcscta/hebden\%20demos/chemical\%20inventory.pdf.

[94] W. Menges. The BABAR muon system upgrade. IEEE Nucl. Sci. Symp. Conf. Rec., 5:1470-1474, 2006.

[95] et al R. Bartoldus. Trigger and Filter Document for Run1. BaBar Analysis Document 194, April 9, 2002.

[96] B. F. L. Ward, S. Jadach, and Z. Was. Precision calculation for $e^{+} e^{-} \rightarrow 2 f$ : The K K MC project. Nucl. Phys. Proc. Suppl., 116:73-77, 2003.

[97] S. Jadach, B. F. L. Ward, and Z. Was. The precision Monte Carlo event generator KK for two- fermion final states in e+ e- collisions. Comput. Phys. Commun., 130:260-325, 2000.

[98] Z. Was. TAUOLA the library for tau lepton decay, and KKMC/KORALB/KORALZ/... status report. Nucl. Phys. Proc. Suppl., 98:96-102, 2001. 
[99] D. M. Asner et al. Resonance structure of $\tau^{-} \rightarrow K^{-} \pi^{+} \pi^{-} \nu_{\tau}$ decays. Phys. Rev., D62:072006, 2000.

[100] Markus Finkemeier and Erwin Mirkes. Tau decays into kaons. Z. Phys., C69:243-252, 1996.

[101] Torbjorn Sjostrand. The Lund Monte Carlo for Jet Fragmentation and $e^{+} e^{-}$ Physics: Jetset Version 6.2. Comput. Phys. Commun., 39:347-407, 1986.

[102] Torbjorn Sjostrand and Mats Bengtsson. The Lund Monte Carlo for Jet Fragmentation and $e^{+} e^{-}$Physics. Jetset Version 6.3: An Update. Comput. Phys. Commun., 43:367, 1987.

[103] K. Amako. Present status of Geant4. Nucl. Instrum. Meth., A453:455-460, 2000 .

[104] S. Brandt, C. Peyrou, R. Sosnowski, and A. Wroblewski. The Principal axis of jets. An Attempt to analyze high- energy collisions as two-body processes. Phys. Lett., 12:57-61, 1964.

[105] Edward Farhi. A QCD Test for Jets. Phys. Rev. Lett., 39:1587-1588, 1977.

[106] Swagato Banerjee, Bolek Pietrzyk, J. Michael Roney, and Zbigniew Was. Tau and muon pair production cross-sections in electron-positron annihilations at $\sqrt{s}=10.58 \mathrm{GeV}$. Phys. Rev., D77:054012, 2008.

[107] Luminosity Measurement for Runs 1,2 and 3 Data sample using Release 12 and SP5 Simulation. BaBar Analysis Document 1312 V1, SEPT-2005.

[108] Luminosity Measurement for Run I and II using SP4 Simulation. BaBar Analysis Document 535 V2, 25-NOV-2003. 
[109] Zinkoo Yun. Study of $e^{+} e^{-} \rightarrow \mu^{+} \mu^{-}(\gamma)$ events at BABAR . MSc Dissertation, University of Victoria, 2005.

[110] B. Aubert et al. A measurement of the total width, the electronic width, and the mass of the $\Upsilon(10580)$ resonance. Phys. Rev., D72:032005, 2005.

[111] Robert Kowaleski Ian M. Nugent, J. M. Roney. Tau31 Tracking Efficiency Study. BaBar Analysis Document 931, Version 02, 13-JUL-2004.

[112] http://www.slac.stanford.edu/BFROOT/www/Physics/TrackEfficTaskForce/ TauEff/R22/TauEff.html. Internal Website (BaBar Documentation) Last Accessed: 2008.

[113] http://www.slac.stanford.edu/BFROOT/www/Physics/TrackEfficTaskForce/ TauEff/R22/Tau31ExpertRecipe.html. Internal Website (BaBar Documentation) Last Accessed: 2008.

[114] Aaron Roodman Mark Tiller Allen, Mitchell T. Naisbit. A Study of Pi-zero Efficiency. BaBar Analysis Document 870, Version 03, 01-NOV-2004.

[115] K. Arms et al. Study of $\tau$ decays to four-hadron final states with kaons. Phys. Rev. Lett., 94:241802, 2005.

[116] R. A. Briere et al. Branching fractions of $\tau$ leptons decays to three charged hadrons. Phys. Rev. Lett., 90:181802, 2003.

[117] R. Barate et al. Three-prong $\tau$ decays with charged kaons. Eur. Phys. J., C1:65-79, 1998.

[118] G. Lopez Castro and D. A. Lopez Falcon. VMD description of $\tau \rightarrow(\omega, \phi) \pi^{-} \nu_{\tau}$ decays and the $\omega-\phi$ mixing angle. Phys. Rev., D54:4400-4402, 1996. 
[119] H. Albrecht et al. SEARCH FOR HADRONIC b $\rightarrow$ u DECAYS. Phys. Lett., B241:278-282, 1990.

[120] Roger Barlow. A calculator for confidence intervals. Comput. Phys. Commun., 149:97-102, 2002

[121] Andreas Hocker and Vakhtang Kartvelishvili. SVD Approach to Data Unfolding. Nucl. Instrum. Meth., A372:469-481, 1996.

[122] Volker Blobel. An unfolding method for high energy physics experiments. 2002. hep-ex/0208022.

[123] Johann H. Kuhn. Tau kinematics from impact parameters. Phys. Lett., B313:458-460, 1993.

[124] K. Maltman, C. E. Wolfe, S. Banerjee, J. M. Roney, and I. M. Nugent. Status of the Hadronic Tau Determination of $\left|V_{u s}\right|$. 2008. hep-ph 0807.3195.

[125] F. Ambrosino et al. $V_{u} s$ and lepton universality from kaon decays with the KLOE detector. JHEP, 04:059, 2008.

[126] Elvira Gamiz, Matthias Jamin, Antonio Pich, Joaquim Prades, and Felix Schwab. Theoretical progress on the $V_{u s}$ determination from $\tau$ decays. PoS, KAON:008, 2008.

[127] Swagato Banerjee. Measurement of $\left|V_{u s}\right|$ using hadronic tau decays from BaBar \& Belle. PoS KAON, 2006:009, 2006.

[128] P. Avery et al. Search for $\phi$ mesons in $\tau$ lepton decay. Phys. Rev., D55:1119$1123,1997$. 
[129] K. Abe et al. The first observation of $\tau^{ \pm} \rightarrow \phi K^{ \pm} \nu_{\tau}$ decay. Phys. Lett., B643:510,2006 .

[130] A. Flores-Tlalpa and G. Lopez-Castro. $(\omega, \phi) P^{-}$decays of tau leptons. Phys. Rev., D77:113011, 2008.

[131] Myungkee Sung. A Search for $\phi$ mesons in the decay of the $\tau$ lepton. $\mathrm{PhD}$ Dissertation, The Ohio State University (1996), UMI-97-10664 , and references within.

[132] M. Davier, S. Descotes-Genon, A. Hocker, B. Malaescu, and Z. Zhang. The Determination of $\alpha_{s}$ from Tau Decays Revisited. 2008. hep-ph 0803.0979.

[133] B. Aubert et al. Measurements of $e+e-\rightarrow K^{+} K^{-} \eta, K^{+} K^{-} \pi^{0}$ and $K_{s} K^{+} \pi^{-}$ Cross Sections Using Initial State Radiation Events. Phys. Rev., D77:092002, 2008.

[134] SuperB: A high-luminosity heavy flavour factory. Conceptual design report. Pisa, Italy: INFN (2007) 453 p. www.pi.infn.it/SuperB/?q=CDR.

[135] A. G. Akeroyd et al. Physics at super B factory. 2004.

[136] D. I. Britton et al. Improved search for massive neutrinos in $\pi^{+} \rightarrow e^{+} \nu$ decay. Phys. Rev., D46:885-887, 1992.

[137] G. Czapek et al. Branching ratio for the rare pion decay into positron and neutrino. Phys. Rev. Lett., 70:17-20, 1993.

[138] Roger Decker and Markus Finkemeier. Short and long distance effects in the decay $\tau \rightarrow \pi \nu_{\tau}(\gamma)$. Nucl. Phys., B438:17-53, 1995. 
[139] Luca Fiorini. Measurement of the ratio RK between the Branching Ratio of $K^{ \pm} \rightarrow e^{ \pm} \bar{\nu}_{e}$ and $K^{ \pm} \rightarrow \mu^{ \pm} \bar{\nu}_{\mu}$ dedays at NA48 . PoS, HEP2005:288, 2006

[140] Venelin Kozhuharov. Testing mu-e universality with $K_{l 2}$ decays. PoS, KAON:049, 2008.

[141] F. Ambrosino et al. Preliminary measurement of $\Gamma\left(K^{ \pm} \rightarrow e^{ \pm} \bar{\nu}_{e}\right) / \Gamma\left(K^{ \pm} \rightarrow\right.$ $\left.\mu^{ \pm} \bar{\nu}_{\mu}\right)$ at KLOE. PoS, KAON:050, 2008.

[142] Matteo Palutan. Experimental review on $\left|V_{u s}\right|$ extraction from kaon decays. PoS, KAON:020, 2008.

[143] Rainer Wanke. Lepton Universality Tests with Kaons. PoS, KAON:051, 2008.

[144] J. Alcaraz et al. A combination of preliminary electroweak measurements and constraints on the standard model. 2006. hep-ex/0612034.

[145] Roger Decker and Markus Finkemeier. Radiative $\tau$ decays with one pseudoscalar meson. Phys. Rev., D48:4203-4215, 1993 [Addendum-ibid. D 50, 7079 (1994)].

[146] C. A. Blocker et al. A study of the decay $\tau^{-} \rightarrow \pi^{-} \nu_{\tau}$. Phys. Lett., B109:119, 1982.

[147] A. Drescher et al. The ARGUS electron - photon calorimeter. 3. electron hadron seperation. Nucl. Instrum. Meth., A237:464-474, 1985.

[148] http://www.slac.stanford.edu/BFROOT/www/Physics/Tools/Pid/Selectors/ r22a/selectors.html. Internal Website (BaBar Documentation) Last Accessed: 2008. 
[149] Thorsten Brandt. Likelihood-based Electron Identification. BaBar Analysis Document 396, Version 01, 27-MAR-2002.

[150] Urs Langenegger. Cut-based Electron Identification. BaBar Analysis Document 90, Version 05, 09-MAY-2001.

[151] Luca Lista Francesco Fabozzi. Muon Identification in the BaBar Experiment. BaBar Analysis Document 60, Version 01, 21-JUN-2000.

[152] Joerg Stelzer. Kalman based reconstruction of IFR tracks. BaBar Analysis Document 799, Version 01, 09-JUN-2004.

[153] Ajit Mohapatra Henry Band, Jonathan Hollar. Studies of A Neural Net Based Muon Selector for the BaBar Experiment. BaBar Analysis Document 474, Version 03, 31-AUG-2004.

[154] Stefan M. Spanier Giampiero Mancinelli. Kaon Selection at BaBar. BaBar Analysis Document 116, Version 01, 27-JUL-2001.

[155] Aaron Roodman (BaBar Collaboration). http://www.slac.stanford.edu/ BFROOT/www/Physics/Tools/Pid/Hadrons/Description_of_the_LH_selectors.html. Internal Website (BaBar Documentation) Last Accessed: 2008.

[156] Stephanie Majewski (BaBar Collaboration). http://www.slac.stanford.edu/ BFROOT/www/Physics/Tools/Pid/Hadrons/pid_summarytable.html. Internal Website (BaBar Documentation) Last Accessed: 2008. 


\section{Appendix A}

\section{Particle Identification}

In particle physics, subatomic particles are identified through their interactions with the detector. A summary of the detector parameters utilized to select electrons, muons, pions and kaons in this work are displayed in Table A.1 and A.2.

\begin{tabular}{|c|c|}
\hline Variable & Description \\
\hline & Tracking Detectors Variables (SVT and DCH) \\
\hline$\frac{d E}{d x}$ & The mean energy loss per unit length \\
\hline $\mathrm{P}$ & The momentum of a track $(\mathrm{GeV} / \mathrm{c})$ \\
\hline $\mathrm{P}_{t}$ & $\begin{array}{l}\text { The momentum of a track in the plane perpendicular to the beam axis } \\
(\mathrm{GeV} / \mathrm{c})\end{array}$ \\
\hline$q$ & The charge of the track \\
\hline & DIRC Variables \\
\hline $\mathrm{N}_{\gamma}$ DIRC & The number of DIRC photons \\
\hline$\theta_{c}$ & The Cherenkov angle (rad) \\
\hline$\theta_{c}^{x}$ & The Cherenkov angle for the $\mathrm{x}$ particle hypothesis (rad) \\
\hline & EMC Variables \\
\hline $\mathrm{E}_{\text {raw }}$ & The energy deposited in the EMC (GeV) \\
\hline$\phi_{E M C}$ & The $\phi$ coordinate of the extrapolated track position in the EMC (rad) \\
\hline$\phi_{\text {cluster }}$ & The $\phi$ coordinate of the position of a cluster in the EMC (rad) \\
\hline $\mathrm{N}_{\text {crystal }}$ & The number of EMC crystals associated with a track \\
\hline Lat & The Lateral moment in the EMC [147] \\
\hline $\mathrm{A}_{42}$ & The 42nd Zernike moment in the EMC \\
\hline
\end{tabular}

Table A.1: The definition of the variables used for particle identification Part 1. 


\begin{tabular}{|c|c|}
\hline \multicolumn{2}{|r|}{ IFR Variables } \\
\hline $\mathrm{N}_{L}$ & The number of IFR layers that registered a hit \\
\hline$\lambda$ & The number of interaction lengths ${ }^{1}$ transversed by the track \\
\hline$\lambda_{\exp }$ & $\begin{array}{l}\text { The number of interaction lengths expected to be transversed if the track } \\
\text { is a muon }\end{array}$ \\
\hline$\chi_{\text {fit }}^{2}$ & The $\chi^{2} /$ ndof $^{2}$ for the fit applied to the IFR cluster \\
\hline$\chi_{t r k}^{2}$ & The $\chi^{2} /$ ndof between the extrapolated track and the clusters in the IFR \\
\hline $\mathrm{T}_{c}$ & The continuity of a track in the IFR \\
\hline $\bar{m}$ & The mean number of IFR strips hit per layer \\
\hline$\sigma_{m}$ & The standard deviation on the mean number of IFR strips hit per layer \\
\hline
\end{tabular}

Table A.2: The definition of the variables used for particle identification Part 2.

\section{A.1 Electron selectors}

In this work, there are two electron selectors. One which is used to identify electrons and one which is used to reject electrons. The electron selector, which identifies electrons, has an efficiency of above $90 \%$ and a pion fake rate between 0.05-0.01\% [148]. This selector is referred to as the "PidLHElectrons" selector within the BABAR Collaboration. This selector uses information from the EMC, DIRC and the DCH to identify electrons with the likelihood method after some initial pre-selection. The initial pre-selection requires that the energy deposited in the EMC is consistent with originating from an electron and is described in Table A.3. For the EMC, the Probability Density Function (PDF) are used to model the $E_{\text {raw }} / P$ for the deposited energy, the lateral moment of the deposited energy, and the longitudinal moment of the deposited energy. These PDFs are determined in bins of momentum and $\cos (\theta)$ for the electron and pion control samples and in bins of momentum for the kaon and protons which have lower statistics. For a given track, the probability is determined from the PDFs by a bilinear extrapolation between the PDF values for the two clos-

\footnotetext{
${ }^{1}$ An interaction length is the mean distance between inelastic collisions.

${ }^{2}$ The number of degrees of freedom for a $\chi^{2}$ fit is the number of points minus the number of parameters in the fit.
} 
est bins. The PDFs for the $E_{\text {raw }} / P$ for the deposited energy are determined using a Gaussian like shape which has an exponential decay in the tails for the electrons and a double Gaussian for the hadrons. A similar procedure is used to determine the probabilities of the lateral moment and longitudinal moment of the deposited energy from the track, except that a double Gaussian is used for the electron PDF and the longitudinal moment is binned in terms of transverse momentum instead of the momentum. However, because of correlations in the hadronic shower shape, the $E_{\text {raw }} / P$ for the deposited energy and the lateral moment of the deposited energy are correlated. Therefore, two dimensional PDFs are constructed to include the correlations. For the $\theta_{C}$ angle, the probability is used in the likelihood function only if $N_{\gamma}$ DIRC $>6$ and the momentum is less than $1.5 \mathrm{GeV} / c$. The PDFs for the $\theta_{C}$ angle, which are binned in $\cos (\theta)$, uses a triple Gaussian function for the pions, a double Gaussian function for the electrons and a Gaussian function for the kaons and protons, where the parameters of the Gaussians are fitted functions that take into account affects such as the momentum dependence, delta ray production and particle decay. The PDF, used for the DCH likelihood function, is a normalized Gaussian PDF where the mean value and the momentum dependent relative uncertainty are determined from the $\mathrm{dE} / \mathrm{dx}$ calibration. In this calibration, the resolution is determined in terms of its dependence on the $d E / d x$, the number of hits on the DCH wires, the transverse momentum and the polar angle. From the likelihoods of the individual parameters, a total likelihood function is determined for each of these five variables for the four particle hypotheses, $x=e, \pi, K, p$, and a likelihood fraction is determined,

$$
f_{L}=\frac{p_{e} L_{e}}{p_{e} L_{e}+p_{\pi} L_{\pi}+p_{K} L_{K}+p_{p} L_{p}}
$$

where $p_{x}$ are prior probabilities that a track is selected as a electron if it passes 
pre-selection [149].

\begin{tabular}{|c|}
\hline Preselection Cuts \\
\hline $500<\frac{d E}{d x}<1000$ \\
$0.5 c<\frac{\mathrm{E}_{\text {raw }}}{\mathrm{P}}<1.5 c$ \\
$4 \leq \mathrm{N}_{\text {crystal }}$ \\
$N_{\gamma \text { DIRC }}>6$ and $P<1.5 \mathrm{GeV} / c$ for DIRC to be included \\
Selection Cuts \\
$f_{L}>0.95$ \\
\hline
\end{tabular}

Table A.3: The electron selector criteria "PidLHElectrons" [149].

For rejecting electrons, a selection criteria with a slightly higher efficiency is used. In the BABAR Collaboration, this selector is referred to as the "eMicroVeryTight" selector. The selection criteria of this selector are summarized in Table A.4. The $\Delta \phi=q\left(\phi_{E M C}-\phi_{\text {cluster }}\right)$ cut referred to in Table A.4 is a geometric cut in the $\Delta \phi$ vs $\mathrm{P}_{t}$ plane that separates electrons and pions at $\mathrm{P}_{t}[150]$. An illustration of this cut on both electron and pion distributions can be seen in Figure A.1.

\begin{tabular}{|c|}
\hline Selection Cuts \\
\hline $540<\frac{d E}{d x}<860$ \\
$0.89 c<\frac{\mathrm{E}_{\text {raw }}}{\mathrm{P}}<c 1.2$ \\
$3<\mathrm{N}_{\text {crystal }}$ \\
$0<$ Lat $<0.6$ \\
$-10<\mathrm{A}_{42}<0.11$ \\
$\left|\theta_{c}-\theta_{c}^{e}\right|<3 \sigma_{\theta_{c}}$ if $\mathrm{N}_{\gamma \text { DIRC }}>10$ \\
$\Delta \phi=q\left(\phi_{E M C}-\phi_{\text {cluster }}\right)$ cut \\
\hline
\end{tabular}

Table A.4: The electron criteria used for the electron veto "eMicroVeryTight" [150] . 

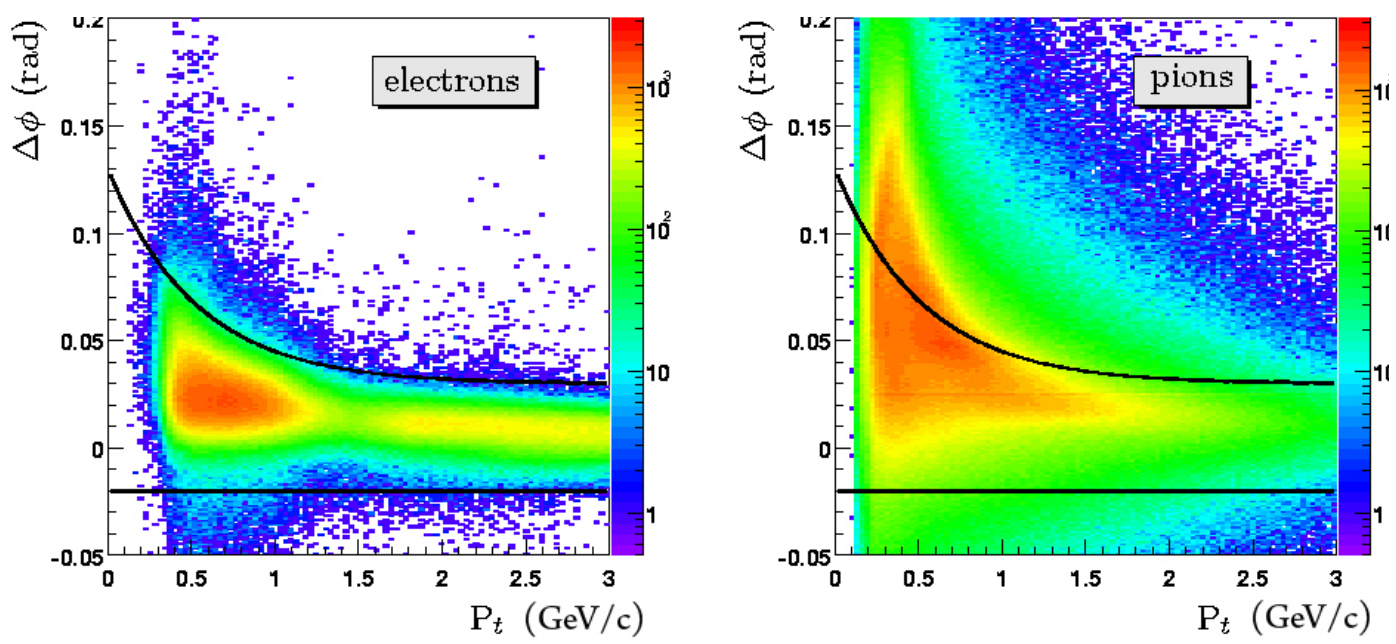

Figure A.1: $\Delta \phi=q\left(\phi_{E M C}-\phi_{\text {cluster }}\right)$ as a function of $\mathrm{P}_{t}$ with the $\Delta \phi$ cut employed in the electron veto superimposed for both electron (left) and pion (right) MC [150]. 


\section{A.2 Muon Selectors}

To select muons, a cut based selector, which employs information from the EMC and the IFR, is used for the $\tau^{-} \rightarrow h^{-} h^{-} h^{+} \nu_{\tau}$ analysis. This selector has a muon selection efficiency of approximately $\sim 60 \%$ and a pion percent mis-id rate of $\sim 5 \%$ [148]. This selector is referred to as the "muMicroTight" selector in the BABAR Collaboration [151,152]. Table A.5 summarizes the muon selection criteria.

\begin{tabular}{|c|}
\hline Selection Cuts \\
\hline $0.05 \mathrm{GeV}<\mathrm{E}_{\text {raw }}<0.4 \mathrm{GeV}$ for tracks in the EMC with $\theta<2.45 \mathrm{rads}$ \\
$\mathrm{N}_{L} \geq 2$ \\
$\lambda>2.2$ \\
$\left|\lambda-\lambda_{\text {exp }}\right|<1$ \\
$\chi_{\text {fit }}^{2}<3$ \\
$\chi_{\text {trk }}^{2}<5$ \\
$\mathrm{~T}_{c}>0.3$ for $0.3 \mathrm{rad}<\theta<1.0 \mathrm{rad}$ \\
$\bar{m}<8$ \\
$\sigma_{m}<4$ \\
\hline
\end{tabular}

Table A.5: The muon selection criteria for the $\tau^{-} \rightarrow h^{-} h^{-} h^{+} \nu_{\tau}$ analysis $[151,152]$.

In the $\frac{\mathcal{B}\left(\tau^{-} \rightarrow \mu^{-} \nu_{\tau} \bar{\nu}_{\mu}\right)}{\mathcal{B}\left(\tau^{-} \rightarrow e^{-} \nu_{\tau} \bar{\nu}_{e}\right)}$ measurement, a neural network selector was used to identify the signal muon. This selector has a muon selection efficiency of approximately $\sim 70 \%$, a pion percent mis-id rate of $\sim 2 \%$ and kaon fake rate of $\sim 2 \%$ [148] and is referred to as the "muNNTight" in the BABAR Collaboration. This selector uses the neural network application in ROOT. The input layer for the muon selector neural network contains eight layers corresponding to the input parameters: $\lambda_{\exp }-\lambda, \chi_{t r k}^{2}$, $\sigma_{m}, T_{C}, E_{c a l}, \lambda, \bar{m}$ and $\chi_{f i t}^{2}$. The input layer connects to a hidden layer with 16 nodes that then output to a single node in the output layer. Each node modifies the input so that it receives into an output using a transfer function which depends on the input activity $\left(A_{i}\right)$ and the weight $\left(W_{i j}\right)$. The transfer function employed in this 
neural net is the "sigmoid" function,

$$
f\left(A_{i}, W_{i j}\right)=\frac{1}{1-e^{-\sum A_{i} W_{i j}}} .
$$

To account for variation in the behaviour of the detector as a function of time, momentum and polar angle, the neural network is trained in bins of momentum, $\theta$ and time. The training utilized a muon control sample from $e^{+} e^{-} \rightarrow \mu^{+} \mu^{-} \gamma$ events and a pion control sample from $\tau^{-} \rightarrow \pi^{-} \pi^{-} \pi^{+} \nu_{\tau}$ events with a lepton tag [153]. These control samples are split into two sets, one to train the neural network and another to validate the neural network. After training and validation, the cut placed on the output of the neural network is tuned to have a constant muon selection efficiency of $70 \%$ for this selector [153].

To reject muons in the measurement of $\frac{\mathcal{B}\left(\tau^{-} \rightarrow \pi^{-} \nu_{\tau}\right)}{\mathcal{B}\left(\tau^{-} \rightarrow e^{-} \nu_{\tau} \bar{\nu}_{e}\right)}$ a looser selection criteria with a substantially higher efficiency, which has been optimized to have a small pion fake rate, is required. This selector is referred to as the "muNNVeryLooseFakeRate" selector in the BABAR Collaboration. This selector has a muon selection efficiency of approximately $\sim 90 \%$, a pion percent mis-id rate of $\sim 5 \%$ and kaon fake rate of $\sim 10 \%$ [148]. This selector uses the same neural network as the "muNNTight" selector, but has the pion mis-id rate tuned to a constant rate of 5\% [153].

For the measurement of $\frac{\mathcal{B}\left(\tau^{-} \rightarrow K^{-} \nu_{\tau}\right)}{\mathcal{B}\left(\tau^{-} \rightarrow e^{-} \nu_{\tau} \bar{\nu}_{e}\right)}$ a looser selection criteria with a substantially higher efficiency was used to reject muons. This selector is referred to as the "muMicroLoose" selector in the BABAR Collaboration. This selector has a muon selection efficiency of approximately $\sim 85 \%$, a pion percent mis-id rate of $\sim 10 \%$ and kaon fake rate of $\sim 10 \%[148,151,152]$. The selection criteria for this selector is summarized in Table A.6

The standard BABAR pion selector used in the $\frac{\mathcal{B}\left(\tau^{-} \rightarrow \pi^{-} \nu_{\tau}\right)}{\mathcal{B}\left(\tau^{-} \rightarrow e^{-} \nu_{\tau} \bar{\nu}_{e}\right)}$ analysis also has a muon veto in it, but it is not sufficient to reduce the contamination from $\tau^{-} \rightarrow$ 


\begin{tabular}{|c|}
\hline Selection Cuts \\
\hline $\mathrm{E}_{\text {raw }}<0.5(\mathrm{GeV})$ for tracks with clusters in the EMC \\
$\mathrm{N}_{L} \geq 2$ \\
$\lambda>2$ \\
$\left|\lambda-\lambda_{\text {exp }}\right|<2$ \\
$\chi_{\text {fit }}^{2}<4$ \\
$\chi_{\text {trk }}^{2}<7$ \\
$0.3 \mathrm{rad}<\theta<1.0 \mathrm{rad}$ \\
$\bar{m}<10$ \\
$\sigma_{m}<6$ \\
$\mathrm{~T}_{c}>0.2$ for \\
\hline
\end{tabular}

Table A.6: The muon selection criteria for the $\frac{\mathcal{B}\left(\tau^{-} \rightarrow K^{-} \nu_{\tau}\right)}{\mathcal{B}\left(\tau^{-} \rightarrow e^{-} \nu_{\tau}\right)}$ measurement $[151,152]$.

$\mu^{-} \nu_{\tau} \bar{\nu}_{\mu}$ events. This selector is referred to as "muMicroVeryTight" in the BABAR Collaboration. This selector has a muon selection efficiency of approximately $\sim 60 \%$, a pion percent mis-id rate of $\sim 1 \%$ and kaon fake rate of $\sim 0.5 \%[148,151,152]$. The selection criteria for this selector is summarized in Table A.7

\begin{tabular}{|c|}
\hline Selection Cuts \\
\hline $0.05<\mathrm{E}_{\text {raw }}<0.4(\mathrm{GeV})$ for tracks in the EMC with $\theta<2.45 \mathrm{rads}$ \\
$\mathrm{N}_{L} \geq 2$ \\
$\lambda>2.2$ \\
$\left|\lambda-\lambda_{\text {exp }}\right|<0.8$ \\
$\chi_{\text {fit }}^{2}<3$ \\
$\chi_{\text {trk }}^{2}<5$ \\
$\bar{m}<8$ \\
$\sigma_{m}<4$ \\
$\mathrm{~T}_{c}>0.34$ for $0.3 \mathrm{rad}<\theta<1.0 \mathrm{rad}$ \\
\hline
\end{tabular}

Table A.7: The muon veto included in the standard BABAR pion selector [151,152]. 


\section{A.3 Kaon Selectors}

The kaon identification is based on a likelihood approach and is referred to as the "MicroVeryTightKaon" selector. The MicroVeryTightKaon selector utilizes information from the SVT, DCH and DIRC to obtain a total likelihood function upon which the selection criteria are applied. For the DCH and the SVT, the likelihood function is a normalized Gaussian PDF, where the mean value and the momentum dependent relative uncertainty are determined from the $\mathrm{dE} / \mathrm{dx}$ calibration. The DIRC likelihood consists of two components: $g_{i}$ the normalized Gaussian PDF for the Cherenkov angle, where the mean value is the theoretical prediction for the given mass hypothesis and the uncertainty is determined from the fit of the Cherenkov angle; and $p_{i}$ the normalized Poisson PDF for the fitted number of photons signal with background and the expected number of photons with background. The $\mathrm{dE} / \mathrm{dx}$ information from the SVT is considered when the momentum of the track is between $0.025 \mathrm{GeV} / c<P<0.6 \mathrm{GeV} / c$ or $P>1.5 \mathrm{GeV} / c$ and there are at least three $\mathrm{dE} / \mathrm{dx}$ SVT hits. The DCH likelihood information is used when $0.090 \mathrm{GeV} / c<P<0.6 \mathrm{GeV} / c$ or $P>1.5 \mathrm{GeV} / c$ and there are at least ten $\mathrm{dE} / \mathrm{dx} \mathrm{DCH}$ hits. The likelihood information from the DIRC is utilized when $0.6 \mathrm{GeV} / c<P<10 \mathrm{GeV} / c$ and the expected number of photons for the electron hypothesis is greater than 0 and the $\theta_{C}$ vs $\mathrm{P}$ is located outside region $\mathrm{A}$, as defined in

Figure 4.2. The total likelihood function, $L_{x}=L_{x}^{D I R C} \times L_{x}^{D C H} \times L_{x}^{S V T}$, is determined for the $x=K, \pi, p$. To be identified as a pion the particle must satisfy the selection criteria in Table A.8. This selector has an efficiency of $\sim 80 \%$, a pion fake rate of $\sim 1-2 \%$, an electron fake rate of $<2 \%$ and a muon fake rate of $<1 \%[148,154]$. 


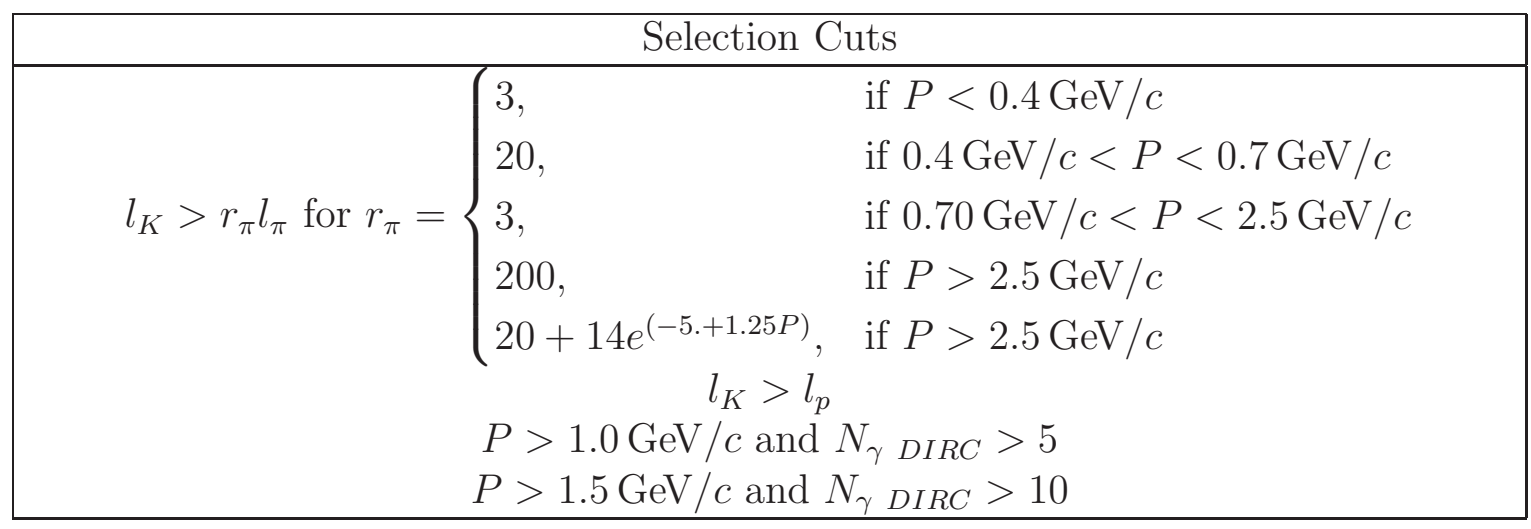

Table A.8: The Kaon Selection Criteria [154]. 


\section{A.4 Pion Selectors}

The pion selector, used to select pions in the $\tau \rightarrow \pi^{-} \nu$ measurement, is referred to as the "piLHVeryTight" selector in the BABAR Collaboration. As the name suggests, this selector is based on a likelihood approach. The total likelihood function, used in the pion selector, is determined from the likelihood functions from the individual detectors, namely the SVT, the DCH and the DIRC. For the DCH the likelihood function is a normalized Gaussian PDF where the mean value and the momentum dependent relative uncertainty are determined relative to the Bethe-Bloch parameterization. For the SVT the likelihood function is a normalized Bifurcated Gaussian PDF where the mean value and the momentum dependent relative uncertainty are determined relative to the Bethe-Bloch parameterization. For the DIRC, the likelihood is determined from the MC simulation in a binned likelihood table. This table is binned in terms of the $\mathrm{P}, \theta_{C}$ and $N_{\gamma}$ DIRC. The total likelihood function, $L_{x}=L_{x}^{D I R C} \times L_{x}^{D C H} \times L_{x}^{S V T}$, is determined for the $x=K, \pi, p$ [155]. To be identified as a pion the particle must satisfy the selection criteria in Table A.9. This selector has an efficiency of $\sim 85 \%$, a kaon fake rate of $\sim 2-4 \%$, an electron fake rate of $\sim 5 \%$, and a muon fake rate of $\sim 20 \%$ [148].

\begin{tabular}{|c|}
\hline Selection Cuts \\
\hline$L_{K} /\left(L_{K}+L_{\pi}\right)<0.2$ \\
$L_{p} /\left(L_{p}+L_{\pi}\right)<0.5$ \\
fail the "eLHTight" selector defined in Section A.1 \\
fail the "MicroVeryTight" selector defined in Section A.2 \\
\hline
\end{tabular}

Table A.9: The Pion Selection Criteria [156]. 


\section{Appendix B}

\section{Dalitz Weighting in the $\tau^{-} \rightarrow h^{-} h^{-} h^{+} \nu_{\tau}$ Analysis}

In the $\tau^{-} \rightarrow h^{-} h^{-} h^{+} \nu_{\tau}$ analysis Section 4.4, it was found that the kinematics of the decay structure needed to be corrected in the MC to model the data. The bin configurations used for this 3D Dalitz weighting of the MC were: $20 \times 20$ bins for the $\tau^{-} \rightarrow \pi^{-} \pi^{-} \pi^{+} \nu_{\tau}$ decay; $10 \times 10$ bins for the $\tau^{-} \rightarrow K^{-} \pi^{-} \pi^{+} \nu_{\tau}$ decay; $10 \times 10$ bins for the $\tau^{-} \rightarrow K^{-} \pi^{-} K^{+} \nu_{\tau}$ decay; 10 bins for the invariant mass only in the $\tau^{-} \rightarrow K^{-} K^{-} K^{+} \nu_{\tau}$ decay. The number of bins for each decay mode was limited by the statistics. Two iterations of the Dalitz weighting were applied to account for the cross-feed between the channels. Figure B.1 to B.4 show the $\tau^{-} \rightarrow h^{-} h^{-} h^{+} \nu_{\tau}$ $M_{123}, M_{13}$, and $M_{23}$ invariant mass distributions with the Dalitz weighted MC. The background subtracted Dalitz plots can be seen in Figure B.5. 

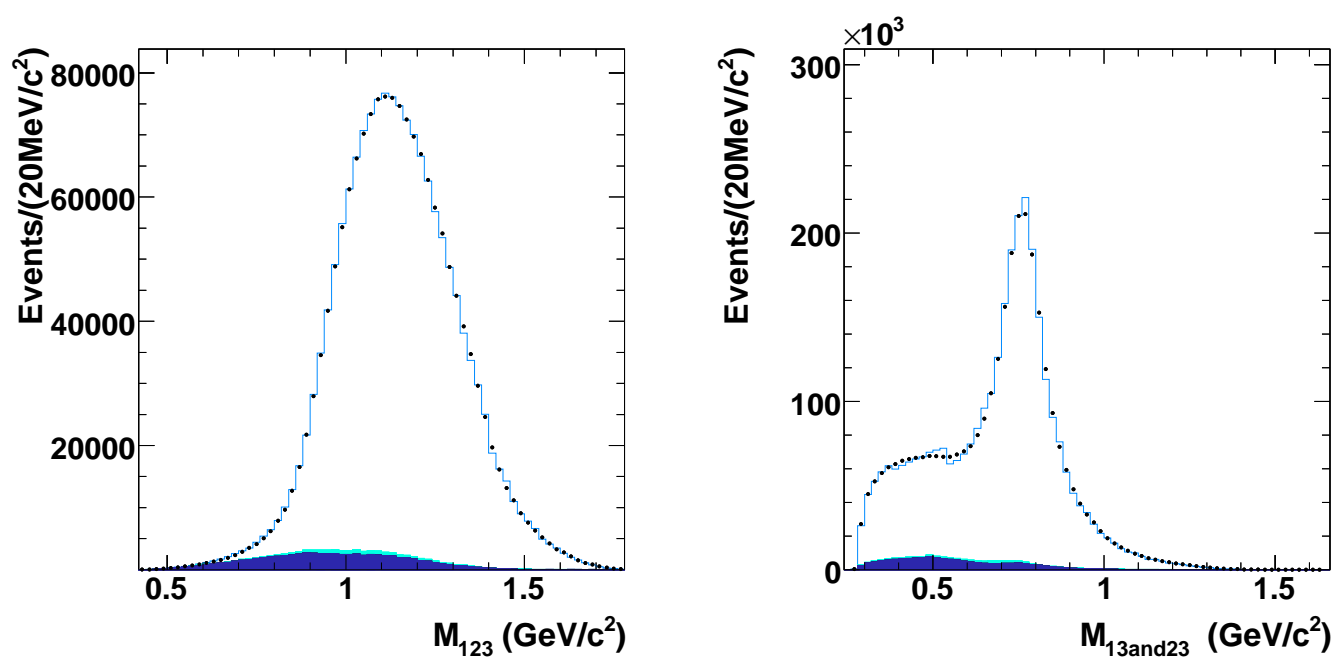

Figure B.1: The Raw $\tau^{-} \rightarrow \pi^{-} \pi^{-} \pi^{+} \nu_{\tau}$ invariant mass distributions for $M_{123}$ (left), $M_{13}$ and $M_{23}$ (right) with Dalitz Weighted MC. The data is represented by the points with the error bars representing the statistical uncertainty. The open histogram represents the signal MC for the respective channels, while the lightly shaded histogram represents the cross-feed from the other $\tau^{-} \rightarrow$ $h^{-} h^{-} h^{+} \nu_{\tau}$ channels. The remaining $\tau$ backgrounds and the non- $\tau$ backgrounds are represented by the dark histogram. 

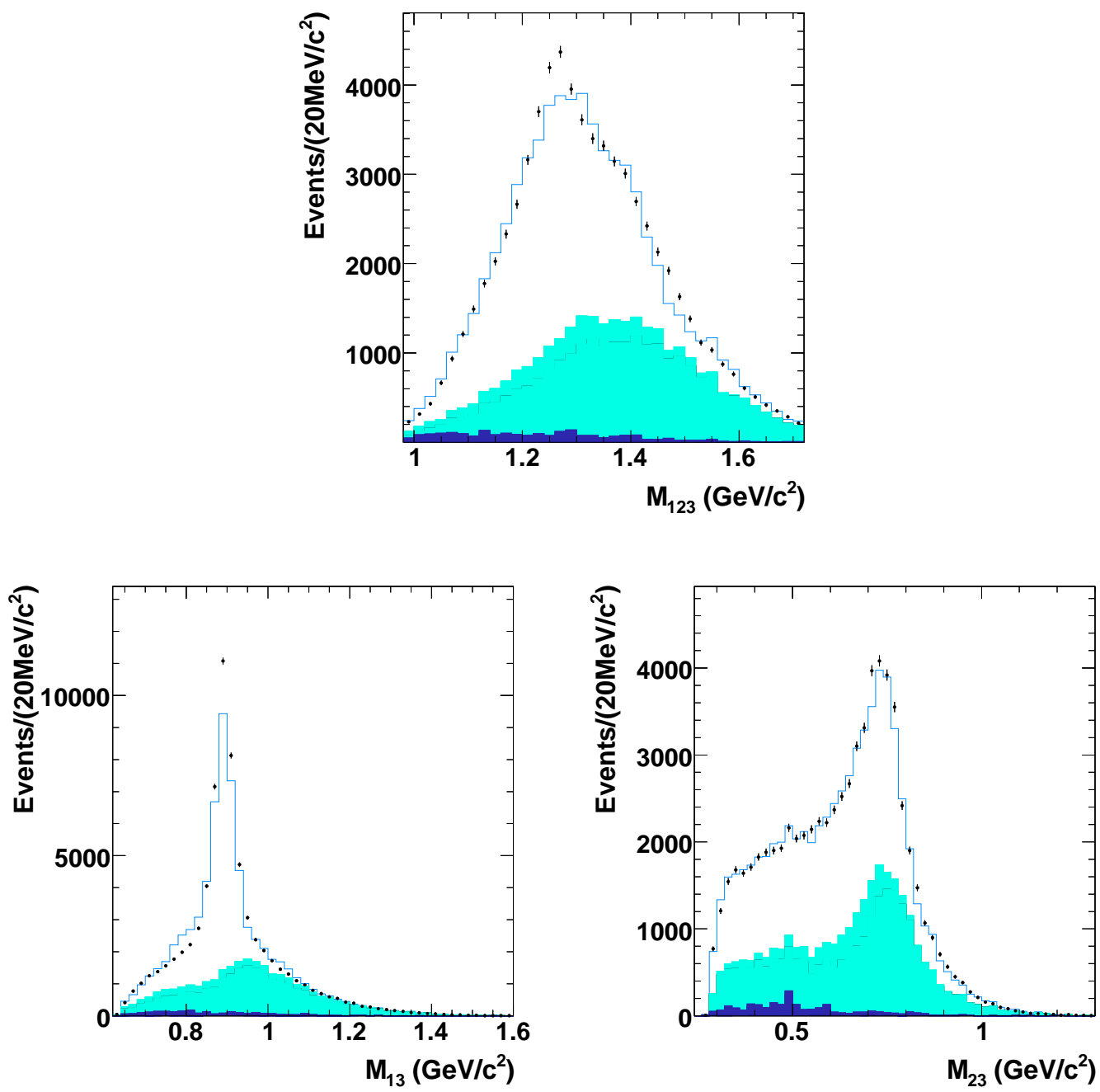

Figure B.2: The Raw $\tau^{-} \rightarrow K^{-} \pi^{-} \pi^{+} \nu_{\tau}$ invariant mass distributions for $M_{123}$ (top), $M_{13}$ (bottom-left), and $M_{23}$ (bottom-right with Dalitz Weighted MC). The data is represented by the points with the error bars representing the statistical uncertainty. The open histogram represents the signal MC for the respective channels, while the lightly shaded histogram represents the cross-feed from the other $\tau^{-} \rightarrow h^{-} h^{-} h^{+} \nu_{\tau}$ channels. The remaining $\tau$ backgrounds and the non- $\tau$ backgrounds are represented by the dark histogram. 

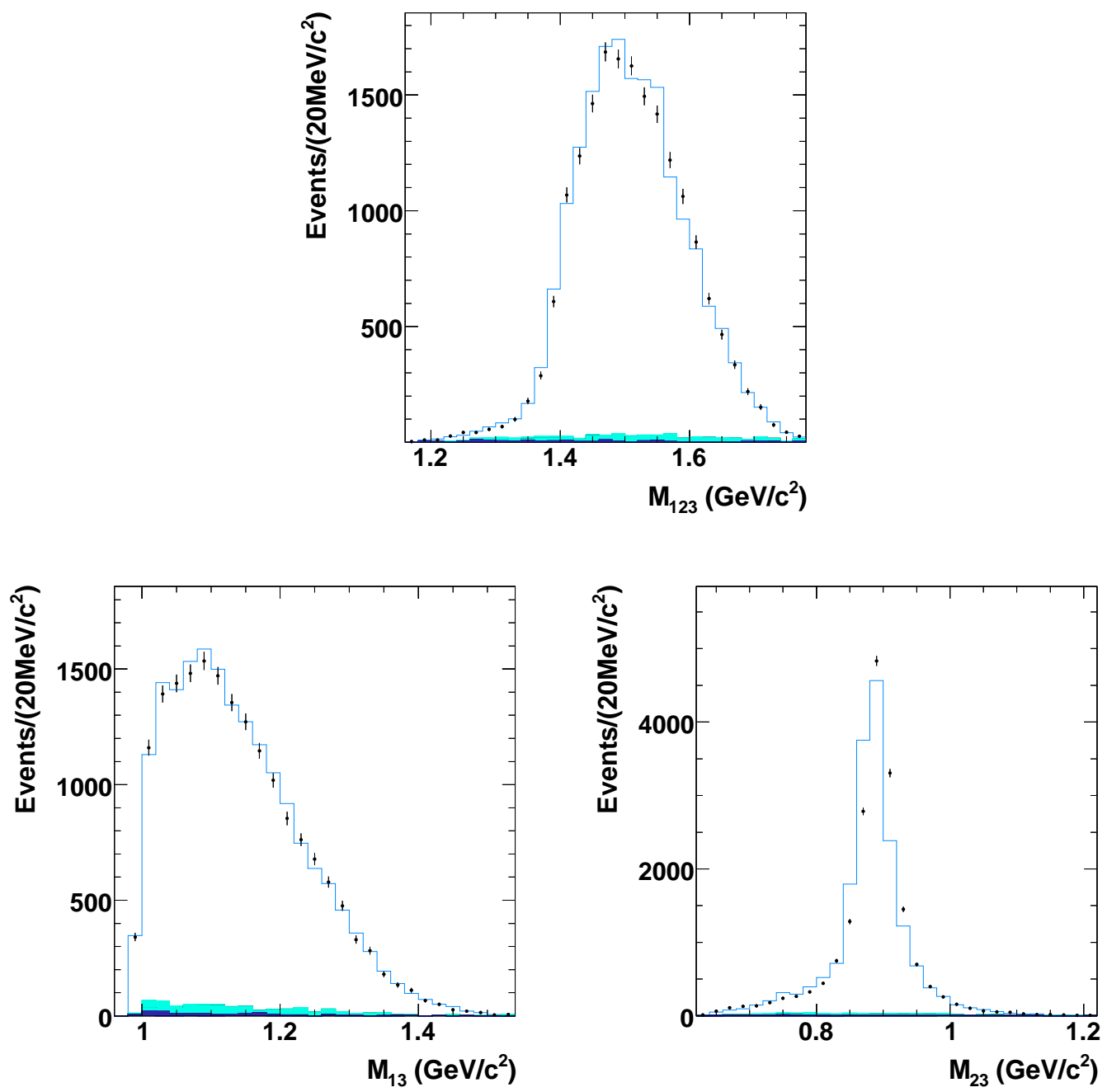

Figure B.3: The Raw $\tau^{-} \rightarrow K^{-} \pi^{-} K^{+} \nu_{\tau}$ invariant mass distributions for $M_{123}$ (top), $M_{13}$ (bottom-left), and $M_{23}$ (bottom-right) with Dalitz Weighted MC. The data is represented by the points with the error bars representing the statistical uncertainty. The open histogram represents the signal MC for the respective channels, while the lightly shaded histogram represents the cross-feed from the other $\tau^{-} \rightarrow h^{-} h^{-} h^{+} \nu_{\tau}$ channels. The remaining $\tau$ backgrounds and the non- $\tau$ backgrounds are represented by the dark histogram. 

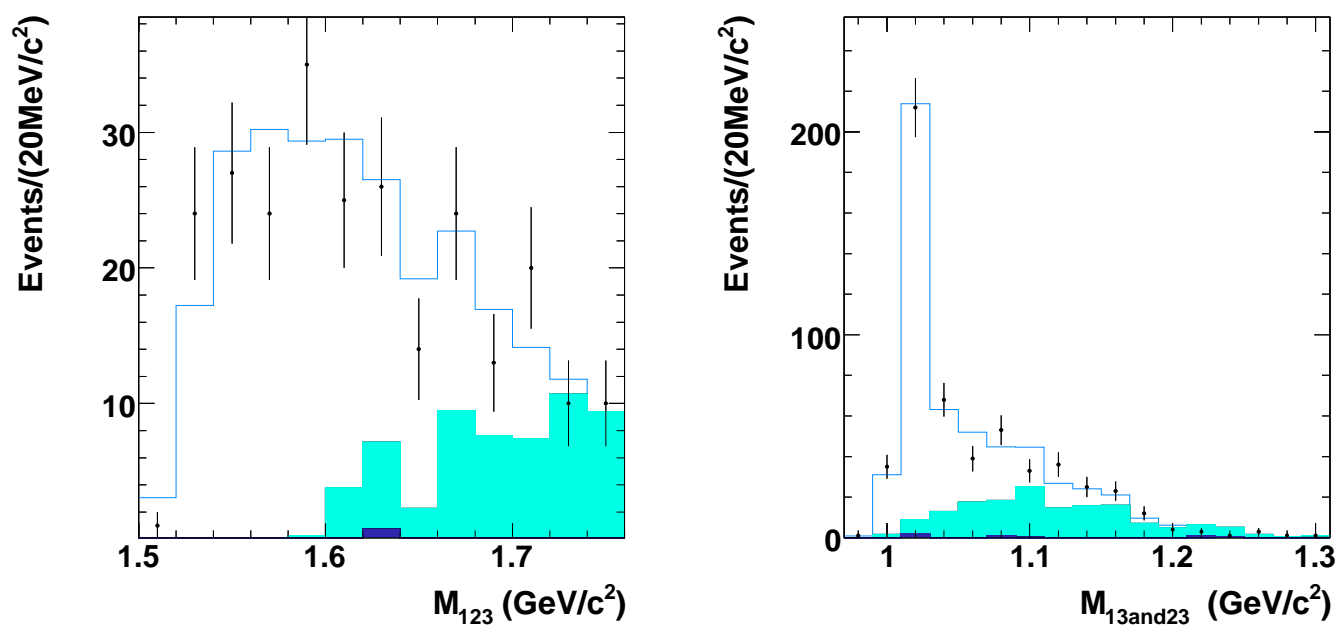

Figure B.4: The Raw $\tau^{-} \rightarrow K^{-} K^{-} K^{+} \nu_{\tau}$ invariant mass distributions for $M_{123}$ (left), $M_{13}$ and $M_{23}$ (right) with Dalitz Weighted MC. The data is represented by the points with the error bars representing the statistical uncertainty. The open histogram represents the signal MC for the respective channels, while the lightly shaded histogram represents the cross-feed from the other $\tau^{-} \rightarrow$ $h^{-} h^{-} h^{+} \nu_{\tau}$ channels. The remaining $\tau$ backgrounds and the non- $\tau$ backgrounds are represented by the dark histogram. 

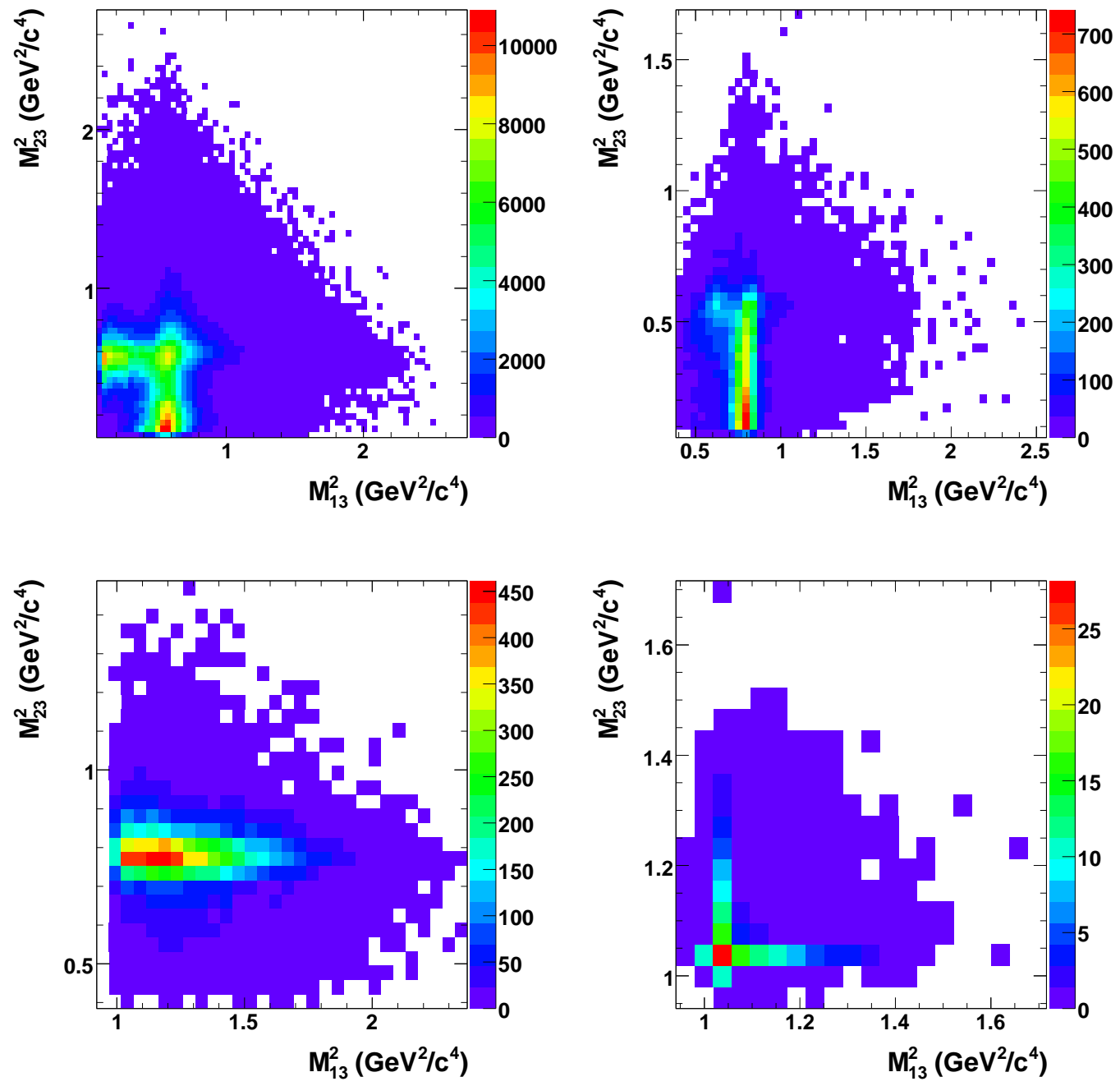

Figure B.5: The background subtracted Dalitz plots for: $\tau^{-} \rightarrow \pi^{-} \pi^{-} \pi^{+} \nu_{\tau}$ (top-left), $\tau^{-} \rightarrow K^{-} \pi^{-} \pi^{+} \nu_{\tau}$ (top-right), $\tau^{-} \rightarrow K^{-} \pi^{-} K^{+} \nu_{\tau}$ (bottom-left), and $\tau^{-} \rightarrow K^{-} K^{-} K^{+} \nu_{\tau}$ (bottom-right) channels. 
Appendix C

Unfolded $\tau^{-} \rightarrow \pi^{-} \pi^{-} \pi^{+} \nu_{\tau}$ Invariant Mass Spectra

C.1 The Unfolded $\tau^{-} \rightarrow \pi^{-} \pi^{-} \pi^{+} \nu_{\tau} M_{123}$ Invariant Mass Spectra 

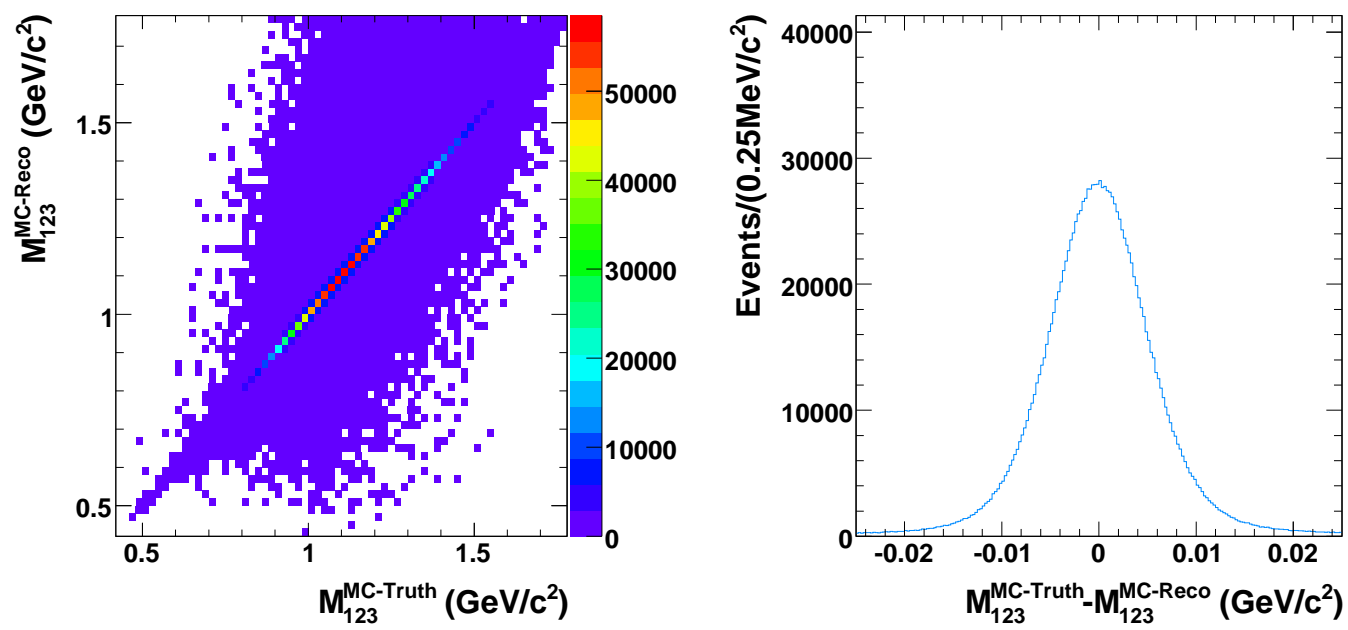

Figure C.1: A comparison of the $\tau^{-} \rightarrow \pi^{-} \pi^{-} \pi^{+} \nu_{\tau}$ MC Truth and reconstructed MC for the $M_{123}$ invariant mass spectra. The reconstructed MC $M_{123}$ invariant mass as a function of MC Truth $M_{123}$ invariant mass (left) is used to describe the response of the BABAR Detector in the unfolding of the $\tau^{-} \rightarrow \pi^{-} \pi^{-} \pi^{+} \nu_{\tau} M_{123}$ invariant mass spectrum. The reconstructed MC $M_{123}$ invariant mass minus the MC Truth $M_{123}$ invariant mass (right) illustrates the average response of the BABAR Detector for the $\tau^{-} \rightarrow \pi^{-} \pi^{-} \pi^{+} \nu_{\tau} M_{123}$ invariant mass spectrum. 
Appendix C. Unfolded $\tau^{-} \rightarrow \pi^{-} \pi^{-} \pi^{+} \nu_{\tau}$ Invariant Mass Spectra

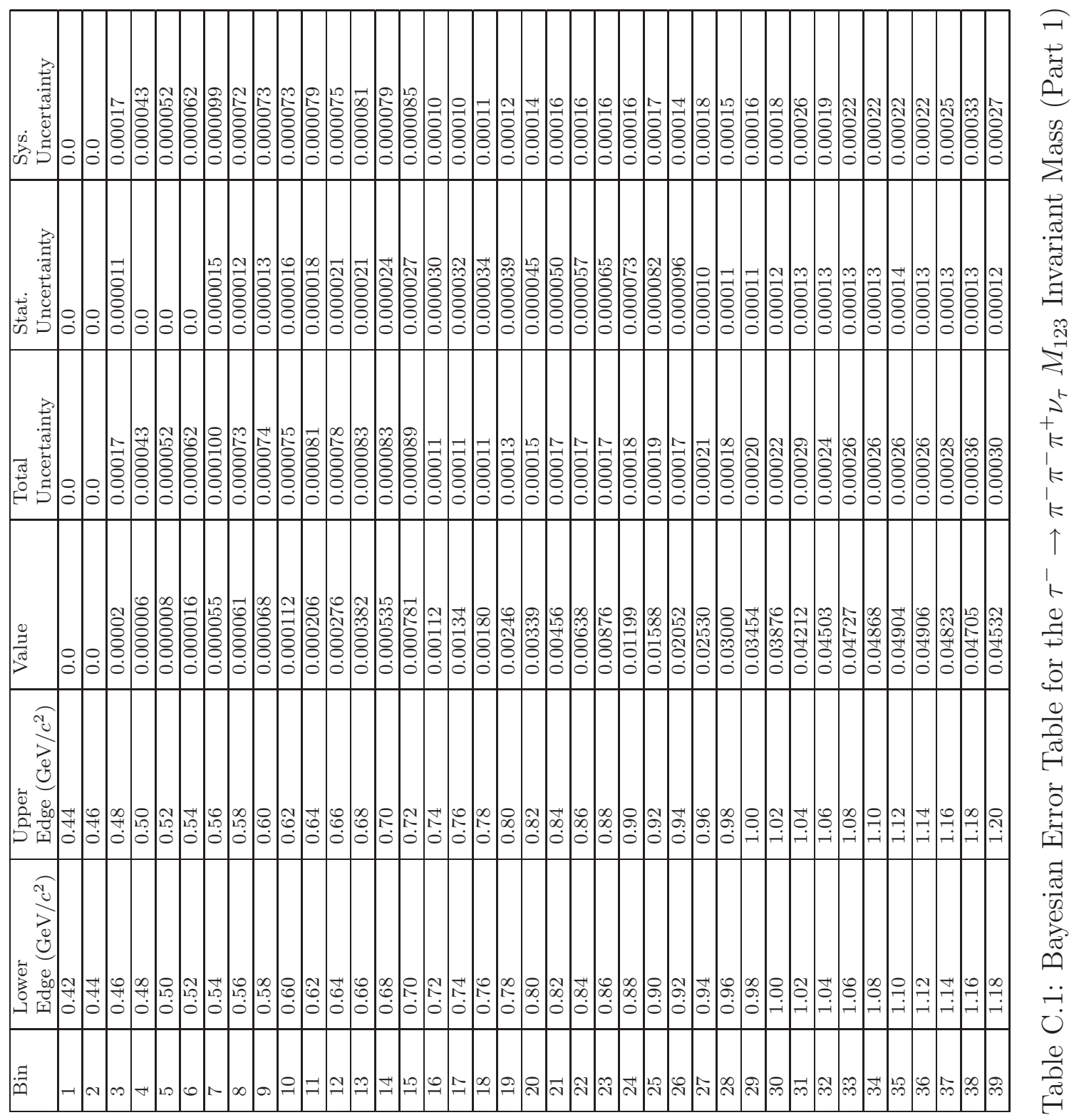


Appendix C. Unfolded $\tau^{-} \rightarrow \pi^{-} \pi^{-} \pi^{+} \nu_{\tau}$ Invariant Mass Spectra

191

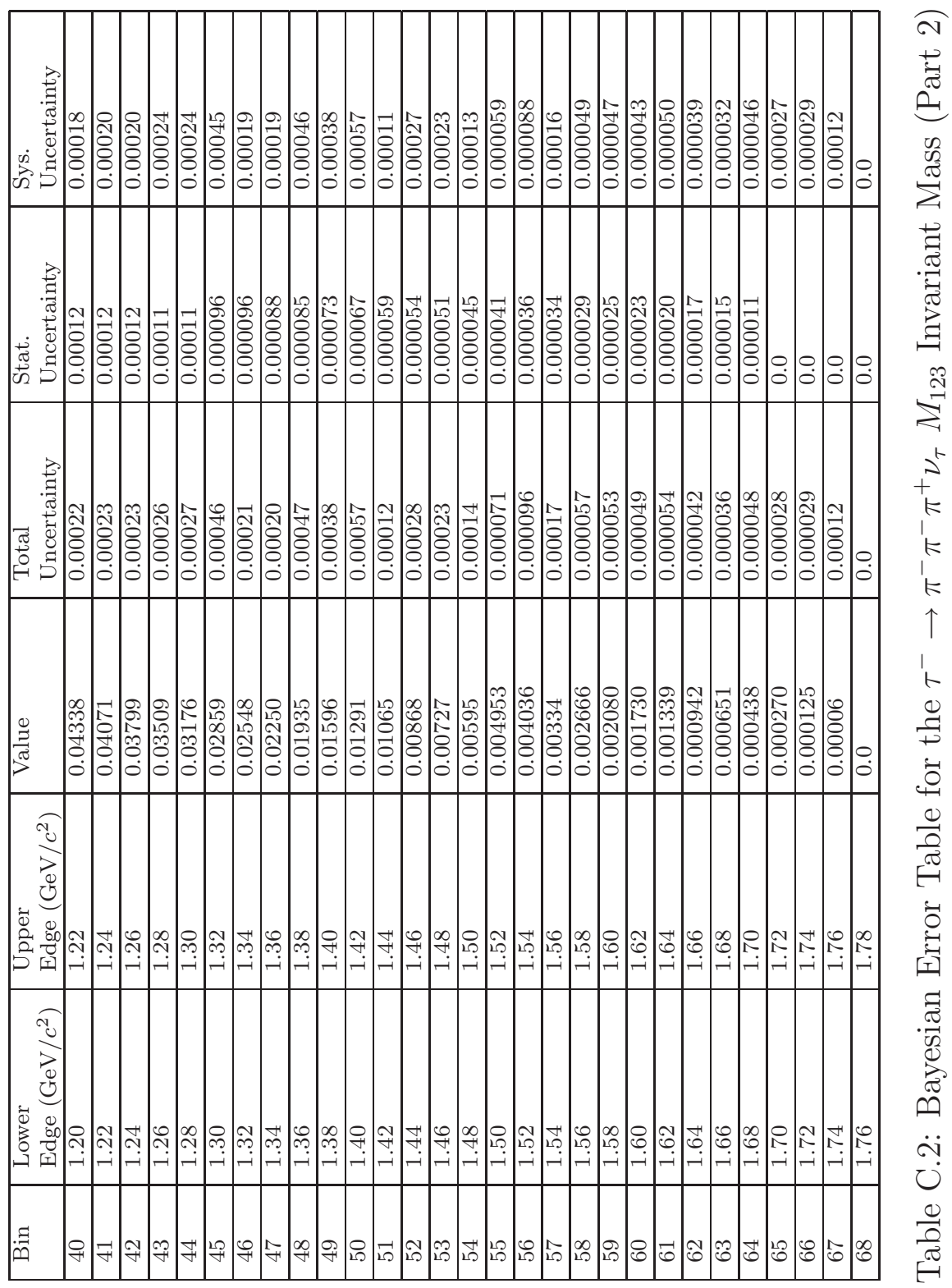




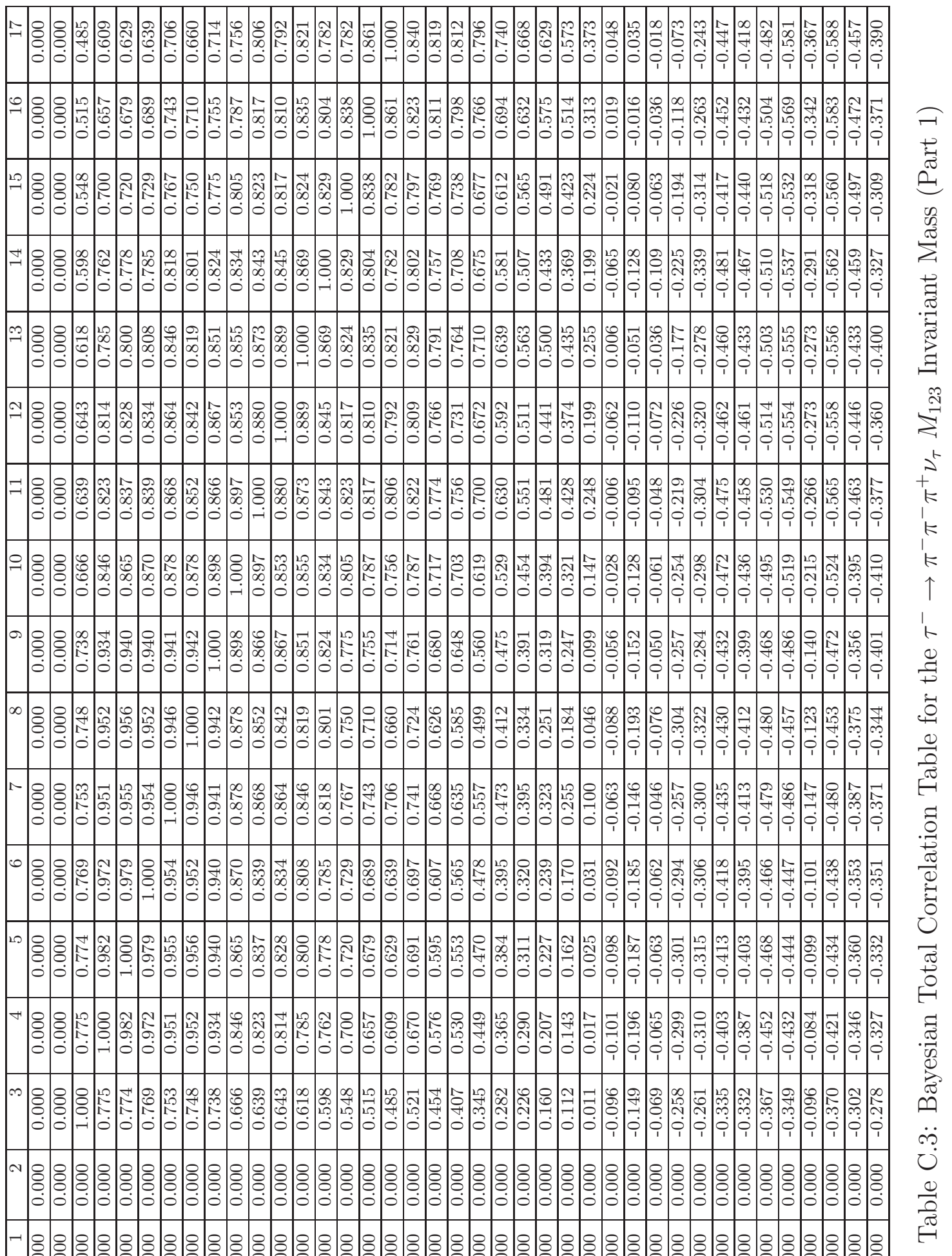




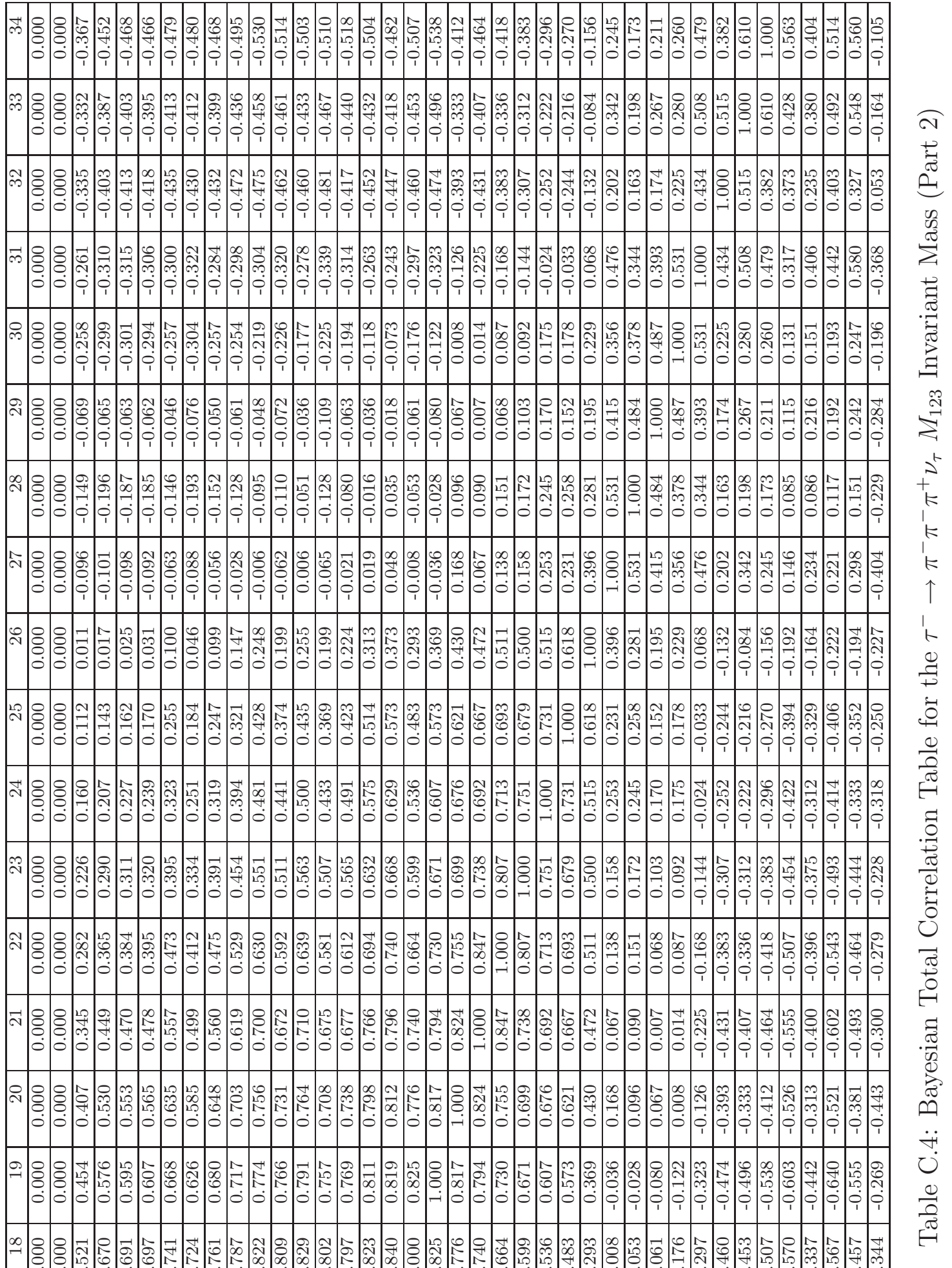

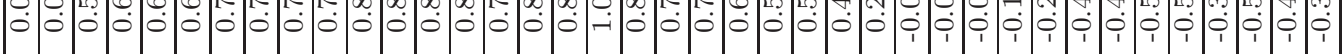

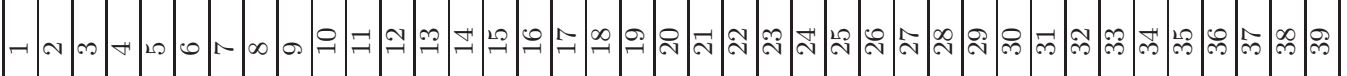




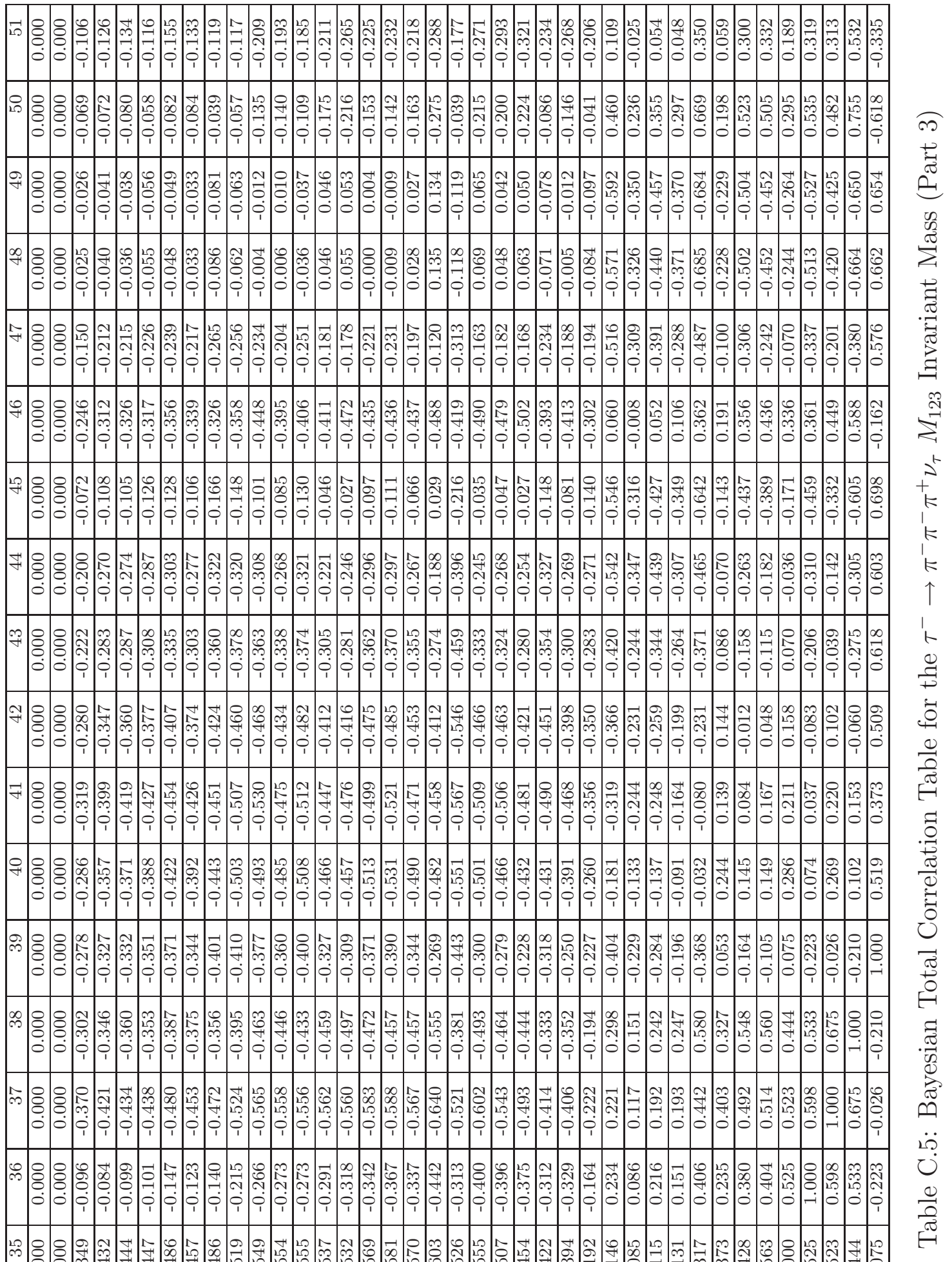
80 .

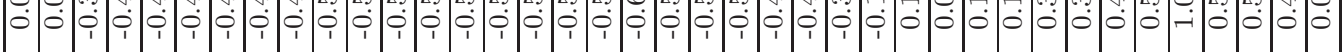

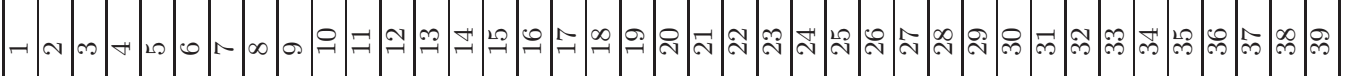




\begin{tabular}{|c|c|c|c|c|c|c|c|c|c|c|c|c|c|c|c|c|c|c|c|c|c|c|c|c|c|c|c|c|c|c|c|c|c|c|c|c|c|c|c|}
\hline \multicolumn{2}{|r|}{ 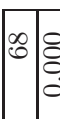 } & & 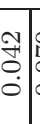 & & & & & & 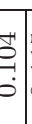 & & & & $\mid \begin{array}{l}12 \\
\stackrel{+}{1} \\
0 \\
0 \\
0\end{array}$ & & $\mid \begin{array}{l}-7 \\
0 \\
0 \\
0 \\
1\end{array}$ & & $\left|\begin{array}{l}\dot{2} \\
\dot{8} \\
\dot{0}\end{array}\right|$ & $\mid \begin{array}{l}-1 \\
0 \\
0 \\
1 \\
1\end{array}$ & & $\begin{array}{l}0 \\
0 \\
0 \\
0 \\
0\end{array}$ & $\mid \begin{array}{l}8 \\
\stackrel{8}{0} \\
0 \\
0 \\
1\end{array}$ & $\mid \begin{array}{l}\mathcal{N} \\
\mathscr{O} \\
0 \\
\dot{0} \\
1\end{array}$ & & & & 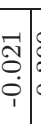 & & & & & \begin{tabular}{l}
$\vec{J}$ \\
\multirow{\leftrightarrow}{*}{} \\
$\dot{0}$
\end{tabular} & & & & & & & 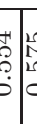 & 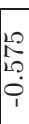 \\
\hline$\widehat{6}$ & $\mid$ & \begin{tabular}{l}
8 \\
$\Xi$ \\
\hdashline \\
$\vdots$
\end{tabular} & 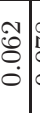 & $\begin{array}{c}\infty \\
1 \\
0 \\
0 \\
0\end{array}$ & & & 5 & & & & & & $\mid \begin{array}{l}+ \\
\stackrel{3}{0} \\
0 \\
0\end{array}$ & $\begin{array}{l}\infty \\
2 \\
0 \\
0 \\
0\end{array}$ & $\mid \begin{array}{l}0 \\
\stackrel{2}{0} \\
0 \\
0\end{array}$ & $\mid \begin{array}{l}\infty \\
2 \\
0 \\
0 \\
0 \\
0\end{array}$ & $\mid \begin{array}{l}10 \\
10 \\
0 \\
0 \\
0\end{array}$ & $\left|\begin{array}{l}1 \\
0 \\
0 \\
0 \\
0\end{array}\right|$ & & $\mid \begin{array}{l}1 \\
0 \\
0 \\
0 \\
0\end{array}$ & 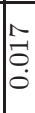 & $\mid \begin{array}{l}1 \\
\stackrel{0}{0} \\
0 \\
0 \\
1\end{array}$ & రె & & $\begin{array}{l}0 \\
0 \\
0 \\
0 \\
1 \\
1\end{array}$ & $\begin{array}{l}10 \\
\stackrel{1}{0} \\
0 \\
0 \\
1 \\
1\end{array}$ & & & & & $\begin{array}{l}\stackrel{1}{2} \\
0 \\
0 \\
0\end{array}$ & $\mid \begin{array}{l}\infty \\
+ \\
0 \\
0 \\
0 \\
1 \\
1\end{array}$ & $\left|\begin{array}{c}10 \\
\overrightarrow{0} \\
0 \\
1 \\
1\end{array}\right|$ & $\mid \begin{array}{l}-1 \\
0 \\
0 \\
0 \\
1\end{array}$ & $\mid$ & & & & \\
\hline & $\mid$ & $\begin{array}{l}8 \\
8 \\
0 \\
\vdots\end{array}$ & 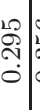 & 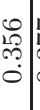 & & & 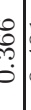 & & & $\begin{array}{l}\dot{+} \\
\dot{0} \\
\dot{0}\end{array}$ & & & 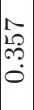 & & & & 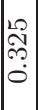 & 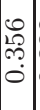 & & & & 离 & & $\stackrel{\infty}{=}$ & & $\begin{array}{l}0 \\
\stackrel{2}{0} \\
0 \\
0 \\
1\end{array}$ & \begin{tabular}{c}
$\infty$ \\
+1 \\
\hdashline
\end{tabular} & 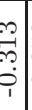 & & & 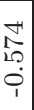 & $\mid \begin{array}{l}0 \\
0 \\
0 \\
0 \\
0 \\
1\end{array}$ & $\mid \begin{array}{l}0 \\
0 \\
0 \\
0 \\
0 \\
1\end{array}$ & 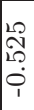 & & 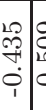 & & & 䍃 \\
\hline ڤִ & $\left|\begin{array}{l}8 \\
\varnothing \\
0 \\
0\end{array}\right|$ & $\begin{array}{l}8 \\
8 \\
\vdots \\
0\end{array}$ & 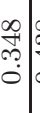 & $\begin{array}{c}\infty \\
\stackrel{2}{+} \\
\vdots \\
0\end{array}$ & \begin{tabular}{c}
0 \\
\multirow{2}{2}{} \\
0 \\
0
\end{tabular} & $\stackrel{1}{6}$ & 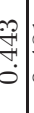 & $\begin{array}{c}\vec{\infty} \\
\stackrel{\infty}{+} \\
0 \\
0\end{array}$ & $\forall$ & 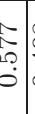 & \begin{tabular}{l}
$\infty$ \\
\multirow{F}{*}{}
\end{tabular} & E & $\begin{array}{l}\exists \\
\vec{F} \\
0 \\
0\end{array}$ & 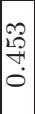 & & 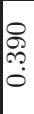 & 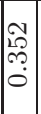 & 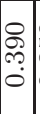 & 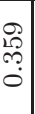 & 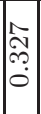 & $\begin{array}{l}\underset{N}{*} \\
\text { Na } \\
0\end{array}$ & 官 & : & $\begin{array}{l}10 \\
= \\
\\
0\end{array}$ & $\begin{array}{l}\infty \\
\infty \\
0 \\
0 \\
0\end{array}$ & $\begin{array}{l}1 \\
0 \\
0 \\
0 \\
1 \\
1\end{array}$ & 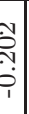 & $\stackrel{N}{N}$ & 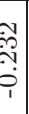 & ? & 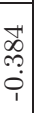 & 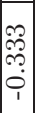 & $\mid$\begin{tabular}{l}
$\infty$ \\
0 \\
0 \\
\hdashline \\
0 \\
1
\end{tabular} & 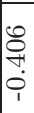 & $\mid$ & $\begin{array}{l}\infty \\
\stackrel{1}{N} \\
\vdots \\
1 \\
1\end{array}$ & & & \\
\hline ఈ & $\mid$ & $\begin{array}{l}8 \\
\Xi \\
\vdots \\
\vdots\end{array}$ & 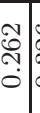 & 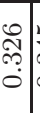 & & 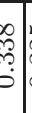 & مै & & & & & P & 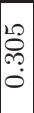 & & & 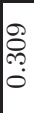 & $\begin{array}{l}\infty \\
\stackrel{1}{1} \\
\stackrel{2}{0} \\
0\end{array}$ & 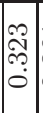 & 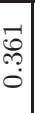 & $\mid \begin{array}{c}\curvearrowright \\
\infty \\
-1 \\
0 \\
0\end{array}$ & & 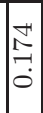 & $\begin{array}{l}0 \\
\stackrel{0}{0} \\
\stackrel{-}{0}\end{array}$ & 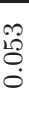 & $\begin{array}{l}-1 \\
0 \\
0 \\
0\end{array}$ & $\begin{array}{c}\vec{a} \\
0 \\
\dot{0} \\
1\end{array}$ & \begin{tabular}{l}
0 \\
0 \\
\hdashline \\
\\
\\
1 \\
\end{tabular} & 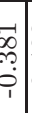 & $\mathscr{Z}$ & 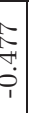 & $\begin{array}{l}1 \\
\infty \\
0 \\
0 \\
0 \\
1\end{array}$ & 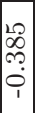 & $\left|\begin{array}{l}0 \\
0 \\
0 \\
0 \\
0 \\
1\end{array}\right|$ & & & 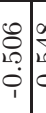 & & & \\
\hline 8 & $\mid$ & $\begin{array}{l}8 \\
8 \\
\vdots \\
\vdots\end{array}$ & 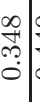 & \begin{tabular}{c}
0 \\
+ \\
\hdashline \\
\hdashline \\
0
\end{tabular} & 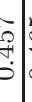 & 8 & 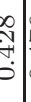 & 最 & 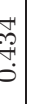 & 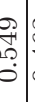 & ְै & 8 & 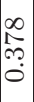 & $\mid \begin{array}{l}\infty \\
\overrightarrow{7} \\
\vdots \\
0\end{array}$ & 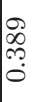 & 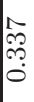 & 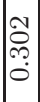 & 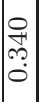 & 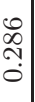 & $\mid \begin{array}{l}20 \\
\stackrel{2}{2} \\
\stackrel{2}{2} \\
0\end{array}$ & 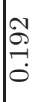 & 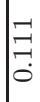 & 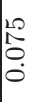 & 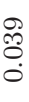 & 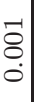 & 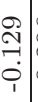 & $\begin{array}{l}0 \\
0 \\
0 \\
0 \\
1 \\
1\end{array}$ & 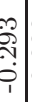 & 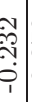 & 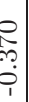 & 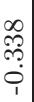 & 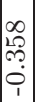 & \begin{tabular}{l}
0 \\
0 \\
0 \\
\hdashline \\
0 \\
1
\end{tabular} & $\mid \begin{array}{l}\overrightarrow{0} \\
0 \\
0 \\
0 \\
1\end{array}$ & 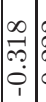 & 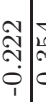 & & & 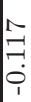 \\
\hline ชิ & $\mid \begin{array}{l}8 \\
8 \\
0 \\
0\end{array}$ & $\begin{array}{l}8 \\
8 \\
\vdots \\
\vdots\end{array}$ & $\begin{array}{l}\stackrel{R}{N} \\
N \\
0\end{array}$ & \begin{tabular}{l}
$\infty$ \\
$\cdots$ \\
$\infty$ \\
\hdashline \\
0
\end{tabular} & $\begin{array}{l}0 \\
0 \\
0 \\
0 \\
0\end{array}$ & & $\begin{array}{l}0 \\
0 \\
0 \\
0\end{array}$ & 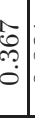 & $\begin{array}{l}- \\
0 \\
0 \\
0\end{array}$ & $\begin{array}{l}F \\
\\
0\end{array}$ & 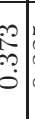 & 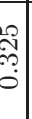 & 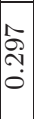 & $\mid \begin{array}{c}0 \\
0 \\
2 \\
ٌ \\
0 \\
0\end{array}$ & $\mid \begin{array}{l}m \\
\dddot{m} \\
\dddot{m} \\
0 \\
0\end{array}$ & & $\mid \begin{array}{l}\underset{+}{\sim} \\
\stackrel{\sim}{\sim} \\
0\end{array}$ & 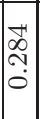 & & & 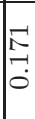 & ஓ & \begin{tabular}{l}
$\infty$ \\
\multirow{8}{8}{} \\
$\dot{0}$
\end{tabular} & $\begin{array}{l}\mathscr{8} \\
\mathscr{8} \\
\dot{0}\end{array}$ & $\begin{array}{l}\overrightarrow{1} \\
0 \\
0 \\
0 \\
1\end{array}$ & 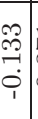 & 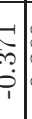 & : & 8 & 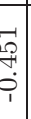 & 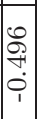 & $\mid$\begin{tabular}{c}
$\Re$ \\
$\infty$ \\
$\infty$ \\
\hdashline \\
0 \\
0 \\
1
\end{tabular} & $\mid \begin{array}{l}0 \\
0 \\
0 \\
0 \\
1 \\
1\end{array}$ & 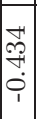 & & 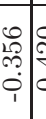 & & & \\
\hline$\overline{6}$ & $\mid \begin{array}{l}8 \\
0 \\
0 \\
0\end{array}$ & $\begin{array}{l}8 \\
8 \\
\vdots \\
0\end{array}$ & 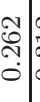 & \begin{tabular}{c}
$\mathcal{N}$ \\
\hdashline \\
\hdashline \\
\hdashline \\
\hdashline
\end{tabular} & .2 & 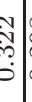 & $\infty$ & 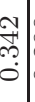 & 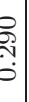 & $\begin{array}{l}5 \\
6 \\
0 \\
0\end{array}$ & & 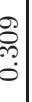 & 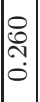 & 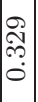 & 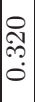 & 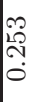 & 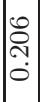 & 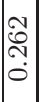 & $\begin{array}{l}10 \\
\\
\\
0\end{array}$ & 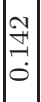 & 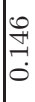 & $\mid \begin{array}{l}10 \\
0 \\
0 \\
0\end{array}$ & $\begin{array}{l}10 \\
0 \\
0 \\
0 \\
0 \\
0\end{array}$ & \begin{tabular}{l}
0 \\
\hdashline \\
0 \\
$\dot{0}$ \\
\end{tabular} & $\begin{array}{l}2 \\
\stackrel{2}{0} \\
0 \\
\vdots \\
1 \\
1\end{array}$ & 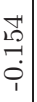 & $\begin{array}{l}2 \\
\stackrel{2}{7} \\
\\
1 \\
1\end{array}$ & $\begin{array}{l}\infty \\
\infty \\
0 \\
0 \\
\vdots \\
1 \\
1\end{array}$ & $\stackrel{8}{\stackrel{8}{\prime}}$ & $\begin{array}{l}R \\
7 \\
\end{array}$ & $\begin{array}{l}3 \\
0 \\
0 \\
0 \\
0 \\
1 \\
1\end{array}$ & 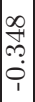 & 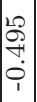 & $\mid$\begin{tabular}{|c}
2 \\
0 \\
0 \\
0 \\
0 \\
1
\end{tabular} & $\mid \begin{array}{c}0 \\
0 \\
0 \\
0 \\
0 \\
1 \\
1\end{array}$ & $\begin{array}{l}1 \\
\infty \\
\infty \\
0 \\
0 \\
\vdots \\
1\end{array}$ & & & \\
\hline 8 & $\mid$ & $\begin{array}{l}8 \\
\varrho \\
\vdots \\
\vdots\end{array}$ & $\begin{array}{c}\infty \\
\infty \\
-1 \\
0\end{array}$ & \begin{tabular}{l}
$\stackrel{1}{N}$ \\
\multirow{2}{0}{} \\
0
\end{tabular} & 0 & 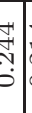 & $\underset{v}{+}$ & $\stackrel{0}{7}$ & 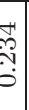 & 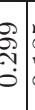 & $\infty$ & $F$ & 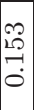 & $\mid$\begin{tabular}{l}
0 \\
$\stackrel{\leftrightarrow}{\oplus}$ \\
\hdashline \\
0
\end{tabular} & $\mid$\begin{tabular}{l}
$\stackrel{2}{A}$ \\
-1 \\
\hdashline \\
0
\end{tabular} & $\underset{\exists}{\exists}$ & $\mid \begin{array}{l}2 \\
\infty \\
0 \\
0 \\
0\end{array}$ & \begin{tabular}{c}
1 \\
$\stackrel{0}{0}$ \\
\hdashline \\
0
\end{tabular} & 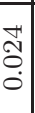 & $\mid$\begin{tabular}{l}
$\stackrel{2}{2}$ \\
\hdashline \\
\hdashline \\
\hdashline \\
0
\end{tabular} & 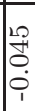 & $\mid \begin{array}{l}\mathcal{N} \\
\dot{O} \\
\dot{0} \\
\dot{0}\end{array}$ & 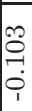 & 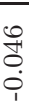 & 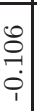 & $\begin{array}{l}0 \\
0 \\
0 \\
0 \\
0 \\
1\end{array}$ & $\begin{array}{l}0 \\
0 \\
0 \\
0\end{array}$ & $\begin{array}{l}\infty \\
0 \\
0 \\
0 \\
1 \\
1\end{array}$ & 8 & $\begin{array}{l}0 \\
0 \\
0 \\
\\
1 \\
1\end{array}$ & 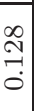 & $\mid \begin{array}{l}0 \\
\dot{0} \\
\dot{0} \\
\dot{1} \\
1\end{array}$ & $\begin{array}{l}\widehat{1} \\
\stackrel{0}{0} \\
0 \\
0\end{array}$ & $\mid \begin{array}{l}\infty \\
+ \\
0 \\
0 \\
0\end{array}$ & $\mid \begin{array}{l}3 \\
3 \\
0 \\
0 \\
1\end{array}$ & \begin{tabular}{c}
$\mathfrak{N}$ \\
\hdashline \\
0
\end{tabular} & & & \\
\hline is & $\left|\begin{array}{c}8 \\
\hdashline \\
0 \\
0\end{array}\right|$ & & 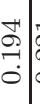 & 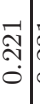 & 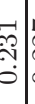 & 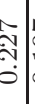 & $\hat{\sigma}$ & 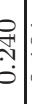 & $\begin{array}{c}\vec{\sigma} \\
\overrightarrow{0} \\
0\end{array}$ & $\begin{array}{c}-1 \\
0 \\
0 \\
0 \\
0\end{array}$ & $\begin{array}{l}\infty \\
\infty \\
\stackrel{1}{\prime} \\
0\end{array}$ & 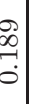 & $\mid \begin{array}{l}\stackrel{2}{+} \\
\stackrel{4}{-} \\
0\end{array}$ & $\begin{array}{l}\exists \\
\vec{ज} \\
0 \\
0\end{array}$ & 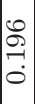 & 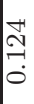 & $\begin{array}{l}1 \\
1 \\
0 \\
0 \\
0\end{array}$ & 焉 & in & $\mid \begin{array}{l}2 \\
0 \\
0 \\
0 \\
0\end{array}$ & $\underset{0}{-1}$ & $\begin{array}{l}1 \\
10 \\
0 \\
0 \\
0 \\
1\end{array}$ & 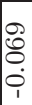 & 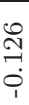 & \begin{tabular}{l}
$\stackrel{1}{N}$ \\
\hdashline \\
$\vdots$ \\
1 \\
1
\end{tabular} & 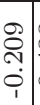 & 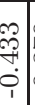 & 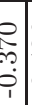 & $\stackrel{\circ}{\circ}$ & 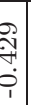 & $\begin{array}{l}8 \\
\stackrel{8}{+} \\
\vdots \\
1 \\
1\end{array}$ & 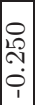 & $\mid \begin{array}{l}0 \\
0 \\
+ \\
0 \\
0 \\
1\end{array}$ & 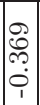 & & 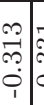 & & & \\
\hline$\left[\begin{array}{l}\infty \\
10\end{array}\right.$ & $\mid$ & $\begin{array}{l}8 \\
8 \\
\vdots \\
0\end{array}$ & $\begin{array}{l}\Omega \\
\vec{z} \\
0\end{array}$ & $\begin{array}{c}\stackrel{9}{-} \\
\stackrel{-}{-} \\
\end{array}$ & 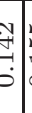 & 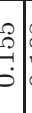 & $\begin{array}{c}0 \\
0 \\
\end{array}$ & 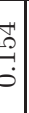 & 워 & 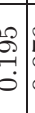 & $\begin{array}{l}0 \\
0\end{array}$ & 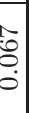 & $\begin{array}{l}\stackrel{g}{+} \\
\stackrel{0}{0} \\
\dot{0}\end{array}$ & $\mid \begin{array}{l}0 \\
0 \\
0 \\
0 \\
0\end{array}$ & $\begin{array}{l}- \\
\overrightarrow{0} \\
0 \\
0 \\
0\end{array}$ & $\begin{array}{l}2 \\
\stackrel{2}{2} \\
0 \\
0 \\
0\end{array}$ & $\mid \begin{array}{l}\stackrel{2}{2} \\
\dot{O} \\
\dot{0} \\
\dot{1}\end{array}$ & 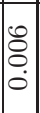 & 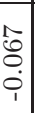 & $\mid \begin{array}{l}\mathbb{1} \\
10 \\
0 \\
0 \\
1 \\
1\end{array}$ & 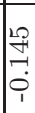 & 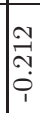 & $\begin{array}{l}\mathscr{N} \\
\stackrel{\leftrightarrow}{2}\end{array}$ & 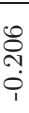 & 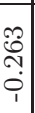 & 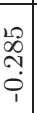 & $\begin{array}{l}\vec{H} \\
\overrightarrow{4} \\
\dot{1} \\
\end{array}$ & 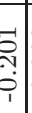 & ֶे & 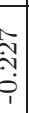 & $\begin{array}{l}-\overrightarrow{3} \\
3 \\
0 \\
0 \\
1\end{array}$ & 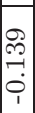 & \begin{tabular}{|l}
-1 \\
0 \\
0 \\
0 \\
1 \\
1
\end{tabular} & $\mid \begin{array}{l}0 \\
0 \\
0 \\
0 \\
1\end{array}$ & $\begin{array}{l}\mathbb{1} \\
0 \\
0 \\
1 \\
1\end{array}$ & $\begin{array}{l}1 \\
10 \\
0 \\
0 \\
0\end{array}$ & & & 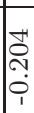 \\
\hline is & $\mid$ & \begin{tabular}{l}
8 \\
$\Xi$ \\
\hdashline \\
$\vdots$
\end{tabular} & $\begin{array}{l}\mathcal{N} \\
\delta \\
\vdots \\
\dot{0}\end{array}$ & 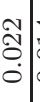 & $\frac{0}{b}$ & 0 & 8 & 6 & & $\begin{array}{l}\stackrel{8}{0} \\
0 \\
\end{array}$ & ֶै & \begin{tabular}{l}
$\infty$ \\
\hdashline
\end{tabular} & $\begin{array}{l}\vec{F} \\
0 \\
0 \\
0 \\
1 \\
1\end{array}$ & $\mid \begin{array}{l}1 \\
0 \\
0 \\
0 \\
1 \\
1\end{array}$ & 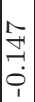 & $\begin{array}{l}\mathscr{8} \\
\mathscr{8} \\
0 \\
0 \\
1\end{array}$ & $\mid$\begin{tabular}{l}
0 \\
0 \\
\hdashline \\
0 \\
1 \\
1
\end{tabular} & 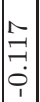 & 10 & $\mid \begin{array}{l}+ \\
0 \\
0 \\
0 \\
1\end{array}$ & \begin{tabular}{l}
$\stackrel{0}{2}$ \\
\multirow{2}{*}{} \\
0 \\
\\
1
\end{tabular} & 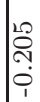 & $\stackrel{\leftrightarrow}{\stackrel{2}{2}}$ & $\begin{array}{l}8 \\
8 \\
0 \\
0 \\
1\end{array}$ & $\begin{array}{l}n \\
N \\
\vdots \\
0 \\
1 \\
1\end{array}$ & $\begin{array}{l}0 \\
0 \\
0 \\
0 \\
1 \\
1\end{array}$ & $\begin{array}{l}-1 \\
\\
0 \\
0\end{array}$ & $\frac{1}{6}$ & 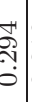 & 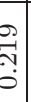 & 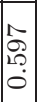 & $\mid$\begin{tabular}{l}
$\infty$ \\
1 \\
\hdashline \\
\hdashline \\
0 \\
0
\end{tabular} & 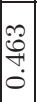 & 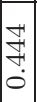 & & & & & \\
\hline$\stackrel{10}{\circ}$ & $\mid$ & $\begin{array}{l}8 \\
\varrho \\
\vdots\end{array}$ & \begin{tabular}{c}
+1 \\
0 \\
\hdashline \\
0
\end{tabular} & \begin{tabular}{c}
$\sigma$ \\
- \\
\hdashline \\
0
\end{tabular} & $\stackrel{f}{\mathcal{H}}$ & $\exists$ & 8 & $\underset{\sigma}{.}$ & $\stackrel{\mathbb{Z}}{\mathbf{E}}$ & $\delta$ & ठ゙ & $\underset{\sim}{\infty}$ & & & & $\mid \begin{array}{l}\hat{1} \\
\infty \\
0 \\
0 \\
0\end{array}$ & $\mid \begin{array}{l}0 \\
10 \\
0 \\
0 \\
0 \\
0\end{array}$ & $\mid \begin{array}{l}\overrightarrow{1} \\
\infty \\
0 \\
0\end{array}$ & $\begin{array}{c}\infty \\
10\end{array}$ & $\mid \begin{array}{l}\mathbb{1} \\
\dot{0} \\
0 \\
0 \\
0 \\
1\end{array}$ & & 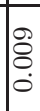 & $\begin{array}{l}8 \\
\\
0\end{array}$ & so & $\begin{array}{l}1 \\
0 \\
0 \\
0 \\
1 \\
1\end{array}$ & $\begin{array}{c}0 \\
0 \\
0 \\
0 \\
0 \\
1 \\
1\end{array}$ & $\begin{array}{l}\text { in } \\
\text { in } \\
\text { ? }\end{array}$ & e & 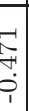 & $x$ & 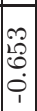 & 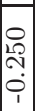 & 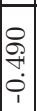 & $\mid \begin{array}{l}0 \\
0 \\
0 \\
0 \\
0 \\
1 \\
1\end{array}$ & & \begin{tabular}{l}
8 \\
$\stackrel{0}{0}$ \\
+ \\
\hdashline \\
1
\end{tabular} & & & \\
\hline 180 & $\mid$ & $\begin{array}{l}8 \\
\Xi \\
\vdots \\
\vdots\end{array}$ & $\begin{array}{l}\infty \\
+ \\
0 \\
0\end{array}$ & 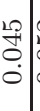 & $\begin{array}{l}v^{2} \\
\text { है. }\end{array}$ & 8 & $\frac{0}{0}$ & 8 & ¿̂. & $\begin{array}{l}0 \\
0 \\
0 \\
0\end{array}$ & & $\stackrel{I}{-1}$ & $\begin{array}{l}\hat{N} \\
\hat{N} \\
0 \\
0 \\
1\end{array}$ & $\mid \begin{array}{l}20 \\
0 \\
0 \\
0 \\
0\end{array}$ & 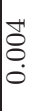 & 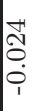 & $\mid \begin{array}{l}1 \\
12 \\
0 \\
0 \\
0 \\
1 \\
1\end{array}$ & $\begin{array}{l}8 \\
8 \\
0 \\
\dot{0} \\
1\end{array}$ & & & 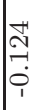 & $\begin{array}{c}2 \\
\infty \\
0 \\
0 \\
0\end{array}$ & 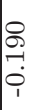 & 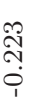 & 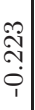 & 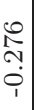 & 6 & N & & & 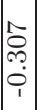 & $\mid \begin{array}{l}\overrightarrow{1} \\
0 \\
-0 \\
0 \\
1 \\
1\end{array}$ & 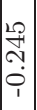 & \begin{tabular}{|l|}
$\infty$ \\
$\infty$ \\
$-\infty$ \\
0 \\
1 \\
1
\end{tabular} & & $\begin{array}{c}1 \\
\infty \\
-1 \\
0 \\
1 \\
1\end{array}$ & & & \\
\hline is & $\mid$ & $\begin{array}{l}8 \\
8 \\
0 \\
\circ\end{array}$ & $\begin{array}{l}0 \\
0 \\
0 \\
\dot{1}\end{array}$ & $\begin{array}{l}- \\
0 \\
0 \\
0 \\
1\end{array}$ & T. & 3 & 0 & 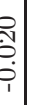 & & & & & $\mid \begin{array}{l}\mathfrak{N} \\
\mathbf{1} \\
0 \\
0 \\
1\end{array}$ & 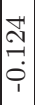 & 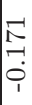 & 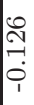 & 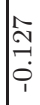 & $\begin{array}{l}0 \\
0 \\
-1 \\
0 \\
1 \\
1\end{array}$ & & & 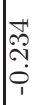 & 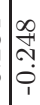 & & $\stackrel{2}{2}$ & 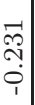 & \begin{tabular}{l}
0 \\
0 \\
10 \\
\hdashline \\
0 \\
1 \\
1 \\
1
\end{tabular} & $\underset{\sim}{\stackrel{H}{*}}$ & 5 & 8 & & 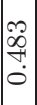 & $\begin{array}{l}\overrightarrow{0} \\
\overrightarrow{0} \\
0 \\
0 \\
0\end{array}$ & $\mid \begin{array}{c}0 \\
0 \\
0 \\
0 \\
0 \\
0 \\
0\end{array}$ & 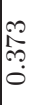 & $\left(\begin{array}{c}\curvearrowright \\
\mathscr{\sigma} \\
\hdashline \\
\hdashline \\
\hdashline\end{array}\right.$ & & & & 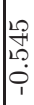 \\
\hline is & $\left|\begin{array}{l}8 \\
8 \\
0 \\
0\end{array}\right|$ & 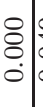 & $\begin{array}{l}9 \\
\dot{+} \\
\dot{1} \\
\dot{1}\end{array}$ & $\begin{array}{c}m \\
+ \\
0 \\
\dot{P} \\
1\end{array}$ & $\underset{+}{*}$ & $g$ & 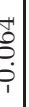 & & $\infty$ & & & & $\begin{array}{l}0 \\
0 \\
0 \\
0 \\
0 \\
1\end{array}$ & $\mid \begin{array}{l}\mid 7 \\
0 \\
0 \\
0 \\
1 \\
1\end{array}$ & 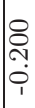 & 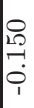 & $\mid \begin{array}{l}0 \\
0 \\
0 \\
0 \\
1\end{array}$ & $\mid \begin{array}{l}1 \\
0 \\
0 \\
0 \\
0 \\
1 \\
1\end{array}$ & & $\mid$\begin{tabular}{l}
\multirow{2}{*}{} \\
$\dot{0}$ \\
0 \\
0 \\
0 \\
1
\end{tabular} & 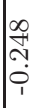 & $\mid$\begin{tabular}{l}
$\mathcal{N}$ \\
N \\
\multirow{O}{*}{}
\end{tabular} & & & $\begin{array}{l}\stackrel{2}{2} \\
\vdots \\
\vdots \\
1 \\
1\end{array}$ & $\begin{array}{l}\mathbb{N} \\
8 \\
0 \\
\vdots \\
1 \\
1\end{array}$ & $\infty$ & 8 & & & $\mid \begin{array}{l}\infty \\
0 \\
0 \\
0 \\
0\end{array}$ & $\mid \begin{array}{l}\overrightarrow{1} \\
- \\
-1 \\
0 \\
0\end{array}$ & 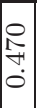 & 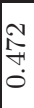 & & & & & $\begin{array}{l}\infty \\
\infty \\
20 \\
10 \\
0 \\
1\end{array}$ \\
\hline iิ & $\mid \begin{array}{l}8 \\
8 \\
0 \\
0\end{array}$ & $\begin{array}{l}8 \\
8 \\
\vdots \\
0\end{array}$ & $\begin{array}{l}\Omega \\
0 \\
0 \\
0\end{array}$ & $\begin{array}{l}0 \\
0 \\
0 \\
0\end{array}$ & & & & & & & & & $\begin{array}{l}10 \\
0 \\
0 \\
0 \\
0\end{array}$ & 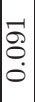 & 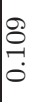 & 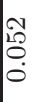 & 令 & $\begin{array}{l}\hat{1} \\
\hat{\theta} \\
0 \\
0\end{array}$ & & $\mid \begin{array}{l}0 \\
\hat{R} \\
0 \\
0 \\
1 \\
1\end{array}$ & $\begin{array}{l}\overrightarrow{0} \\
\stackrel{1}{\circ} \\
\stackrel{0}{0}\end{array}$ & $\mid \begin{array}{l}\mathcal{N} \\
0 \\
0 \\
0\end{array}$ & & I & $\begin{array}{l}\infty \\
8 \\
0 \\
0\end{array}$ & $\begin{array}{l}1 \\
\infty \\
0 \\
0 \\
1 \\
1\end{array}$ & & & & & $\mid \begin{array}{l}\overrightarrow{1} \\
\mathbb{1} \\
0 \\
1 \\
1\end{array}$ & 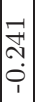 & 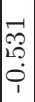 & $\mid \begin{array}{l}0 \\
\stackrel{2}{2} \\
+ \\
0 \\
1 \\
1\end{array}$ & \begin{tabular}{c}
$\Omega$ \\
$D$ \\
$N$ \\
\hdashline \\
1 \\
1
\end{tabular} & & & & \\
\hline & - & & & & & & & & & & & & & & & & & & & & & & & & & & & & & & m & $n$ & & & & & & & \\
\hline
\end{tabular}




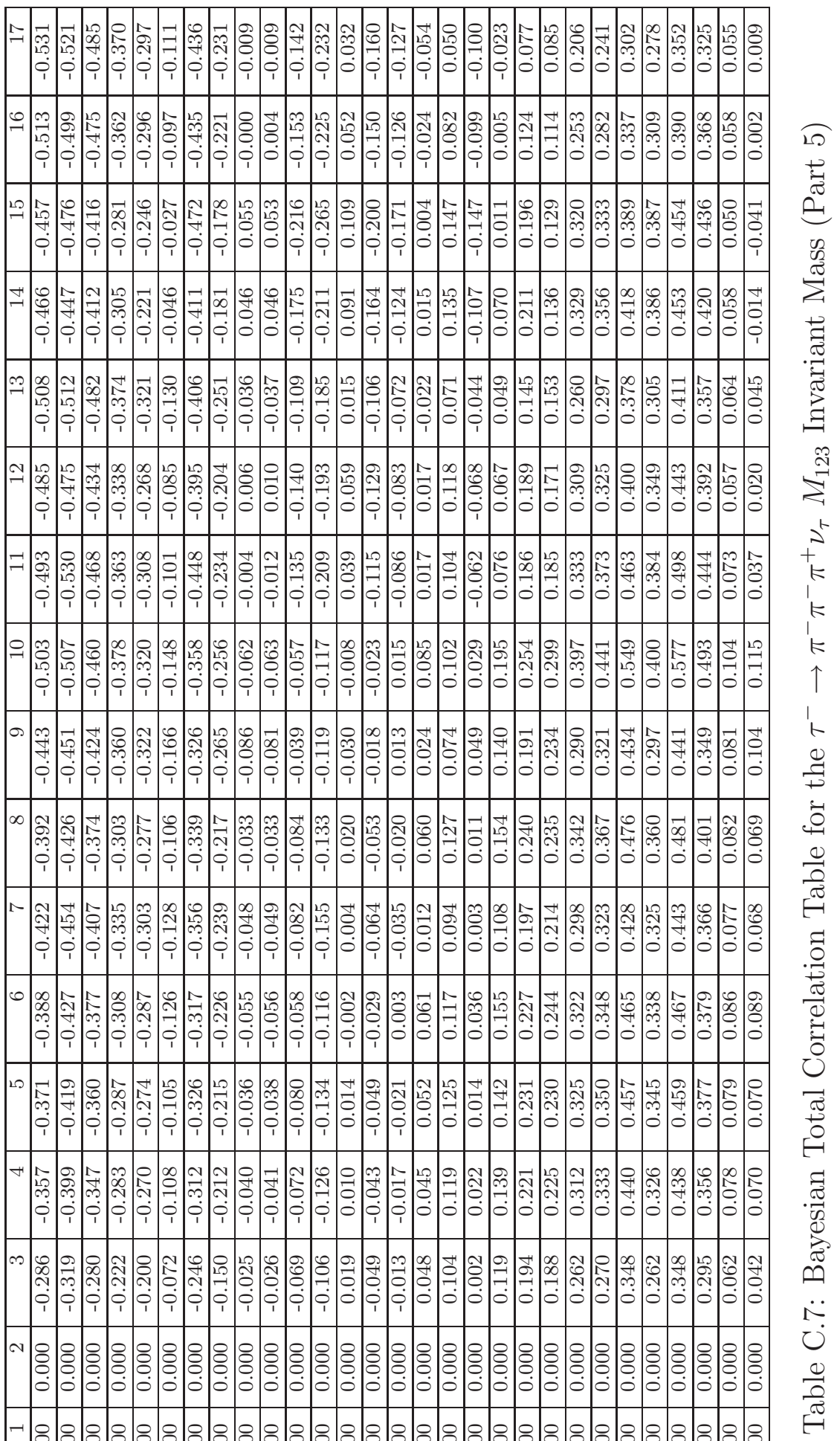


Appendix C. Unfolded $\tau^{-} \rightarrow \pi^{-} \pi^{-} \pi^{+} \nu_{\tau}$ Invariant Mass Spectra

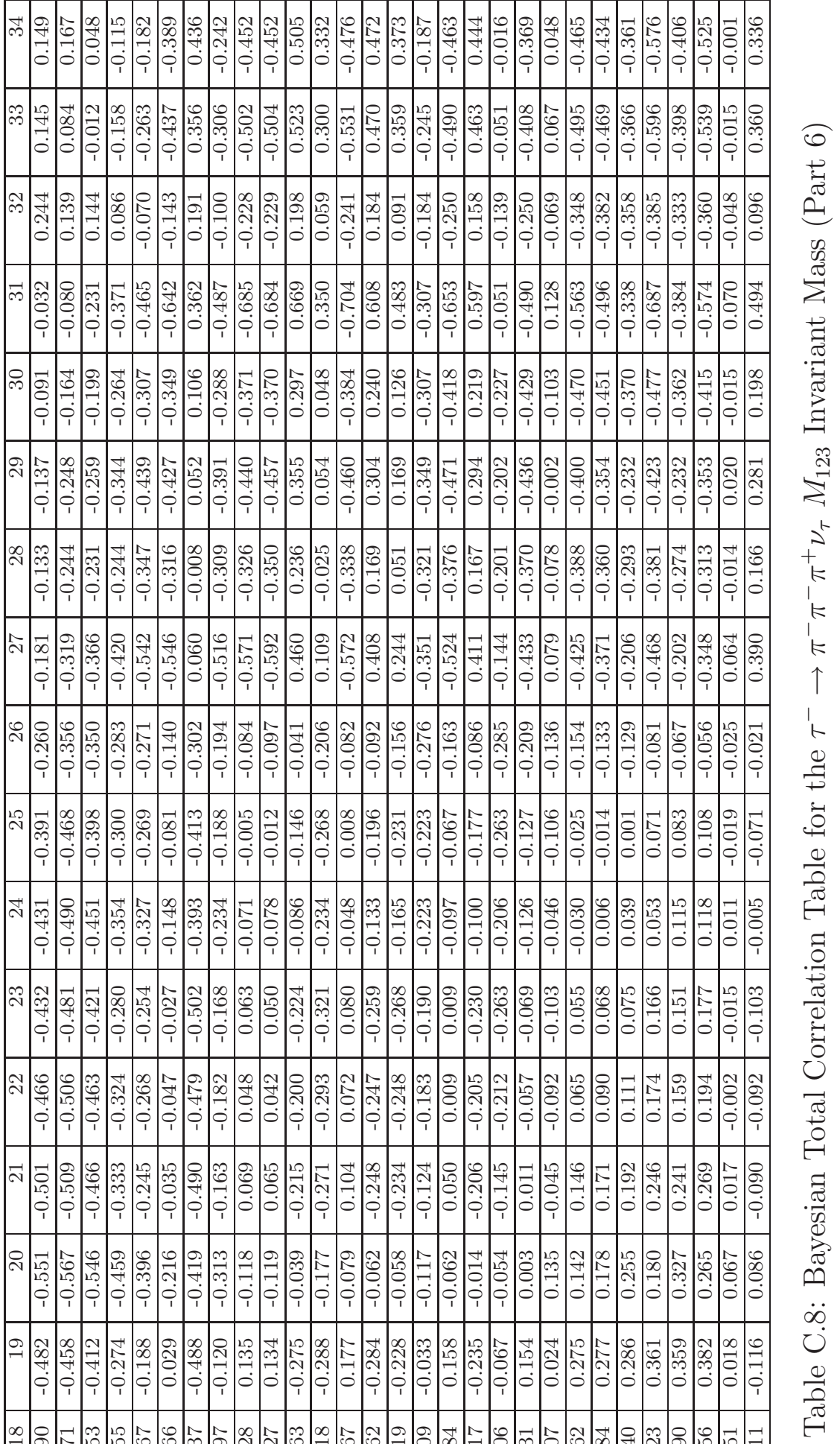

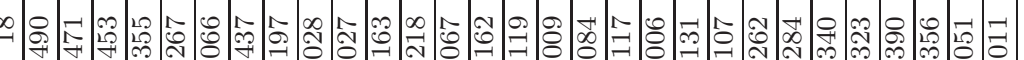

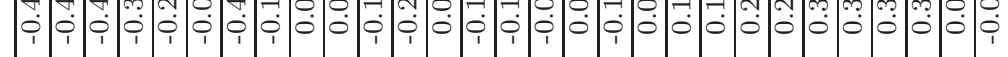

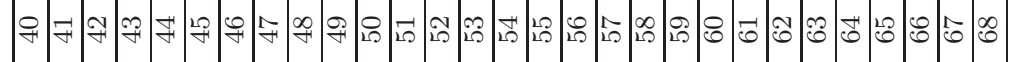




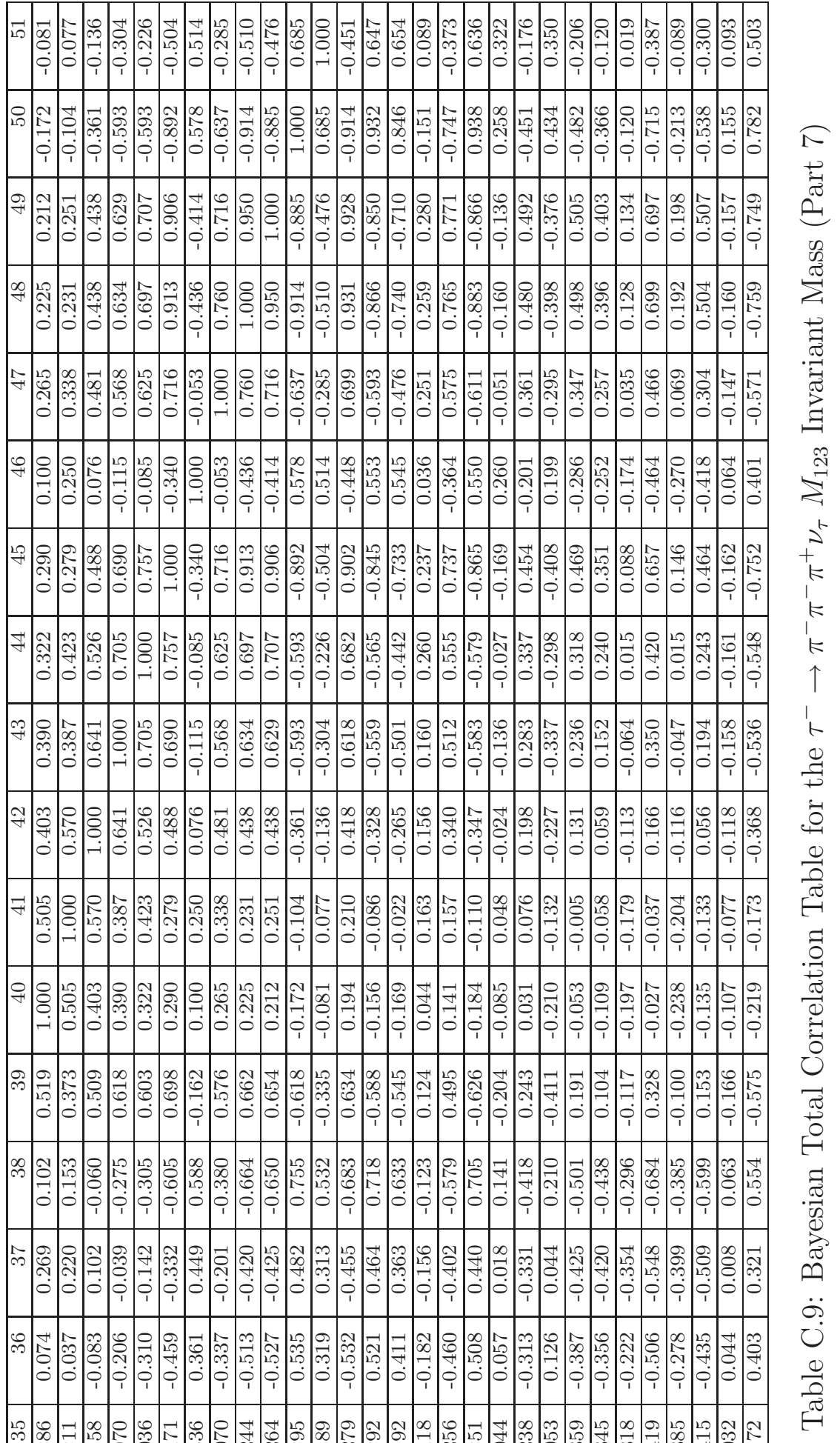


Appendix C. Unfolded $\tau^{-} \rightarrow \pi^{-} \pi^{-} \pi^{+} \nu_{\tau}$ Invariant Mass Spectra

199

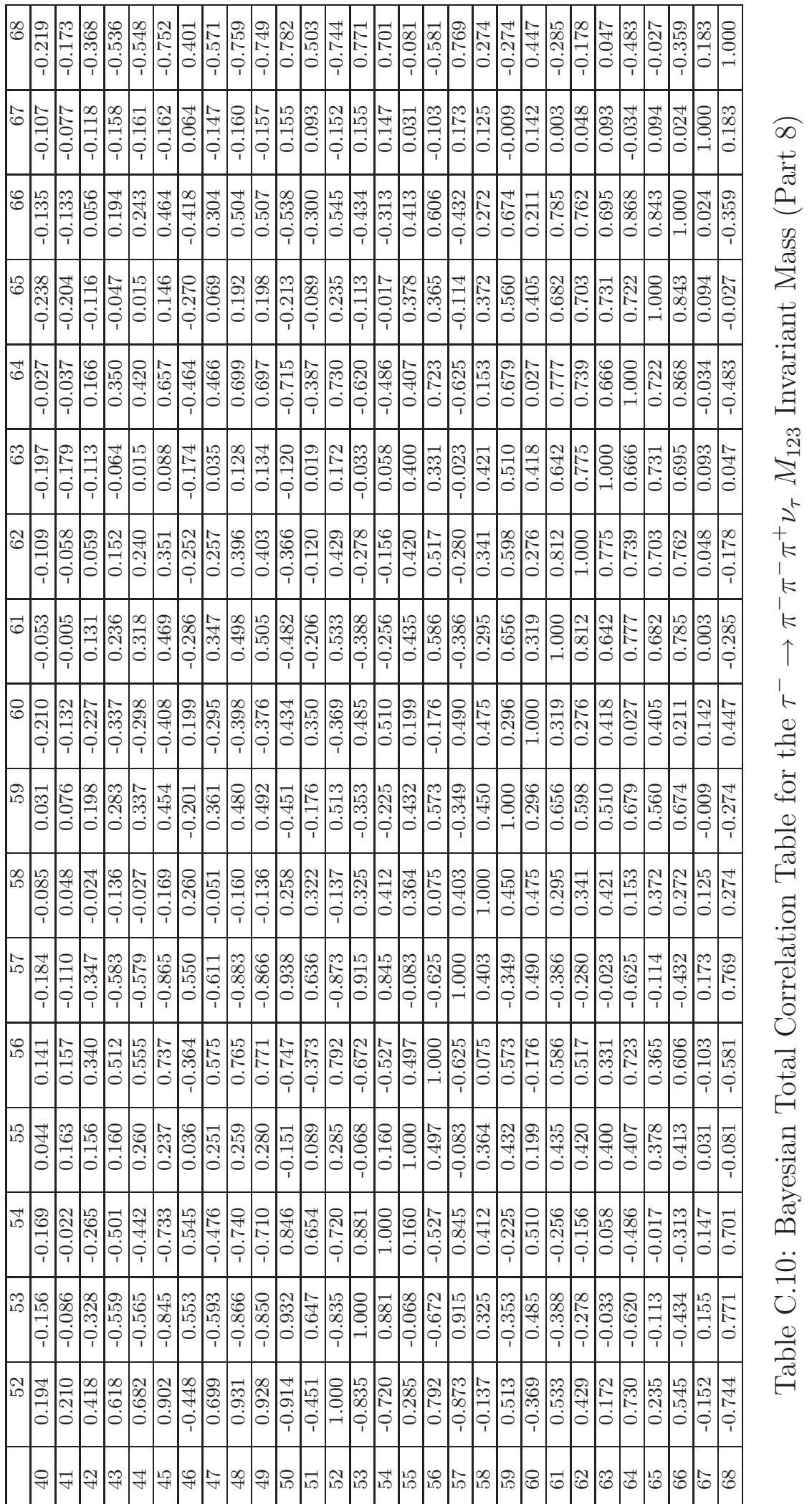




\section{C.2 The Unfolded $\tau^{-} \rightarrow \pi^{-} \pi^{-} \pi^{+} \nu_{\tau} M_{13 a n d 23}$ Invari- ant Mass Spectra}
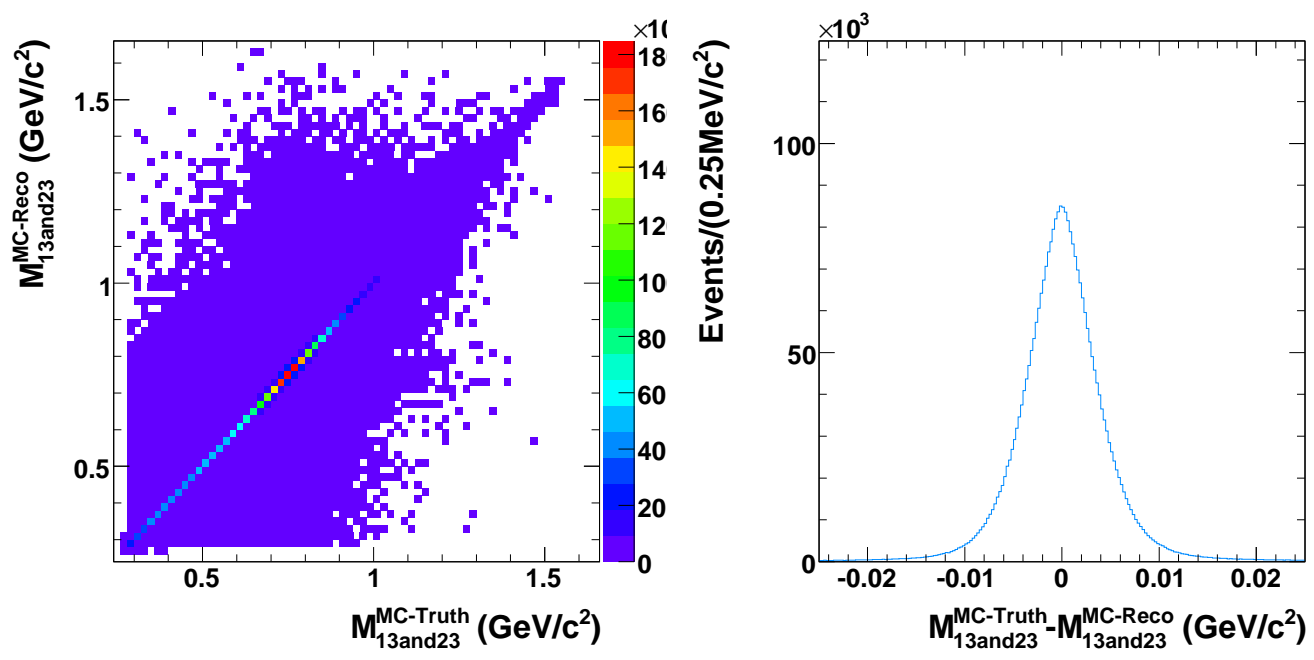

Figure C.2: A comparison of the $\tau^{-} \rightarrow K^{-} \pi^{-} \pi^{+} \nu_{\tau}$ MC Truth and reconstructed MC for the $M_{13 a n d 23}$ invariant mass spectra. The reconstructed MC $M_{13 a n d 23}$ invariant mass as a function of MC Truth $M_{13 a n d 23}$ invariant mass (left) is used to describe the response of the BABAR Detector in the unfolding of the $\tau^{-} \rightarrow K^{-} \pi^{-} \pi^{+} \nu_{\tau} M_{13 a n d 23}$ invariant mass spectrum. The reconstructed $\mathrm{MC} M_{13 a n d 23}$ invariant mass minus the MC Truth $M_{13 a n d 23}$ invariant mass (right) illustrates the average response of the BABAR Detector for the $\tau^{-} \rightarrow K^{-} \pi^{-} \pi^{+} \nu_{\tau} M_{13 a n d 23}$ invariant mass spectrum. 
Appendix C. Unfolded $\tau^{-} \rightarrow \pi^{-} \pi^{-} \pi^{+} \nu_{\tau}$ Invariant Mass Spectra

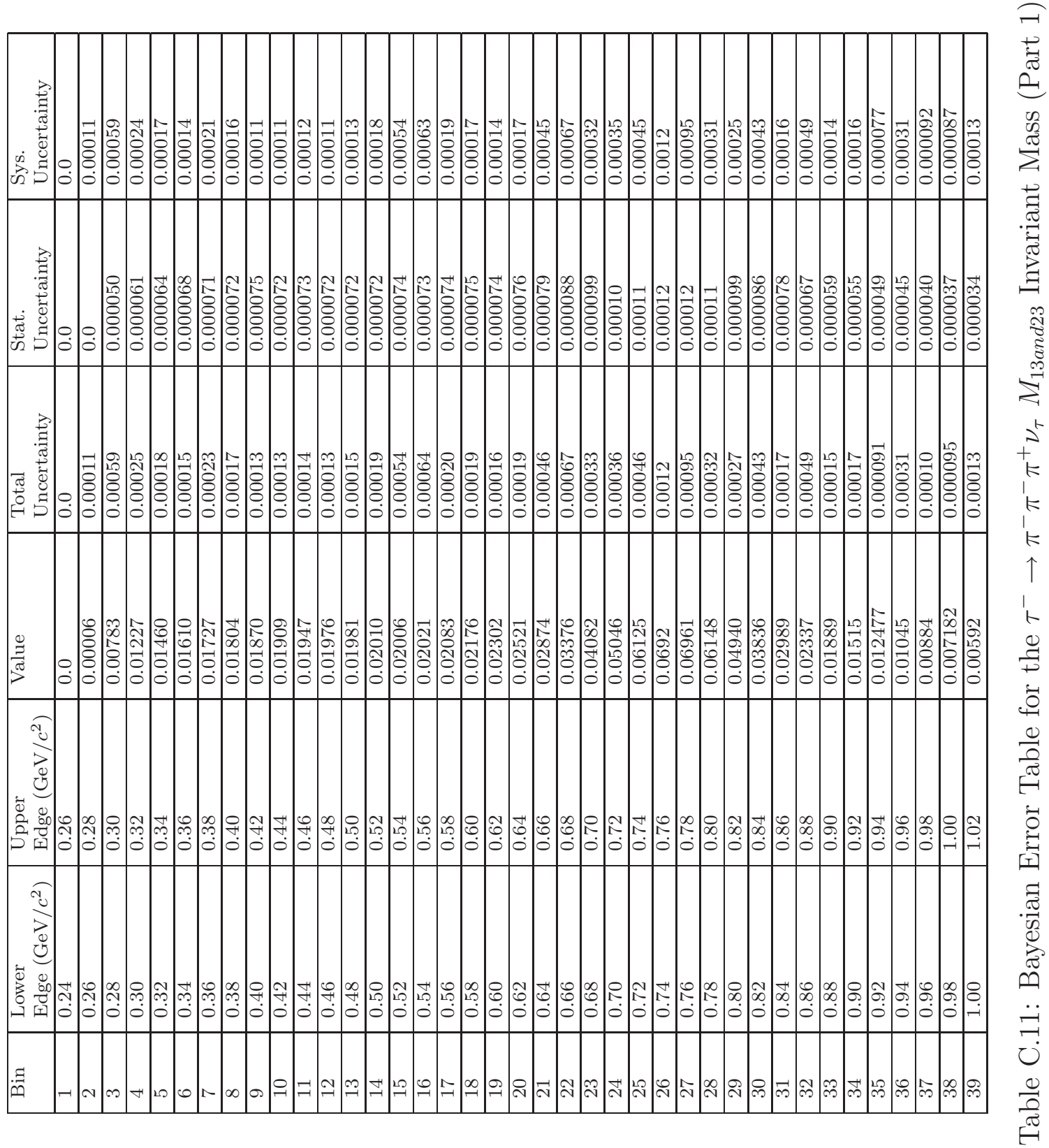




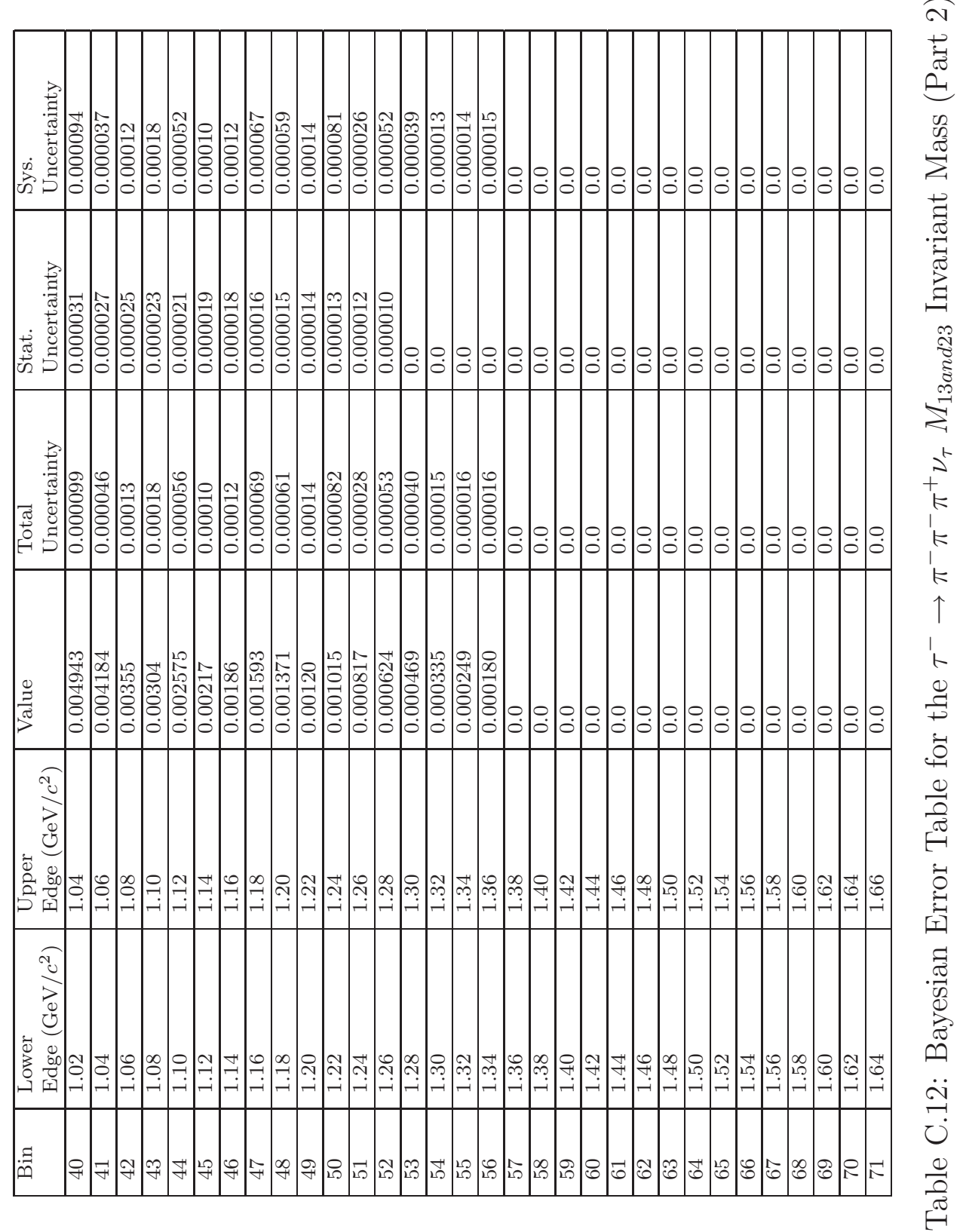




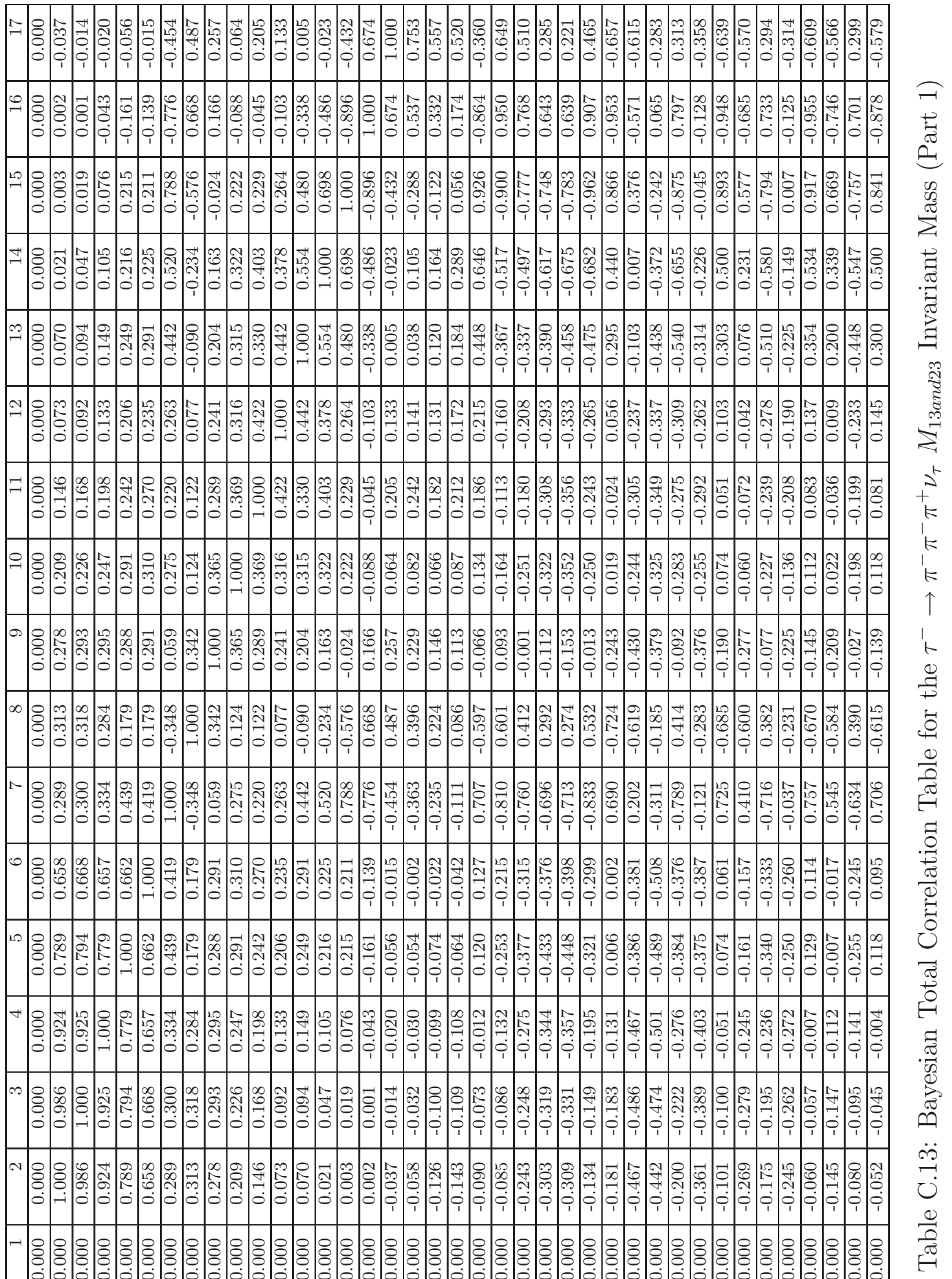




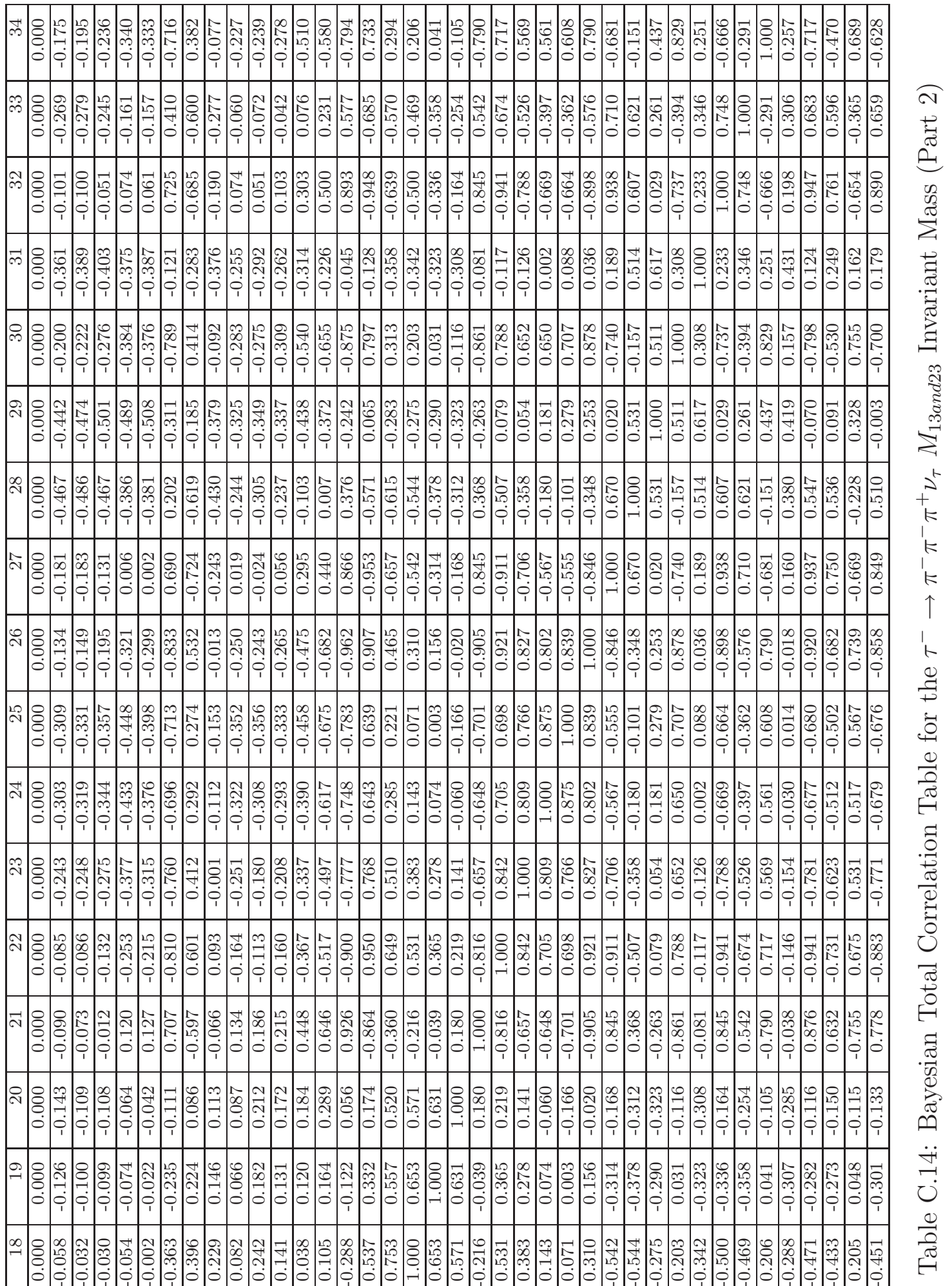




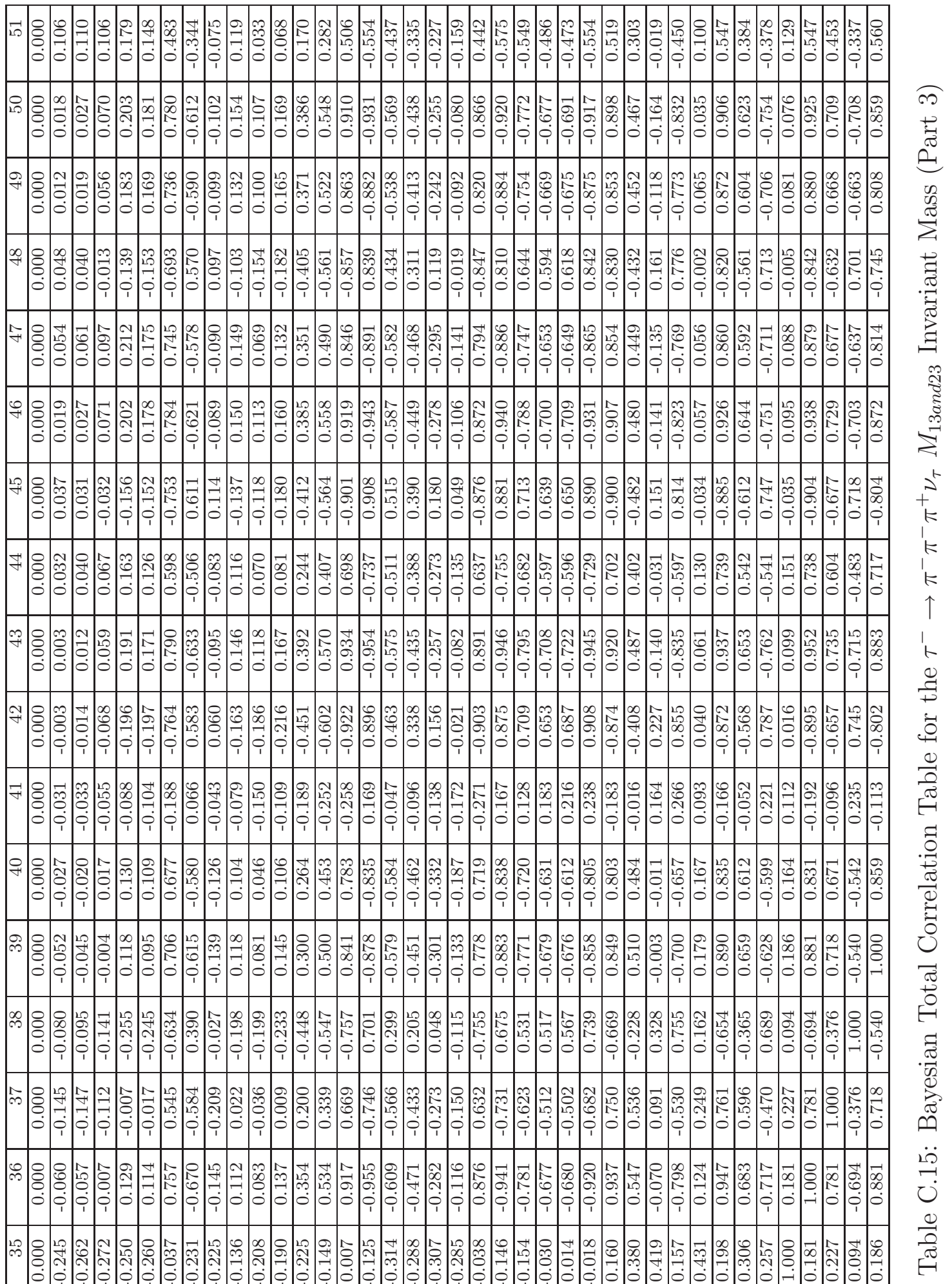




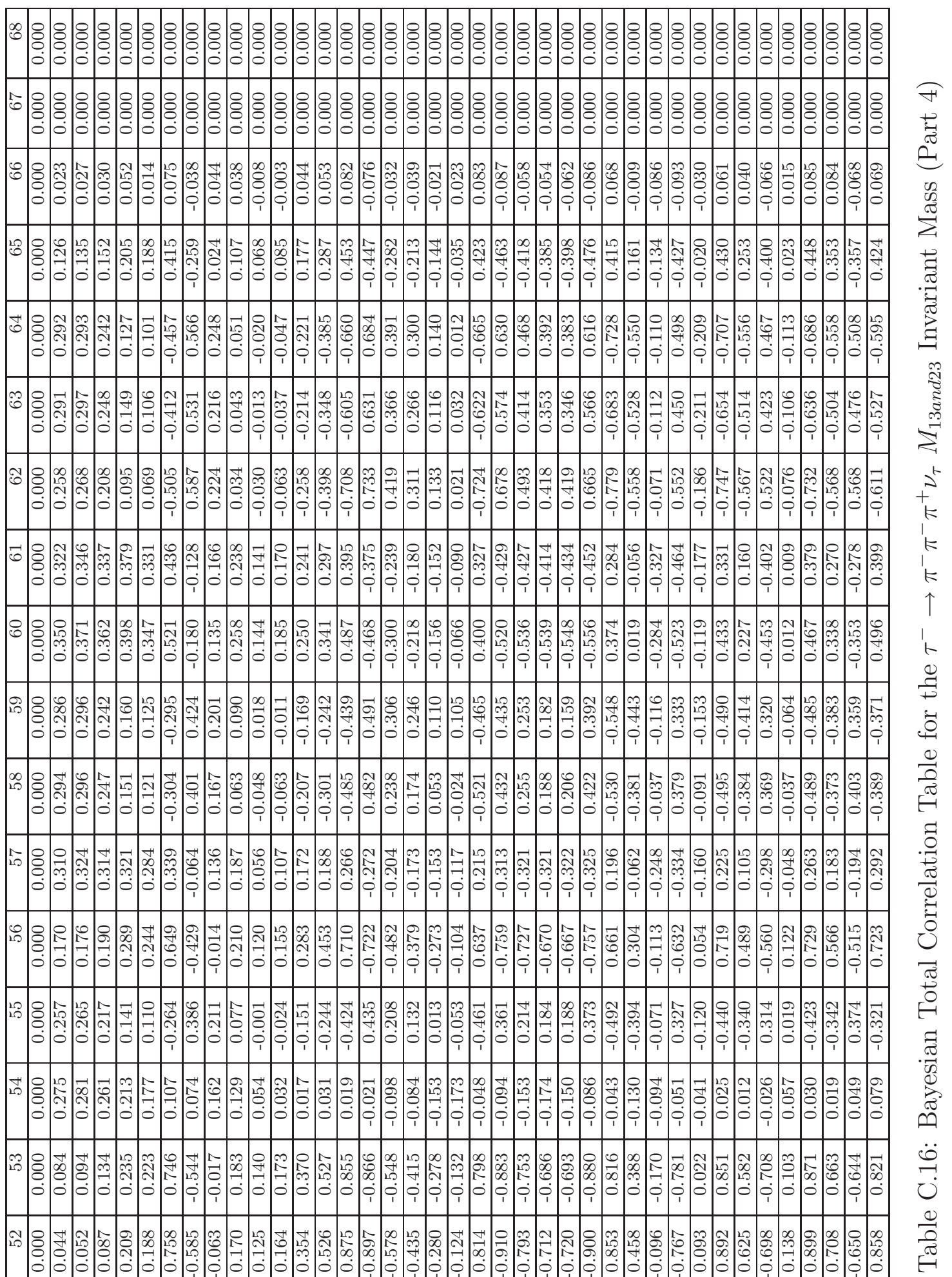


Appendix C. Unfolded $\tau^{-} \rightarrow \pi^{-} \pi^{-} \pi^{+} \nu_{\tau}$ Invariant Mass Spectra

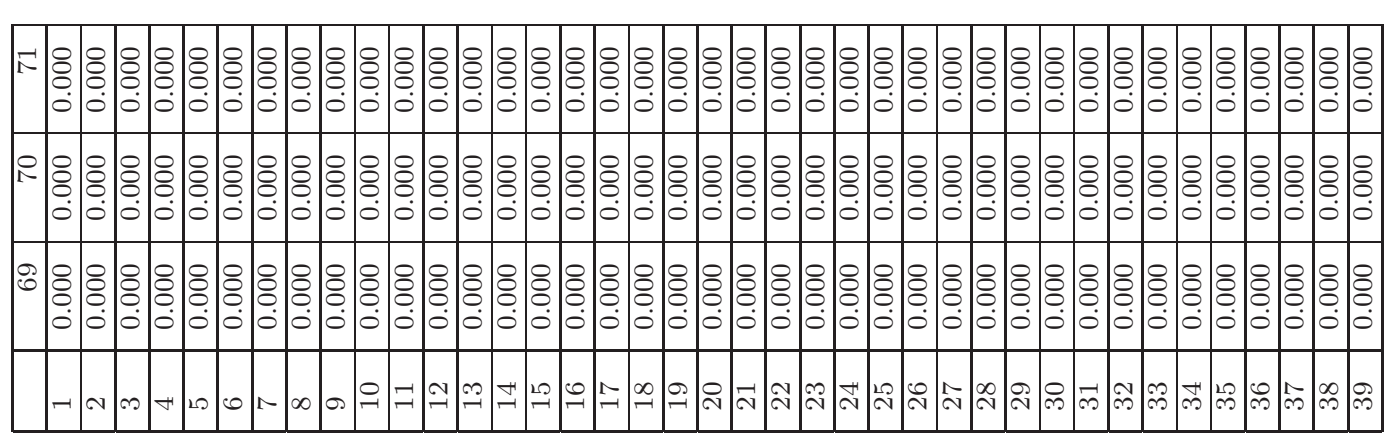

20

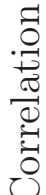

ت્త్ర

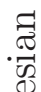

ติ

$\ddot{0}$

$\frac{0}{0}$ 
Appendix C. Unfolded $\tau^{-} \rightarrow \pi^{-} \pi^{-} \pi^{+} \nu_{\tau}$ Invariant Mass Spectra

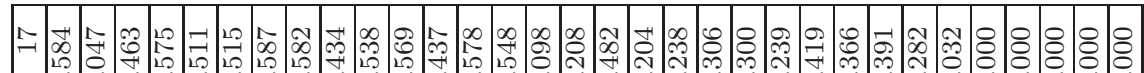

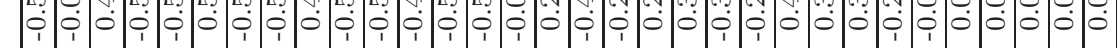

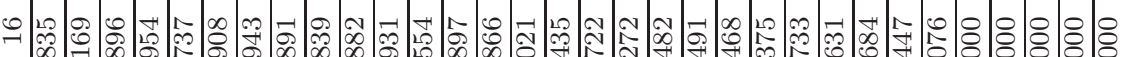

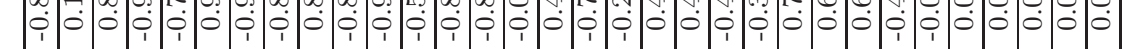

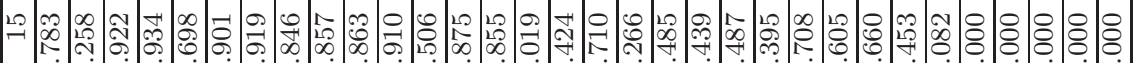

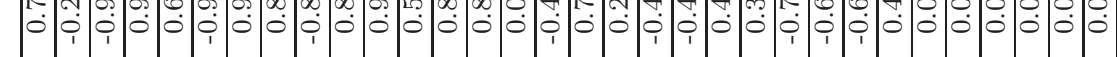

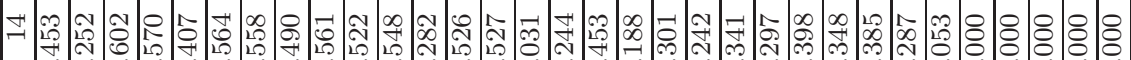

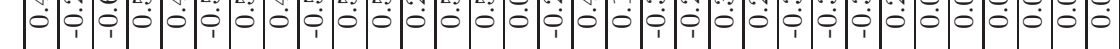

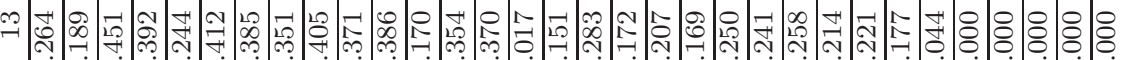

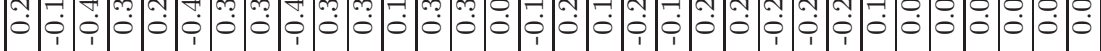

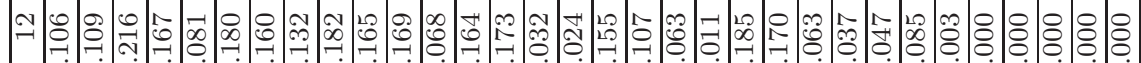

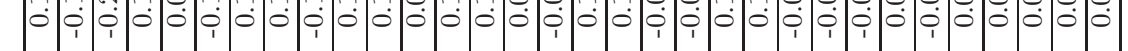

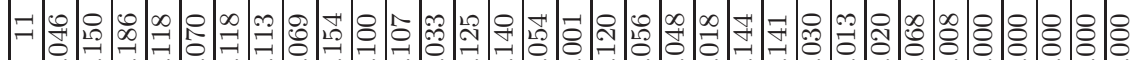

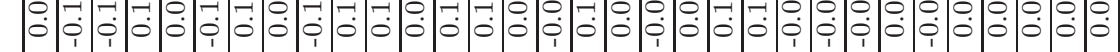

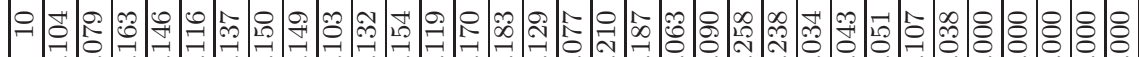

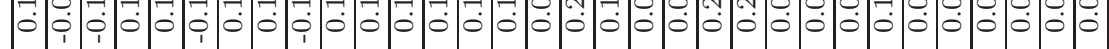

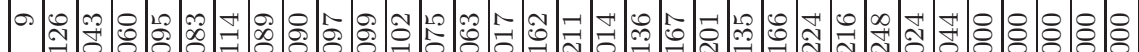

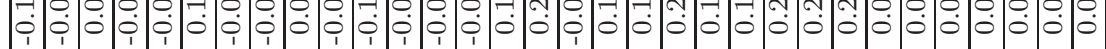

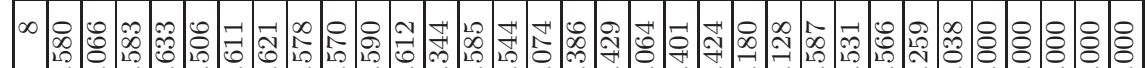

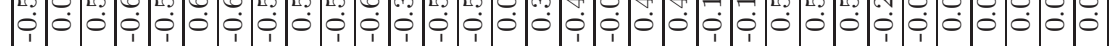

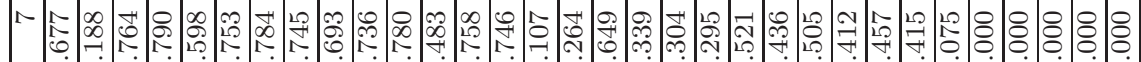

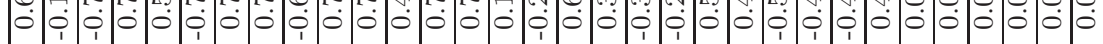

0 2 \&

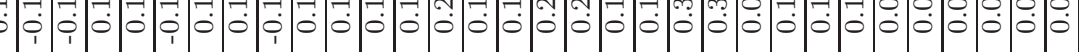

బ

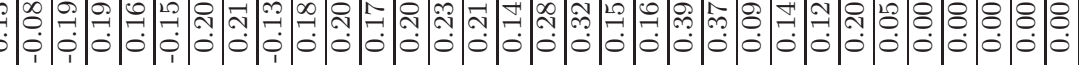

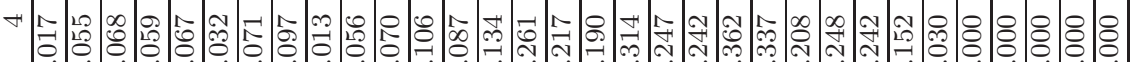

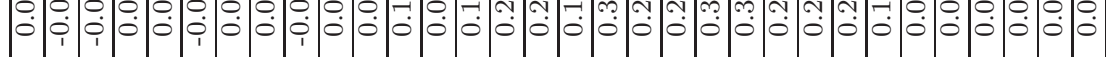

$m$ 定

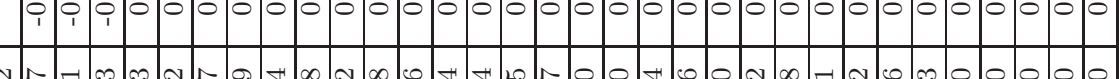

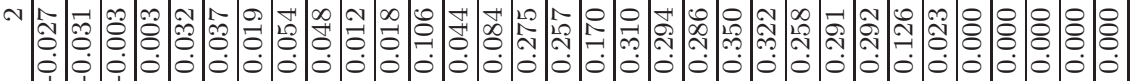
-

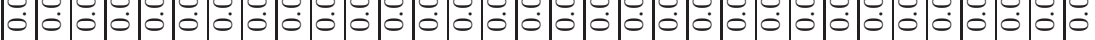


Appendix C. Unfolded $\tau^{-} \rightarrow \pi^{-} \pi^{-} \pi^{+} \nu_{\tau}$ Invariant Mass Spectra

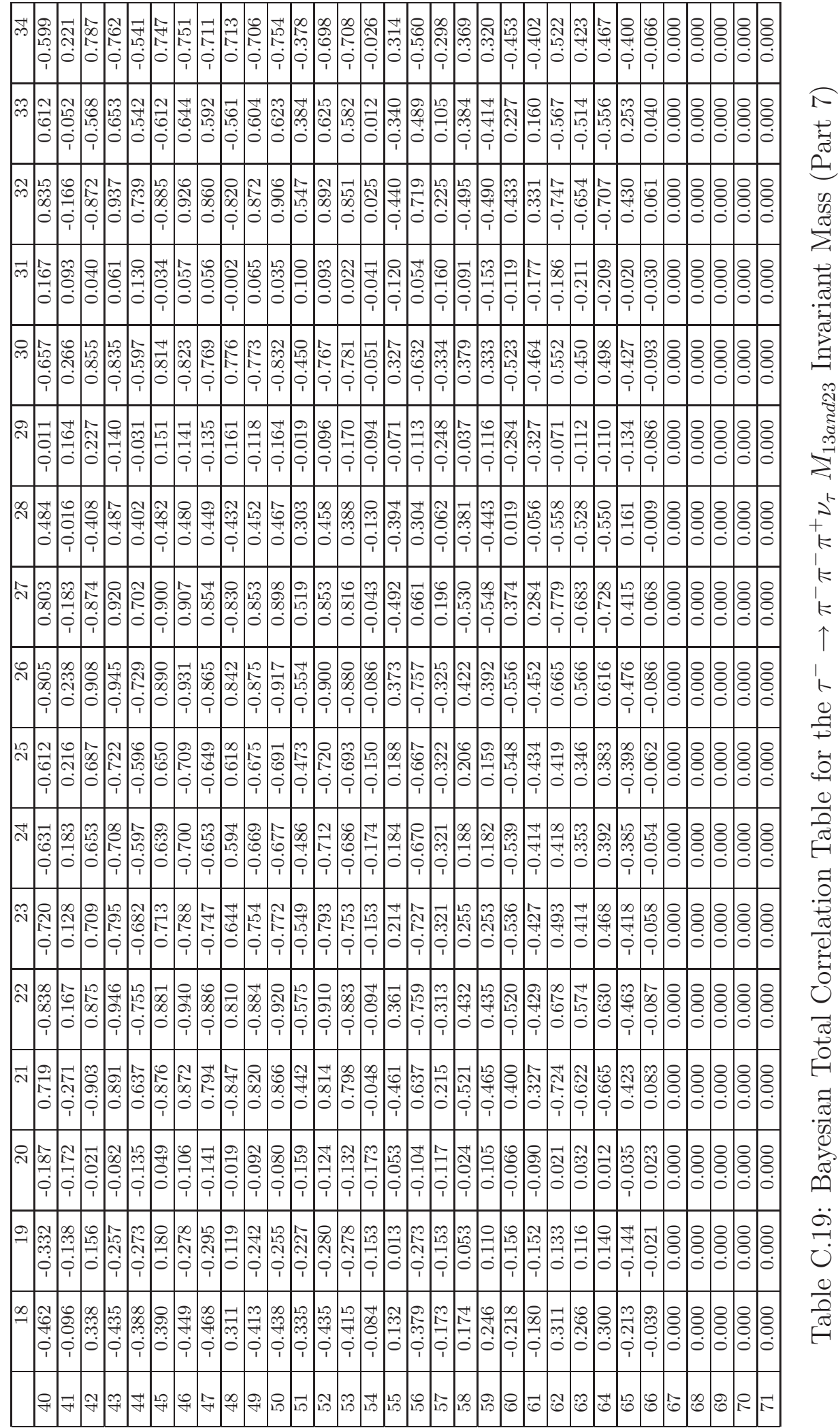


Appendix C. Unfolded $\tau^{-} \rightarrow \pi^{-} \pi^{-} \pi^{+} \nu_{\tau}$ Invariant Mass Spectra

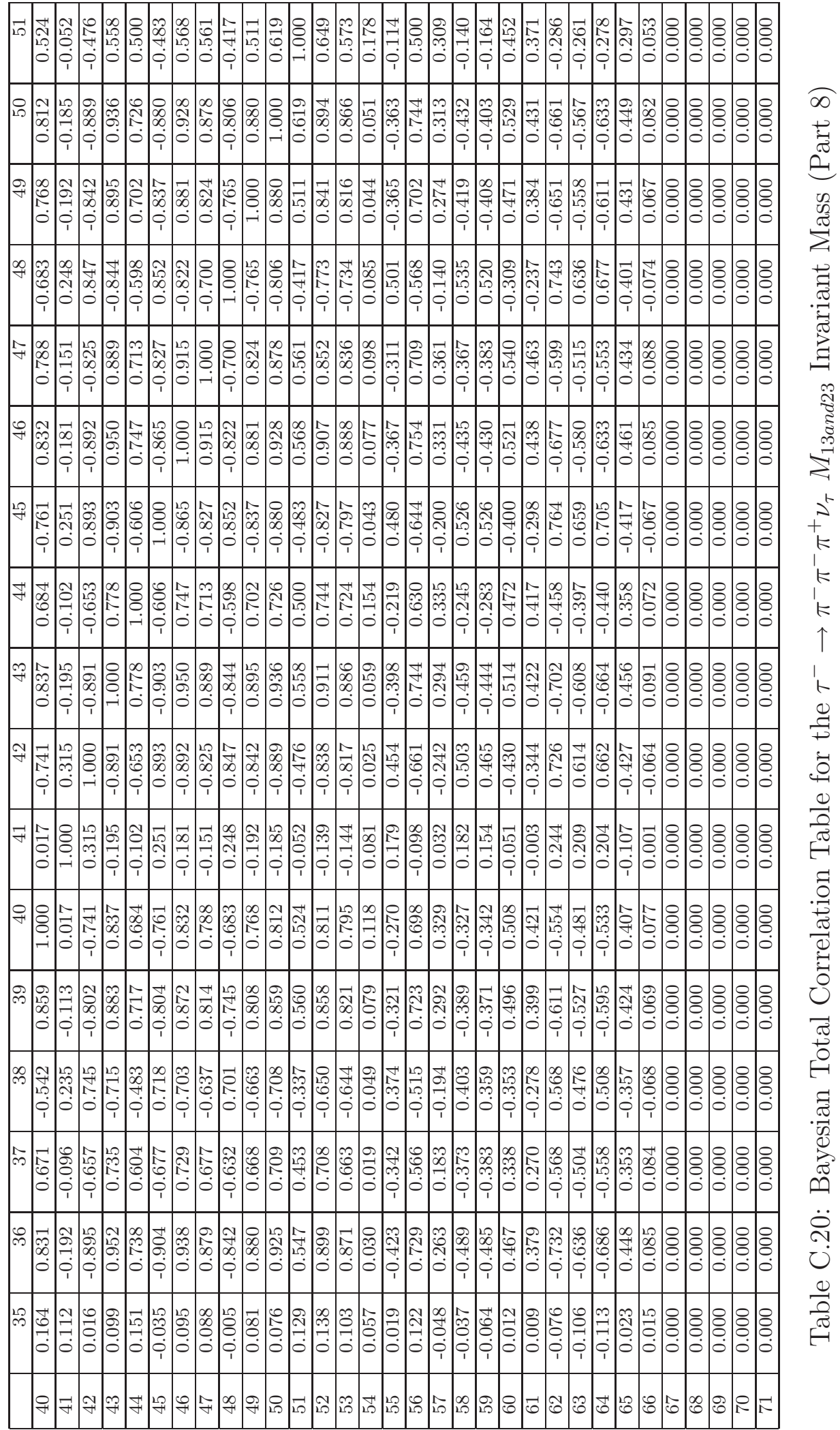


Appendix C. Unfolded $\tau^{-} \rightarrow \pi^{-} \pi^{-} \pi^{+} \nu_{\tau}$ Invariant Mass Spectra

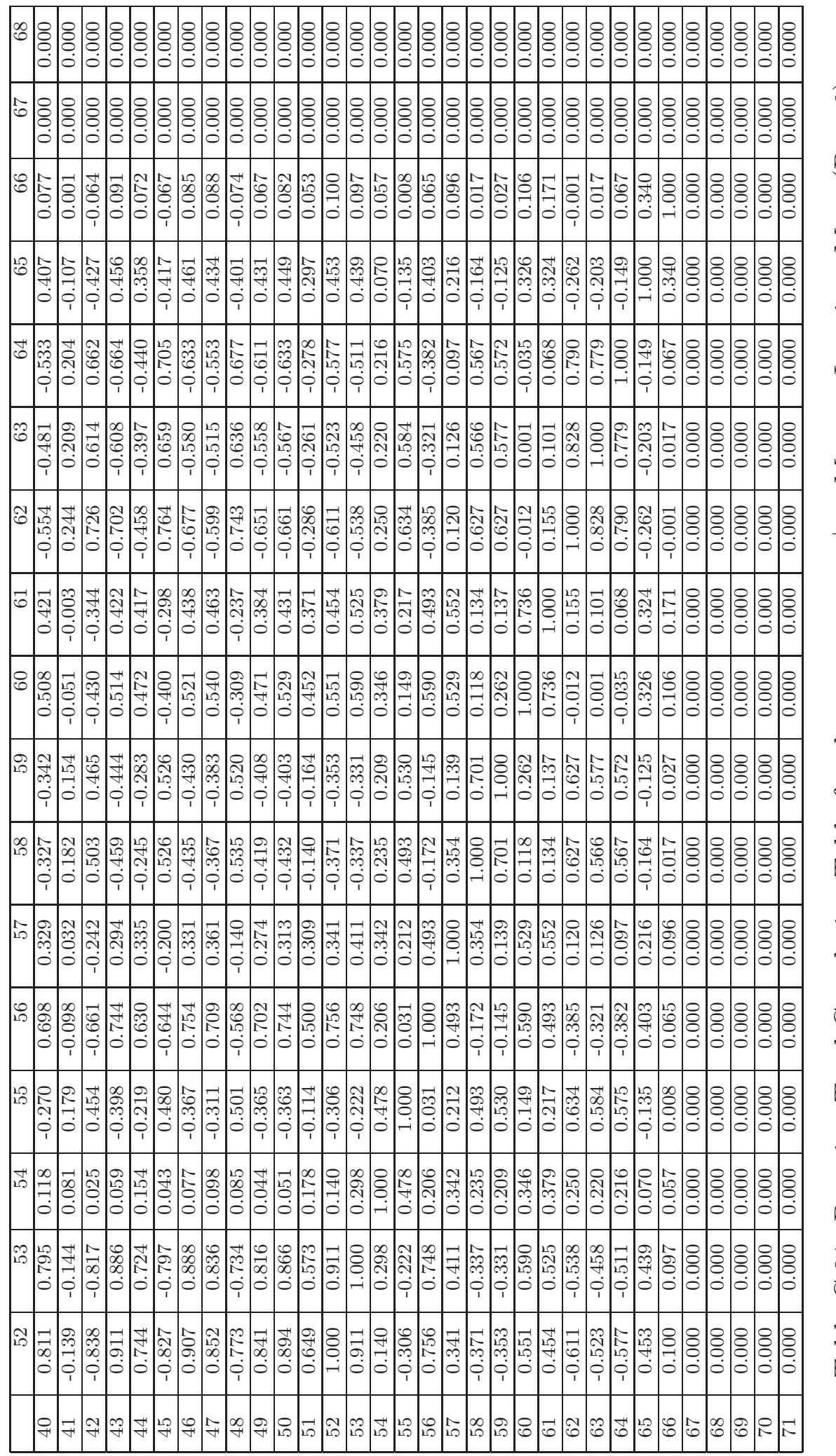


Appendix C. Unfolded $\tau^{-} \rightarrow \pi^{-} \pi^{-} \pi^{+} \nu_{\tau}$ Invariant Mass Spectra

212
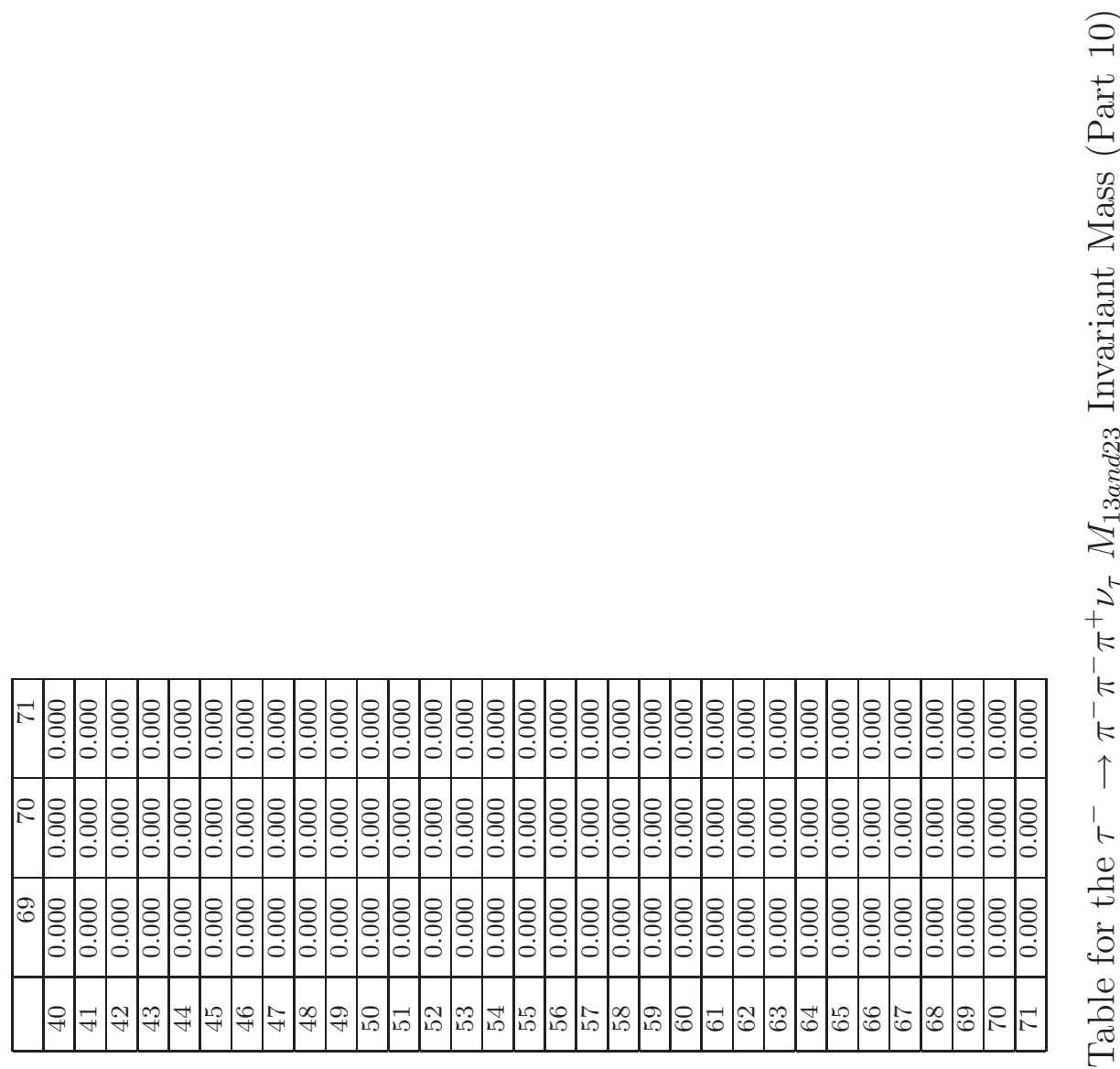

의

赵

旅

苨

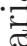

$\Xi$

भ

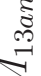

5

$+1$

E

it

宽

[

.

స్తు

$\ddot{\sim}$

ช

$\frac{0}{\frac{\pi}{\pi}}$ 


\section{Appendix D}

\section{Unfolded $\tau^{-} \rightarrow K^{-} \pi^{-} \pi^{+} \nu_{\tau}$ Invariant Mass Spectra}

D.1 The Unfolded $\tau^{-} \rightarrow K^{-} \pi^{-} \pi^{+} \nu_{\tau} M_{123}$ Invariant Mass Spectra 

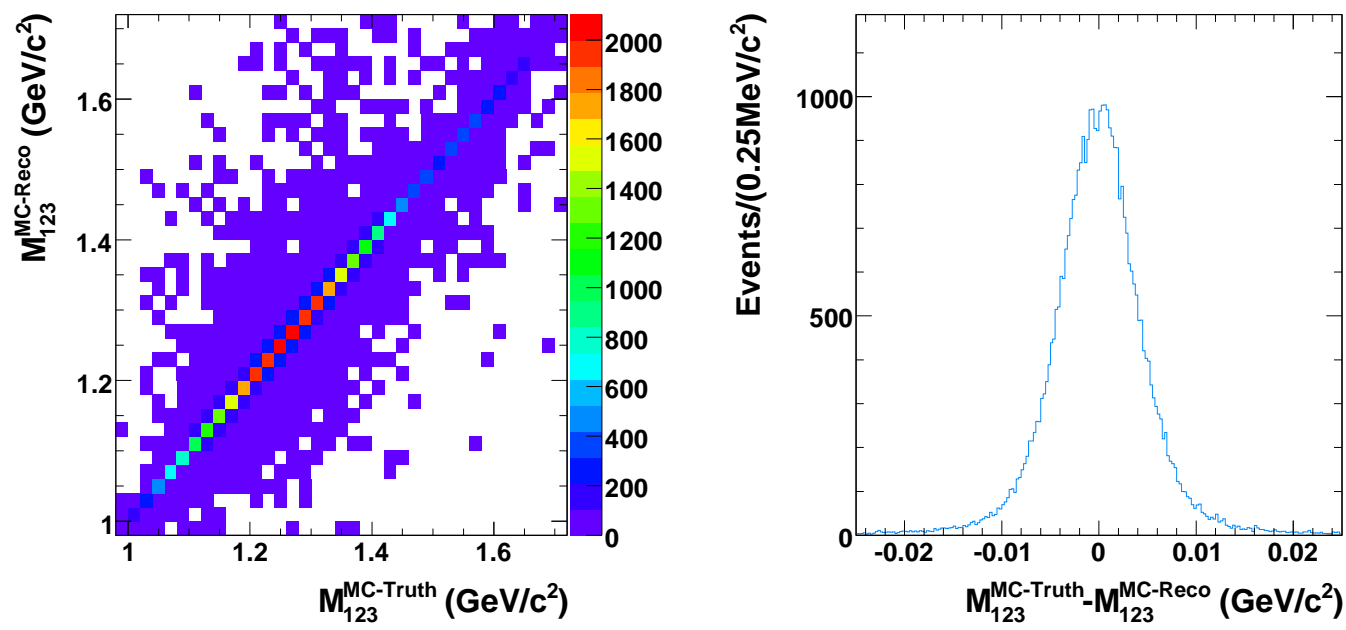

Figure D.1: A comparison of the $\tau^{-} \rightarrow K^{-} \pi^{-} \pi^{+} \nu_{\tau}$ MC Truth and reconstructed MC for the $M_{123}$ invariant mass spectra. The reconstructed MC $M_{123}$ invariant mass as a function of MC Truth $M_{123}$ invariant mass (left) is used to describe the response of the BABAR Detector in the unfolding of the $\tau^{-} \rightarrow K^{-} \pi^{-} \pi^{+} \nu_{\tau} M_{123}$ invariant mass spectrum. The reconstructed MC $M_{123}$ invariant mass minus the MC Truth $M_{123}$ invariant mass (right) illustrates the average response of the BABAR Detector for the $\tau^{-} \rightarrow K^{-} \pi^{-} \pi^{+} \nu_{\tau} M_{123}$ invariant mass spectrum. 


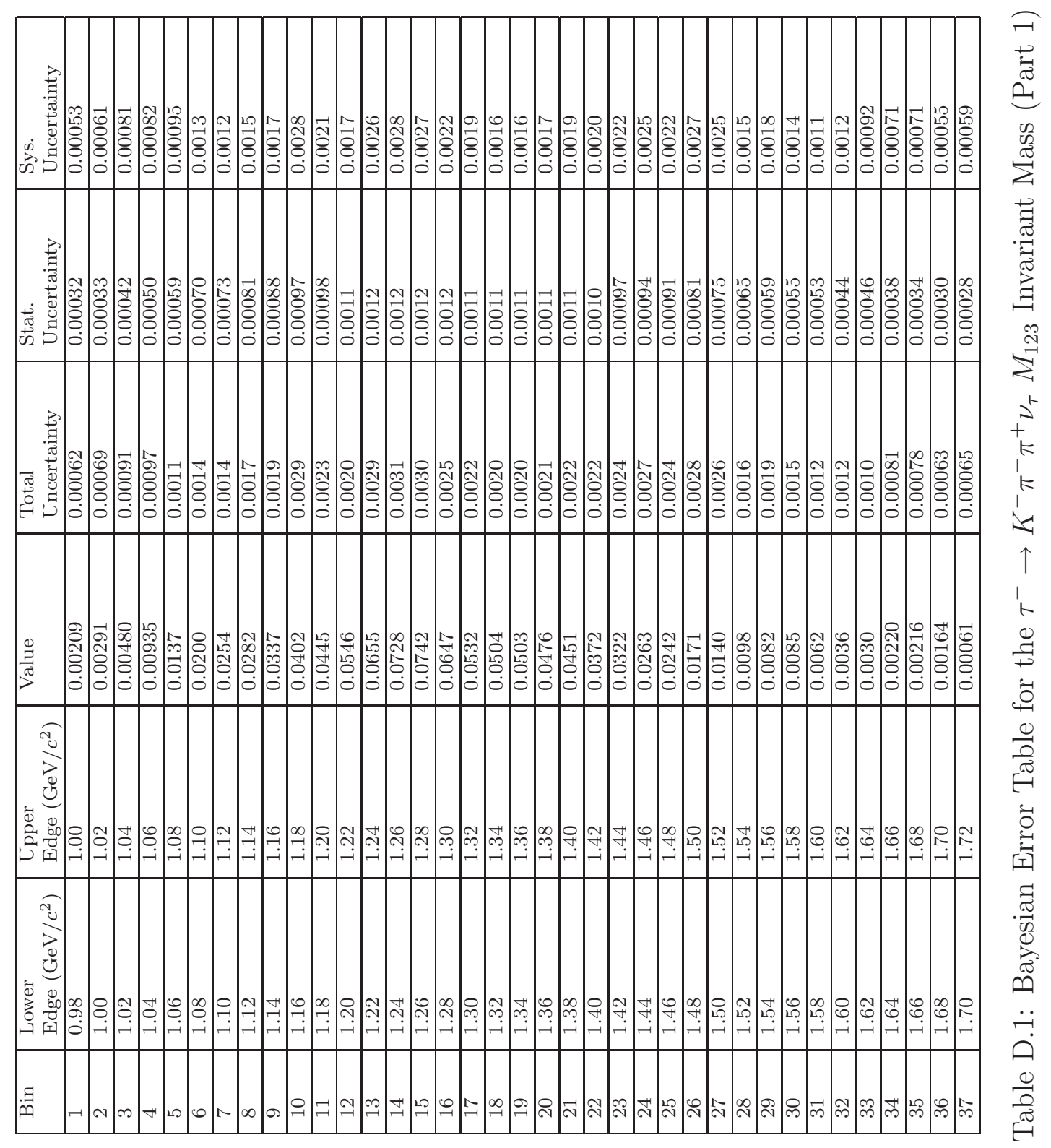




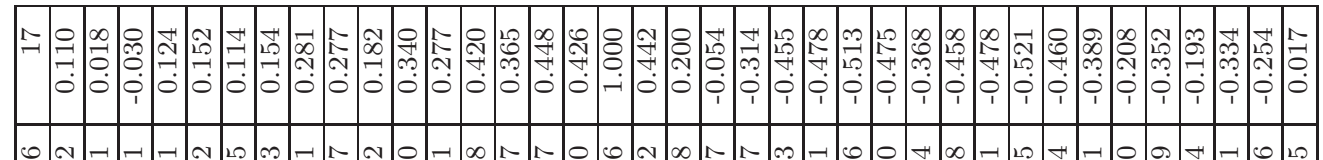

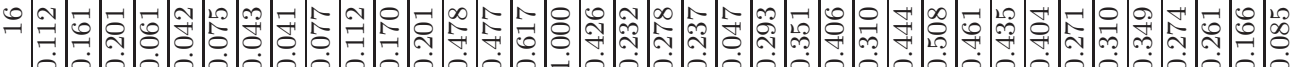

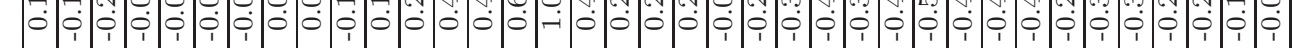

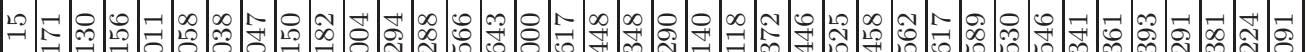

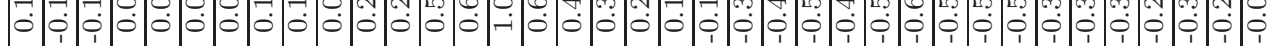

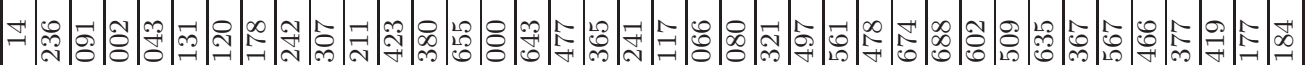

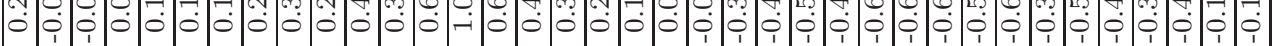

m

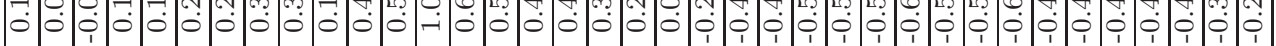

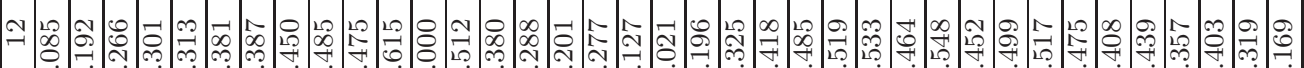

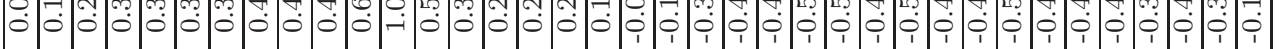

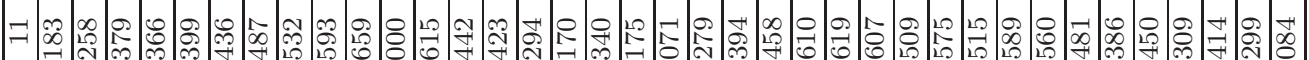

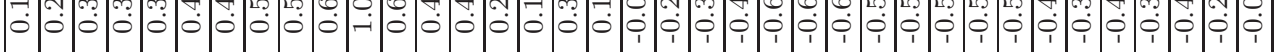

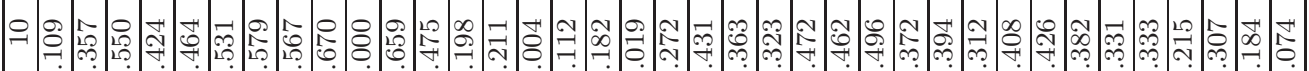

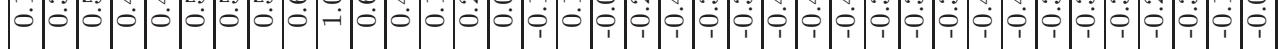

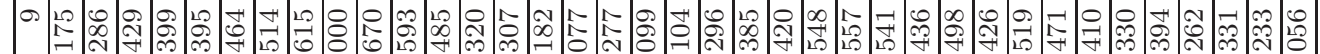

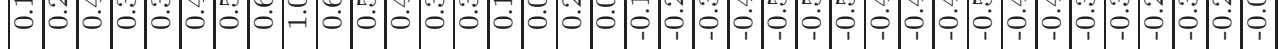

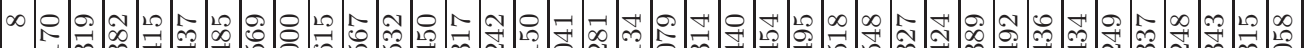

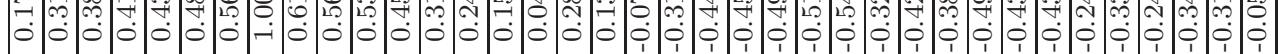

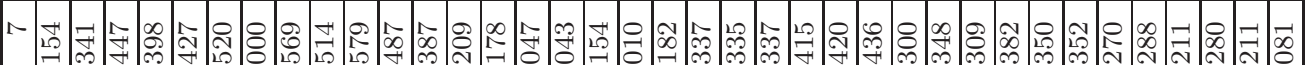

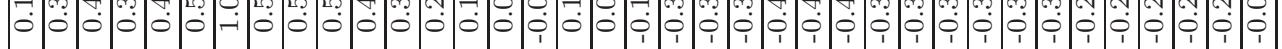

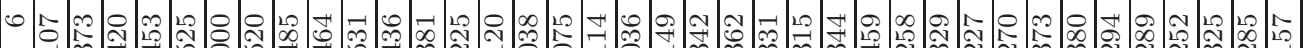

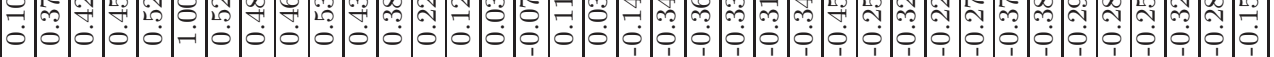

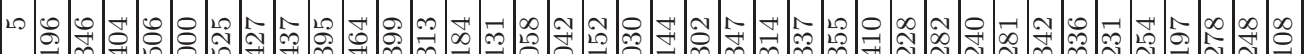

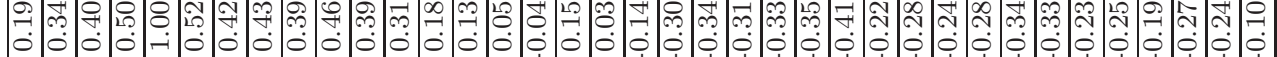

म సै

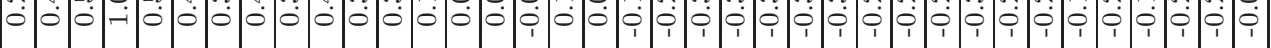

$m$ m $\infty$ \&

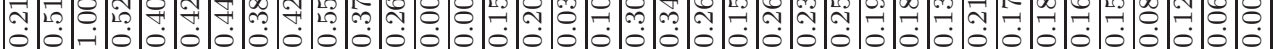

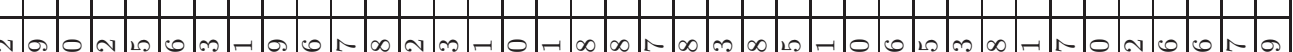

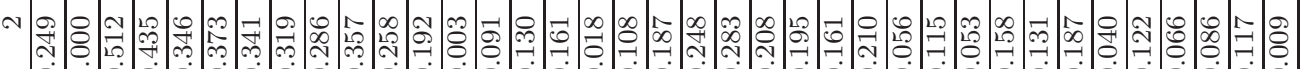

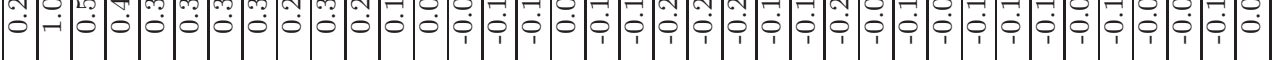

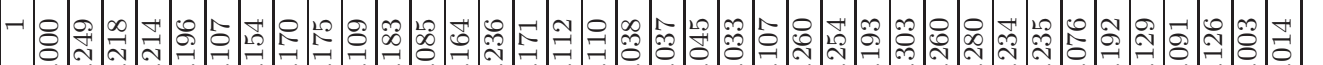

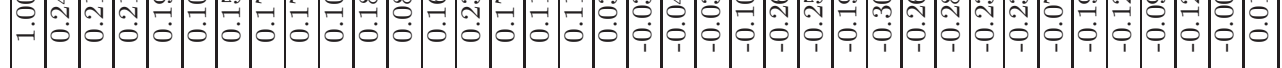


m

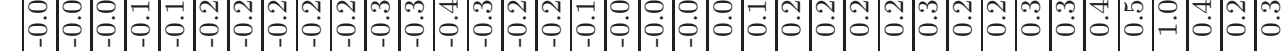

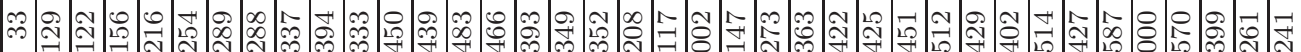

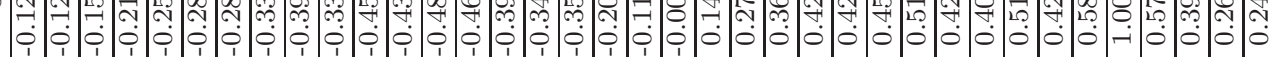

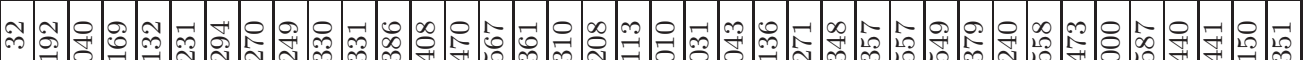

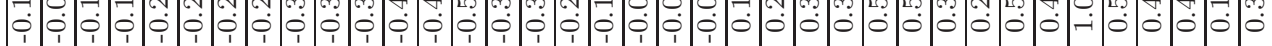

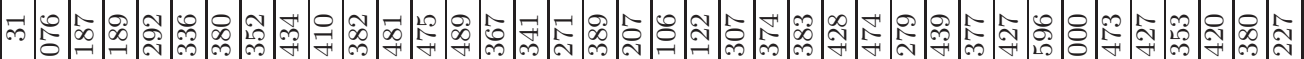

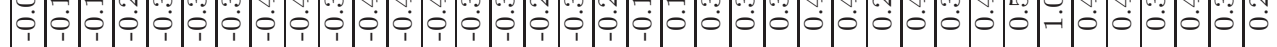

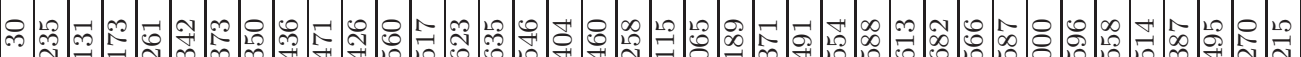

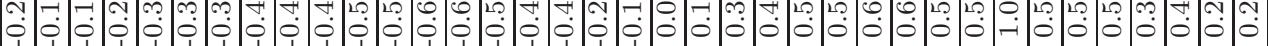

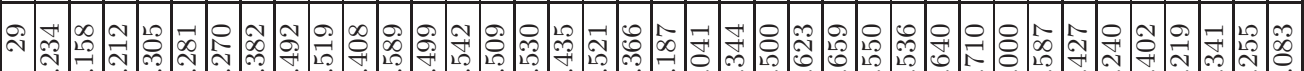

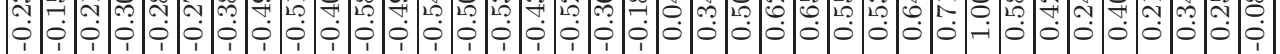

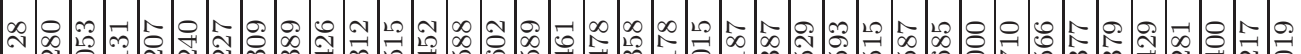

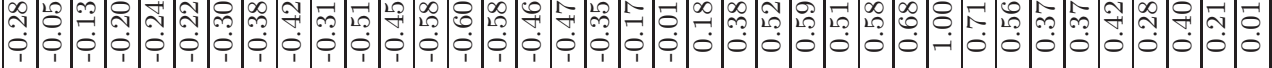

N

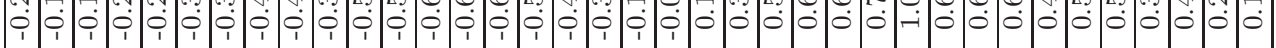

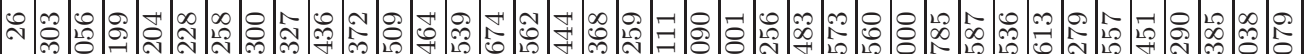

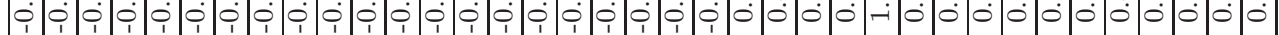

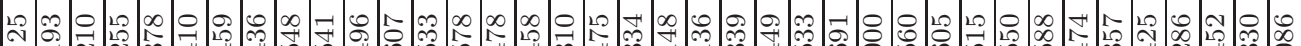

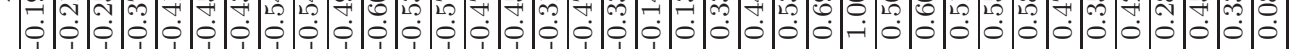

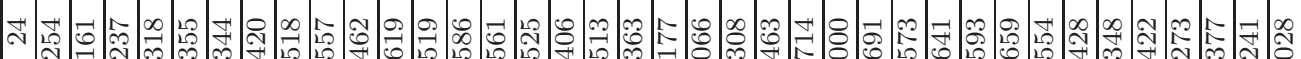

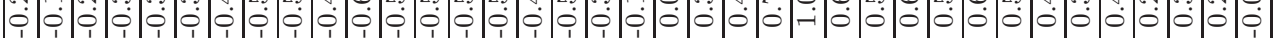

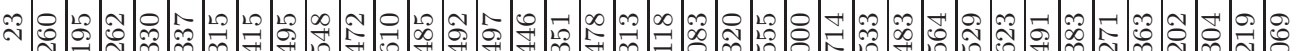

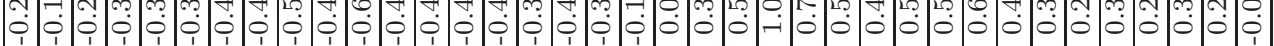

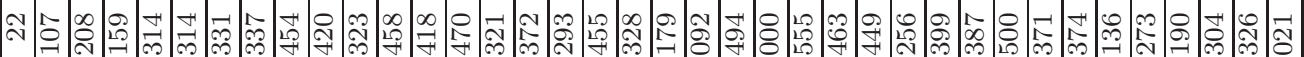

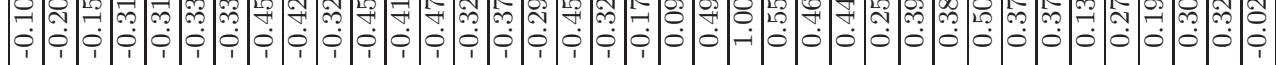

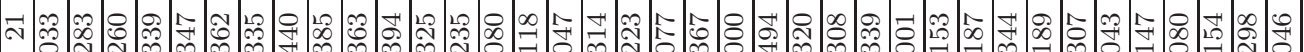

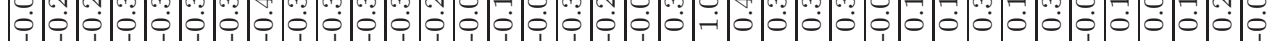

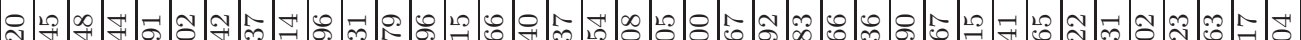

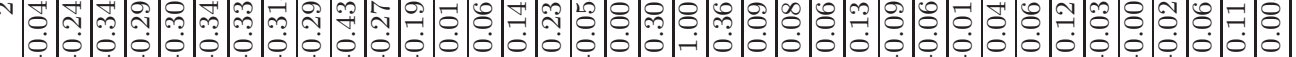

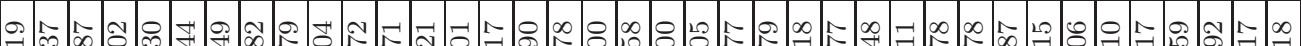

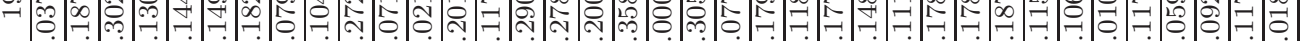

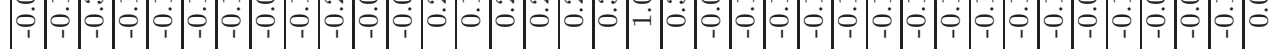

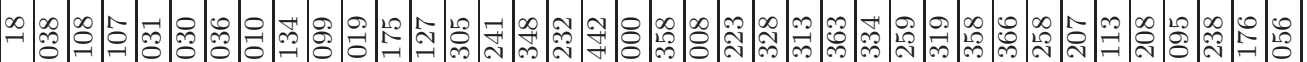

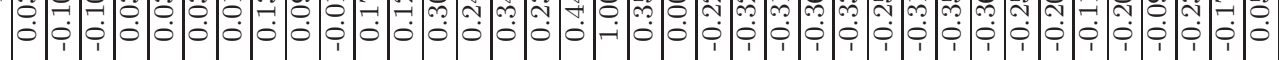


Appendix D. Unfolded $\tau^{-} \rightarrow K^{-} \pi^{-} \pi^{+} \nu_{\tau}$ Invariant Mass Spectra 


\section{D.2 The Unfolded $\tau^{-} \rightarrow K^{-} \pi^{-} \pi^{+} \nu_{\tau} M_{13}$ Invariant Mass Spectra}
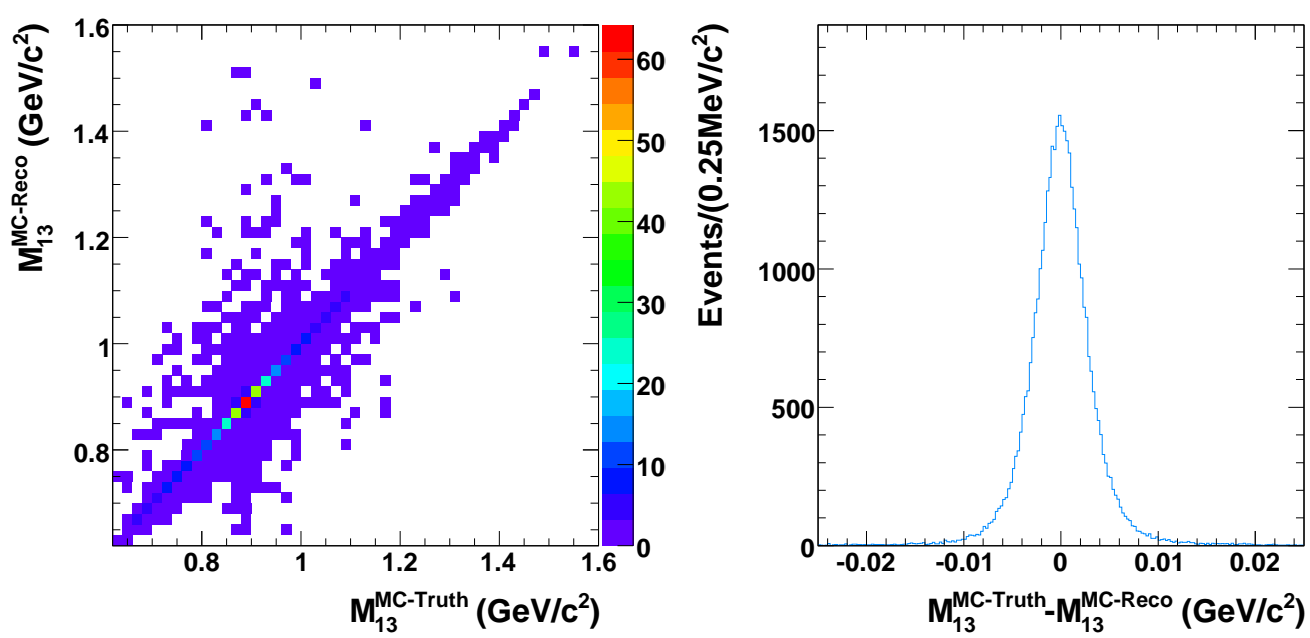

Figure D.2: A comparison of the $\tau^{-} \rightarrow K^{-} \pi^{-} \pi^{+} \nu_{\tau}$ MC Truth and reconstructed MC for the $M_{13}$ invariant mass spectra. The reconstructed MC $M_{13}$ invariant mass as a function of MC Truth $M_{13}$ invariant mass (left) is used to describe the response of the BABAR Detector in the unfolding of the $\tau^{-} \rightarrow K^{-} \pi^{-} \pi^{+} \nu_{\tau} M_{13}$ invariant mass spectrum. The reconstructed MC $M_{13}$ invariant mass minus the $\mathrm{MC}$ Truth $M_{13}$ invariant mass (right) illustrates the average response of the BABAR Detector for the $\tau^{-} \rightarrow K^{-} \pi^{-} \pi^{+} \nu_{\tau} M_{13}$ invariant mass spectrum. 
Appendix D. Unfolded $\tau^{-} \rightarrow K^{-} \pi^{-} \pi^{+} \nu_{\tau}$ Invariant Mass Spectra

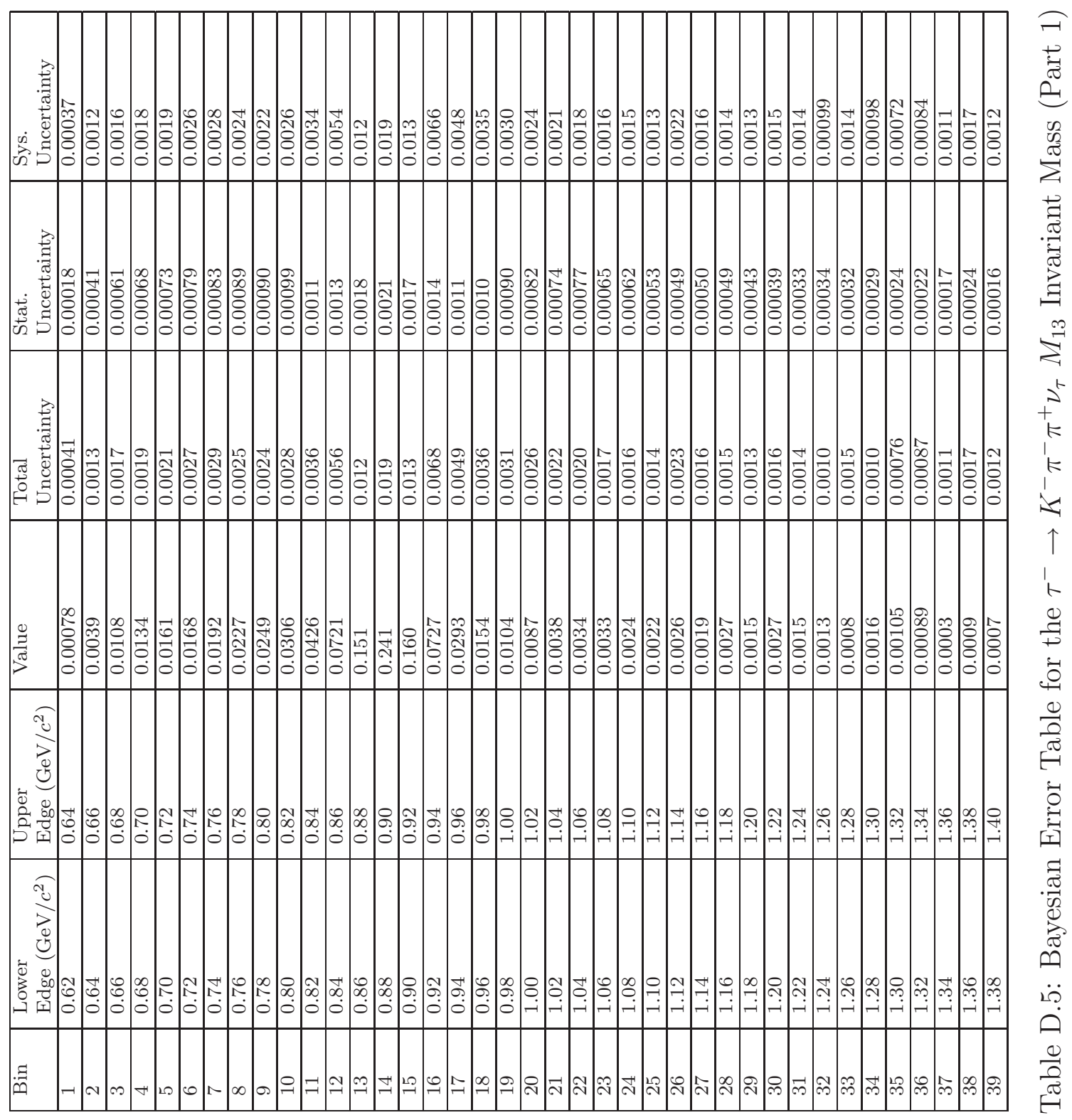




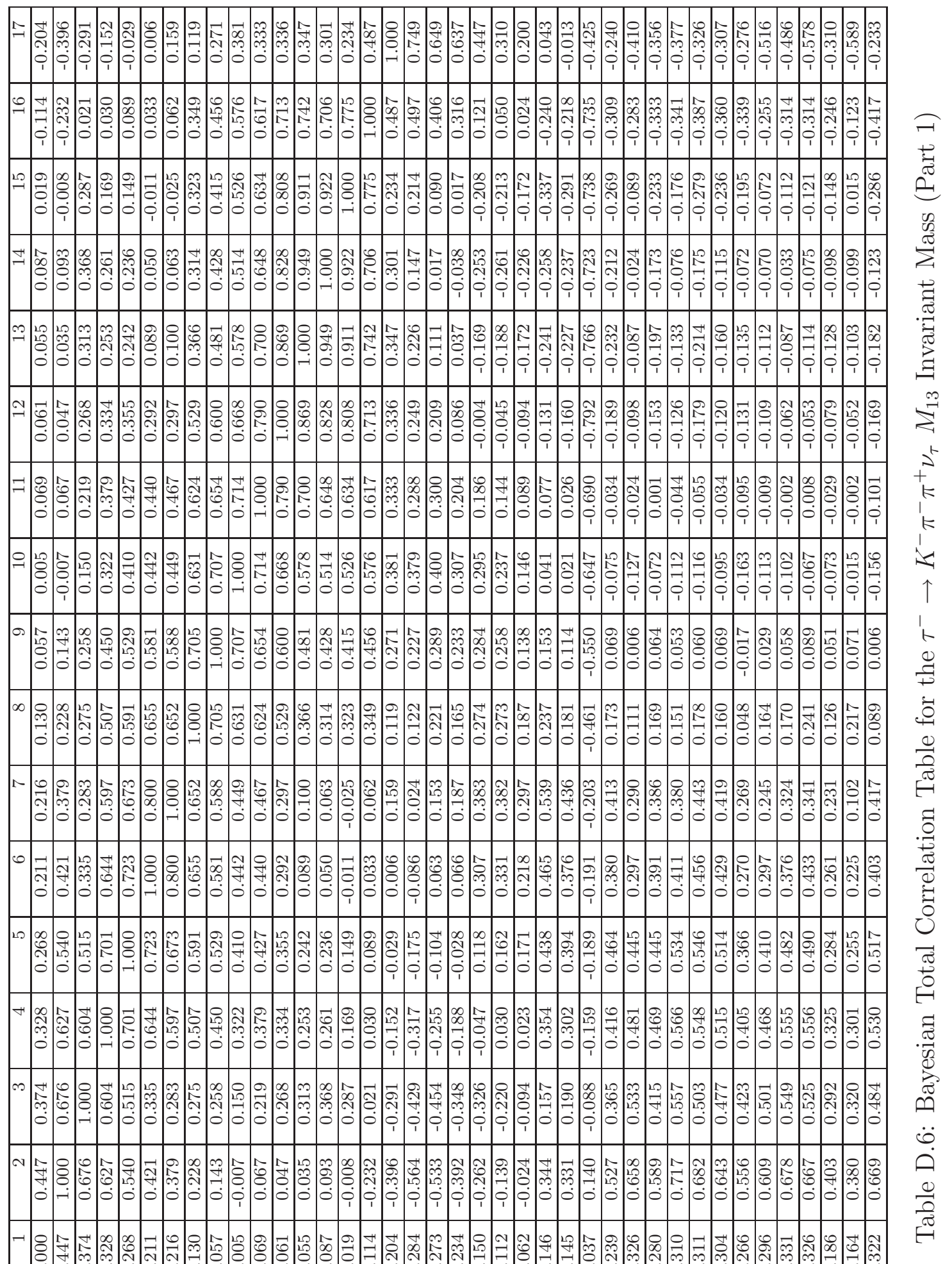

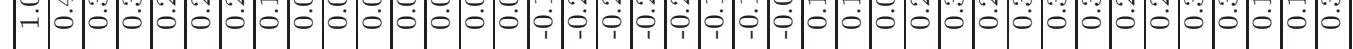

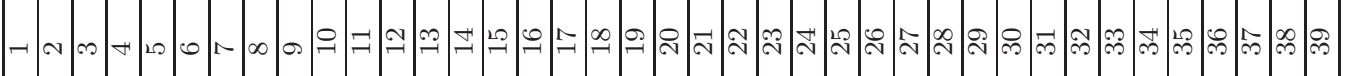




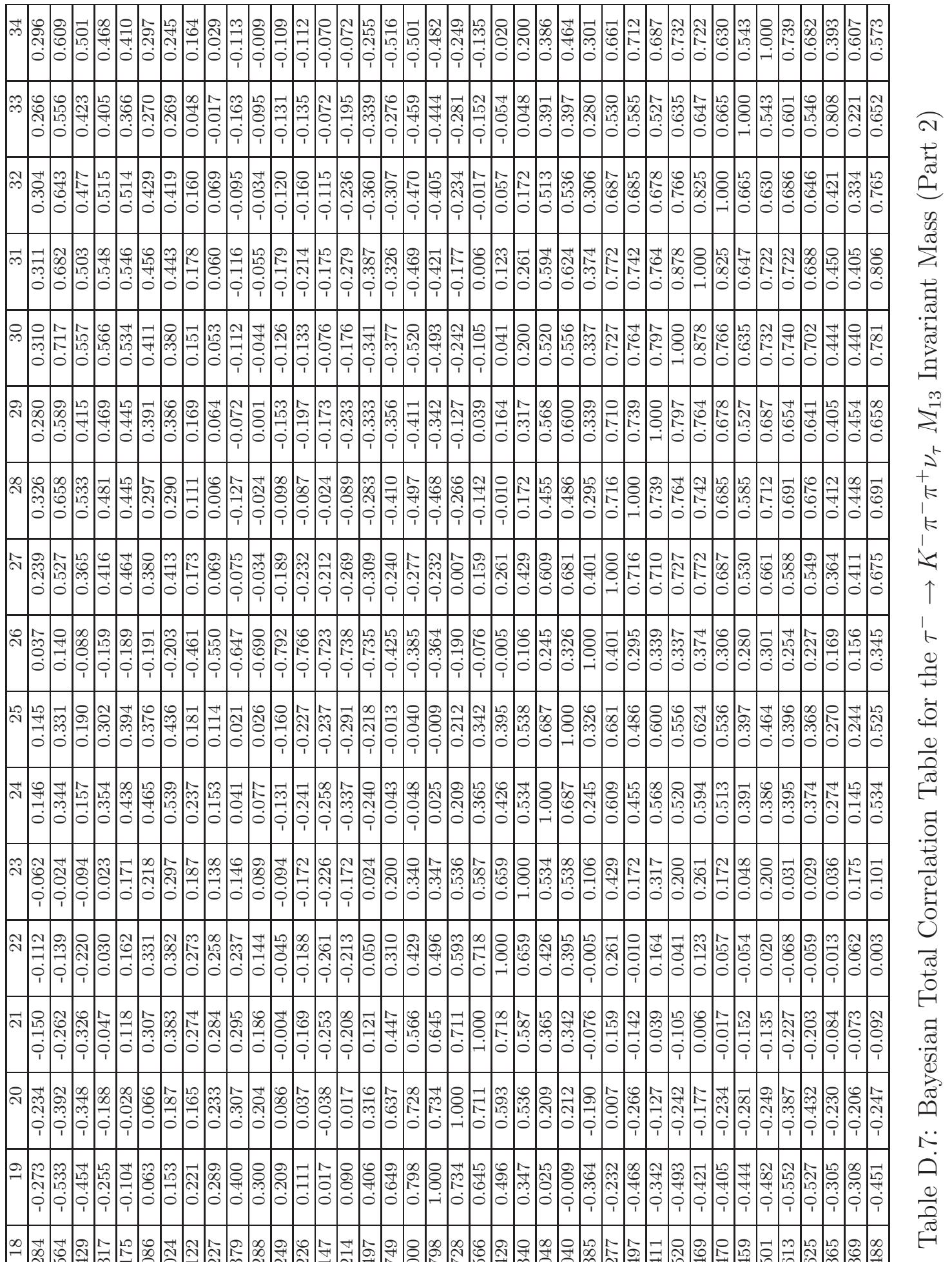

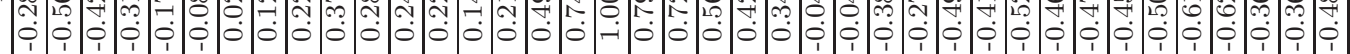

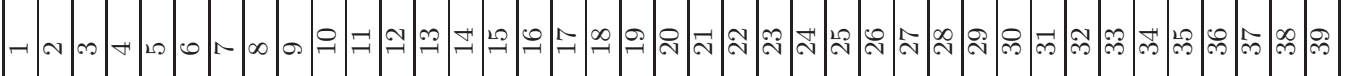


Appendix D. Unfolded $\tau^{-} \rightarrow K^{-} \pi^{-} \pi^{+} \nu_{\tau}$ Invariant Mass Spectra

ก

䓂

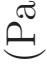

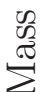

국

.

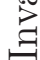

$\stackrel{m}{\geq}$

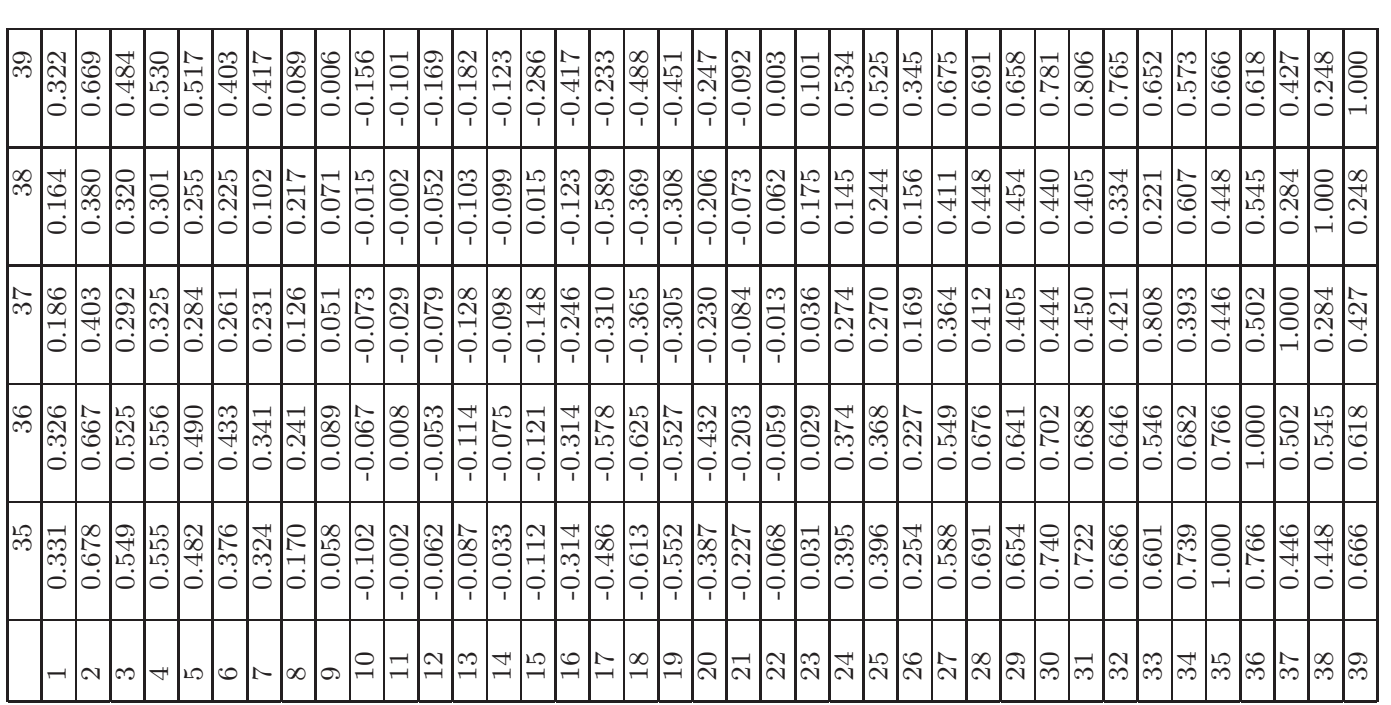

$+\frac{1}{2}$

I

อี

$\frac{0}{2}$

.

ֻั0

ن

疋

.

๑

$\infty$

$\frac{0}{2}$ 


\section{D.3 The Unfolded $\tau^{-} \rightarrow K^{-} \pi^{-} \pi^{+} \nu_{\tau} M_{23}$ Invariant Mass Spectra}
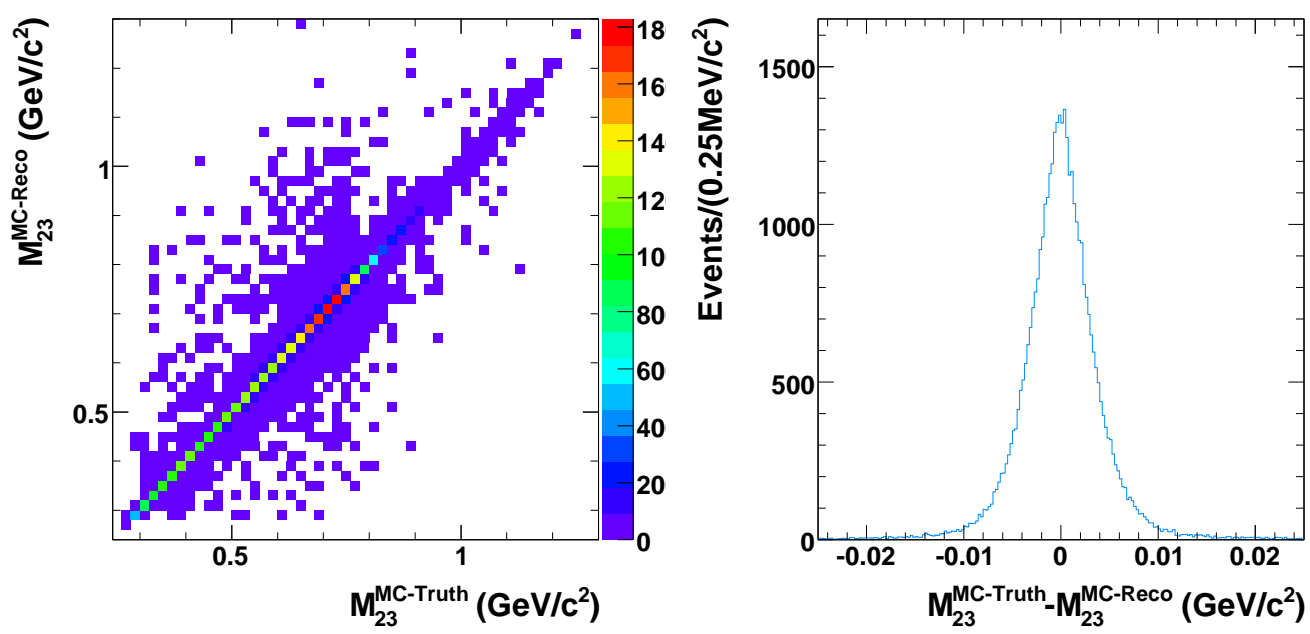

Figure D.3: A comparison of the $\tau^{-} \rightarrow K^{-} \pi^{-} \pi^{+} \nu_{\tau}$ MC Truth and reconstructed MC for the $M_{23}$ invariant mass spectra. The reconstructed MC $M_{23}$ invariant mass as a function of MC Truth $M_{23}$ invariant mass (left) is used to describe the response of the BABAR Detector in the unfolding of the $\tau^{-} \rightarrow K^{-} \pi^{-} \pi^{+} \nu_{\tau} M_{23}$ invariant mass spectrum. The reconstructed MC $M_{23}$ invariant mass minus the $\mathrm{MC}$ Truth $M_{23}$ invariant mass (right) illustrates the average response of the BABAR Detector for the $\tau^{-} \rightarrow K^{-} \pi^{-} \pi^{+} \nu_{\tau} M_{23}$ invariant mass spectrum. 
Appendix D. Unfolded $\tau^{-} \rightarrow K^{-} \pi^{-} \pi^{+} \nu_{\tau}$ Invariant Mass Spectra

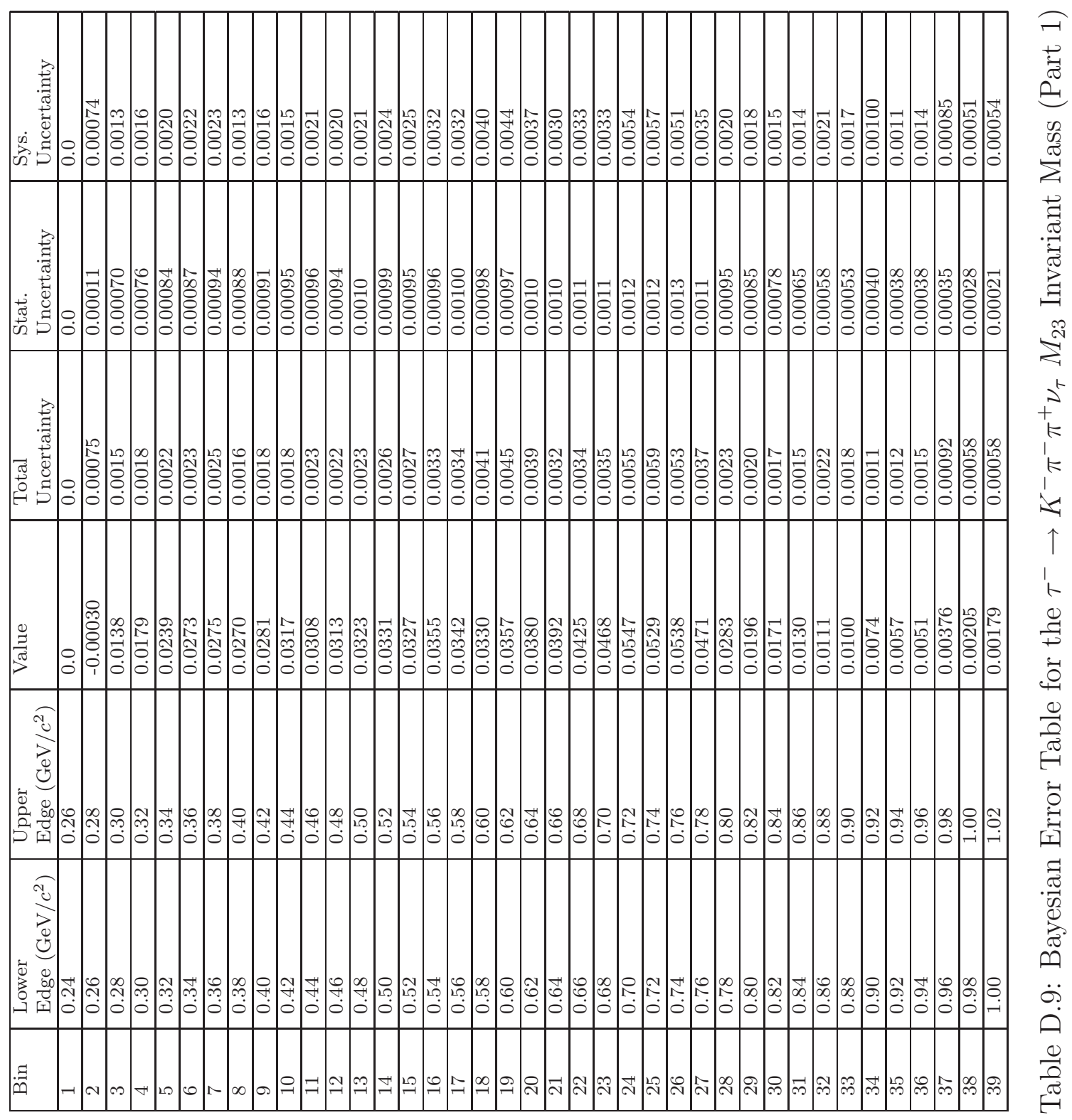




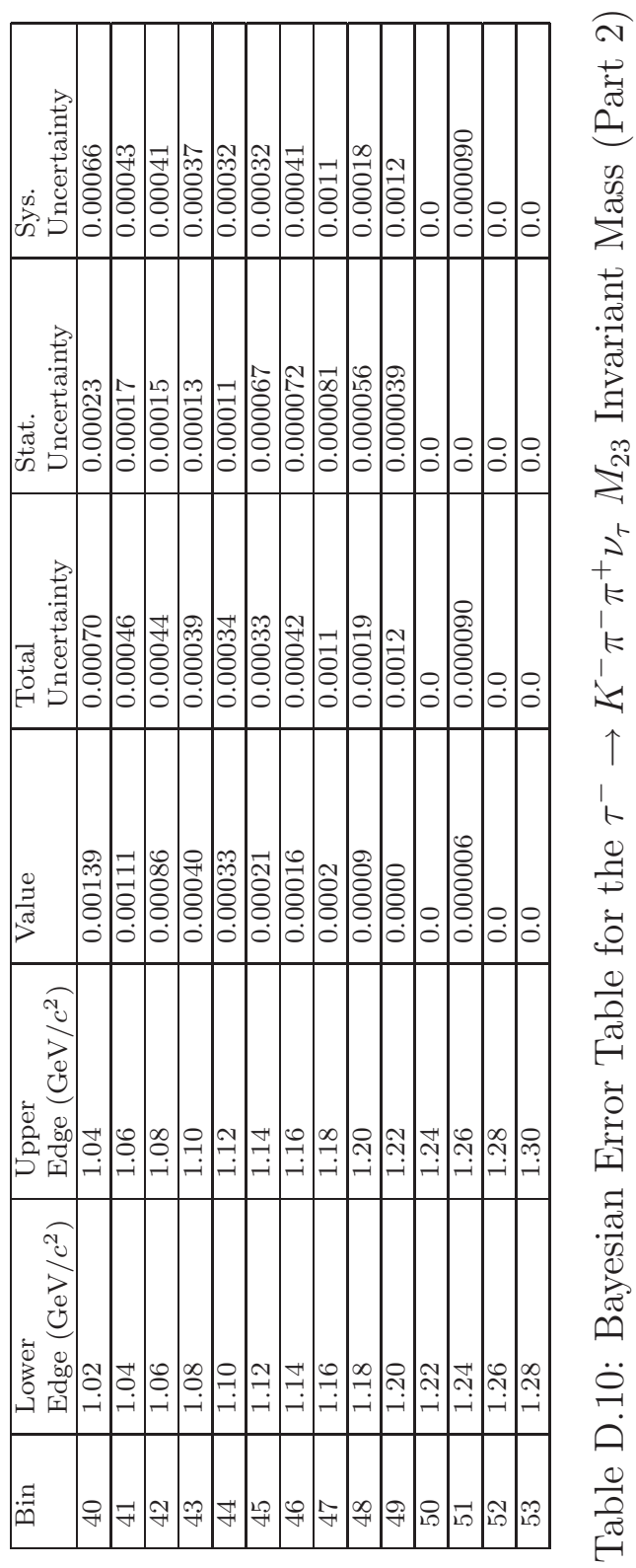




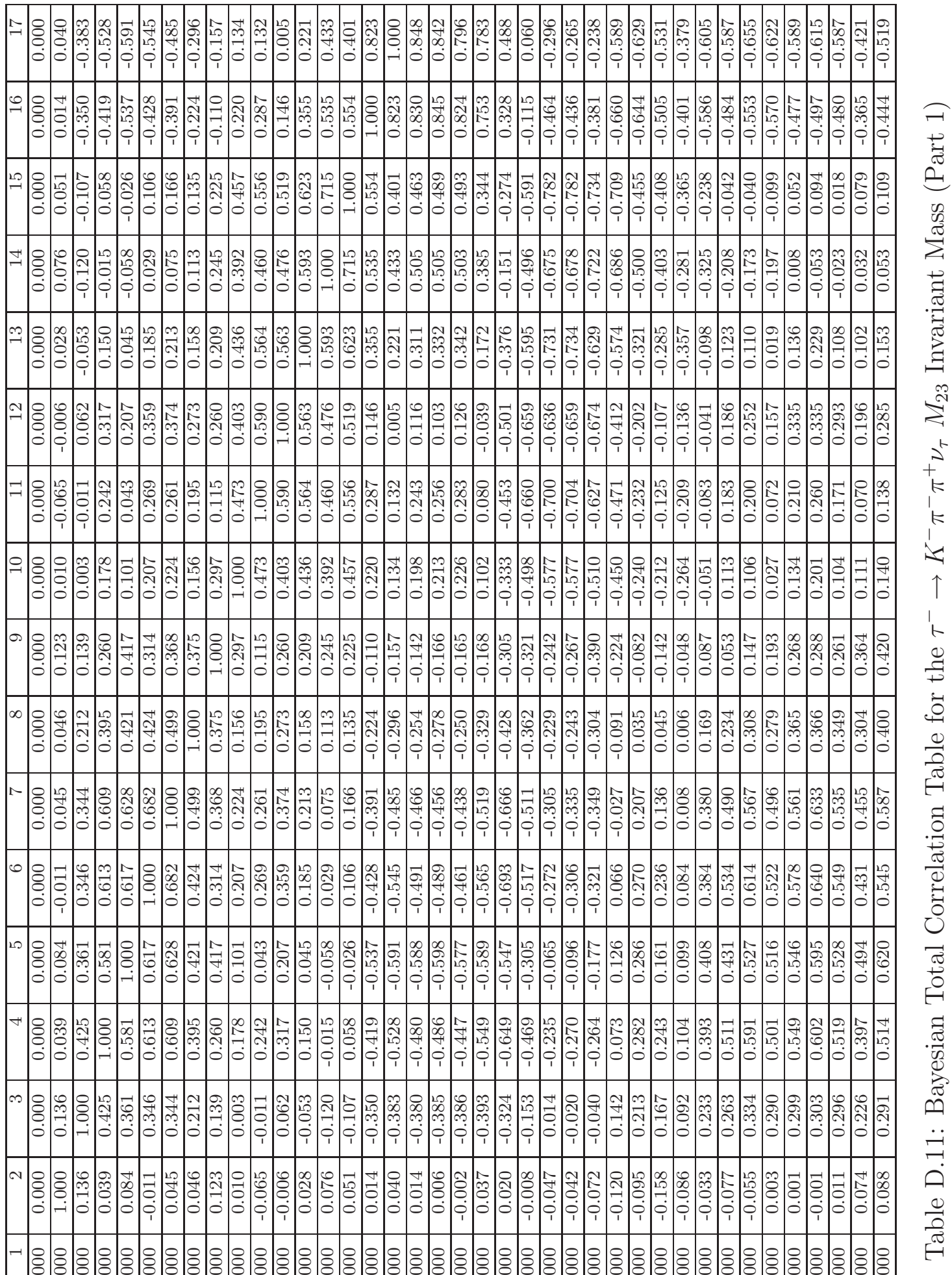




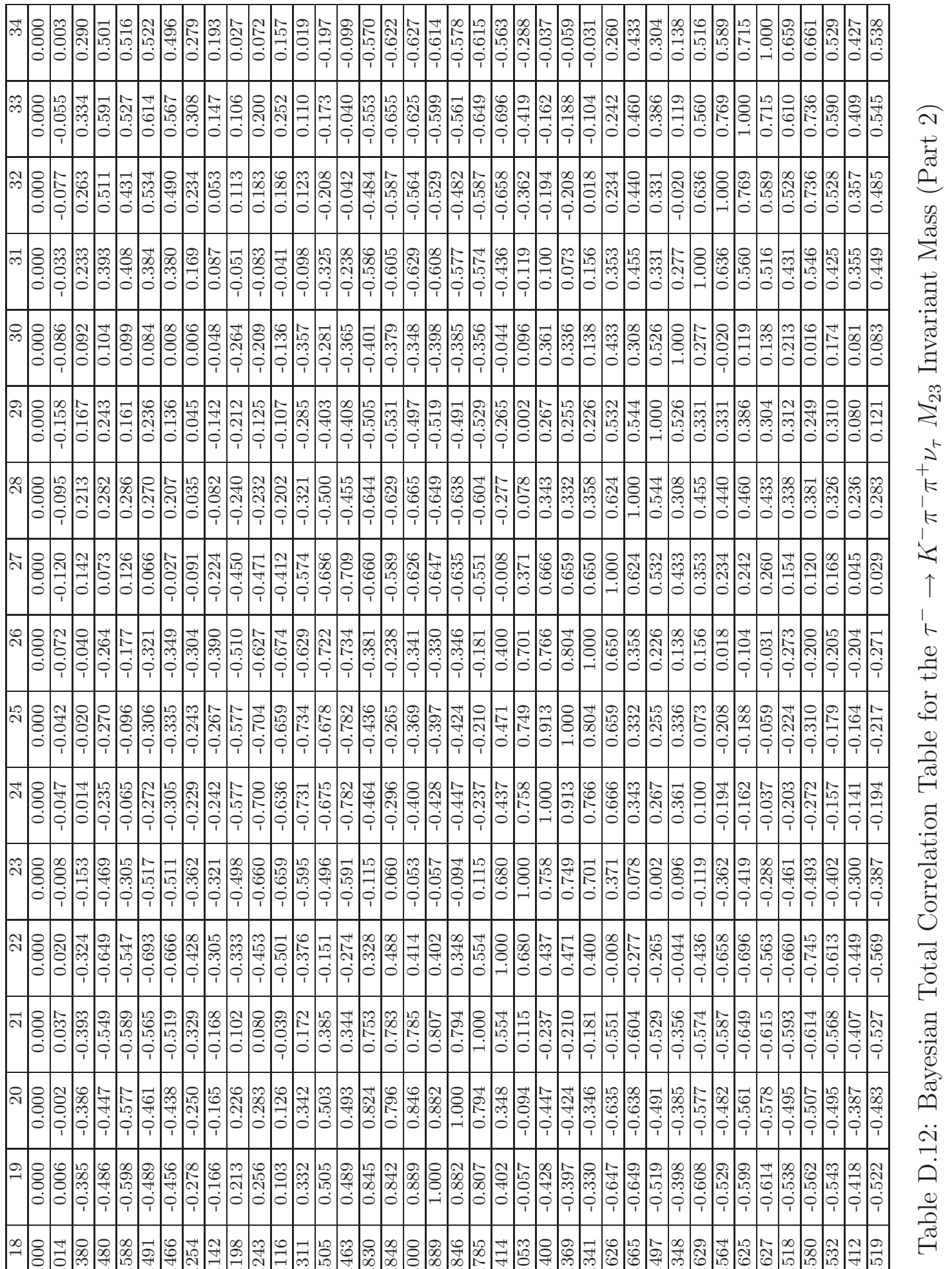




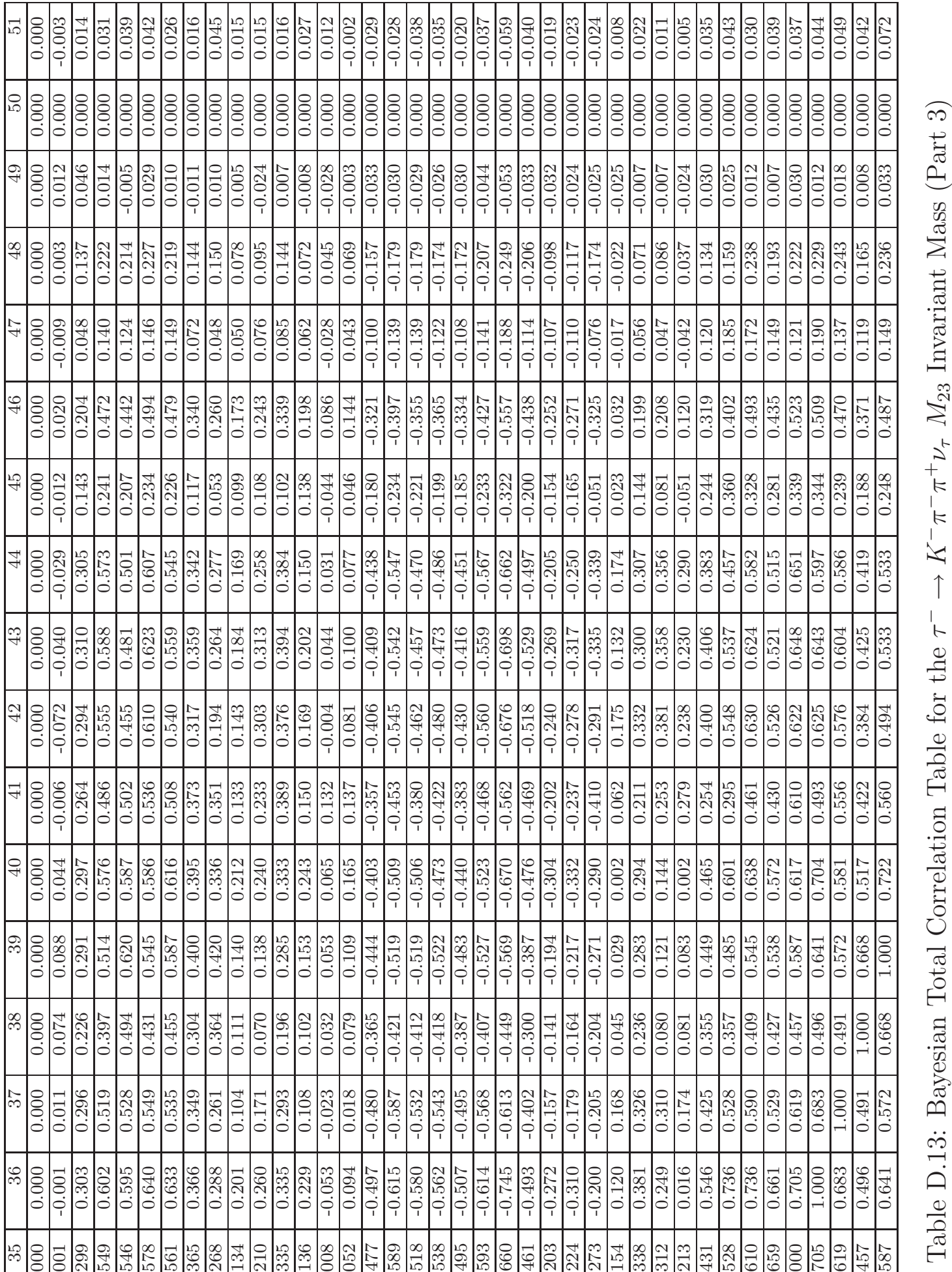


Appendix D. Unfolded $\tau^{-} \rightarrow K^{-} \pi^{-} \pi^{+} \nu_{\tau}$ Invariant Mass Spectra

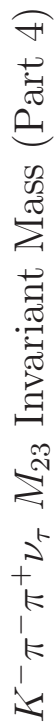

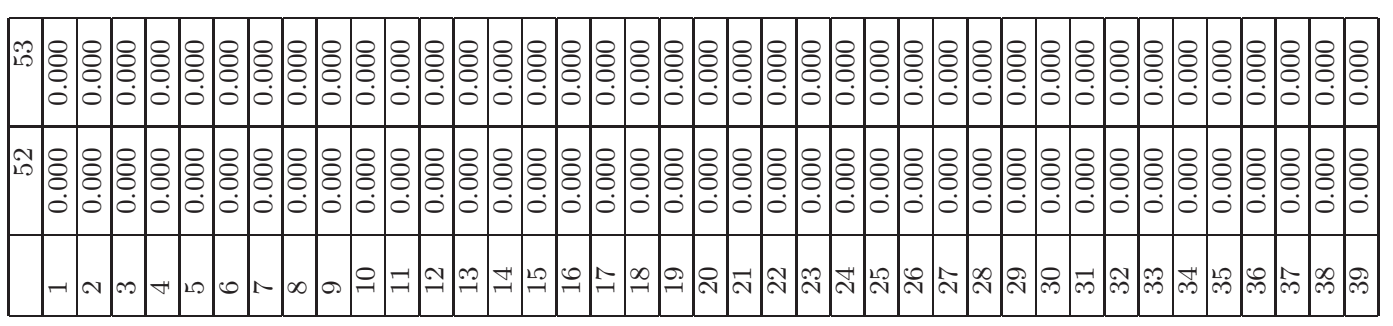

$\uparrow$

t

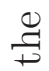

苛

$\frac{\frac{0}{2}}{\frac{0}{6}}$



$\frac{0}{\stackrel{\pi}{0}}$

E

.

赵

$\ddot{\vec{\square}}$

$\stackrel{+}{\oplus}$

$\frac{0}{\frac{0}{0}}$ 
Appendix D. Unfolded $\tau^{-} \rightarrow K^{-} \pi^{-} \pi^{+} \nu_{\tau}$ Invariant Mass Spectra

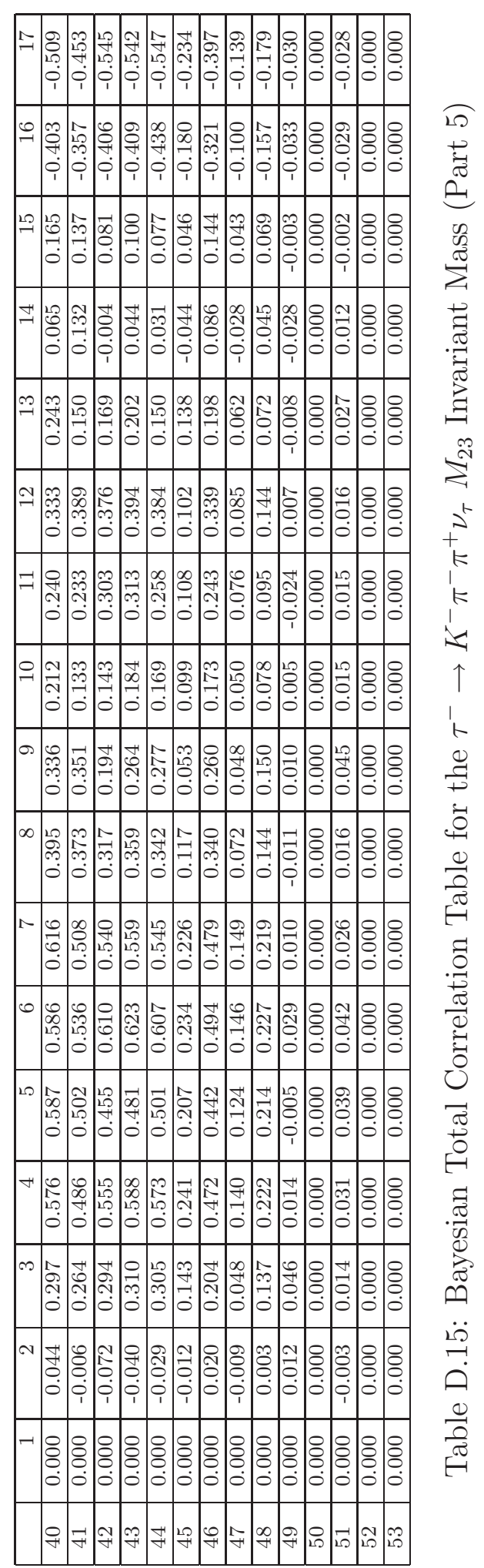


Appendix D. Unfolded $\tau^{-} \rightarrow K^{-} \pi^{-} \pi^{+} \nu_{\tau}$ Invariant Mass Spectra

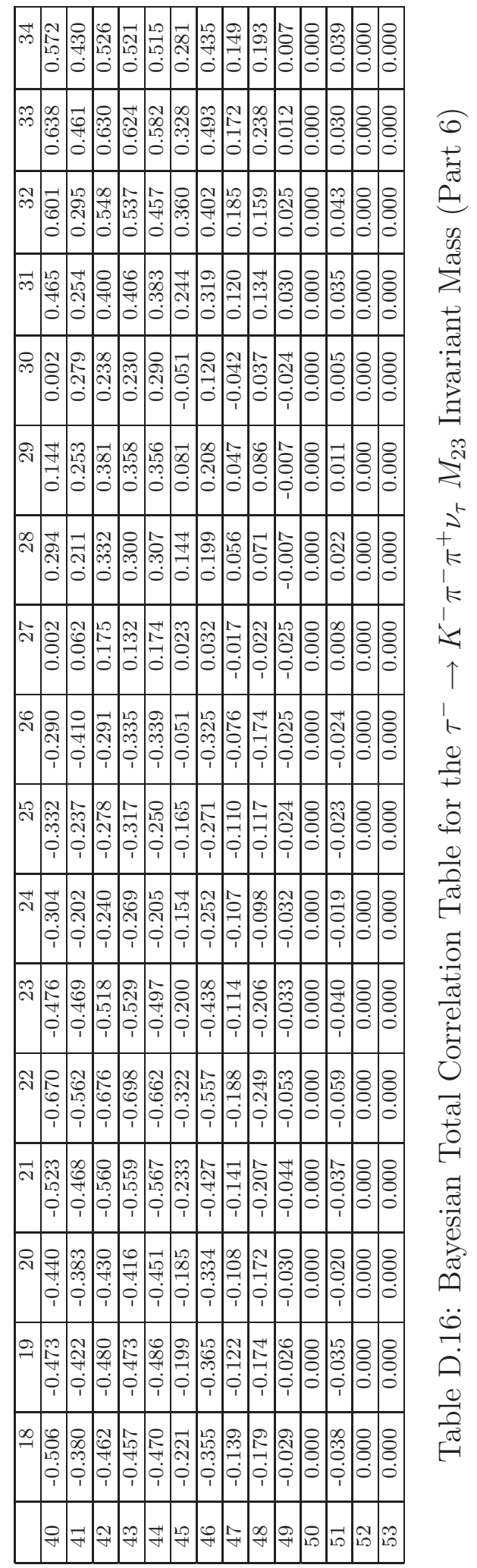


Appendix D. Unfolded $\tau^{-} \rightarrow K^{-} \pi^{-} \pi^{+} \nu_{\tau}$ Invariant Mass Spectra

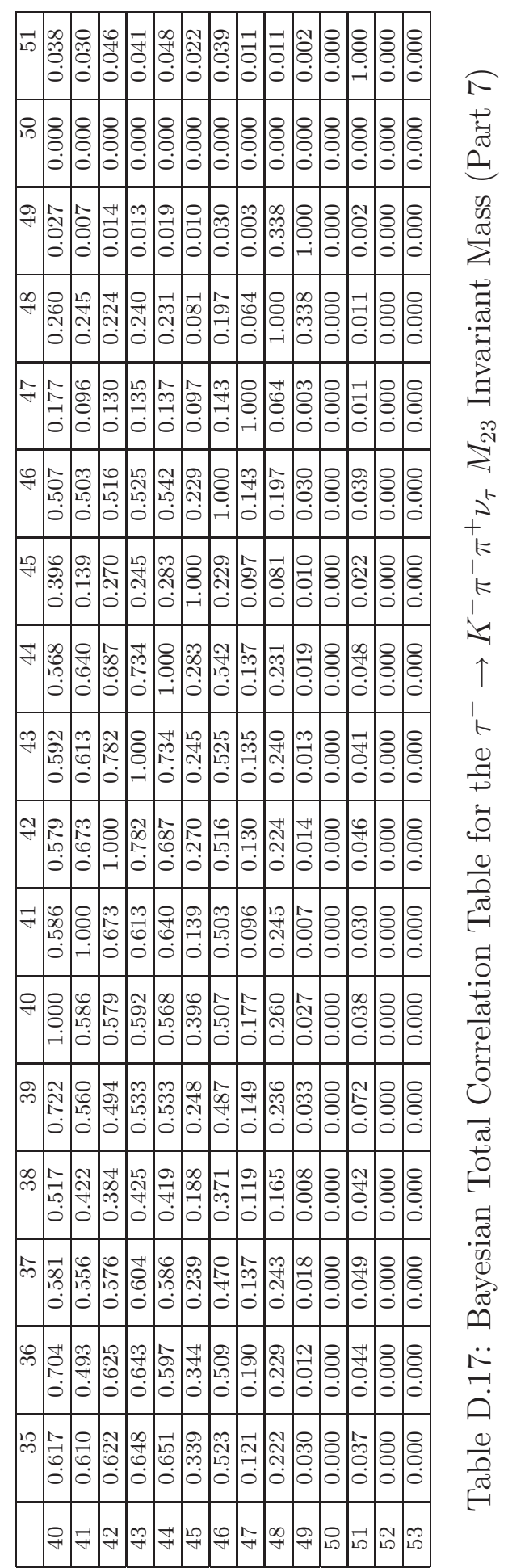




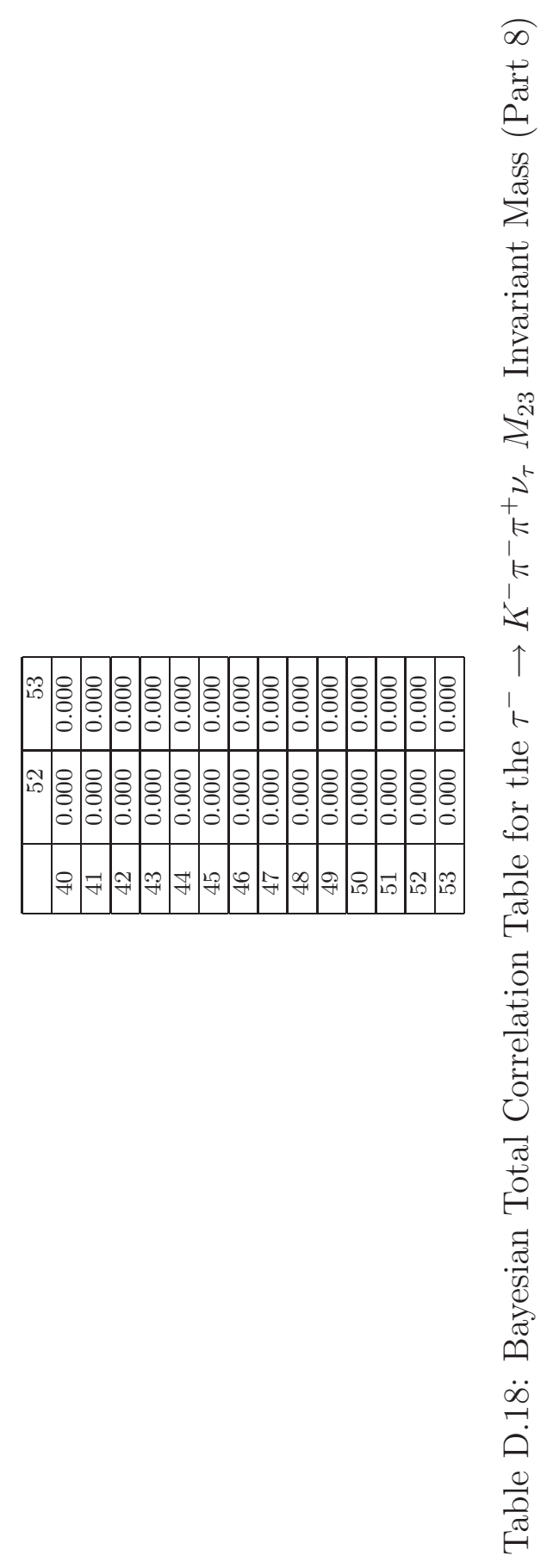




\section{Appendix E}

\section{Unfolded $\tau^{-} \rightarrow K^{-} \pi^{-} K^{+} \nu_{\tau}$ Invariant Mass Spectra}

E.1 The Unfolded $\tau^{-} \rightarrow K^{-} \pi^{-} K^{+} \nu_{\tau} M_{123}$ Invariant Mass Spectra 

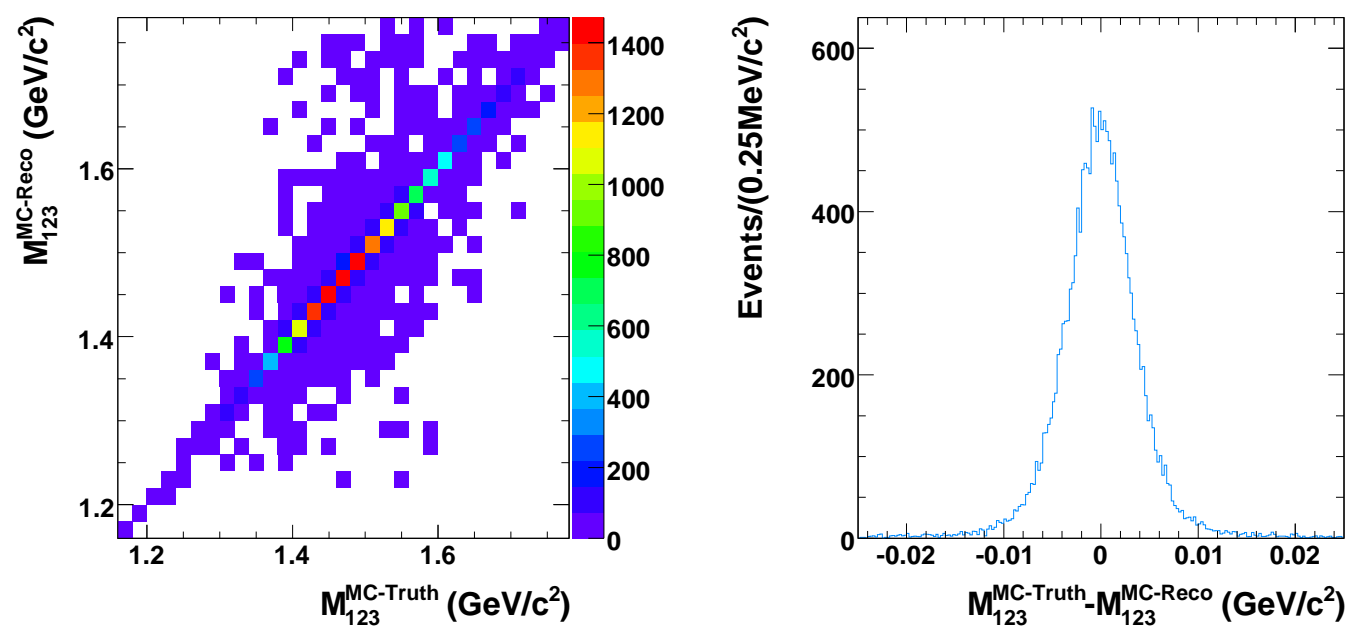

Figure E.1: A comparison of the $\tau^{-} \rightarrow K^{-} \pi^{-} K^{+} \nu_{\tau}$ MC Truth and reconstructed MC for the $M_{123}$ invariant mass spectra. The reconstructed MC $M_{123}$ invariant mass as a function of MC Truth $M_{123}$ invariant mass (left) is used to describe the response of the BABAR Detector in the unfolding of the $\tau^{-} \rightarrow K^{-} \pi^{-} K^{+} \nu_{\tau} M_{123}$ invariant mass spectrum. The reconstructed $\mathrm{MC} M_{123}$ invariant mass minus the $\mathrm{MC}$ Truth $M_{123}$ invariant mass (right) illustrates the average response of the BABAR Detector for the $\tau^{-} \rightarrow K^{-} \pi^{-} K^{+} \nu_{\tau} M_{123}$ invariant mass spectrum. 
Appendix E. Unfolded $\tau^{-} \rightarrow K^{-} \pi^{-} K^{+} \nu_{\tau}$ Invariant Mass Spectra

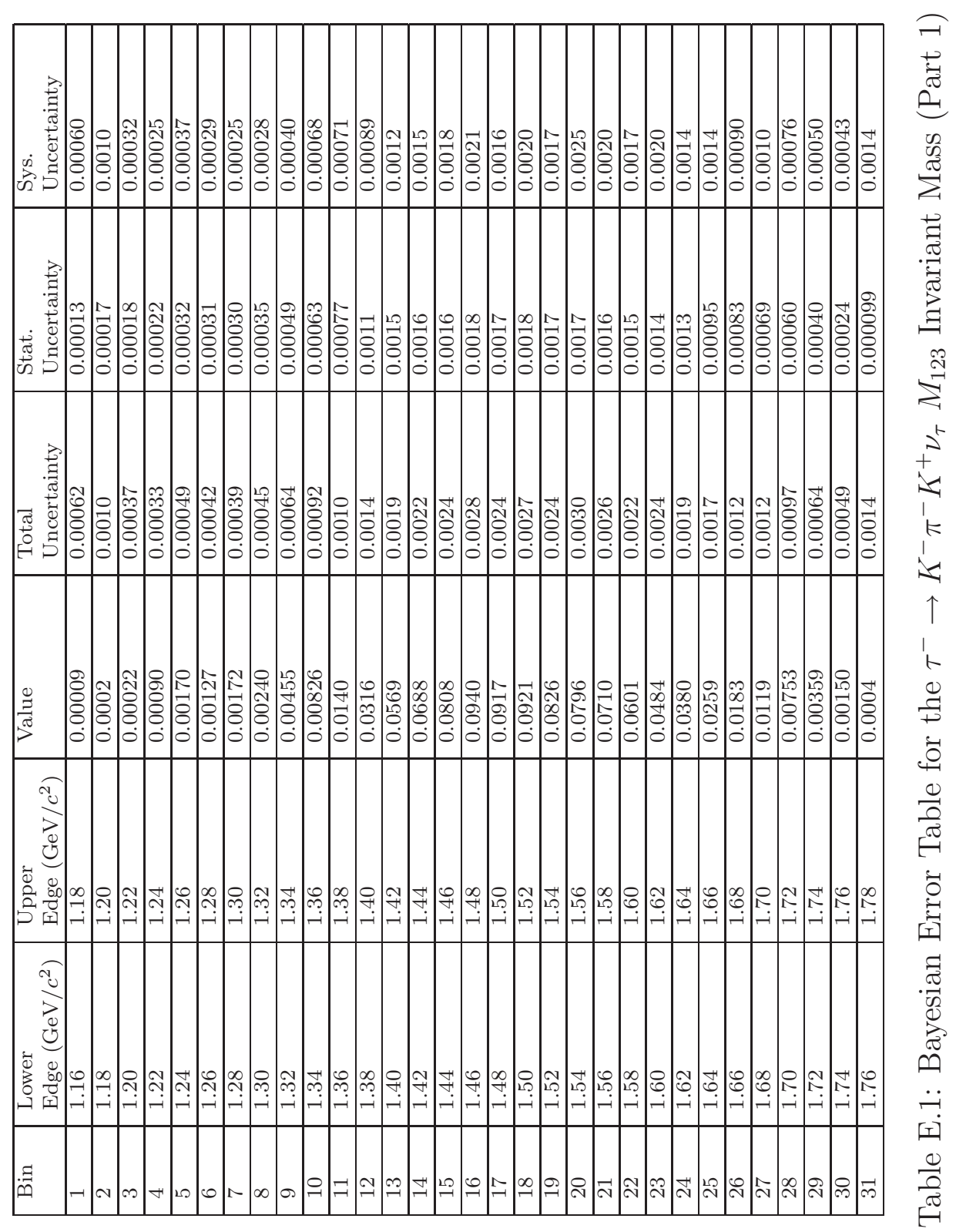


Appendix E. Unfolded $\tau^{-} \rightarrow K^{-} \pi^{-} K^{+} \nu_{\tau}$ Invariant Mass Spectra

238

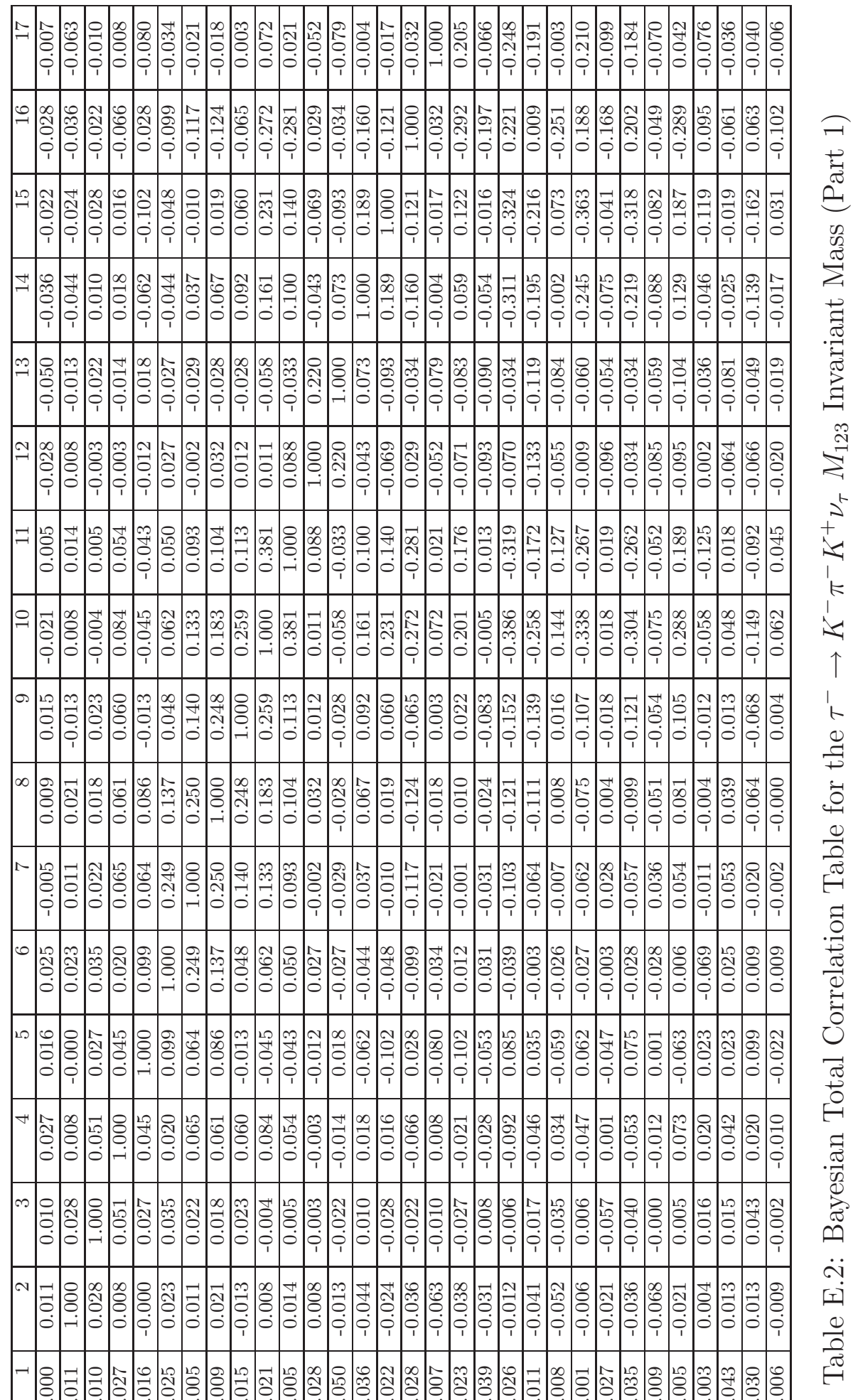


Appendix E. Unfolded $\tau^{-} \rightarrow K^{-} \pi^{-} K^{+} \nu_{\tau}$ Invariant Mass Spectra

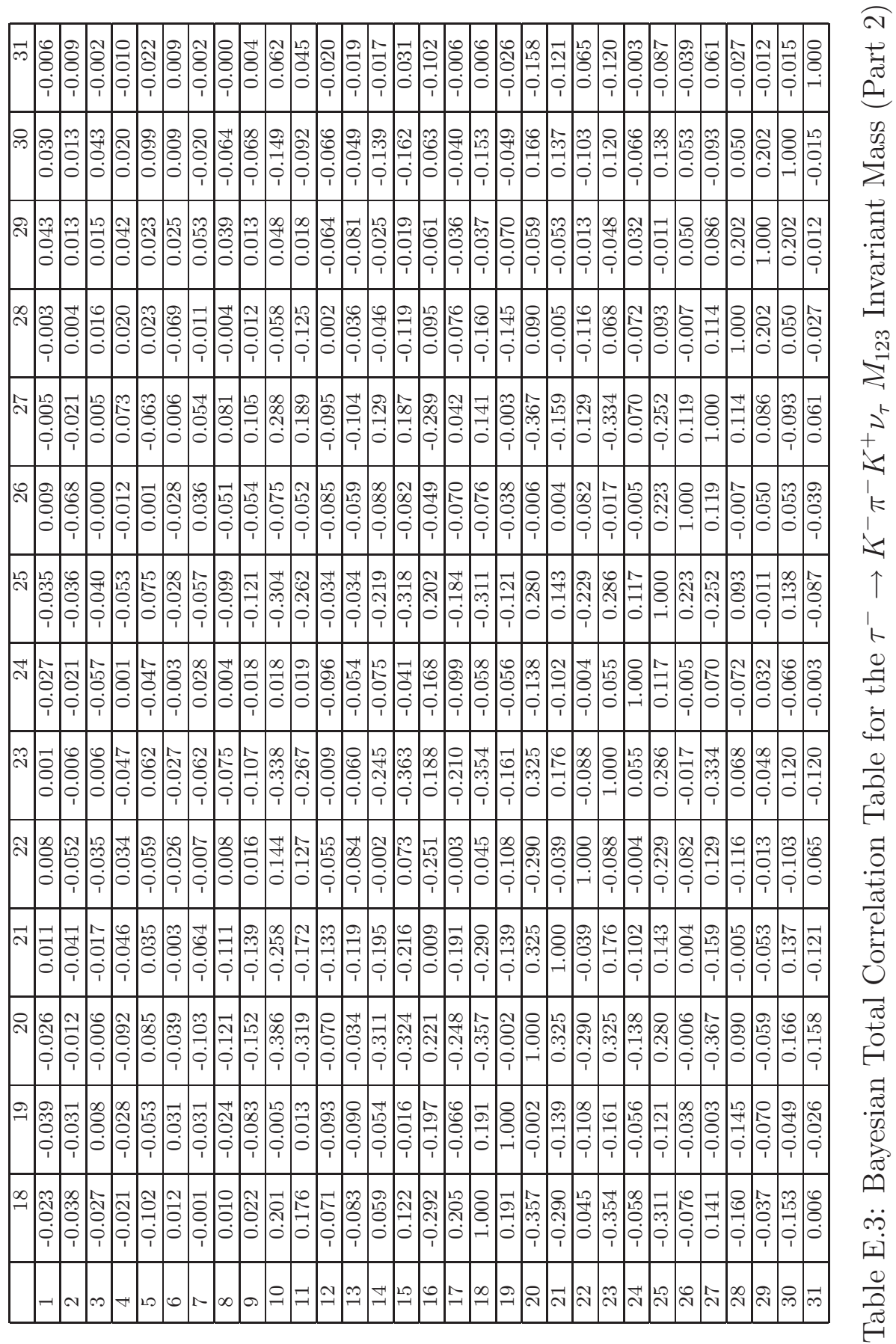




\section{E.2 The Unfolded $\tau^{-} \rightarrow K^{-} \pi^{-} K^{+} \nu_{\tau} M_{13}$ Invariant Mass Spectra}
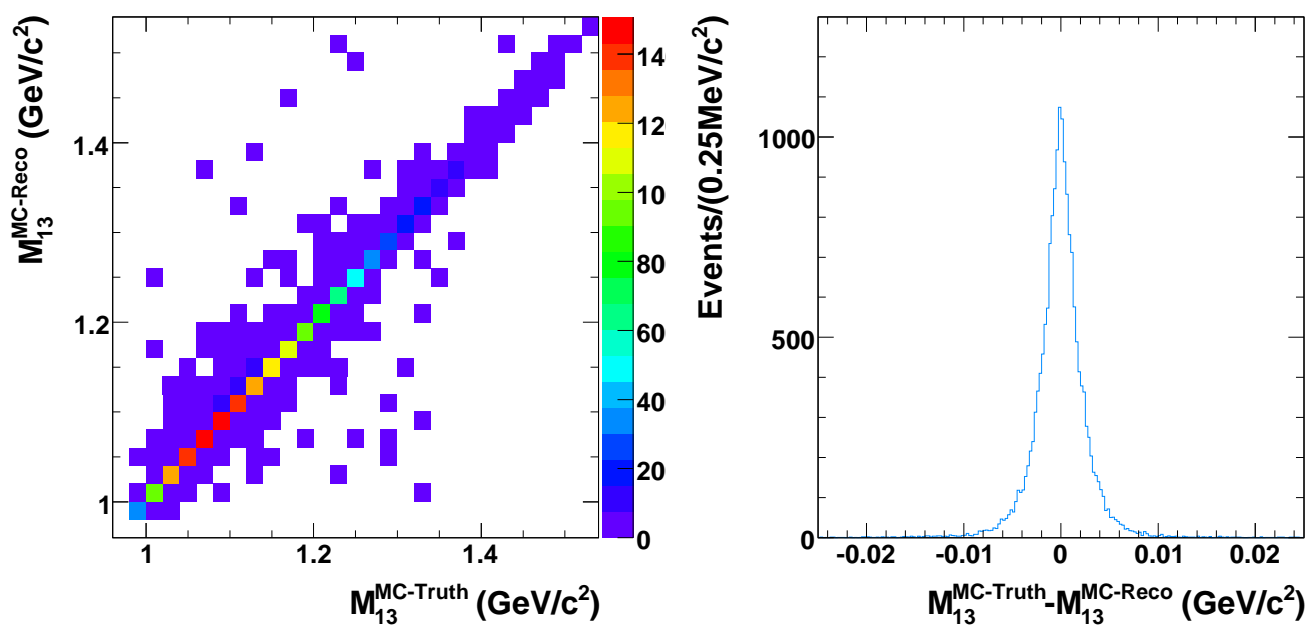

Figure E.2: A comparison of the $\tau^{-} \rightarrow K^{-} \pi^{-} K^{+} \nu_{\tau}$ MC Truth and reconstructed $\mathrm{MC}$ for the $M_{13}$ invariant mass spectra. The reconstructed $\mathrm{MC} M_{13}$ invariant mass as a function of MC Truth $M_{13}$ invariant mass (left) is used to describe the response of the BABAR Detector in the unfolding of the $\tau^{-} \rightarrow K^{-} \pi^{-} K^{+} \nu_{\tau} M_{13}$ invariant mass spectrum. The reconstructed MC $M_{13}$ invariant mass minus the $\mathrm{MC}$ Truth $M_{13}$ invariant mass (right) illustrates the average response of the BABAR Detector for the $\tau^{-} \rightarrow K^{-} \pi^{-} K^{+} \nu_{\tau} M_{13}$ invariant mass spectrum. 
Appendix E. Unfolded $\tau^{-} \rightarrow K^{-} \pi^{-} K^{+} \nu_{\tau}$ Invariant Mass Spectra

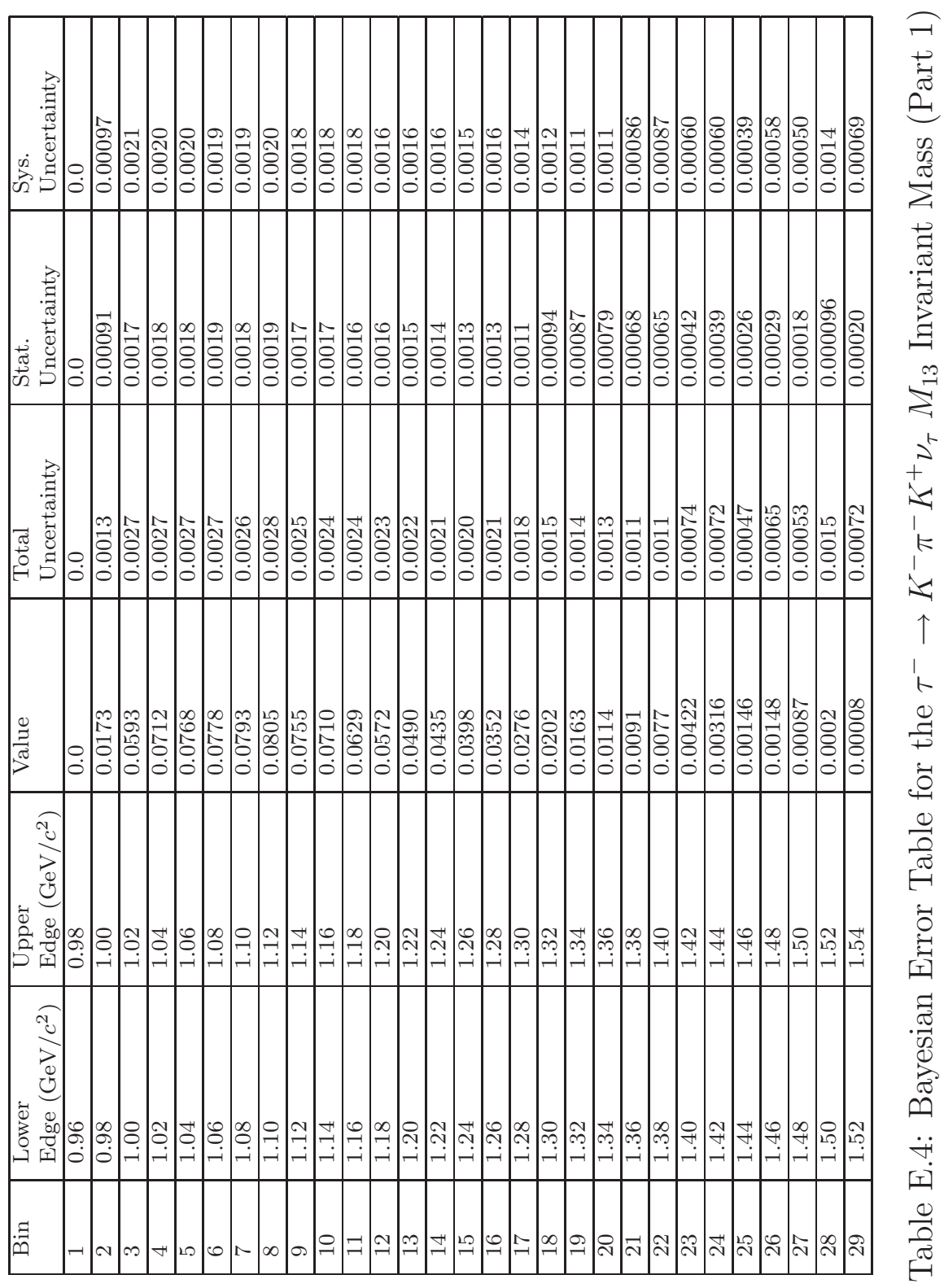


Appendix E. Unfolded $\tau^{-} \rightarrow K^{-} \pi^{-} K^{+} \nu_{\tau}$ Invariant Mass Spectra

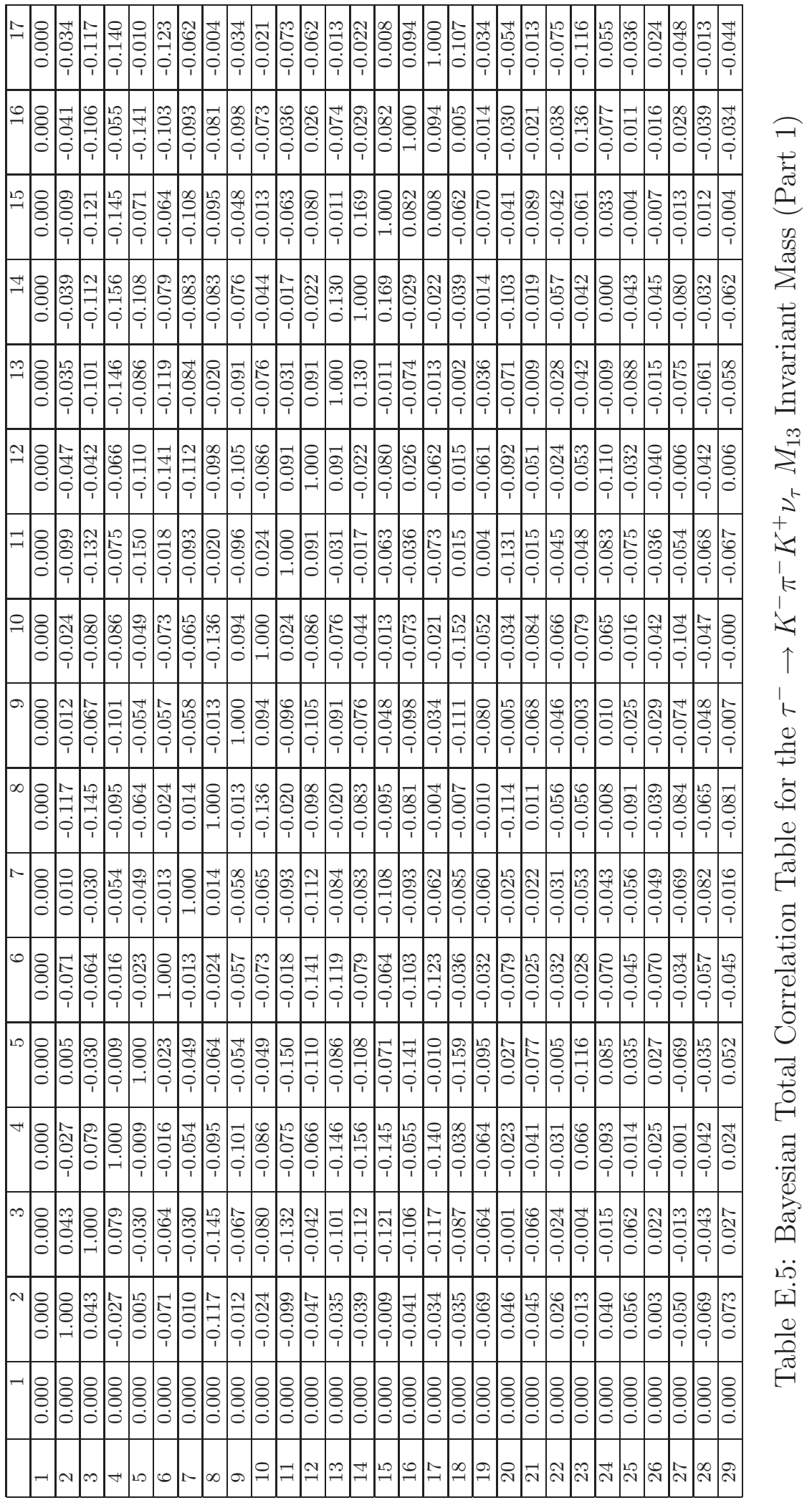


Appendix E. Unfolded $\tau^{-} \rightarrow K^{-} \pi^{-} K^{+} \nu_{\tau}$ Invariant Mass Spectra

243

$\overparen{(1}$

Е

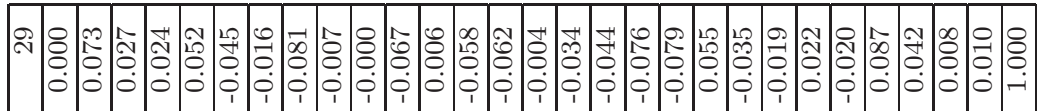

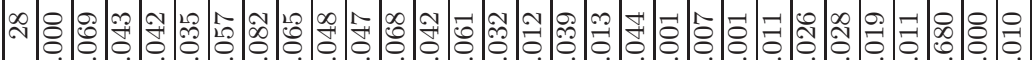

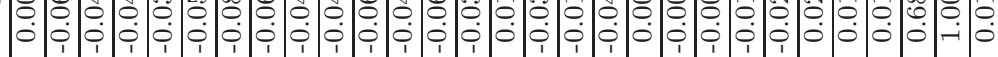
N $\begin{gathered}0 \\ 0\end{gathered}$

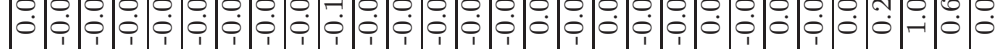

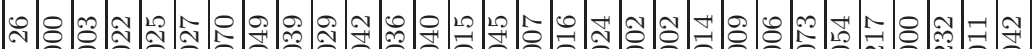

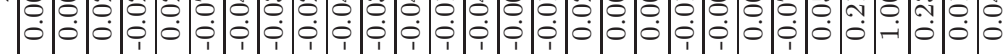

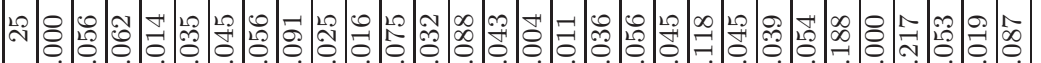

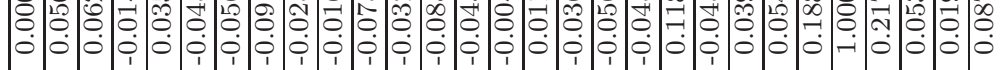

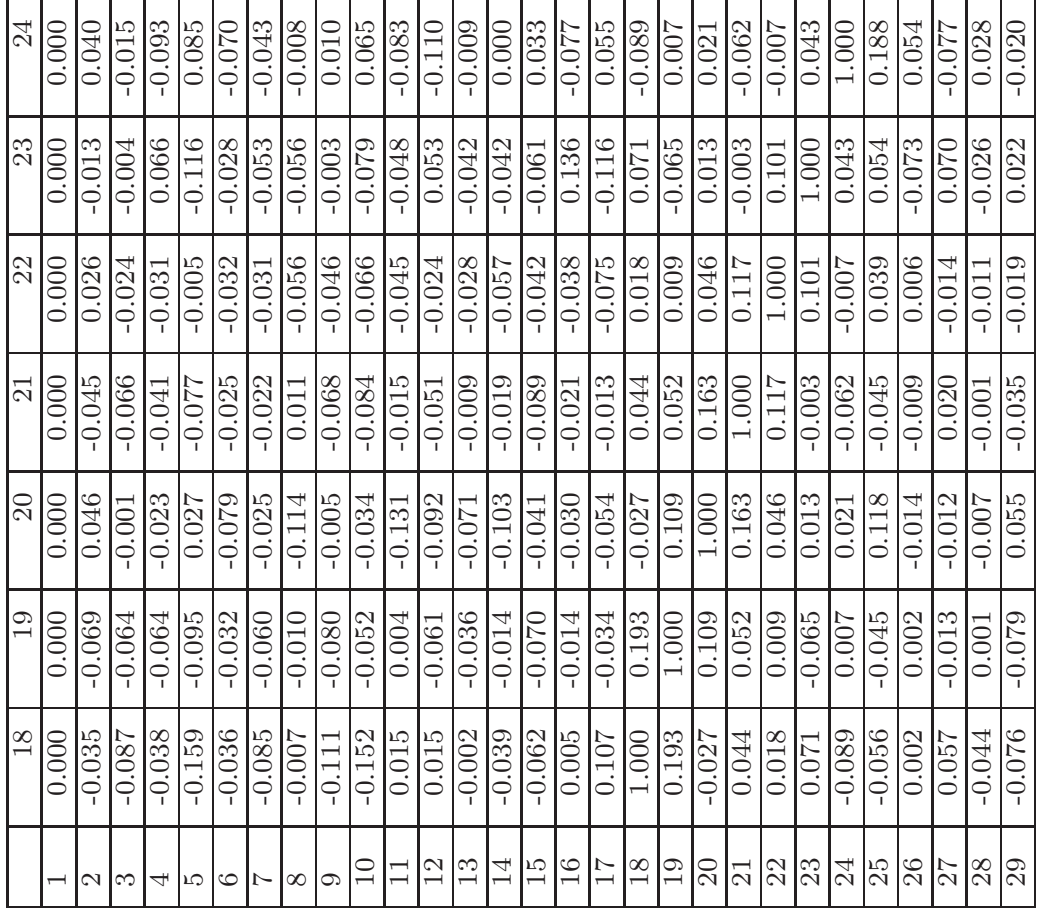
r i $\uparrow$ t

$\stackrel{0}{+}$

菊

$\frac{0}{\frac{0}{\pi}}$

.

$\frac{\pi}{9}$

过

2

西

莍

๘ี

के

๑ึ

$\ddot{0}$

$\frac{0}{\frac{0}{6}}$ 


\section{E.3 The Unfolded $\tau^{-} \rightarrow K^{-} \pi^{-} K^{+} \nu_{\tau} M_{23}$ Invariant Mass Spectra}
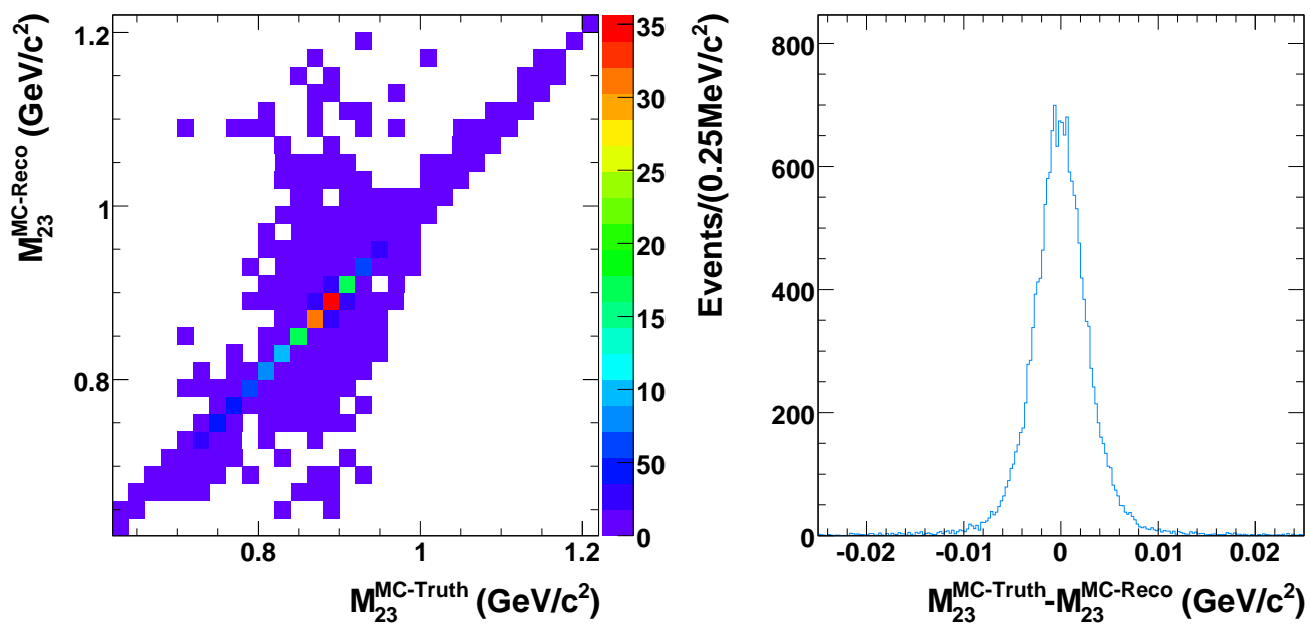

Figure E.3: A comparison of the $\tau^{-} \rightarrow K^{-} \pi^{-} K^{+} \nu_{\tau}$ MC Truth and reconstructed MC for the $M_{23}$ invariant mass spectra. The reconstructed MC $M_{23}$ invariant mass as a function of MC Truth $M_{23}$ invariant mass (left) is used to describe the response of the BABAR Detector in the unfolding of the $\tau^{-} \rightarrow K^{-} \pi^{-} K^{+} \nu_{\tau} M_{23}$ invariant mass spectrum. The reconstructed MC $M_{23}$ invariant mass minus the MC Truth $M_{23}$ invariant mass (right) illustrates the average response of the BABAR Detector for the $\tau^{-} \rightarrow K^{-} \pi^{-} K^{+} \nu_{\tau} M_{23}$ invariant mass spectrum. 
Appendix E. Unfolded $\tau^{-} \rightarrow K^{-} \pi^{-} K^{+} \nu_{\tau}$ Invariant Mass Spectra

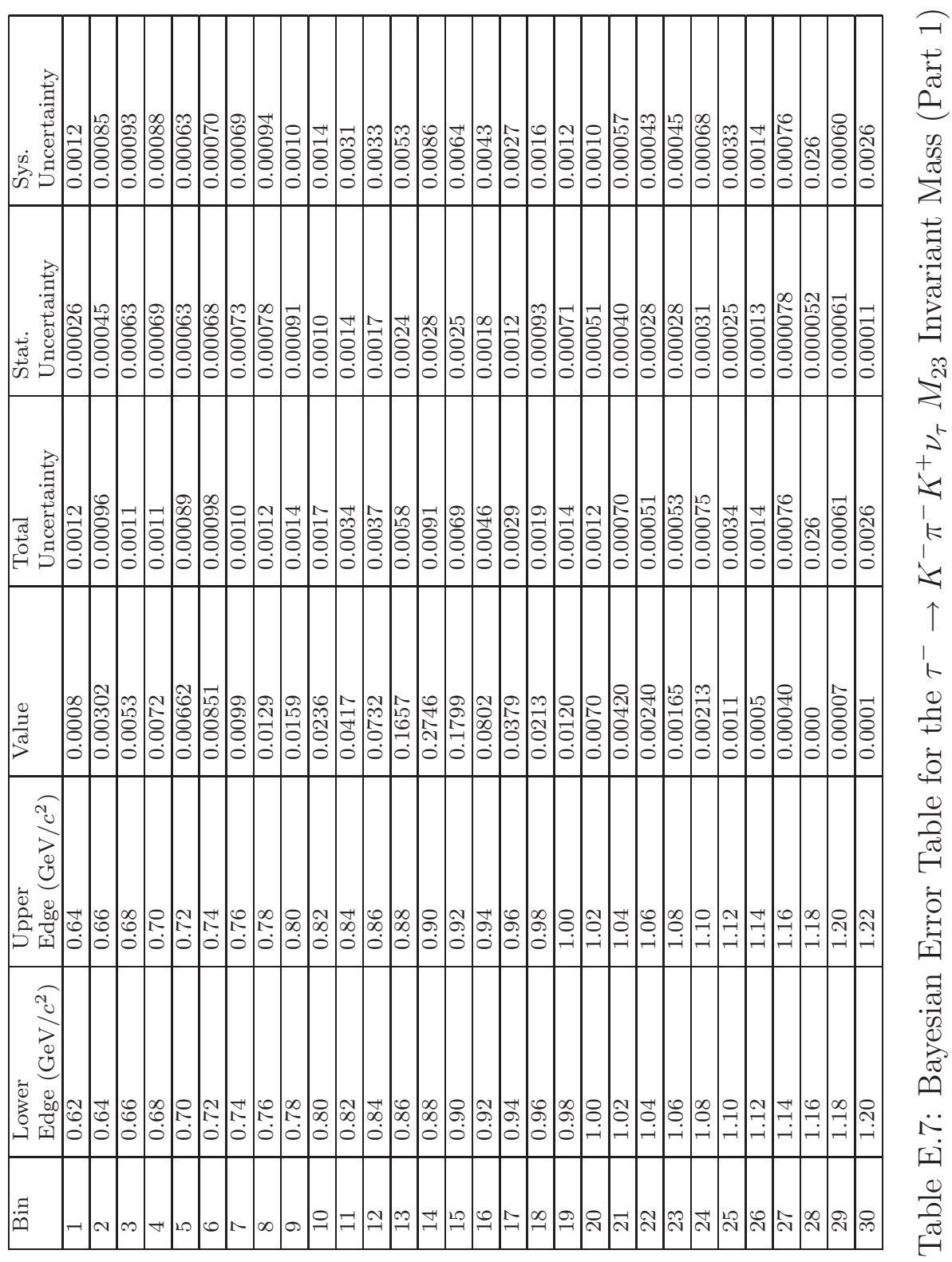


Appendix E. Unfolded $\tau^{-} \rightarrow K^{-} \pi^{-} K^{+} \nu_{\tau}$ Invariant Mass Spectra

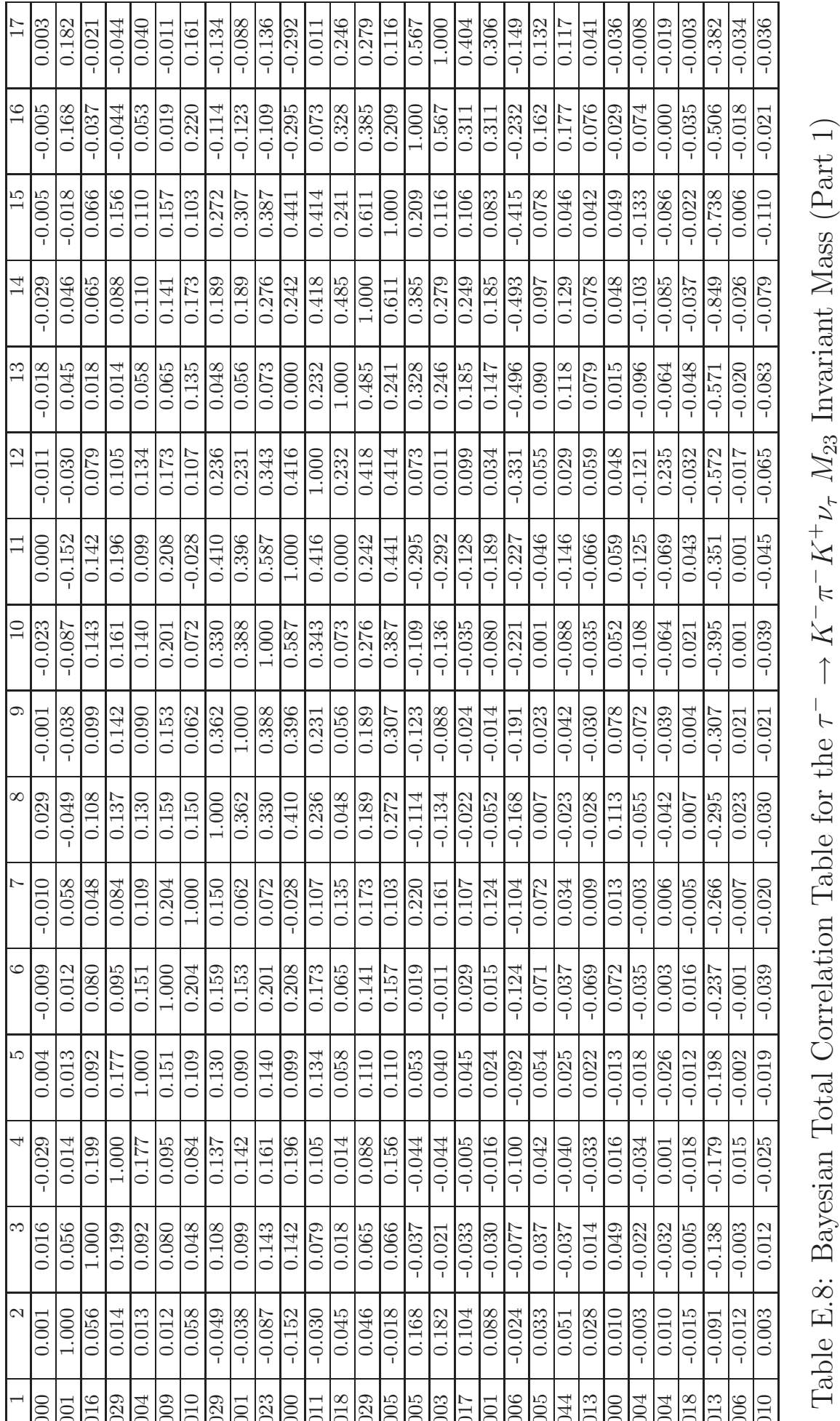


Appendix E. Unfolded $\tau^{-} \rightarrow K^{-} \pi^{-} K^{+} \nu_{\tau}$ Invariant Mass Spectra

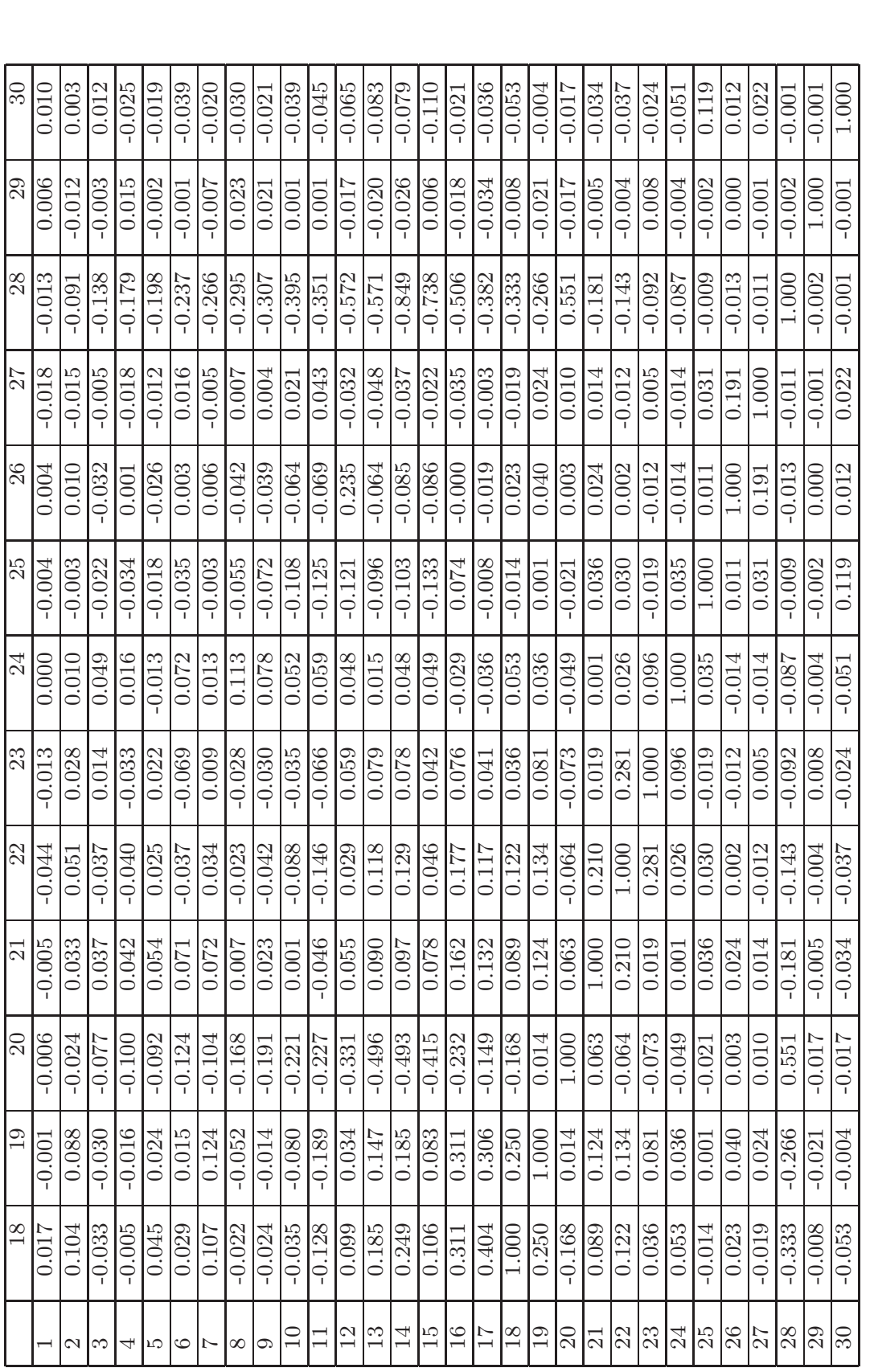

ล

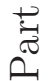

$E$

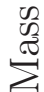

.

莡

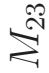

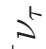

is

t

$\leqslant$

$\uparrow$

t

$\stackrel{9}{\Phi}$

.0

$\frac{0}{\pi}$

$\stackrel{-\pi}{-1}$

.0ี

胥

०్.

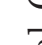

5

$$
\text { . }
$$$$
5
$$

.

ค

壬 


\section{Appendix F}

\section{Unfolded $\tau^{-} \rightarrow K^{-} K^{-} K^{+} \nu_{\tau}$ Invariant Mass Spectra}

F.1 The Unfolded $\tau^{-} \rightarrow K^{-} K^{-} K^{+} \nu_{\tau} M_{123}$ Invariant Mass Spectra 

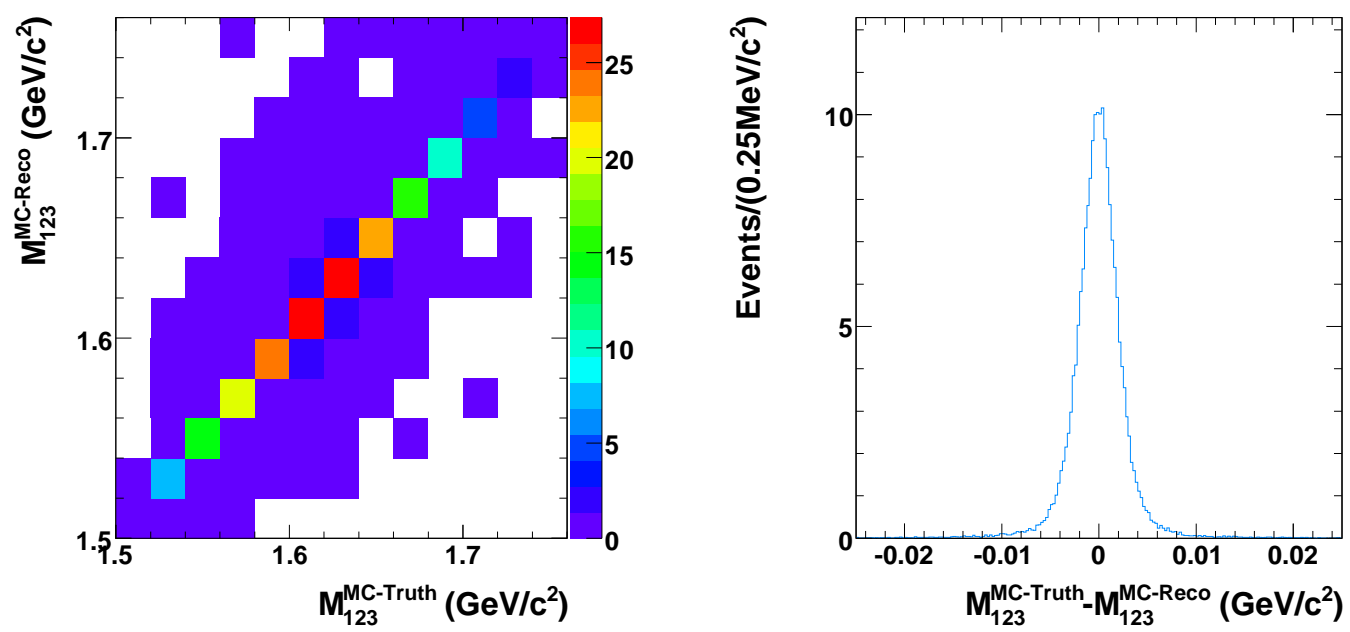

Figure F.1: A comparison of the $\tau^{-} \rightarrow K^{-} K^{-} K^{+} \nu_{\tau}$ MC Truth and reconstructed MC for the $M_{123}$ invariant mass spectra. The reconstructed MC $M_{123}$ invariant mass as a function of MC Truth $M_{123}$ invariant mass (left) is used to describe the response of the BABAR Detector in the unfolding of the $\tau^{-} \rightarrow K^{-} K^{-} K^{+} \nu_{\tau} M_{123}$ invariant mass spectrum. The reconstructed MC $M_{123}$ invariant mass minus the MC Truth $M_{123}$ invariant mass (right) illustrates the average response of the BABAR Detector for the $\tau^{-} \rightarrow K^{-} K^{-} K^{+} \nu_{\tau} M_{123}$ invariant mass spectrum. 


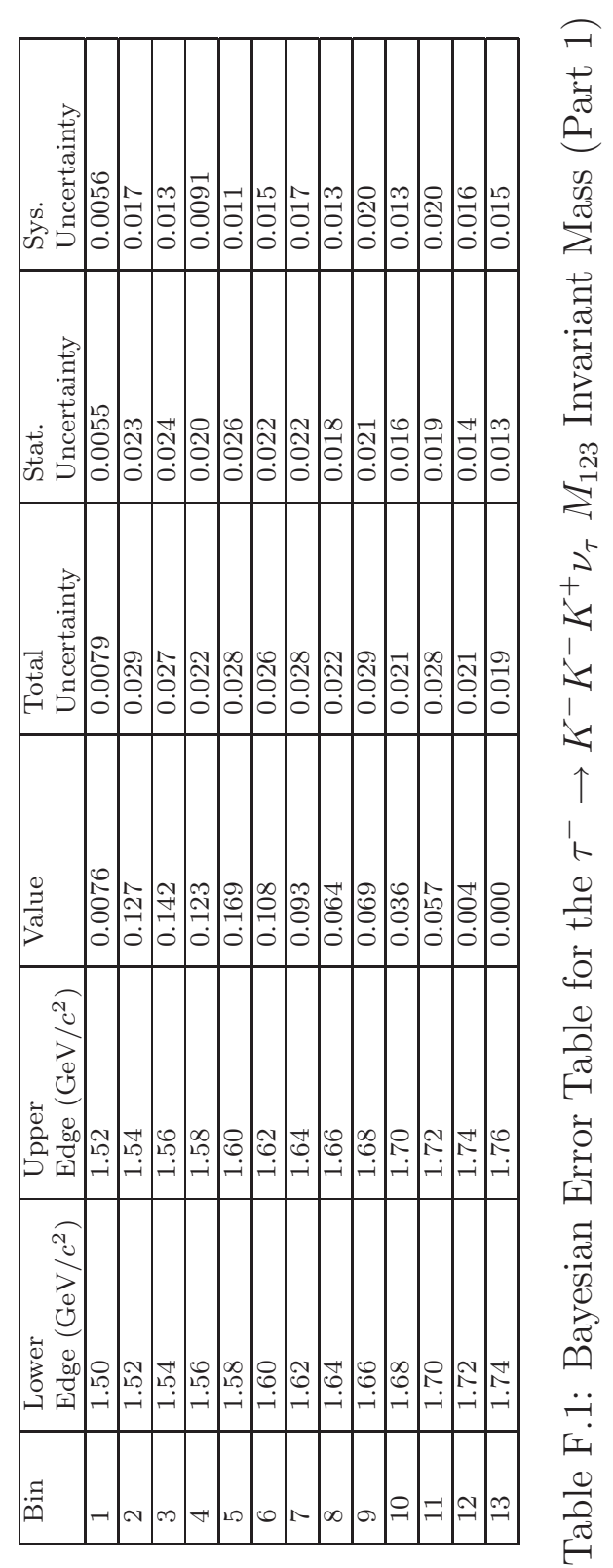


Appendix F. Unfolded $\tau^{-} \rightarrow K^{-} K^{-} K^{+} \nu_{\tau}$ Invariant Mass Spectra

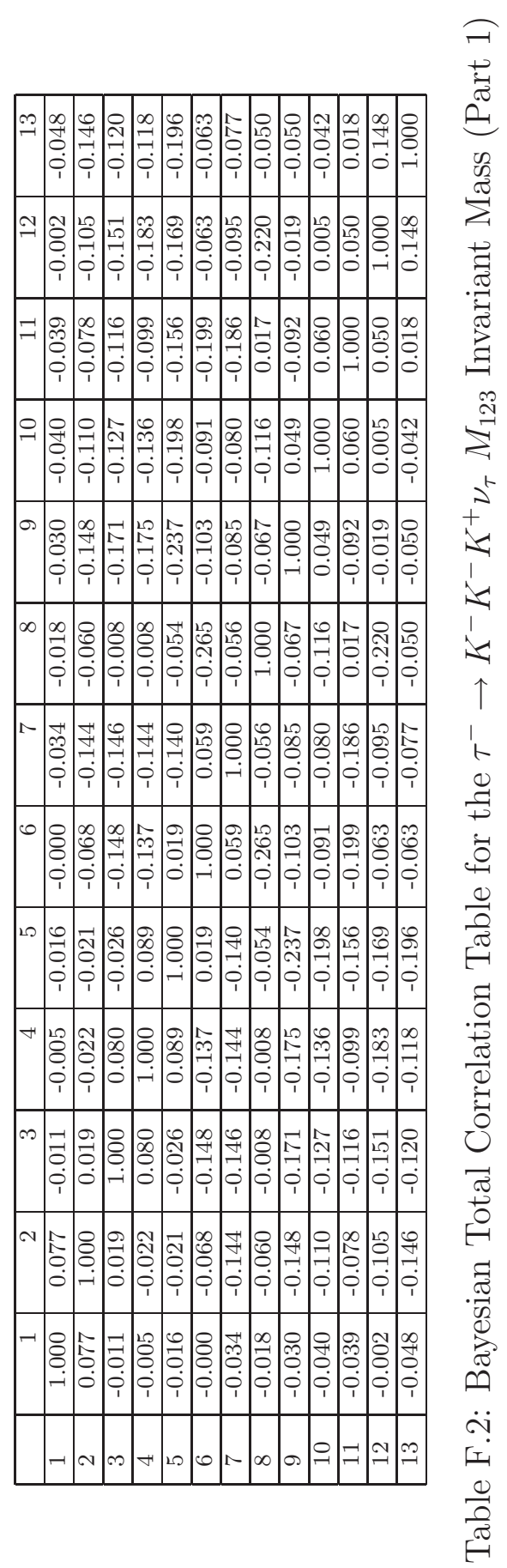




\section{F.2 The Unfolded $\tau^{-} \rightarrow K^{-} K^{-} K^{+} \nu_{\tau} M_{13 a n d 23}$ Invari- ant Mass Spectra}
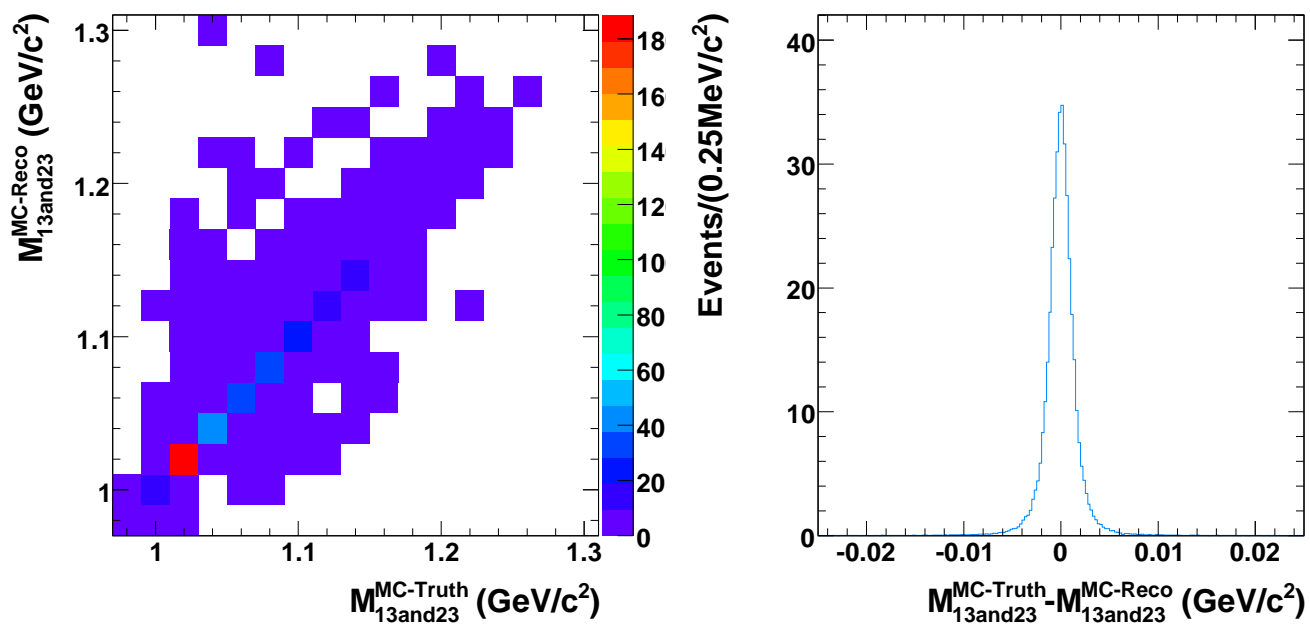

Figure F.2: A comparison of the $\tau^{-} \rightarrow K^{-} K^{-} K^{+} \nu_{\tau}$ MC Truth and reconstructed MC for the $M_{13 a n d 23}$ invariant mass spectra. The reconstructed MC $M_{13 a n d 23}$ invariant mass as a function of MC Truth $M_{13 a n d 23}$ invariant mass (left) is used to describe the response of the BABAR Detector in the unfolding of the $\tau^{-} \rightarrow K^{-} K^{-} K^{+} \nu_{\tau} M_{13 a n d 23}$ invariant mass spectrum. The reconstructed $\mathrm{MC} M_{13 a n d 23}$ invariant mass minus the MC Truth $M_{13 a n d 23}$ invariant mass (right) illustrates the average response of the BABAR Detector for the $\tau^{-} \rightarrow K^{-} K^{-} K^{+} \nu_{\tau} M_{13 a n d 23}$ invariant mass spectrum. 


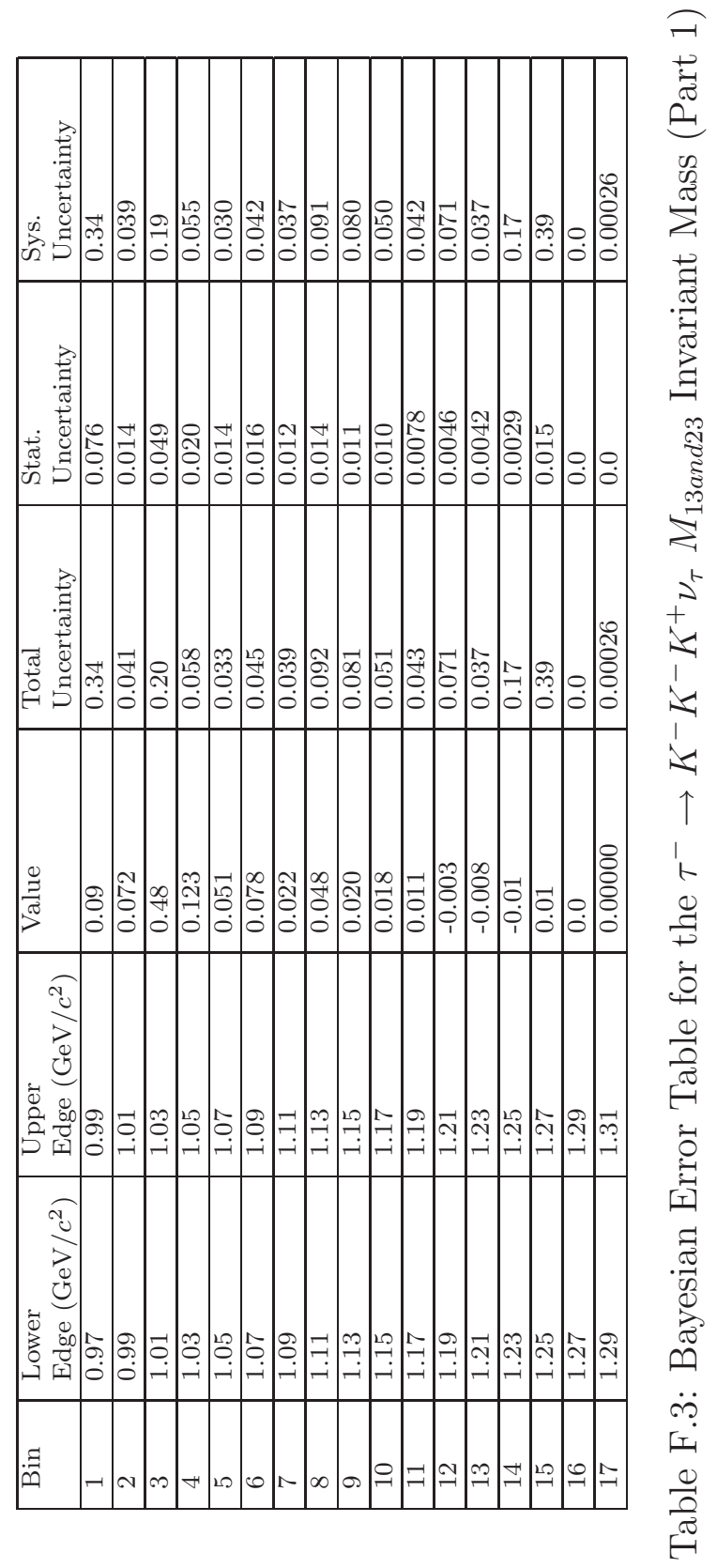


Appendix F. Unfolded $\tau^{-} \rightarrow K^{-} K^{-} K^{+} \nu_{\tau}$ Invariant Mass Spectra

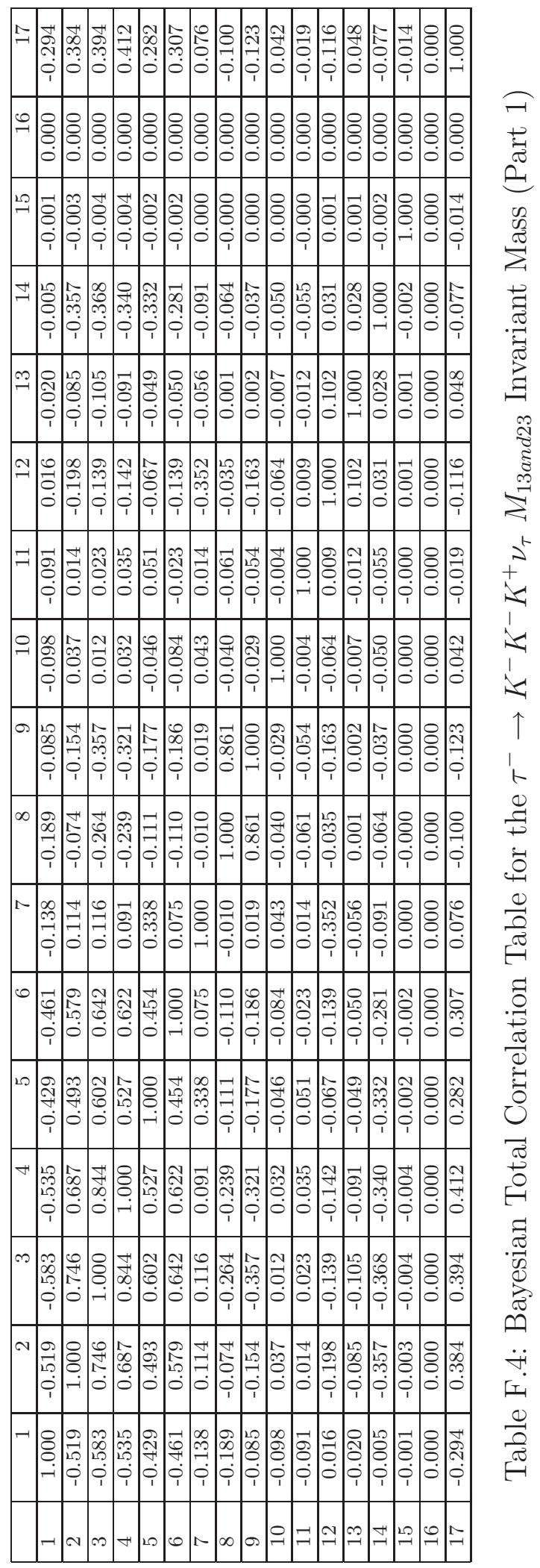




\section{Appendix G}

\section{PID Control Samples}

\section{G.1 Kaon Control Sample: $\tau^{-} \rightarrow K^{-} \pi^{-} K^{+} \nu$}

The Kaon control sample uses $\tau^{+} \rightarrow e^{+} \nu \nu-\tau^{-} \rightarrow K^{-} \pi^{-} K^{+} \nu$ and $\tau^{+} \rightarrow \mu^{+} \nu \nu-$ $\tau^{-} \rightarrow K^{-} \pi^{-} K^{+} \nu$ events to select a pure sample of Kaons. The like charged particle, which is not a pion in the 3 prong hemisphere, is the control track. The event selection for this control sample is based on the event selection from the $\frac{\mathcal{B}\left(\tau^{-} \rightarrow K^{-} \nu_{\tau}\right)}{\mathcal{B}\left(\tau^{-} \rightarrow \pi^{-} \nu_{\tau}\right)}$, $\frac{\mathcal{B}\left(\tau^{-} \rightarrow K^{-} \nu_{\tau}\right)}{\mathcal{B}\left(\tau^{-} \rightarrow e^{-} \nu_{\tau} \bar{\nu}_{e}\right)}, \frac{\mathcal{B}\left(\tau^{-} \rightarrow \pi^{-} \nu_{\tau}\right)}{\mathcal{B}\left(\tau^{-} \rightarrow e^{-} \nu_{\tau} \bar{\nu}_{e}\right)}$, and $\frac{\mathcal{B}\left(\tau^{-} \rightarrow \mu^{-} \nu_{\tau} \bar{\nu}_{\mu}\right)}{\mathcal{B}\left(\tau^{-} \rightarrow e^{-} \nu_{\tau} \bar{\nu}_{e}\right)}$ analysis.

- 4 well reconstructed tracks, which do not originate from conversions in the material of the detectors, with 3 tracks in one hemisphere and one isolated track. The hemispheres are defined by the thrust axis which is calculated from the 4 tracks and neutrals.

- The net charge is zero.

- All the tracks are required to be within the acceptance of the calorimeter $(-0.76<\cos (\theta)<0.80)$.

- DIRC acceptance $P_{t}>0.250 \mathrm{GeV} / c$. 
- $p_{t}$ balance cut:

$$
-\log \frac{2 \sqrt{\left(p_{x}^{\text {tot,cm }}\right)^{2}+\left(p_{y}^{\text {tot,cm }}\right)^{2}}}{E_{c m}^{P E P}}<4.0 .
$$

- thrust $>0.90$.

- $0.1<E_{c m}^{\text {miss }} / E_{c m}^{P E P}<0.70$.

- $\left|\cos \left(\theta^{\text {miss }}\right)\right|<0.7$.

- $K_{S}$ cut

$$
\frac{R_{x y}^{K_{S}^{0}}}{\sigma_{R_{x y}^{K_{S}^{0}}}}<6.0
$$

- In the 1 prong hemisphere the event is vetoed if the residual neutral energy is $E_{\text {res }}^{\text {Tag }} \geq 1.0 \mathrm{GeV}$ for an electron signal track or $E_{\text {res }}^{\text {Tag }} \geq 0.5 \mathrm{GeV}$ for a muon signal track.

- $2 p_{c m} / E_{P E P, c m}<0.8 c$ for the 1 prong track.

- The 1 prong track must be identified as an electron or a muon .

- In the 3 prong hemisphere, there are two "like charged" tracks and one "oppositely charged" track. One like charged track in the 3 prong hemisphere is required to be a pion and not an electron, while the oppositely charged track in the 3 prong hemisphere is required to be a kaon and not a electron.

- In the 3 prong hemisphere the event is vetoed if the total residual energy is $E_{\text {res }}^{3 p r o n g} \geq 0.200 G e V$.

- $M_{123}<1.82 \mathrm{GeV} / c^{2}$. 
The kaon control sample has a significant contamination mainly $\tau^{-} \rightarrow \pi^{-} \pi^{-} \pi^{+} \nu$ events $(\sim 20-30 \%)$. However, the background is known to the $1.4 \%$ level and can be removed at a precision of about $\sim 0.5 \%$. This control sample has the same Bhabha and two photon vetoes as the default analysis and therefore has a similar background, $\sim 0.069 \%$ Bhabha in the electron tag $<0.069 \%$ for the muon tag and $<0.025 \%$ two-photon, before applying the kaon and pion particle-ID.

\section{G.2 Pion Control Sample: $\tau^{-} \rightarrow \pi^{-} \pi^{-} \pi^{+} \nu$}

The Pion control sample uses $\tau^{+} \rightarrow e^{+} \nu \nu-\tau^{-} \rightarrow \pi^{-} \pi^{-} \pi^{+} \nu$ and $\tau^{+} \rightarrow \mu^{+} \nu \nu-\tau^{-} \rightarrow$ $\pi^{-} \pi^{-} \pi^{+} \nu$ events to select a pure sample of pions. The oppositely charged particle in the 3 prong hemisphere is the control track. The event selection for this control sample is based on the event selection from the $\frac{\mathcal{B}\left(\tau^{-} \rightarrow K^{-} \nu_{\tau}\right)}{\mathcal{B}\left(\tau^{-} \rightarrow \pi^{-} \nu_{\tau}\right)}, \frac{\mathcal{B}\left(\tau^{-} \rightarrow K^{-} \nu_{\tau}\right)}{\mathcal{B}\left(\tau^{-} \rightarrow e^{-} \nu_{\tau} \bar{\nu}_{e}\right)}, \frac{\mathcal{B}\left(\tau^{-} \rightarrow \pi^{-} \nu_{\tau}\right)}{\mathcal{B}\left(\tau^{-} \rightarrow e^{-} \nu_{\tau} \bar{\nu}_{e}\right)}$, and $\frac{\mathcal{B}\left(\tau^{-} \rightarrow \mu^{-} \nu_{\tau} \bar{\mu}_{\mu}\right)}{\mathcal{B}\left(\tau^{-} \rightarrow e^{-} \nu_{\tau} \bar{\nu}_{e}\right)}$ analysis.

- 4 well reconstructed tracks, which do not originate from conversions in the material of the detectors, with 3 tracks in one hemisphere and one isolated track. The hemispheres are defined by the thrust axis which is calculated from the 4 tracks and neutrals.

- The net charge is zero.

- All the tracks are required to be within the acceptance of the calorimeter $(-0.76<\cos (\theta)<0.80)$.

- DIRC acceptance $P_{t}>0.250 \mathrm{GeV} / c$.

- $p_{t}$ balance cut: 


$$
-\log \frac{2 \sqrt{\left(p_{x}^{t o t, c m}\right)^{2}+\left(p_{y}^{t o t, c m}\right)^{2}}}{E_{c m}^{P E P}}<4.0 .
$$

- thrust $>0.90$.

- $0.1<E_{c m}^{\text {miss }} / E_{c m}^{P E P}<0.70$.

- $\left|\cos \left(\theta^{\text {miss }}\right)\right|<0.7$.

- $K_{S}$ cut

$$
\frac{R_{x y}^{K_{y}^{0}}}{\sigma_{R_{x y}^{K_{y}^{0}}}}<6.0 .
$$

- In the 1 prong hemisphere the event is vetoed if the residual neutral energy is $E_{r e s}^{T a g} \geq 1.0 \mathrm{GeV}$ for an electron signal track or $E_{r e s}^{T a g} \geq 0.5 \mathrm{GeV}$ for a muon signal track.

- $2 p_{c m} / E_{P E P, c m}<0.8 c$ for the 1 prong track.

- The 1 prong track must be identified as an electron or a muon.

- In the 3 prong hemisphere, there are two "like charged" tracks and one "oppositely charged" track. The two like charged track in the 3 prong hemisphere are required to both be selected as pions and not electrons.

- In the 3 prong hemisphere the event is vetoed if the total residual energy is $E_{\text {res }}^{3 \text { prong }} \geq 0.200 \mathrm{GeV}$.

- $M_{123}<1.82 \mathrm{GeV} / c^{2}$. 
This control sample has a non- $\tau$ background of $0.25 \%$ (this $0.25 \%$ contains pions and kaons) and a $\tau$ background of $0.19 \%$. After correcting for the pion non- $\tau$ background, this gives a total purity of about $99.8 \%$. This control sample has the same Bhabha and two-photon vetoes as the default analysis and therefore has a similar background, $\sim 0.069 \%$ Bhabha in the electron tag $<0.069 \%$ for the muon tag and $<0.025 \%$ two-photon, before applying the pion particle-ID.

\section{G.3 Electron Control Sample: $e^{+} e^{-} \rightarrow e^{-} e^{+} \gamma(\rightarrow$ $\left.e^{+} e^{-}\right)$}

The electron control sample uses $e^{+} e^{-} \rightarrow e^{-} e^{+} \gamma\left(\rightarrow e^{+} e^{-}\right)$events for selecting electrons in the data and the signal electron from the $\tau^{-} e^{-} \nu \nu$ channel in the default analysis for the electron in MC. The 3 prong track not associated with the conversion is used as the conversion track. This is because it is the only track which has a sufficient number of events in the momentum range $1 \mathrm{GeV}_{i} \mathrm{P}_{i} 4 \mathrm{GeV}$.

- 4 well reconstructed tracks with 3 tracks in one hemisphere and one isolated track. The hemispheres are defined by the thrust axis which is calculated from the 4 tracks and neutrals.

- The net charge is zero.

- $\left|P_{1 \text { prong }} c-E_{P E P, c m}\right|<1.5 \mathrm{GeV}$.

- The 1 prong track must be identified as an electron .

- Two of the oppositely charged 3 prong tracks have an invariant mass less than $100 \mathrm{MeV} / \mathrm{v}^{2}$ (electron mass is assumed for the two tracks). 
- The control track is required to be within the acceptance of the calorimeter $(-0.76<\cos (\theta)<0.80)$.

- The control track has $1 \mathrm{GeV} / c<P<4 g e v / c$.

\section{G.4 Muon Control Sample 1: $e^{+} e^{-} \rightarrow \mu^{-} \mu^{+} \gamma(\rightarrow$ $\left.e^{+} e^{-}\right)$}

The muon control sample uses $e^{+} e^{-} \rightarrow \mu^{-} \mu^{+} \gamma\left(\rightarrow e^{+} e^{-}\right)$events for selecting a high purity sample of muon. In this sample, the muon in the 3 prong hemisphere is the control track.

- 4 well reconstructed tracks with 3 tracks in one hemisphere and one isolated track. The hemispheres are defined by the thrust axis which is calculated from the 4 tracks and neutrals.

- The net charge is zero.

- thrust $>0.90$

- $\left|P_{1 \text { prong }} c-E_{P E P, c m}\right|<0.2 \mathrm{GeV}$.

- In the 1 prong hemisphere the event is vetoed if the residual neutral energy is $E_{r e s}^{\text {Tag }} \geq 0.5 \mathrm{GeV}$.

- $\mathrm{E} / \mathrm{P}<0.1 \mathrm{c}$ for the 1 prong track.

- One of the muon candidates is required to be selected as a muon and not an electron.

- 2 of the oppositely charged 3 prong tracks have an invariant mass less than $100 \mathrm{MeV}$ (electron mass is assumed for the two tracks). 
- The control track has $1 \mathrm{GeV} / \mathrm{c}<P<4 \mathrm{GeV} / \mathrm{c}$.

- The control track is required to be within the acceptance of the calorimeter $(-0.76<\cos (\theta)<0.80)$.

This control sample has a $0.89 \%$ Bhabha contamination (upper limit), while the other impurities are less than $0.0093 \%$.

\section{G.5 Muon Control Sample 2: $e^{+} e^{-} \rightarrow \mu^{-} \mu^{+} \gamma$}

- A 1-vs-1 topology.

- the total visible energy in the event, calculated by summing up all the tracks and the neutral bumps above $50 \mathrm{MeV}$ energy threshold with lateral moment less than 0.8 , to be within $500 \mathrm{MeV}$ from the total beam energy in the laboratory frame.

- One of the tracks is required to have the signature of a minimum ionizing particle with energy deposited in the electromagnetic calorimeter between $100 \mathrm{MeV}$ and $500 \mathrm{MeV}$.

- $\left|P_{1 \text { prong }} c-E_{P E P, c m}\right|<0.2 \mathrm{GeV}$.

- $\mathrm{E} / \mathrm{P}<0.1 \mathrm{c}$ for the 1 prong track.

- The control track has $1 \mathrm{GeV} / \mathrm{c}<P<4 \mathrm{GeV} / \mathrm{c}$.

- The control track is required to be within the acceptance of the calorimeter $(-0.76<\cos (\theta)<0.80)$.

This control sample has a purity of $99.9278 \%$, where $\sim 0 \%$ comes from Bhabha events, $0.00555705 \%$ comes from $\tau$ pair events, $0.0664345 \%$ comes from $e^{+} e^{-} \rightarrow u \bar{u}$, $e^{+} e^{-} \rightarrow d \bar{d}$, and $e^{+} e^{-} \rightarrow s \bar{s}$ events and $0.000173436 \%$ comes from $e^{+} e^{-} \rightarrow c \bar{c}$ events. 


\section{G.6 Data MC Agreement with Control Samples}

The control samples were used to validate the standard BABAR particle-ID tables and to apply additional corrections when there was a significant deviation. The corrections were applied to: pions selected as muons; muons selected as pions; muons selected as kaons; pions selected as pions; and pions selected as kaons. The complete discussion of the systematic uncertainties is described in Section 7.3.2. Figures G.1 and G.2 illustrate the data MC agreement after the particle identification corrections from these control samples have been applied. 

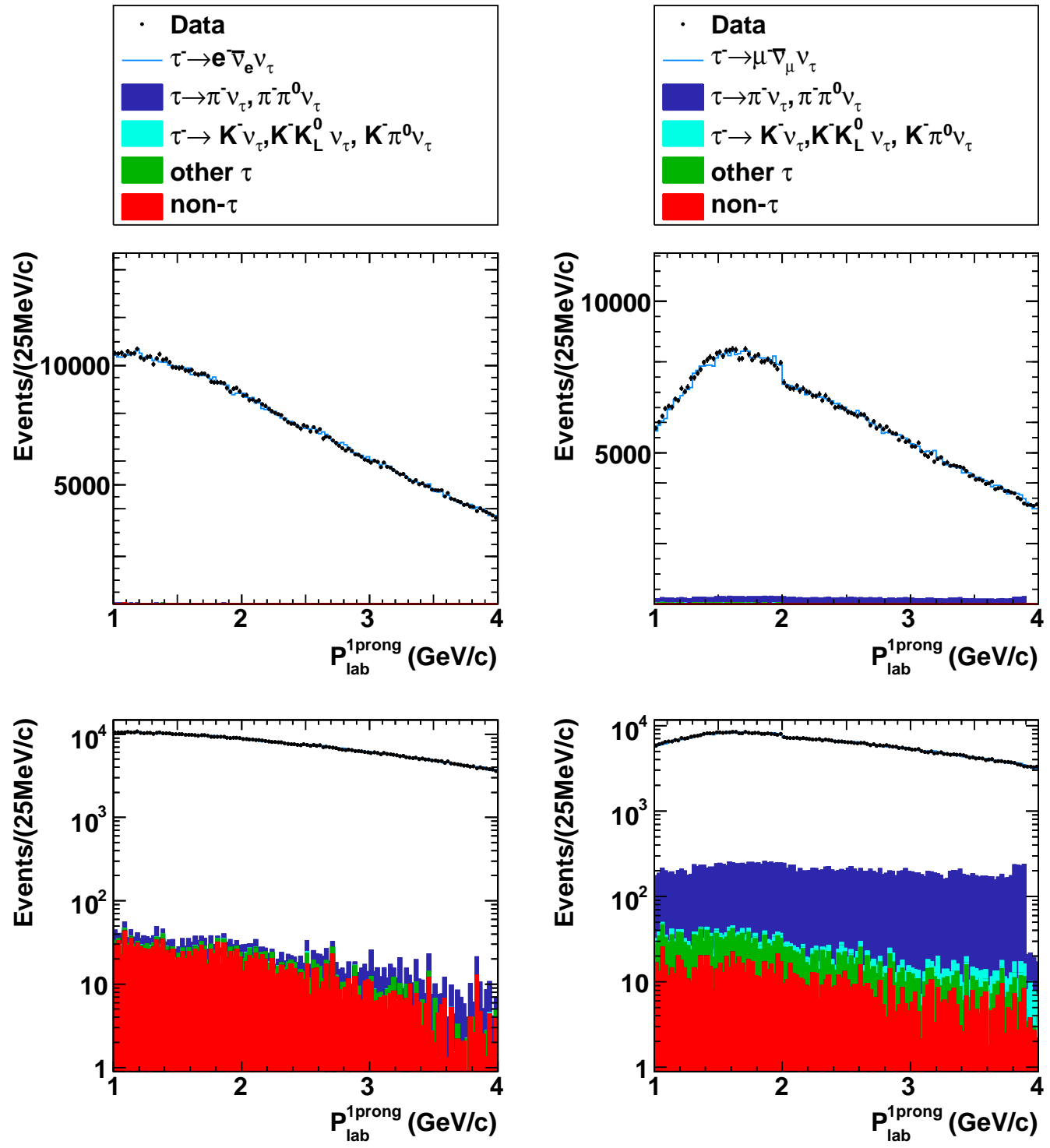

Figure G.1: The $\tau^{-} \rightarrow e^{-} \nu_{\tau} \bar{\nu}_{e}$ (left) and $\tau^{-} \rightarrow \mu^{-} \nu_{\tau} \bar{\nu}_{\mu}$ (right) laboratory momentum shown both linear (top) and logarithmic scale(bottom). The legends for the $\tau^{-} \rightarrow$ $e^{-} \nu_{\tau} \bar{\nu}_{e}$ and $\tau^{-} \rightarrow \mu^{-} \nu_{\tau} \bar{\nu}_{\mu}$ channels are shown above the respective plots. This plot illustrated the data MC agreement after the Corrections from the Control Samples were applied. 

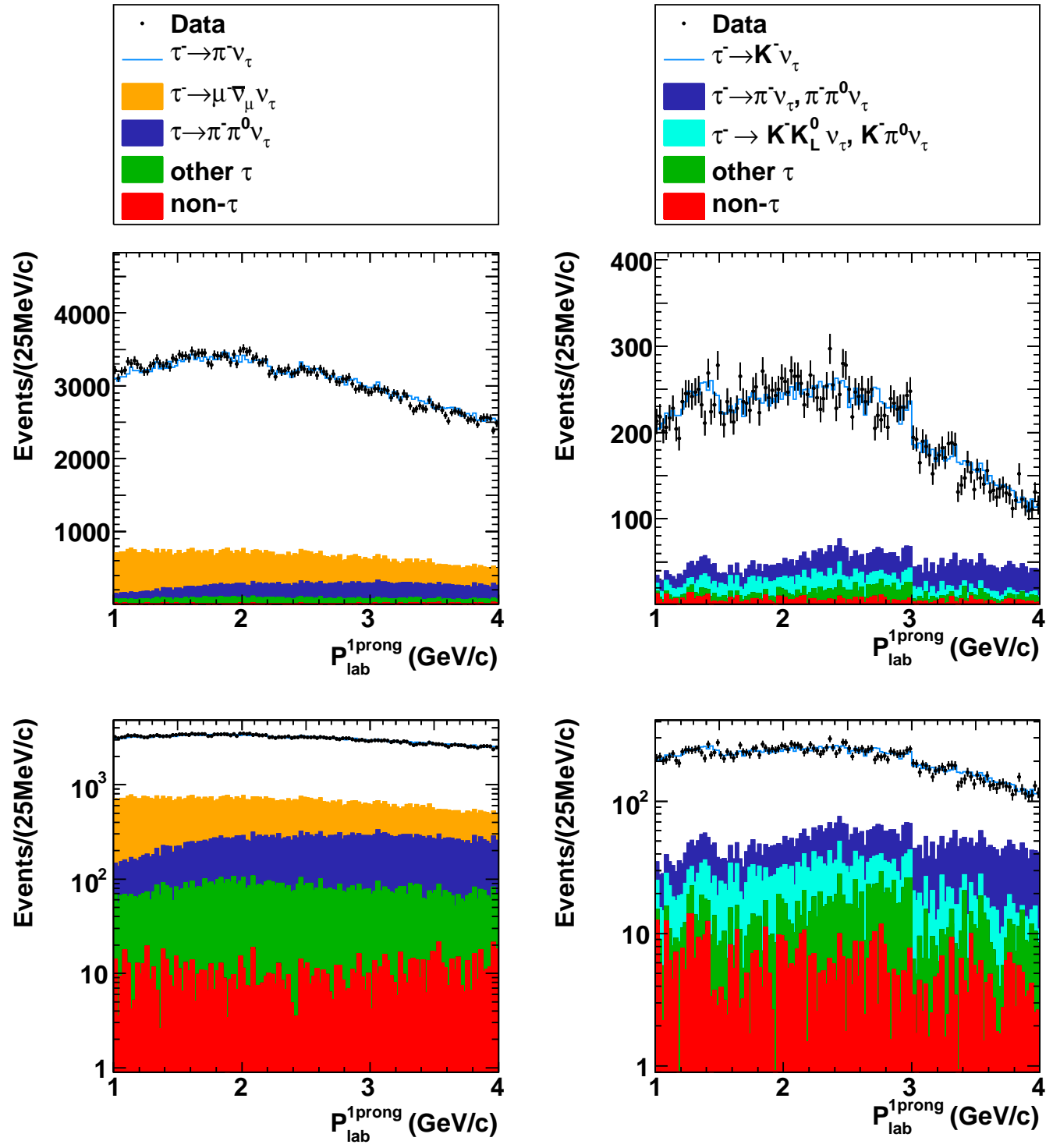

Figure G.2: The $\tau^{-} \rightarrow \pi^{-} \nu_{\tau}$ (left) and $\tau^{-} \rightarrow K^{-} \nu_{\tau}$ (right) laboratory momentum shown both linear (top) and logarithmic scale(bottom). The legends for the $\tau^{-} \rightarrow$ $\pi^{-} \nu_{\tau}$ and $\tau^{-} \rightarrow K^{-} \nu_{\tau}$ channels are shown above the respective plots. This plot illustrated the data MC agreement after the Corrections from the Control Samples were applied. 


\section{Appendix $\mathbf{H}$}

\section{Neutrino Pseudo-mass}

The neutrino mass is calculated using a kaon 4-vector and the 4-vector of the mother $\tau$ particle which is calculated from a cone matching algorithm [123]. First, the 4-vector of the 3 prong hadronic system is calculated, and the angle between the hadronic 3 -vector and the 3 -vector of its mother $\tau$ particle in calculated in the c.m. frame. A diagram of this can be seen in Figure H.1.

$$
\cos \left(\alpha_{1}\right)=\frac{E_{\text {Beam }}^{c m} E_{h h h}^{c m}-m_{\tau}^{2} c^{4}-m_{h h h}^{2} c^{4}}{2 P_{\tau}^{c m} P_{h h h}^{c m} c^{2}}
$$

where $E_{\text {Beam }}^{c m}$ is collision energy of the $e^{+} e^{-}$in the c.m. frame, $E_{h h h}$ is the energy of the hadronic system in the c.m. frame, $m_{\tau}$ is the mass of the $\tau, m_{h h h}$ is the mass of the hadronic system, $P_{\tau}^{c m}$ is the momentum of the $\tau$ in the c.m. frame, and $P_{h h h}^{c m}$ is the momentum of the hadronic system in the c.m. frame. Similarly, the angle between the kaon and its mother $\tau$ particle is

$$
\cos \left(\alpha_{2}\right)=\frac{E_{\text {Beam }}^{c m} E_{K}^{c m}-m_{\tau}^{2} c^{4}-m_{K}^{2} c^{4}}{2 P_{\tau}^{c m} P_{K}^{c m} c^{2}}
$$

where the index $h h h$ has been replaced with an index representing the kaon, $K$. The angle between the kaon 3-vector and the 3-vector of the hadronic system is called $\beta . \cos (\beta)$ is determined from the dot product. If any of these angles are unphysical 
$\left(\left|\cos \left(\alpha_{1}\right)\right|>1,\left|\cos \left(\alpha_{2}\right)\right|>1\right.$, or $\left.|\cos (\beta)|>1\right)$ the direction of the mother $\tau$ of the kaon is defined from the 3 -vector of the hadronic system, by normalizing it to unity and inverting the direction. The occurrence of unphysical angles is primarily a result of the initial state radiation. If the angles are physical, one can define an orthogonal axis relative to the hadronic 3-vector in the c.m. frame which can be used to express the direction of the mother $\tau$. Using the 3-vector of the hadronic system as the $z$ axis and the vector, perpendicular to the $z$ axis in the plane of the kaon and hadronic system, as the $x$ axis, one can write the direction of the mother $\tau$ of the hadronic system as:

$$
\hat{\tau_{1}}=\left[\begin{array}{r}
\sin \left(\alpha_{1}\right) \cos (\delta) \hat{x} \\
\sin \left(\alpha_{1}\right) \sin (\delta) \hat{y} \\
\cos \left(\alpha_{1}\right) \hat{z}
\end{array}\right]
$$

where $\delta$ is the unknown azimuthal angle. Similarly, if one defines the an orthogonal axis relative to the kaon in the same manner to that of the hadronic system, the direction of the mother $\tau$ for the kaon becomes:

$$
\hat{\tau_{2}^{\prime}}=\left[\begin{array}{r}
\sin \left(\alpha_{2}\right) \cos \left(\delta^{\prime}\right) \hat{x}^{\prime} \\
\sin \left(\alpha_{2}\right) \sin \left(\delta^{\prime}\right) \hat{y}^{\prime} \\
\cos \left(\alpha_{2}\right) \hat{z}^{\prime}
\end{array}\right]
$$

where $\delta^{\prime}$ is the unknown azimuthal angle for the signal decay. Using an Euler rotation of $\beta$ about the $y$ axis, the latter equations become

$$
\hat{\tau_{2}}=\left[\begin{array}{r}
\mp \sin (\beta) \cos \left(\alpha_{2}\right)+\cos (\beta) \sin \left(\alpha_{2}\right) \cos \left(\delta^{\prime}\right) \hat{x} \\
\sin \left(\alpha_{2}\right) \sin \left(\delta^{\prime}\right) \hat{y} \\
\cos (\beta) \cos \left(\alpha_{2}\right) \pm \sin (\beta) \sin \left(\alpha_{2}\right) \cos \left(\delta^{\prime}\right) \hat{z}
\end{array}\right]
$$

where the signs $(\mp, \pm)$ depend on whether the rotation is counter clockwise or clock- 
wise. Since the two $\tau$ are back to back, $\hat{\tau_{1}}=-\hat{\tau_{2}}$, in the events, there are three equations:

$$
\begin{array}{r}
\sin \left(\alpha_{1}\right) \cos (\delta)=-\left(\mp \sin (\beta) \cos \left(\alpha_{2}\right)+\cos (\beta) \sin \left(\alpha_{2}\right) \cos \left(\delta^{\prime}\right)\right) \\
\sin \left(\alpha_{1}\right) \sin (\delta)=-\left(\sin \left(\alpha_{2}\right) \sin \left(\delta^{\prime}\right)\right) \\
\cos \left(\alpha_{1}\right)=-\left(\cos (\beta) \cos \left(\alpha_{2}\right) \pm \sin (\beta) \sin \left(\alpha_{2}\right) \cos \left(\delta^{\prime}\right)\right) .
\end{array}
$$

From Equation H.7 we have

$$
\frac{\sin \left(\alpha_{1}\right)}{\sin \left(\alpha_{2}\right)} \sin (\delta)=\sin \left(\delta^{\prime}\right)
$$

or

$$
1-\left(\frac{\sin \left(\alpha_{1}\right)}{\sin \left(\alpha_{2}\right)} \sin (\delta)\right)^{2}=\cos \left(\delta^{\prime}\right)^{2}
$$

Rearranging and squaring Equation H.8 one obtains

$$
\begin{aligned}
\left(\cos \left(\alpha_{1}\right)+\cos (\beta) \cos \left(\alpha_{2}\right)\right)^{2}= & \sin (\beta)^{2} \sin \left(\alpha_{2}\right)^{2} \cos \left(\delta^{\prime}\right)^{2} \\
= & \sin (\beta)^{2} \sin \left(\alpha_{2}\right)^{2} \\
& \times\left(1-\left(\frac{\sin \left(\alpha_{1}\right)}{\sin \left(\alpha_{2}\right)} \sin (\delta)\right)^{2}\right) .
\end{aligned}
$$

Therefore,

$$
\frac{\sin (\beta)^{2} \sin \left(\alpha_{2}\right)^{2}-\left(\cos \left(\alpha_{1}\right)+\cos (\beta) \cos \left(\alpha_{2}\right)\right)^{2}}{\sin (\beta)^{2} \sin \left(\alpha_{1}\right)^{2}}=\sin (\delta)^{2} .
$$

From $\hat{z}$ and $\hat{z}^{\prime}$ the orthogonal basis of the non-primed system may be defined as $\hat{z}$, $\hat{x}=\frac{\hat{z}^{\prime}-\hat{z} \cos (\beta)}{\left|\bar{z}^{\prime}-\hat{z} \cos (\beta)\right|}$, and $\hat{y}=\hat{z} \times \hat{x}^{\prime}$. From this, one can compute the $\tau$ direction, $\hat{\tau}_{1}$, where the sign of $\sin \left(\alpha_{1}\right)$ can be determined by the constraint that $\hat{\tau_{1}}=\hat{\tau_{2}}$. In other words $\pm \alpha_{1} \cos (\delta), \pm \alpha_{2} \cos \left(\delta^{\prime}\right)$ and $\beta$ must add to $\pi$.

Unfortuntely, this method is sensitive to the modelling of the IFSR and the 
angular resolution of the detector. This results in data-MC discrepancies that limit the $\tau^{-} \rightarrow K^{-} \nu_{\tau}$ measurement. Therefore, another method that is less sensitive to the modelling of the IFSR and the angular resolution is required.

The sensitivity to the angular resolution and modelling imperfections of the IFSR can be avoided if we use a pseudo-mass that does not depend on the calculation of $\delta$. Using the single cone from the 3-prong decay reduces the sensitivity to the angular resolution because it does not depend on the difference between small angles. The dependence on the IFSR is reduced with this method, since the differences in $\alpha_{1}$ and $\alpha_{2}$, due to the IFSR, are amplified when the difference is taken. If a fixed value of $\delta$ is assumed then the $\tau$ direction can be estimated allowing for a pseudo-mass, $m_{\nu}^{\text {Cone-hhh }}$, of the neutrino to be calculated. A diagram illustrating the pseudomass method be seen in Figure H.2. When the $\tau$ direction is solved with this method one only obtains the magnitude for the angles which define the $\tau$ direction relative to the 3 prong system. The sign ambiguity of the angle $\alpha_{1}$ is solved from the $\tau$ cone matching algorithm [123]. The sign ambiguity of $\delta$, the angle of projection out of the $\alpha_{1}$ plane, is not solved; instead the direction of the $y$ axis is determined from the cross product of the 3 prong momentum and the kaon momentum in the c.m. frame. The optimal value of $\delta$ and pseudo-mass cut values are the values that minimize the combined statistical uncertainty, the $K_{L}^{0}$ uncertainty and the non- $\tau$ uncertainty. The optimal value of $\delta$ and the associated cut are $\delta=58.5^{\circ}$ with $m_{\nu}^{h h h-C o n e}=0.56$. The neutrino pseudo-mass distribution for $\delta=58.5^{\circ}$ can be seen in Figure H.3. 


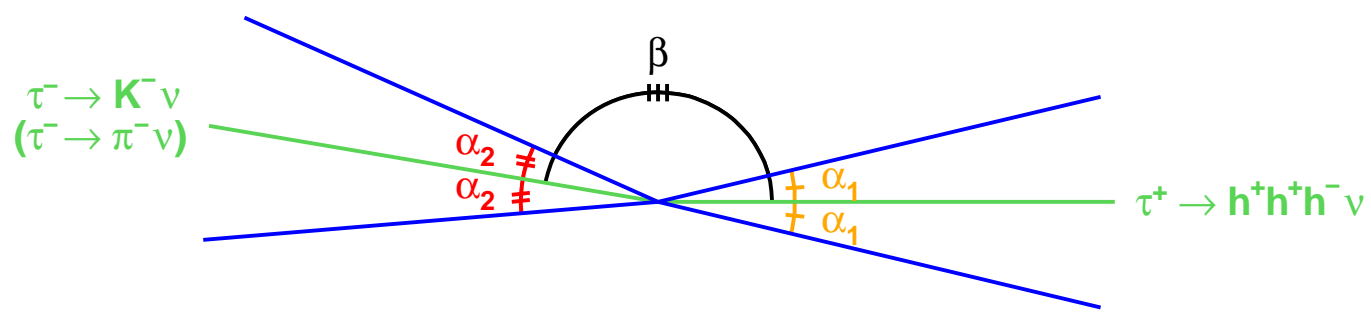

Figure H.1: A 2D projection of the $k / \pi-h h h$ plane used for determining the $\tau$ direction in the c.m. frame. The green lines represent the direction of the hadronic systems, the signal particle is on the left and $h h h$ is on the right. The blue lines represent the projection of the cone for the $\tau$ direction about each of the hadronic systems. For the hhh system, the angle of the cone about the hadronic system is $\alpha_{1}$ which is defined in Equation 7.2, and for the signal particle the angle of the cone about the hadron is $\alpha_{2}$ which is defined in Equation H.2. $\beta$ is the angle between the signal particle the hadronic system. 


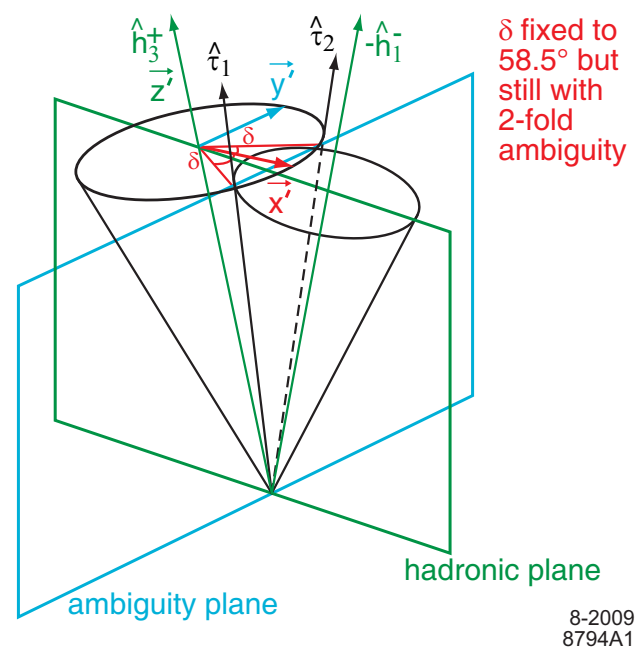

Figure H.2: A powerful discriminator against background $\tau$ decays containing an unmeasured neutral hadron or photon is the pseudo missing mass associated with the signal decay. This requires an estimator of the signal $\tau$ momentum which, owing to the presence of neutrinos, is imperfect. The diagram provides the definitions of the quantities in the c.m. frame of the annihiliating $e^{+} e^{-}$system used to calculate the estimators for the unmeasured $\tau$ momenta. As we assume both $\tau$ 's are backto-back and have half the c.m. energy, we only require a method for estimating the direction of the momentum of the signal $\tau$, denoted by the unit vector $\hat{\tau}$. The unit vector along the measured momentum of the 3-prong tag-side hadronic system is denoted by $\hat{h}_{3}^{+}$. The unit vector opposite to the measured momentum of the 1-prong signal-side system is denoted by $-\hat{h}_{1}^{-}$. The kinematics for a $\tau$ decaying to a hadronic system of a particular mass determines the opening angle between momentum of the $\tau$ and that of the hadronic system to which it decays thereby forcing $\hat{\tau}$ to lie on a cone whose axis is along the momentum of the hadronic system. These are depicted in the figure by the cones having axes $\hat{h}_{3}^{+}$and $-\hat{h}_{1}^{-}$and represent all $\hat{\tau}$ which are kinematically possible. When both $\tau$ leptons decay hadronically, there are only two kinematically allowed values of $\hat{\tau}$ which are given by the intersection of the two cones: $\hat{\tau}_{1}$ and $\hat{\tau}_{2}$. We construct the $z^{\prime}$ axis along the direction of $\hat{h}_{3}^{+}$with the $y^{\prime}$ axis given by $\hat{h}_{1}^{-} \times \hat{h}_{3}^{+} /\left|\hat{h}_{1}^{-} \times \hat{h}_{3}^{+}\right|$. The $x^{\prime}$ axis is then determined in the right-handed coordinate system, as shown. We label the plane containing $\hat{h}_{3}^{+}$and $-\hat{h}_{1}^{-}$as the 'hadronic plane' and the plane perpendicular to the hadronic plane containing $\hat{\tau}_{1}$ and $\hat{\tau}_{2}$ as the 'ambiguity plane'. To estimate $\hat{\tau}$ we only use the intersections of the two cones to specify the orientation of the co-ordinate system and, rather than constraining it to lie on either $\hat{\tau}_{1}$ or $\hat{\tau}_{2}$, we choose to fix the azimuthal angle $\delta$, as measured from the $x^{\prime}$ axis, shown in the diagram, to an optimized value of $58.5^{\circ}$. This renders the missing mass estimator less sensitive to initial and final state radiation as well as to the details of the detector modelling. Note that both $+58.5^{\circ}$ and $-58.5^{\circ}$ yield the same pseudo missing mass value. 


\begin{tabular}{|l|}
\hline Data \\
$\tau^{-} \rightarrow \pi^{-} v_{\tau}$ \\
$\tau \rightarrow \mu \nabla_{\mu} v_{\tau}$ \\
$\tau \rightarrow \pi^{-} \pi^{0} v_{\tau}$ \\
other $\tau$ \\
non- $\tau$ \\
\hline
\end{tabular}

\begin{tabular}{|c|}
\hline $\begin{array}{l}\text { Data } \\
\tau^{-} \rightarrow \mathbf{K}^{-} v_{\tau} \\
\tau \\
\tau^{-} \rightarrow \pi^{-} v_{\tau}, \pi^{-} \pi^{0} v_{\tau} \\
\tau^{-} \rightarrow \mathbf{K}^{-} \mathbf{K}_{\mathbf{L}}^{0} v_{\tau}, K^{-} \pi^{0} v_{\tau} \\
\text { other } \tau \\
\text { non- } \tau\end{array}$ \\
\hline
\end{tabular}
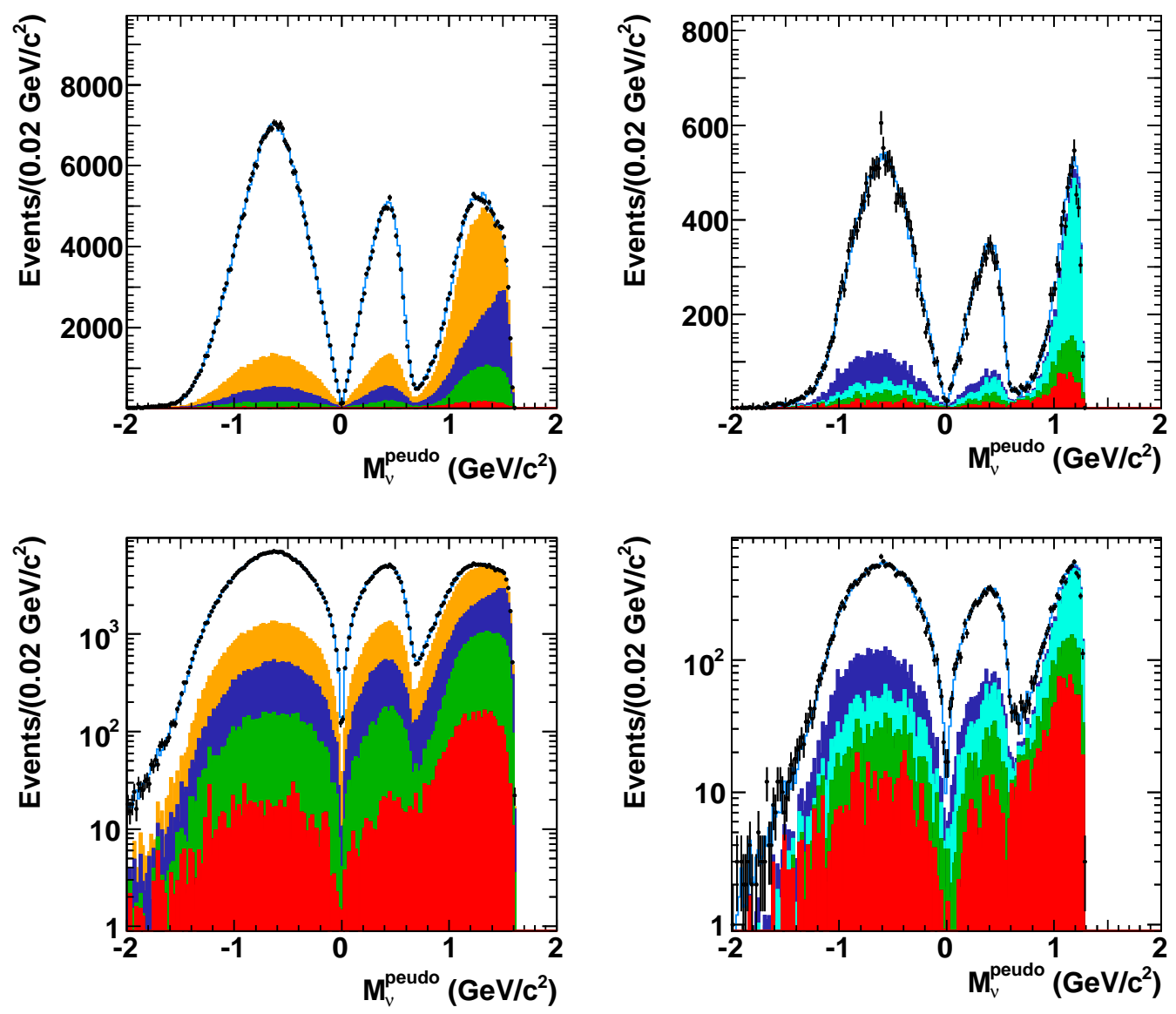

Figure H.3: The neutrino pseudo-mass distribution $\left(\delta=58.5^{\circ}\right)$ for the $\tau^{-} \rightarrow \pi^{-} \nu_{\tau}$ (left) and $\tau^{-} \rightarrow K^{-} \nu_{\tau}$ (right) channels shown both in linear (top) and logarithmic scale(bottom). The legends for the $\tau^{-} \rightarrow \pi^{-} \nu_{\tau}$ and $\tau^{-} \rightarrow K^{-} \nu_{\tau}$ channels are shown above the respective plots. 


$\begin{aligned} & \cdot \text { Data } \\ & \tau^{-} \rightarrow \mathbf{e}^{-} \nabla_{\mathbf{e}} v_{\tau} \\ & \tau \rightarrow \pi^{-} v_{\tau}, \pi^{-} \pi^{0} v_{\tau} \\ & \tau^{-} \rightarrow \mathbf{K}^{-} v_{\tau}, K^{-} \mathbf{K}_{\mathbf{L}}^{0} v_{\tau}, \mathbf{K}^{-} \pi^{0} v_{\tau} \\ & \text { other } \tau \\ & \text { non- } \tau\end{aligned}$

\begin{tabular}{|c|}
\hline 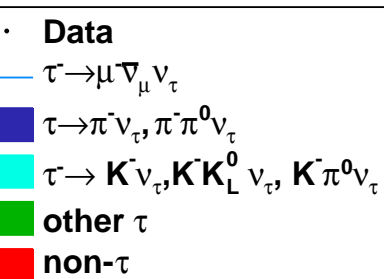 \\
\hline
\end{tabular}
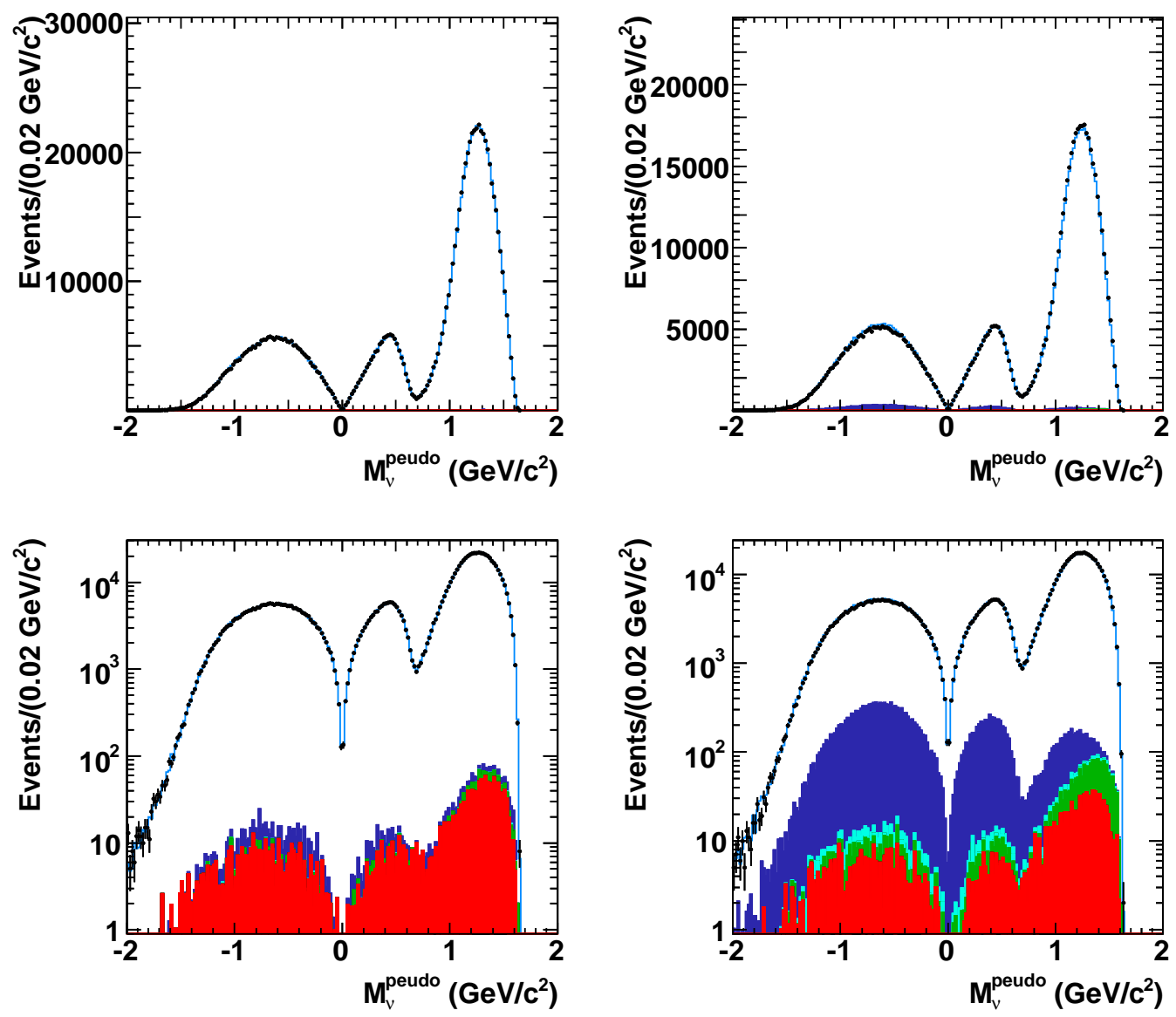

Figure H.4: The neutrino pseudo-mass distribution $\left(\delta=58.5^{\circ}\right)$ for the $\tau^{-} \rightarrow e^{-} \nu_{\tau} \bar{\nu}_{e}$ (left) and $\tau^{-} \rightarrow \mu^{-} \nu_{\tau} \bar{\nu}_{\mu}$ (right) channels shown both in linear (top) and logarithmic scale(bottom). The legends for the $\tau^{-} \rightarrow e^{-} \nu_{\tau} \bar{\nu}_{e}$ and $\tau^{-} \rightarrow \mu^{-} \nu_{\tau} \bar{\nu}_{\mu}$ channels are shown above the respective plots. 


\section{Appendix I}

$$
\begin{aligned}
& \frac{\mathcal{B}\left(\tau^{-} \rightarrow K^{-} \nu_{\tau}\right)}{\mathcal{B}\left(\tau^{-} \rightarrow \pi^{-} \nu_{\tau}\right)}, \frac{\mathcal{B}\left(\tau^{-} \rightarrow K^{-} \nu_{\tau}\right)}{\mathcal{B}\left(\tau^{-} \rightarrow e^{-} \nu_{\tau} \bar{\nu}_{e}\right)}, \\
& \frac{\mathcal{B}\left(\tau^{-} \rightarrow \pi^{-} \nu_{\tau}\right)}{\mathcal{B}\left(\tau^{-} \rightarrow e^{-} \nu_{\tau} \bar{\nu}_{e}\right)}, \text { and } \frac{\mathcal{B}\left(\tau^{-} \rightarrow \mu^{-} \nu_{\tau} \bar{\nu}_{\mu}\right)}{\mathcal{B}\left(\tau^{-} \rightarrow e^{-} \nu_{\tau} \bar{\nu}_{e}\right)} \\
& \text { Backgrounds }
\end{aligned}
$$

\begin{tabular}{|l|l|l|l|l|}
\hline Channel & $\tau^{-} \rightarrow K^{-} \nu$ & $\tau^{-} \rightarrow \pi^{-} \nu$ & $\tau^{-} \rightarrow e^{-} \nu \nu$ & $\tau^{-} \rightarrow \mu^{-} \nu \nu$ \\
\hline \multicolumn{5}{|c|}{ non- $\tau$ Background } \\
\hline Scale Factor & $1.64 \pm 0.23$ & $1.34 \pm 0.21$ & $1.20 \pm 0.11$ & $1.26 \pm 0.14$ \\
\hline$u \bar{u}, d d$, and $s \bar{s}$ & $319 \pm 18$ & $826 \pm 33$ & $86 \pm 14$ & $298 \pm 18$ \\
$c \overline{\mathrm{c}}$ & $75 \pm 6.7$ & $122.7 \pm 9.3$ & $1326 \pm 28$ & $638 \pm 20$ \\
$\mathrm{~B}^{0} \overline{\mathrm{B}}^{0}$ & $3.7 \pm 1.13$ & $13.6 \pm 5.0$ & $86.7 \pm 7.1$ & $48.1 \pm 5.2$ \\
$\mathrm{~B}^{+} \mathrm{B}^{-}$ & $3.9 \pm 1.2$ & $9.6 \pm 2.7$ & $45.9 \pm 4.3$ & $21.0 \pm 3.0$ \\
$\mu$-pair & $0 \pm 0$ & $0 \pm 0$ & $0 \pm 0$ & $0 \pm 0$ \\
\hline \multicolumn{5}{|c|}{$\tau$-pair Background } \\
\hline$\tau^{-} \rightarrow e^{-} \nu \nu$ & $106 \pm 8.0$ & $4339 \pm 67$ & na & $1.04 \pm 0.74$ \\
$\tau^{-} \rightarrow \mu^{-} \nu \nu$ & $702 \pm 28$ & $48732 \pm 220$ & $20 \pm 3.3$ & na \\
$\tau^{-} \rightarrow \pi^{-} \nu$ & $2565 \pm 25$ & na & $239.5 \pm 8.8$ & $10880 \pm 41$ \\
$\tau^{-} \rightarrow K^{-} \nu$ & na & $605 \pm 10$ & $6.6 \pm 1.2$ & $335.5 \pm 5.4$ \\
$\tau^{-} \rightarrow K^{-} K^{0} \nu$ & $987 \pm 23$ & $36.9 \pm 5.4$ & $0.96 \pm 0.67$ & $56.5 \pm 6.7$ \\
$\tau^{-} \rightarrow K^{-} \pi^{0} \nu$ & $503 \pm 17$ & $20.6 \pm 4.5$ & $3.9 \pm 1.6$ & $66.5 \pm 7.1$ \\
$\tau^{-} \rightarrow \rho^{-} \nu$ & $273 \pm 15$ & $22274 \pm 117$ & $412 \pm 20$ & $6289 \pm 60$ \\
other & $168 \pm 11$ & $3388 \pm 45$ & $240 \pm 16$ & $1241 \pm 26$ \\
\hline
\end{tabular}

Table I.1: Background Summary for the $\frac{\mathcal{B}\left(\tau^{-} \rightarrow K^{-} \nu\right)}{\mathcal{B}\left(\tau^{-} \rightarrow e^{-} \nu \bar{\nu}\right)}, \frac{\mathcal{B}\left(\tau^{-} \rightarrow \pi^{-} \nu\right)}{\mathcal{B}\left(\tau^{-} \rightarrow e^{-} \nu \bar{\nu}\right)}$ and $\frac{\mathcal{B}\left(\tau^{-} \rightarrow \mu^{-} \nu \overline{\mathcal{D}}\right)}{\mathcal{B}\left(\tau^{-} \rightarrow e^{-} \nu \bar{\nu}\right)}$ analysis. 